\title{
Opioid receptor protein expression during development of the rat brainstem
}

\author{
By \\ Bronwyn Maree Kivell
}

\begin{abstract}
A thesis
Submitted to Victoria University of Wellington

In fulfilment of the requirements for the degree of

Doctor of Philosophy

In Cell Biology
\end{abstract}

Victoria University of Wellington 


\section{Abstract}

Few satisfactory protocols exist for primary culture of postnatal brainstem neurons, and commonly used procedures often give poor survival rates in older foetal (>E16) and early postnatal brainstem cultures. The present study describes the first reliable method for establishing stable in vitro cultures of foetal and postnatal brainstem neurons up to six days postnatal age in a defined, serum-free culture medium. This novel culture method was used to study opioid receptor expression and distribution in developing brainstem cells. Opioids play an important role in brainstem functions, being involved in respiratory and cardiovascular modulation and pain control (Olsen et al., 1995; Olson et al., 1997; Vaccarino et al., 1999; Vaccarino and Kastin, 2001). These brainstem functions are particularly important for survival at birth, and opioid receptor distribution patterns and sensitivities to opioid ligands change during development. Using cultured cells and frozen sections of brainstem tissue, mu (MOR) and delta (DOR) opioid receptor localisation in neuronal and glial cells at different stages of foetal and postnatal development in the rat were examined by immunocytochemistry and confocal microscopy. Bipolar and multipolar neurons showed similar immunoreactivities; whereas, glial cells were more lightly stained than neurons. Developmentally advanced stages were more intensely stained for MOR $(\mathrm{P}<0.006$, Mann-Whitney test); whereas, DOR immunoreactivity did not change during development. These developmental expression patterns observed in culture for MOR were similar to those obtained from Western blots of electrophoreses brainstem lysates. DOR, however, decreased in expression in brainstem lysates with increased developmental age, even though there was no difference in DOR expression in cultured cells. MOR and DOR were colocalised in specific brainstem regions and in the cerebellum of foetal and postnatal animals, although the distribution of both opioid receptors in the foetal brain was more diffuse than in the older animals. The intracellular distributions of MOR and DOR were investigated by confocal microscopy. In addition to plasma membrane staining, a population of internalised cytoplasmic receptors was present in neurons. MOR was down-regulated after exposure of either cultured brainstem cells or transfected or non-transfected SH-SY5Y neuroblastoma cells to the MOR agonist DAMGO. From the above investigation, it was concluded that opioid receptors are developmentally regulated during maturation of the brainstem of the rat, and that primary 
cell culture, immunocytochemistry, and immunoblotting of cell lysates are suitable techniques for investigating opioid systems in the foetal, postnatal, and adult rat. 


\section{Acknowledgements}

I would like to acknowledge, with appreciation the help I have received from the following people:

Dr John Miller, for being a great supervisor, for his enthusiasm, encouragement and proofreading. Thanks for the considerable help and guidance you have given.

Dr Fiona McDonald for proofreading and ideas.

Dr Darren Day for his enthusiasm, ideas and proofreading.

The students who have passed through the ' $6{ }^{\text {th }}$ floor lab' over the years, who have helped give support, shared expertise, equipment, office space, advice, and coffee breaks especially Dr Atalie Colman; Dr Stephanie Hughes; Dr Sarah Coddington-Lawson; Collette Bromhead; Joy McIntosh; Eli Mrkusich; Genna Flanagan; Tom Gaitanos; Meino Rolfs; and Bruce Norris.

To the administration staff and technicians: Mary Murray; Peter Watson; Chris Thorn; Margaret Crimp; Janet Butler; Richard Moore; and Alan Hoverd.

To Liz McAvoy and students in the $8^{\text {th }}$ floor DNA sequencing lab for electrophoresis of sequencing samples.

To Dr Geoff Rickards for introducing me to confocal microscopy.

To the School of Biological Sciences, for being a flexible and supportive workplace.

I would like to thank my family, with special thanks to my partner Terry for his patience, tolerance, support, and for encouraging me to follow my goals.

This project was funded by research grants from the Wellington Medical Research Foundation and a VUW postgraduate scholarship. 


\section{Table of Contents}

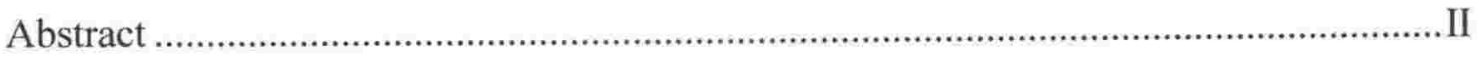

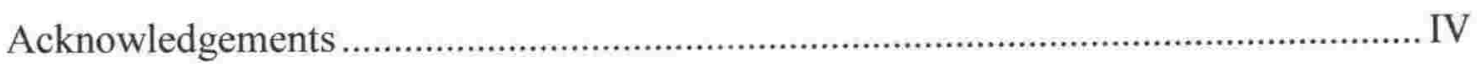

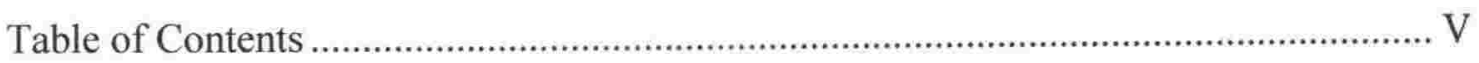

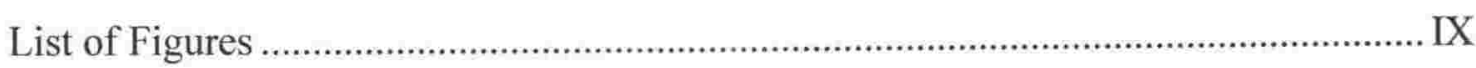

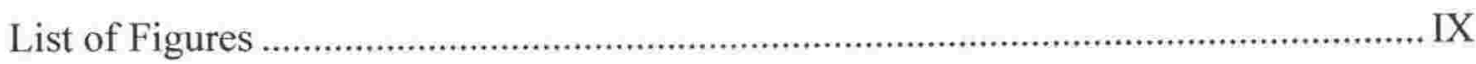

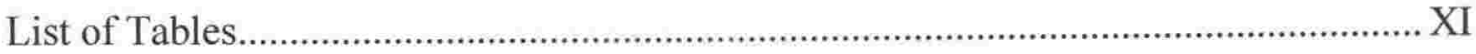

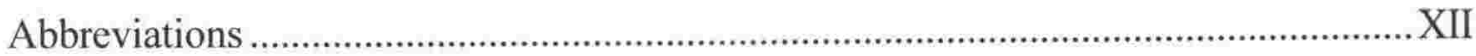

$1 \quad$ Introduction and Literature Review ............................................................... 1

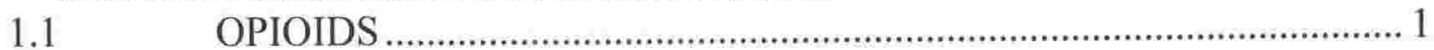

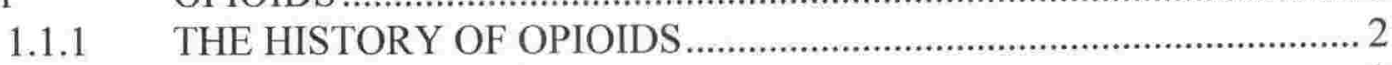

1.1.2 ENDOGENOUS OPIOIDS ................................................................. 3

1.1.3 SELECTIVITY OF LIGANDS FOR OPIOID RECEPTORS................ 3

$1.2 \quad$ OPIOID RECEPTORS

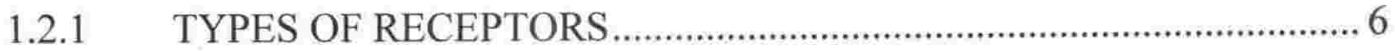

1.2.2 OPIOID RECEPTOR STRUCTURE …................................................ 7

1.2.3 OPIOID RECEPTOR REGULATION .................................................... 8

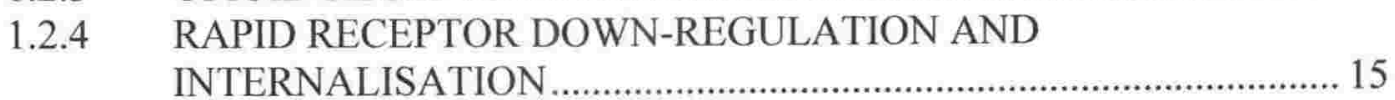

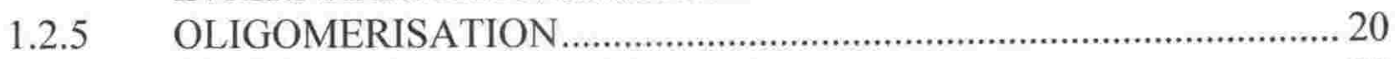

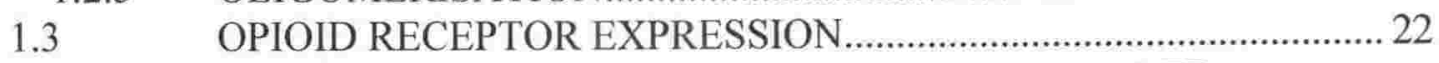

1.3.1 OPIOID RECEPTOR COLOCALISATION WITH OTHER RECEPTORS AND NEUROTRANSMITTERS ..................................2 27

1.3.2 DEVELOPMENTAL OPIOID RECEPTOR EXPRESSION AND

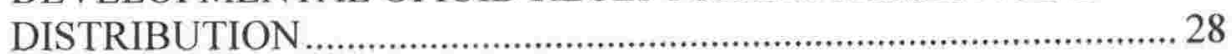

1.3.3 DEVELOPMENT OF THE RAT BRAINSTEM …...............................2 29

1.3.4 FUNCTION OF OPIOIDS IN THE BRAINSTEM................................ 32

1.3.5 EFFECTS OF OPIOIDS ON THE CARDIOVASCULAR SYSTEM.. 35

1.3.6 EFFECT OF OPIOIDS ON RESPIRATION ........................................... 36

1.3.7 EFFECT OF OPIOIDS DURING DEVELOPMENT .......................... 42

1.4 ROLE OF OPIOIDS IN HEALTH AND DISEASE ............................. 44

1.4.1 RESPIRATORY RELATED DISEASES.............................................. 44

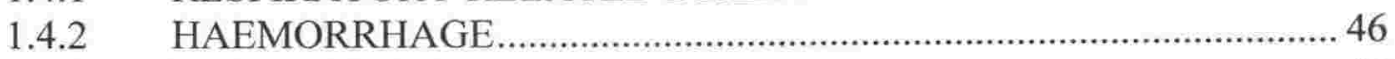

1.4.3 DRUG ADDICTION ......................................................................... 46

1.4 .4 THE ROLE OF THE BRAINSTEM AND OPIOIDS IN PAIN
CONTROL $\ldots \ldots \ldots \ldots \ldots \ldots \ldots \ldots \ldots \ldots \ldots \ldots \ldots \ldots \ldots \ldots \ldots \ldots \ldots \ldots \ldots \ldots \ldots \ldots \ldots \ldots \ldots \ldots \ldots \ldots \ldots \ldots \ldots \ldots \ldots \ldots \ldots \ldots \ldots \ldots \ldots \ldots \ldots \ldots \ldots \ldots \ldots \ldots$

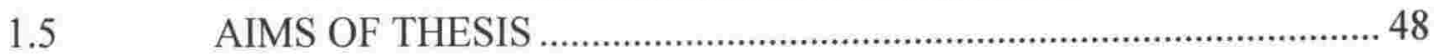

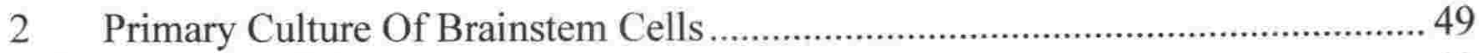

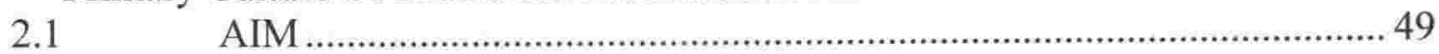

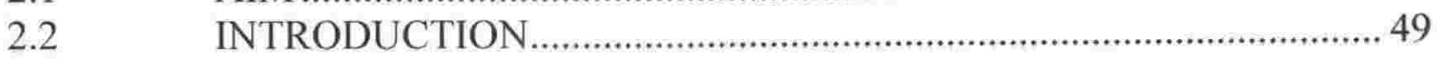

2.2.1 USE OF PRIMARY CULTURE IN INVESTIGATION OF BRAINSTEM FUNCTION …............................................................ 49

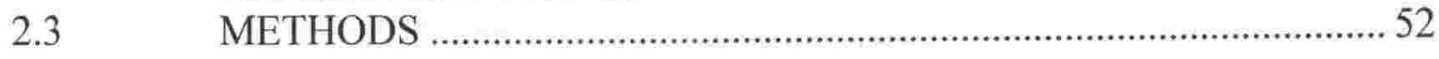


2.3.1 PRIMARY CELL CULTURE ….................................................... 52

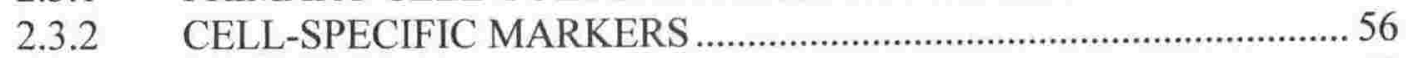

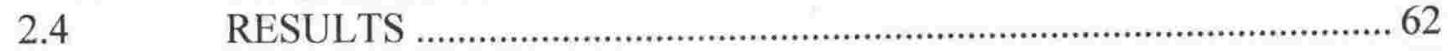

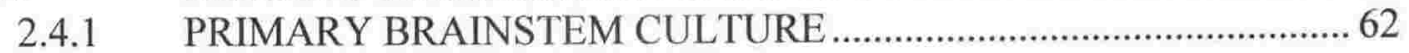

2.4.2 EFFECTS OF SEEDING DENSITY ON NEURONAL SURVIVAL.. 65

2.4.3 CELL MORPHOLOGY AND IMMUNOREACTIVITY IN

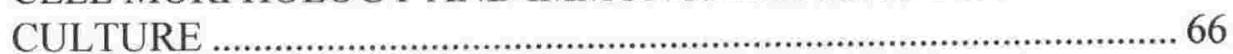

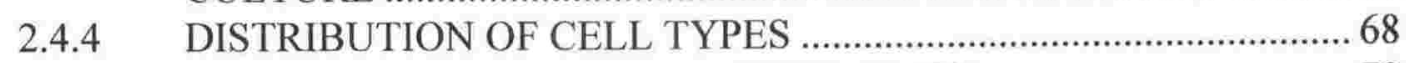

2.4.5 CELL MORPHOMETRY MEASUREMENTS …............................... 70

2.4.6 PRESENCE OF CHOLINERGIC NEURONS IN PRIMARY BRAINSTEM CULTURES OF THE RAT …...................................... 70

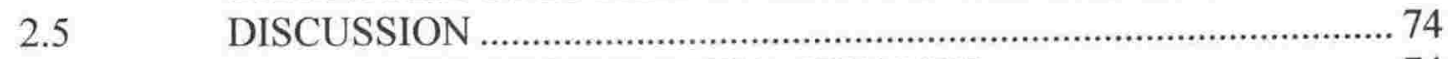

2.5.1 PRIMARY BRAINSTEM CELL CULTURE ....................................... 74

2.5.2 METHOD DEVELOPMENT …............................................................. 74

2.5.3 SEEDING DENSITY AND CELL ATTACHMENT ........................... 77

2.5.4 CULTURE CHARACTERISTICS ...................................................... 78

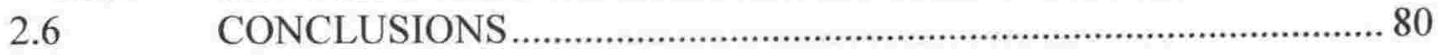

3 Opioid Receptor Distribution In Cultured Cells ….......................................... 81

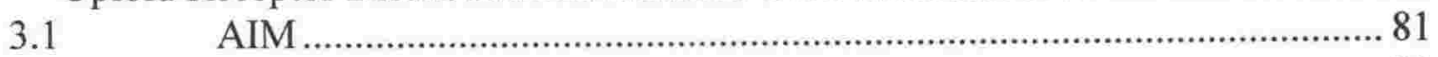

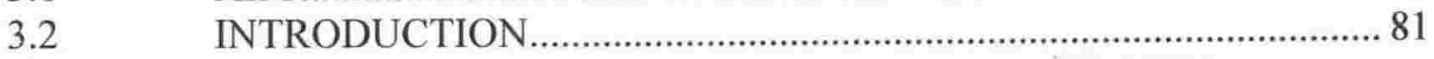

3.2.1 A NOVEL BRAINSTEM CELL CULTURE SYSTEM FOR

STUDYING OPIOID RECEPTOR EXPRESSION DURING

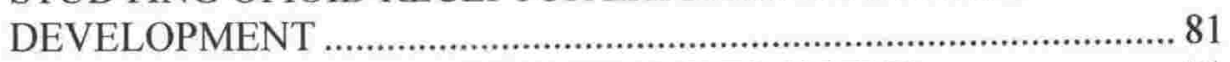

3.2.2 OPIOID RECEPTOR IMMUNOCYTOCHEMISTRY …..................... 81

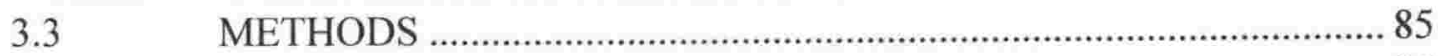

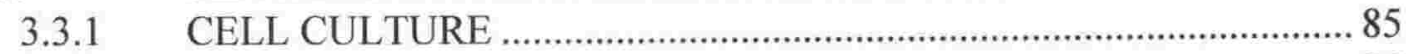

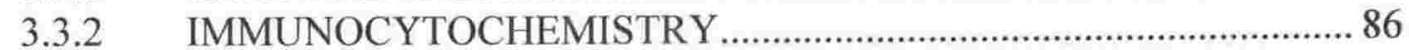

3.3.3 AMPLIFICATION AND DETECTION METHODS …........................ 87

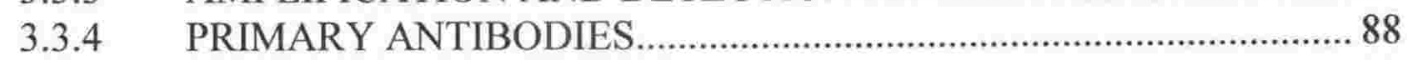

3.3.5 DOUBLE-LABELLING EXPERIMENTS ......................................... 90

3.3.6 COUNTERSTAINING OF THE NUCLEUS ....................................... 91

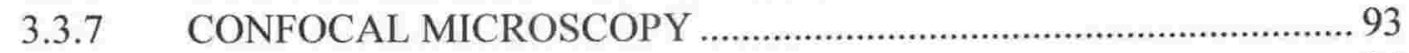

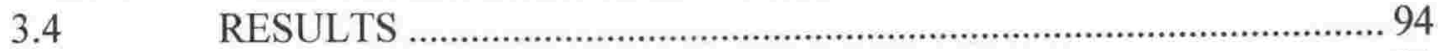

3.4.1 SELECTION OF ANTIBODIES ....................................................... 94

3.4.2 DETERMINING ANTIBODY SPECIFICITY .................................... 94

3.4.3 OPIOID LABELLING OF DIFFERENT CELL TYPES IN

PRIMARY CULTURES OF THE RAT BRAINSTEM .......................99

3.4.4 OPIOID RECEPTOR EXPRESSION IN PRIMARY CULTURES

DURING DEVELOPMENT ........................................................... 100

3.4.5 INTRACELLULAR DISTRIBUTION OF OPIOID RECEPTORS ... 107

3.4.6 COLOCALISATION OF MOR AND DOR IN CELLS CULTURED

FROM THE RAT BRAINSTEM............................................................ 108

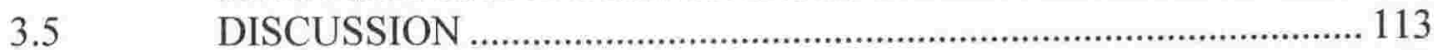

3.5.1 ANTIBODY TESTING FOR IMMUNOCYTOCHEMISTRY .......... 113

3.5.2 CONTROL FOR ANTIBODY SPECIFICITY …................................. 114

3.5.3 CELL TYPE-SPECIFIC OPIOID EXPRESSION IN CULTURE,..... 115

3.5.4 OPIOID RECEPTOR EXPRESSION DURING DEVELOPMENT .. 117

3.5.5 INTRACELLULAR DISTRIBUTION OF OPIOID RECEPTORS ... 119

3.5.6 COLOCALISATION OF MOR AND DOR....................................... 121 
3.5.7 SUMMARY AND CONCLUSIONS …......................................... 122

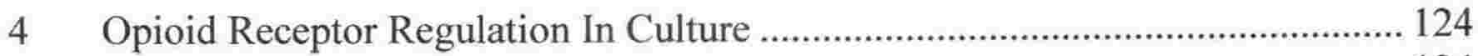

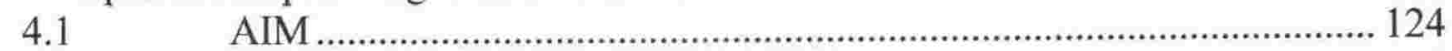

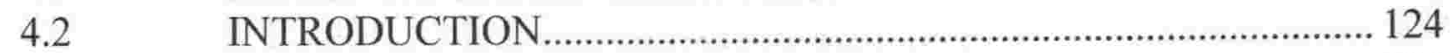

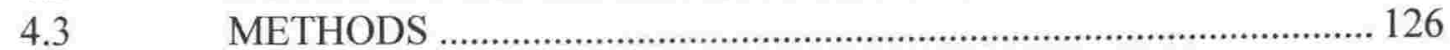

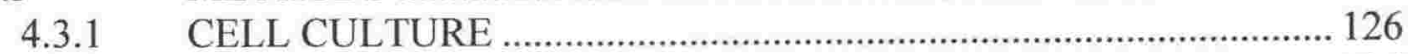

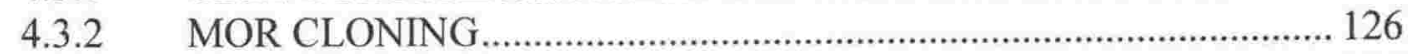

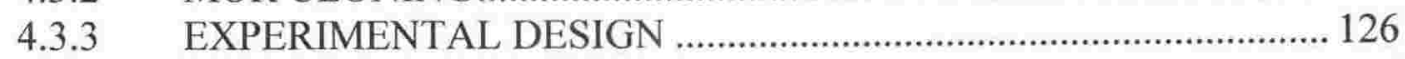

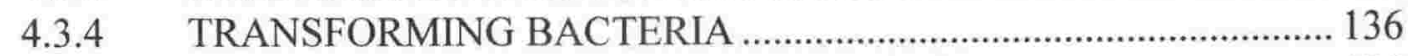

4.3.5 MINIPREP ISOLATION OF PLASMID DNA …................................ 136

4.3.6 MIDIPREP ISOLATION OF PLASMID DNA …................................ 138

4.3.7 TRANSFECTION OF MAMMALIAN CELL LINES ........................ 144

4.3.8 IMMUNOCYTOCHEMISTRY.......................................................... 145

4.3.9 ANTI-FLAG ANTIBODY LABELLING OF TRANSFECTED

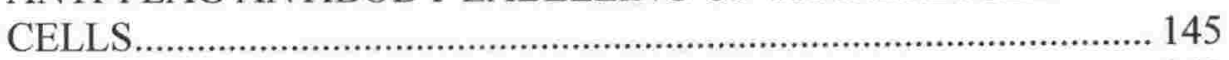

4.3.10 MOR RECEPTOR IN VITRO REGULATION …............................. 145

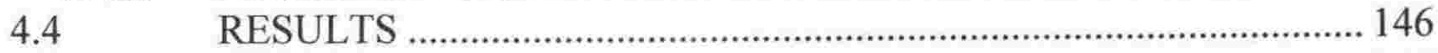

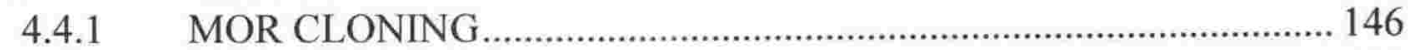

4.4.2 MOR TRANSFECTION OF MAMMALIAN CELL LINES ............. 146

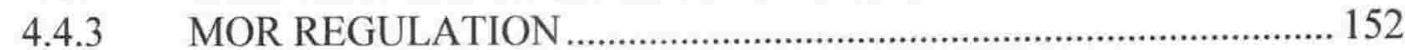

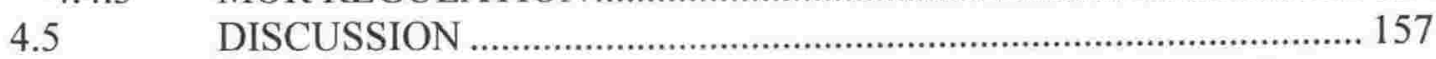

4.5.1 MOR CLONING AND TRANSFECTION OF MAMMALIAN

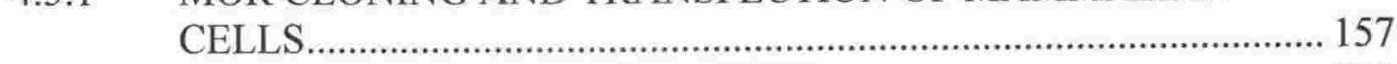

4.5.2 OPIOID RECEPTOR REGULATION .............................................. 158

4.5.3 SUMMARY AND CONCLUSIONS ….............................................. 161

$5 \quad$ MOR and DOR Expression In Purkinje Cells Of The Rat Cerebellum .............. 162

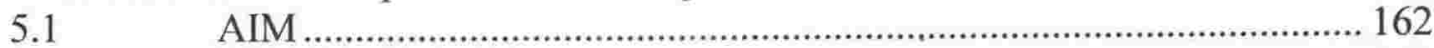

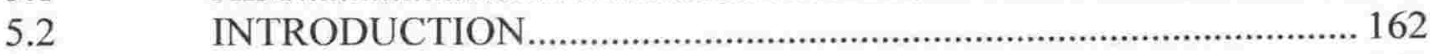

5.2.1 MOR AND DOR EXPRESSION IN THE BRAIN ............................ 162

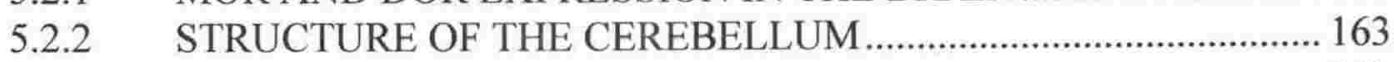

5.2.3 CEREBELLAR FUNCTION ........................................................... 163

5.2.4 SPECIES DIFFERENCES IN MOR AND DOR DISTRIBUTIONS

IN THE CEREBELLUM ................................................................. 164

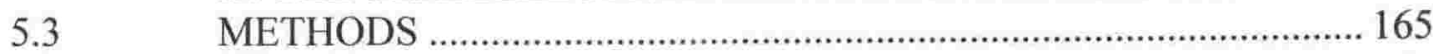

5.3.1 IMMUNOHISTOCHEMISTRY ...................................................... 165

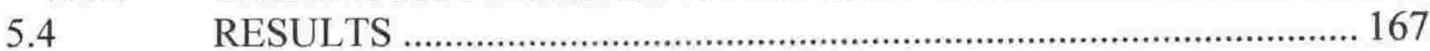

5.4.1 DISTRIBUTION OF MOR AND DOR IN THE RAT BRAIN .......... 167

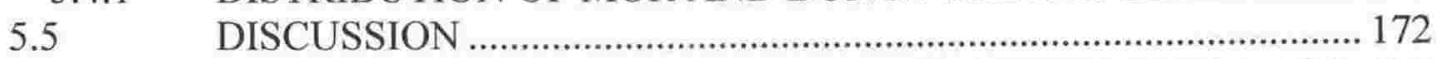

5.5.1 MOR AND DOR DISTRIBUTION IN THE ADULT RAT BRAIN.. 172

5.6 SUMMARY AND CONCLUSIONS …............................................ 175

6 Developmental Expression and Distribution Of Opioid Receptors In The Rat

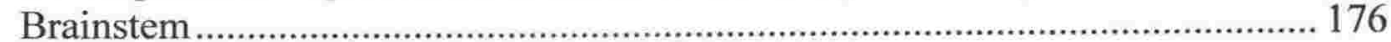

AIM $6.1 \quad 176$

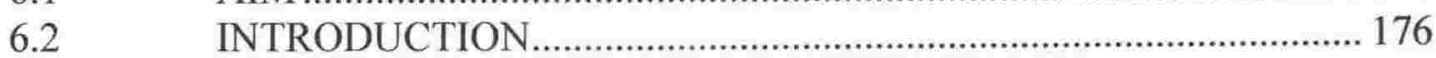

6.2.1 MOR AND DOR EXPRESSION IN THE RAT BRAINSTEM

DURING DEVELOPMENT ........................................................... 176

6.2.2 MOR AND DOR DISTRIBUTION IN THE ADULT BRAIN........... 177 
6.2.3 THE EXPRESSION AND DISTRIBUTION OF MOR AND DOR DURING DEVELOPMENT ............................................................ 178

6.2.4 DISTRIBUTION OF MOR AND DOR DURING RAT DEVELOPMENT . 179

6.2.5 FUNCTIONAL IMPLICATIONS OF MOR AND DOR EXPRESSION IN THE BRAINSTEM …........................................ 180

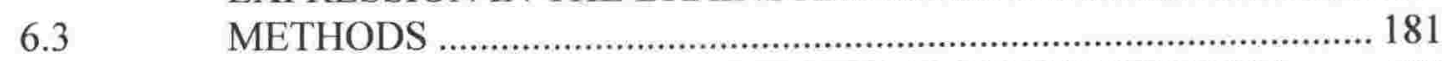

6.3.1 QUANTIFICATION OF OPIOID RECEPTOR EXPRESSION ........ 181

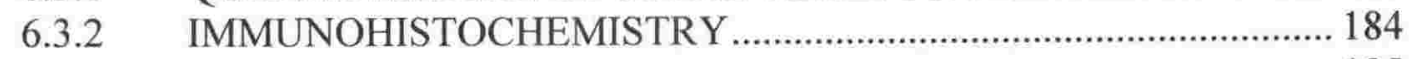

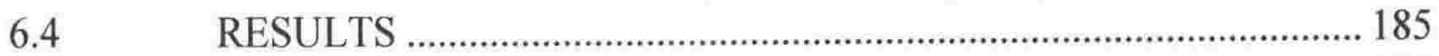

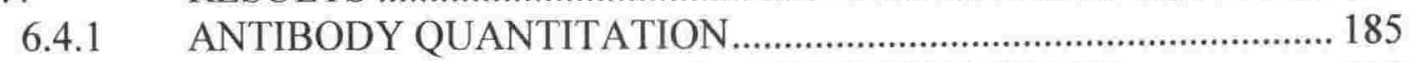

6.4.2 MOR AND DOR EXPRESSION IN SH-SY5Y CELLS ..................... 185

6.4.3 MOR AND DOR PROTEIN EXPRESSION DURING BRAINSTEM DEVELOPMENT IN VIVO ….................................... 188

6.4.4 MOR AND DOR EXPRESSION IN CULTURED BRAINSTEM CELLS FROM LATE FOETAL AND EARLY POSTNATAL ANIMALS .................................................................................. 192

6.4.5 MOR AND DOR DISTRIBUTION DURING DEVELOPMENT OF RAT BRAINSTEM IN VIVO …..................................................... 198

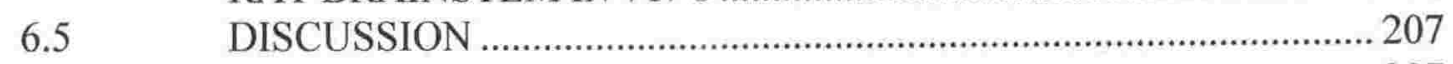

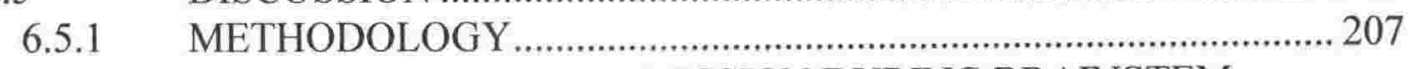

6.5.2 OPIOID RECEPTOR EXPRESSION DURING BRAINSTEM DEVELOPMENT …..................................................................... 209

6.5.3 MOR AND DOR DISTRIBUTION IN THE ADULT RAT BRAIN.. 214

6.5.4 DEVELOPMENTAL DISTRIBUTION OF MOR AND DOR RECEPTORS IN THE RAT BRAINSTEM ...................................... 216

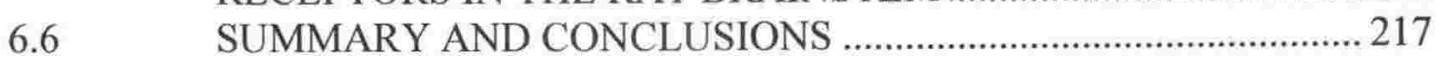

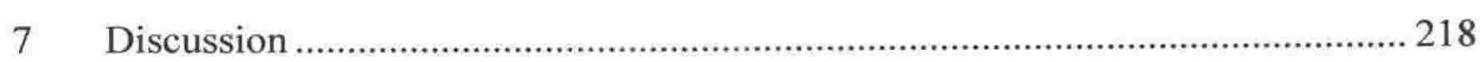

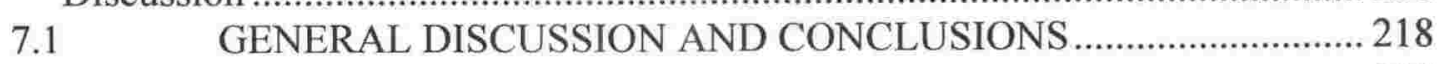

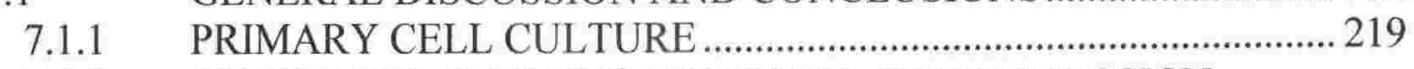

7.1.2 OPIOID RECEPTOR DISTRIBUTION AND EXPRESSION DURING BRAINSTEM DEVELOPMENT ...................................... 223

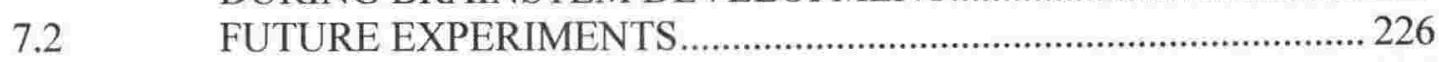

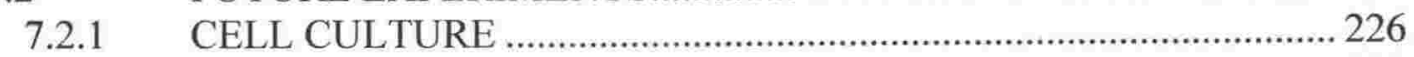

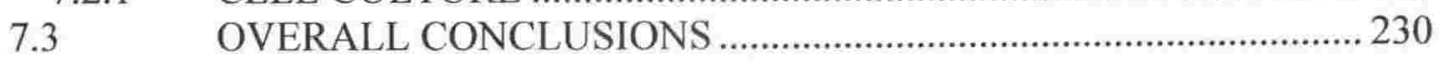

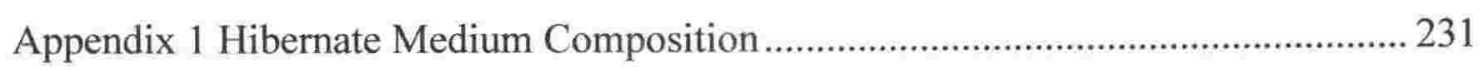

Appendix 2 Solutions, Buffers and Miscellaneous Methods ..................................... 232

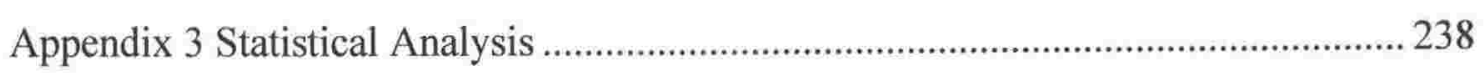

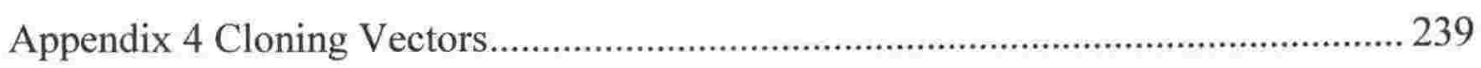

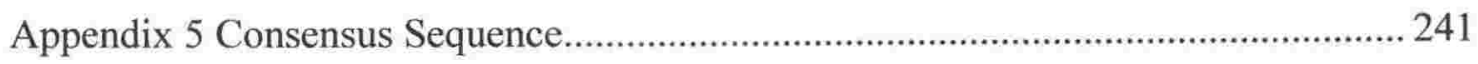

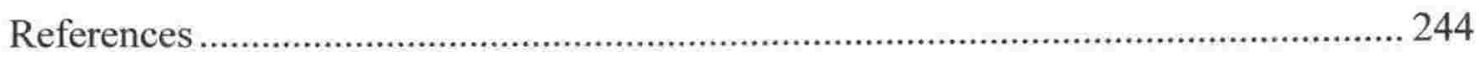




\section{List of Figures}

Figure 1.1 Opioid receptor structure, binding, and regulation properties .......................... 12

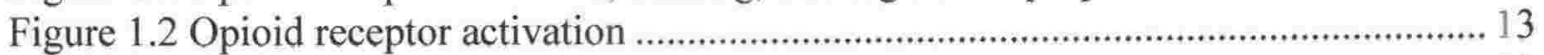

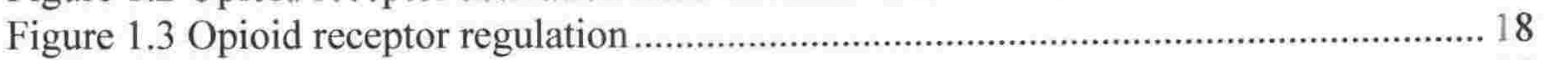

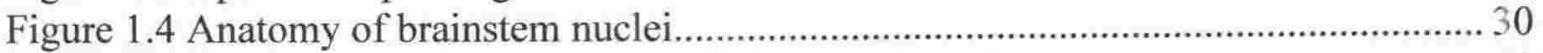

Figure 1.5 Brainstem respiratory centers of the ventral respiratory group ......................... 40

Figure 2.1 Diagram of E16 foetal rat showing the brainstem region taken for culture ....... 54

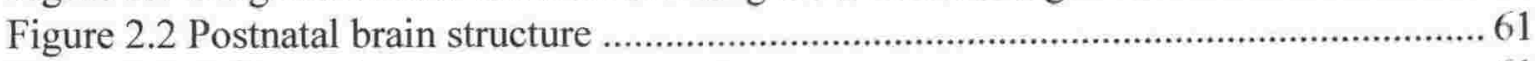

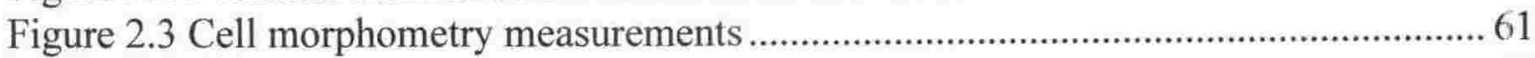

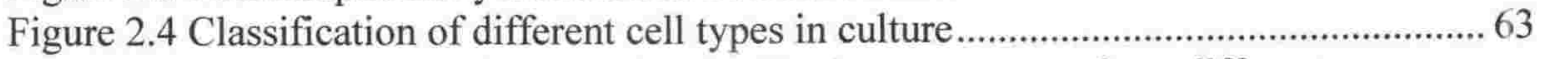

Figure 2.5 Morphology of primary cultures of brainstem neurons from different developmental ages of foetal and postnatal rats........................................... 64

Figure 2.6 Cultured brainstem neurons positively stained for MAP2 .............................. 67

Figure 2.7 Proportion of different cell types present in brainstem cultures of foetal

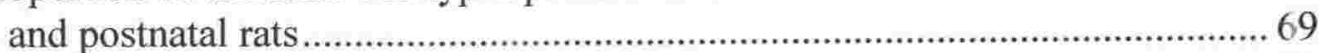

Figure 2.8 Frequency histograms of neurite length ........................................................ 71

Figure 2.9 Frequency histograms of cell body diameter................................................ 72

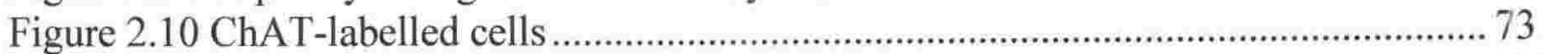

Figure 3.1 Method for double-labelling using TSA ........................................................... 92

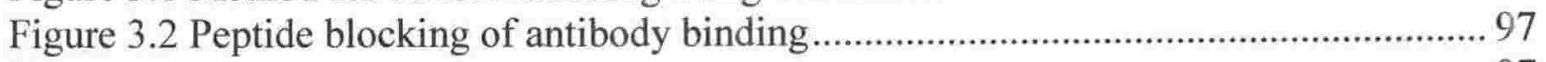

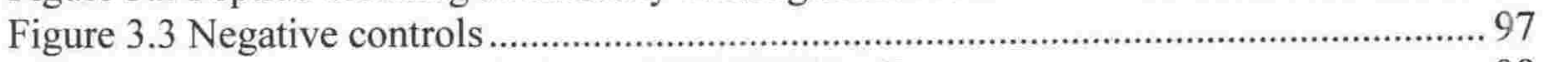

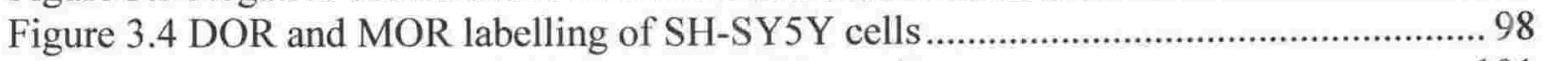

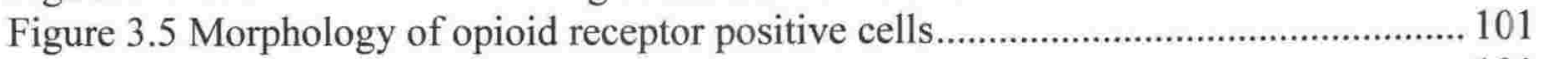

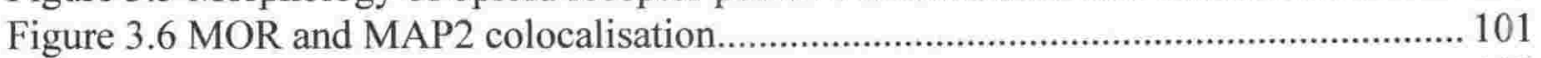

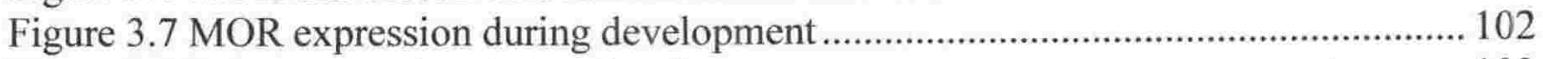

Figure 3.8 DOR expression during development............................................................. 103

Figure 3.9 MOR and DOR staining intensity of neurons and astrocytes in primary rat brainstem cultures from different developmental ages ................................ 104

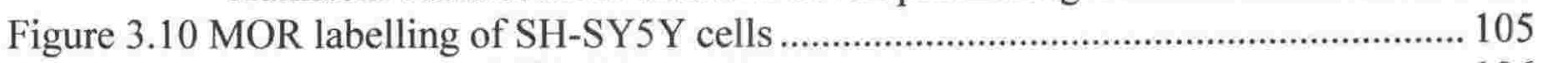

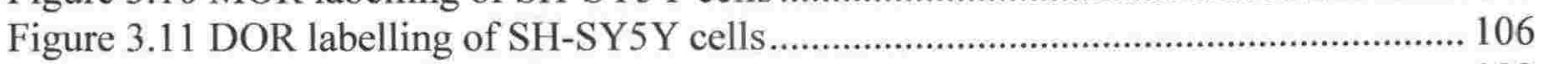

Figure 3.12 Double-labelling of MOR and DOR in culture ............................................ 109

Figure 3.13 Three-dimensional, confocal analysis of MOR and DOR staining during primary culture .................................................................................... 110

Figure 3.14 MOR and DOR double-labelling .................................................................. 111

Figure 3.15 Serial confocal sections through a group of P1 cells expressing MOR and DOR

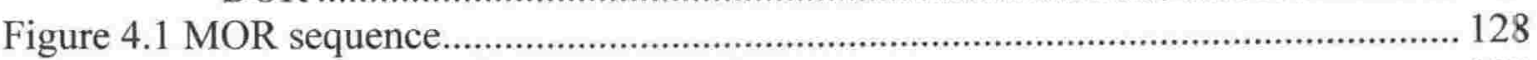

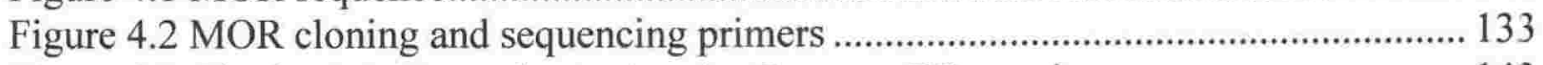

Figure 4.3 Cloning a PCR product using the Gateway BP reaction ................................. 143

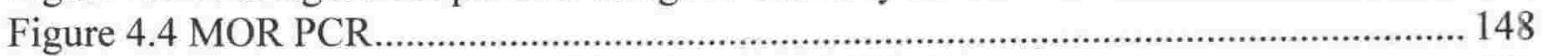

Figure 4.5 Sequence alignment of the cloned rat MOR-1 receptor ................................... 150

Figure 4.6 Labelling of transfected SH-SY5Y cells ........................................................ 151

Figure 4.7 MOR regulation in non-transfected SH-SY5Y cells (A) ................................. 153

Figure 4.8 MOR regulation in non-transfected SH-SY5Y cells (B) ................................ 154

Figure 4.9 OpR regulation in dissociated brainstem cell cultures ................................... 156

Figure 5.1 DOR and MOR labelling in brain regions ................................................ 169 
Figure 5.2 Distribution of DOR and MOR immunoreactivity in the adult rat

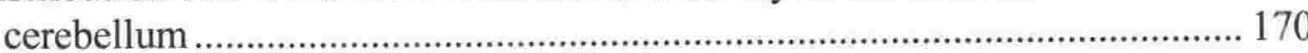

Figure 5.3 DOR and MOR labelled Purkinje cells ........................................................ 171

Figure 5.4 Distribution of DOR and MOR immunoreactivity in the postnatal P6 rat

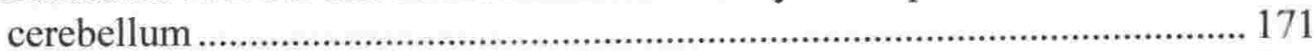

Figure 6.1 DOR and MOR labelling of adult brainstem tissue lysates .............................. 186

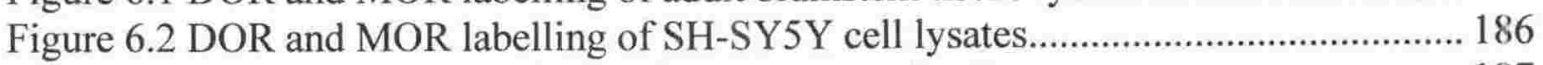

Figure 6.3 Western blot comparisons of two MOR antibodies......................................... 187

Figure 6.4 MOR protein expression during development in aged-matched samples ........ 189

Figure 6.5 Western blot showing developmental expression of MOR .............................. 190

Figure 6.6 Combined sample data of MOR expression during development ................... 191

Figure 6.7 DOR expression during development in aged-matched samples ..................... 194

Figure 6.8 Western blot showing developmental expression of DOR............................ 195

Figure 6.9 Combined sample data of DOR expression during development.................... 196

Figure 6.10 MOR and DOR expression in cells cultured from the rat brainstem.............. 197

Figure 6.11 Regions of brainstem used for immunolabelling of MOR and DOR in

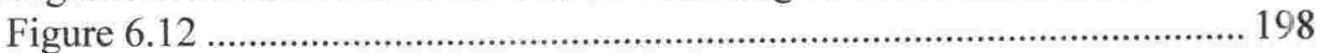

Figure 6.12 Distribution of DOR and MOR immunoreactivity in the adult rat brainstem ....................................................................................... 200

Figure 6.13 High power images of adult DOR and MOR immunoreactivity in the

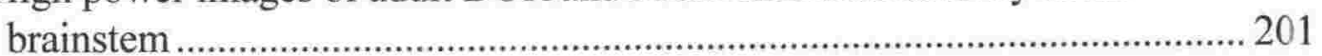

Figure 6.14 DOR and MOR labelling in the postnatal rat brainstem................................. 203

Figure 6.15 High power images of postnatal DOR and MOR labelled brainstem

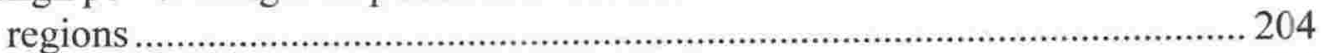

Figure 6.16 DOR and MOR immunoreactivity in the foetal brainstem........................... 205

Figure 6.17 High power images of DOR and MOR labelled brainstem regions .............. 206 


\section{List of Tables}

Table 1.1 Selectivity of ligands for opioid receptors …..................................................... 5

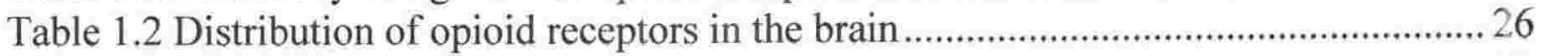

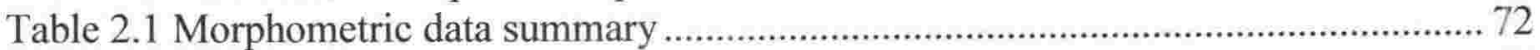

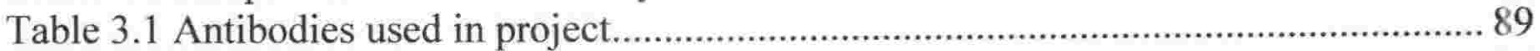

Table 3.2 Double-labelling method validation................................................................. 92

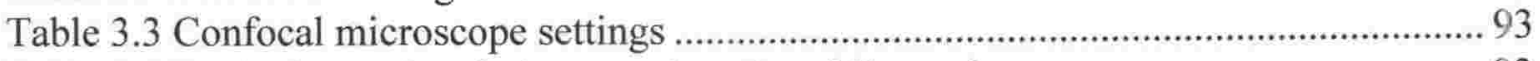

Table 3.4 Excitation and emission wavelengths of fluorophores .................................. 93

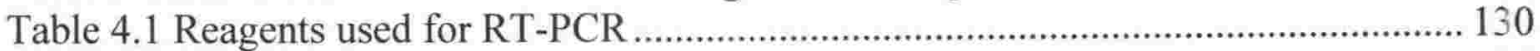

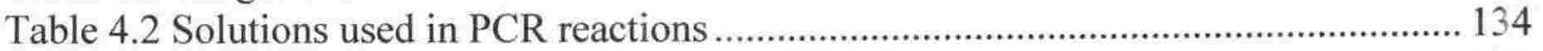

Table 4.3 Reagents used to ligate MOR into pGEM-T vector......................................... 135

Table 4.4 Reagents required for the GW BP reaction................................................... 140

Table 4.5 Reagents required for the GW LR reaction ................................................... 141

Table 4.6 MOR regulation in non-transfected, monensin pretreated SH-SY5Y cells ....... 155

Table Appendix 2 Arrangement of western transfer apparatus ........................................ 237 


\section{Abbreviations}

\begin{tabular}{|c|c|}
\hline $\mathrm{AC}$ & Adenylyl cyclase \\
\hline $\mathrm{AP}$ & Area postrema \\
\hline Ara C & $\beta$-D-cytosine arabinoside \\
\hline ARB & Autoradiography of ligand binding \\
\hline BötC & Bötzinger complex \\
\hline BRET & Bioluminescence resonance energy transfer \\
\hline BSA & Bovine serum albumin \\
\hline BSS & Balanced salt solution \\
\hline $\mathrm{CaM}$ & $\mathrm{Ca}^{2+} /$ calmodulin \\
\hline ChAT & Choline acetyltransferase \\
\hline CNS & Central nervous system \\
\hline CSF & Cerebrospinal fluid \\
\hline DAB & Diaminobenzidine \\
\hline DALDE & {$\left[\mathrm{D}-\mathrm{Ala}^{2}, \mathrm{D}-\mathrm{Leu}^{5}\right]$ enkephalin } \\
\hline DAMGO & {$\left[\mathrm{D}-\mathrm{Ala}^{2}, \mathrm{~N}\right.$-methyl-Phe ${ }^{4}, \mathrm{Gly}^{5}$-ol]enkephalin } \\
\hline DEPC & Diethyl-pyro carbonate \\
\hline DH & Dorsal horn of the spinal cord \\
\hline DIG & Digoxigenin \\
\hline DMEM & Dulbecco's modified Eagles medium \\
\hline DOR & Delta opioid receptor \\
\hline DPDPE & {$\left[\mathrm{D}-\mathrm{Pen}^{2,5}\right.$ ]enkephalin } \\
\hline DRG & Dorsal respiratory group \\
\hline DSLET & {$\left[\mathrm{D}-\mathrm{Ser}^{2}\right]$ Leu-enkephalin-Thr } \\
\hline $\mathrm{ECu}$ & External cuneate nucleus \\
\hline FCS & Foetal calf serum \\
\hline FDU & 5-fluro-2-deoxyuride \\
\hline FISH & Fluorescent in situ hybridisation \\
\hline FITC & Fluoresecin isocyanate \\
\hline FRET & Time resolved fluorescence resonance energy transfer \\
\hline g & Gila \\
\hline GABA & Gamma-aminobutyric acid \\
\hline GASP & G protein-coupled receptor-associated sorting protein \\
\hline GASP & G-protein coupled receptor-associated sorting protein \\
\hline GFAP & Glial fibrillary acidic protein \\
\hline $\mathrm{Gi}$ & Gigantocellular reticular nucleus \\
\hline GIRK & G-protein activated potassium channel \\
\hline GP & Guinea pig \\
\hline GPCR & G-protein coupled receptor \\
\hline $\mathrm{Gr}$ & Gracile nucleus \\
\hline GrL & Granular layer of the cerebellum \\
\hline H441 & Human lung bronchoalveolar adenocarcinoma cell line \\
\hline H441 & Human lung bronchoalveolar adenocarcinoma cell line \\
\hline HRP & Horseradish peroxidase \\
\hline ICC & Immunocytochemistry \\
\hline IHC & Immunohistochemisty \\
\hline
\end{tabular}




\begin{tabular}{ll} 
IO & Inferior olive \\
ip & Intraperitoneal \\
ISH & In situ hybridisation \\
KOR & Kappa opioid receptor \\
Li & Linear nucleus \\
LLC-PK 1 & Pig kidney cell line \\
LPGi & Lateral parpagigantocellular nucleus \\
LR & Lateral reticular nucleus \\
M & Molecular layer of cerebellum \\
MAP2 & Microtubule-associated protein-2 \\
MAPK & Mitogen-activated protein kinase \\
mlf & Medial longitudinal fasciculus \\
MOR & Mu opioid receptor \\
MVe & Medial vestibular nucleus \\
MW & Molecular weight \\
n & Neuron \\
NA & Nucleus ambiguus \\
NGS & Normal goat serum \\
NK1r & Neurokinin 1 receptor \\
NMDA & N-methyl-D-aspartate \\
NTS & Nucleus of the solitary tract \\
nu & Nucleus \\
OGFr & Opioid growth factor receptor \\
OP & Opioid peptide \\
OpR & Opioid receptor \\
P & Punctate \\
PAG & Periaqueductal grey \\
PBS & Phosphate buffered saline \\
PCR & Polymerase chain reaction \\
PDL & Poly-D-lysine \\
PEI & Polyethyenimine \\
PI & Propidium iodide \\
Pk & Purkinje cell \\
PKA & Protein kinase A \\
POMC & Pro-opiomelanocortin \\
preBötC & Pre-Bötzinger complex \\
PVDF & Polyvinylidene difluoride \\
Py & Pyramidal tract \\
R & Raphe nuclei \\
RA & All-trans retinoic acid \\
RT & Reverse transcription \\
RVLM & Rostral ventrolateral medulla \\
S & Soma \\
SDS & Sodium dodecyl sulphate \\
SEM & Standard error of the mean \\
SH-SY5Y & Human neuroblastoma cell line \\
SNP & Single nucleotide polymorphism \\
SpVe & Spinal vestibular nucleus \\
STT & Spinal trigeminal tract and nucleus \\
TBS & Tris buffered saline \\
& \\
\hline
\end{tabular}


TSA Tyramine signal amplification

T-TBS Tris buffered saline containing tween

VLM Ventrolateral medulla

VRG

RPMI- 1640

Ventral respiratory group

COS-7

ORL-1

Roswell Park Memorial Institute solution-1640

10

African green monkey kidney cell line

Opioid receptor like

12

Hypoglossal nucleus

Dorsal motor nucleus of the vagus 


\section{CHAPTER 1}

\section{Introduction and Literature Review}

This chapter summarises the physiological function of opioids in the central nervous system (CNS), their localisation and that of their receptors, their regulation and expression during mammalian development, the functional implications of differential distributions of opioid receptors within the rat brainstem during development and the overall role the opioid system plays in health and disease.

\subsection{OPIOIDS}

Opioid signalling is coupled to many physiological processes within the body (Olsen et al., 1989; Olsen et al., 1995; Olson et al., 1997; Vaccarino et al., 1999; Vaccarino and Kastin, 2001). Opioids modulate respiratory, cardiovascular, and gastrointestinal systems and are implicated in body temperature regulation (Adler and Geller, 1988; Takita et al., 1998), analgesia (Paul et al., 1989; Pasternak, 1993), antinociception (Fields and Heinricher, 1985; Porreca et al., 1992; Gutstein et al., 1998; Commons et al., 2001) immune cell signalling (Bidlack, 2000; Tefano et al., 2000; Kraus et al., 2001; Sun, 2002; Suzuki et al., 2002), cognitive functions (Olsen et al., 1989), locomotion (Vaccarino et al., 1999), and the central control of endocrine function (Olson et al., 1997; Vaccarino et al., 1999; Vaccarino and Kastin, 2001). Opioid peptides bind to receptors on the plasma membrane and exert their effects on the cell through G-protein signalling pathways (Lefkowitz, 1998; Wang et al., 1999; Remmers et al., 2000; Wang et al., 2000; Tsao et al., 2001) (for review see) (Standifer and Pasternak, 1997).

There are three main groups of endogenous opioid peptides: enkephalins, $\beta$-endorphins, and dynorphins. These peptides are derived from proteolysis of their larger precursor proteins, pro-enkephalin, pro-opiomelanocortin (POMC), and pro-dynorphin, respectively. Pro-enkephalin is the precursor for several active peptides, all of which have opioid activity. Within its structure, it contains 7 peptides with the [Met]- or [Leu]-enkephalin active core. POMC has at its carboxyl terminus the 31 amino acid $\beta$-endorphin and its 91 amino acid precursor beta-lipotropin. POMC is also the precursor for adrenocorticotropic 
hormone. $\beta$-endorphin is the only peptide from this precursor to have opioid functions. Pro-dynorphin produces three main [Leu]-enkephalin-containing peptides, including dynorphin-A and -B. All endogenous opioid peptides contain the opioid core Tyr-GlyGly-Phe-Met (or Leu). Endomorphin-1 and endomorphin-2 are proposed endogenous ligands for mu opioid receptors, although they are the product of an as yet unidentified precursor. For a review on endogenous opioids, see Akil et al. (1984) (Akil et al., 1984). Opioid peptides bind with different affinities to the five main classes of opioid receptors: mu receptor (MOR), delta receptor (DOR), kappa receptor (KOR) (Martin et al., 1976; Barnard and Demoliou-Mason, 1983; Kieffer et al., 1992; Pasternak, 1993; Minami and M., 1995; Reisine, 1995; Satoh and Minami, 1995; Standifer and Pasternak, 1997; Law and Loh, 1999), opioid receptor-like receptor (ORL-1) (Pan et al., 1998; Meunier et al., 2000; Mollereau and Mouledous, 2000; Slowe et al., 2001), and opioid growth factor receptor (OGFr) (Zagon et al., 1999; Zagon et al., 2002), all of which have been cloned. Each class of opioid receptor (OpR) has distinct expression and functional profiles within the body, especially within the brain, where they are developmentally and spatially regulated (Liu et al., 1999). The distributions of opioid peptides and their receptors in the adult brain and within the foetal brain during early development are functionally important. To better understand opioid action at the cellular level, Xenopus oocyte expression systems (Appleyard et al., 1999; Koch et al., 2000), transfected cells (Cvejic and Devi, 1997; Martin and PL., 2001; McVey et al., 2001; He et al., 2002), and cells endogenously expressing opioid receptors (Prathert et al., 1994; Kramer and Simon, 1999) have been studied.

\subsubsection{THE HISTORY OF OPIOIDS}

Opiates are alkaloid compounds that have been used for over 4000 years in religious rituals because of their ability to evoke euphoria. The first active substance was isolated in 1806 from opium extracted from poppy seeds (Papaver somniferum) and was named morphine, after Morpheus, the god of dreams. In the 1850 s, morphine was introduced into medicine for the treatment of acute pain during surgery and for the relief of chronic pain. It was also used as a cough suppressant and for the treatment of diarrhoea. Since this time, research efforts have sought molecules that mimic the action of morphine without causing the unwanted side effects of nausea, constipation, respiratory depression, immune suppression, addiction, and tolerance (Olson et al., 1997; Vaccarino et al., 1999; Vaccarino and Kastin, 
2001). In 1898, heroin, a diacetylated derivative of morphine, was synthesised for this purpose but later discovered to be even more addictive than morphine because it crosses the blood-brain barrier more readily. OpR antagonists are often used in drug therapy to block the effects of opioids (Gonzalez and Brogden, 1988). Opioids are now one of the most dangerous and costly drugs of abuse and are estimated to cost the USA over 100 billion US dollars/pa in lost earnings, medical expenses and crime associated with drug abuse [www.nida.nih.gov/EconomicCosts].

The development of cellular and molecular techniques with which to study OpRs has dramatically increased the knowledge of how the opioid system works, although the exact cellular mechanisms that lead to desensitisation and addiction still remain unknown. Unfortunately, little progress has been made in separating the desired anti-pain effects of opioids with their unwanted side effects.

\subsubsection{ENDOGENOUS OPIOIDS}

The term opioid refers to all drugs with morphine-like actions, regardless of their molecular structure. Endogenous opioid ligands were first identified by Hughes et al. (1975), who isolated met-enkephalin and leu-enkephalin from the pig brain (Hughes et al., 1975). Two other classes of endogenous opioid peptides, dynorphins and $\beta$-endorphin, were discovered soon after from porcine and camel pituitary glands, respectively (Li and Chang, 1976; Goldstein et al., 1981). The endorphins tend to have a peripheral site of action; whereas, the enkephalins and dynorphins are widely distributed in the central nervous system and are considered to be the predominant central opioid peptide neurotransmitters (for reviews see (Carr, 1988; Lutz and Pfister, 1992; Dhawan et al., 1996; Vaccarino et al., 1999; Vaccarino and Kastin, 2001)). Opioid receptors were first identified in the mammalian brain in the 1970's by autoradiography of binding sites of radiolabelled opioid peptides (Pert et al., 1973). Multiple OpRs were characterised by the different effects of different OpR agonists or partial agonists in the morphine-dependent and nondependent chronic spinal dog (Martin et al., 1976).

\subsubsection{SELECTIVITY OF LIGANDS FOR OPIOID RECEPTORS}

Binding experiments have shown that DOR preferentially binds enkephalin, KOR preferentially binds dynorphin, and MOR shows high affinity for endomorphins, $\beta$ - 
endorphin and enkephalin. Endogenous opioid peptides have only moderate selectivity for one OpR type over another and it is likely that they activate more than one OpR. The interaction between opioid peptides and OpRs involves both the binding selectivity of the ligands and receptor availability in the vicinity of peptide release. The pharmacological profiles of each opioid receptor have been well characterised (for reviews see (Iwamoto and Martin, 1981; Smith and Low, 1981)). Most of the opiates used clinically such as morphine, codeine, and methadone, selectively interact with MOR. The pharmacology of the three groups of classical opioid receptors is distinct and well characterised. OpR ligands have been synthesised with varying specificities for each class of opioid receptor, summarised in Table 1.1. [D-Ala ${ }^{2}, \mathrm{~N}$-methyl-Phe ${ }^{4}, \mathrm{Gly}^{5}$-ol] enkephalin (DAMGO) and dermorphin have a high selectivity and affinity for MOR (Goldstein and Naidu, 1989; Negri et al., 1992; Attila et al., 1993). The DOR agonists [D-Ser ${ }^{2}$ Leu-enkephalin-Thr (DSLET) and $\left[\mathrm{D}-\mathrm{Ala}^{2}, \mathrm{D}-\mathrm{Leu}^{5}\right]$ enkephalin (DALDE) have only slight selectivity for DOR; whereas, [D-Pen ${ }^{2,5}$ ]enkephalin (DPDPE) is highly selective. DSLET has been reported to have 20- to 600-fold selectivity for DOR over MOR (Gacel et al., 1980); whereas, DALDE has a 2- to 20-fold selectivity for DOR over MOR (James and Goldstein, 1984). DPDPE is 100- to 1000-times more potent at DOR than MOR (Mossberg et al., 1983; Toth et al., 1990) and is the preferred DOR agonist for use in binding studies. The specificity of the ligands has been mapped to specific regions of the opioid receptor, using chimeric or mutated OpRs (Meng et al., 1996; Capeyrou et al., 1997; Gouldson et al., 1998; Chaturvedi et al., 2000; El Kouhen et al., 2000). 
Table 1.1 Selectivity of ligands for opioid receptors

\begin{tabular}{|c|c|c|c|}
\hline Receptor & Agonists & Antagonists & Natural Transmitter \\
\hline \multirow[t]{5}{*}{ MOR } & DAMGO & CTOP & Endomorphin-1 \\
\hline & Morphine & Naloxonazine & Endomorphin-2 \\
\hline & \multirow[t]{2}{*}{ Dermorphin } & $\beta$-FNA & Enkephalin \\
\hline & & SNC 80 & Endorphin \\
\hline & \multicolumn{3}{|c|}{ Morphine-6-glucuronide } \\
\hline \multirow[t]{5}{*}{ DOR } & DPDPE & Naltrindole & Enkephalin \\
\hline & DALDE & NTB & Endorphin \\
\hline & DSLET & BNTX & \\
\hline & Deltorphin I & ICI 174864 & \\
\hline & Deltorphin II & & \\
\hline \multirow[t]{3}{*}{ KOR } & U50,488 & nor-BNI & Dynorphin A \\
\hline & U69,593 & & Dynorphin B \\
\hline & Enadoline & & \\
\hline
\end{tabular}




\subsection{OPIOID RECEPTORS}

\subsubsection{TYPES OF RECEPTORS}

In the 1970s, the three main classes of OpR, $\mu, \delta$, and $\kappa$, were identified (Martin et al., 1976). These three classes were further subdivided according to their pharmacological properties into $\mu_{1} / \mu_{2} / \mu_{3}, \delta_{1} / \delta_{2}$, and $\kappa_{1} / \kappa_{2} / \kappa_{3}$ receptors. Although these eight isoforms of opioid receptors (OpRs) have been recognised pharmacologically, only three OpR genes have been cloned. DOR is located on chromosome 1 , MOR on chromosome 6 , and KOR on chromosome 8 (Befort et al., 1994; Wang et al., 1994; Yasuda et al., 1994). The seven MOR, DOR, and KOR isoforms are all products of these three cloned genes. This has recently been confirmed by production of knockout mice in which the pharmacological responses are lost for each particular OpR gene deleted (Matthes et al., 1996; Kieffer, 1999; Chen et al., 2000; Kieffer and Gavériaux-Ruff, 2002). The correlation between the biological activity of the encoded proteins and the pharmacological subtypes remains to be established.

Recent studies have shown that some pharmacological heterogeneity arises from the three known MOR, DOR and KOR genes by alternative splicing mechanisms. At present fifteen different $\mathrm{C}$ - and $\mathrm{N}$ - terminal splice variants have been reported for the MOR-1 receptor in rodents (Koch et al., 1998; Pan et al., 1999; Koch et al., 2001; Pan et al., 2001). These splice variants, classified as MOR-1 and MOR-1A to MOR-1N, are differentially distributed in the brain and undergo region-specific processing (Schulz et al., 1998; Abbadie et al., 2000b; Abbadie et al., 2000a). For example, MOR-1C is absent or sparse in the nucleus ambiguous (NA), locus coeruleus, and striatum, regions where MOR-1 is expressed at high levels. MOR-1C and MOR-1 are both found in the periaqueductal gray (PAG) and laminae I and II of the spinal cord ( $\mathrm{SpC})$; whereas, MOR-1C expression is higher in the hypothalamus than MOR-1 (Abbadie et al., 2000a). MOR-1D is expressed at high levels in the nucleus of the solitary tract (NTS), area postrema, inferior olive (IO), NA, and spinal trigeminal nucleus; whereas, it is absent from the striatum and loecus coeruleus (Abbadie et al., 2000b). In the mouse, five splice variants of KOR have been described, and each of these splice variants has distinct expression patterns within the brain (Pan et al., 1998). KOR-3A is expressed at high levels in the striatum, a region where KOR-3 is absent. KOR-3C is found in the PAG and hypothalamus in the same regions as KOR-3. KOR-3B levels were low throughout the brain. KOR-3D was most predominant 
in the cerebellum; whereas, the brainstem had similar expression levels of KOR-3, KOR3A, and KOR-3D (Pan et al., 1998). Two DOR splice variants have also been identified in mouse brain by RT-PCR. The DOR splice variants are present at low levels (approximately 5\%) when compared to total DOR-1 expression levels (Gavériaux-Ruff et al., 1997). Most of the splice variants express a truncated form of the receptor protein, and in the case of the KOR and DOR splice variants, the receptors are unlikely to couple with G-proteins. The precise biological functions of these splice variants remains to be determined.

\subsubsection{OPIOID RECEPTOR STRUCTURE}

A major breakthrough in the understanding of OpR structure and function was achieved with the cloning of the different OpRs (Evans et al., 1992; Kieffer et al., 1992; Chen et al., 1993b; Fukuda et al., 1993; Li et al., 1993; Thompson et al., 1993; Abood et al., 1994; Zimprich et al., 1995; Akil et al., 1996; Zagon et al., 1999; Meunier et al., 2000). The sequence of the cloned genes indicated that opioid receptors belonged to the superfamily of G-protein coupled receptors (GPCR), lying within the subfamily of rhodopsin receptors. The three classical OpRs share about 60\% sequence identity (Knapp et al., 1995; Satoh and Minami, 1995), and each OpR is approximately $90 \%$ identical to its counterpart in other mammalian species (Befort and Kieffer, 1997). The N-terminus shares the least homology (around $10 \%$ ), with the transmembrane regions and intracellular loops showing the greatest homology (Blake et al., 1996) for review see (Satoh and Minami, 1995).

OpRs have seven transmembrane helices (Figure 1.1) connected by three intracellular and three extracellular loops, a cytoplasmic C-terminus, which has a highly conserved potential palmitoylation site, and an extracellular N-terminus that contains multiple glycosylation sites. Two cysteine residues located in the first and second extracellular loops are highly conserved between different OpRs and form a disulfide bond in the properly folded, functional protein. Multiple sites exist for protein kinase phosphorylation, and these sites are present in the $2^{\text {nd }}$ and $3^{\text {rd }}$ intracellular loops and in the C-terminal domain (Chen et al., 1993a), (for review, see (Minami and M., 1995)). Mutation analysis of the OpRs has been carried out by construction of opioid receptor chimeras (Befort and Kieffer, 1997; Gouldson et al., 1998; Law and Loh, 1999; Law et al., 2000a) and site-directed mutagenesis. (Meng et al., 1996; Capeyrou et al., 1997; Chaturvedi et al., 2000; El Kouhen 
et al., 2000). Mutation analysis has increased the understanding of ligand binding selectivity and identified the receptor regions responsible for activation and regulation of the receptors. For example, the C-terminal of DOR and MOR appears to be involved in receptor trafficking, since loss of the C-terminal prevents polarised insertion of the receptor in the plasma membrane (Trapaidze et al., 1996; Trapaidze et al., 2000). KORs require the $\mathrm{C}$-terminal region for agonist-induced receptor down-regulation (Appleyard et al., 1999). A summary of opioid receptor regions important for binding and regulation is shown in Figure 1.1 (Minami and M., 1995; Satoh and Minami, 1995; Wang et al., 1995; Blake et al., 1996; Meng et al., 1996; Trapaidze et al., 1996; Befort and Kieffer, 1997; Koch et al., 2000; Law et al., 2000a; Wang et al., 2000). Extracellular loops have a role in determining ligand selectivity. These extracellular loops probably do not form the actual binding pocket for the peptide, but act as filters for the ligands, regulating the ability of the ligands to interact with the binding pocket within the core of the receptor. The orientation of the amino acid side chains greatly affects the ability of the extracellular loop to filter opioid selective ligands, since mutation of a single amino acid can alter the tertiary structure of the receptor protein. For example, when an OpR agonist binds to its receptor, a small conformational change exposes the intracellular loop, allowing its interaction with G-proteins (Javitch et al., 1997).

The resolution of the atomic structure of GPCRs has not yet been carried out, but several models have been presented in the literature (Knapp et al., 1995; Wess, 1998). In one proposed model, the seven transmembrane helices are tightly packed and arranged in a sequential clockwise order in a tight bundle. The position of the transmembrane helices is important in determining the interaction of the side chains. A schematic diagram of the organisation of the transmembrane helices has been presented (Law et al., 2000a). The structure of the OpR in the membrane has been modelled in detail for rat MOR (Minami and M., 1995; Satoh and Minami, 1995) and DOR (Law et al., 1999).

\subsubsection{OPIOID RECEPTOR REGULATION}

The OpR signalling pathways described in this section are summarised in Figure 1.2. The cloning of DOR in 1992 by Evans et al. (1992) (Evans et al., 1992) and Kiefer et al. (1992) (Kieffer et al., 1992) dramatically increased the understanding of OpR regulation. Regulation has now been extensively studied using cell lines (Elliott et al., 1994; Prathert 
et al., 1994; Gies et al., 1997; Yabaluri and Medzihradsky, 1997; Kramer and Simon, 1999; Law et al., 2000c; Remmers et al., 2000; Jenab and Inturrisi, 2002), brain membrane preparations (Satoh and Minami, 1995; Gray et al., 2001), Xenopus oocyte expression systems (Appleyard et al., 1999; Koch et al., 2000), and yeast expression systems (Lagane et al., 2000). Cloning and transfection techniques have allowed the function of individual OpR amino acid sequences to be determined by selective mutation (Meng et al., 1996), thus, permitting identification of the receptor domains responsible for opioid ligand selectivity, agonist activation, and receptor regulation. Transfected cells and cells endogenously expressing OpRs are often used to study receptor regulation because they consist of homogeneous populations of cells that are more easily characterised than the heterogeneous population of cells within the brain that express OpRs. Studies of OpR function within the brain are further complicated by differences in regional distribution, function, and regulatory mechanisms (Mansour et al., 1987, 1988). These regional differences are further complicated by species differences that exist in the type, localisation, and expression levels of different OpRs. Species variability is described in detail later in this chapter.

Activation of OpRs causes a reduction in pre- and postsynaptic neuronal excitability. OpRs, through GPCR signalling pathways, inhibit adenylyl cyclase activity. The resultant decrease in cAMP in the cells, through alteration of numerous protein kinase activities, leads to changes in receptor phosphorylation, activation of inward-rectifying $\mathrm{K}^{+}$channels, and inhibition of voltage-gated $\mathrm{Ca}^{2+}$ channels. (Xiang et al., ; Fan and Crain, 1995; Kovoor et al., 1995; Belcheva et al., 1996; Trapaidze et al., 1996; Blake et al., 1997; Chen et al., 1997; Chu et al., 1997; Gaudriault et al., 1997; Pak et al., 1997; Yabaluri and Medzihradsky, 1997; Yu et al., 1997; Koch et al., 1998; Zhang et al., 1998; Kramer and Simon, 1999; Law and Loh, 1999; Liu et al., 1999; Pak et al., 1999; Bohn et al., 2000; Kramer and Simon, 2000; Petäja-Repo et al., 2000; Remmers et al., 2000; Tryoen-Toth et al., 2000; Wang et al., 2000; Yoshikawa et al., 2000; Wei and Low, 2002). Exposure of cultured cells that express OpRs, or cells transfected with OpRs, to opioid agonists causes an inhibition of adenylyl cyclase activity, similar to that seen in vivo (Law et al., 1983; Kieffer et al., 1992; Chan et al., 1995). Chronic exposure causes a decrease in the ability of the OpR to regulate adenylyl cyclase activity (receptor desensitisation) and a decrease in receptor density (receptor down-regulation) (Law et al., 1983; Law et al., 2000b). Exposure of OpRs to opioid antagonists in cultures of spinal cord dorsal root ganglion 
causes an up-regulation of receptors to the plasma membrane (up to a $40 \%$ increase); whereas, incubation with $\mathrm{OpR}$ agonists causes a down-regulation of OpR function (up to a $70 \%$ reduction in function) (Chen et al., 1997). 
Figure 1.1 Opioid Receptor Structure, Binding and Regulation Properties 


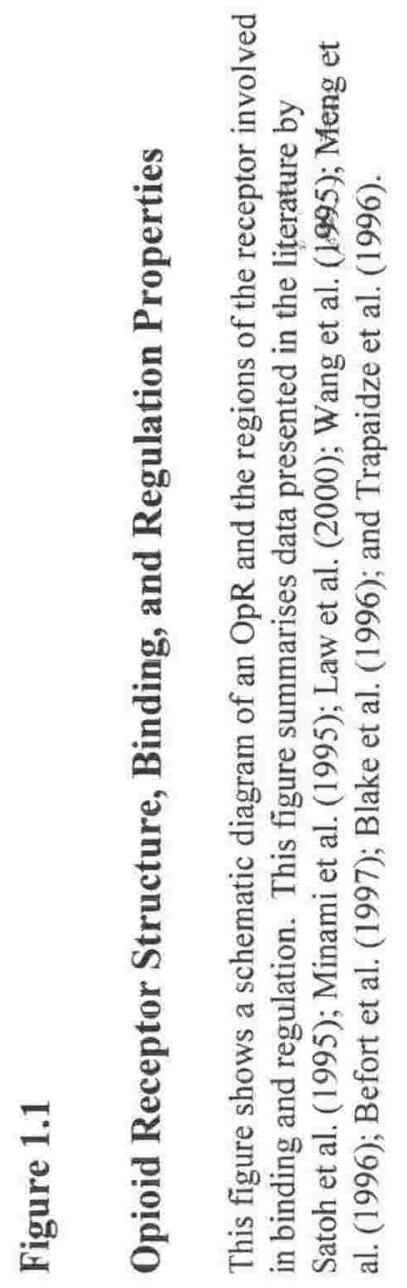




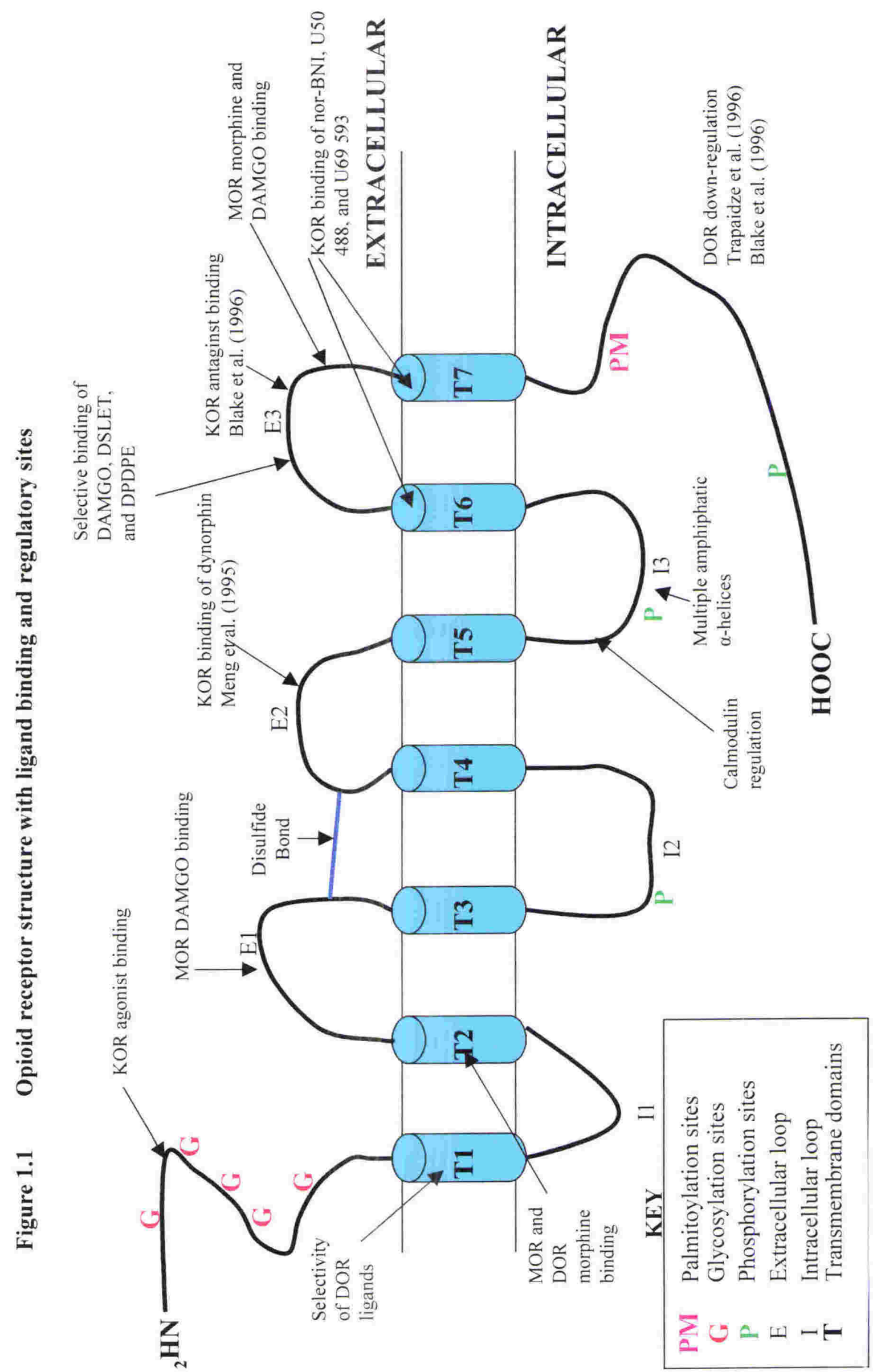




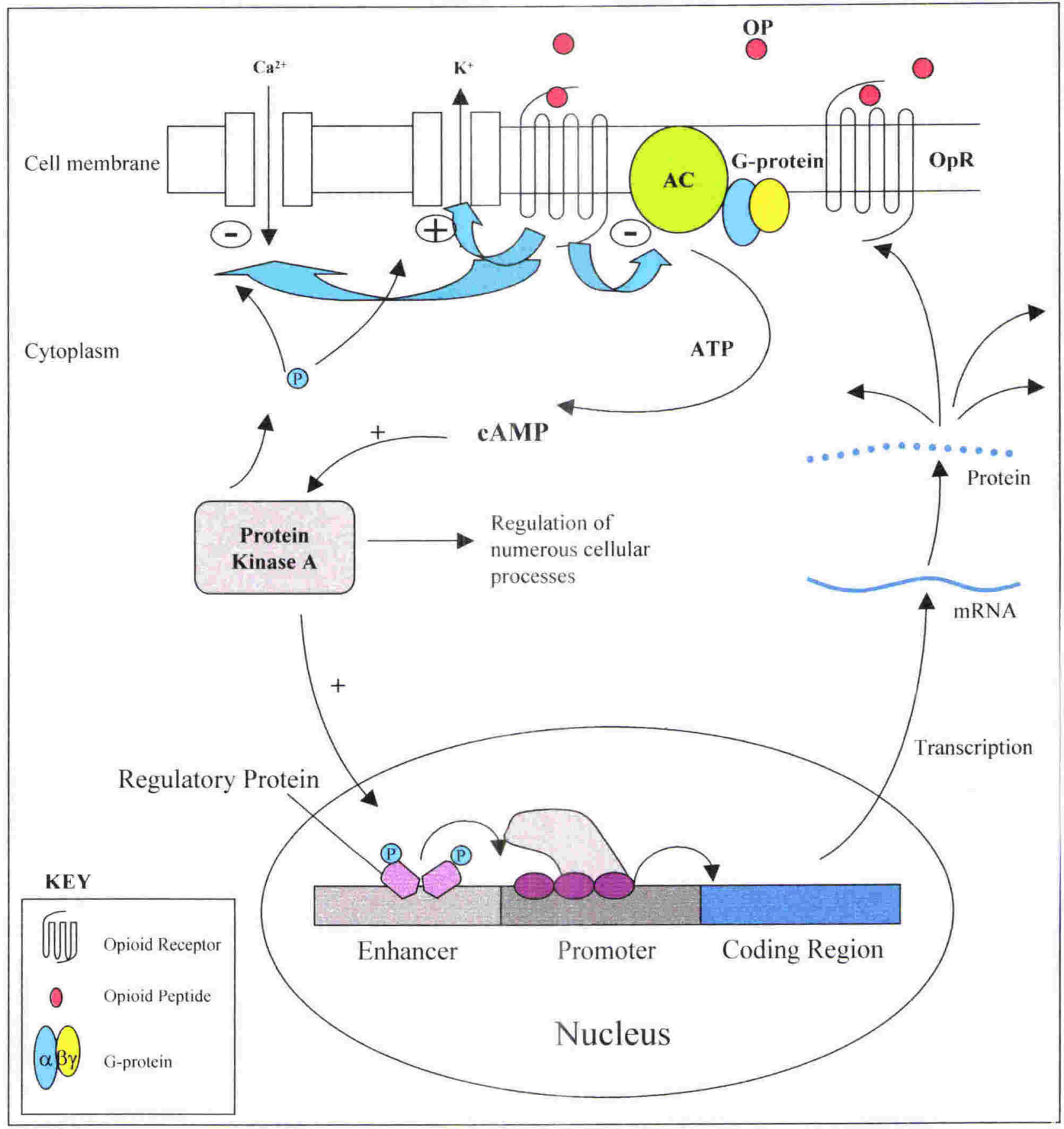

Figure 1.2

\section{Opioid Receptor Activation}

Binding of opioid peptides (OP) at opioid receptors (OpR) causes G-protein mediated inhibition of adenylyl cyclase (AC) which causes a decrease in intracellular cAMP levels. The $\mathrm{K}^{+}$channel is activated and the $\mathrm{Ca}^{2+}$ channel inhibited, causing hyperpolarisation of the cell membrane. Decreased cAMP levels causes a decrease in protein kinase activity (PKA) which modulates numerous cellular processes, including reduced OpR transcription and changes in channel activities. Modified from Nestler (1996) and Kandel et al. (2000). 
Opioid receptors, through coupling to G-proteins, inhibit (Chen et al., 1993b) or activate (Chan et al., 1995) different isoforms of adenylyl cyclases (Standifer and Pasternak, 1997). There are many different types of G-proteins, and there are differences in subunit conformation between these G-proteins. Each of the 19 different $\alpha$ subunits, the $5 \beta$ subunits, and the $6 \gamma$ subunits described in the literature has unique functional properties (reviewed by Harrison, 1998 and Conner 1999) (Harrison et al., 1998; Connor and Christie, 1999). G- protein coupling differs between different brain regions; cell lines, transfected cells, and between OpR subtypes (Chan et al., 1995; Appleyard et al., 1999). There are also differences in the sensitivity of G-proteins to toxins (Standifer and Pasternak, 1997). For example, in SH-SY5Y cells, MOR couples to the G-protein $\mathrm{G}_{\mathrm{i} 3}$; whereas, DOR couples to the G-protein $G \alpha_{i 1}$, suggesting that G-protein specificity exists for different OpRs (Remmers et al., 2000) (reviewed in Standifer and Pasternak (1997) (Standifer and Pasternak, 1997). The different types of adenylyl cyclases, of which eight have been described (Standifer and Pasternak, 1997), vary in their sensitivity to calcium/calmodulin and the different G-protein subunits (Harrison et al., 1998; Connor and Christie, 1999), as well as their regulation by different protein kinases (Xiang et al., ; Koch et al., 1997; Wilson, 1997; Zhang et al., 1998; Appleyard et al., 1999; Kramer and Simon, 1999, 2000; Tso et al., 2000; Wang et al., 2000). All these factors affect the overall regulation of OpRs in neuronal cells.

Receptors within the GPCR class share similarities in their regulatory properties (Dohlman, 1991; Lefkowitz, 1998; Jordan and Devi, 1999; Tsao and von Zastro, 2000), and G-protein signalling has been well described in the literature. Briefly, when GPCRs are not stimulated by agonists, the G-protein exists as a heterotrimer with $\alpha, \beta$, and $\gamma$ subunits. GDP is bound to the $\alpha$ subunit. When the ligand binds to the receptor, the receptor associates with the G-protein, and GDP is exchanged for GTP. The G-protein $\alpha$ and $\beta \gamma$ subunits separate, and the OpR dissociates from the G-protein subunits. The $\alpha \mathrm{G}$ protein subunit then acts on adenylyl cyclase, GTP is hydrolysed to GDP, and the $\alpha$ subunit reassociates with the $\beta \gamma$ subunit (Connor and Christie, 1999).

Activation of OpRs causes a reduction in cellular excitability and neurotransmitter release. This can occur by several different mechanisms that are described below and illustrated in Figure 1-2. The OpR, on exposure to an agonist, inhibits voltage-dependent $\mathrm{Ca}^{2+}$ channels, 
including N-type (Tallent et al., 1994) and L-type $\mathrm{Ca}^{2+}$ channels (Piros et al., 2000). OpR stimulation reduces neuronal excitability by hyperpolarising the plasma membrane by increasing $\mathrm{K}^{+}$conductance out of the cell, and inhibiting $\mathrm{Ca}^{2+}$ entry into the cell (Piros et al., 2000). This has been confirmed by studies in Xenopus oocytes that coexpress either MOR or KOR along with the G-protein activated $\mathrm{K}^{+}$channel (GIRK1) (Kovoor et al., 1995). In the brain, MOR also couples to the G-protein-activated, inward-rectifying $\mathrm{K}^{+}$ channel (Bausch et al., 1995b). The presence of coupling between MOR and GIRK1 has been directly supported by studies that demonstrate that MOR and $\mathrm{K}^{+}$channels colocalise in some regions of the brain, including the olfactory nucleus, cerebral cortex, thalamus, locus coeruleus, and the dorsal raphe nucleus (Bausch et al., 1995b).

Agonist-induced receptor phosphorylation is critical in the regulation of GPCRs (Arden et al., 1995; Yu et al., 1997; El Kouhen et al., 2001) and involves phosphorylation of the Cterminal of MOR (Pak et al., 1997). Several different protein kinases are involved in OpR phosphorylation, including protein kinase C (Xiang et al., ; Kramer and Simon, 1999), $\mathrm{Ca}^{2+} /$ calmodulin (CaM)-dependent protein kinases (Koch et al., 1997), G-protein-coupled receptor kinases (Zhang et al., 1998; Appleyard et al., 1999), and mitogen-activated protein kinases (MAPK) such as Erk-1 and Erk-2. Activation of MAPK occurs via the G-protein

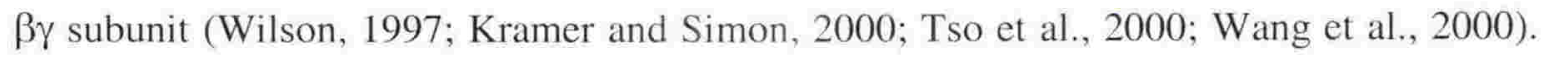
GPCR regulation by phosphorylation has been recently reviewed by Law (2000) (Law et al., 2000a). A G-protein independent, tyrosine-kinase-dependent pathway may also be involved in MOR down-regulation (Pak et al., 1999). DOR may also be partially regulated by G-protein independent and phosphorylation independent mechanisms. For example, mutant C-terminal truncated DOR, in the absence of phosphorylation, showed downregulation on exposure to DOR agonist DALDE. The DOR internalisation observed in this study was via a dynamin-dependent mechanism involving clathrin-coated pits (Murray et al., 1998). The study of DOR down-regulation carried out by Murray et al. (1998) was the first to show GPCR down-regulation that does not require phosphorylation to undergo dynamin-dependent endocytosis.

\subsubsection{RAPID RECEPTOR DOWN-REGULATION AND INTERNALISATION}

Opioid receptor signal transduction is closely associated with receptor down-regulation or desensitisation, a phenomenon characterised by a rapid loss of receptor function upon 
sustained exposure to an opioid agonist. Several cellular systems, including cell lines endogenously expressing these receptors and transfected cell lines, provide useful models to study these functions in vitro. In vivo systems are complicated further by long-term adaptive changes, tolerance, and addiction (Nestler and Aghajanian, 1997; White and Irvine, 1999). Recent findings suggest that MOR down-regulation during agonist stimulation is the sum of both accelerated receptor degradation and decreased receptor biosynthesis (Afify, 2002). Numerous mechanisms operate at the transcriptional, translational, and protein levels to lead to the desensitisation of opioid receptors.

The current model for OpR desensitisation (summarised in Figure 1.3) follows the same model as for other GPCRs (recently reviewed by (Tsao et al., 2001)). Briefly, the receptor is phosphorylated by protein kinases on exposure to its agonist, allowing $\beta$-arrestin to bind to the receptor. The binding of $\beta$-arrestin competes with $\mathrm{G}$-protein binding for the attachment sites, resulting in termination of receptor function. Addition of G-protein receptor kinase and $\beta$-arrestin to a Xenopus oocyte KOR expression system showed increased KOR down-regulation on exposure to KOR agonists (Appleyard et al., 1999). The internalisation of the receptor is also $\beta$-arrestin-dependent, and therefore phosphorylation-dependent, and occurs via endocytosis mediated by clathrin-coated pits (Xiang et al., ; Chu et al., 1997). The internalised receptor is resensitised by dephosphorylation and is then recycled to the membrane, or, alternatively, the receptor is degraded within lysosomes (Ko et al., 1999; Shapira et al., 2001; Tanowitz and von Zastrow, 2002). The endocytosis of GPCRs usually requires ubiquitination. Cytoplasmic lysine residues are covalently modified by ubiquitin to signal MOR and DOR removal from the membrane to endosomes (Chaturvedi et al., 2001). Another study, however, has described ubiquitination-independent trafficking of DOR to lysosomes (Tanowitz and von Zastrow, 2002). Several pathways for down-regulation of OpRs probably exist. Recently, a G-protein-coupled receptor-associated sorting protein (GASP) has been implicated in the modulation of lysosomal sorting (Whistler et al., 2002). 
Figure 1.3 Opioid Receptor Regulation 


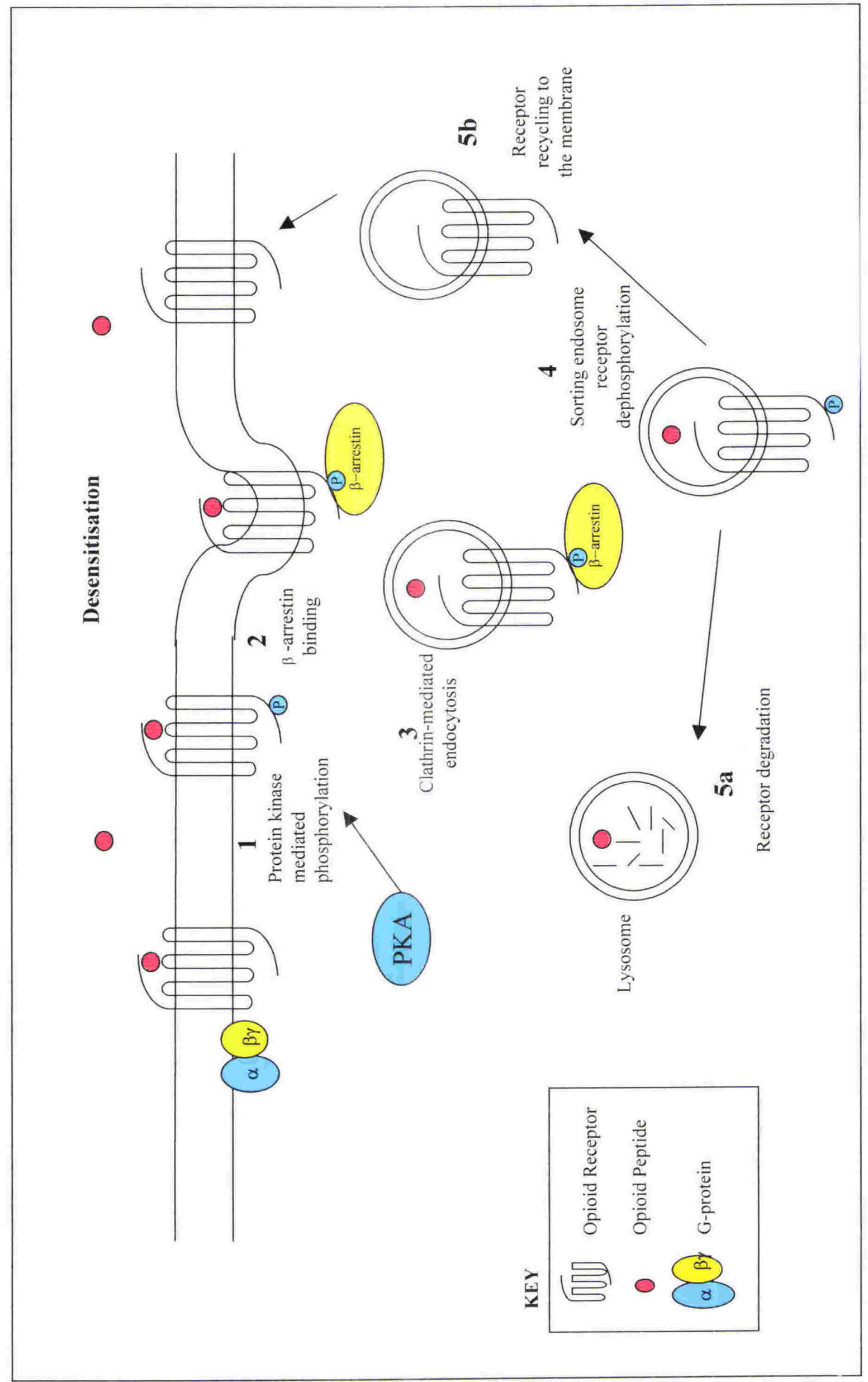


Whistler et al. (2001) using a C-terminal truncated DOR suggested that phosphorylation was required for wild-type DOR internalisation, but not for internalisation of the truncated DOR. This suggests that the DOR C-terminal region acts as a brake for receptor internalisation and that the break is removed by receptor phosphorylation (Whistler et al., 2001). Another protein, dynamin, has also been implicated in OpR internalisation in addition to $\beta$-arrestin (Chu et al., 1997). There are differences in the specific pathways of down-regulation and internalisation for different types of receptor. For example, Gauderiault et al. (1997), using cells transfected with MOR and DOR showed that OpRs were down-regulated and internalised through partially distinct endocytotic pathways (directly visualised by confocal microscopy) (Gaudriault et al., 1997). This suggests that each receptor interacts with distinct proteins that mediate intracellular trafficking and sorting. OpR splice variants also display different patterns of insertion and removal from the membrane, and the rates of down-regulation with different agonists vary (Koch et al., 1998; Koch et al., 2000; Abbadie and Pasternak, 2001; Koch et al., 2001).

Despite all this direct experimental evidence of OpR down-regulation and desensitisation, some studies indicate that other factors may be involved, since inhibition of phosphatase activity does not enhance $\mathrm{OpR}$ desensitisation and internalisation, and protein kinase activation, or over-expression of $\beta$-arrestin, does not enhance phosphorylation (El Kouhen et al., 1999). Numerous factors may therefore be involved in regulation, and regulatory pathways may be unique for each OpR subtype, splice variant, G-protein subtype, or adenylyl cyclase isoform (Pak et al., 1999; Tso et al., 2000). Indeed, not all OpR regulation directly involves G-protein-mediated pathways. To complicate matters further, opioids are not always inhibitory. It has been shown, for example, that KOR acts in an excitatory manner to oppose the actions of MOR (summarised in a review by Pan 1998) (Pan, 1998). KOR was recently found to reduce $\mathrm{K}^{+}$currents via activation of stimulatory $\mathrm{G}_{\mathrm{s}}$ proteins in cultured hippocampal neurons, supporting the concept of an excitatory opioid system (Fan et al., 1992; Fan and Crain, 1995; Hampson et al., 2000). In addition, it has been proposed that morphine, which does not cause rapid internalisation of MOR, may have a different mechanism for down-regulation that leads to opioid tolerance (Keith et al., 1996; Keith et al., 1998). 
In summary, activation of OpRs generally results in hyperpolarisation of the membrane via altered ion channel activity and G-protein-mediated inhibition of adenylyl cyclase activity. After activation, receptor phosphorylation causes OpR desensitisation (reduced coupling to signaling pathways) or down-regulation (removal of OpRs from the membrane), processes mediated by protein kinases and $\beta$-arrestin. Opioid receptor regulation is complicated by the involvement of different isoforms of adenylyl cyclase (Standifer and Pasternak, 1997) as well as G-protein subunits (Harrison et al., 1998; Connor and Christie, 1999), and the interactions they have with each subtype of receptor are unique.

\subsubsection{OLIGOMERISATION}

GPCRs form oligomers in a tissue, regional, and species-specific fashion (Rios et al., 2001; Salim et al., 2002). The formation of oligomers alters the function of individual receptors and influences signal amplification. Oligomerisation has therefore been postulated to be a mechanism for increasing the functional repertoire of G-protein coupled receptors (Gouldson et al., 1998). OpRs, as members of the family of GPCRs, also form oligomers. Using time-resolved fluorescence resonance energy transfer (FRET) techniques (Cvejic and Devi, 1997; Levac et al., 2001), DOR has been shown to form homo-oligomers when transfected into HEK 293, CHO, or COS cells. The structural basis for this association remains unclear but may involve the formation of disulfide bonds, hydrophobic interactions, and intracellular coiled-coil interactions (Bouvier, 2001). The presence of the DOR C-tail is reported to be necessary for receptor dimerisation (Cvejic, 1997). Indirect evidence also exists for MOR homodimer formation. In a study of mouse brain tissue, MOR homodimers were observed by protein coimmunoprecipitation techniques (Garzón et al., 1995), and Western blots of electrophoretic extracts of the periaqueductal grey from mouse brain demonstrated the presence of low $(50-58 \mathrm{kD})$ and high molecular weight (100-114 kD) MOR bands. The high molecular weight bands were twice the size of the MOR monomers (Garzón et al., 1995).

Hetero-oligomerisation has been reported between MOR-DOR (George et al., 2000; Gomes et al., 2000; Martin and PL., 2001) and DOR-KOR (Jordan and Devi, 1999; Ramsay et al., 2002) and between DOR and KOR with $\beta_{2}$-adrenoceptors (another GPCR) (Jordan and Devi, 2001; McVey et al., 2001; Ramsay et al., 2002). MOR-1 also forms heterodimers with the GPCR, somatostatin-2a (Pfeiffer et al., 2002). Recently, MOR, 
DOR, and KOR were found to oligomerise with the chemokine receptor CCR5, and the heterodimers were postulated to have a role in modulating immune responses (Suzuki et al., 2002). The DOR-KOR heterodimer displays differences in ligand binding profiles when exposed to the KOR selective agonists dynorphin A and U69593 and the DOR selective agonist DPDPE. In the case of DPDPE, a decreased affinity for the heterodimer was seen relative to the DOR monomer. On the other hand, the non-specific opioid antagonists naloxone and diprenorphine showed an increased affinity for the DOR-KOR heterodimer receptor complex (Jordan and Devi, 1999).

Interactions between MOR and DOR have been postulated for years (Traynor and Elliott, 1993), although direct interactions have only been discovered recently (George et al., 2000). Like the DOR-KOR heterodimer, the $150 \mathrm{kD}$ MOR-DOR heterodimer has novel binding sites compared with the MOR and DOR monomers, showing reduced binding affinity for some agonists (DALDE, DPDPE, DAMGO, and morphine), and increased affinity for some endogenous opioid peptides (endomorphin-1 and leu-enkephalin). Differences in $\mathrm{OpR}$ regulation, desensitisation, and receptor internalisation were also seen with heterodimers (Gomes et al., 2000; Martin and PL., 2001). An intact MOR C-terminal region is not required for MOR-DOR hetero-oligomerisation to occur (Gomes et al., 2000). The functional relevance of $\mathrm{OpR}$ hetero-oligomerisation is still being investigated. MORDOR hetero-oligomers do not closely match any MOR or DOR pharmacological subtype. At present, hetero-oligomerisation has only been seen in vitro, mainly in OpR transfected cell lines that grossly over-express OpRs. It still remains to be established if heterooligomers are present in vivo, although some indirect evidence exists for their formation. For example, in studies using knockout mice, MOR is needed to obtain full DOR-mediated analgesia of the tail withdrawal response (Matthes et al., 1996; Matthes et al., 1998). MOR knockout mice also do not display place preference, a test designed to measure reward and drug seeking behaviour in response to a selective DOR agonist such as deltorphin II (Hutcheson et al., 2001). MOR knockout mice are unresponsive to morphine, including the effects of morphine on pain relief, addiction, and tolerance. The expected functional consequences of OpR knockout are not always observed. The maintenance of normal function in these mice is not a result of increases in expression of the remaining OpRs, since their expression and distribution remain unchanged (Kitchen et al., 1997). For example, OpRs are believed to have a role in developmental maturation and in the modulation of respiration. OpR knockout mice, however, seem to develop and mature 
normally and have normal breathing rhythms. MOR and DOR have some overlapping functions in vivo and act synergistically to increase the response to MOR and DOR agonists (Cahill et al., 2001a; Martin and PL., 2001). Recent investigations have indirectly linked these enhancements of function to heterodimer formation (Levac et al., 2001).

Both MOR and DOR homodimers on their own cause adenylyl cyclase inhibition, MAPK activation via pertussis toxin-sensitive GPCRs, and undergo agonist-dependent internalisation and desensitisation. The MOR-DOR heterodimer, however, is coupled via pertussis toxin-insensitive G-proteins. Inhibition of adenylyl cyclase and MAPK activation in the MOR-DOR heterodimer only occurs if both MOR and DOR selective ligands are added together. In the MOR-DOR heterodimer no desensitisation was seen with the MOR-selective agonist DAMGO or the DOR-selective agonist DPDPE. As stated earlier, the MOR-DOR heterodimer has increased affinity for endomorphim-1 and leuenkephalin. The DOR-KOR heterodimer shows an increased affinity for non-selective agonists. The properties of OpR dimers have been recently reviewed by Levac et al. (2001) (Levac et al., 2001).

\subsection{OPIOID RECEPTOR EXPRESSION}

The anatomical distribution and expression levels of specific OpRs within the brain can provide important clues on the sites of action and functional significance of the different opioid systems within the brain. For example, OpRs located in parts of the limbic system and cerebral cortex have been implicated in modulating the opioid effects of euphoria and drowsiness (Wamsley, 1983); whereas, activation of OpRs within the spinal cord leads to spinal analgesia and desensitisation to pain (Fields and Heinricher, 1985; Gamboa-Esteves et al., 2001). OpRs in brainstem areas modulate cardiovascular and respiratory functions (Denavit-Saubíe et al., 1978; Pasternak et al., 1980; Hassen et al., 1982; Sessle and Henry, 1985; Yeadon and Kitchen, 1989; Shook et al., 1990; Gray et al., 1999; Liu et al., 2000; Moss and Laferrière, 2000; Aicher et al., 2001; Stornetta et al., 2001). These regional distributions show species variations in both receptor expression levels and in the specific sites of localisation within the brain (Yoburn et al., 1991; George et al., 1994). Differences can also be seen in receptor expression and localisation patterns at different stages of development (Beland and Fitzgerald, 2001) and in disease states (Delay-Goyet et al., 1987; Barg et al., 1993b). 
The distribution of OpRs generally matches the pharmacological site of action and production of opioid peptides. For example, met-enkephalin immunoreactivity is found in the medulla (Ceccatelli et al., 1989) and dorsal horn of the rat spinal cord (SpC) where it is colocalised within the same $\mathrm{SpC}$ regions as DOR (Cheng et al., 1995b). Leu-enkephalin is also found in the rat NTS where it is found closely associated with MOR-positive neurons (Cheng et al., 1996). Radioimmunoassay experiments have shown that met-enkephalin, $\beta$ endorphin, and dynorphin are present in brainstem respiratory regions of the piglet (Zhang and Moss, 1995). Met-enkephalin is also present in the NTS and medulla of newborn rabbits (Gingras-Leatherman et al., 1986) and human foetal brain tissue (Yew et al., 1990). These regions all express high levels of both MOR and DOR receptors (Delfs et al., 1994; Arvidsson et al., 1995a; Arvidsson et al., 1995b; Bausch et al., 1995a; Ding et al., 1996a). No single OpR, however, exists in the same brain regions as a single opioid peptide. More often, several opioid peptides are found in the same regions along with multiple OpRs (Loughlin et al., 1985). It is difficult to compare or correlate the distributions of peptides and receptors reported in the literature, because of the differences in techniques used in each study and the fact that opioid peptides can diffuse away from their site of production. Zadinia et al. (1997) showed that endomorphin had a high affinity for MOR (Zadina et al., 1997) and was found in the CNS in regions of high MOR density (Zadina et al., 1999). Both the level of expression and receptor distribution can help investigators gain better insight into opioid functions, interactions, and regulation in vivo since the physiological function of any neuromodulatory system depends on the ratio of active receptors to available ligands.

The distribution of endogenous opioid peptides (Olson et al., 1997; Vaccarino et al., 1999; Vaccarino and Kastin, 2001) and the OpRs they interact with (Xia and Haddad, 1991) are developmentally regulated and spatially distributed in the brain. The anatomical distribution of OpRs in the rodent brain has been examined by radioligand binding in the foetal (Tsang et al., 1982), postnatal (Tsang et al., 1982; Kornblum et al., 1987; Xia and Haddad, 1991), and adult rat (Kornblum et al., 1987; Mansour et al., 1988; Xia and Haddad, 1991; Bunzow et al., 1995), and in the adult mouse (Matthes et al., 1998; Goodman et al., 1999; Chen et al., 2000). Immunocytochemistry (ICC) has been used to investigate OpR populations in the adult rat (Dado et al., 1993; Elde et al., 1995; Ding et 
al., 1996b; Kalyuzhny et al., 1996; Cheng et al., 1997; Gutstein et al., 1998; Schulz et al., 1998; Abbadie et al., 2000b; Abbadie et al., 2000a; Cahill et al., 2001b; Wessendorf and Dooyema, 2001; Abeyta et al., 2002; Wang and Wessendorf, 2002) and adult mouse (Pan et al., 1998; Abbadie et al., 2000b). The ICC studies have been limited, until recently, by the unavailability of suitable antibodies against OpRs (Elde et al., 1995). With the cloning of the OpR genes, mRNA expression has also been examined in various regions of the CNS and SpC of foetal rats (Georges et al., 1998) and mice (Zhu et al., 1998), postnatal rats (Georges et al., 1998; Gulledge et al., 2000), and adult rats (Mansour et al., 1988; George et al., 1994; Maekawa et al., 1994; Zastawny et al., 1994; Mansour et al., 1995; Wittert et al., 1996; Georges et al., 1998; Bouret et al., 2000; Tong et al., 2000; Cahill et al., 2001b; Wang and Pickel, 2001; Wang and Wessendorf, 2002) and adult mice (Pan et al., 1998; Pan et al., 1999). In all these in situ hybridisation studies, radiolabelled, rather than fluorescent probes, were used for visualisation of receptor binding sites. The resolution and specificity of radiolabelled probes are not as good as those obtained with fluorescent or colourimetric antibody or nucleic acid probe labelling techniques.

OpR expression is primarily regulated by transcription. OpR expression levels are controlled by both proximal and distal promoters (Kraus et al., 1995; Andria and Simon, 1999; Liu et al., 1999), and several transcriptional regulatory factors have been discovered (Kraus et al., 2001; Sun and Loh, 2001). For example, DOR is regulated by both transcription promoters and suppressors (Andria, 2001). An example of this is Ets-1, a DOR transcriptional activation factor (Sun and Loh, 2001). Transcriptional regulation is known to vary during development (Ko et al., 2002). OpR expression and spatial distributions in the adult rat brain are summarised in Table 1.2. 
Table 1.2 Distribution of Opioid Receptors in the Brain 


\begin{tabular}{|c|c|c|c|c|c|c|c|c|}
\hline Animal & Rat & Rat & Rat & Mouse & Rat & Rat & RAT & RAT \\
\hline Reference number & 7 & 8 & 7 & 9 & 7 & 4 & \multicolumn{2}{|c|}{ THE PRESENT STUDY } \\
\hline Method & $\mathrm{IHC}$ & $\mathrm{IHC}$ & $\mathrm{IHC}$ & $\mathrm{IHC}$ & mRNA & ARB & $\mathrm{IHC}$ & $\mathrm{HHC}$ \\
\hline Opioid Receptor & DOR & DOR & DOR & DOR & DOR & KOR & MOR & DOR \\
\hline \multicolumn{9}{|l|}{ Telencephalon } \\
\hline olfactory bulb & & & & $\mathrm{P}$ & & & +++ & +++ \\
\hline olfactory tubercle & & & & $\mathrm{P}$ & & +++ & + & + \\
\hline \multicolumn{9}{|l|}{ CORTEX } \\
\hline Ant cingulate & +++ & & ++ & & ++ & & + & + \\
\hline frontal parietal & & & & & & ++ & + & + \\
\hline piriform & & & & $\mathrm{P}$ & & ++ & + & + \\
\hline Striatum & & & & $\mathrm{P}$ & & & ++ & ++ \\
\hline \multicolumn{9}{|l|}{ Nucleus accumbens } \\
\hline HIPPOCAMPUS & & & & $\mathrm{P}$ & & + & + & + \\
\hline CA1 & & & & $P$ & & & & \\
\hline $\mathrm{CA} 2$ & & & & $\mathrm{P}$ & & & & \\
\hline CA3 & & & & $\mathrm{P}$ & & & & \\
\hline Dendate gyrus & & & & $\mathrm{P}$ & & + & & \\
\hline Amygdala & & & & $\mathrm{P}$ & & & & \\
\hline Globus pallidus & & & & & & + & & \\
\hline \multicolumn{9}{|l|}{ Diencephalon } \\
\hline Hypothalamus & & & & $\mathrm{P}$ & & +++ & & \\
\hline THALAMUS & & & & $\mathrm{P}$ & & & & \\
\hline \multicolumn{9}{|l|}{ Anterior group } \\
\hline Medial group & & & & & & ++ & & \\
\hline \multicolumn{9}{|l|}{ Midline group } \\
\hline \multicolumn{9}{|l|}{ Lateral group } \\
\hline \multicolumn{9}{|l|}{ Ventral group } \\
\hline \multicolumn{9}{|l|}{ Lateral geniculate } \\
\hline \multicolumn{9}{|l|}{ Medial geniculate } \\
\hline \multicolumn{9}{|l|}{ Mesencephalon } \\
\hline \begin{tabular}{|l} 
Substantia nigra \\
\end{tabular} & +++ & $\mathrm{P}$ & +++ & & & + & & \\
\hline Vental tegmental area & +++ & & +++ & & & + & & \\
\hline Interpeduncular nucleus & +++ & $\mathrm{P}$ & +++ & & & +++ & & \\
\hline Superior colliculus & ++ & & ++ & & & ++ & & \\
\hline Inferior colliculus & +++ & & +++ & & & ++ & & \\
\hline Periqueductal gray & & $\mathrm{P}$ & & & & ++ & & \\
\hline Cerebellum & & & & $P$ & & & ++ & ++ \\
\hline \multicolumn{9}{|l|}{ Rhombencephalon } \\
\hline \begin{tabular}{|l} 
Pontine reticular nucleus \\
\end{tabular} & ++ & $\mathrm{P}$ & + & & ++++ & & & \\
\hline \begin{tabular}{|l|} 
Locus coeruleus \\
\end{tabular} & 0 & $\mathrm{P}$ & 0 & & 0 & & & \\
\hline Facial motor nucleus & & $\mathrm{P}$ & & $\mathrm{P}$ & & & & \\
\hline Parabrachial nucleus & ++ & $\mathrm{P}$ & ++ & & ++ & ++ & & \\
\hline \begin{tabular}{|l|} 
Gigantocellular nucleus \\
\end{tabular} & +++ & $\mathrm{P}$ & +++ & & +++ & + & + & + \\
\hline \multicolumn{9}{|l|}{ Intermediate reticular nucleus } \\
\hline \multicolumn{9}{|l|}{ Parvocellular reticular nucleus } \\
\hline Medullary reticular nucleus & & & & & & & & \\
\hline Raphe nuclei & + & $\mathrm{P}$ & + & & + & + & + & + \\
\hline \begin{tabular}{|l|} 
Nucleus ambiguus \\
\end{tabular} & & $\mathrm{P}$ & & & & & ++++ & ++++ \\
\hline Nucleus of the solitary tract & & $\mathrm{P}$ & & & & +++ & ++++ & ++++ \\
\hline Gracile & & & & & & & + & + \\
\hline Cuneate & & & & & & & + & + \\
\hline Extemal cuneate & & & & & & & + & + \\
\hline Spinal trigeminal nucleus & ++ & $\mathrm{P}$ & +++ & $\mathrm{P}$ & ++++ & ++ & +++ & +++ \\
\hline Hypogiossal Nuclei & & $\mathrm{P}$ & & $\mathrm{P}$ & & & +++ & +++ \\
\hline \begin{tabular}{|l} 
Vagal nuclei \\
\end{tabular} & & $\mathrm{P}$ & & $\mathrm{P}$ & & & ++ & ++ \\
\hline Inferior olive & & $\mathrm{P}$ & & & & & +++ & +++ \\
\hline Medial reticular formation & ++ & $\mathrm{P}$ & +++ & & +++ & & & \\
\hline Lateral reticular nuclei & & $\mathrm{P}$ & & & & + & ++ & ++ \\
\hline Area postrema & & & & & & & +++ & +++ \\
\hline
\end{tabular}

Data from Table 1-2 has been collected from the following reference; (1) Delfs et al. (1994); (2) Morwaki et al. (1996); (3) Diaz et al. (2000); (4) Mansour et al. (1995); (5) Zastawny et al. (1994); (6) Delay-Goyet et al. (1990); (7) Cahill et al. (2001); (8) Arvidson et al. (1995); (9) Bausch et al (1995); (10) Goody et al. (2002); (11) Ding et al. (1996). 


\subsubsection{OPIOID RECEPTOR COLOCALISATION WITH OTHER RECEPTORS AND NEUROTRANSMITTERS}

OpRs colocalise with opioid peptides, other neurotransmitters, and ion channels (Bausch et al., 1995b; Ding et al., 1995; Gracy et al., 1997b; Gray et al., 1999; Aicher et al., 2000a; Huang et al., 2000; Stasinopoulos et al., 2000; Taki et al., 2000; Barry et al., 2001). Colocalisation in specific brain regions helps to elucidate the specific functions of OpRs in these areas. For example, in the cerebral cortex, olfactory nucleus, nucleus accumbens, substantia nigra, hippocampus, thalamus, locus coeruleus, reticular nucleus, vestibular nucleus, and trigeminal nucleus, MOR is colocalised with $\mathrm{K}^{+}$channels (Bausch et al., 1995b) and acts on postsynaptic sites to inhibit neuronal firing through the opening of these channels (Hampson et al., 2000). Recently, GIRK knockout mice were shown to have impaired morphine-induced analgesia (Ikeda et al., 2002). Knowledge of the spatial distribution and regulatory properties of OpRs may contribute to the development of an opioid analgesic with fewer adverse side effects, provided that opioid peptides can be targeted to specific regions.

MOR is also often colocalised with neurokinin 1 (or substance P) receptors (NK1r) in the dorsal horn of the SpC (Ding et al., 1995; Aicher et al., 2000a) where the dorsal horn receives input from the PAG (Fields and Heinricher, 1985). This input modulates antinociception and plays an important role in opioid-mediated analgesia (Kalyuzhny et al., 1996). Brainstem regions showing colocalisation of NK1r and MOR include the spinal trigeminal tract (STT) (Aicher et al., 2000a), nucleus of the solitary tract (NTS) (Laferrière et al., 2003), and the pre-Bötzinger complex (preBötC) of the brainstem (Gray et al., 1999), the latter a region believed to be responsible for generating respiratory rhythm (for review see)(Rekling and Feldman, 1998; St. John, 1998; Lipski et al., 2002). Usually, NK1r and MOR are colocalised at postsynaptic sites on dendrites. Opioids have also been implicated in the release of substance P from cultured dorsal root ganglion neurons (Suarez-Roca and Maixner, 1995). MOR colocalises with N-methyl-D-aspartate (NMDA)-type glutamate receptors in the rat NTS (Huang et al., 2000) and in other brain regions (Gracy et al., 1997a) and is believed to have a role in the presynaptic modulation of glutamate release in these areas (Aicher et al., 2000b). The colocalisation of MOR with glutamate receptors may be involved in the decrease in arterial pressure and heart rate produced on exposure to opioid ligands (Miyawaki et al., 2002). In the cerebral cortex of the rat, MOR is expressed on GABAergic neurons (Taki et al., 2000), and MOR is present in the NTS in areas where 
acetylcholinesterase-positive neurons are located (Barry et al., 2001). In the rostral ventrolateral medulla (RVLM) of the rat, DOR immunoreactive boutons appose catecholaminergic neurons (Stasinopoulos et al., 2000).

In addition to colocalising with other receptors and neurotransmitters, OpRs also colocalise with sites of opioid peptide synthesis in many brain regions. In the periaqueductal gray (PAG) of the rat $\mathrm{SpC}, \mathrm{DOR}$ is present in enkephalin-positive neurons (Commons et al,, 2001) and is postulated to act as a presynaptic autoreceptor. In the caudate putaman nucleus of the basal ganglion, MOR colocalises with leu-enkephalin in both axons and dendrites (Wang et al., 1996). In the cerebral cortex, it colocalises with preproenkephalinproducing neurons (Taki et al., 2000).

\subsubsection{DEVELOPMENTAL OPIOID RECEPTOR EXPRESSION AND DISTRIBUTION}

OpRs are expressed in different regions of the brain and at different expression levels during growth and maturation. Opioid receptors appear first in the brainstem and later in the striatum, midbrain and cortex (Coyle and Pert, 1976). Studies of OpR expression in the foetus and early postnatal rodent have shown that MOR and KOR first appear around E1 1 to E15. DOR appears later, not normally being detected until early postnatal development (Spain et al., 1985; Tavani et al., 1985; Petrillo et al., 1987), although in one study in the mouse, DOR was first detected at E13 (Zhu et al., 1998). Detection of OpR expression varies with the sensitivity of the techniques used (Tsang et al., 1982; Villiger et al., 1982; Kornblum et al., 1987; Attali et al., 1990; Xia and Haddad, 1991; Zhu et al., 1998). More recent techniques that make use of specific antibody binding and nucleic acid hybridisation are more sensitive and can pick up lower expression levels than radioligand binding techniques. These distinct and repeatable ontogenic profiles of OpR expression in different brain regions suggest that opioids are important in the development of neural pathways. A naloxone-reversible $\beta$-endorphin effect on circulating growth hormone has been described in the foetal ovine brain, suggesting the presence of functional opioid receptors in the foetus that control release of growth hormone (Gluckman et al., 1980). Opioids are expressed in the CNS of all mammals at birth, and administration of opioid agonists in neonates causes marked effects on respiratory and cardiovascular activities (Moss et al., 1993a, b; Murphey and Olsen, 1994; Greer et al., 1995; Takita et al., 1998; Colman and Miller, 2001; Colman and Miller, 2002). This suggests that OpR modulation 
is present and functional at birth. Opioid agonist exposure before birth has important implications in cardiorespiratory function with regard to the increased risk of respiratory arrest in newborns (Chavez et al., 1979; Kandall et al., 1993). Variations in OpR sensitivities to particular agonists and antagonists occur during development, especially in the first few weeks after birth (Spain et al., 1985). These variations in sensitivity may be related to OpR expression levels and neuronal maturation of brainstem nuclei. The detailed effect that opioids have on respiration during development is discussed later in this chapter.

\subsubsection{DEVELOPMENT OF THE RAT BRAINSTEM}

At birth, the rat brain is very similar in morphology to that of the adult rat, and nearly all structures can be recognised from a familiarity with the adult rat brain. In the foetal brain, however, fewer nuclei are distinguishable, and the boundaries of the nuclei are less distinguishable in stained sections. Figure 1.4 compares brainstem structures of the rat at E16 with the adult (Paxinos and Watson, 1986; Paxinos et al., 1994). In the medullary raphe nucleus, a region that shows OpR expression in the adult (Delfs et al., 1994; Mansour et al., 1995; Moriwaki et al., 1996; Cahill et al., 2001b; Díaz et al., 2002), 5-HTpositive neurons synthesise neurotransmitter at E13 and receive synaptic contacts by E20 (Lidov and Molliver, 1982). At E12, the posterior neuropore closes and several brainstem regions, including the reticular formation and medullary regions, send projections to the SpC (de Boer-van Huizen and ten Donkelaar, 1999). In intact brainstem preparations from E12 foetuses, spontaneous action potentials can be detected in the medial and lateral brainstem regions. These action potentials are believed to arise from motorneuronal responses in the dorsal motor nucleus of the vagus and sensory nerve inputs from the NTS (Momose-Sato et al., 2001). 


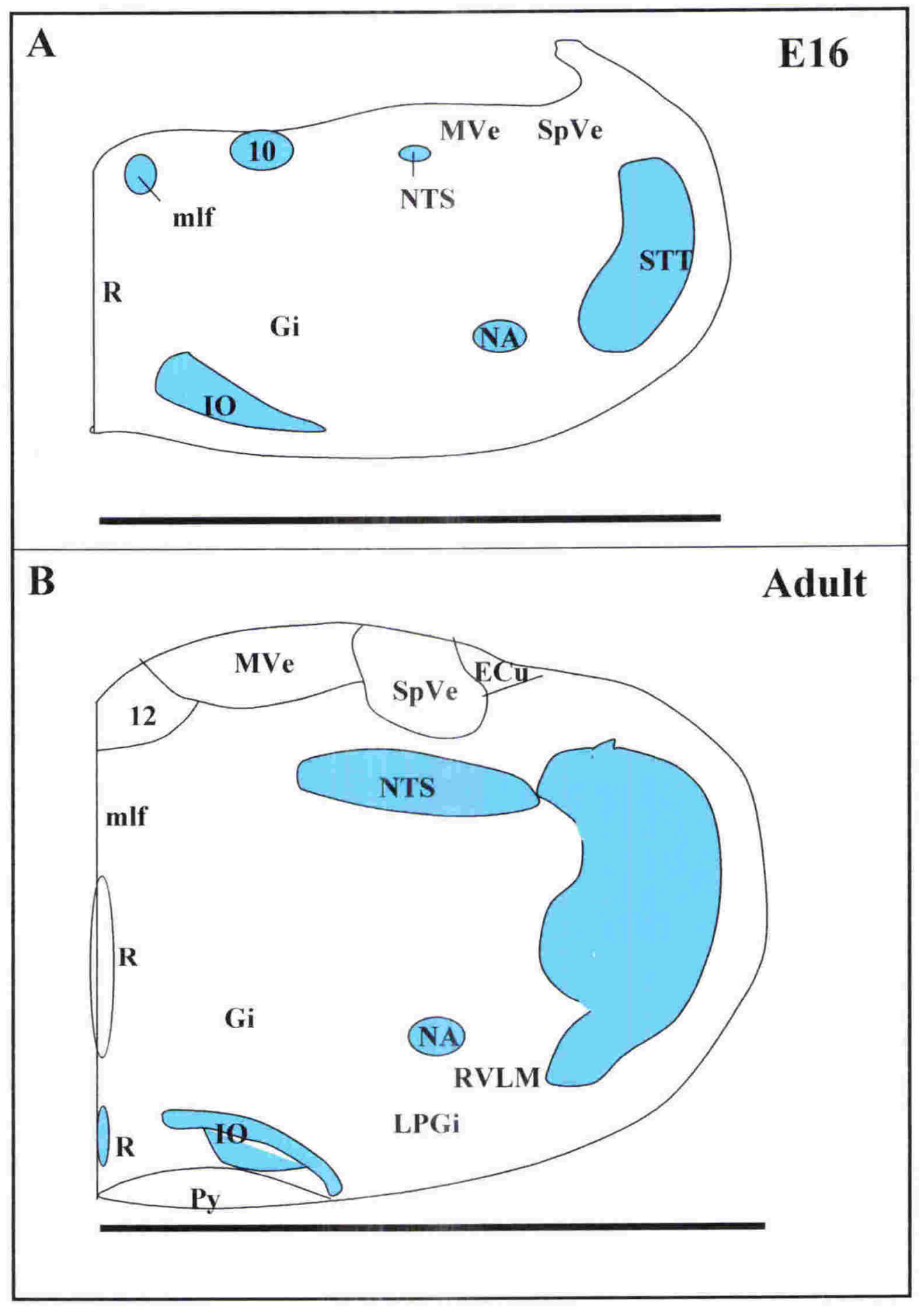

\section{Figure 1.4}

\section{Anatomy of brainstem nuclei}

Schematic pictures of E16 (A), and adult (B) coronal sections of the rat brainstem, showing locations of specific brainstem regions. Scale Bars $=1.3 \mathrm{~mm}(\mathrm{~A})$ and $4 \mathrm{~mm}$ (B). Abbreviations: nucleus of the solitary tract (NTS); dorsal motor nucleus of the vagus (12); medial longitudinal fasiculus (mlf); raphe nucleus (R); gigantocellular reticular nuclei (Gi); pyramidal tract (Py); inferior olive ( $\mathrm{IO})$; lateral paragigantocellular nuclei (LPGi); spinal trigeminal tract and nuclei (STT); nucleus ambiguus (NA); spinal vestibular nuclei (SpVe); external cuneate nucleus (ECu); rostral ventrolateral medulla (RVLM); and medial vestibular nucleus (MVe). 
Most neurons in the NTS and vagal nucleus undergo their final mitotic division between E10 and E15 (Altman and Bayer, 1980; Phelps et al., 1990; Lakke, 1997) with a peak at E13; whereas, neurons in the NA finish dividing at E15. The acquisition of neuronal properties during development is described by Momose-Sato et al. (2001) (Momose-Sato et al., 2001). As E13 and E14 neurons mature, more multipolar cells appear and the cells become larger in size (de Boer-van Huizen and ten Donkelaar, 1999). At developmental age E15 in the rat, the neural tube undergoes a marked increase in thickness, especially in the rostral brainstem, and neural migration is apparent. At E15, several nuclei are easily identifiable, including the hypoglossal and trigeminal nuclei. There is also a marked daily increase in cell volume and cell number. At E16, more brainstem nuclei can be identified in the spinal trigeminal tract and nucleus (STT), inferior olive, NA, NTS, and facial nucleus, although the characteristic shapes and boundaries of these brainstem nuclei do not become apparent until E19 (Lidov and Molliver, 1982). The caudal medullary regions are more highly innervated early in development than the more rostral brainstem regions, such as the pons, although the STT and reticular areas appear to be devoid of terminals at E16 (Lidov and Molliver, 1982).

In late embryonic development there is an increase in growth of neuronal processes. Brainstem projections can be seen in the lumbar $\mathrm{SpC}$ of the $\mathrm{E} 17$ to $\mathrm{E} 21$ rat, emanating from many brainstem nuclei, including the NA and NTS (Lakke, 1997). After E19, there is an increase in dendritic growth and a decrease in cellular packing density (Lidov and Molliver, 1982). In the neonate there are postnatal changes in neurotransmitter and receptor densities that influence neuronal functions (Bayliss et al., 1997; Moss and Laferrière, 2002). At birth it is necessary for many brainstem nuclei to be functional for survival, including the brainstem nuclei responsible for breathing (Mitchel and Berger, 1975), swallowing, and suckling (Sessle, 1973; Nakamura et al., 1999). The rat at birth is relatively immature; hence, other functions such as eye and limb movements develop later in postnatal development (Vinay et al., 2000; Freeman and Nicholson, 2001). The development and maturation of breathing and the respiratory system will be discussed later in this chapter. 


\subsubsection{FUNCTION OF OPIOIDS IN THE BRAINSTEM}

The brainstem has an important role in the integration and control of numerous essential physiological functions, including control of the somatic and autonomic nervous systems. These functions are modulated by endogenous opioid peptides but can also be affected by administration of exogenous opioids (Denavit-Saubíe et al., 1978; Harlan et al., 1987). Brainstem functions that are affected by the opioid system include the generation and modulation of respiratory movements and chemoreception, cardiovascular activity, gastrointestinal tract motility and digestion, transmission of pain, modulation of arousal and consciousness, modulation of sensory motor pathways, control of reflexes mediated by cranial nerves, taste, hearing and maintenance of balance, temperature regulation, and swallowing and control of the face and neck muscles (Sessle, 1973; Feuerstein, 1985; Fields and Heinricher, 1985; Punnen and Sapuru, 1986; Adler and Geller, 1988; Wang and Li, 1988; Shook et al., 1990; Wind and Kuhin, 1995; Szilagyi, 1998; Llewellyn-Smith, 2000; Tershner and Helmstetter, 2000; Vanderah et al., 2001). For reviews on the functional role of opioids in these physiological systems, see Vaccarino (1999; 2001) and Olson (1995; 1997) (Olsen et al., 1995; Olson et al., 1997; Vaccarino et al., 1999; Vaccarino and Kastin, 2001).

Studying brainstem areas that express OpRs helps in the understanding of the functional implications of these receptors and their peptides and provides a rationale for the differential distribution of receptors and their peptides in different brain regions. Further information on the role OpRs play in regulating function can be gained from genetically modified mice that lack specific OpRs (knockout mice). Genes encoding all the components of the opioid system have been disrupted in mice by homologous recombination (Matthes et al., 1998; Kieffer, 1999; Chen et al., 2000; Kieffer and Gavériaux-Ruff, 2002). All these mutated mice, including double-knockouts as well, reach adulthood with no obvious abnormalities, suggesting that OpRs are not essential for normal development. It is possible that redundancy is built into opioid functions in vivo, such that the action of one receptor type can be taken over by another. As described earlier, in the single-knockout mouse, the remaining $\mathrm{OpR}$ expression levels remain unchanged, suggesting that the absence of one OpR is not compensated for by an increase in expression levels of other OpR types (Kitchen et al., 1997; Simonin et al., 2001). 
Increased receptor coupling of the other OpRs (Matthes et al., 1998), or enhanced ligand expression levels, also appear not to be involved (Matthes et al., 1996).

Respiratory function in mice lacking MOR showed no significant differences in respiratory rhythm generation, ventilatory pattern, or chemosensitivity control when compared with wild-type mice (Matthes et al., 1998; Morin-Surun et al., 2001). These results suggest that MOR does not play a direct or essential role in the development and maturation of the neural respiratory pathways. Respiratory parameters, however, have not been evaluated in DOR or KOR knockouts or in double-knockouts. Another possibility is that gene knockout can lead to a compensatory upregulation of other genes that can maintain the function normally associated with the gene of interest. MOR and KOR action are known to influence analgesia, and, as expected, both MOR and KOR single-knockout mice show a reduced threshold to pain (Matthes et al., 1996; Matthes et al., 1998; Park et al., 2001). In a study of MOR knockout mice, Matthes et al. (1996) found that morphine was unable to induce analgesia, reward, or physical dependence (Matthes et al., 1996). The effects of morphine on respiratory depression and immunosuppression were also absent in these mice, demonstrating that these effects of morphine are encoded for by the MOR gene (Matthes et al., 1996). In these same mice, it was also shown that the response of mice to KOR agonist-induced analgesia and KOR adenylyl cyclase inhibition were maintained in the MOR knockout mouse. Reduced DOR analgesia and the absence of DOR-mediated respiratory depression, however, were observed in the MOR knockout mouse, suggesting a link between the actions of MOR and DOR; whereas, MOR and KOR act independently of each other in vivo (Matthes et al., 1998).

Brainstem areas with high expression patterns for both MOR and DOR include the NTS (Atweh and Kuhar, 1977; Mansour et al., 1994; Mansour et al., 1995; Cheng et al., 1996; Gamboa-Esteves et al., 2001), NA (Atweh and Kuhar, 1977; Nomura et al., 1996; Stasinopoulos et al., 2000), rostral ventrolateral medulla (RVLM) (Gray et al., 1999; Stasinopoulos et al., 2000; Aicher et al., 2001; Guyenet et al., 2002; Wang and Wessendorf, 2002), and STT (Kandel et al., 2000; Stasinopoulos et al., 2000), as described earlier in this chapter and summarised in Table 1.2. The functions of these brainstem regions and the implications of $\mathrm{OpR}$ localisation are discussed below, with emphasis on opioid systems involved in cardiorespiratory modulation and pain control. The SpC shows high levels of MOR and DOR in the dorsal horn (Cheng et al., 1997), which sends 
projections to brainstem areas. The outer layer of the STT contains fibre tracts that are a continuation of the superficial laminae of the dorsal horn extended into the medulla. The spinal trigeminal nucleus receives sensory fibres from all of the cranial nerves of the head, including the facial nerve, glossopharyngeal nerve, and vagal nerve (Kandel et al., 2000), and in these areas opioids may be involved in modulation of such functions as taste, tongue movements and swallowing (Sessel et al., 1981; Sessle and Henry, 1989).

\subsubsection{Opioid Receptor Function in the RVLM and NA}

The RVLM is located around the NA and contains a pool of mixed motor neurons that have functions in respiration, vocalisation, and vomiting. This brainstem region contains the decrementing expiratory neurons of the Bötzinger complex (BötC). When these neurons are labelled with neurobiotin in the ventral respiratory group of the rat, they show axonal projections to other respiratory areas in the medulla and SpC (Saito et al., 2002), supporting the findings of previous electrophysiological and morphological studies (Bryant et al., 1993). The central control of breathing has been extensively reviewed (Mitchel and Berger, 1975; Loeschcke, 1982; Yeadon and Kitchen, 1989; Bianchi et al., 1995; Bonham, 1995; Rekling and Feldman, 1998; St. John, 1998; Butera et al., 1999; Ballantyne and Scheid, 2000; Lipski et al., 2002). Opiates like morphine, commonly used in the relief of pain, often cause nausea and vomiting as unwanted side effects (Olson et al., 1997; Vaccarino et al., 1999; Vaccarino and Kastin, 2001), and it is possible these opioid effects are modulated by neurons in these brainstem areas. The NA contains motorneurons that innervate pharyngeal muscles through branches of the vagus nerve and the larynx via laryngeal nerves (Iscoe, 1988); whereas, hypoglossal motorneurons are involved in tongue protrusion during inspiration (Iscoe, 1988). Axons from the NA motorneurons typically run dorsally toward the vagus nerve (No. 10) and hypoglossal nerve (No. 12) before turning and heading ventrally (Saito et al., 2002). The vagus and hypoglossal nuclei in the brainstem express MOR (Delfs et al., 1994; Nomura et al., 1996) and DOR (Arvidsson et al., 1995a; Bausch et al., 1995a) and may be a site for opioid effects on swallowing (Sessle and Henry, 1989).

\subsubsection{Function of Opioids in the NTS}

The NTS shows marked opioid peptide (Ceccatelli et al., 1992; Cheng et al., 1996) and OpR immunoreactivity (Mansour et al., 1987, 1988; Ceccatelli et al., 1989; Cheng et al., 
1996). The NTS is part of the dorsal respiratory group and receives projections from spinal cord fibers of the dorsal horn (Gamboa-Esteves et al., 2001). The NTS is a sensory nucleus dedicated to processing visceral input from all the major organs, including the heart, lungs, and abdominal visera (Lawrence and Jarrott, 1996). The peripheral afferent information from baroreceptors and chemoreceptors, together with inputs from higher brain centers, is integrated in the NTS, and the final reflex response is determined and directed to medullary neurons that regulate sympathetic and parasympathetic outflow. The NTS is also involved in descending pain control via neurons that send projections to the NA (Lewis, 1998). OpRs in the NTS affect cardiopulmonary, gastrointestinal, and cortical arousal activities (Cheng et al., 1996), and these functions are mainly modulated via the MOR receptor (Ruegg et al., 1994). In the NTS, immunogold-labelling experiments showed that MOR was present in both presynaptic vagal afferent axon terminals and postsynaptic dendrites but was rarely found in both sites in the same neuron (Aicher et al., 2000b). These results suggest that MOR ligands modulate either the presynaptic release from or the postsynaptic response to vagal afferents in the NTS. The vagal afferents are likely to include the inhibitory neurons and gastric and cardiorespiratory afferents that terminate in regions of the NTS that have MOR immunoreactivity (Cheng et al., 1996). Electron microscope studies indicate that MOR is colocalised with NMDA receptors in the rostral NTS where MOR acts postsynaptically to modulate excitation via these NMDA receptors (Huang et al., 2000). In the intermediate NTS, MOR activation inhibits presynaptic release of glutamate from visceral afferents, thus, reducing neuron excitability (Huang et al., 2000). Opioid administration reduces the urge to cough (Rutherford et al., 2002), causes vomiting (Flórez and Hurlé, 1993), and influences taste and feeding responses in rats via MOR activation in the NTS (Kotz et al., 1997). These opioid effects possibly occur via sensory fibres in the throat that terminate in the NTS, as well as via connections to the vomiting centre in the hypothalamus.

\subsubsection{EFFECTS OF OPIOIDS ON THE CARDIOVASCULAR SYSTEM}

\subsubsection{Cardiovascular Effects of Opioids in the RVLM and NA}

The opioid signalling system in the brainstem plays a role in the medullospinal network that controls sympathetic tone and arterial pressure. In the RVLM, this occurs via both pre- and postsynaptic modulation of neurons on exposure to MOR agonists (Huang et al., 2000), and presynaptic modulations on exposure to DOR agonists (Guyenet et al., 2002). 
The presynaptic OpR effects are inhibitory, causing a reduction in glutamate release that leads to reduced neuronal firing. DOR activation in the RVLM modulates cardiovascular reflexes by inhibiting neurotransmitter release from axon terminals of neurons that synapse with presympathetic neurons. Microinjection of various opioid receptor agonists into the RVLM produces hypotension (Punnen and Sapuru, 1986), and the overall action of opioids in this area is to inhibit presynaptic sites, causing a fall in blood pressure (Feuerstein, 1985; Punnen and Sapuru, 1986; Miyawaki et al., 2002; Pugsley, 2002). A recent study by Miyawaki et al. (2002) has shown that microinjection of a MOR-selective agonist (DAMGO) into the RVLM selectively blocks baroreceptor reflexes. DOR activation with a selective agonist (DPDPE) in some cells inhibits respiratory-related neuronal burst patterns; whereas, no effect on baroreceptor or chemoreceptor reflexes was seen in other cells (Miyawaki et al., 2002). These results suggest that opioid peptides in the RVLM act on distinct OpR populations at presynaptic sites.

\subsubsection{Cardiovascular Effects of Opioids in the NTS}

Opioid peptides in the NTS mediate complex cardiovascular events. Almost all visceral afferent projections that relay information to the brain about the status of peripheral cardiovascular functions terminate in the NTS (Brody, 1986). Met-enkephalin, when injected into the NTS, causes bradycardia and a decrease in blood pressure (Rabkin, 1991). In this brainstem region there seems to be opposing actions of KOR and MOR on the control of the baroreceptor heart rate reflex when selective agonists are microinjected into the NTS. Agonist activation of DOR causes a vagus-dependent bradycardia (Randich et al., 1992); whereas, the MOR selective agonist, DAMGO, when injected into the NTS produces hypertension and tachycardia (Hassen et al., 1982). In whole cell patch clamp experiments, MOR agonists were shown to cause hyperpolarisation of NTS neurons by acting at presynaptic sites, leading to a depression of glutamate-mediated excitatory postsynaptic potentials (Rhim et al., 1993)(for review see) (Lawrence and Jarrott, 1996).

\subsubsection{EFFECT OF OPIOIDS ON RESPIRATION}

OpRs are highly expressed in certain brainstem regions responsible for the generation and/or modulation of respiratory rhythm (for review, see (Bianchi et al., 1995)). Exogenous administration of opioids directly affects the rate of respiration in all mammals studied [Gray, 1999 \#321; Liu, 2000 \#567; Moss, 2000 \#568; Shook, 1990 \#743; (Denavit- 
Saubíe et al., 1978; Ling et al., 1985; Sessle and Henry, 1985; Neubauer et al., 1987; Yeadon and Kitchen, 1989; Paakkari et al., 1990; Moss et al., 1993a, b; Murphey and Olsen, 1994; Kato, 1998; Laferrière et al., 1999; Colman and Miller, 2001; Morin-Surun et al., 2001). The specific effects opioids have on respiration depended on the OpR subtype activated (Yeadon and Kitchen, 1989). KORs appear to be devoid of respiratory depressant activity, DOR exerts modest respiratory depressant effects, and MOR exerts a strong inhibitory influence on ventilation. Studies have shown that administration of opioids in neonatal, juvenile and adult rats causes a decrease in the rate of respiration and a fall in tidal volume via activation of both $\mu_{1}$ and $\mu_{2}$ receptors (Colman and Miller, 2001).

\subsubsection{Respiratory Rhythm Generation}

The pons and medulla of the brainstem contains several areas with respiratory function. The medullary respiratory regions can be further divided into the dorsal respiratory group (DRG) and ventral respiratory group (VRG). For a review, see (St. John, 1998). The DRG group is made up of the ventrolateral NTS and adjacent parts of the reticular formation including the nuclei of the cranial nerves and contains neurons that display an inspiratory discharge pattern. The VRG, shown in sagittal section in Figure 1.5, is made up of the NA, RVLM, preBötC, BötC, and facial nuclei and contains neurons with mixed discharge patterns (St. John, 1996). The BötC is a group of neurons located near the rostral end of the NA in the VRG, and motorneurons and interneurons within this region are active during expiration (Bryant et al., 1993). The preBötC is located in the ventrolateral medulla (VLM) caudal to the BötC and is the postulated site of the respiratory rhythm generator in mammals (Smith et al., 1991). Others, however, suggest that the discharge pattern from this area more closely resembles gasping (St. John, 1996, 1998). An alternative theory of respiratory rhythm generation, the network theory, suggests that a network of interconnecting, pre-sympathetic RVLM neurons are involved. It is possible that a combination of these two systems may generate the respiratory rhythm. Respiratory rhythm generation has been reviewed by Funk and Feldman (1995) and Lipski et al. (2002) (Funk and Feldman, 1995; Lipski et al., 2002). A recent study by Del Negro et al. (2002) shows that abolishing pacemaker neurons with low doses (less than $20 \mu \mathrm{M}$ ) of riluzole (a sodium channel antagonist used to abolish bursting activity) has no effect on the respiratory rhythm in neonatal slice preparations of the mouse and rat, thus, suggesting an emergent network property (Del Negro et al., 2002). The preBötC, in tissue slice 
preparations taken from neonatal rats, generates respiratory-related motor nerve output (Smith et al., 1991). Excitatory interneurons also show intrinsic pacemaker-like properties (Rekling and Feldman, 1998). Respiratory related areas, especially the preBötC, do not have precise anatomical boundaries. A study by Gray et al. (1999) reported that preBötC neurons responsible for rhythmogenesis were immunoreactive for both MOR and neurokinin-1 receptors (NK1r). This finding has helped to anatomically define the location of the preBötC (Gray et al., 1999). MOR agonists applied to the preBötC in tissue slice preparations cause a decrease in respiratory frequency (Johnson et al., 1996) by hyperpolarising the membrane via an increase in $\mathrm{K}^{+}$conductance (Gray et al., 1999) in a similar way to that observed in the intact animal (Bonham, 1995). MOR and NK1r positive neurons in the preBötC are depolarised by substance $\mathrm{P}$ and thyrotrophic hormone (Rekling et al., 1996) and hyperpolarized by MOR agonists such as DAMGO. These neurons are believed to be responsible for both rhythm generation and respiratory frequency control (Rekling and Feldman, 1998).

\subsubsection{Modulation of Respiration}

The endogenous opioid peptides, enkephalin and dynorphin, have been implicated in central respiratory control (Rutherfurd and Gundlach, 1993). The NTS is part of the dorsal respiratory group (DRG), which has for a long time been implicated in respiration. Different types of respiratory neurons in this region have been classified according to their firing properties (Berger, 1977; Berger et al., 1984). Discrete areas within the brainstem are linked via neural processes, and modulation of neurons in one area transmits signals to modulate neuronal activity in other areas. This is especially apparent in the brainstem areas involved in respiratory and cardiovascular control. In the rat, inspiratory neurons in the VRG project to the NTS (Lipski et al., 1994). Respiratory related neurons in the NA receive chemoreceptor inputs from other VRG nuclei, the NTS, and the caudal raphe nucleus (Smith et al., 1989; Lewis, 1998; Wang et al., 1998), and have been implicated in NA-mediated respiratory modulation. Both the NTS and the raphe nuclei also provide OpR inputs to the RVLM (Stasinopoulos et al., 2000).

The primary muscle of inspiration is the diaphragm, a flat sheet of muscle that forms the lower boundary of the thorax and is innervated by the phrenic nerves. Motorneurons projecting to muscles of the chest wall and abdomen are located in the $\mathrm{SpC}$ and are 
controlled by respiratory centers in the brainstem. Ventilatory activity can be modulated in multiple regions of the brainstem. One group of regions having neurons with respiratorymodulated discharge patterns are the nuclei of the cranial nerves V, VII, IX, X, and XII. These cranial nerves innervate muscles of the upper airway and larynx (for review see) (Iscoe, 1988). Some of the cranial nuclei, as mentioned above, also express OpRs, including the facial nuclei (VII), vagus (X) and hypoglossal nuclei (XII) (Delfs et al., 1994; Arvidsson et al., 1995a; Bausch et al., 1995a), thus, providing more putative locations for possible OpR modulation of brainstem function. 


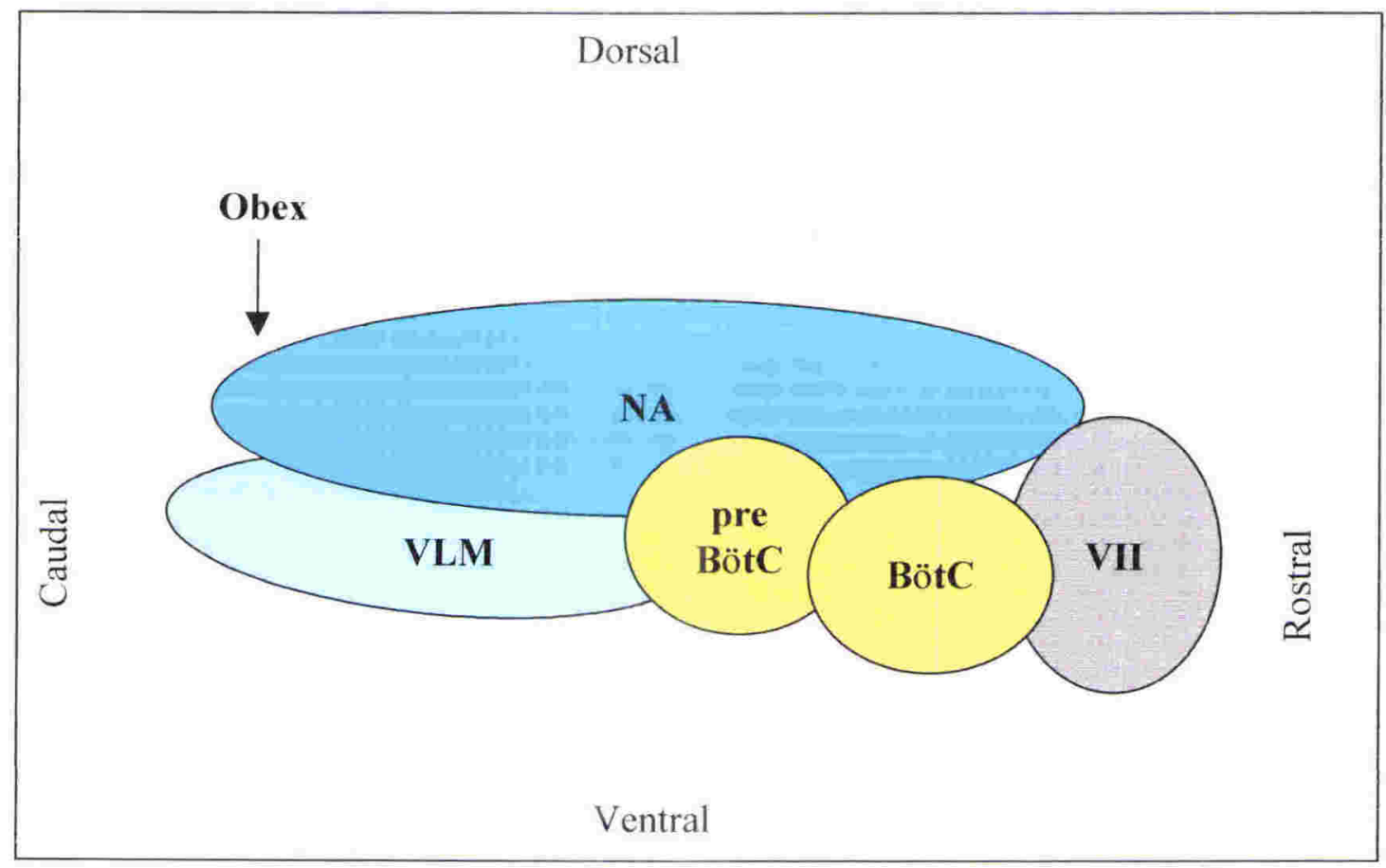

Figure 1.5

\section{Brainstem Respiratory Centers of the Ventral Respiratory Group}

Schematic diagram of the rat brainstem in sagittal section, showing the location of respiratory areas. Modified from Gray et al. (1999). 


\subsubsection{Chemosensitivity}

The respiratory system is extremely responsive to acid/base changes in the plasma and cerebrospinal fluid (CSF). Chemosensitivity arises at the cellular level from the activity of specialised glomus cells in the carotid body and via central chemoreceptors located within brainstem nuclei and in the ventral floor of the medulla. These peripheral and central chemoreceptors can communicate with each other. For example, efferent fibres in the carotid nerve innervate glomus cells within the carotid body, and sensory afferents synapse with relay neurons in the NTS and nuclei within the VRG (Finley and Katz, 1992). In vitro experiments using brainstem slices have identified neurons within many brainstem nuclei that increase their firing rate when exposed to acidosis. These neurons are found in the NTS, locus coeruleus, medullary raphe nucleus, and the NA (Rigatto et al., 1992). This arrangement suggests that central respiratory chemoreception is a distributed property of the respiratory network; although, in some cases, $\mathrm{CO}_{2}$ changes needed to elicit a response have been outside the concentration range expected to occur in vivo (Wang et al., 1998). Chemosensitive neurons show unique morphologies, with neurons stimulated by $\mathrm{CO}_{2}$ having large multipolar somas, whereas, $\mathrm{CO}_{2}$-inhibited neurons having small fusiform somas. Only neurons in a limited number of brainstem regions respond to the small changes in $\mathrm{CO}_{2}$ that typically occur under physiological conditions. These findings suggest that not all chemoreceptors play an equal role in respiratory chemoreception (Wang et al., 1998).

Medullary raphe nuclei contain putative central respiratory chemoreceptors and also express OpRs (Delfs et al., 1994; Mansour et al., 1994; Moriwaki et al., 1996; Díaz et al., 2000; Cahill et al., 2001b). Medullary raphe neurons project to several respiratory nuclei, including the NTS, NA, and RVLM, and all these regions express MOR and DOR. It seems likely, therefore, that OpRs modulate the chemosensitivity of neurons within the brainstem. Abnormalities in the human homologue of the medullary raphe have been reported in infants who died of sudden infant death syndrome (Kinney et al., 1995). These findings have been linked to a decrease in respiratory drive and/or arousal in response to a hypercapnic stimulation during sleep. A decrease in respiratory drive and response to hypoxia is also a characteristic of OpR activation, suggesting a possible link between the two systems. For example, OpR activation by morphine in awake, unrestrained rats causes 
a decrease in sensitivity of the $\mathrm{CO}_{2}$ controller. This was observed as a blunted respiratory response to a hypercapnic stimulus (Van Den Hoogen and Colpaert, 1996).

In the rostral pons and medulla oblongata, MOR activation causes respiratory depression by decreasing chemoreceptor sensitivity to hypercapnia (Murphey and Olsen, 1994). Long-term exposure to hypoxia can also cause alterations in $\mathrm{OpR}$ expression patterns. For example, rats exposed to hypoxia for 7 days show decreased DOR expression in the brain. This finding suggests that DOR agonists play a protective role in chronic hypoxia (Mayfield et al., 1996). A significant role for endogenous opioids in the modulation of respiration is also supported by studies in the piglet by Moss et al. (1981) who showed that $\beta$-endorphin caused a naloxone-reversible decrease in respiratory drive and reduces the sensitivity of breathing to hypercapnic stimuli (Moss and Scarpelli, 1981).

\subsubsection{EFFECT OF OPIOIDS DURING DEVELOPMENT}

The respiratory neural network in the brainstem undergoes maturation after birth (Funk and Feldman, 1995), but the circuitry for generating respiration is established before birth (St. John, 1998). Spinal and brainstem motorneurons are coupled in the NA in early development of the newborn mouse (Rekling and Feldman, 1997) in a similar way to the adult, suggesting that this circuitry develops early.

Centrally administered morphine at low doses stimulates, and at high doses decreases, foetal breathing movements (Szeto et al., 1988). At birth, breathing must become continuous, and several mechanisms have been postulated to be involved in this process, including the loss of a placental inhibitor of respiration at birth or a sudden alteration in blood/gas levels. The individual importance of these two factors remains controversial (Rigatto, 1996; Kuipers et al., 1997). Respiratory output patterns are irregular at birth, and regular rhythmicity is not established until days or weeks after birth, depending on the species under investigation (Mortola, 1984).

Both MOR and DOR help maintain normal foetal (Cheng et al., 1993a) and neonatal (Grunstein and Grunstein, 1982) breathing movements, although the particular receptor sub-types involved in stimulatory and depressant effects are controversial (Moss anc Scarpelli, 1979; Cheng et al., 1991; Cheng et al., 1992; Cheng et al., 1993b). In neonates 
opioids are associated with respiratory depression, mainly via MOR, although some DOR modulation has also been reported, similarly to that seen in adult animals. For example, in conscious neonatal rabbits the DOR agonist DALDE reduces ventilation (Grunstein and Grunstein, 1982). Similar DOR effects were shown in conscious neonatal rats in one study (Greer et al., 1995) but were not seen in conscious neonatal rats in another study (Atalie Colman, unpublished observations). MOR respiratory effects in young mammals are more clearly established. In conscious neonatal dogs, the MOR-selective agonist fentanyl caused a decrease in the frequency of breathing (Bragg et al., 1995). In conscious, unanaesthetised, neonatal rats, fentanyl administration led to a frequency change that resembled a gasping response, and this change was shown to involve $\mu_{1}$ receptors; whereas, a general reduction in tidal volume occurred via $\mu_{2}$ receptors. A blunted response to hypoxia was also seen in conscious rat pups (Colman and Miller, 2002). The OpR antagonist, naloxone, when administered to conscious neonatal rats, caused an enhancement in breathing at rest (Hazinski et al., 1981); whereas, the DOR-specific antagonist, naltrindole, had no effect in neonatal piglets (Moss et al., 1993a), suggesting that endogenous MOR-selective agonists are modulating breathing shortly after birth in the pig.

The magnitude of the effects of opioids on respiration is increased during hypoxia. This was demonstrated in a study by Moss et al. (1993), who showed that naltrexone enhanced the respiratory response to hypoxia in piglets (Moss et al., 1993a). Endogenous opioid levels, both in the CNS and peripherally, are increased in neonates exposed to hypoxia (Moss and Inman, 1989; Armstead, 1995; Yan et al., 1995). Long-term hypoxic treatment (7 days) of adult mice also alters OpR expression levels. DOR expression (but not MOR or KOR) decreased by down-regulation of receptors as a result of release of endogenous DOR agonists (Mayfield et al., 1996). Evidence has accumulated that opioids have a major role in breathing during hypoxia during neonatal development. This hypoxic modulation presumably occurs via the OpR-expressing regions of the DRG and VRG in a manner similar to adults. OpR expression changes during development, and modulation of OpR functions during development may reflect these changes. Exposure to morphine during foetal or early neonatal rat development causes a decrease in OpR expression in the brain. Tolerance to the analgesic effects of morphine appears similarly to that seen in the 
adult, suggesting that the mechanisms for OpR down-regulation are present and functional at birth (Temple et al., 1988).

\subsection{ROLE OF OPIOIDS IN HEALTH AND DISEASE}

\subsubsection{RESPIRATORY RELATED DISEASES}

The effects of opioids on the respiratory system, although known for a long time, are only currently being linked to specific physiological responses and pathological conditions. As stated above, endogenous opioid peptides are involved in the modulation of normal breathing (Yeadon and Kitchen, 1989; Shook et al., 1990; Morin-Surun et al., 1992; Flórez and Hurlé, 1993) but have also been linked to stress responses in diseases such as sudden infant death syndrome (SIDS) (Chavez et al., 1979; McMillen, 1986; Coqueral et al., 1992; Storm et al., 1994), sleep apnoea (Ferber et al., 1988; Myer et al., 1990; Shook et al., 1990), asthma (Rogers and Barnes, 1989), and other chronic obstructive pulmonary disorders (Flórez and Hurlé, 1993). Opioids have also been studied in relation to cardiovascular diseases; in particular, haemorrhage (Bedell et al., 1998; Vaccarino et al., 1999; Cavun et al., 2001; Henderson et al., 2002).

\subsubsection{SIDS}

The risk of SIDS increases when infants are unable to, or have compromised ability to, respond to a stressful stimulus, such as hypoxia. These risks increase with exposure to opioids and nicotine. A postulated cause of SIDS is the abnormal regulation of opioid transmission (Morin-Surun et al., 1992). Evidence supporting the role of opioids in SIDS includes a study on infants at risk for SIDS. These infants have a reduced central chemosensitivity and display alveolar hypoventilation (Shannon and Kelly, 1977). During the first day of life, partially asphyxiated preterm infants have higher plasma $\beta$-endorphin levels than nonasphyxiated preterm infants. An elevated $\beta$-endorphin concentration in the CSF may be responsible for an increased risk of infant apnoea syndrome and its associated SIDS (Shook et al., 1990; Pokela, 1993; Storm et al., 1994). $\beta$-endorphin injected into the CSF of dogs' causes a respiratory depression that is mediated by central vagal pathways, shown to be important in the timing of respiration (Moss and Scarpelli, 1981). It has been suggested that incidences of SIDS may be related to exposure to opiates in breast milk, either arising from opiates prescribed for the mother, or possibly from naturally occurring opioids present in breast milk (Shook et al., 1990). Foetal bradycardia may also arise from intrathecal opioids prescribed for labour analgesia (Mardirosoff et al., 2002), and this may 
further complicate the opioid induction of SIDS symptoms. Prenatal methadone or heroin exposure also increases SIDS risk (Chavez et al., 1979; Kandall et al., 1993). The true role of opioids in increasing the risk of SIDS is unknown. OpR distributions within the brainstem cardiorespiratory nuclei of victims of SIDS appear to be normal, suggesting that OpR dysfunction itself does not play a role (Kinney et al., 1998).

\subsubsection{Sleep Apnoea}

A disease related to SIDS, infant apnoea, is often referred to as near-miss SIDS. There are many forms of infant apnoea syndrome that can be attributed to abnormally high endorphin levels, and prenatal exposure to methadone is generally associated with a depressed response to $\mathrm{CO}_{2}$ after birth in human infants (Olsen and Less, 1980). In adult sleep apnoea sufferers, a reduction in number, duration, and intensity of hypoxic events occurs if the patients are given the opioid antagonist naltrexone, suggesting that opioids play some role in the development of sleep apnoea (Ferber et al., 1988).

\subsubsection{Asthma}

Opioids are believed to influence responses to asthma, although the mechanism and extent of their role in this disease remains largely unknown. For recent reviews of opioids and asthma, see Mazzone and Canning (2002) and Groneberg and Fischer (2001) (Groneberg and Fischer, 2001; Mazzone and Canning, 2002). Opioids can be detected in the healthy lung. For example, met-enkephalin immunoreactivity exists in the rat and guinea pig lung in nerve fibres in the trachea and bronchi (Shimosegawa et al., 1990). In asthma sufferers, there is damage to the epithelial lining of airways that exposes C-fibres in the bronchial mucosa. Stimulation of these C-fibres by inflammatory mediators may subsequently lead to bronchoconstriction and inflammation (Barnes, 1986). In animal models, inhalation of a MOR agonist inhibits C-fiber activation, although this agonist was not effective in humans (Pavord et al., 1994). Opioid effects on the airways also include inhibition of smooth muscle contraction and inhibition of neurally-mediated mucous secretion in human bronchi (Rogers and Barnes, 1989). New studies with the non-classical opioid receptor ORL-1 have shown that activation of this receptor prevents airway constriction; however, effective therapeutic strategies employing ORL-1 drugs have not yet been developed (Groneberg and Fischer, 2001). Chronic exposure to opioids by pharmaceutical workers increases the incidence of bronchial asthma, probably as a result of effects of opioids on the immune 
system (Górski and Ulinski, 1996). Thus, opioids may influence the progression of asthma by directly altering mucous secretion and airway responsiveness, or they may indirectly affect asthma through their effects on immune responses.

\subsubsection{HAEMORRHAGE}

In addition to their involvement in respiratory disease, endogenous opioids may also play a role in cardiovascular disease. Opioid receptors are found in the heart, vessels, and areas of the CNS that regulate cardiovascular function (Hassen et al., 1982; Pfeiffer et al., 1984; Feuerstein, 1985; Lawrence and Jarrott, 1996; Miyawaki et al., 2002; Pugsley, 2002). For example, severe haemorrhage reduces blood volume (direct effect) and suppresses the activity of the sympathetic nervous system leading to a marked bradycardia (decompensatory response) and a fall in blood pressure. The exact central mechanism for the decompensatory response is not understood, although opioids are believed to play a role. Opioid involvement in haemorrhage was first observed by Faden and Holaday (1979) who discovered that intravenous injection of naloxone inhibited the fall in arterial blood pressure caused by haemorrhage in rats (Faden and Holaday, 1979). In the periaqueductal gray of the $\mathrm{SpC}$, this response is mediated by DOR (Cavun et al., 2001). Fentanyl, a MOR agonist, when infused into a cat with brain trauma injuries, also produces a decrease in blood pressure (Bedell et al., 1998; Vaccarino et al., 1999). Another study has shown that KOR activity in the medulla inhibits sympathetic activity in severe haemorrhage (Henderson et al., 2002). Thus, all three classical receptors, MOR, DOR, and KOR, can influence the sympathetic control of the cardiovascular system, particularly affecting its response to haemorrhage. These effects are clinically important with regard to haemorrhage during surgery because opioids are often used in anaesthesia and for reducing postoperative pain

\subsubsection{DRUG ADDICTION}

Opioids are often used as drugs of abuse because of their ability to produce euphoria. Chronic use, however, results in tolerance and addiction, and opioid overdose often results in death from severe respiratory depression (White and Irvine, 1999). OpR downregulation and desensitisation on exposure to opioids have been discussed earlier in this chapter. Briefly, chronic exposure to opioids causes down-regulation and reduced OpR density (Prathert et al., 1994; Trapaidze et al., 1996; Gaudriault et al., 1997; Standifer and 
Pasternak, 1997; Massotte and Kieffer, 1998; Law and Loh, 1999). There is also a decrease in the synthesis of endogenous opioids [Fang, 1998 \#1120, suggesting a negative feedback system, as well as alterations in gene regulation. Behaviourally, chronic opioid administration produces tolerance through the action of N-methyl-D-aspartate (NMDA) receptors and serotonergic systems (Vaccarino et al., 1999). Chronic opioid administration also results in dependence and addiction, as observed by opiate withdrawal symptoms that include aches and pains, changes in respiration, sweating, and tremors. MOR mediates most of the analgesic effects of morphine, although DOR also plays a role (Quock et al., 1999). The exact mechanism of addiction and tolerance are not well understood and involve both cellular and behavioural changes (Koob and Moal, 1997; Nestler and Aghajanian, 1997; Vaccarino et al., 1999; Vaccarino and Kastin, 2001).

There appears to be a genetic predisposition for drug abuse. People who show a predisposition to drug abuse sometimes have polymorphisms in genes for the opioid peptides and receptors (Bond et al., 1998; Mayer and Höllt, 2001). In a study by Bond et al. (1998) five single nucleotide polymorphisms (SNPs) in the MOR coding region were found in heroin addicts. One of the SNPs in the MOR gene showed enhanced responses to $\beta$-endorphin when the mutated protein was expressed in cell lines, due to increased binding affinity of $\beta$-endorphin for MOR (Bond et al., 1998; Mayer and Höllt, 2001).

\subsubsection{THE ROLE OF THE BRAINSTEM AND OPIOIDS IN PAIN CONTROL}

Opioids play an important role in stress-induced analgesia, being implicated in childbirth, exercise, and conflict or threat (Olson et al., 1997; Vaccarino et al., 1999; Vaccarino and Kastin, 2001). In a study in which mice were exposed to biting flies, opioid-mediated analgesia (measured by naloxone sensitivity) developed within $30 \mathrm{~min}$ (Kavaliers et al., 1998), although not all stress-induced analgesia was reversible by naloxone (Fields and Heinricher, 1985). The degree of opioid-mediated analgesia varied with the sex of the subject, being greater in males than females (Kavaliers et al., 1998). The neural pathways by which stress-induced analgesia occurs have been extensively studied, although the exact mechanisms remain unknown. Several brain regions and OpR subtypes have been implicated in pain control (Vaccarino et al., 1999; Vaccarino and Kastin, 2001). Stimulation of the PAG, an area that expresses high levels of MOR, DOR and KOR (Gutstein et al., 1998; Commons et al., 2001) reduces the severity of clinical pain in 
humans (Fields and Heinricher, 1985). The PAG receives input from the cortex and sends projections to the RVLM, which in turn sends projections to the dorsal horn of the SpC. At each of these integrative levels, there are enkephalin-containing interneurons that express MOR and DOR (Commons et al., 2001). SpC neurons also project rostrally to terminate at medullary and midbrain regions and play an important role in analgesia. The $\mathrm{SpC}$ is the point of entrance of nociceptive sensory information and is an important site for antinociception by MOR and DOR agonists (Cheng et al., 1995b). Clinically, morphine is one of the most widely used drugs for the relief of pain, and is often used for pain relief after surgery, during labour, and for cancer sufferers. Opioids modulate analgesia by their actions on MOR, DOR, and KOR subtypes. Within these receptor classifications, $\kappa 1, \kappa 2$, $\kappa 3$, and $\delta 1$ receptors mediate supraspinal morphine analgesia and $\mu 2, \delta 1$, and $\kappa 1$ receptors are responsible for spinal analgesia (Pasternak, 1993). The use of opioids in controlling pain is problematic, due to the effect of opioids on other systems, including respiratory, gastrointestinal, and cardiovascular systems. As previously stated, opioids injected into the CSF of women in labour causes foetal bradycardia (see review by Mardirosoff et al. 2002) (Mardirosoff et al., 2002), and such treatment may therefore be undesirable because of this adverse effect on the late-term foetus.

\subsection{Aims of Thesis}

The aim of the present study was to establish a primary culture model for investigating rat brainstem cells isolated from late foetal and early postnatal developmental stages. This culture method will be used, along with studies on frozen sections from rat brain, to examine developmental changes in MOR and DOR OpR expression and distribution in the rat brainstem. 


\section{Chapter 2}

\section{Primary Culture Of Brainstem Cells}

\subsection{AIM}

The aim of this study was to develop a method for growing primary brainstem neurons from rats at different stages of foetal and early postnatal development. The development of a method for culturing postnatal brainstem neurons was important because it would allow study of cultured brainstem neurons at later developmental ages than had previously been possible. This method could then be used to investigate opioid receptor expression during development (Chapter 3).

\subsection{INTRODUCTION}

\subsubsection{USE OF PRIMARY CULTURE IN INVESTIGATION OF BRAINSTEM FUNCTION}

Primary cultures of brainstem neurons from foetal and postnatal rats are useful models for in vitro studies of brainstem function, because they more closely mimic the normal situation than cultures of established cell lines. In both primary cultures and established cell cultures, more control exists over the cell environment. Greater experimental control is beneficial because it allows single components of the medium to be changed and their effects evaluated. The use of a chemically defined, serum-free medium is recommended for studies in which complete control of the environment in the nutrient medium is desired, for example, studies involving electrophysiological recordings (Rigatto et al., 1994), immunocytochemical staining (Fitzgerald et al., 1992), neurotransmitter release (Shimoda et al., 1992), and growth factor responses (Copray and Leim, 1993; Miller and Azmitia, 1999). Culture in a defined medium provides a more stable and consistent environment because it removes the variability between different batches of serum. The advantages of studying receptor expression in culture, as opposed to whole brain slices, is the ability to trace individual neuronal processes and observe interactions with other cells. The disadvantages of using a cell culture model may include the formation of abnormal cell architecture, changes in gene expression, and the formation of abnormal glial-neuronal, neuronal-neuronal, and neuronal-glial communication. 
The brainstem integrates many functions of the somatic and autonomic nervous systems. For example, the brainstem plays a role in the suckling response, swallowing, respiration, cardiovascular control, the maintenance of the arousal state, and the mediation of stress responses [Vaccarino, 1999 \#877; de Boer-van Huizen, 1999 \#549; Lehnert, 1998 \#548; (Pilowsky et al., 1990a; Rigatto et al., 1992). The brainstem is composed of many heterogeneous neurons having different morphologies and functional properties. Neurons from the brainstem send projections to wide areas of the brain and spinal cord, and the specific morphology of cells relates to their functional properties (Berger et al., 1984; Pilowsky et al., 1990b; Pilowsky et al., 1993). Rostral brain regions are relatively easily maintained in culture because they are at an earlier developmental stage than caudal regions; and thus fewer connections are broken during tissue dissociation (Brewer, 1997).

No satisfactory protocols for primary culture of postnatal brainstem neurons in defined medium have been reported. Even with serum, commonly used procedures often give poor survival rates in older foetal (>E16) and postnatal brainstem cultures (Azmitia, 1990; Minamoto et al., 1991). Only two papers, Masuko et al. (Masuko et al., 1986), and Ternaux and Portalier (Ternaux and Portalier, 1993) have reported the successful culture of brainstem neurons from the postnatal rat. Masuko et al. (1986) cultured brainstem cells from neonatal mice up to P5 using a glial feeder layer and FCS. Neurons were not labelled by neuron-specific markers but were identified solely on morphology, a criterion shown to be unreliable (Raff et al., 1979). Some brainstem cultures from neonatal rats were also used, although no actual data were presented for these cultures. The study by Ternaux and Portalier (1993) provided more comprehensive culture data; however, their culture medium generally contained serum and was therefore undefined (Ternaux and Portalier, 1993). Although some of their cultures were maintained without serum, detailed results in defined medium were not reported.

The present study describes the first reliable method for establishing stable in vitro cultures of foetal and early postnatal brainstem neurons in a defined culture medium. Optimal neuron survival of both late foetal and early postnatal brainstem cultures was achieved with a commercially available, serum-free culture medium specifically designed for support of neurons in culture. This medium consisted of Neurobasal ${ }^{\mathrm{TM}}$, a medium from Gibco (Invitrogen, Gathersburg, MD, USA) for foetal cultures, and Neurobasal ${ }^{\mathrm{TM}}$-A for postnatal cultures. Both of these were used with a defined supplement (B27) high in 
antioxidants, also from Gibco. Neuron and glial cells present in the cultures were identified using immunocytochemical staining with antibodies against microtubuleassociated protein-2 (MAP2) or glial fibrillary acidic protein (GFAP), respectively. Neuron populations were stable for up to 9 days in culture and showed significant neurite outgrowth with time. This culture system will be useful for studying brainstem neuron function in vitro at later developmental ages than previously possible, thus providing a more complete picture of neuronal maturation during development of the rat brainstem. 


\subsection{METHODS}

\subsubsection{PRIMARY CELL CULTURE}

\subsubsection{Animals}

All procedures were approved by the Victoria University of Wellington Animal Ethics Committee (JM95R2, JM98R2 and JM2000R4).

Foetal brainstem neurons were cultured from pooled embryos of time-mated Wistar rats. Pro-oestrous rats were mated overnight. Successful mating was confirmed by the presence of sperm in vaginal smears the next morning and termed EO according to Paxinos et al. (1994). Smears were taken using damp cotton wool wrapped around curved forceps. Smears were dabbed onto microscope slides and stained for several minutes with a drop of toluidine blue $(0.05 \%$ in water) (The General Chemical Company Ltd, Budbury, Middlesex, UK). Excess toluidine blue was removed by blotting the edge of the slide with a paper towel. Vaginal epithelial cells were observed using a compound microscope (Zeiss, Oberkochen, Germany).

Neonatal Wistar rats aged 0 to 6 days postnatal (P0 to P6) were anaesthetised with Nembutal (50 mg/kg; Virbac, Otahuhu, Auckland) intraperitoneal (ip) injection, and then sacrificed by cervical dislocation. The external surface of the animal was sprayed with $70 \%$ ethanol before aseptic removal of the brain.

\subsubsection{Culture Reagents and Equipment}

Pasteur pipettes were siliconised by aspirating Siliclad solution (Sigma, St Louis, MO, USA), into the barrel of the pipette, then expelling it once. The pipettes were allowed to air dry before autoclaving at $120^{\circ} \mathrm{C}$ for $15 \mathrm{~min}$. Circular $13 \mathrm{~mm}$ diameter coverslips (BDH, Palmerston North, NZ) were cleaned for $10 \mathrm{~min}$ in a sonicator bath (L\&R, Kearney, NJ, USA) with warm water containing a small amount of detergent. The coverslips were then washed in 7 changes of $\mathrm{dH}_{2} \mathrm{O}$ and two changes of $70 \%$ ethanol, and stored in a sealed container in $70 \%$ ethanol until needed. The day prior to setting up the cultures, the coverslips were flame-sterilised, and four coverslips were placed in each $35 \mathrm{~mm}$ diameter cell culture dish (Nunc; Invitrogen). Each coverslip was coated with $50 \mu \mathrm{L}$ of $10 \mu \mathrm{g} / \mathrm{mL}$ poly-D-lysine (PDL) (Sigma) by incubation at room temperature for $5 \mathrm{~min}$. Excess coating solution was removed, and the coverslips were allowed to dry overnight. Coated 
coverslips could be stored at $4^{\circ} \mathrm{C}$ for up to one week. On the day of plating, the coverslips were washed $3 \mathrm{x}$ in sterile $\mathrm{dH}_{2} \mathrm{O}$ and pre-treated with $10 \%$ heat-inactivated foetal calf serum (FCS) (Invitrogen) for at least $2 \mathrm{~h}$ at $37^{\circ} \mathrm{C}$ to assist cell attachment. The FCS solution was removed prior to plating the cells.

\subsubsection{Foetal Brainstem Culture}

Primary brainstem cultures were established from E14, E16, E18 and E20 rat foetuses by a modification of the method of Cheung et al. (1997) (Cheung et al., 1997) who used serumfree conditions to culture E14-E16 foetal rat mesencephalic neurons. Pregnant rats were anaesthetised by ip injection of Nembutal (Virbac). Foetuses were removed from the uterus and placed in sterile ice-cold balanced salt solution (BSS) (pH 7.4) (Appendix 2: Section 1). All dissections were performed with sterile instruments, and solutions were kept on ice in a laminar flow cabinet (Gelman Sciences, Australia). Foetal rat brains were removed and placed in Hibernate-E medium (Invitrogen), a medium that allows prolonged storage of tissue without loss of viability. Both Hibernate-E (for foetal tissue) and Hibernate-A (for postnatal tissue) media are no longer commercially available through Invitrogen. The appropriate Hibernate formulae are given in Appendix 1. Recently, Hibernate medium has been made commercially available again through the original designer of the medium, Dr Gregory Brewer (email:gbrewer@simed.edu, Springfield IL, USA) at a cost of approximately $\$ 60$ (USA) for $500 \mathrm{~mL}$.

Brainstems were dissected from the whole brain with the aid of a dissecting microscope (Olympus, Tokyo, Japan), and blood vessels, meninges, and the cerebellum were carefully removed using fine-tipped, sterile watchmaker's forceps. Detailed foetal brain dissections complete with photographs and diagrams are described in the following references, (Brewer and Cotman, 1989; Azmitia, 1990; Fitzgerald et al., 1992; Copray and Leim, 1993). Brainstem regions taken for culture included the rostral brainstem region, consisting of the pons and medulla, extending posteriorly to approximately $2 \mathrm{~mm}$ caudal to the obex (Figure 2.1). 


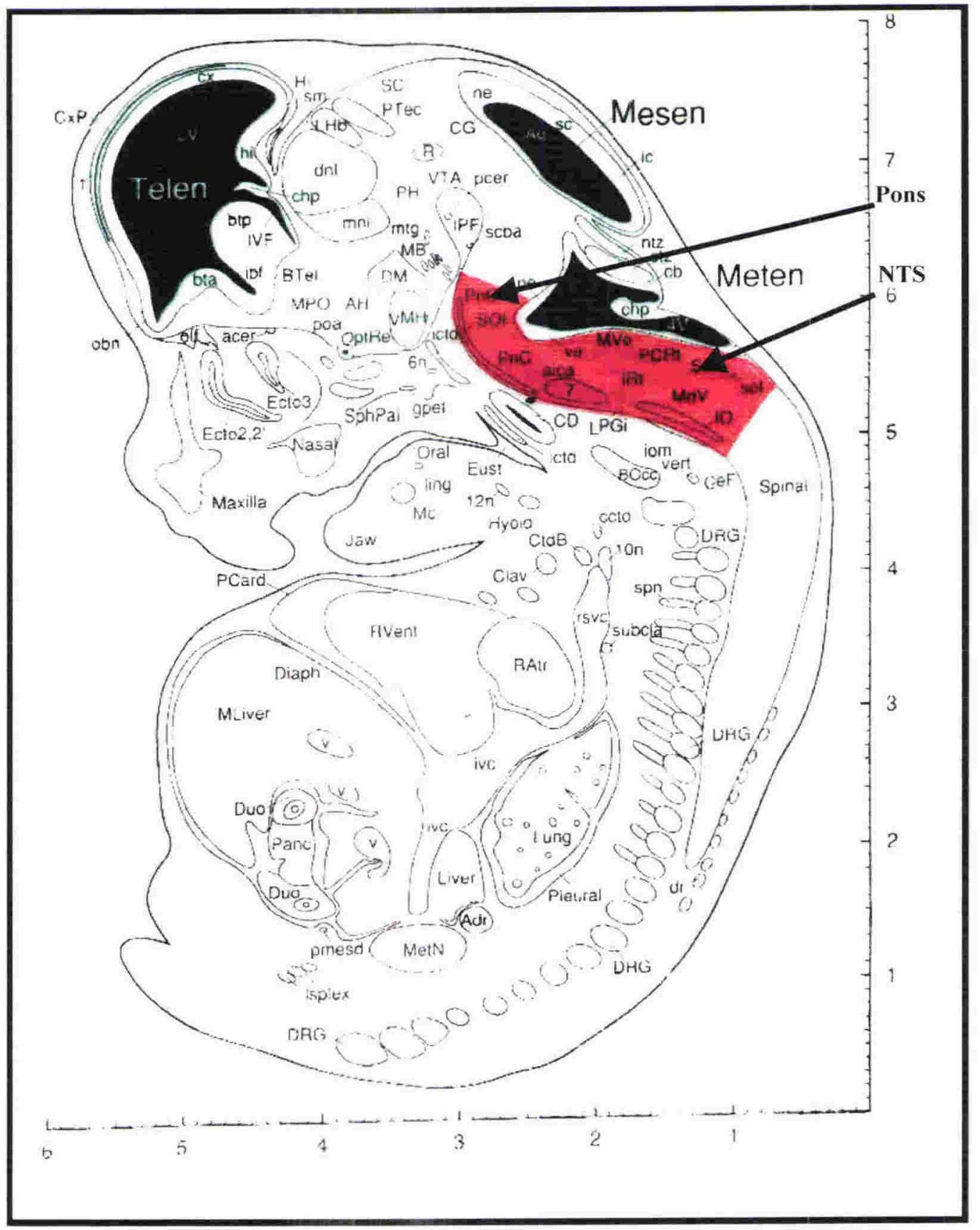

Figure 2.1

\section{Diagram of E16 Foetal Rat Showing the Brainstem Region} Taken for Culture

The area shown in red is the area taken for primary brainstem cultures. This region includes the pons, medulla, nucleus of the solitary tract (NTS), and nucleus ambiguus (NA). From Paxinos et. al. (1994). Scale bar $=\mathrm{mm}$. 
Pooled brainstems from a single litter ( $8-15$ fetuses) were transferred to fresh Hibernate ${ }^{\mathrm{TM}}$ E medium. The tissue pieces (approx $2 \mathrm{~mm}^{3}$ ) could be left at this stage for up to a week at $4^{\circ} \mathrm{C}$ without loss of viability although for all experiments discussed in this thesis, they were never stored for more than $30 \mathrm{~h}$. The tissue was then transferred to a sterile $15 \mathrm{~mL}$ centrifuge tube and mechanically dissociated by triteration using a fire-polished, siliconised, long-stem Pasteur pipette. Trituration involved aspirating the tissue suspension in and out of the pipette approximately $10 \mathrm{x}$. Tissue pieces were allowed to settle, and the supernatant containing the dissociated cells was removed and retained. This procedure was repeated $3 x$, and the combined supernatants were centrifuged in a non-refrigerated bench-top centrifuge ( $300 \mathrm{~g}, 5 \mathrm{~min}$, Clements, GS200, Sydney, Australia) and resuspended in complete growth medium. The complete growth medium consisted of Neurobasal medium with 2\% B-27 supplement (Invitrogen), $0.25 \mathrm{mM} \mathrm{L-glutamine} \mathrm{(Sigma),} 0.25 \mathrm{mM}$ GlutaMax I (Invitrogen), penicillin G (50 U/mL) (Invitrogen), and streptomycin sulphate (50 $\mu \mathrm{g} / \mathrm{mL}$ ) (Invitrogen). A mixture of glutamine and GlutaMax I was used initially due to the cells' inability to metabolise GlutaMax I when first seeded (personal communication, Dr Stephanie Hughes), although when the culture medium was changed (every 5-7 days) $50 \mathrm{mM}$ GlutaMax I was used instead of the glutamine/glutaMax mixture. This complete medium, with an osmolality of $240 \mathrm{mosm} / \mathrm{kg} \mathrm{H} 2 \mathrm{O}$, was used for E14 and E16 cultures. Osmolality was determined in a Fiske OSTM osmometer (Fiske Associates, Needham Heights, MA, USA). To enhance neuron survival in E20 cultures, a mixture of $50 \%$ Neurobasal medium and $50 \%$ Neurobasal-A medium containing identical supplements as for the E14 and E16 cultures was used. The osmolality of this modified medium was 260 $\operatorname{mosm} / \mathrm{kg} \mathrm{H}_{2} \mathrm{O}$.

The percentage of viable cells was determined by trypan blue dye exclusion. A $50 \mu \mathrm{I}$. sample of suspended cells was diluted in $450 \mu \mathrm{L}$ of $0.02 \%$ trypan blue in BSS. The percentage of viable cells was determined microscopically using a haemocytometer. The cells were seeded onto previously cleaned coverslips that had been treated with poly-Dlysine and serum at a density of $0.5-1.0 \times 10^{6}$ viable cells $/ \mathrm{cm}^{2}$ in a total of $2 \mathrm{~mL}$ complete growth medium per $35 \mathrm{~mm}$ dish. Cells were incubated in a controlled atmosphere of 95\% air, $5 \% \mathrm{CO}_{2}$ and $97 \%$ humidity at $37^{\circ} \mathrm{C}$ for up to 3 weeks. To keep cellular debris to a minimum in the cultures, half the medium was replaced after 1 day in culture with fresh, 
pre-warmed culture medium. Following this, cultures were fed every 5-7 days with half changes of medium.

\subsubsection{Postnatal Brainstem Culture}

Neonatal Wistar rats aged up to 6 days (P0 to P6) were anaesthetised with pentobarbital by ip injection $(50 \mathrm{mg} / \mathrm{kg})$, and then sacrificed by cervical dislocation. The animals were sprayed with $70 \%$ ethanol before aseptic removal of the brain. Whole brains from rats of the same litter were removed and placed in a $100 \mathrm{~mm}$ diameter culture dish containing 10 $\mathrm{mL}$ of Hibernate-A medium (Invitrogen) on ice. The meninges and blood vessels were carefully removed with the aid of a dissecting microscope. A separate $60 \mathrm{~mm}$ culture dish containing Hibernate-A medium was used for every two animals. Brainstems were identified using the same criteria as for foetuses (Figure 2.2), removed, pooled and placed in petri dishes containing Hibernate ${ }^{\mathrm{TM}}-\mathrm{A}$ medium. The tissue was cut into $2 \mathrm{~mm}$ cubes and the tissue matrix loosened by treating with trypsin stock (Invitrogen) to give a final concentration of $0.05 \%$ trypsin in Hibernate-A medium. Tissue was then incubated for 15 min at $37^{\circ} \mathrm{C}$ in a shaking waterbath. Alternatively, the tissue pieces could be stored overnight in Hibernate-A medium containing B-27 supplement (without trypsin), as described for embryonic tissue. The tissue pieces were allowed to settle for 5 min and the supernatant removed. The pellet was resuspended in Hybernate-A medium containing $10 \%$ FCS (to inhibit trypsin activity) and $0.01 \mathrm{mg} / \mathrm{mL}$ DNase (Sigma). The tissue was then mechanically dissociated in the same way as for foetal cultures, substituting Hibernate-A medium and Neurobasal-A medium for the embryonic media, Hibernate-E and Neurobasal. The supernatant containing the dissociated cells was centrifuged at $300 \mathrm{~g}$ for $5 \mathrm{~min}$, and the cell pellet resuspended in Neurobasal-A medium containing $2 \% \mathrm{~B}-27$ supplement, $0.25 \mathrm{mM}$ L-glutamine, $0.25 \mathrm{mM}$ GlutaMax I, penicillin G (50 U/mL), and streptomycin sulphate $(50 \mu \mathrm{g} / \mathrm{mL})$. The osmolality of this medium was $280 \mathrm{mosm} / \mathrm{kg}$ $\mathrm{H}_{2} \mathrm{O}$. Cell viability was determined by trypan blue dye exclusion as described above. Cells in $2 \mathrm{~mL}$ complete growth medium were seeded onto prepared coverslips in $35 \mathrm{mrn}$ culture dishes at a density of $0.5-1.0 \times 10^{6}$ viable cells $/ \mathrm{cm}^{2}$.

\subsubsection{CELL-SPECIFIC MARKERS}

Immunocytochemistry (see below for methods) was used to identify and characterise the cell types present in the cultures. GFAP was used to specifically label astrocytes (Raff et 
al., 1979), and MAP2 was used to specifically label neurons (Sarnat et al., 1997). Cholinergic neurons in some cultures were labelled using antibodies to choline acetyltransferase (ChAT) (Knusel and Hefti, 1988; Burton et al., 1995; Chen et al., 2001).

\subsubsection{Fixation of Cultured Cells}

Coverslips with their attached cells were removed from the culture medium and washed $3 x$ in phosphate buffered saline (PBS) ( $\mathrm{pH} \mathrm{7.4)} \mathrm{(Appendix} \mathrm{2:} \mathrm{Section} \mathrm{2)} \mathrm{before} \mathrm{being} \mathrm{fixed} \mathrm{in}$ a mixture of $50 \%$ acetone: $50 \%$ methanol for $8 \mathrm{~min}$ at $-20{ }^{\circ} \mathrm{C}$. The coverslips were washed $3 x$ in cold PBS ( 5 min each) and stored at $4^{\circ} \mathrm{C}$ (for up to 3 weeks before staining) in PBS containing $0.1 \%$ sodium azide in a culture dish sealed with Parafilm (American National Can ${ }^{\mathrm{TM}}$ Chicago, IL, USA).

\subsubsection{Permeabilisation and Removal of Endogenous Peroxidase Activity}

Prior to immunostaining, cells were permeabilised for $30 \mathrm{~min}$ at room temperature in PBS containing $0.2 \%$ Triton X100. Endogenous peroxide activity was removed by incubation in $0.1 \% \mathrm{H}_{2} \mathrm{O}_{2}, 50 \%$ methanol in $\mathrm{dH}_{2} \mathrm{O}$ (Appendix 2: Section 3) for $15 \mathrm{~min}$, and washed $3 \times 5$ $\min$ in PBS.

\subsubsection{Colourmetric Visualisation with DAB}

$\mathrm{DAB}$ colourmetric visualisation of immunostaining was used as an alternative to fluorescence signal amplification. The colourmetric method was used to obtain permanent mounted samples that permitted a comparison to be made between samples of differen developmental ages after varying times in culture, without the problems of photobleaching generally associated with fluorescence. DAB visualisation used secondary antibodies conjugated to Horseradish peroxidase (HRP), as described above.

\subsubsection{MAP2 Immunocytochemistry}

To identify neurons, microtubule-associated protein-2 (MAP2) immunostaining was carried out using a modification of the method of Hsu et al. (1981) (Hsu et al., 1981). Biotin/avidin amplification was performed, followed by visualisation using 3,3'diaminobenzidine (DAB) (Volsen, 1984). Briefly, cells were fixed, permeabilised, endogenous peroxidase activity was removed and coverslips were washed. Non-specific binding sites were then blocked at room temperature for $30 \mathrm{~min}$ in PBS containing $2 \%$ 
normal goat serum (NGS) (Invitrogen), 4\% bovine serum albumin (BSA), $0.2 \%$ Triton, and $0.01 \%$ sodium azide. Coverslips were incubated with mouse monoclonal anti-MAP2 primary antibody (specific for MAP2a, b and c) (1:250; Sigma) in PBS containing 0.2\% Triton, $1 \%$ NGS, and $2 \% \mathrm{BSA}$, at $4{ }^{\circ} \mathrm{C}$ overnight. Coverslips were then washed $3 \mathrm{x}$ in PBS containing $0.2 \%$ Triton, incubated with biotin-labelled goat anti-mouse IgG (1:250 in PBS containing $0.2 \%$ Triton; Sigma) for $2 \mathrm{~h}$ at room temperature, washed $3 \mathrm{x}$ in cold PBS (5 min each), and then incubated for $2 \mathrm{~h}$ at room temperature in ExtrAvidin Peroxidase (1:500 in PBS; Sigma). After incubation, the coverslips were washed $3 x$ as before. Immunostaining was visualised with DAB developing solution $(0.5 \mathrm{mg} / \mathrm{mL}$; Sigma $), 0.1 \mathrm{M}$ phosphate buffer, and $0.01 \% \mathrm{H}_{2} \mathrm{O}_{2}$. Coverslips were washed $3 \mathrm{x}$ in cold PBS and mounted on microscope slides with glycerin jelly. Negative controls were incubated identically but without primary antibody. Some coverslips were counterstained with $3 \%$ toluidine blue or Delafield's haematoxylin to improve visualisation of non-immunostained, non-neuronal cells. Cells were observed using a compound microscope (Zeiss, Oberkochen, Germany) at $400 \mathrm{X}$ magnification by phase contrast or brightfield optics. Cell types were identified according to immunostaining characteristics and morphology.

\subsubsection{GFAP Immunocytochemistry}

Astrocytes were positively identified using a rabbit primary antibody to GFAP (1:100; Sigma) and a sheep anti-rabbit secondary antibody conjugated to FITC (1:100; Sigma). Non-specific binding sites were blocked and the coverslips washed between antibody treatments as described above for MAP2. Fixed cells were incubated in primary and secondary antibodies for $1 \mathrm{~h}$ each at room temperature in the dark. The coverslips were mounted in Antifade (Molecular Probes, Eugene, OR, USA) and examined for fluorescence on a confocal microscope (TCS 4D, Leica Lasertechnik Gmbh, Germany). In some cases, cells were double-labelled with antibodies against MAP2 and GFAP using secondary antibodies conjugated to Rhodamine Red and FITC, respectively.

\subsubsection{Choline Acetyltransferase (ChAT) Immunocytochemistry}

Some primary brainstem cultures were immunostained for the presence of cholinergic neurons using antibodies specific for ChAT (Knusel and Hefti, 1988; Burton et al., 1995; Chen et al., 2001). ChAT antibody was kindly donated by Dr Gregory Funk (Department of Physiology, University of Auckland, NZ). Cell culture samples were fixed, 
permeabilised, endogenous peroxidase activity removed, and non-specific binding sites blocked as described previously. Primary antibody (rabbit anti-ChAT) (1:500; Sigma) was incubated overnight at $4^{\circ} \mathrm{C}$ followed by goat anti- rabbit IgG Biotin (1:500, Sigma). Samples were washed 3x 5 min, then incubated with ExtrAvidin HRP (1:500, Sigma) for DAB visualisation, or ExtrAvidin-FITC (1:1000, Sigma) for detection by fluorescence.

\subsubsection{Colour Development and Mounting}

Antibody-labelled cells were washes $3 \times 5 \mathrm{~min}$, then incubated in DAB colour development reagent (Appendix 2: Section 4) for 1-20 min until the colour had developed. They were then washed $3 \times 5$ min in PBS and mounted onto glass slides using glycerin jelly mounting medium (3.8\% gelatin (Sigma), 50\% pure glycerin (Sigma), and $0.2 \%$ phenol crystals $(\mathrm{BDH})$ in $\mathrm{dH}_{2} \mathrm{O}$ (Appendix 2: Section 6)).

\subsubsection{Cell Type Classification}

Neurons, positively stained for MAP2, were classified as either bipolar or multipolar according to the number of processes extending out from the soma (Figure 2.4C \& D). Glial cells were classified by morphology as either type I astrocytes (large flat cells with an epitheloid shape and a prominent nucleus) or type II astrocytes (smaller stellate-shaped cells morphologically similar to neurons)(Raff et al., 1983) (Figure 2.4A \& B). Cells not fitting within these categories four were classified as 'other' and presumably consisted of fibroblasts, macrophages, oligodendrocytes, and endothelial cells.

\subsubsection{Morphometric Analysis}

Morphometric analysis was carried out on MAP2 immunoreactive neurons, using the Semper 6 image acquiring software program (Synoptics, Cambridge, UK). Coverslips were counterstained with toluidine blue to identify cell nuclei. Measurements of cell body length were obtained using Image Pro software (Media Cybernetics, Silver Spring, MD, USA). Fifteen cell body diameters and total neurite length for each cell were analysed from foetal (E16) and postnatal (P6) cultures after 4 and 7 days in culture. Only nerve cells in sparsely populated areas of the coverslip were morphometrically analysed because of the difficulty in distinguishing cell and neurite boundaries in densely populated regions (Figure 2.3). 



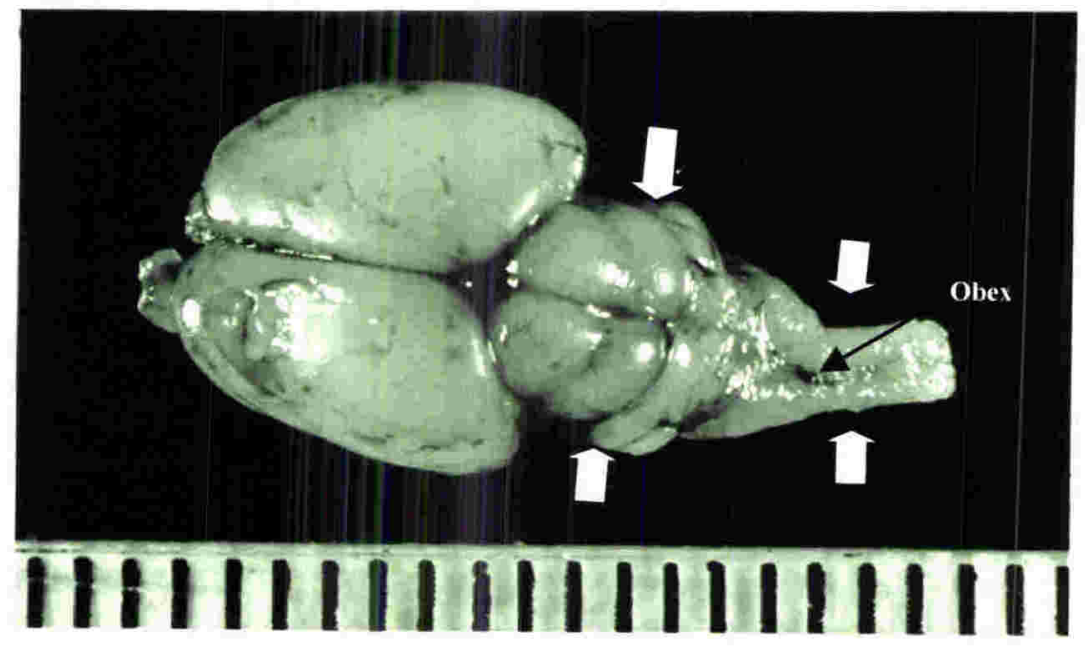

Figure 2.2

\section{Postnatal Brain Structure}

Three-day-old postnatal rat brain (P3), showing lower brainstem regions taken for culture (highlighted by arrows). Scale bar divisions equal $1 \mathrm{~mm}$.

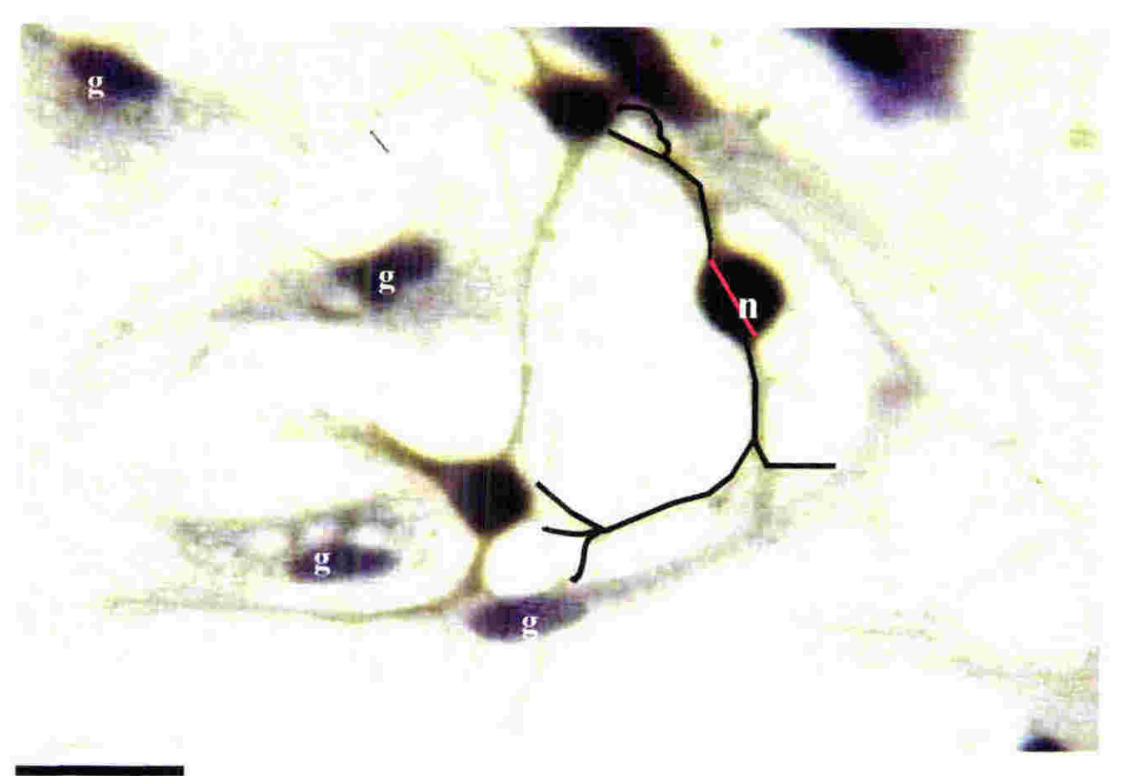

Figure 2.3

\section{Cell Morphometry Measurements}

A MAP2-positive neuron (n) showing cell body length (red) and neuronal process measurements (black). Total neurite length from black outlined neuronal processes was calculated using Image Pro software. Note the presence of toluidine blue stained glial cells (g). Scale bar $=20 \mu \mathrm{m}$. 


\subsection{RESULTS}

\subsubsection{PRIMARY BRAINSTEM CULTURE}

During the development of the brainstem culture method a number of variations in culture conditions were tried. The results of these experiments are described below.

\subsubsection{Brainstem Cell Dissociation}

Brainstem tissue was dissociated in Hibernate $^{\mathrm{TM}}$ medium/B27, which is high in antioxidants and contains $\mathrm{Ca}^{2+}$ and $\mathrm{Mg}^{2+}$ ions. (Brewer and Price, 1996). In the present study Hibernate ${ }^{\mathrm{TM}}$ medium/B27 increased cell viability during dissection and dissociation (60-80\% viability) compared with $\left(\mathrm{Ca}^{2+}, \mathrm{Mg}^{2+}\right.$ free) BSS (40-60\% viability). Cell viability, immediately before plating, was typically between $65-80 \%$, with younger foetal cultures having the highest proportion of viable cells.

\subsubsection{Establishment of Primary Cultures}

Dissociated brainstem cells attached to the coverslip within 1 day in culture and nerve cells began to produce neurites within the first few days. After 4 days in culture, neurons visualised by phase contrast optics, had a phase-bright soma (Figure 2.5A-D) and stained positively for MAP2 (Figure 2.5E \& F). Neurons were often seen growing on top of type I astrocytes, although a small proportion of neurons were not directly associated with glial cells.

\subsubsection{Cell Attachment in Culture}

In addition to serum pre-treatment of coverslips, several cell attachment substrates were tested for their ability to promote neuron survival. In the absence of added substrate, cell attachment was very poor. Coverslips treated with poly-D-lysine showed better attachment than those treated with poly-L-lysine. Polyethyenimine (PEI) $(50 \% \mathrm{w} / \mathrm{v})$ (Sigma) gave good neuron attachment, survival and process outgrowth initially, but the PEI solution obtained from Sigma proved to be unstable, and therefore results were inconsistent. PolyD-lysine gave the most successful and consistent cell attachment and neurite outgrowth of the three substrates tested. In some cultures poly-D-lysine-treated coverslips were not pretreated with serum, and this prevented most neurons from attaching to the surface. 


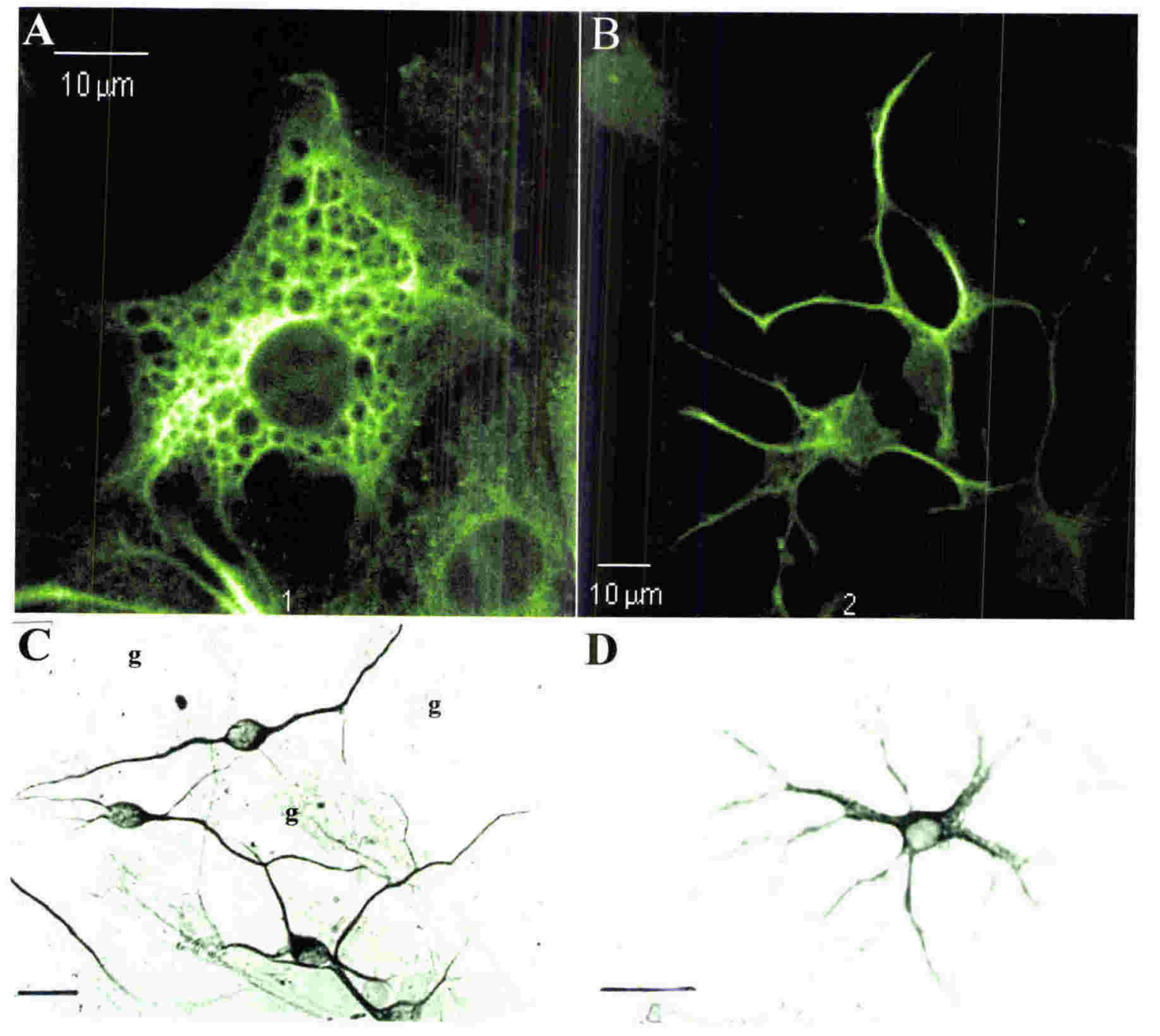

Figure 2.4

\section{Classification of Different Cell Types in Culture}

The main cell types observed in brainstem cultures were type I astrocyte (A) and type II astrocyte (B), staining positively for GFAP (FITC) and observed by confocal microscopy. Scale bar $=10 \mu \mathrm{m}$. Bipolar neurons (C) and multipolar neurons (D), staining positively for MAP2 (DAB) were observed using brightfield microscopy. Note the absence of staining of type I glial cells by anti-MAP2 antibody (g). Scale bar $=20 \mu \mathrm{m}$. 

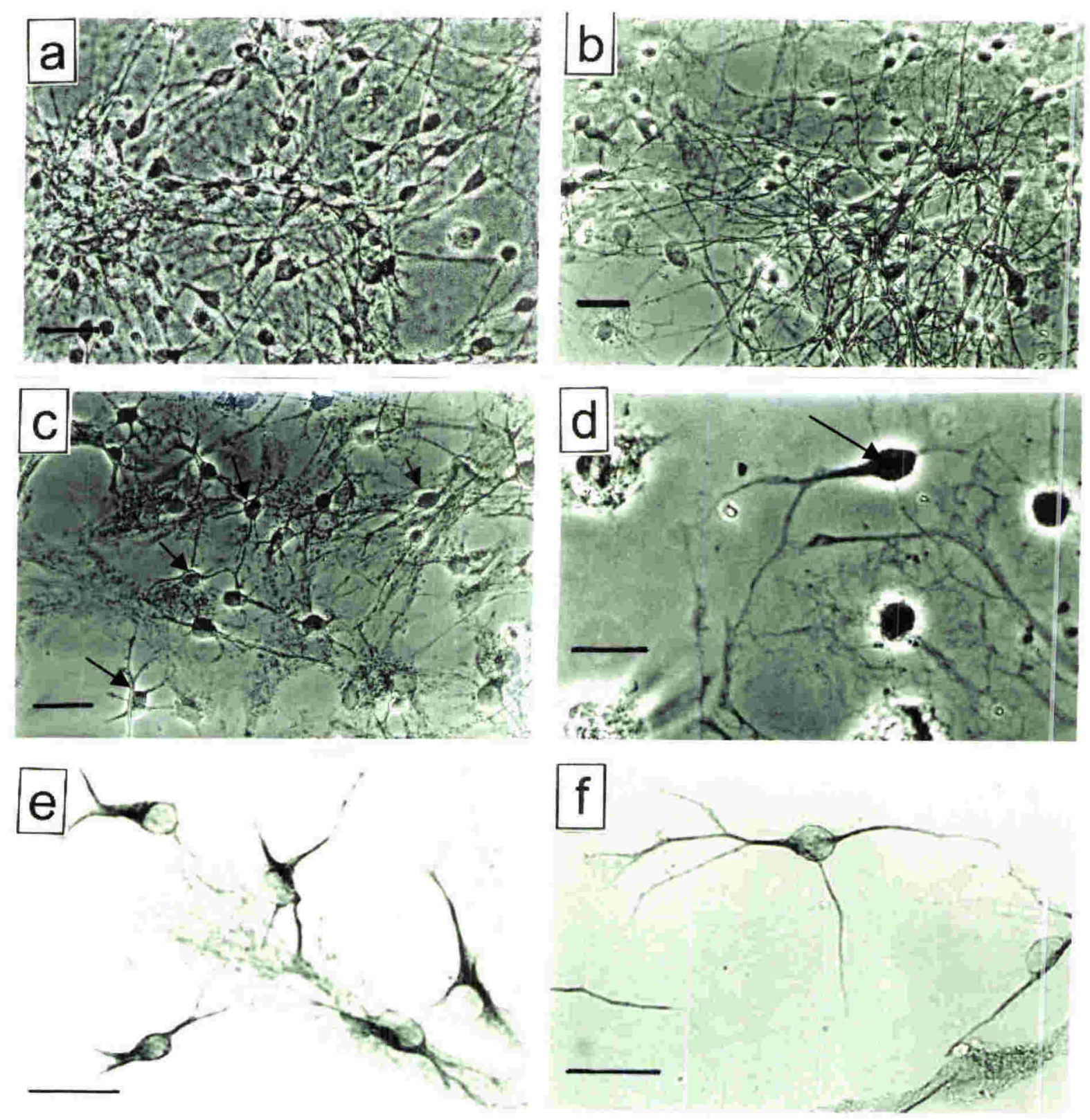

\section{Figure 2.5}

\section{Morphology of Primary Cultures of Brainstem Neurons from Different Developmental Ages of Foetal and Postnatal Rats.}

Brainstem cultures from E14 (a) and E16 (b) rats were grown in Neurobasal medium/B27 supplement. Cultures from 3-day old postnatal rats $\mathbb{C}$, and 6-day old postnatal rats (d), were grown in Neurobasal-A medium/B27 supplement. All cultures were seeded at $0.5-1.0 \times 10^{6} \mathrm{cells} / \mathrm{cm}^{2}$. Photomicrographs were taken after 7 days in culture using phase contrast microscopy. Some phase bright neurons are shown with arrows. Brainstem cultures stained for MAP2 from 6-day old postnatal rats are shown after 4 days in culture (e) and after 7 days in culture (f). Note the increased neurite length at 7 days (f) compared with 4 days (e). Scale bars $=20 \mu \mathrm{m}$. 


\subsubsection{Effect of Different Culture Media on Cells}

Several variations of culture media were tested to determine the optimal conditions for culturing brainstem neurons. In postnatal cultures, qualitative estimates of neuron survival were made. Quantitative comparisons between neurons cultured in DMEM medium and DMEM-F12 medium, or Neurobasal medium have been reported previously by Brewer et al. (1993) (Brewer et al., 1993). In our study DMEM-F12 with 10\% FCS gave poor neuron survival; whereas, DMEM-F12 with B27 supplement gave moderate neuron survival. Neurobasal/B27 supplement for foetal cultures and Neurobasal-A/B27 for postnatal cultures gave the best neuron survival and were chosen as the standard culture media for brainstem neurons.

\subsubsection{GlutaMax I Replacement of Glutamine}

Glutamine and GlutaMax I supplements were directly compared in P1 cultures. Cultures were seeded at $1 \times 10^{5}$ cells $/ \mathrm{cm}^{2}$ with either $0.5 \mathrm{mM}$ L-glutamine or with $0.25 \mathrm{mM}$ GlutaMax I/0.25 mM L-glutamine. The density of the cultures was estimated using a compound microscope equipped with phase contrast optics at $160 \mathrm{x}$ magnification. Cultures were scored on a scale of 1 to 3 according to cell density, without regard to the type of cells. A score of 3 was given when cells covered $>85 \%$ of a typical field of view, a score of 2 when $50-85 \%$ of the field of view was covered, and a score of 1 when $<50 \%$ of the field of view was covered. After 6 and 12 days in culture there were significantly more cells in cultures containing GlutaMax I supplement (mean score $=2.1$ ) than in cultures containing glutamine only (mean score $=1.5)(\mathrm{P}<0.03$, Mann-Whitney test, $\mathrm{n}=33$ fields of view for glutamine: $\mathrm{n}=159$ fields of view for GlutaMax I from counts of two coverslips and six coverslips, respectively). Others have reported similar results with GlutaMax I in neuronal cultures (Minamoto et al., 1991). Neither the replacement of glutamine with GlutaMax I nor the seeding density had any obvious effect on the percentage of neurons in the cultures relative to the total number of cells.

\subsubsection{EFFECTS OF SEEDING DENSITY ON NEURONAL SURVIVAL}

In preliminary experiments a range of seeding densities from $1 \times 10^{5}$ to $1 \times 10^{6}$ cells $/ \mathrm{cm}^{2}$ were tested. Good neuron survival was observed at each density within this range. In one culture, 13 pooled brainstems from P1 rat pups were seeded at either $1 \times 10^{5}$ cells $/ \mathrm{cm}^{2}(n=8$ coverslips) or $5 \times 10^{5}$ cells $/ \mathrm{cm}^{2}$ ( $\mathrm{n}=6$ coverslips). There was no statistically significant 
difference in the percentage of neurons staining positively for MAP2 after 6 or 12 days in culture relative to the total number of cells present in the cultures (50-100 cells were counted on each coverslip and classified as being either bipolar or multipolar neurons, or type I or type II astrocytes).

All other cultures were initially seeded at the same density of $5 \times 10^{5}$ cells $/ \mathrm{cm}^{2}$. Neuron density was not directly measured over time in culture, although neuron survival appeared to be reduced in cultures established from older developmental ages, as evidenced by a decrease in overall cell density with time and an increase in the number of unattached cells and debris after one day in culture. Some cell divisions could be seen in the cultures. One older postnatal culture (P15) was attempted; however, few neurons survived more than one day in culture.

\subsubsection{CELL MORPHOLOGY AND IMMUNOREACTIVITY IN CULTURE}

Cells classified as bipolar and multipolar neurons (Figure 2.4C \& D) were confirmed to be neurons by their positive MAP2 immunoreactivity (Figure 2.6). It was necessary to use a specific marker to identify neurons since their morphology was very similar to the stellateshaped type II astrocytes that stained positive for GFAP (Figure 2.4A \& B), but negative for MAP2 (Figure 2.4C \& D). The negative control for MAP2 (no primary antibody) showed no staining (Figure. 2.6F). In a single experiment, a culture of brainstem neurons and glial cells was double-labelled using antibodies against GFAP (FITC-labelled) and MAP2 (Rhodamine Red-labelled). No cells stained with both GFAP and MAP2 when observed by confocal microscopy, thus ensuring that MAP2-stained multipolar neurons were not incorrectly identified as type II astrocytes, and vice versa. This immunostaining distinction has been well-established in other culture systems (Marriott et al., 1995). 

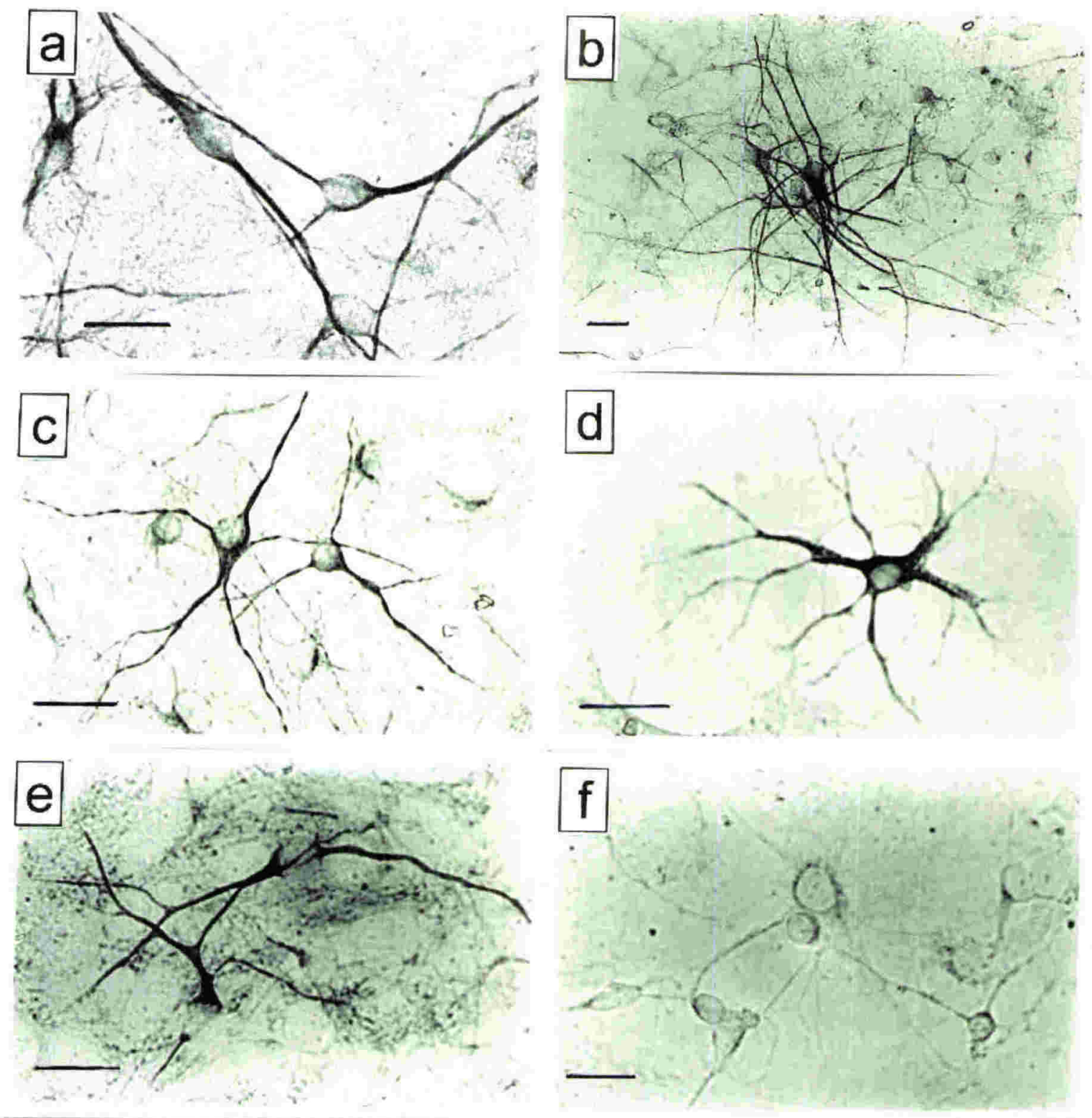

Figure 2.6

\section{Cultured Brainstem Neurons Positively Stained for MAP2}

E14 neurons after 7 days in culture (a); E16 neurons after 9 days in culture (b); E20 neurons after 7 days in culture (b); P3 neurons after 7 days in culture (d); P6 neurons after 9 days in culture (e); Negative MAP2 control for P6 neurons after 7 days in culture (no MAP2 antibody) (f). Note the absence of MAP2 staining in the cell body and nucleus in a-e. Brightfield microscopy. Scale bar = $20 \mu \mathrm{m}$. 


\subsubsection{DISTRIBUTION OF CELL TYPES}

Cell type distributions on coverslips from each developmental age, embryonic E14, E16, E20 and postnatal P3 and P6, were counted after 4, 7, and 9 days in culture (Figure, 2.7). Between 50 and 250 cells on each coverslip were counted and classified into one of the five groups. The ratio of neurons to glial cells remained constant with time in culture in E16, E20, P3 and P6 cultures. Type I astrocytes made up a greater proportion of the total cells (50-72\%) in more advanced developmental ages compared with the youngest E14 cultures (17-20\%). There was no significant change in the distributions of cell types with time in culture from 4-9 days, suggesting good neuron survival and maintenance between 4 and 9 days in culture.

The more adherent, flattened type I astrocytes were more common than the neuronal like type II astrocytes in both foetal and postnatal cultures. The marked difference in the frequency distribution of cell types in E14 cultures compared with the developmentally more advanced cultures was highly significant $(\mathrm{P}<0.001, \mathrm{n}=5$, Kruskal-Wallis $)$. Comparing cell types at different developmental ages (average value from the pooled 4, 7 and 9 day results of Figure. 2.7), E14 cultures had a higher proportion of neurons than glial cells (61\% compared with $35 \%$ ), and most of the neurons were bipolar ( $40 \%$ of total cells) rather than multipolar (21\% of total cells). Late embryonic (E16 and E20) and early postnatal cultures (P3 and P6) had a higher proportion of astrocytes than neurons $(67-73 \%$ compared with $25-31 \%$ ), and most of the neurons were multipolar (18-25\%) rather than bipolar (5-9\%) (range of values averaged from pooled results of E16, E20, P3 and P6 cultures after 4, 7 and 9 days in culture). There were significant differences between the bipolar/multipolar neuron ratios in cultures from foetal E14, E16 and E20 and postnatal P3 and P6 stages. More advanced cultures had disproportionately more multipolar neurons. This difference was statistically significant between E14, E16, E20, P3 and P6 cultures at 4, 7 and 9 days in culture $(\mathrm{P}<0.0019, \mathrm{n}=5$, two-factor, Kruskal-Wallis test). Thus E14 cultures showed significantly more neurons than glial cells; however, by E16, type 1 glial cells predominated in the cultures. E14 also showed more bipolar than multipolar neurons compared with later stage cultures in which multipolar neurons were more common. 


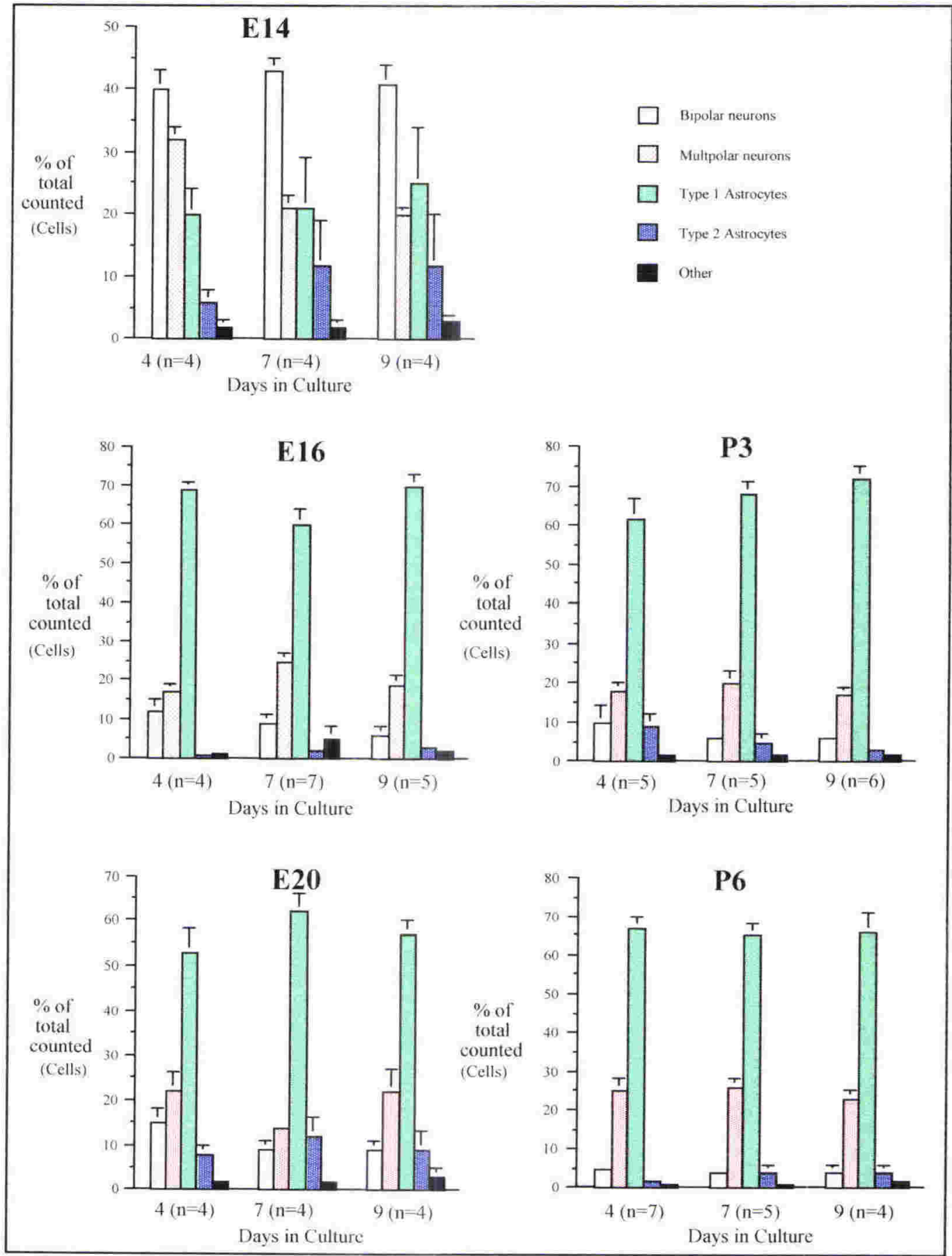

Figure 2.7

\section{Proportion of different cell types present in brainstem cultures of foetal and postnatal rats}

Proportion of different cell types in cultures from foetal and postnatal rat brainstems. Coverslips were removed, stained, and cell type distributions determined after 4, 7, and 9 days in culture. Mean \pm S.E.M of the percentage of total cells counted at each age vs. time in culture. Multiple cultures for each developmental age of rat were used, and the results were pooled ( $\mathrm{n}=$ number of pooled coverslips from two to four different culture preparations). 


\subsubsection{CELL MORPHOMETRY MEASUREMENTS}

Neuronal processes increased in length during culture (Figure 2.8 and Table 2.1) and made connections with cell bodies or processes of other neurons, but more commonly with glial cells. The mean cell body diameter was the same for foetal and postnatal cultures, with no size difference over time in culture or between bipolar and multipolar neurons (Figure 2.9). Neurite length increased in E16 foetal cultures between 4 and 7 days in culture $(P<0.005$, $\mathrm{n}=29$, Mann-Whitney test). In P6 cultures, neurite length increased from zero at day 1 to $162 \pm 20 \mu \mathrm{m}$ by 7 days in culture; however, there was no significant increase in neurite length between days 4 and 7 in these cultures. Total neurite length in foetal E16 cultures was $45 \%$ greater than in P6 cultures after 7 days $(\mathrm{P}<0.02, \mathrm{n}=31$, Mann-Whitney test).

\subsubsection{PRESENCE OF CHOLINERGIC NEURONS IN PRIMARY BRAINSTEM CULTURES OF THE RAT}

ChAT-positive cells were present in all developmental ages of primary brainstem cultures from E16 to P6. All ChAT-positive cells showed neuronal morphology, and no staining was observed on glial cells. The percentage of cells stained positive for ChAT was determined by counting ChAT-positive cells as a percentage of total cell nuclei from 3 fields of view from 2 separate coverslips from developmental ages E16, E20, P3, and P6 primary brainstem cultures of the rat. Approximately $5 \%$ of cell nuclei were found to be ChAT positive (not shown in Figure). Usually, ChAT-positive cells appeared in small clusters of 2-3 neighbouring ChAT-positive cells (Figure 2.10). Staining was diffuse in the cytoplasm of some cells (Figure 2.10A) and punctate in others (Figure 2.10B \& C). Cel processes were also stained (Figure 2.10A). 

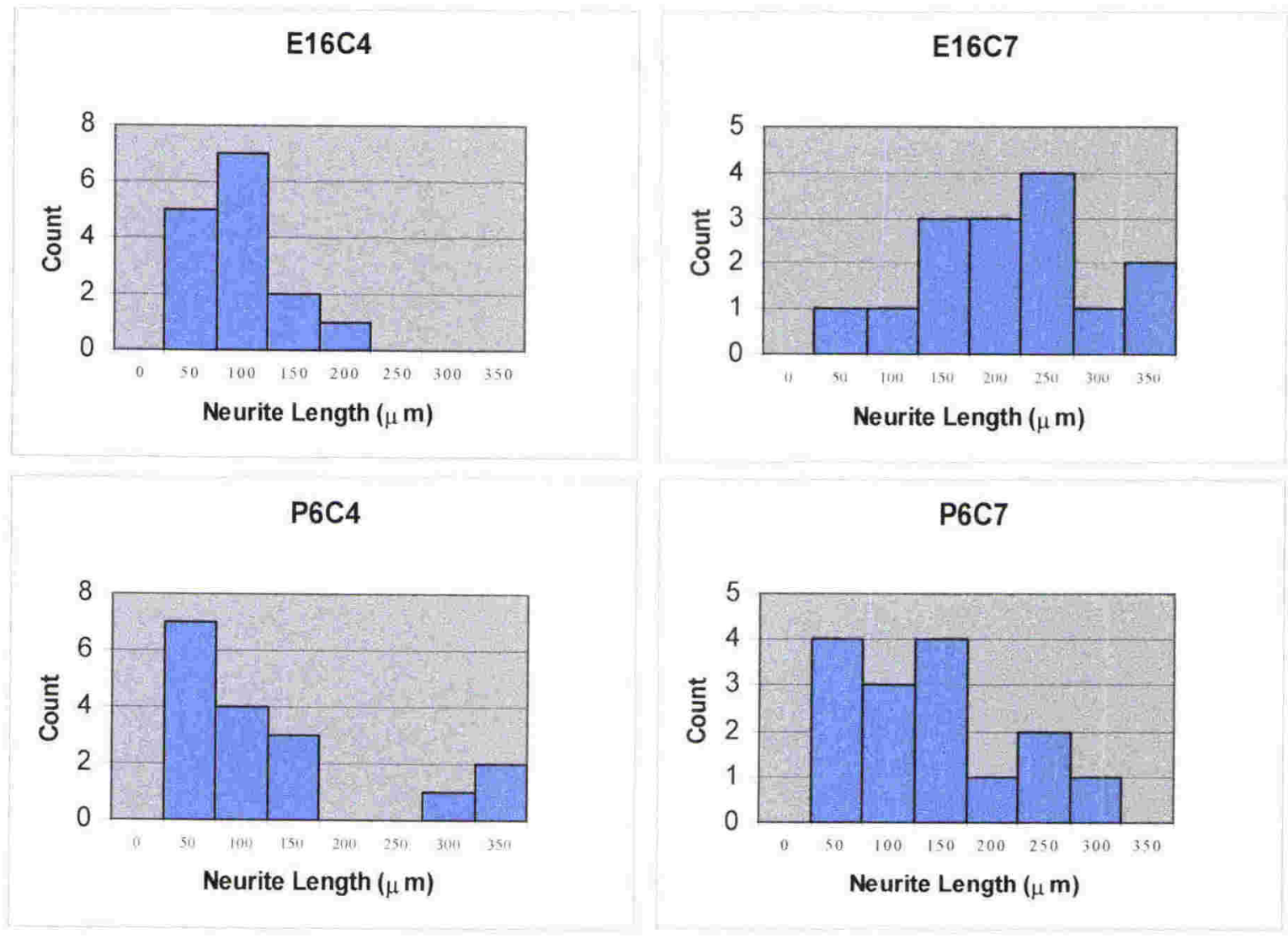

Figure 2.8

\section{Frequency Histograms of Neurite Length}

Frequency histograms of MAP2 positive neurons (count) and total neurite length for E16 and P6 cultures after 4 and 7 days in culture. E16C4 stands for E16 cells cultured for 4 days. 
E16C4

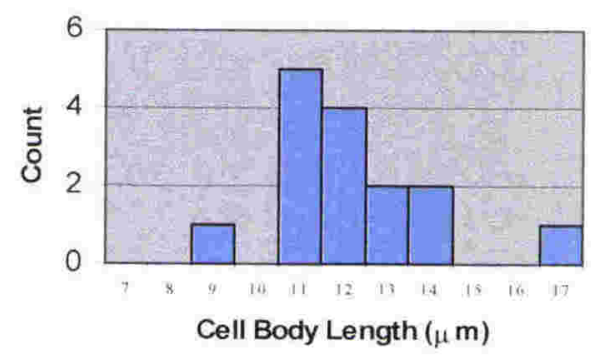

P6C4

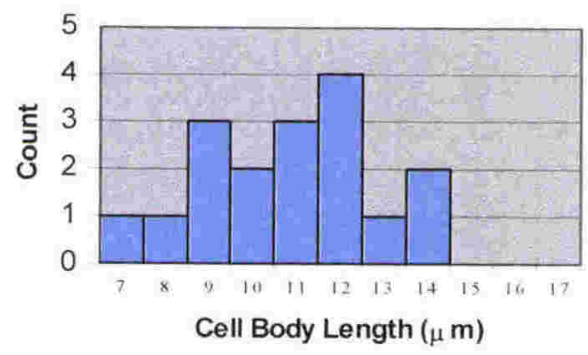

$\mathrm{E} 16 \mathrm{C} 7$

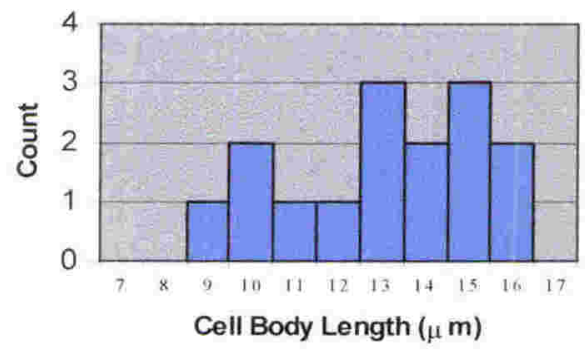

$\mathrm{P} 6 \mathrm{C} 7$

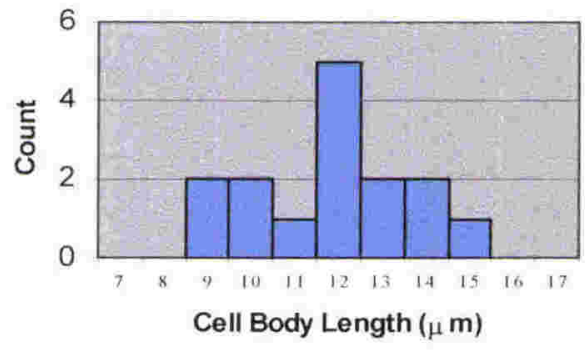

Figure 2.9

\section{Frequency Histograms of Cell Body Diameter}

Frequency histograms of MAP2 positive neurons showing cell body length for E16 and P6 cultures after 4 and 7 days in culture. E16C4 stands for E16 cells cultured for 4 days.

\begin{tabular}{|cccccc|}
\hline Culture type & Neuron type & Days in culture & $\begin{array}{c}\text { Cell Body diameter } \\
(\mathrm{mm})\end{array}$ & $\begin{array}{c}\text { Total neurite length } \\
(\mathrm{mm})\end{array}$ & Number of cells \\
\hline E16 & Multipolar & 4 & $12.4 \pm 0.3$ & $142 \pm 5$ & 5 \\
\hline & Bipolar & 4 & $12.1 \pm 0.6$ & $118 \pm 3$ & 10 \\
\hline & All Neurons & 4 & $12.2 \pm 0.5$ & $126 \pm 11$ & 15 \\
\hline E16 & Multipolar & 7 & $13.7 \pm 0.6$ & $252 \pm 6$ & 6 \\
\hline & Bipolar & 7 & $12.7 \pm 0.7$ & $224 \pm 6$ & 9 \\
\hline & All neurons & 7 & $13.1 \pm 0.6$ & $235 \pm 23$ & 15 \\
\hline P6 & Multipolar & 4 & $11.3 \pm 0.5$ & $168 \pm 11$ & 12 \\
\hline & Bipolar & 4 & $10.9 \pm 0.7$ & $116 \pm 3$ & 5 \\
\hline P6 & All Neurons & 4 & $11.2 \pm 0.5$ & $152 \pm 28$ & 17 \\
\hline & Muthipolar & 7 & $12.0 \pm 0.5$ & $173 \pm 6$ & 12 \\
\hline & Bipolar & 7 & $11.3 \pm 0.6$ & $118 \pm 3$ & 3 \\
\hline & All Neurons & 7 & $11.9 \pm 0.5$ & $162 \pm 20$ & 15 \\
\hline
\end{tabular}

\section{Table 2.1 Morphometric Data Summary}

Average values of cell body diameter and total neurite length (mean \pm SEM) for the data of Figure 2.8 and Figure 2.9. Cell body diameter was determined for the long axis of ellipsoid cells. Total neurite length was calculated as the sum of the lengths of individual neurites of a single cell. 

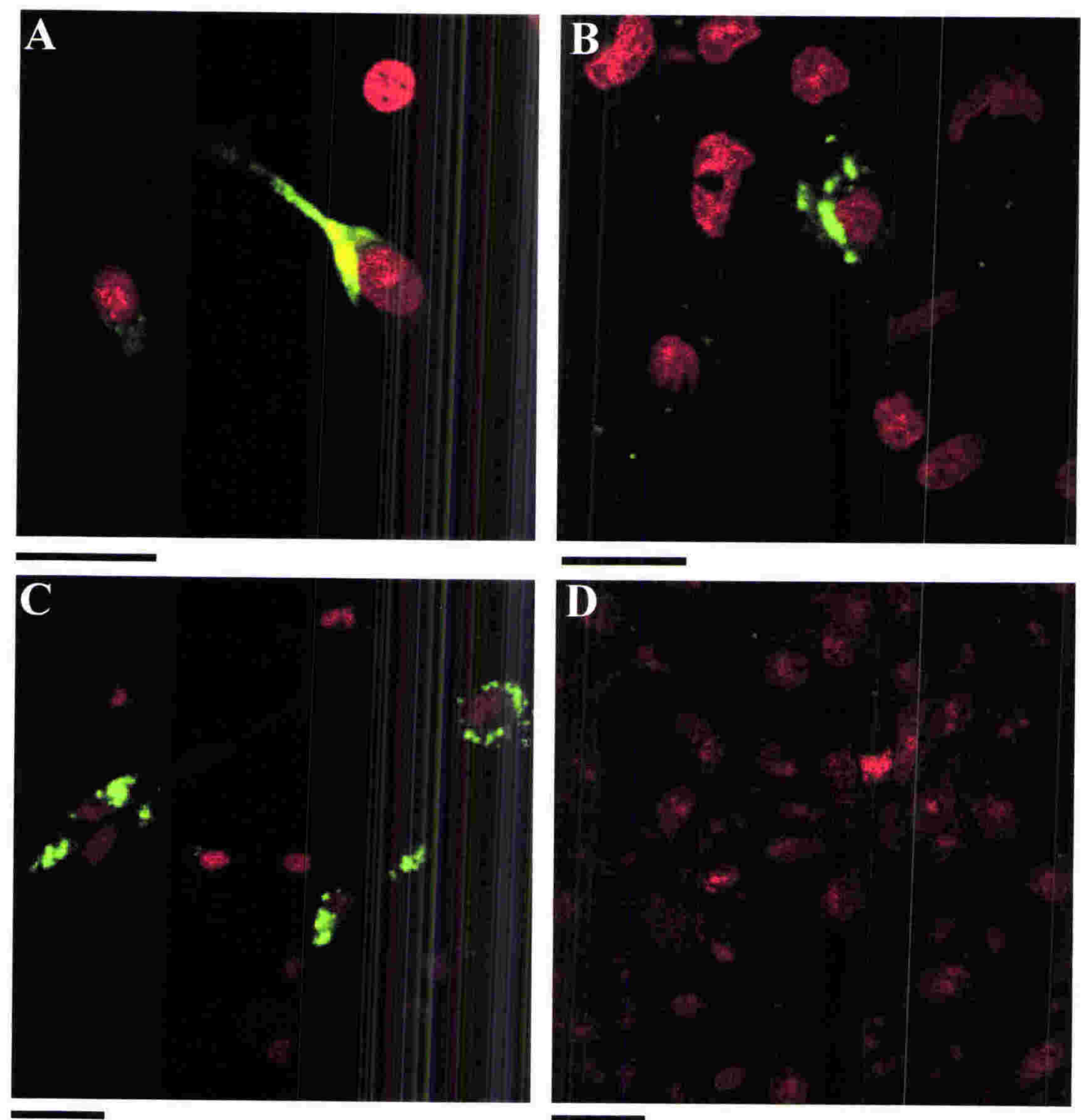

Figure 2.10

\section{ChAT-Labelled Cells}

Cells labelled positive for ChAT (green) from primary brainstem cultures from E16 (A), E20 (B), and P3 (C) rats, counterstained with propidium iodide (red) to visualise cell nuclei. The negative control cell line, LLC-PK 1 showed no ChAT staining (D). Scale Bars $=20 \mu \mathrm{m}$. 


\subsection{DISCUSSION}

\subsubsection{PRIMARY BRAINSTEM CELL CULTURE}

This study describes the first successful method for culture of late foetal and early postnatal animals in defined medium, using a commercially available neuronal medium containing supplements high in antioxidants. Few satisfactory protocols for primary culture of postnatal brainstem neurons have been reported, and commonly used procedures often give poor survival rates in older foetal and early postnatal brainstem cultures, despite the presence of serum (Azmitia, 1990; Miller and Azmitia, 1999). The use of primary culture as a model for in vitro studies of brainstem function is important because it more closely mimics the normal situation when compared with cultures of established cell lines. In a culture system, it is possible to trace individual neurons and neuronal processes and observe interactions between cells that would not be possible in brainstem slice studies. This method has been used successfully to study the survival of neurons, outgrowth of neurites, and to determine the proportion of different cell types in cultures from foetal and postnatal rats of different ages (Kivell et al., 2000, 2001). This culture system supports brainstem neuron survival in the absence of FCS, present only in the initial seeding to promote cell attachment. Glial cell feeder layers are also not necessary, thus reducing the amount of advance preparation required to set up the cultures. In addition, the use of Hibernate medium removes the time constraints normally faced when establishing primary cultures from brain tissue.

\subsubsection{METHOD DEVELOPMENT}

\subsubsection{Cell Dissociation}

A problem that arises when culturing cells from various brain regions of the neonatal rat is that some areas, such as the brainstem, are proportionally more mature than others, such a; the more rostral cortex, and are therefore difficult to establish and maintain in culture. Mature, developing neurons require increased interactions with trophic factors released from their target fields (Barde, 1989; Levi-Montalcini, 1995). These trophic factors, including nerve growth factor, brain-derived neurotrophic factor, and basic and acidic fibroblast growth factor, tend to be selective for different types of neurons (Varon and Adler, 1980; Barde, 1989; Barbacid, 1995; Levi-Montalcini, 1995). One study, using foetal (E18-19) rat cells cultures from the rostral ventrolateral medulla (RVLM), tested the effect of trophic factors on neurons expressing tyrosine hydroxylase. Glial derived 
neurotrophic factor, brain-derived neurotrophic factor, neurotrophin 3, neurotrophin-4/5, and ciliary neurotrophic factor increased the number of tyrosine hydroxylase positive cells in culture. However, nerve growth factor, transforming growth factor-beta, and basic fibroblast factor did not (Copray et al., 1999). The more immature a tissue is the more easily it can be dissociated, thus yielding higher numbers of viable cells. In the present cultures, there was better cell survival at E14 than later developmental ages. This may have been due to increased plasticity and immaturity of the younger neurons and their reduced requirements for trophic support. It is also possible that some of the cells present at E14 were either stem cells, progenitor or precursor cells that can give rise to neurons or astrocytes, depending on culture conditions. The increased requirement for trophic support in developmentally older cultures may also explain why neuronal processes from P6 cultures were significantly shorter than those from E16 cultures (Table 2.1) (P<0.02, Mann-Whitney test). This may also be due to the fact that trypsin was not used in tissue dissociation of foetal cultures, whereas trypsin was used in cultures prepared from developmentally older brainstems.

\subsubsection{Tissue Storage}

Hibernate ${ }^{\mathrm{TM}}$ is a serum-free medium containing $\mathrm{Ca}^{2+}$ and $\mathrm{Mg}^{2+}$. The use of Hibernate medium was a significant improvement in the present culture system because it allowed foetal tissue to be dissociated without the need for harmful exposure to $\mathrm{Ca}^{2+}-\mathrm{Mg}^{2+}$-free conditions, as well as permitting long-term storage of dissected tissue prior to culturing (Brewer and Price, 1996). Hibernate has been used for storage of living brain tissue for up to 4 weeks at $2-8{ }^{\circ} \mathrm{C}$ and ambient $\mathrm{CO}_{2}(0.2 \%)$ (Brewer and Price, 1996). Using Hibernate, the viability of dissociated cells was improved compared with use of the more common $\mathrm{Ca}^{2+}-\mathrm{Mg}^{2+}$-free tissue dissociation solutions (Copray and Leim, 1993). It was noted in the present study that undissociated foetal and postnatal brainstem tissue pieces $\left(2 \mathrm{~mm}^{3}\right)$ could be maintained in this storage medium for several days at $4^{\circ} \mathrm{C}$ without noticeable loss of viability. This reduced the time constraints of setting up cultures. Literature reports emphasise the necessity of rapid ( $1-2 \mathrm{~h}$ ) tissue dissociation and plating procedures to obtain good neuron viability and attachment (Buse, 1985). The two variations of Hibernate medium, E (embryonic) and A (adult), differ only in their osmolality (240 and $280 \mathrm{mOsm} / \mathrm{kg} \mathrm{H}_{2} \mathrm{O}$, respectively). Neurons are particularly sensitive to changes in osmolality, and the optimal osmolality for survival of mid-foetal neurons (E14-E18) is 
lower than that of late foetal neurons (E20). Early postnatal neurons tend to swell at lower osmolalities. Unfortunately, soon after the present method was developed and published, Hibernate medium was deleted from the Invitrogen range of cell culture reagents. It is now necessary to either produce the medium from its individual components or purchase it from its originator, Dr Gregory Brewer:

[gbrewer@simed.edu<mailto:gbbrewer@simed.edu]. The protocols for making Hibernate-A, and -E are described in the Appendix of this thesis (Appendix 1).

\subsubsection{Choice of Medium}

Neurobasal $^{\text {TM }}$ medium is a serum-free, bicarbonate-buffered medium that was originally developed for optimising growth of foetal rat hippocampal neurons (Brewer, 1997). Neurobasal medium also supports neuron growth and differentiation in cultures of rat cells derived from other brain regions, including cortex, cerebellum, dendate gyrus, striatum, and substantia nigra (Brewer, 1995). The composition of Neurobasal medium is based on DMEM (Brewer et al., 1994). DMEM medium has a higher osmolality, however, than other reported media that have been optimised for the culture of foetal neurons (Brewer, 1997). In the present study it was shown that Neurobasal medium effectively supported the maintenance of E14-E18 rat brainstem neurons, and Neurobasal-A medium supportec the maintenance of more advanced E20 and postnatal rat brainstem regions, wher supplemented with B27. B27 is an antioxidant-rich, serum-free medium supplement (Brewer et al., 1993). B27 supplement can replace the obligatory requirement for serum, once neurons have attached to the substrate. In Neurobasal/B27, our neuron survival rate was higher than that in Neurobasal containing 10\% FCS. Coverslips were coated with poly-D-lysine and FCS to promote attachment, but no serum was added to the mediun itself during the actual cultures. Thus, culture conditions were completely defined after plating. Defined media are important for providing complete control over in vitro conditions. Studies that use FCS-containing medium, such as those of Ternaux and Portalier (1993) (Ternaux and Portalier, 1993) and Masuko et al. (1986) (Masuko et al., 1986), report good neuron stability, however, FCS content is unknown and variable between batches and FCS supports astrocyte division. In the present study, culture in a defined medium without FCS showed similar neuron survival without the inherent disadvantages of serum use. 


\subsubsection{Glutamine/Glutamate Toxicity}

A major problem encountered when growing neurons in culture is their susceptibility to glutamine toxicity, yet cultures without glutamine tend to grow poorly. L-glutamine produces ammonia, a toxic breakdown product (Minamoto et al., 1991). GlutaMax I (Lalynyl-L-glutamine) does not produce ammonia and was used to replace half of the glutamine to maximise neuronal survival. Neurotoxicity can also occur from glutamate, which is thought to play a role in neurodegenerative disorders, including Alzheimer's disease (Arias et al., 1997). Glutamate excitotoxicity has been extensively studied in cell culture (Kassof et al., 1995; MacManus et al., 1997). Using glutamate-free media containing serum, Brewer et al. (Brewer et al., 1994), have shown that DMEM-F12 provides better neuronal survival than DMEM in cultures of some brain regions (brainstem not reported). Neurobasal medium, although DMEM based, is glutamate-free. The present study used a modification of the method of Cheung et al. (1997), originally developed for mesencephalic cultures (Cheung et al., 1997). Neurobasal medium with B27 supplement gave better neuron survival than glutamate-containing DMEM-F12 with B27 supplement. This improved survival in Neurobasal compared with DMEM-F12 or DMEM has been attributed to the omission of the excitatory amino acids, glutamate and aspartate, as well as the lower osmolality of the culture medium (Brewer et al., 1994). The addition of B27 supplement to the medium during cell dissociation and culture also reduces cell damage caused by free radical production.

\subsubsection{SEEDING DENSITY AND CELL ATTACHMENT}

Primary culture of post-mitotic neurons from distinct brain regions requires the use of large numbers of animals in order to have enough cells to plate at high densities $\left(1 \times 10^{6}\right.$ cells $/ \mathrm{cm}^{2}$ or higher) required for viable cultures (Azmitia, 1990). In the present study, survival was excellent at seeding densities as low as $1 \times 10^{5} \mathrm{cell} / \mathrm{s} / \mathrm{cm}^{2}$. The seeding density used here allowed between 4 to 10 culture dishes ( $35 \mathrm{~mm}$ diameter, each containing 4 coverslips) to be seeded from one litter of rat pups.

Poly-D-lysine gave the most successful and consistent cell attachment and neurite outgrowth in culture. This conclusion is supported by the findings of Brewer and Cotman (1989) (Brewer and Cotman, 1989) who also found that poly-D-lysine reduces the aggregation of neurons compared with poly-L-lysine. Other commonly described 
attachment substrates include collagen and fibronectin (Mains and Patterson, 1973; Masuko et al., 1986). These were not tested in the present study. Failure to pre-treat the coverslips with serum prevented most neurons from attaching to the coverslip surface, indicating the need for attachment factors present in serum. A review by Barnes (Barnes and Sato, 1980) describes the role of serum in providing specific attachment factors in neuronal cultures. These attachment factors include fibronectin-like molecules, cell adhesion and spreading factor, and cold-insoluble globulin.

\subsubsection{CULTURE CHARACTERISTICS}

\subsubsection{Cell Specific Markers}

In the present study, different types of cells in culture were identified using phase contrast morphology and staining profiles with antibodies against MAP2 and GFAP. GFAP is only present in glial cells (Raff et al., 1979). MAP2 localisation is specific for neurons and neural precursor cells (Sarnat et al., 1997). Distributions of MAP2 during normal development, (Cáceres et al., 1983; Binder et al., 1984; Matus, 1988; Chauhan and Siegel, 1997) and during development in culture have been well established (De Lima et al., 1997). In mature neurons in situ and in cultured cells after several days in culture, MAP2 staining is found predominantly in dendrites, and the axons are unstained (Kadota et al., 1997). In the present study, all neurites, whether dendrites or axons, stained positively for MAP2 for at least the first 9 days in culture.

Neurite growth occurred by an increase in length as well as an increase in the number of branches with time in culture. This was observed for all developmental ages tested, although at younger developmental stages, neurite growth tended to be faster, especially immediately after plating. Cultures could be successfully maintained for up to 3 weeks with little neuronal cell death. The number and type of neurons present in culture were unchanged between 3-9 days for all developmental ages tested. A few cultures were also observed for longer time periods with little differences seen. In cultures established from older developmental ages (P3 and P6), the neurons tended to be multipolar, whereas at younger developmental ages (E14 and E16), the neurons were mostly bipolar. These results support the findings of de Boer-Van Huisen et al. (de Boer-van Huizen and ten Donkelaar, 1999) who showed that brain tissue from E14 foetuses contained more mature multipolar cells than tissue from E12 foetuses, suggesting that the number of neurite 
connections increases with age. Mature neurons require more trophic support from their target tissues than immature neurons. The increased neurite complexity of developmentally older neurons and the complex connections formed with other neurons and glial cells may in part explain why postnatal and late foetal tissues are more difficult to establish in culture.

\subsubsection{Neural-Glial Associations}

Many multipolar and bipolar neurons at all developmental ages tested and after 4, 7 and 9 days in culture tended to be associated with glial cells. Usually neurons were observed on top of type 1 astrocytes, although there were always some isolated neurons present in the same cultures. This observation is consistent with findings in other culture systems established from different brain regions (Trenkner, 1992). Miller and Azmitia (1999) reported neuronal-glial connections in E14 and E16 cultures of cholinergic neurons from ventral forebrain. Neurons connected to glial cells stained more intensely with ChAT antibody than unconnected neurons (Miller and Azmitia, 1999). In the present study, the proportion of glial cells from all developmental ages remained constant between days 4 and 9 in culture (Figure. 2.7). Since the proportion of astrocytes did not change with time in culture, it is clear that this Neurobasal/B27 medium inhibits the proliferation and overgrowth of astrocytes, although astrocytes remained viable. It is well-established that serum-free culture conditions inhibit astrocyte cell division (Marriott et al., 1995). The lack of glial cell proliferation means that cultures did not need to be treated with an agent such as 5-fluoro-2-deoxyuridine (FDU) or $\beta$-D-cytosine arabinoside (Ara C) to inhibi division of non-neuronal cells. This is advantageous for setting up healthy cultures because addition of Ara $\mathrm{C}$ has been shown to cause neuronal cell death (Wallace and Johnson, 1989), and exposure to FDU for greater than $24 \mathrm{~h}$ can also be neurotoxic.

\subsubsection{Presence of Cholinergic cells in primary brainstem cultures of the rat}

ChAT-positive cells were present in cultures from the rat brainstem. The choline acetyl transferase (ChAT) antibody is commonly used as a marker for cholinergic neurons (Knusel and Hefti, 1988; Burton et al., 1995; Chen et al., 2001). The presence of cholinergic neurons was tested to help determine if brainstem neurotransmitter characteristics were maintained in culture. ChAT-positive neurons are found in many brainstem areas including the NTS and spinal trigeminal tract (Barry et al., 1993; Barry et 
al., 2001), areas also shown to express opioid receptors (Delfs et al., 1994; Mansour et al., 1994; Arvidsson et al., 1995a; Arvidsson et al., 1995b; Bausch et al., 1995a; Mansour et al., 1995; Moriwaki et al., 1996; Cahill et al., 2001b). In the present study ChAT-positive neurons were found in primary cultures of the rat brainstem at all stages of development tested from E16 to P6.

\subsection{CONCLUSIONS}

A serum-free primary culture system was successfully developed for establishing and maintaining foetal brainstem cells in culture. In addition, it was possible to culture postnatal brainstem neurons up to age P6 in a completely defined medium. These studies reinforce several key factors that must be considered when culturing late foetal and early postnatal brainstem neurons: (1) optimal osmolalities must be provided for different developmental ages of neurons; (2) antioxidant-rich medium supplements should be included in culture media; (3) glutamine cytotoxicity should be controlled by partial replacement with GlutaMax I; (4) culture media that do not include glutamate should be used, and (5) Hibernate medium/B27 (Invitrogen) should be considered to increase neuron survival during tissue dissociation. Taking account of these factors, this study has provided the first reliable method, using a defined culture medium, that supports the culture of brainstem neurons from late foetal and early postnatal stages of the rat. A wellcharacterised cell culture system allows developmental studies of the rat brainstem to be carried out at a later developmental age than has previously been possible. 


\section{CHAPTER 3}

\section{Opioid Receptor Distribution In Cultured Cells}

\subsection{AIM}

The aim of the present study was to characterise mu opioid receptor (MOR) and delta opioid receptor (DOR) expression during late foetal and early postnatal development of the rat using primary cultures of dissociated brainstem cells. Using the culture technique described in Chapter 2, changes in the expression patterns of MOR and DOR during development were investigated.

\subsection{INTRODUCTION}

\subsubsection{A NOVEL BRAINSTEM CELL CULTURE SYSTEM FOR STUDYING OPIOID RECEPTOR EXPRESSION DURING DEVELOPMENT}

Using the dissociated brainstem cell culture method previously developed (Chapter 2) (Kivell et al., 2000, 2001), brainstem cells from late foetal and early postnatal stages were used to study opioid receptor expression during early development, a critical time when neuronal control of cardiovascular and respiratory function is established (Moss and Laferrière, 2002). MOR and DOR action in the brainstem has been shown to modulate cardiovascular and respiratory functions (Laferrière et al., 1999) (for reviews see (Guyenet et al., 1994; Vaccarino and Kastin, 2001; Pugsley, 2002)). Primary cell cultures were used because they more closely resemble the in vivo brainstem than established neural cell lines in part because of the heterogeneous population of neurons and astrocytes present in the cultures. A secondary advantage of using primary cultures is that established cell line often have random loss or gain of genotypes and phenotypes. Thirdly, an established cell line that models brainstem cells does not exist. A better understanding of opioid receptor expression during early brainstem development is thus possible using primary culture techniques coupled with immunocytochemistry (ICC) and confocal microscopy (Ojcius et al., 1996).

\subsubsection{OPIOID RECEPTOR IMMUNOCYTOCHEMISTRY}

Several different methods were used in the present study to achieve optimal opiod receptor visualisation in cultured cell lines and in primary brainstem cell cultures. When 
this project was first started, there were few commercially available opioid receptor antibodies. The antibodies that were available were of questionable quality, presumably due to the difficulty generally encountered in making good antibodies to G-protein-coupled receptors. Problems with detection of antibody signal as a result of low expression levels were also common. These difficulties made it necessary to pre-test opioid receptor $(\mathrm{OpR})$ antibodies from different sources in order to choose the one with the highest specificity and to evaluate that antibody with different signal amplification strategies. One amplification method that was used was Tyramide Signal Amplification (TSA ${ }^{\mathrm{TM}}$ ) (Perkin Elmer Life Sciences Inc., Boston, MA, USA). This reagent is available in a complete kit designed to amplify weak signals [Buki, 2000 \#893; Shindler, 1996 \#938]. TSA uses HRP enzyme to catalyse the deposition and covalent cross-linking of haptens (FITC, Rhodamine, Biotin), or other detection reagents to the HRP-conjugated antibody. TSA allows a lower concentration of primary antibody to be used, thus, minimising non-specific primary antibody binding that can occur at high concentrations, while generating the high signal amplification that is necessary for the receptors to be detected (De Haas et al., 1996; Hunyady et al., 1996).

\subsubsection{Opioid Receptor Localisation in SH-SY5Y Cells}

Previous studies have shown that SH-SY5Y human neuroblastoma cells endogenously express MOR, DOR and KOR (Yu et al., 1986; Cheng et al., 1995a). The exact expression level of opioid receptors on these cells varies according to the methods used to detect OpR expression and whether the cells are differentiated or not. Kazmi and Mishra (1986) repor a 2/1 ratio of MOR/DOR (Kazmi and Mishra, 1986); whereas, Yu et al. (1988) report a ratio of 5/1 (Yu and Sadée, 1988). When SH-SY5Y cells are differentiated with all-tran; retinoic acid (RA), the neuroblastoma cells become more like mature neurons (Påhlman et al., 1984). The density of MOR increases by $32-40 \%$, whereas DOR density remains the same (Yu and Sadée, 1988). The levels of MOR mRNA have also been shown to increase on differentiation (Jenab and Inturrisi, 2002). The amount of MOR protein at the SHSY5Y cell surface has been reported to be in the range of $43 \mathrm{fmol} / \mathrm{mg}$ protein (Law et al., 2000c). MOR expression on SH-SY5Y cells has been further classified to be of the $\mu_{2}$ subtype because the receptors reversibly bind naloxonazine (Elliott et al., 1994). Naloxonazine is known to irreversibly bind and block $\mu_{1}$ opioid receptors (Ling et al., 1986; Pick et al., 1991); thus, reversible binding suggests that $\mu_{2}$ receptors are present. The 
SH-SY5Y cell line has become a well-characterised, reproducible cell model for studying opioid receptor regulation and function (Påhlman et al., 1990; Gies et al., 1997) and provides an ideal positive control for opioid receptor immunoreactivity.

\subsubsection{Opioid Receptor Localisation in Primary Culture}

Many studies have used ligand binding, autoradiography and biochemical responses to confirm the presence of OpRs in primary cultures of dissociated cells from rat brain (Vaysse et al., 1990), brainstem (Miller and Azmitia, 1999), and dorsal root ganglion (Fan and Crain, 1995; Chen et al., 1997). Few studies, however, have used ICC to look at opioid peptides or opioid receptors in primary cultures, with the exception of He (1992) who detected dynorphin and enkephalin presence in dendate gyrus cell cultures, KulkarniNarla (2001) who studied DOR and KOR expression in ileal myenteric neurons (He et al., 1992; Kulkarni-Narla and Brown, 2001) and Lee et al. (2002) who studied fluorescent ligand binding in cortex cultures (Lee 2002). No ICC localisation of opioid receptors has been reported in primary cultures of the brainstem. Most studies on OpR functions in primary culture use rat dorsal root ganglion cells (MacDonald and Werz, 1986; Werz et al., 1987), striatal cells (Maus et al., 1990), hippocampal cells (Vaysse et al., 1990), hypothalamic cells (Di Scala-Guenot et al., 1990; Vaysse et al., 1990), and cells cultured from the cerebral cortex (Erikson et al., 1990; Erikson et al., 1992). Cells cultured from different brain regions may regulate OpRs differently due to expressions of different forms of adenylyl cyclase, and g-protein coupled receptors in these regions. The present study provides a good immunocytochemistry based model for further studies on opioid receptor expression in cultured cells of both primary and established cell lines.

\subsubsection{Developmental expression of MOR and DOR}

Previous studies have shown that $\mathrm{OpR}$ expression changes during development. These changes have been described in detail in Chapter 1 (Section: 3.2.1) and Chapter 6. Briefly, OpR binding studies show that MOR and DOR expression levels increase during late foetal and early postnatal development (Tsang et al., 1982; Villiger et al., 1982; Kornblum et al., 1987; Attali et al., 1990; Xia and Haddad, 1991; Zhu et al., 1998). The level of expression is dependent on the brain region studied. Opioid receptor development occurs earliest in the brainstem and later in the striatum, midbrain and cortex (Coyle and Pert, 
1976). The functional implications of changes in expression during development have been discussed in Chapter 1 (Section: 1.3.7 and 1.4). 


\subsection{METHODS}

\subsubsection{CELL CULTURE}

\subsubsection{Dissociated Brainstem Cell Culture}

Primary cultures from brainstems of foetal and early postnatal rats were grown on poly-Dlysine-coated coverslips in serum-free Neurobasal culture medium supplemented with B27, as described in Chapter 2 (Section: 2.3.1).

\subsubsection{SH-SY5Y Cell Culture}

The human neuroblastoma cell line SH-SY5Y was obtained from Dr Mark Grimes (Massey University, Palmerston North, New Zealand). The cell line was grown in Roswell Park Memorial Institute 1640 medium (RPMI-1640, GIBCO, Invitrogen) containing 5 $\mathrm{mg} / \mathrm{L}$ phenol red, $2 \mathrm{~g} / \mathrm{L} \mathrm{Na} \mathrm{CO}_{3}, 30 \mathrm{nM}$ selenium, 10\% FCS (v/v), $50 \mathrm{U} / \mathrm{ml}$ penicillin $\mathrm{G}$, and $50 \mu \mathrm{g} / \mathrm{ml}$ streptomycin sulphate. The cell line was grown either directly on the polystyrene surface of the culture dish or on circular coverslips (13 mm diameter). Coverslips had previously been cleaned and then coated with $50 \mu \mathrm{L}$ of $10 \mu \mathrm{g} / \mathrm{mL}$ poly-Dlysine (Sigma) in the same way as described for primary brainstem cultures (Chapter 2, Section: 2.3.1).

Cell differentiation was induced by addition to the culture medium of $1 \mu \mathrm{L} / \mathrm{mL}$ all-trans retinoic acid (RA) (Sigma) (10 $\mathrm{M}$ stock in 100\% ethanol). The $1000 \mathrm{x}$ stock solution of $10 \mu \mathrm{M}$ RA was kept at $-20^{\circ} \mathrm{C}$ in the dark. Cells were differentiated for between $7-14$ days and fed twice weekly by removing the entire medium by aspiration and replacing it with fresh medium containing RA.

Stock cultures of SH-SY5Y cells were passaged every 5-7 days by washing the attached cells with sterile PBS, then adding trypsin solution to dissociate the cells $(0.05 \%$ trypsin; $5.3 \mathrm{mM}$ EDTA; Invitrogen). Culture medium containing FCS was added to the culture dish, the cell suspension was transferred to a $15 \mathrm{~mL}$ sterile centrifuge tube (Becton Dickinson, Christchurch, NZ) and centrifuged in a bench-top centrifuge for $3 \mathrm{~min}$ at 1200 rpm. The supernatant was discarded, the cell pellet resuspended in fresh medium, and the cell suspension transferred into new culture dishes. 


\subsubsection{LLC-PK1 Cell Culture}

A pig kidney cell line, LLC-PK 1 , obtained from Flow Laboratories (Ervine, Ayrshire, Scotland) was cultured in RPMI-1640 medium (Invitrogen). Cells were grown on coverslips and maintained and passaged in the same way as described above for SH-SY5Y cells. LLC-PK, cells differentiate spontaneously upon reaching confluence, as evidenced by the formation of domes in cultures. Domes are raised areas of epithelium that form in culture on non-porous surfaces as a result of vectorial (apical to basal) fluid transport under the cell monolayer (Mullin et al., 1980).

\subsubsection{IMMUNOCYTOCHEMISTRY}

\subsubsection{General Immunocytochemistry Methods}

All cell culture samples were fixed, washed, and had endogenous peroxidase activity removed as described in Chapter 2 (Section: 2.3.2.2) and Appendix 2 (Section 3). The samples were washed between all steps for $3 \times 5$ min each in PBS containing $0.2 \%$ Triton, except after blocking. Non-specific primary antibody binding sites were blocked at room temperature for 30-60 $\mathrm{min}$ in the appropriate blocking solution. For non-TSA-amplified samples, the blocking solution consisted of PBS containing $2 \%$ normal goat serum (NGS), $4 \%$ bovine serum albumin (BSA), and $0.2 \%$ Triton. For TSA amplification, the blocking solution was supplied in the TSA kit. Blocking solutions were stored in $25 \mathrm{~mL}$ aliquots at $-20^{\circ} \mathrm{C}$. All antibodies were also diluted in blocking solution to the appropriate concentration. Primary antibodies were incubated with cells at $4^{\circ} \mathrm{C}$ overnight. Secondary antibodies were incubated for $2 \mathrm{hrs}$ at room temperature, unless otherwise stated. $\mathrm{Al}$ samples labelled with fluorescence probes were mounted onto slides cell-side up using anti-fade mounting medium (Appendix 2: Section 7) and covered with number 0 coverslips $(\mathrm{BDH})$. The edge of the coverslips was sealed with clear nail varnish or Repair Vulcanising Rubber Solution (bicycle tyre repair glue) (Weldtite Products, Barton on Humber, England) and protected from light. Samples were viewed, scanned and photographed immediately after fluorescent staining, although fluorescently labelled cells could be stored for 1-2 days at $4^{\circ} \mathrm{C}$ in the dark with only minor loss of fluorescence intensity. Some DAB-stained coverslips were counterstained using $3 \%$ toluidine blue by immersion in the solution for several minutes, washing and mounting. Toluidine blue stains cell nuclei and improves visualisation of non-labelled cells, thus allowing all cells to be counted. 
To study OpR expression during development, all paired samples from each developmental age and each time in culture were collected and stained at the same time as a matched set. $\mathrm{DAB}$ colour development was stopped after the same duration of staining in these agematched samples. This procedure was used to prevent any differences arising from variations in $\mathrm{DAB}$ colour development when the same samples were tested on different days.

\subsubsection{AMPLIFICATION AND DETECTION METHODS}

\subsubsection{Digoxigenin Secondary Antibody Amplification Method}

Digoxigenin (DIG) (1:10, anti-rabbit DIG), 1:10, anti-mouse DIG), Chemicon), followed by anti-DIG HRP (1:500, Roche), was used as an alternative to the biotin/avidin amplification method. DIG-conjugated antibodies have the advantage of forming few nonspecific interactions with animal tissues, unlike avidin/biotin, which may bind nonspecifically to animal tissues.

\subsubsection{Tyramide Signal Amplification}

FITC- or Rhodamine- conjugated: FITC or Rhodamine labelled TSA was used to amplify and visualise HRP-conjugated secondary antibodies. After incubation in secondary antibody, samples were washed $(3 \times 5 \mathrm{~min})$ as previously described, then incubated with TSA solution containing 1:50 dilution of amplification reagent in the supplied buffer, according to the manufacturer's instructions. Coverslips were incubated for $4 \mathrm{~min}$ at room temperature, washed as described previously, and mounted onto slides using anti-fade mounting medium.

Biotin-conjugated: TSA ${ }^{\mathrm{TM}}$-Biotin was used as an alternative to biotin labelled secondary antibodies because it increases the amplification of the signal. Primary antibodies were incubated with cells as described previously, washed, incubated in HRP-conjugated secondary antibodies (anti-guinea pig IgG HRP; 1:2000 for MOR, and anti-rabbit IgG HRP; 1:5000 for DOR), washed, then incubated in TSA-Biotin (1:50), as per manufacturer's instructions. After incubation in TSA TM- Biotin (3-10 min) the tissue was washed, then incubated in ExtrAvidin-HRP (1:1000) and washed again before DAB colour development, as described previously in Chapter 2 (Section: 2.3.2.3) and Appendix 2 (Section: 4). 


\subsubsection{PRIMARY ANTIBODIES}

\subsubsection{Rabbit anti-MOR (Chemicon)}

Several different antibody staining protocols and combinations of antibodies were used for MOR ICC (Table 3.1). Initially rabbit polyclonal MOR antibody against the C-terminal of the rat MOR receptor was used (Chemicon International, 1:2000-1:5000; Temecula, CA, USA). The secondary antibody was FITC-coupled, sheep anti-rabbit IgG (1:50-1:150; Sigma).

\subsubsection{Rabbit anti-MOR (gift of Dr Stephan Schulz)}

This anti-MOR antibody, raised against the amino terminal of the rat MOR, was kindly supplied by Dr Stephan Schulz from the laboratory of Professor Völker Höllt (Otto-von Guerich University, Germany) (Schulz et al., 1998). Briefly, rabbit anti-MOR primary antibody was used at a dilution of 1:3000 for TSA-FITC, TSA-Rhodamine or TSA-Biotin amplification. A dilution of 1:1000 was used for non-TSA amplification for either DIG or Biotin/ExtrAvidin amplification, as described in amplification and detection methods (Section: 3.3 .3$)$.

\subsubsection{Rabbit anti-MOR (Research and Diagnostics Antibodies)}

Rabbit anti-MOR serum, specific for the carboxyl terminal of the rat MOR (Research and Diagnostic Antibodies (R\&D), Berkeley, CA, USA) (1:500) was incubated with either cultured brainstem cells, LLC-PK 1 cells, undifferentiated or RA-differentiated SH-SY5Y cells. Signal amplification was carried out using anti-rabbit IgG biotin for $2 \mathrm{~h}$ at room temperature, followed by ExtrAvidin-HRP (Sigma). Antibody binding was visualisec using both colourmetric (DAB) and fluorescence methods. Immunofluorescence was achieved by substituting ExtrAvidin-FITC (1:1000; Sigma) for ExtrAvidin-HRP. 


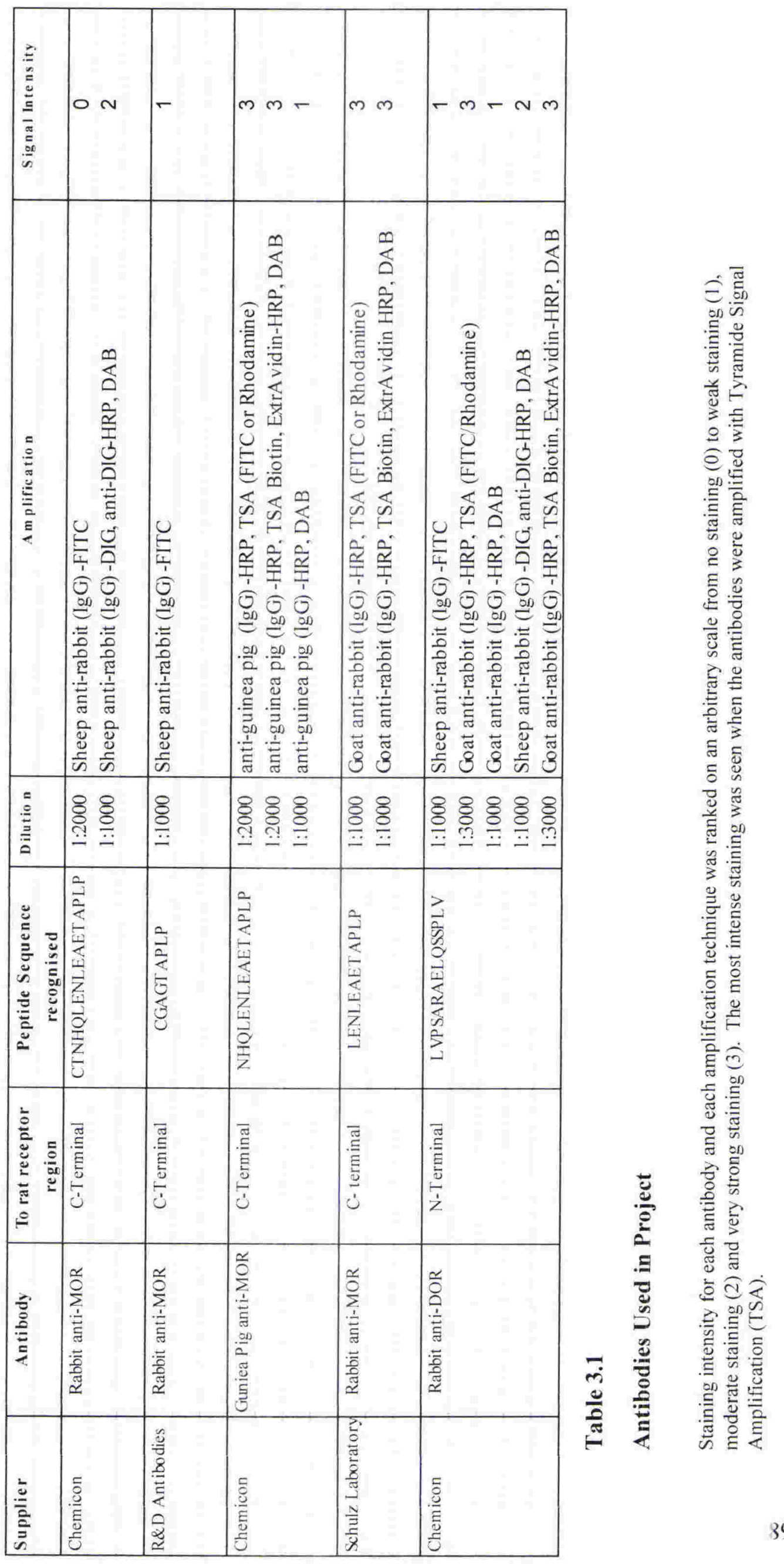


Negative controls were set up by: i) incubation in the absence of primary antibody or ii) incubation with primary antibody in a cell line (LLC-PK $)_{1}$ that does not express MOR and DOR or iii) pre-incubation of the primary antibody with an excess of the MOR peptide used to generate the antibody $(250 \mu \mathrm{g} / \mathrm{mL}$; Research and Diagnostic Antibodies, Berkeley, $\mathrm{CA}$ ) in order to compete with the tissue epitope sites. The peptide was added to the diluted antibody solution $45 \mathrm{~min}$ prior to incubation with the tissue in order to allow binding of the peptide to the primary antibody.

\subsubsection{Guinea Pig anti-MOR (Chemicon)}

The guinea pig (GP) anti-MOR antibody corresponds to amino acid residues 384-398 of the carboxyl-terminus of the cloned rat MOR-1. Briefly, samples were incubated with primary antibody, GP anti-MOR (1:2000) followed by anti-GP HRP (1:2000, Zymed Laboratories Inc., San Francisco, CA, USA) and then amplified by the TSA (FITC, Rhodamine or Biotin) amplification protocol.

\subsubsection{Rabbit anti-DOR (Chemicon)}

To detect DOR in cultured cells using colourmetric DAB reagent, rabbit anti-DOR antibody (1:1000) against the rat receptor was incubated with cells, the cells were washed, and then incubated with anti-rabbit DIG (1:10), washed again, then incubated with antiDIG HRP (1:500). To detect DOR by fluorescence, primary antibody (rabbit anti-DOR) was incubated at a lower concentration (1:3000) than for colourmetric detection, the cells were washed, then incubated in anti-rabbit IgG DIG (1:10), washed again, then incubated in anti-DIG HRP (1:500), washed once more, then treated with TSA (FITC or Rhodamine) reagent.

\subsubsection{DOUBLE-LABELLING EXPERIMENTS}

Double-labelling experiments were carried out using a method modified from Rocheville et al. (2000) and Pecci Saavedra et al. (1993) (Pecci Saavedra et al., 1993; Rocheville et al., 2000). Double-labelling experiments were carried out using a neuronal specific marker, mouse anti-MAP2 (1:1000, Sigma), and guinea pig anti-MOR (1:2000, Chemicon) or rabbit anti-DOR (1:2000, Chemicon). Alternatively, samples were double-labelled with MOR and DOR antibodies instead of OpR and MAP2 antibodies. Double-labelling was possible because the primary antisera were raised in different animal species (Figure 3.1). 
Double-labelling was carried out in the same way as for single-labelling, except where indicated. Primary antibodies were incubated together on the same samples in blocking solution overnight, washed, incubated in one HRP-conjugated secondary antibody (for example for MAP2 detection, anti-mouse IgG-HRP) for $1 \mathrm{hr}$ at $37^{\circ} \mathrm{C}$, washed $(3 \mathrm{x} 5 \mathrm{~min})$, amplified using TSA-FITC, and washed again. Peroxidase activity was then removed by incubating the cells for $15 \mathrm{~min}$ in hydrogen peroxide solution (Appendix 2: Section 3) but replacing $50 \%$ methanol with $\mathrm{ddH}_{2} \mathrm{O}$ since methanol causes a reduction in the fluorescent signal. The sample, labelled in this case for MAP2, was then washed and incubated with the other secondary antibody specific for the second primary antibody (for example, for MOR detection, anti-GP-HRP) as described above, washed ( $3 \times 5 \mathrm{~min}$ ), then incubated with TSA-Rhodamine, washed, then mounted onto slides in anti-fade mounting medium. Care was taken after fluorescence labelling to protect the samples from light during washes and subsequent incubations. When using this double-labelling method, it was necessary to carry out multiple controls to ensure specificity of the staining. These controls are described in Table 3.2 .

\subsubsection{COUNTERSTAINING OF THE NUCLEUS}

Propidium iodide (PI) was used to label the nucleus in some fluorescently labelled samples to help pinpoint antibody localisation within the cell body and identify the proportion of positively labelled MOR and DOR cells. Without PI labelling of the nucleus, it is difficult to identify individual cell bodies in cell clumps. PI stock $(100 \mu \mathrm{g} / \mathrm{mL}$ in anti-fade solution and stored in the dark at $4^{\circ} \mathrm{C}$ ) was diluted immediately before use in PBS washing buffer to a final concentration of $1 \mu \mathrm{g} / \mathrm{mL}$. Samples were then counterstained with PI after fluorescent labelling was complete, then washed and mounted as previously described. 


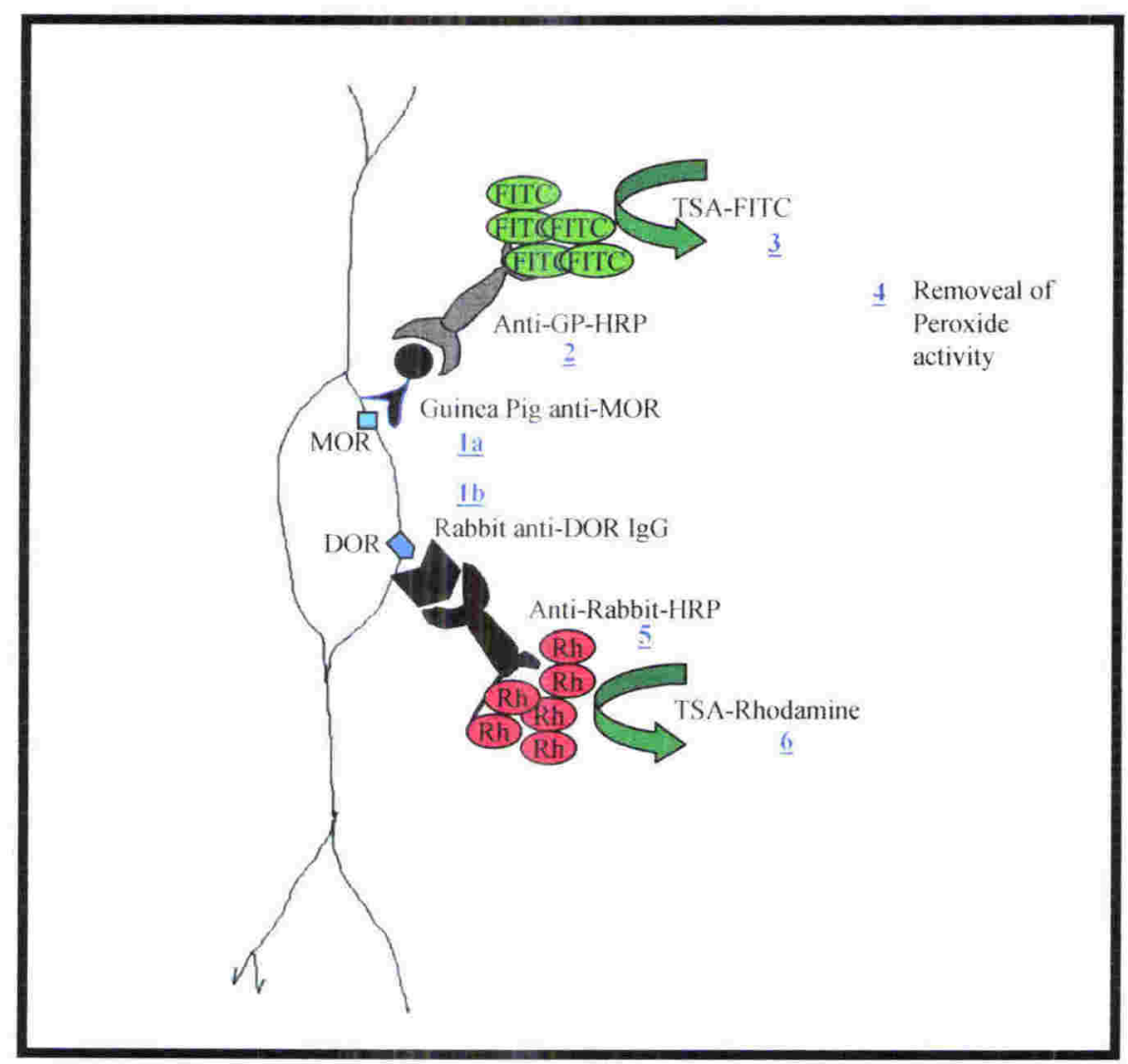

Figure 3.1

\section{Method for Double-Labelling using TSA}

Double-labelling of MOR and DOR using Tyramide signal amplification (TSA). Cells were incubated with primary antibodies (1a, 1b), followed by an HRP-labelled secondary antibody (Step 2). TSA amplification was then carried out, depositing a fluorophore (FITC) (MOR labelling) (Step 3), and the HRP activity of the first secondary antibody for the MOR signal was removed (Step 4). to allow independent detection of the second primary-secondary antibody combination (DOR labelling) (Step 5), followed by TSA amplification of the DOR signal using TSA-Rhodamine (Step 6). Hence, MOR is labelled with FITC (green) and DOR with Rhodamine (red) in the same sample.

\begin{tabular}{|c|c|c|c|c|c|}
\hline Primary antibody & $\begin{array}{c}\text { First } \\
\text { Secondary Antibody }\end{array}$ & $\begin{array}{c}\text { First } \\
\text { TSA detection }\end{array}$ & $\begin{array}{c}\text { Second } \\
\text { Secondary Antibody }\end{array}$ & $\begin{array}{c}\text { Second } \\
\text { TSA detection }\end{array}$ & $\begin{array}{c}\text { Final Signal } \\
\text { Observed }\end{array}$ \\
\hline guinea pig anti-MOR & anti-GP (lgG) HRP & FITC & anti-Rabbit (lgG) HRP & Rhodamine & FITC \\
\hline rabbit anti-DOR & anti-GP (lgG) HRP & FITC & anti-Rabbit (lgG) HRP & Rhodamine & Rhodamine \\
\hline MOR + DOR & anti-GP (lgG) HRP & FITC & anti-Rabbit (IgG) HRP & Rhodamine & FITC and Rhod amine \\
\hline rabbit anti-DOR & anti-Rabbit (lgG) HRP & FITC & anti-GP (lgG) HRP & Rhodamine & FITC \\
\hline guinea pig anti-MOR & anti-Rabbit (lgG) HRP & FITC & anti-GP (lgG) HRP & Rhodamine & Rhodamine \\
\hline MOR + DOR & anti-Rabbit (lgG) HRP & FITC & anti-GP (lgG) HRP & Rhodamine & FITC and Rhodamine \\
\hline
\end{tabular}

\section{Table 3.2}

\section{Double-Labelling Method Validation}

The controls used to ensure that: a) peroxidase removal was complete after staining with the first antibody and b) there was no loss of the fluorescent signal from the first antibody during staining for the second antibody binding sites. 


\subsubsection{CONFOCAL MICROSCOPY}

Fluorescence was visualised using a Leica TCS 4D confocal microscope with a krypton/argon laser fitted with a $63 x / 1.4$ NA or $40 x / 1.4$ NA objective. Phase contrast images were generated under transmitted light using a 40x/1.4 NA phase 3 lens. The computer software programmes NIH Image (Wayne Rasband; http://zippy.nimh.nih.gov) and Adobe Photoshop $^{\mathrm{TM}}$ (Adobe Systems Inc., 1996) were used to analyse and display the images. As an alternative to confocal microscopy, an Olympus AX70 photomicroscope (Olympus Optical Co., Tokyo, Japan) equipped with fluorescence and brightfield optics was used to obtain images. The confocal microscope settings are shown in Table 3.3. The excitation and emission wavelengths for the fluorescent probes used in this study are presented in Table 3.4 .

Table 3.3 Confocal microscope settings

\begin{tabular}{|c|c|c|c|c|}
\hline Fluorophore & $\begin{array}{c}\text { Excitation } \\
\text { Filter }\end{array}$ & $\begin{array}{c}\text { Excitation Beam } \\
\text { Splitter }\end{array}$ & $\begin{array}{c}\text { Detection Beam } \\
\text { Splitter }\end{array}$ & Barrier Filters \\
\hline FITC & BP 488 & DD & Mirror & BP FITC \\
\hline Rhodamine & BP 568 & DD & RSP 580 & LP 590 \\
\hline PI & BP 568 & DD & RSP 580 & LP 590 \\
\hline DAB & BP 488 & DD & DD & LP 590 \\
\hline
\end{tabular}

Confocal filter settings allows the excitation, emission, and splitting of the wavelengths of light to be carefully controlled for each fluorophore. Abbreviations: Double dichroic mirror (DD); long pass filter (LP) (transmits wavelengths above the given value); band pass filter (BP) (allows transmission of light around the given wavelength); second beam splitter (RSP).

Table 3.4 Excitation and emission wavelengths of fluorophores

\begin{tabular}{|l|c|c|}
\hline Fluorophore & $\begin{array}{c}\text { Excitation } \\
\text { Wavelength (nm) }\end{array}$ & $\begin{array}{c}\text { Emission } \\
\text { Wavelength (nm) }\end{array}$ \\
\hline FITC & 490 & 520 \\
\hline Rhodamine & 580 & 605 \\
\hline PI & 535 & 620 \\
\hline
\end{tabular}

Excitation and emission wavelengths of fluorophores. 


\subsection{RESULTS}

\subsubsection{SELECTION OF ANTIBODIES}

Primary cultures from brainstems of all developmental ages tested (E16 to P6) and after varying times in culture all showed positive staining for both MOR and DOR. Positive labelling was seen with both colourmetric (DAB) and fluorescent labelling methods. Differences were observed in the intensity of the staining with the antibodies tested from different sources and with the use of different amplification techniques (Table 3.1). Very weak staining was observed in cells in which the primary antibody was directly bound to FITC-labelled secondary antibody. This staining pattern was usually very diffuse and required high voltage settings on the confocal microscope. Greater amplification was achieved using DAB colourmetric detection, although this signal was also very weak without the use of TSA-Biotin in the amplification protocol. No differences in the staining patterns of membranes versus cytoplasm were observed with the different primary antibodies used. In all cultures regardless of the primary antibody used, a mixture of membrane and cytoplasmic staining was seen in different populations of neurons on the same coverslip. Specific subcellular localisations were not easily detected in DAB-stained neurons due to the nature of the deposition of the coloured product. Often, staining of the cell membrane obstructed the view of cytoplasmic vesicle staining because the light microscope could not focus through the deposited coloured product on the plasma membrane. For this reason most cellular OpR distribution patterns were determined by fluorescent detection on the confocal microscope. General staining intensity for each antibody and each amplification technique was ranked on an arbitrary scale from no staining (0) to weak staining (1), moderate staining (2), and very strong staining (3) (Table 3.1). The most specific staining, and the most intense, of both MOR and DOR occurred with TSA amplification, presumably because lower primary antibody concentrations were possible, thus, preventing the non-specific binding seen at higher concentrations with conventional amplification methods (Table 3.1).

\subsubsection{DETERMINING ANTIBODY SPECIFICITY}

SH-SY5Y cells were used as a positive control for both DAB and fluorescent labelling methods. These cells showed both MOR and DOR immunoreactivity and the staining intensity was similar to that seen in primary cultures of brainstem cells. Some primary 
antibodies were supplied with a blocking peptide that was used to test the specificity of the antibody. The peptides for the rabbit anti-MOR antibody from R\&D Antibodies and from Chemicon blocked most of MOR antibody binding at antibody dilutions greater than 1:1000 (Figure 3.2).

\subsubsection{Negative Controls}

LLC-PK 1 pig kidney cells showed no immunoreactivity when incubated with MOR (Figure 3.3A) or DOR (not shown) antibodies, and, thus, this cell line was used as a negative control to test antibody specificity in each experiment. Cultured brainstem cells were incubated with blocking solution containing no primary antibody and were developed the same as MOR and DOR antibody-labelled cells. This second type of negative control also showed no immunoreactivity (Figure $3.3 \mathrm{~B} \& \mathrm{C}$ ). At MOR primary antibody concentrations higher than 1:1000, the MOR blocking peptide was unable to completely block MOR antibody binding when used at a concentration of $250 \mu \mathrm{g} / \mathrm{mL}$. Numerous amplification techniques were attempted (Table 3.1). Some of these, such as FITClabelled secondary antibodies failed to adequately label the cells. The strongest signal development was always achieved with TSA.

\subsubsection{Positive Controls}

The neuroblastoma cell line, SH-SY5Y, has been reported in the literature to express both MOR and DOR (Yu et al., 1986) and was used in this study as a positive control to help establish the immunofluorescent procedures. Differentiated SH-SY5Y cells showed an increase in both MOR and DOR immunoreactivity (Figure 3.4B \& D) relative to nondifferentiated SH-SY5Y cells (Figure 3.3A \& C). Staining intensity was greater in cell; displaying a more neuronal morphology, in particular, cells with neurite outgrowths. Staining of neuronal processes could be seen using both fluorescent and colourmetric labelling methods. Fluorescent labelling observed with the confocal microscope was best for determining the cellular localisation of OpRs within single cells when the cells were scanned in three-dimensions. DAB colour development was easier for determining the intensity of staining within cell populations, and for comparing cultures from different developmental ages. MOR and DOR staining was observed on the plasma membrane of the cell body and on neuronal processes, as well as in the cytoplasm. Both membrane and cytoplasmic staining was observed in both bipolar and multipolar neurons, and no 
morphologically distinct cellular characteristic could be determined from the staining pattern observed in any one neuron. No nuclear staining of MOR and DOR was found in any neurons or astrocytes. In some cultures, lymphocytes could be seen that were stained positive for both MOR and DOR, and these lymphocytes often showed nuclear staining. Both MOR and DOR are known to be expressed in lymphocytes (for review see) (Bidlack, 2000); therefore, the immunolabelling of both the cytoplasm and the nucleus of lymphocytes seen in the present study are likely to be specific. This result adds support to the validation of the OpR immunostaining protocol used in this study. 

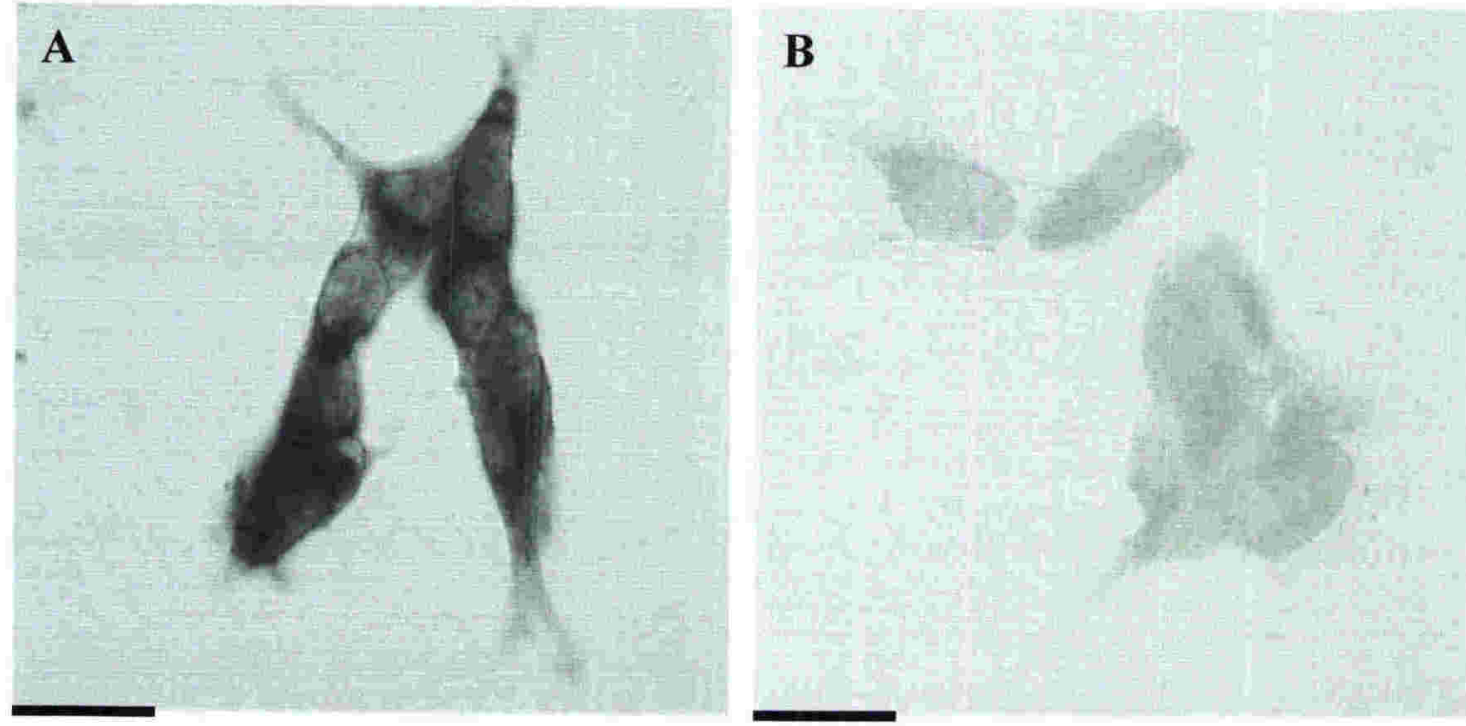

\section{Figure 3.2}

\section{Peptide Blocking of Antibody Binding}

MOR labelling of SH-SY5Y cells using DAB colour development without (A) and with (B) preincubation with the MOR peptide used to make the antibody (anti-MOR rabbit antibody and peptide from R\&D Antibodies). Note that the MOR immunostaining has been partially blocked by the peptide. These figures were scanned at the same voltage and laser settings on the confocal microscope. Scale bars $=20 \mu \mathrm{m}$.
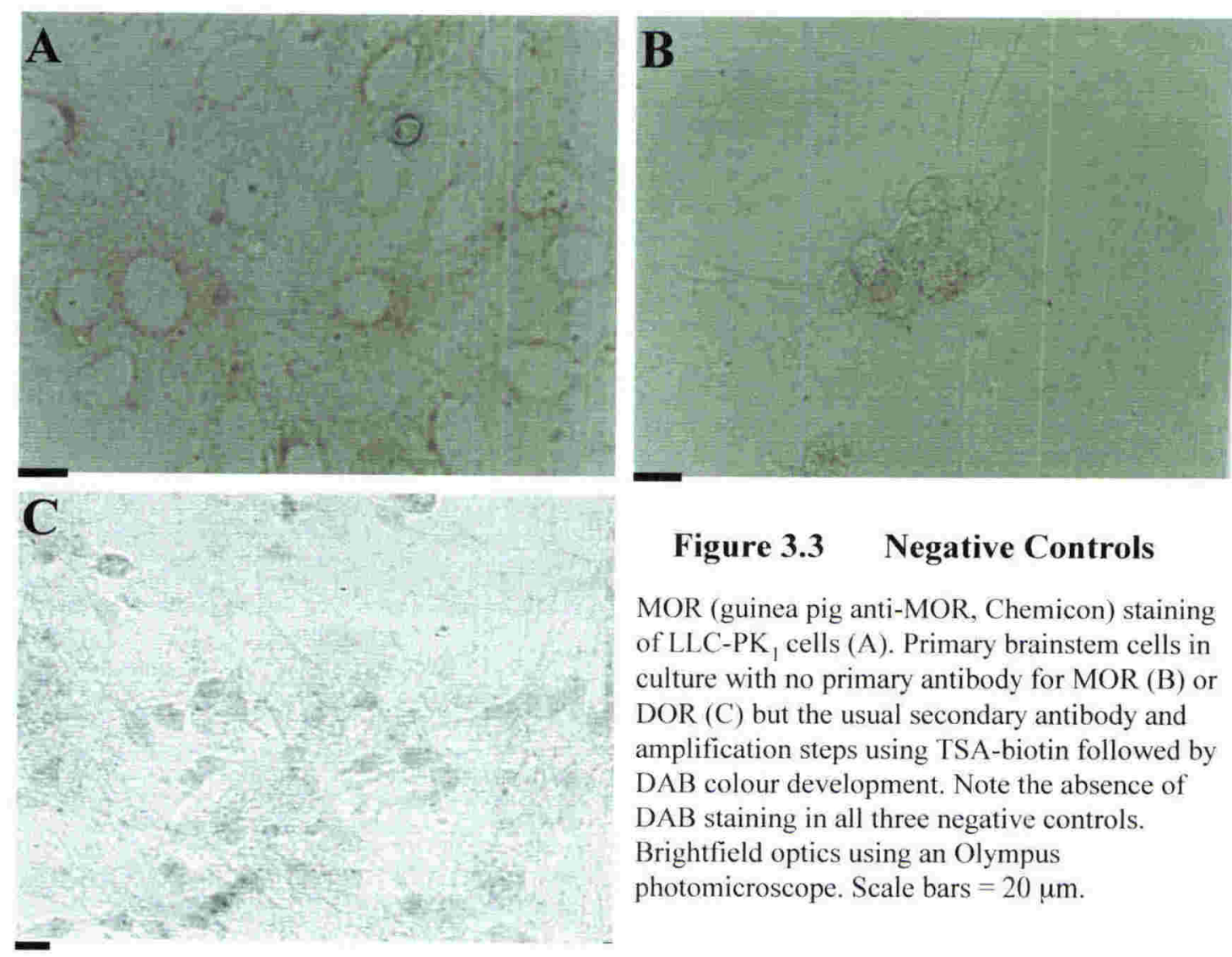

\section{Figure 3.3 Negative Controls}

MOR (guinea pig anti-MOR, Chemicon) staining of LLC-PK 1 cells (A). Primary brainstem cells in culture with no primary antibody for MOR (B) or DOR (C) but the usual secondary antibody and amplification steps using TSA-biotin followed by DAB colour development. Note the absence of $\mathrm{DAB}$ staining in all three negative controls. Brightfield optics using an Olympus photomicroscope. Scale bars $=20 \mu \mathrm{m}$. 


\section{Growing cells}

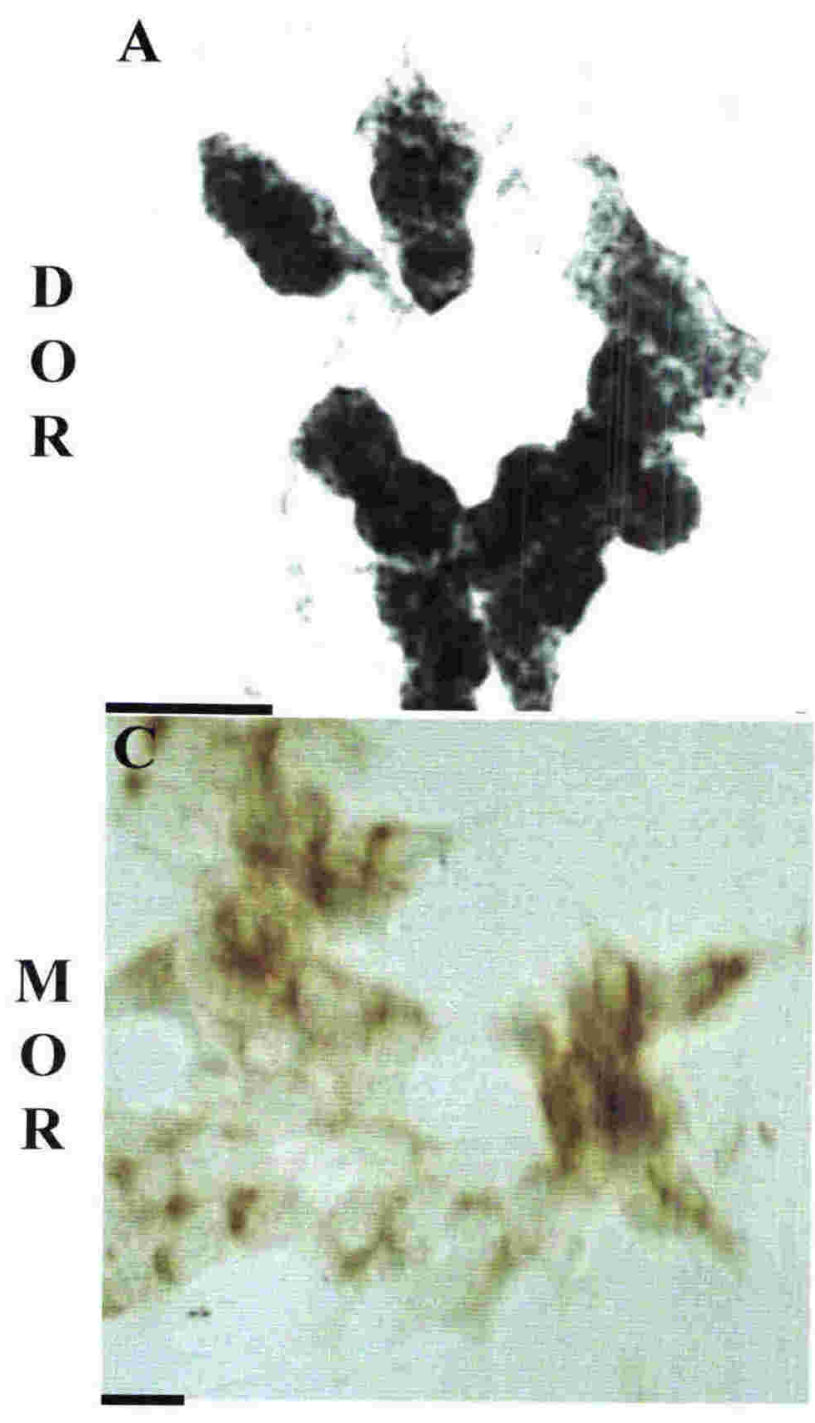

\section{Differentiated cells}

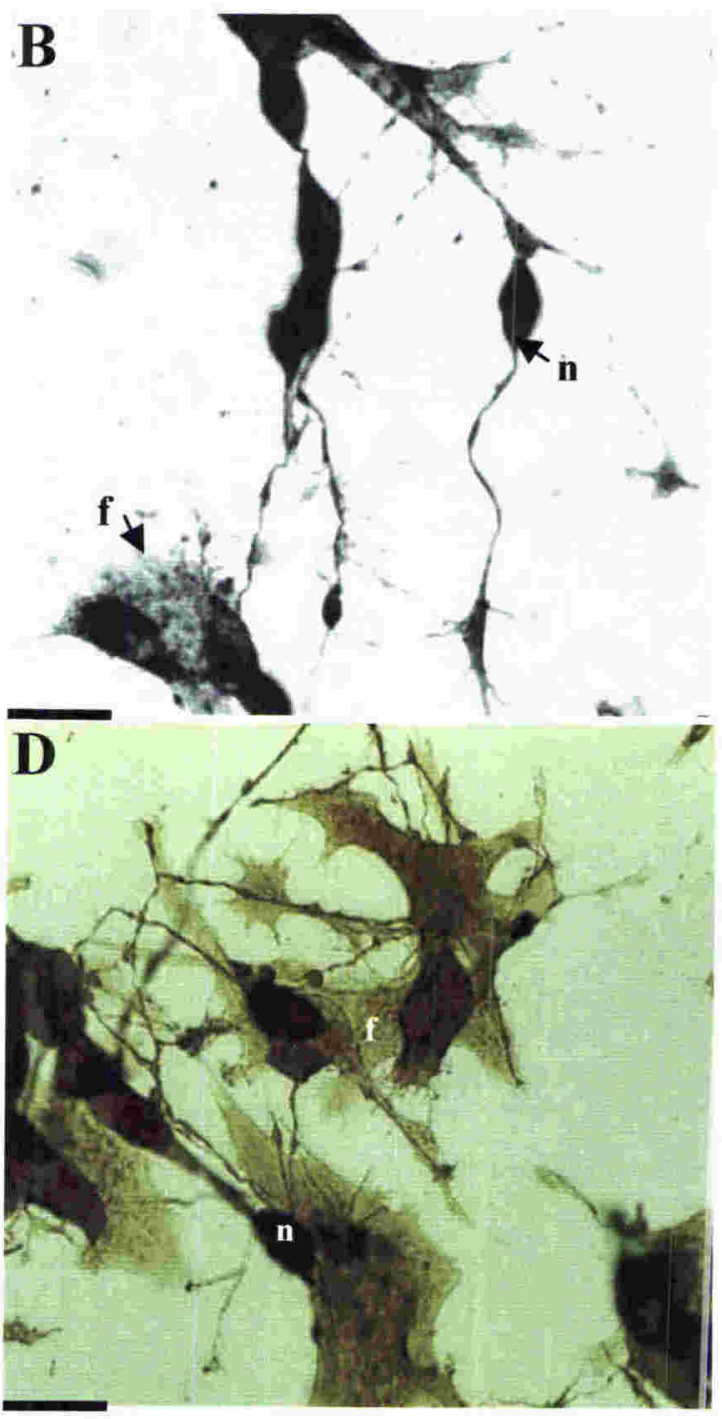

Figure 3.4

\section{DOR and MOR Labelling of SH-SY5Y Cells}

DOR (A) (rabbit anti-DOR, Chemicon) and MOR (C) (guinea pig anti-MOR, Chemicon) labelling of growing undifferentiated SH-SY5Y cells, and DOR (B) and MOR (D) labelling of retinoic acid-differentiated SH-SY5Y cells. Cells were treated in culture with $10 \mathrm{nM}$ retinoic acid for 10 days. Note the darker opioid receptor staining on cells displaying a more neuronal morphology $(n)$ than the flat, less differentiated cells present in the same culture dish (f). Scale bars $=20 \mu \mathrm{m}$. 


\subsubsection{OPIOID LABELLING OF DIFFERENT CELL TYPES IN PRIMARY CULTURES OF THE RAT BRAINSTEM}

MOR and DOR staining of brainstem cells in primary culture were examined using DAB staining (Figure 3.5, Figure 3.7 and Figure 3.8). MOR and DOR positive cells in culture were classified according to their morphology as either bipolar or multipolar neurons, type 1 or type 2 astrocytes, or other cells, using a photomicroscope equipped with brightfield and phase-contrast optics. Neurons could be seen as phase-bright cells, often growing on a layer of astrocytes (Figure 3.5B). Astrocytes were commonly closely associated with MOR- and DOR-positive neurons and, with the light microscope, looked as if they were connected to each other (Figure 3.5A). The most intense MOR and DOR staining was seen on neurons whose identity was confirmed by double-labelling with the neuron specific marker MAP2. Fluorescent double-labelling of MOR and MAP2, or DOR and MAP2, with FITC and Rhodamine showed colocalisation (Figure 3.6). Fluorescence was observed by confocal microscopy. Combined images showed that most $(>95 \%)$ of the opioid receptor labelling was on MAP2 positive neurons.

OpR staining intensity was also partially quantified by brightfield microscopy using DAB (Figure 3.7 and 3.8). Staining intensity of cells was classified on a scale ranging from 0 to $3(0=$ unstained, and $3=$ intensely stained $)$ for each cell type described above. Primary brainstem cultures were labelled with the rabbit anti-MOR antibody (Chemicon) or the rabbit anti-DOR antibody (Chemicon) and amplified using anti-rabbit IgG DIG and antiDIG HRP before colour development using DAB. Differences in opioid receptor expression between the different cell types present in culture were assessed. Both MOR (Figure 3.7) and DOR (Figure 3.8) labelled cells were seen at all developmental ages tested (Figure 3.9). Data pooled from all developmental ages for MOR $(\mathrm{P}<0.002, \mathrm{n}=3$ coverslips from each developmental age, Kruskal-Wallis test) and DOR $(\mathrm{P}<0.0001, \mathrm{n}=3$ coverslips from each developmental age, Kruskal-Wallis test) showed significantly greater staining intensity of neurons compared with glial cells. The actual intensities are difficult to compare directly because glial cells are flattened cells, whereas the soma of neurons is rounded, and thus, may appear darker because of its thickness. There were no significant differences in the staining intensities between bipolar and multipolar neurons or between type 1 and type 2 astrocytes (data not presented). Not all neurons showed OpR immunoreactivity. No difference in the percent of $\mathrm{OpR}$ positive neurons was seen in cultures from the different developmental ages. In all cultures, 40-70\% of the neurons 
were OpR positive (with the same percent of MOR and DOR positive cells present in all cultures). Large variations in the percent neurons, however, were seen between cultures prepared on different days.

In Summary, it was concluded that: a) OpR expression was greater in neurons than in glial cells, but that there was no difference between bipolar and multipolar neurons; b) there was no obvious difference between the number of MOR and the number of DOR expressing neurons in the cultures; c) astrocytes expressed both MOR and DOR, but at very low levels; e) There were no obvious differences in the number of cells stained or the proportion of MOR- versus DOR-positive cells between cultures prepared from different developmental ages.

\subsubsection{OPIOID RECEPTOR EXPRESSION IN PRIMARY CULTURES DURING DEVELOPMENT}

\subsubsection{Neuronal MOR and DOR Expression During Development}

MOR expression changed during late foetal and early postnatal development in primary cultures of the rat brainstem. Data pooled from MOR-labelled neurons of foetal (E16-E18) and postnatal (P1-P6) cultures showed that developmentally more advanced postnatal stages were more intensely stained $(\mathrm{P}<0.006, \mathrm{n}=423$ cells, Mann-Whitney test) than foetal cultures (Figure 3.9A). There were no significant differences in DOR expression levels during development (Figure 3.9B). 

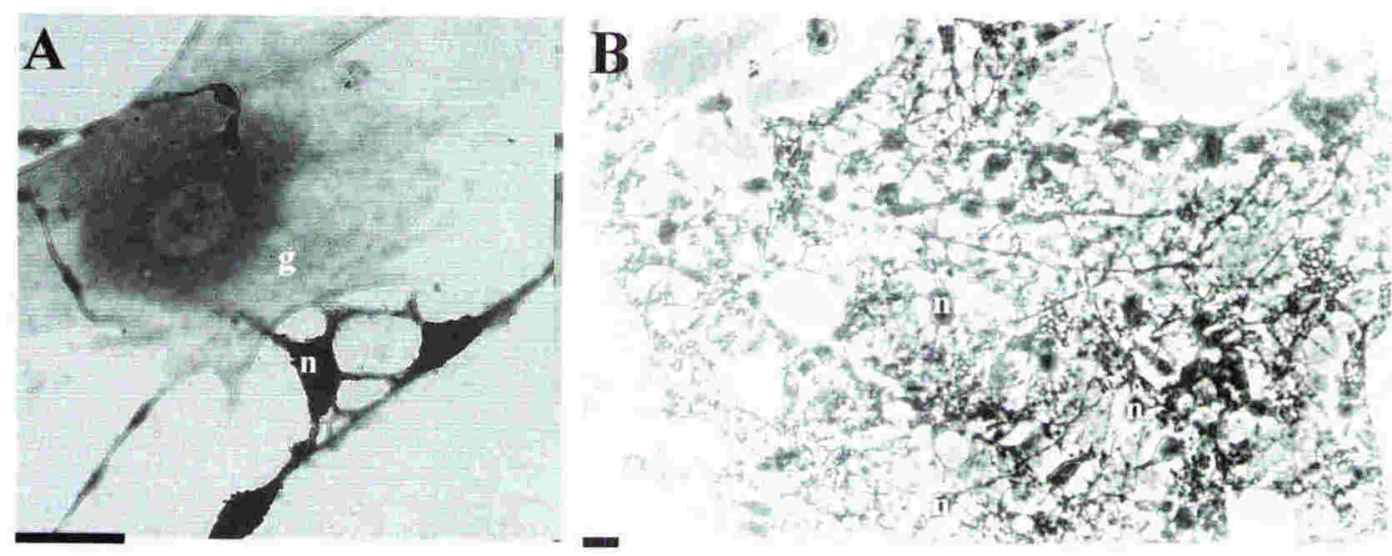

Figure 3.5

\section{Morphology of Opioid Receptor Positive Cells}

DOR-labelled (rabbit anti-DOR, Chemicon) E18 primary brainstem cells (A) visualised by DAB. Note the darkly stained DOR-positive neurons (n) and the less intensely stained type 1 astrocyte (g). Astrocyte/neuronal and neuronal/neuronal connections can be seen. (B) Phase-contrast microscopy of a foetal E16 primary brainstem culture shows phase-bright neurons (n) lying on top of a layer of astrocytes. MOR staining of this same photo using brightfield optics can be seen in Figure 4.7A. Brightfield (A) and phase-contrast microscopy (B). Scale bars $=20 \mu \mathrm{m}$.
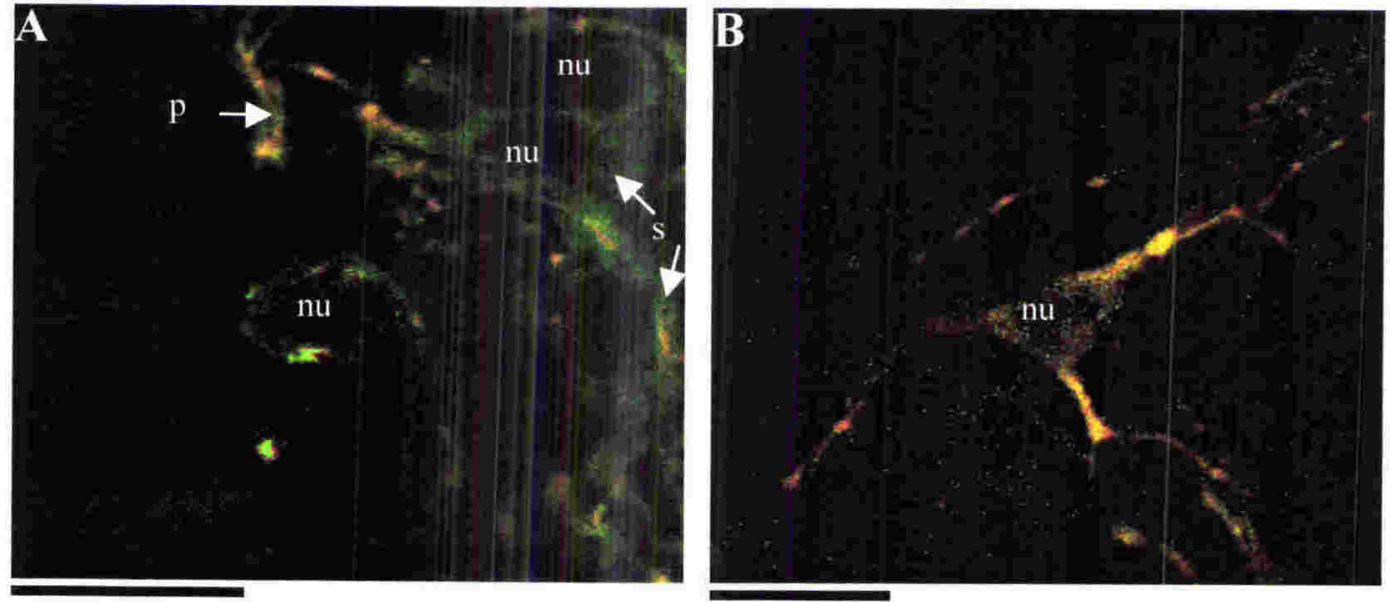

Figure 3.6

\section{MOR and MAP2 Colocalisation}

Confocal images of MOR (guinea pig anti-MOR) and MAP2 double-labelled rat brainstem cells in culture showing MOR-positive cells (green) and MAP2 positive neurons (red). Some cells show MOR labelling of the cytoplasm of the soma (s); whereas, MAP2 localisation is usually in the cell processes (p). No nuclear (nu) staining is seen. Some single neurons show double-labelling of both MOR and MAP2 (B) (yellow). Similar results were obtained for DOR and MAP2 staining in culture (results not shown). Scale bars $=20 \mu \mathrm{m}$. 


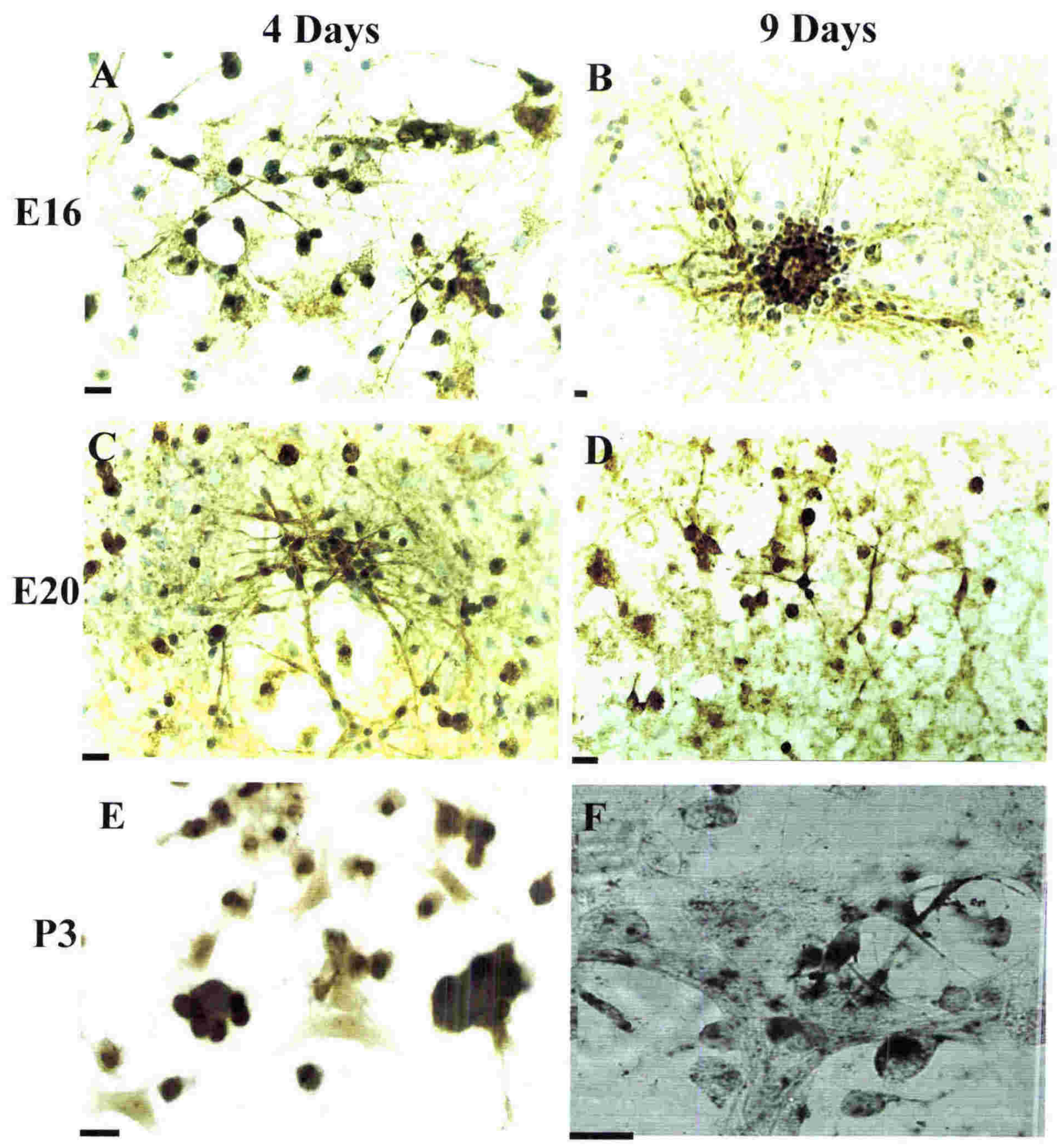

Figure 3.7

MOR Expression During Development

Foetal E16 (A, B), E20 (C, D) and postnatal P3 (E, F) cultures showing the distribution of MOR (rabbit anti-MOR, Chemicon) visualised by DAB. Some cultures (A, B, \& C) have been counter-stained using toluidine blue to visualise the nucleus and aid in the counting of cells. MOR expression is shown during development after 4 (A, C, \& E) and 9 (B, D, \& F) days in culture. No significant differences were observed in the staining pattern over time in culture. Brightfield microscopy (A-E), Transmitted light confocal microscopy (F). Scale bars $=20 \mu \mathrm{m}$. 

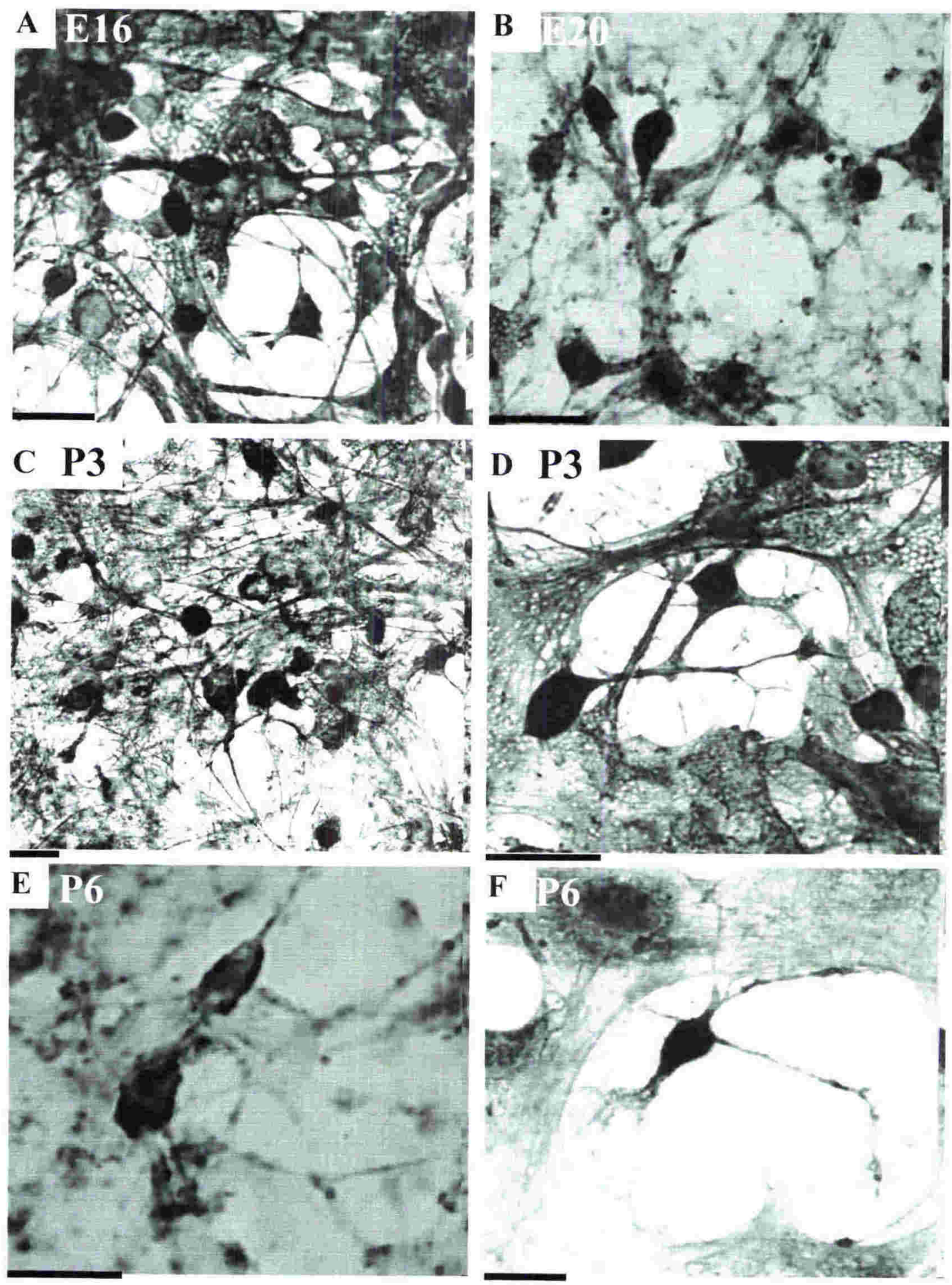

Figure 3.8

DOR Expression During Development

Confocal pictures (B\&W) showing DOR expression (using DAB) in foetal E16 (A), E20 (B), and postnatal P3 (C \& D), and P6 (E \& F) cells during development, and after 4 (A, B, C, \& E) and 9 (D \& F) days in culture. Confocal transmitted light images. Scale bars $=20 \mu \mathrm{m}$. 


\section{A MOR staining in rat primary brainstem cultures during development}

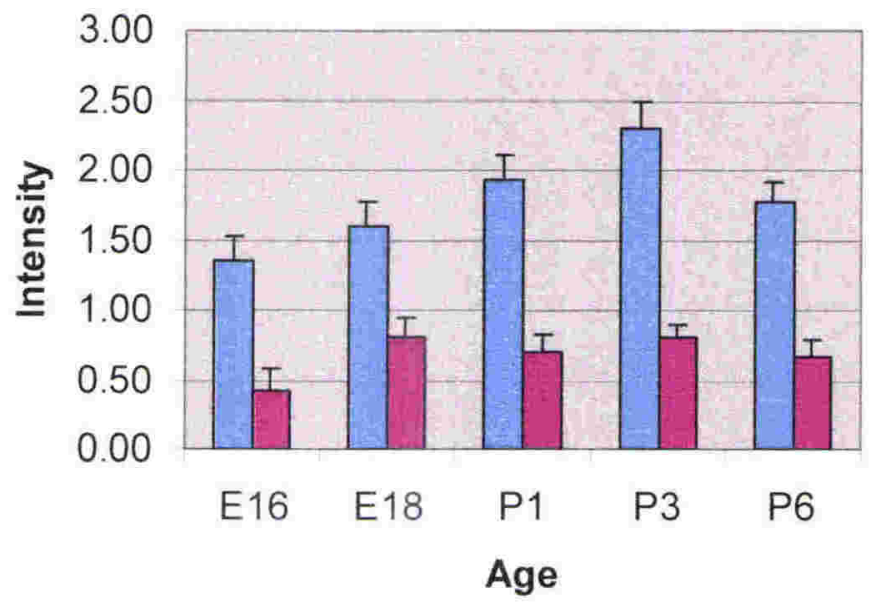

B

\section{DOR staining in rat primary brainstem cultures during development}

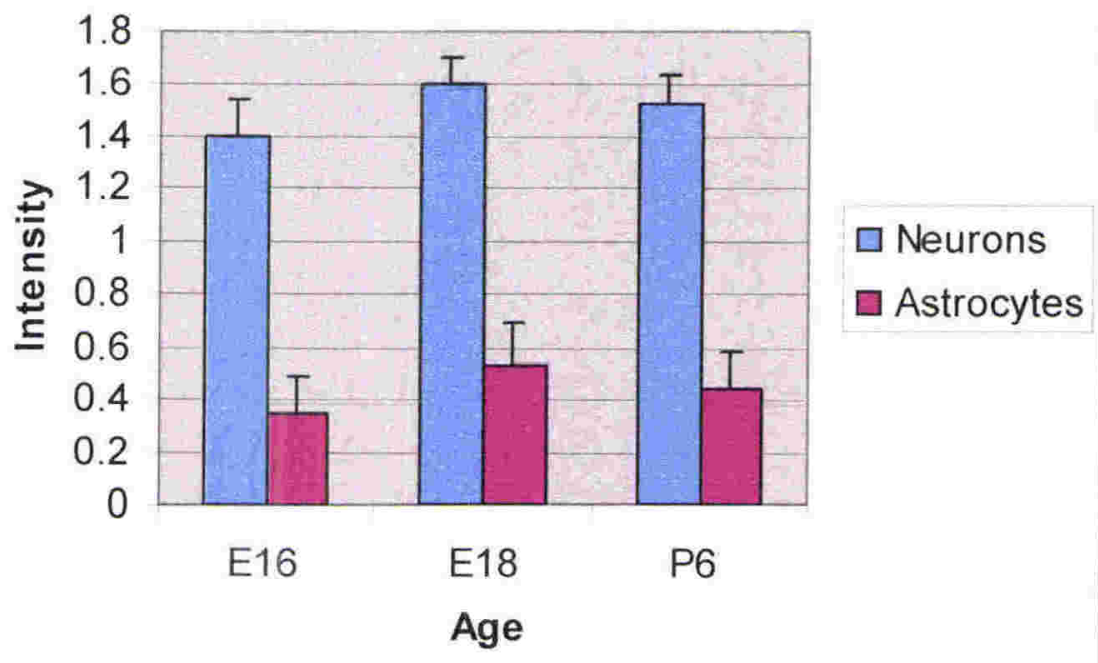

Figure 3.9

MOR and DOR Staining Intensity of Neurons and Astrocytes in Primary Rat Brainstem Cultures from Different Developmental Ages

Staining of neurons and astrocytes in primary brainstem cells cultured from late foetal and early postnatal rats (mean + SEM). There is an increase in staining intensity of MOR during development (A), but no change in the staining intensity of DOR (B). Neurons stain more intensely for both MOR and DOR than astrocytes. 

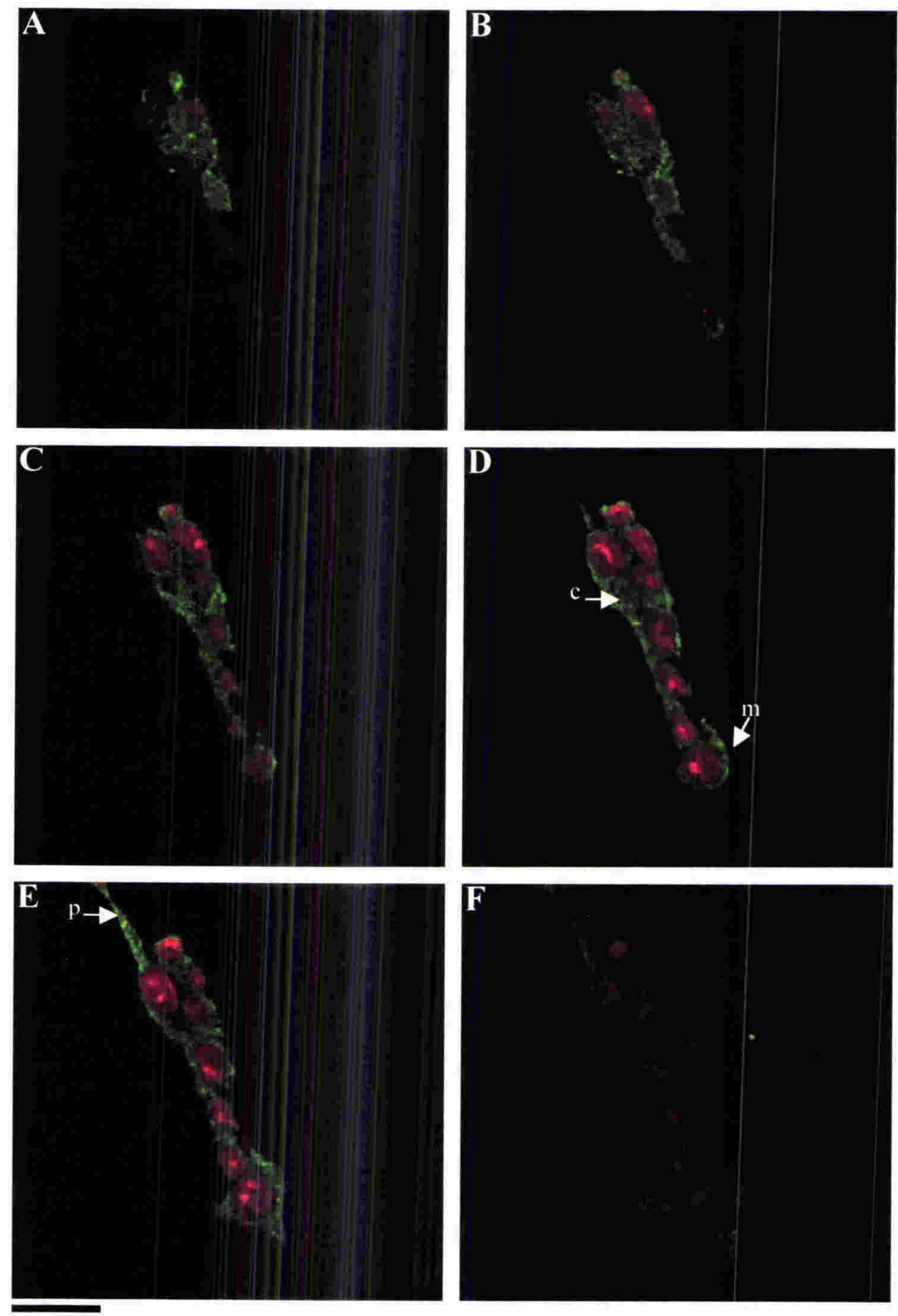

\section{Figure 3.10}

\section{MOR Labelling of SH-SY 5 Y Cells}

Confocal serial sections (A-F) through MOR-labelled SH-SH5Y cells (guinea pig antiMOR, Chemicon) (green) using tyramide amplification (FITC). Cells were counter stained with propidium iodide (red). Note the labelling of the cell membrane (m), cytoplasm (c), and processes (p) as shown by the arrows. Scale bar $=20 \mu \mathrm{m}$. 

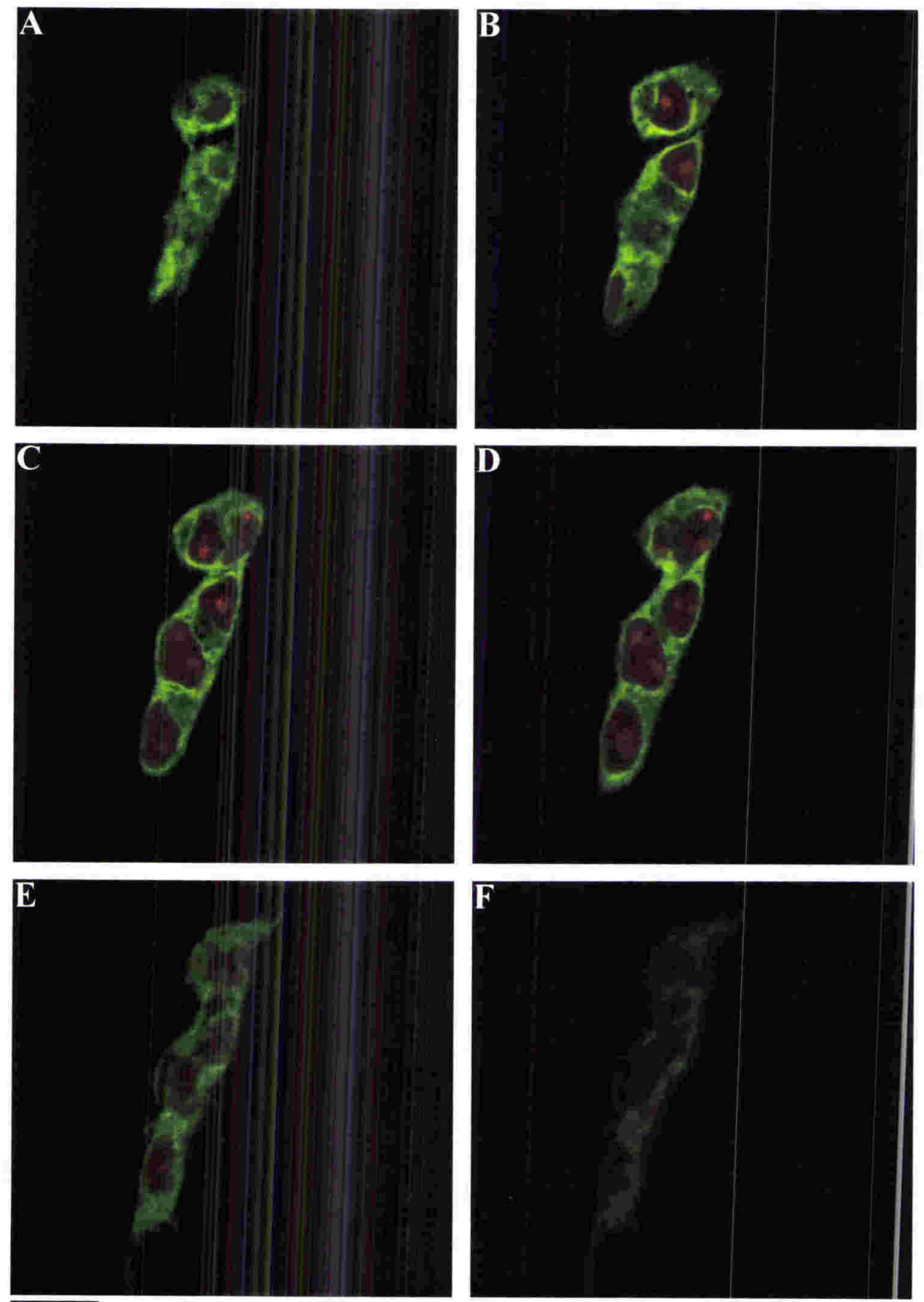

Figure 3.11

\section{DOR Labelling of SH-SY5Y Cells}

Confocal serial sections (A-F) through DOR-labelled SH-SH5Y cells (rabbit anti-DOR, Chemicon) (green) using tyramide amplification (FITC). Cells were counter stained with propidium iodide (red). Note the similar staining of both MOR (see Figure 3.9) and DOR (this figure) in SH-SY5Y cells. Scale bar $=20 \mu \mathrm{m}$. 


\subsubsection{Glial Cell Expression of MOR and DOR During Development}

Staining intensities of glial cells in the cultures were similar at all developmental ages. In all cultures labelled with either MOR or DOR, glial cells showed only weak staining that was difficult to distinguish from background. As mentioned above, the thinness of the flattened glial cells made it difficult to compare staining intensities directly with neuronal staining. Type II astrocytes, however, were only lightly stained, despite having a rounded soma similar to multipolar neurons. Astrocytes clearly stained more intensely than the negative control cell line, LLC-PK density of OpRs of both the MOR and DOR subtype.

\subsubsection{Stability of Opioid Receptor Expression in Culture}

Opioid receptor expression was measured in cultures established from foetal (E16) and postnatal (P6) brainstems after 4, 7, and 12 days in culture. There were no significant differences in either MOR or DOR immunoreactivities with time in culture (data not presented).

\subsubsection{INTRACELLULAR DISTRIBUTION OF OPIOID RECEPTORS}

The three-dimensional, intracellular distribution of opioid receptors was examined by confocal microscopy. In addition to plasma membrane receptor staining, a population of internalised receptors was present in neurons. Internalised OpRs were seen as punctate or diffuse staining within the cytoplasm of the soma and in the neurites. Membrane-bound and internalised opioid receptors were present in both neuronal and glial cells cultured from the rat brainstem (Fig 3.12, 3.13, 3.14, and 3.15) and in SH-SY5Y cells (Figure 3.10, 3.11 , and 3.14C). Nuclear staining was only seen in the occasional lymphocyte that was present as a contaminant in the cultures.

All SH-SY5Y cells stained positive for MOR (Figure 3.10) and DOR (Figure 3.11). Staining was strongest in the plasma membrane, neurites, and cytoplasm but was absent in the nucleus. Sometimes the staining was diffuse; whereas, other times it was punctate. Double-labelling experiments showed colocalisation of MOR and DOR in SH-SY5Y cells. This colocalisation of opioid receptors was found in the membrane, cytoplasmic vesicles, and on processes. Not all MOR were colocalised with DOR receptors, and not all DOR were colocalised with MOR. Distinct MOR or DOR stained regions of the cell showed up 
as red (MOR) or green (DOR) staining, rather than yellow (MOR and DOR) (Figure 3.14C). Often MOR and DOR staining was found in the axon hillock region of neurons. Here the staining was usually intense and mostly intracellular. In other neurons intense staining was seen in the axon and dendrites. There was no clear pattern or trend to the staining pattern except the absence of nuclear staining in neurons and glia.

\subsubsection{COLOCALISATION OF MOR AND DOR IN CELLS CULTURED FROM THE RAT BRAINSTEM}

Similar to SH-SY5Y cells, MOR and DOR were colocalised in some cells in primary cultures of the rat brainstem from both foetal (Figure 3.14A) and postnatal cultures (Figure 3.12, Figure 3.14B and Figure 3.15). Colocalisation was not present in all areas of a cell or in all cells. As with SH-SY5Y cells (Figure 3.14C), there were regions within a cell that had only MOR, or only DOR, immunoreactivity. Serial optical sections through a group of MOR and DOR double-labelled cells in a Pl culture using confocal microscopy revealed some predominantly MOR-positive cells adjacent to predominantly DOR-positive cells. Other cells showed colocalisation of MOR and DOR in the same cluster (Figure 3.15). One region showing high MOR and DOR colocalisation is where the two cell membranes and processes meet. In these regions it is possible that MOR and DOR are present in boutons and synaptic junctions although the resolution in the present study is not sufficient. 

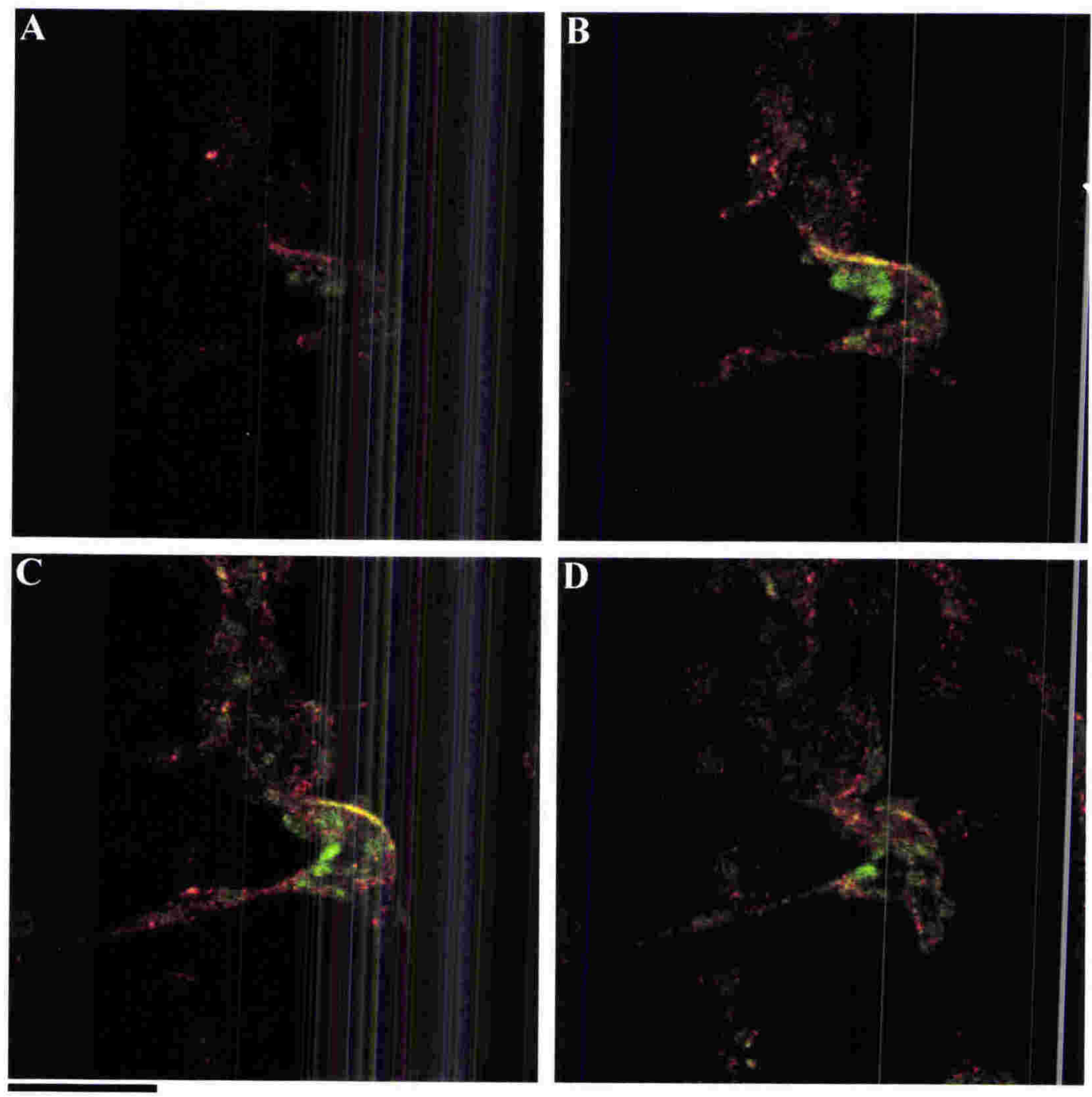

Figure 3.12

\section{Double-Labelling of MOR and DOR in Culture}

Confocal serial sections through MOR (guinea pig anti-MOR, Chemicon) (red) and DOR (rabbit anti-DOR, Chemicon) (green) double-labelled cells from postnatal (P3) brainstem cultures after 4 days in culture. Opioid receptor colocalisation is shown in yellow. Scale bar $=20 \mu \mathrm{m}$. 

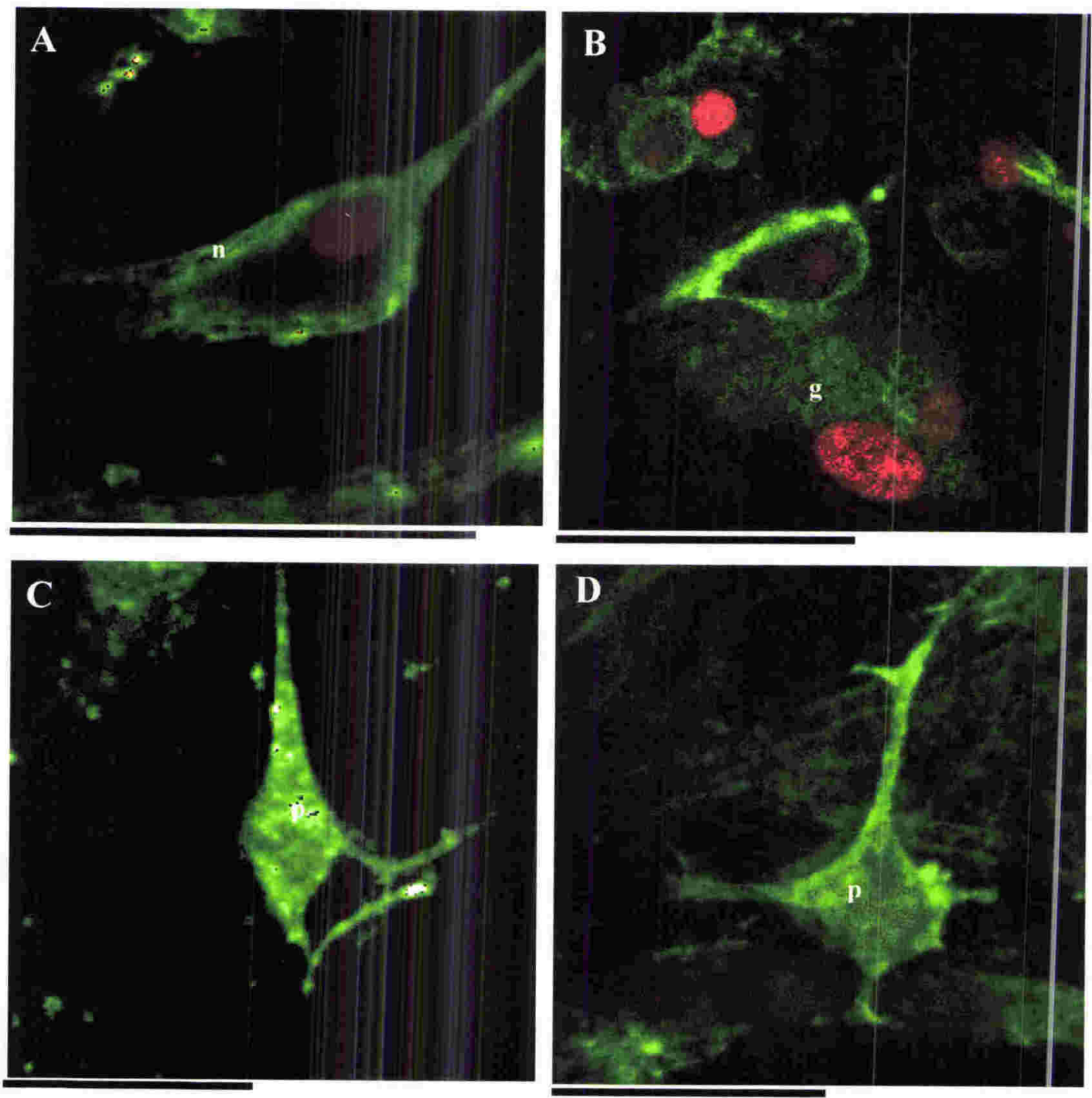

Figure 3.13

Three-Dimensional, Confocal Analysis of MOR and DOR Staining in Primary Culture

Confocal images showing similar staining patterns of MOR (A \& C) (guinea pig anti-MOR, Chemicon) and DOR (B \& D) (rabbit anti-DOR, Chemicon) (green). Diffuse intense MOR (A) and DOR (B) membrane staining of neurons (n) and diffuse weaker staining of nearby type 1 astrocytes for DOR (g). Some MOR (C) and DOR (D) labelling of neurons was also punctate (p) and cytoplasmic. Cells are counterstained with propidium iodide (red). Scale bars $=20 \mu \mathrm{m}$. 

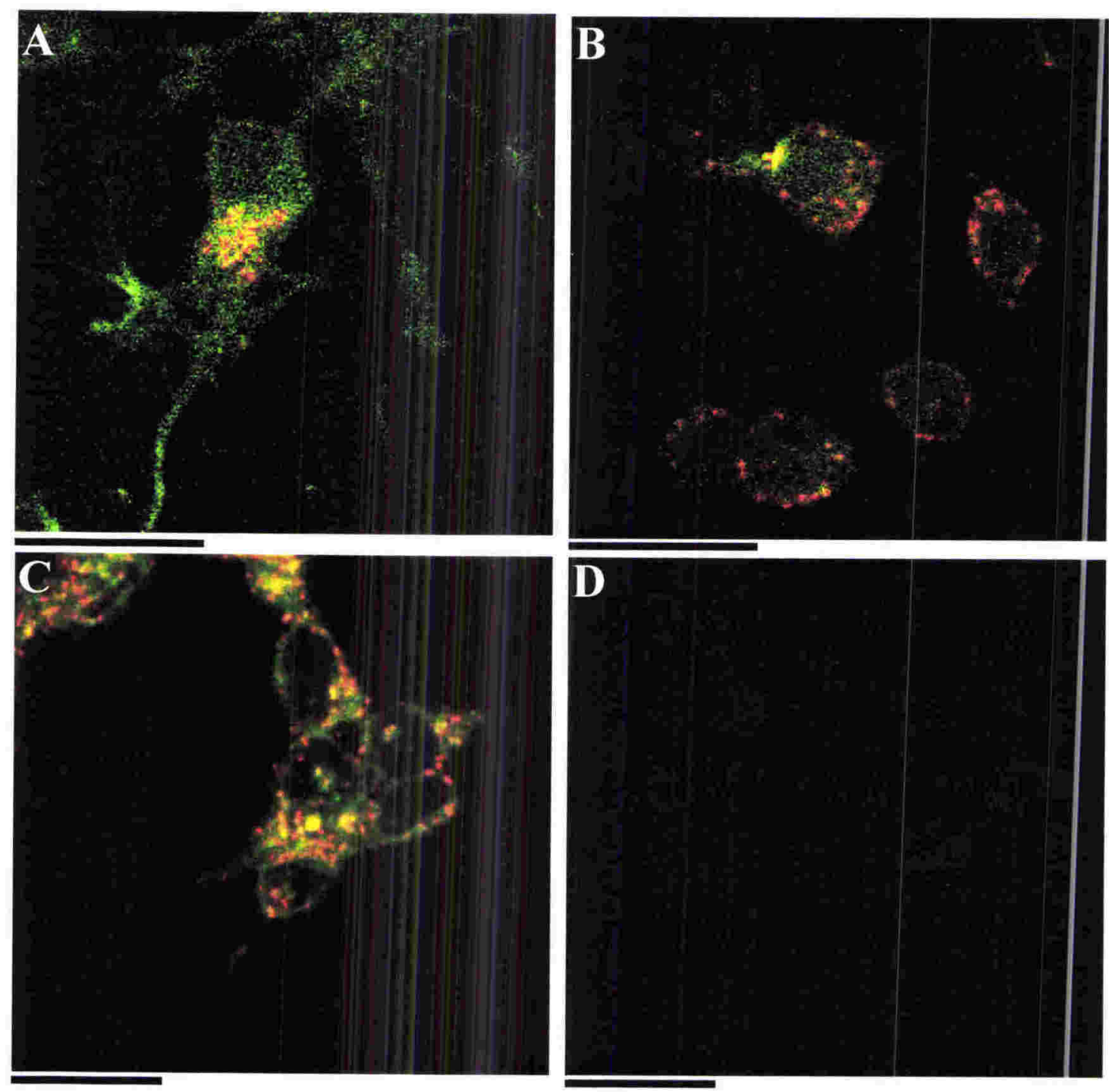

Figure 3.14

\section{MOR and DOR Double-Labelling}

Confocal images of MOR (guinea pig anti-MOR, Chemicon) (red) and DOR (rabbit anti-DOR, Chemicon) (green) double-labelled cells from foetal E16 (A) and postnatal P1 (B) primary brainstem culture, and SH-SY5Y cells (C). Colocalisation (yellow) can be seen in some areas. Negative control with no primary antibodies (D) shows no staining at the same confocal laser setting used in $\mathrm{A}, \mathrm{B}, \& \mathrm{C}$. Scale bars $=20 \mu \mathrm{m}$. 

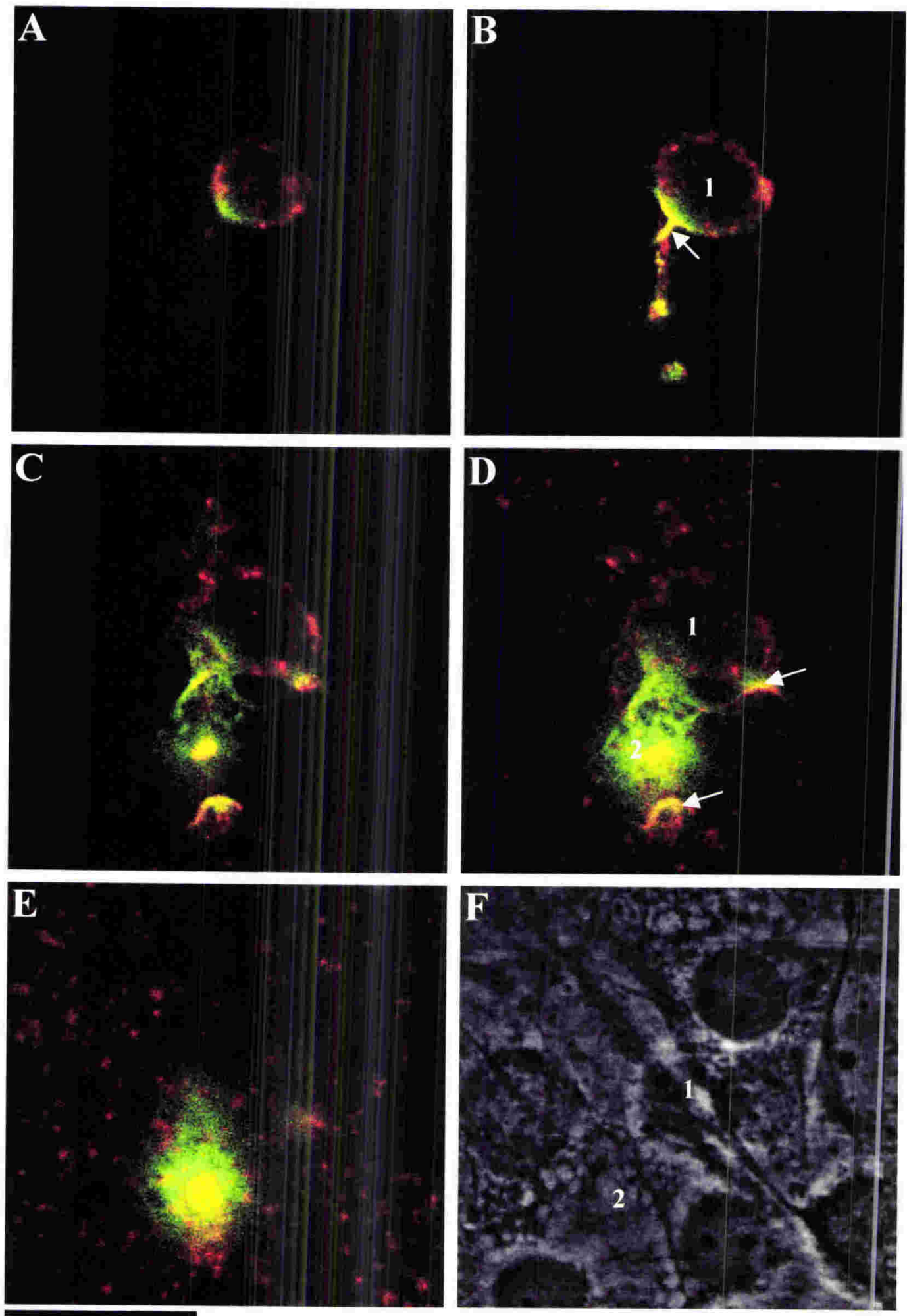

\section{Figure 3.15}

\section{Serial Confocal Sections Through a Group of P1 Cells Expressing MOR and DOR}

Confocal serial sections through a group of cells cultured from postnatal (P1) brainstem after 8 days in culture (A-E). Cells are double-labelled for MOR (guinea pig anti-MOR, Chemicon) (green) and DOR (red). A phase contrast confocal transmitted light image $(F)$ shows the same field of view as A, B, C, D, $\&$ E. Note the punctate DOR staining (red) in several cells surrounding A MOR-positive (green) cell (2). Also note that not all cells show OpR immunoreactivity. Arrows show areas of colocalisation where neuronal processes of the neighboring cells meet. Scale bar $=20 \mu \mathrm{m}$. 


\subsection{DISCUSSION}

\subsubsection{ANTIBODY TESTING FOR IMMUNOCYTOCHEMISTRY}

Antibody quality and specificity was a problem early on in the study. Few opioid receptor antibodies were commercially available at the start of the research, with the exception of one rabbit anti-MOR antibody from Chemicon. This antibody proved to have undesirable high background staining properties when used at the high primary antibody concentration initially required to give an adequate signal. TSA signal amplification however, helped overcome the low signals initially observed using standard biotin/avidin amplification techniques and a lower primary antibody concentration with lower background staining levels could be used. Early studies by others involving opioid receptor localisation used ligand-binding assays to determine OpR distributions in the brain (Mack et al., 1984; Xia and Haddad, 1991). Some investigations used fluorescently-tagged opioid receptor peptides, demonstrating specific binding by competition, but these studies were unable to detect opioid receptors directly by microscopy because of the weakness of the signal (Kolb et al., 1983; Kolb et al., 1985; Koman et al., 1985; Mihara et al., 1985). The peptides often bound to more than one type of opioid receptor, and the covalent attachment of the fluorophore to the peptide in many cases altered binding activity, making it difficult to determine specific OpR distributions. In the mid to late 1980's, antibodies were made to peptide fragments of OpRs, and the antibody binding was monitored (Glasel et al., 1983; Carr et al., 1986; Myers and Glasel, 1986). These early immunostaining protocols, however, were only suitable for labelling transfected cell lines in which there was massive over-expression of receptors (Madsen et al., 2000). In 1988, Maneckjee et al. annealed one of the first polyclonal antibodies to MOR and showed ICC fluorescent labelling of MOR on a non-transfected neurotumour cell line, NCB-20 (Maneckjee et al., 1988). This was the first study to use ICC for visual detection of MOR in a non-transfected cell line. MOR staining was seen on both the membrane and within the cytoplasm, and the staining pattern was similar to that seen in the present study with SH-SY5Y cells.

Due to the initial unknown quality of the antibodies chosen in the present study, and the weak staining patterns observed, numerous positive and negative controls were essential to ensure receptor specificity, while amplifying the signal adequately to visualise staining. Four different MOR antibodies were selected in our study, all targeting the cytoplasmic carboxyl-terminal region of MOR. Several different splice variants of MOR have been 
described in the literature (Gavériaux-Ruff et al., 1997; Koch et al., 1998; Pan et al., 1999; Abbadie et al., 2000a; Koch et al., 2001), and all of these MOR antibodies have been demonstrated to be specific for the MOR-1 receptor splice variant (Schulz et al., 1998). In the present study all of the antibodies gave adequate staining, provided TSA amplification was used. The DOR antibody from Chemicon was the only DOR antibody tested in the present study, because it gave good, reliable staining, and was generally easier to work with than the four MOR antibodies. To some extent, the ease of using the DOR antibody was due to the experience gained with the earlier development of staining techniques for MOR antibodies.

\subsubsection{CONTROL FOR ANTIBODY SPECIFICITY}

Throughout this study, LLC-PK pig kidney cells showed no immunoreactivity for either MOR or DOR using ICC and Western blotting techniques and were used as negative controls. No previously studies, however, have mentioned the presence or absence of OpRs on LLC-PK I cells. These cells were used as a negative control to ensure that immunoreactivity was only correlated with the presence of opioid receptors. Controls using no primary antibody were also used to ensure that there was no reaction with endogenous HRP and that no non-specific DAB colour deposition occurred. DAB car produce a brown colour on non-labelled cells if incubated for too long during the colou development step. These negative controls were incorporated into every staining experiment and were incubated and developed for the same amount of time as the experimental samples.

SH-SY5Y cells were used as positive controls because they have both MOR and DOR opioid receptors present both before and after RA-induced differentiation (Kazmi and Mishra, 1986; Yu et al., 1986; Yu and Sadée, 1988; Cheng et al., 1995a). Differentiation could be confirmed by the morphological changes that occurred during neuronal maturation, including the formation of long neuritic outgrowths with networks of interconnecting processes (Påhlman et al., 1984; Påhlman et al., 1990). It has been shown by others from radiolabelled binding experiments (Yu and Sadée, 1988) that differentiation of SH-SY5Y cells causes an increase in expression of MOR. This increase in expression could clearly be seen in the present study, which employed ICC detection protocols. Differentiated SH-SY5Y cells with long neuronal processes showed increased staining for 
both MOR and DOR when compared to the more strongly attached, undifferentiated SHSY5Y cells with shorter processes (Figure 3.4A \& C). There have been no reports in the literature from radioligand binding to suggest that DOR levels are increased in differentiated SH-SY5Y, although an increase was clearly seen in our cultures using ICC. The increase in MOR and DOR staining in RA differentiated SH-SY5Y cells suggests that OpR ICC using the amplification techniques described in the present study, is in fact quantitative. However, the increase in staining must be large enough to be visualised. The increase in MOR with RA differentiation in SH-SY5Y cells has been reported by others to be approximately 32-40\% (Yu and Sadée, 1988). Taking these findings into account, the present study would expect to observe changes in OpR expression above this $30 \%$ range. Changes in OpR expression below this level are unlikely to show statistically significant changes in expression levels unless large sample numbers are used. The use of positive (SH-SY5Y cells) and negative (LLC-PK 1 cells) controls in conjunction with peptide blocking confirmed that the antibodies used were specifically labelling their purported targets. The best single negative control would be to use tissues from MOR and DOR knockout mice to be absolutely certain that the primary antibodies were binding specifically. These knockout models were not available in New Zealand at the time these experiments were performed.

\subsubsection{CELL TYPE-SPECIFIC OPIOID EXPRESSION IN CULTURE}

MOR and DOR are known to be present on neurons and glial cells within the brain (Hauser et al., 1990; Stiene-Martin and Hauser, 1991; Mansour et al., 1994; Chao et al., 1997). Changes in astrocyte opioid receptor expression levels with the cell cycle have been reported (Thorlin et al., 1999; Anders et al., 2000), with increased expression occurring in mitosis. The neurons and astrocytes in the present study, however, are unlikely to be undergoing mitosis. Brainstem neurons undergo their last cell division between E10 and E15 (Altman and Bayer, 1980; Phelps et al., 1990; Lakke, 1997), whereas, the astrocytes in serum-free culture do not divide significantly (Marriott et al., 1995). In the primary cultures of the present study, MOR and DOR staining was significantly greater on neurons than on glial cells. This was true for each developmental age tested, and in general, staining of most astrocytes was no higher than background levels. In our culture model it is possible that the occasional stronger staining of astrocytes seen in some cultures cou d be attributed to their stage in the cell cycle, although this parameter was not monitored. 
The serum-free Neurobasal medium used in this study has been reported by others to reduce cell proliferation (Brewer et al., 1993); thus, astrocyte cycling through the cell cycle should be minimal. Wilkin et al. (1995) has reviewed the functional significance of OpR expression in astrocytes (Wilkin et al., 1995). In general, opioids play a role in inhibiting cell division in astrocytes (Stiene-Martin and Hauser, 1990, 1991).

The present study is the first to describe OpR expression in cultures of brainstem neurons during late foetal and early postnatal development. A large number of neurons in culture showed positive immunoreactivities in single labelling studies of both MOR and DOR (between 40-70\% of total neurons in culture). It is difficult to ascertain whether the number of $\mathrm{OpR}$ positive neurons present in the present cell culture study using ICC technique is within the expected in vivo expression levels of MOR and DOR within the rat brainstem.

Previous cell culture studies, by others, have measured the amount of opioid ligand binding in cells cultured from regions other than the brainstem. Comparisons between OpR expression levels in cell cultures isolated from different brain regions are difficult to due to OpR expression being different in different brain areas (Mansour et al., 1988; Delfs et al., 1994; Mansour et al., 1994; Ding et al., 1996b; Cahill et al., 2001b). In vivo MOR immunoreactivity levels in the middle layers of the adult rat cortex have been described as moderate, whereas in the same study, brainstem staining was described as 'especially abundant' (Moriwaki et al., 1996). These results support the MOR immunoreactivity found in the present study (Chapter 6). One study of pig myenteric neurons in culture, although not stating the percentage of $\mathrm{OpR}$ positive neurons, did state that a small percentage $(10 \%)$ of the cultured neurons were positive for both DOR and KOR (Kulkarni-Narla and Brown, 2001), implying that single DOR labelled cells were much greater than $10 \%$. Another study measuring dynorphin-immunoreactivity (endogenous KOR ligand) in cultured dendate gyrus cells showed between $80 \%$ to $90 \%$ of neurons were OpR positive (He et al., 1992). A recent study by Lee et al. (2002) using cultured rat cortical neurons showed around $6.3 \%$ of neurons were positively labelled with fluoro-dermorphin, a fluorescent $y$ tagged MOR-specific ligand. The staining pattern observed was also punctate and within the soma and processes, similar to the staining patterns seen in the present study. In the same study by Lee et al. (2002), DOR labelling with fluoro-deltorphin, labelled approximately $11.5 \%$ of cultured cortical neurons (Lee 2002). Although some regions of 
the cortex have high expression levels of MOR and DOR, the expression levels within the brainstem are greater (Ding et al., 1996b; Moriwaki et al., 1996) especially when the density of OpR positive neurons were measured (Delfs et al., 1994). Taking these findings into account we would expect neurons cultured from the brainstem to show proportionally more neurons stained positive for MOR and DOR than cultures from the cortex. The $40 \%$ to $70 \%$ of neurons expressing MOR or DOR in brainstem cultures seen in the present study is therefore within realistic levels. The high proportion of MOR and DOR positive neurons is not likely to be due to staining artefacts because of the rigorous controls used to test for non-specific staining. It is likely that the new TSA amplification techniques allowed detection of very small receptor numbers compared with other more limited traditional methods that use radioactive binding or avidin/biotin ICC amplification methods.

Studies investigating opioid receptor expression in tissue slices have shown that the brainstem has a high density of both MOR- and DOR-positive cells in particular regions of the spinal trigeminal tract (STT), nucleus of the solitary tract (NTS) and nucleus ambiguus (NA) (Atweh and Kuhar, 1977; Mansour et al., 1988; Delfs et al., 1994; Ding et al., 1996b: Cahill et al., 2001b). Cells in primary culture may have altered receptor expression that differs from in vivo cell expression due to changes in the cellular environment and/or' changes in receptor-receptor and receptor-glial interactions. However, the number of OpR expressing cells in the present study remains high, suggesting no significant loss of OpR. expression.

\subsubsection{OPIOID RECEPTOR EXPRESSION DURING DEVELOPMENT}

Using ICC with DAB colour development, MOR immunoreactivity was shown to increase with developmental age of the brainstem tissue used for establishing the primary cultures. These findings are supported by radioligand binding experiments that show an increase in MOR expression between late foetal and early postnatal development in spinal cord (Attali et al., 1990; Rahman et al., 1998), hindbrain (Spain et al., 1985), and whole brain (Petrillo et al., 1987). In the present study, the estimation of MOR levels during development by quantitation of Western-transferred MOR immunolabelled protein (described in Chapter 6 (Section: 6.4.4)) also supports the visual observations of staining intensity in culture (Figure 3.9). From the present results, it can be concluded that MOR expression increases 
between E16 and P6. Unlike the clear changes seen with MOR, DOR immunoreactivity was similar in cultures established from late foetal and early postnatal animals. An increase in expression of DOR during early postnatal development has been reported, based on radioligand binding in the spinal cord, (Attali et al., 1990; Rahman et al., 1998) and whole brain, (Petrillo et al., 1987), although results from the present study do not support this. Using ICC (Chapter 3), no changes in DOR expression were seen. In brainstem homogenates from different developmental ages (Chapter 6), quantitation of Western-transferred immunolabelled protein indicated a decrease in the expression of DOR in the brainstem during development. Changes in expression levels that are too small to detect using ICC methods may account for these discrepancies. In addition, the normal variation commonly seen between cultures established from similar stages may mask small changes that do occur. Some papers report an increase in DOR expression at a later developmental age (P14) (Spain et al., 1985) than was possible to test in our primary culture model, since brainstem neurons older than P6 were difficult to establish in culture. Some older cultures were attempted, however, the results were not encouraging.

The increased expression of MOR during development is well known and accepted (Spain et al., 1985; Tavani et al., 1985; Petrillo et al., 1987), and may be related to the development of opioid related functions during early development. For example, in the maturation of opioid responses to respiration, which have been described in detail in Chapter 1 (Section: 1.3.7). Opioids are involved in the control of foetal, neonatal and adult breathing movements (Grunstein and Grunstein, 1982; Shook et al., 1990; Cheng et al., 1993a; Gray et al., 1999; Liu et al., 2000; Moss and Laferrière, 2000), with changes in the degree of response to opioid ligands occurring during development. These changes in sensitivity may relate to changes in OpR expression. The significance of this is discussed in more detail in Chapter 6.

The expression of DOR is generally accepted to increase during development (Spain et al., 1985; Tavani et al., 1985; Petrillo et al., 1987). However, the data presented in the present chapter shows DOR expression in cells cultured from the rat brainstem does not change significantly. Western blot results from brainstem lysates (Chapter 6) show a decrease in DOR expression with development. The significance of these findings is described in detain in Chapter 6. 
Some DAB-stained cultures were scanned on the confocal microscope and the density of staining measured using NIH Image software (data not presented). This gave a numerical value for staining intensity that replaced the visual 4-point scale used for Figure 3.9. The use of image analysis software, although making the data more 'quantitative', it did not alter the overall findings. Because no quantitative density measurements were taken during the early stages of the study and since the re-analysis of all the earlier data by this method would be unlikely to yield any new information, no density analysis results have been presented here. Staining intensity measurements were performed blind, whether they were assessed visually or by NIH image analysis, thus, removing any possible bias.

MOR and DOR staining patterns did not change with time in culture, suggesting that the cells in culture are stable, remaining at the same developmental stage as when they were isolated, with no developmental up- or down-regulation of receptors. The absence of developmental changes in OpR expression with time in cultured cells is probably a consequence of removal of signals controlling the developmental changes when the cells are isolated. When brainstem tissue is isolated, neuronal connections are lost and cannot be re-established in the culture dish. The suspension of the developmental state in culture has some benefits. Primary cultures are typically unstable with time as differentiatior factors are diluted out and chromosomal aberrations occur. A stable culture is an idea system in which to study factors controlling receptor expression levels. Since the contro of $\mathrm{OpR}$ expression during development is not understood, cell culture may provide a model in which specific factors affecting expression can be assessed individually. For example, neurotransmitters and growth factors can be added to cultures in a controlled manner and changes in OpR expression monitored.

\subsubsection{INTRACELLULAR DISTRIBUTION OF OPIOID RECEPTORS}

MOR and DOR have been shown to be present on the plasma membrane, in axonal and dendritic processes (Besse et al., 1990; Cheng et al., 1996; Svingos et al., 1998; Abbadie et al., 2000b), and in cytoplasmic vesicles (Abbadie et al., 2000a; Cahill et al., 2001b). This distribution could clearly be seen in the present study in neurons from primary brainstem cultures and in SH-SY5Y cells. Although differences in staining patterns were seen in individual neurons in culture, there were no overall differences in staining patterns in cultures established form different ages. Neurons from both late foetal and early postnatal 
cultures showed a variety of membrane and cytoplasmic staining patterns, and staining could be diffuse, punctate, or a mixture of both, depending on the individual neuron. Both MOR and DOR undergo rapid down-regulation following exposure to their respective agonists (Chang et al., 1982; Law et al., 1983; Puttfarcken and Cox, 1989). Downregulated receptors presumably give a punctate, non-membranous staining pattern if the receptors are sequestered into intracellular vesicles. The reason for the diffuse cytoplasmic staining is unknown, but may relate to poor antibody resolution of smaller vesicles. Opioid receptor localisation within the cell, specifically down-regulation of opioid receptors from the membrane to cytoplasmic vesicles, is discussed in detail in Chapter 4. Punctate staining of MOR and DOR within the cell body may be due to newly synthesised receptors awaiting insertion in the membrane. MOR and DOR receptors may also be awaiting transport to the neuronal processes, although recent studies suggest that $O p R$ are recycled locally, as no changes in immunoreactivity were observed between the soma and processes with agonist exposure over time (Lee et al., 2002). However, the methods used in the study may not have been sensitive enough to visualise receptor trafficking. Also, the receptors could have been transported retrogradely, as well as anterogradely. Transport of OpRs may be important in cultured neurons with rapidly growing neuritic processes. For example, cytoplasmic OpRs may be transported to neuronal processes for OpRs insertion into these peripheral processes. Future experiments specifically measuring the synthesis of new OpR proteins may help determine if this is the case.

Having a large population of inactive receptors inside the cell has the advantage of providing a mechanism for a fast response or up-regulation. Excess OpR expression within the cell may allow for a sudden increase in opioid function if the cell becomes overstimulated, or they may act to down-regulate a neuron when too much excitatory input comes to it. It remains to be determined if this in fact the case. MOR and DOR double labelling in the present study was often greatest in closely associated neurons or connecting processes. The resolution in the present study, however, is not sufficient to determine if this staining was localised to boutons and synaptic junctions. In future electron microscope studies will be needed to confirm this.

In the present study, different staining patterns were observed, for example, punctate, diffuse, membranous, and cytoplasmic. However, no trend was seen in the staining pattern with cell morphology or the developmental age of the culture. In future experiments, the 
cells could be labelled with antibodies to specific neurotransmitters, or neurotransmitter receptors and the staining pattern of OpRs determined. This would help to correlate OpR staining pattern with cellular functions. Although not examined in the present study, particular cell populations such as GABAergic or cholinergic neurons may be regulated differently by different $O p R$ subtypes and OpR trafficking within these cell types may be different. Colocalisation experiments using cell specific markers may help determine if this is the case.

\subsubsection{COLOCALISATION OF MOR AND DOR}

Double-labelling experiments were possible using standard double-labelling techniques (Pilowsky et al,, 1991; Pecci Saavedra et al., 1993), because the primary antibodies used were raised in different animals (guinea pig anti-MOR, and rabbit anti-DOR). Fluorescent detection of these antibodies used FITC and Rhodamine because these fluorophores emit wavelengths of light that can easily be separated using standard confocal microscope filters that prevent bleed-through of signal (Titus et al., 1982; Parks et al., 1983; Entwistle and Noble, 1992; Matsumoto et al., 1993). This procedure ensures that colocalisation is real and not a bleed-through artefact. Regular laser alignment checks of the confocal microscope were undertaken during the study to ensure that false positive or false negative colocalisation data were not collected as a result of poor laser alignment.

In primary cultures of the brainstem and in SH-SY5Y cells, colocalisation of MOR and DOR was evident in many cells. Primary cultures showed colocalisation at all ages, from late foetal to early postnatal stages. MOR and DOR colocalisation has been reported in several brain regions, including the dorsal horn of the spinal cord (Cheng et al., 1997; Abbadie et al., 2002) and many brainstem regions, including the STT, NTS and NA (Ding et al., 1996b; Cahill et al., 2001b). Colocalisation implies that both MOR and DOR ligands can dually modulate the response of a single neuron. Studies on MOR and DOR cotransfected cells have shown that these receptors form heterodimers that have novel signaling, binding, and functional properties (Jordan and Devi, 1999; George et al., 2000; Bouvier, 2001). Although heterodimers have not yet been described in vivo, there are atypical functional observations that may be explained by heterodimerisation. For example, in MOR knockout mice there is reduced DOR-mediated analgesia and DORmediated respiratory depression (Matthes et al., 1998). Thus, MOR must interact with 
DOR in some manner, possibly by heterodimer formation between the two receptors. The binding and functional properties of each opioid receptor subtype, although extensively studied, cannot be fully explained, and this provides further evidence that heterodimers may exist in cells where MOR and DOR are colocalised. Only one MOR gene has been described (Wang et al., 1994), although numerous splice variants exist (Koch et al., 1998; Pan et al., 1999; Koch et al., 2001; Pan et al., 2001). Thus, receptor subtypes may be generated in part by heterodimerisation and in part by variations in pre- or posttranslational modification of the mRNA.

\subsubsection{SUMMARY AND CONCLUSIONS}

Primary culture provides a good model to study opioid receptor expression during development. There are several advantages of using primary cell cultures to study developmental changes in opioid receptor expression. Cell culture enables threedimensional, intracellular localisation of receptors within the cell, and it is possible to visualise individual neurons and their processes. This detail at the cellular level is not possible using tissue slices, in part because thin sections are required for antibody penetration, and these do not allow observation of long neurite processes that extend through a number of slices. The density of cells in slices also makes the individua characteristics of each cell difficult to visualise due to masking of signals. In culture however, the cell density can be controlled, allowing individual neurons and theiprocesses to be easily observed. The disadvantages of using a tissue culture model are that changes in gene expression may occur after cells are removed from their normal environment, and developmental cues from other brain regions, including humoral regulators (neurotransmitters, glial trophic factors) and synaptic connections are absent.

The present study is the first to measure MOR and DOR expression and distribution in primary brainstem cultures during development. There are several advantages and disadvantages associated with using ICC rather than radioligand binding to detect opioid receptor proteins. Immunocytochemistry measures total protein, including membranebound receptors and internalised receptors; whereas, ligand binding usually measures only functional protein and does not measure internalised receptors. When using membrane homogenates $\mathrm{OpR}$ presence on intracellular vesicles is not taken into account unless they are specifically isolated by centrifugation, or cells are kept intact. When interpreting data 
from binding studies it is also important to consider incubation conditions. Opioid ligands are sensitive to incubation conditions; for example, the presence of sodium in the buffer decreases the binding affinity of OpR agonists to receptors (Pert and Snyder, 1974).

The cell culture model used in the present study did not show any changes in OpR expression with time in culture; therefore, the cells' differentiated phenotype was stable in culture. Unique expression levels, however, were seen with MOR when cells were isolated from different developmental ages, and the patterns of expression were similar to those reported by others for MOR expression during development. Thus, cell culture provides a useful model to study foetal and early neonatal developmental changes in OpR expression. The culture system would not be suitable for studies on maturation in older postnatal $(>\mathrm{P} 9)$ or adult animals. Because OpR expression is stable with time in culture, this method has the advantage that it can be used to study factors that regulate OpR expression. It also allows for a more flexible timing of experimental manipulations. All things considered, the primary culture model described in this study provides a novel system for investigating opioid receptor expression and function during development of the brainstem. 


\section{CHAPTER 4}

\section{Opioid Receptor Regulation In Culture}

\subsection{AIM}

To demonstrate that primary cultures of rat brainstem neurons can be used to model opioid receptor responses, MOR was cloned and transfected into a cell line to generate a positive control that over-expresses MOR, thus providing a strong signal for ICC monitoring. MOR down-regulation in response to an opioid agonist was then assessed. Enhanced expression of the cloned MOR was also used to test the specificity of the opioid receptor antibodies.

\subsection{INTRODUCTION}

MOR was used in this study to evaluate opioid receptor $(\mathrm{OpR})$ regulation in cultured cells. and to observe if cultured brainstem cells express functional OpRs. MOR-transfected cells and SH- SY5Y cells were used as positive controls to study opioid receptor regulation SH-SY5Y cells endogenously express opioid receptors in culture (Kazmi and Mishra, 1986; Yu et al., 1986), and MOR localisation in both MOR-transfected cells and non. transfected SH-SY5Y cells are regulated in culture on exposure to MOR agonists and antagonists (Prathert et al., 1994; Kramer and Simon, 1999; Remmers et al., 2000).

Opioid receptor function and regulation is extremely complicated and not very well understood. The current understanding of OpR regulation has been described in detail in the introduction (Chapter 1: Section: 1.2.3). Most studies have been carried out on transfected cells that over-express MOR or on established cell lines, such as the neuroblastoma cell line SH-SY5Y that endogenously expresses MOR. Primary cultures, however, may provide better models to study opioid receptor function in the brainstem because the cells more closely resemble the natural state. OpR-positive cells in different regions of the brain have unique functions that relate to the location of the receptors (Mansour et al., 1987; George et al., 1994; Arvidsson et al., 1995a; Elde et al., 1995; Monteillet-Agius et al., 1996; Svingos et al., 1996; Wittert et al., 1996). For example, OpRs in the pre-Bötzinger complex, nucleus of the solitary tract (NTS), and nucleus ambiguus (NA) of the brainstem are involved in respiratory modulation (Sessle and Henry, 
1985; Dashwood et al., 1988; Shook et al., 1990; Xia and Haddad, 1991; Takita et al., 1998; Gray et al., 1999; Laferrière et al., 1999); whereas, OpRs in the periaqueductal gray (PAG) play a role in pain modulation (Vaughan and Christie, 1997). OpRs have been shown to colocalise with various other neurotransmitters and neurotransmitter receptors (Ding et al., 1995; Wang et al., 1996; Gong et al., 1997; Commons et al., 1999). The different functions of MOR in different brain regions may be due to unique receptor subtypes, unique splice-variant distributions (Koch et al., 1998; Pan et al., 1999; Abbadie et al., 2000a; Koch et al., 2001), localisation with different neurotransmitters or neurotransmitter receptors (Ding et al., 1995; Wang et al., 1996; Gong et al., 1997; Commons et al., 1999; Aicher et al., 2000a; McDonald and Mascagni, 2001), or formation of heterodimers with novel binding characteristics and/or signal transduction pathways (George et al., 2000; Bouvier, 2001; Levac et al., 2001; Ramsay et al., 2002). A single neuronal cell line model like SH-SY5Y can be used to study OpRs, but may not adequately reflect the properties of all MOR-containing cells in the brain. To better understand MOR function in the brainstem, a brainstem primary cell model has been analysed and compared with the more classical cell culture models of OpR regulation involving transfected cells and established cell lines. 


\subsection{METHODS}

\subsubsection{CELL CULTURE}

Several cell lines were used in the present study to find a cell line with a high efficiency of MOR transfection.

\subsubsection{COS-7}

The COS-7 African green monkey kidney cell line (SV-40 transformed) from American Type Culture Collection (ATCC, VA, USA) was grown in DMEM (Invitrogen) with low bicarbonate $(1.5 \mathrm{~g} / \mathrm{L}), 10 \% \mathrm{FCS}(\mathrm{v} / \mathrm{v}), 50 \mu \mathrm{M}$ selenium, penicillin $\mathrm{G}(50 \mathrm{U} / \mathrm{mL})$, and streptomycin sulphate $(50 \mu \mathrm{g} / \mathrm{mL})$. The cell line was passaged using trypsin as described for SH-SY5Y cells Chapter 3 (Section: 3.3.1.2).

\subsubsection{H441}

H441 cells are a human lung bronchoalveolar adenocarcinoma cell line (ATTC). The cells were maintained in RPMI-1640 medium (Invitrogen) supplemented with 10\% (v/v) FCS, $50 \mu \mathrm{M}$ selenium, penicillin $\mathrm{G}(50 \mathrm{U} / \mathrm{mL})$, and streptomycin sulphate $(50 \mu \mathrm{g} / \mathrm{mL})$. Cells were passaged 1:10 every week using trypsin-EDTA (Invitrogen) in the same way as described for LLC-PK 1 cell lines (Chapter 3, Section: 3.3.1.3).

\subsubsection{SH-SY5Y}

See Chapter 3 (Section: 3.3.1.2) for detailed characteristics and culture methods of theSHSY5Y neuroblastoma cell line.

\subsubsection{MOR CLONING}

All cloning experiments were carried out under the New Zealand Environment Risk Management Authority (ERMA) approval code, GMD01138, in a certified PC2 laboratory.

\subsubsection{EXPERIMENTAL DESIGN}

Primers were designed using Oligo 6 software (Version 6.02, Molecular Biology Insights Inc., Plymouth, MN, USA) spanning the start and stop sites of rat MOR mRNA (GenBank accession number L22455) (Thompson et al., 1993). The MOR forward primer spanned the start codon and the reverse primer spanned the stop codon at site 1366 (Figure 4.1). Optimal calculated annealing temperature of the MOR primers was $57.8^{\circ} \mathrm{C}$, yielding a 
predicted 1196 bp PCR product (Figure 4.1). The lyophilised primers were purchased from Invitrogen. The primers were centrifuged and reconstituted at $100 \mu \mathrm{M}$ in TE buffer (10 mM Tris; $1 \mathrm{mM}$ EDTA, $\mathrm{pH} 8.0$ ) and stored at $-20^{\circ} \mathrm{C}$. At least 17 nucleotides in the region of interest complementary to MOR were included in each primer. Some primers included a terminal attB (+4Gs) Gateway-specific sequence needed for insertion of the PCR product into the Gateway ${ }^{\mathrm{TM}}(\mathrm{GW})$ vectors. The $\mathrm{GW}$ cloning system has the advantage that it maintains gene orientation and reading frame, essential features for successful transfection experiments. This system also allows the gene to be easily moved into different vectors. Some reverse primers were also tagged with the FLAG sequence (CTACTTGTCGTCATCGTCCTTGTAGTC (reverse complement)), which was used to provide antibody-labelling of MOR transfected cells with FLAG-specific antibodies. The pattern of staining of transfected cells labelled with the anti-FLAG antibody was compared with staining by MOR antibody (guinea pig anti-MOR, Chemicon) to ensure its MOR specificity. Anti-FLAG antibody was also used to measure transfection efficiency. 


\section{Figure 4.1 MOR Sequence}

1 cacgagcttc tgcctgccge tcttctctgg ttccactagg getggtccat gtaagaatct

61 gacggagcet agggcagctg tgagaggaag aggctgggge gcgtggaace cgaaaagtct

121 gagtgctctc agttacagcc tacctagtce gcagcaggec ttcagcacca tggacagcag 181 caccggceca gggaacacca gcgactgctc agacccctta gctcaggcaa gttgctcccc 241 agcacctgge tcctggctca acttgtccca cgttgatgge aaccagtccg atccatgcgg 301 tctgaaccgc accgggcttg gcgggaacga cagcetgtgc cotcagaccg gcagccctc 361 catggtcaca gccattacca tcatggccet ctactctatc gtgtgtgtag tgggcctctt 421 cggaaacttc ctggtcatgt atgtgattgt aagatacacc aaaatgaaga ctgccaccaa 481 catctacatt ttcaaccttg ctctggcaga cgccttagcg accagtacac tocctttca

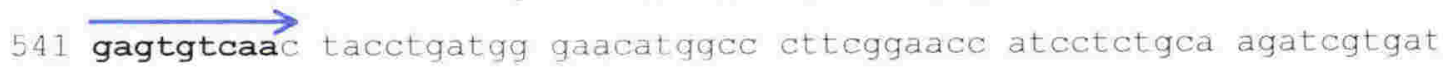
601 ctcaatagat tactacaaca tgttcaccag catattcacc ctctgcacca tgagcgtgga 661 ccgctacatt gctgtetgcc acccagtcaa agccctggat ttccgtaccc cccgaatgc 721 caaatcgtc aacgtctgca actggatcct ctcttctgce atcggtctgc ctgtaatgtt 781 catggcáacc acaaataca ggcaggggtc catagattgc accctcacgt tctcccacc 841 aacctggtac tgggagaacc tgctcaaat ctgtgtcttt atcttcgctt tcatcatgco $901 \overrightarrow{\text { ggtcctcatc atcactgtgt gttacggcet gatgatctta cgactcaaga gegttcgcat }}$ 961 gctatcgggc tccaaagaaa aggacaggaa tctgcgcagg atcacccgga tggtgctggt 1021 ggtcgtgget gtatttatcg tetgctggac ccccatccac atctacgtca tcatcaaagc 1081 gctgatcacg attccagaaa ccacatttca gacggtttcc tggcacttct gcattgctt 1141 gggttacacg aacagctgcc tgaatccagt tctttacgcc ttcctggatg aaaacttcac 1201 gegatgcttc agagagttct geatcccaac ctcgtccacg atcgaacagc aaaactccac 1261 tcgagtccgt cagaacacta gggaacatcc ctccacggct aatacagtgg atcgaactau 1321 ccaccagcta gaaaatctgg aggcagaaac tgctccattg ccctaactgg gtctcacacu 1381 atccagaccc tcgctaagct $t$

\section{$\rightarrow$ Sequencing Primers \\ $\rightarrow$ Cloning Primers \\ - Start and stop codons}

Primer sequences are shown in bold, and indicated by arrows. 


\subsubsection{RNA Isolation}

For RNA isolation experiments, all glassware was baked for 4 hrs at $180^{\circ} \mathrm{C}$, and all solutions were autoclaved and/or made up with diethyl-pyro carbonate (DEPC) treated water (Appendix 2: Section 8). Micropipette filter tips and plasticware were purchased certified RNAse free, and a separate set of pipetters, dedicated only to RNA isolation, was used to prevent RNAse contamination.

A 50-100 mg piece of brainstem tissue from an adult male rat was isolated and immediately transferred to a $1.5 \mathrm{~mL}$ microfuge tube containing $1 \mathrm{~mL}$ of TRIZOL ${ }^{\circledR}$ Reagent $^{-1}$ (Invitrogen). Tissue samples were immediately homogenised using a Polytron PT 1200C power homogeniser (Kinematica AG, Switzerland) for $30 \mathrm{sec}$ at full power, taking care to keep the probe immersed. The sonicator probe was prepared earlier by immersion in RNAse away® solution (Molecular Bio Products Inc., San Diego, CA, USA), air-dried, then washed 3 times in DEPC-treated water before immersion in the sample. The homogenised samples were incubated at room temperature for $5 \mathrm{~min}$ before the addition of $0.2 \mathrm{~mL}$ of chloroform. The tubes were shaken vigorously by hand for $15 \mathrm{sec}$ and incubated at room temp for 2-3 min. Samples were centrifuged at $12,000 \mathrm{rpm}$ for $15 \mathrm{~min}$ at $4^{\circ} \mathrm{C}$. Following centrifugation, the upper, clear aqueous phase containing RNA was transferred into a new tube, and $0.5 \mathrm{~mL}$ of $100 \%$ isopropyl alcohol added. The solution was mixed and incubated at room temperature for $10 \mathrm{~min}$ before centrifugation at $12,000 \mathrm{rpm}$ for 10 min at $4^{\circ} \mathrm{C}$. The supernatant was removed, and precipitated RNA could be seen as a gellike pellet at the bottom and on the sides of the tube. One $\mathrm{mL}$ of $75 \%$ ethanol (prepared with DEPC-treated water) was added and the pellet resuspended using a vortex mixer. The sample was centrifuged at $7500 \mathrm{rpm}$ for $5 \mathrm{~min}$ at $4^{\circ} \mathrm{C}$. The supernatant was discarded and the RNA pellet allowed to air dry for 5-10 min before redissolving in $21 \mu \mathrm{L}$ of water. The concentration of RNA was determined by diluting $1 \mu \mathrm{L}$ of RNA sample in $99 \mu \mathrm{L}$ water and the absorbance measured in a spectrophotometer at $260 \mathrm{~nm}$. The concentration of RNA was determined from the nucleic acid extinction coefficient (50) using the following calculation.

Concentration $(\mu \mathrm{g} / \mu \mathrm{L})=\mathbf{A}_{260} \times$ dilution $\times 50$ 


\subsubsection{Reverse Transcription- PCR (RT-PCR)}

Diluted RNA ( $1 \mu \mathrm{L}$ of $2 \mathrm{ng} / \mu \mathrm{L}$ ) was used immediately after RNA isolation in the RT-PCR reaction. The remaining RNA was stored at $-80^{\circ} \mathrm{C}$. The integrity of RNA was determined by running a sample of RNA ( $2 \mu \mathrm{L}$ of RNA, and $2 \mu \mathrm{L}$ of DNA Loading Dye) on a $0.8 \%$ agarose gel. Care was taken to reduce RNAse contamination by using DEPC-treated solutions. RNAse away solution was used to wash the gel tank. Two-step RT-PCR was performed using C. therm. Polymerase ${ }^{B}$ (Roche Diagnostics GmbH, Mannhein, Germany). Two-step RT-PCR was chosen so that each step could be performed under optimum conditions. The following reagents were pipetted into a thin-walled RNAse/DNAse-free PCR tube (Invitrogen). Reagents were thawed, vortexed, and placed on ice before setting up the reactions (Table 4.1). Oligo dT primers (Invitrogen) were diluted to $1 \mu \mathrm{M}$, and $0.4 \mu \mathrm{L}$ was added to the reaction. The oligo $(\mathrm{dT})$ reaction was carried out at $60^{\circ} \mathrm{C}$ for $45 \mathrm{~min}$ in a PCR machine (Progene Techne, John Morris Scientific, NZ) and placed at $-20^{\circ} \mathrm{C}$ overnight or until the next reaction step.

\section{Table 4.1 Reagents used for RT-PCR}

\begin{tabular}{|c|c|c|}
\hline Component & $\begin{array}{l}\text { Volume per } \\
\text { Reaction }(\mu \mathrm{L})\end{array}$ & Final Concentration \\
\hline 5x RT Buffer & 4.0 & $\begin{array}{l}10 \mathrm{mM} \text { Tris-HCL, } \mathrm{pH} 8.5,10 \mathrm{mM} \mathrm{KCl}, 60 \mathrm{mM} \\
\left(\mathrm{NH}_{4}\right)_{2} \mathrm{SO}_{4} 2 \mathrm{mM} \mathrm{MgCl}\end{array}$ \\
\hline DTT & 1.0 & $5 \mathrm{mM}$ \\
\hline $\begin{array}{l}\text { dNTP mix, } \\
25 \mathrm{mM}\end{array}$ & 0.64 & $0.8 \mathrm{mM}$ each \\
\hline DMSO & 0.6 & $5 \%$ \\
\hline $\begin{array}{l}\text { C. therm. } \\
\text { polymerase }\end{array}$ & 1.5 & 6 units \\
\hline $\begin{array}{l}\text { Primer, } 20 \mu \mathrm{M} \\
\text { oligo dT } \\
\text { or mu reverse } \\
\text { primer }\end{array}$ & $\begin{array}{l}0.4 \\
1.0\end{array}$ & $\begin{array}{l}0.4 \mu \mathrm{M} \\
1.0 \mu \mathrm{M}\end{array}$ \\
\hline RNA (2ng/ $\mu \mathrm{L})$ & $1 \mu \mathrm{L}$ & $1 \mu \mathrm{g} / 20 \mu \mathrm{L}$ \\
\hline $\begin{array}{l}\text { Sterile distilled } \\
\text { water }\end{array}$ & Up to $20 \mu \mathrm{L}$ & Up to $20 \mu \mathrm{L}$ \\
\hline TOTAL & $20 \mu \mathrm{L}$ & \\
\hline
\end{tabular}

As an alternative protocol, the reverse MOR primer (5'-TAG GGC AAT GGA GCA GT $\Gamma$ TC-3') was used in this reaction to specifically amplify MOR cDNA from the total mRNA, in contrast to amplification of total cDNA from total mRNA. The quantity and quality of 
the product was determined by agarose gel electrophoresis, and the product was purified using a DNA gel purification protocol (Section: 4.3.3.5).

\subsubsection{Polymerase Chain Reaction (PCR)}

PCR was performed using the Expand High Fidelity PCR System® (Roche Diagnostics). This system uses a thermostable Taq DNA polymerase. A proofreading polymerase is included to increase the yield and reduce the number of PCR-induced errors. All reactions were performed on ice, taking care not to contaminate samples with foreign DNA. Master mix solutions were made from individual stocks and were added to sterile $0.2 \mathrm{~mL}$ PCR tubes (Invitrogen) as shown in (Table 4.2). After the appropriate amounts of Master $\mathrm{m} x$ were added to each individual reaction tube, forward and reverse primers (Figure 4.2) were added, along with the DNA template.

MOR primers were used both with and without the GW and FLAG tags, and PCR conditions were optimised for annealing temperature and cycle number. A hot start PCR cycle was used consisting of $94^{\circ} \mathrm{C}$ for $60 \mathrm{sec}$ followed by 40 cycles of $95^{\circ} \mathrm{C}$ for $15 \mathrm{sec}$, $57.5^{\circ} \mathrm{C}$ for $90 \mathrm{sec}$, and $72^{\circ} \mathrm{C}$ for $15 \mathrm{sec}$. This 40 -cycle sequence was then followed by $72^{\circ} \mathrm{C}$ for $5 \mathrm{~min}$, and the reaction was then held at $4^{\circ} \mathrm{C}$. 
Figure 4.2 MOR Cloning and Sequencing Primers 


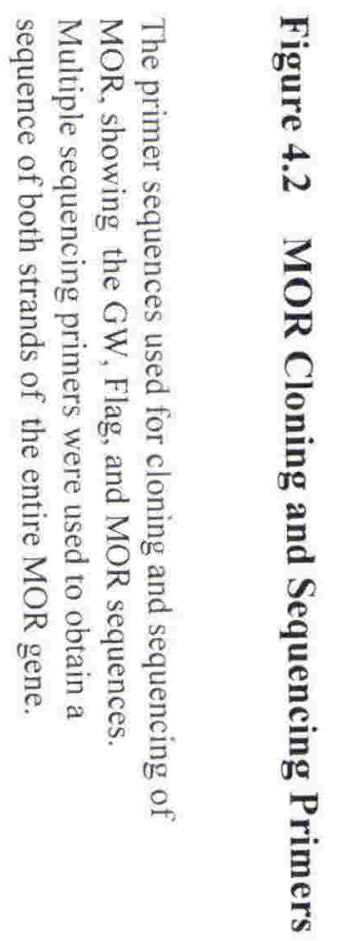




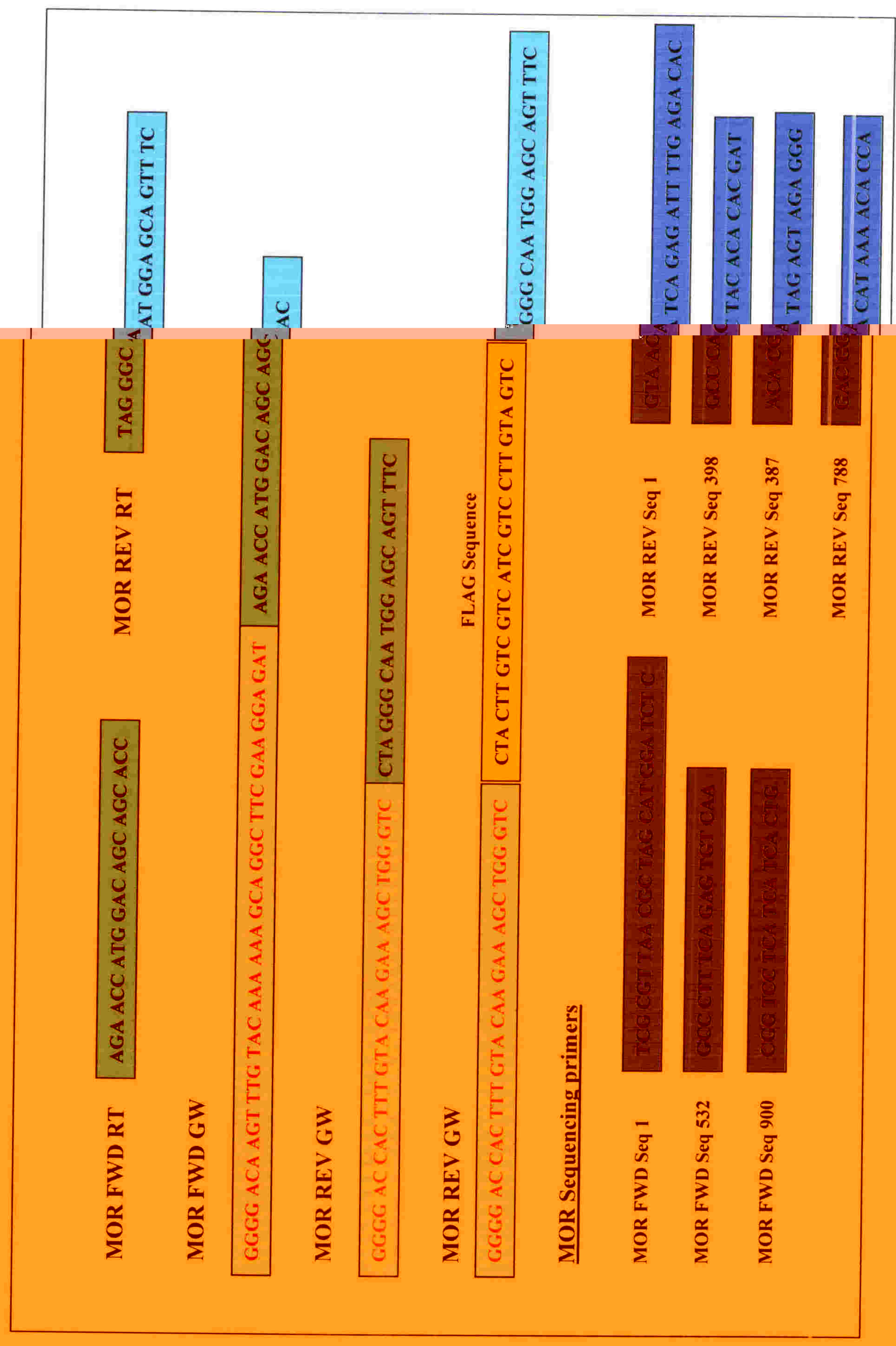


Table 4.2 Solutions used in PCR reactions

\begin{tabular}{|c|c|c|}
\hline \multicolumn{3}{|l|}{ Master mix 1} \\
\hline & Reagent & Dlume \\
\hline & dATP (10 mM) & $1 \mu \mathrm{L}$ \\
\hline & dCTP (10 mM) & $1 \mu \mathrm{L}$ \\
\hline & dGTP (10 mM) & $1 \mu \mathrm{L}$ \\
\hline & dTTP (10 mM) & $1 \mu \mathrm{L}$ \\
\hline & DNA Template (10 ng) & $5 \mu \mathrm{L}$ \\
\hline & 5' Primer $(100 \mu \mathrm{M})$ & $1.5 \mu \mathrm{L}$ \\
\hline & 3' Primer (100 $\mu \mathrm{M})$ & $1.5 \mu \mathrm{L}$ \\
\hline & Sterile dd $\mathrm{H}_{2} \mathrm{O}$ & $13 \mu \mathrm{L}$ \\
\hline & TOTAL & $25 \mu \mathrm{L}$ \\
\hline \multicolumn{3}{|l|}{ Master mix 2} \\
\hline & Reagent & Volume \\
\hline & Sterile dd $\mathrm{H}_{2} \mathrm{O}$ & $19.25 \mu \mathrm{L}$ \\
\hline & $\begin{array}{l}\text { 10x Expand } \mathrm{Hi} \text { Fi Buffer } \\
\text { (with } 15 \mathrm{mM} \mathrm{MgCl}_{2} \text { ) }\end{array}$ & $5 \mu \mathrm{L}$ \\
\hline & Expand Hi Fi Enzyme Mix & $0.75 \mu \mathrm{L}$ \\
\hline & TOTAL VOLUME & $25 \mu \mathrm{L}$ \\
\hline
\end{tabular}

\subsubsection{Agarose Gel Electrophoresis}

DNA samples containing $5 \mu \mathrm{L}$ of DNA loading dye (10\% Ficoll; $0.025 \%$ bromophenol blue) were run on $0.8 \%$ agarose (Invitrogen) gels containing $1 \mu \mathrm{g} / \mathrm{mL}$ of ethidium bromide (Invitrogen) in TAE buffer (0.04 M Tris-acetate, 1 mM EDTA, pH 8.0). A 100 bp DNA ladder (BioRad, CA, USA) was used as a standard. Electrophoresis was performed at 100 $\mathrm{V}$ for $1 \mathrm{hr}$, or until the dye front was $2 / 3$ of the way down the gel. Bands were observed under UV light and polaroid photographs taken. Some samples needed for later experiments were excised from the gel with a clean scalpel and placed in clean microfuge tubes for gel purification of the DNA.

\subsubsection{DNA Gel Purification}

DNA bands of the appropriate size for MOR were cut out of the agarose gel and purif ed using a QIAquick® gel extraction kit (Qiagen, GmbH, Germany). The gel slice was weighed, and $300 \mu \mathrm{L}$ of QG buffer ( $\mathrm{pH} 7.50$ ) was added for every $100 \mathrm{mg}$ of gel. The 
sample was incubated at $50^{\circ} \mathrm{C}$ until the gel slice had dissolved. Isopropanol $(100 \mu \mathrm{L}$ of $100 \%$ ) was added per $100 \mathrm{mg}$ of gel slice, and the sample was mixed. A QIAquick ${ }^{\circledR}$ spin column provided in the kit was placed in a $2 \mathrm{~mL}$ collection tube, and the sample was loaded and centrifuged in a tabletop microfuge for $1 \mathrm{~min}$ at $13,000 \mathrm{rpm}$. Flow-through was discarded and $0.75 \mathrm{~mL}$ of PE buffer added. The solution was incubated for up to $5 \mathrm{~min}$ at room temperature before centrifugation for $1 \mathrm{~min}$ at 13,000 rpm. The flow-through was again discarded, the column centrifuged again, and the residual flow-through discarded. The column was placed in a clean $1.5 \mathrm{~mL}$ microfuge tube, and $30 \mu \mathrm{L}$ of elution buffer ( 10 $\mathrm{mM}$ Tris/ $\mathrm{HCl}, \mathrm{pH} 8.5$ ) was added to the centre of the QIAquick ${ }^{\circledR}$ membrane. The sample was left to stand for $1 \mathrm{~min}$ to increase the DNA yield, and then centrifuged at full speed for $1 \mathrm{~min}$. Approximately $28 \mu \mathrm{L}$ was recovered from the column. Purified DNA was stored at $-20^{\circ} \mathrm{C}$ until needed for later analysis.

\subsubsection{6 pGEM®-T PCR Cloning Method}

The purified MOR DNA PCR product was ligated into the $\mathrm{pGEM}^{\circledR}$-T plasmid (Promega Corporation, Madison, WI, USA), following manufacturer's instructions. Cloning reagents (Table 4.3) were placed in sterile $0.2 \mathrm{~mL}$ PCR tubes, mixed by pipette, and incubated overnight at $4^{\circ} \mathrm{C}$. The attributes of the pGEM-T plasmid are described in Appendix 4.

Table 4.3 Reagents used to ligate MOR into pGEM-T vector

\begin{tabular}{|c|c|c|c|}
\hline Reagent & $\begin{array}{c}\text { Standard } \\
\text { Reaction }\end{array}$ & $\begin{array}{c}\text { Positive } \\
\text { Control }\end{array}$ & $\begin{array}{c}\text { Background } \\
\text { Control }\end{array}$ \\
\hline $\begin{array}{c}\text { 2X Rapid Ligation Buffer } \\
\text { (T4 DNA Ligase) }\end{array}$ & $5 \mu \mathrm{L}$ & $5 \mu \mathrm{L}$ & $5 \mu \mathrm{L}$ \\
\hline pGEM@-T Vector (50 ng) & $1 \mu \mathrm{L}$ & $1 \mu \mathrm{L}$ & $1 \mu \mathrm{L}$ \\
\hline PCR Product & $3 \mu \mathrm{L}$ & - & - \\
\hline Control Insert DNA (4 ng/ $\mu \mathrm{L})$ & - & $2 \mu \mathrm{L}$ & - \\
\hline T4 DNA Ligase (100u/mL) & $1 \mu \mathrm{L}$ & $1 \mu \mathrm{L}$ & $1 \mu \mathrm{L}$ \\
\hline & $0 \mu \mathrm{L}$ & $1 \mu \mathrm{L}$ & $3 \mu \mathrm{L}$ \\
\hline Deionised Water & $10 \mu \mathrm{L}$ & $10 \mu \mathrm{L}$ & $10 \mu \mathrm{L}$ \\
\hline
\end{tabular}


A $5 \mu \mathrm{L}$ aliquot of this mixture was added to transform $50 \mu \mathrm{L}$ of DH5 $\alpha$ competent bacteria (Invitrogen), as described below. This $\mathrm{pGEM}^{\circledR}-\mathrm{T}$ vector has a $\beta$-galactosidase $(\beta \mathrm{GAl}$ ) colour selection feature that causes formation of blue colonies if the $\beta G A L$ gene is not disrupted by an insert of DNA. Transformed bacteria are plated onto LB-amp agar plates (Appendix 2: Section 10) pre-coated with $60 \mu \mathrm{L}$ of X-galactosidase (XGAL) solution ( 50 $\mu \mathrm{L}$ of $40 \mathrm{mg} / \mathrm{mL}$ XGAL; $10 \mu \mathrm{L}$ of $1 \mathrm{M}$ IPTG). White colonies were picked and cultured for DNA miniprep using the alkaline lysis method (see below).

\subsubsection{TRANSFORMING BACTERIA}

Plasmid DNA from the cloning reactions was used to transform library efficiency competent DH5 $\alpha$ cells (Invitrogen). A $10 \mathrm{~cm}$ diameter petri dish containing LB amp agar (LB medium with $1.5 \%$ agar (DIFCO, Becton Dickinson, MD, USA) and $50 \mu \mathrm{g} / \mathrm{mL}$ ampicillin, Appendix 2: Section 10) or LB-kanamycin agar (LB-medium with $1.5 \%$ agar and $50 \mu \mathrm{g} / \mathrm{mL}$ kanamycin) was used to grow the bacteria, depending on the antibiotic resistance characteristics of the plasmid. Fifty $\mu \mathrm{L}$ of competent cells were thawed on ice, placed in a $1.5 \mathrm{~mL}$ microfuge tube, and $1 \mu \mathrm{L}$ of plasmid DNA added. This mixture was gently mixed and placed on ice for $30 \mathrm{~min}$, then heat-shocked at $40^{\circ} \mathrm{C}$ in a waterbath for 2 min, then placed on ice for a further $2 \mathrm{~min}$. SOC medium $(450 \mu \mathrm{L})(2 \%$ tryptone, $0.5 \%$ yeast extract, $0.05 \% \mathrm{NaCl}, 2.5 \mathrm{mM} \mathrm{KCl}, 20 \mathrm{mM}$ glucose, $10 \mathrm{mM} \mathrm{MgCl}, \mathrm{pH} 7.0$, Appendix 2: Section 11) was added and the mixture incubated at $37^{\circ} \mathrm{C}$ for $1 \mathrm{hr}$. This mixture was then plated onto LB plates, left at room temperature for $1 \mathrm{hr}$, then turned upside down and placed in a $37^{\circ} \mathrm{C}$ incubator overnight.

\subsubsection{MINIPREP ISOLATION OF PLASMID DNA}

\subsubsection{Miniprep Standard Alkaline Lysis Method}

Using a sterile toothpick, single colonies were used to inoculate $2 \mathrm{~mL}$ LB medium (Appendix 2: Section 9) containing appropriate selective antibiotic (50 $\mu \mathrm{g} / \mathrm{mL}$ ampicillin or kanamycin) in a sterile $20 \mathrm{~mL}$ test tube with a loose-fitting cap. The tube was placed in a shaking incubator at $37^{\circ} \mathrm{C}$ overnight. The next day, $1.4 \mathrm{~mL}$ of this culture was transferred to a $1.5 \mathrm{~mL}$ microfuge tube, and centrifuged for $1 \mathrm{~min}$ at $13,000 \mathrm{rpm}$. The supernatant was removed and the pellet resuspend in $100 \mu \mathrm{L}$ of ice cold GTE Buffer 50 $\mathrm{mM}$ glucose, $25 \mathrm{mM}$ Tris $\mathrm{HCl} \mathrm{pH} 8.0$, and 10 mM EDTA, pH 8.0) and incubated at room 
temperature for $5 \mathrm{~min}$. The bacteria were then lysed by adding $200 \mu \mathrm{L}$ of $\mathrm{P} 2$ buffer $(0.2 \mathrm{M}$ $\mathrm{NaOH}, 1 \%$ SDS). The tube contents were mixed by inverting the tube several times, followed by incubation on ice for $5 \mathrm{~min}$. Protein in the lysate was precipitated by adding $150 \mu \mathrm{L}$ of ice-cold P3 Buffer ( $3 \mathrm{M}$ potassium acetate, $\mathrm{pH} 5.5$ ). The samples were vortexed gently for $10 \mathrm{sec}$ to resuspend the pellet and then incubated on ice for $10 \mathrm{~min}$. Tubes were centrifuged for $10 \mathrm{~min}$ to pellet the protein. The supernatant was removed and placed in a fresh tube, and $400 \mu \mathrm{L}$ of phenol:chloroform:isoamyl alcohol $(24: 24: 1)$ was added. The sample was vortexed briefly, then centrifuged for $5 \mathrm{~min}$ at $4^{\circ} \mathrm{C}$ to separate the phases. The top aqueous phase was transferred to a fresh tube and $900 \mu \mathrm{L}$ of ethanol added. The sample was then mixed using a vortex mixer and centrifuged for a further 10 min to pellet the DNA. The supernatant was removed, the pellet washed once with $1 \mathrm{~mL}$ of $70 \%$ ethanol. The sample was recentrifuged and the ethanol removed. The DNA pellet was allowed to dry at room temperature for 10-20 min. When the pellet was dry, $30 \mu \mathrm{L}$ of TE buffer containing $50 \mu \mathrm{g} / \mathrm{mL}$ RNAse was added, and the sample was mixed and incubated for $10 \mathrm{~min}$ at room temperature to dissolve the DNA. A $5 \mu \mathrm{L}$ aliquot was removed to determine DNA concentration. The aliquot was added to $495 \mu \mathrm{L}$ of dd $\mathrm{H}_{2} \mathrm{O}$ and the absorbance of the solution measured at $260 \mathrm{~nm}$ in a spectrophotometer. The DNA concentration was calculated using the following calculation.

\section{Concentration $(\mu \mathrm{g} / \mu \mathrm{L})=\mathbf{A}_{260} \times$ dilution $\times 50$}

The purified DNA was PCR-amplified using MOR-specific primers and the product electrophoresed on a $0.8 \%$ agarose gel to confirm the presence of MOR inserts. If the appropriate 1200 bp MOR band was present, the purified plasmid DNA was used in subsequent PCR reactions with MOR primers containing the GW and FLAG sequences inserts that are necessary for cloning into GW vectors. After successful PCR with the GW primers, the DNA was electrophoresed on an agarose gel, the appropriate sized bands were cut out of the gel and the DNA purified using the DNA gel purification protocol described earlier. DNA from this step was then used in the GW BP reaction. 


\subsubsection{MIDIPREP ISOLATION OF PLASMID DNA}

\subsubsection{Midiprep Using Qiagen Plasmid Purification System}

The Qiagen plasmid purification system (Qiagen) was used to purify DNA required for mammalian transfection experiments. This system was used because it removes endotoxins, which are detrimental to transfection experiments. A flask containing $50 \mathrm{~mL}$ of LB-amp was inoculated with a $200 \mu \mathrm{L}$ aliquot of a miniprep culture containing the pDEST 12.2 plasmid with the MOR-FLAG tagged DNA. The culture was placed in a shaking incubator and agitated overnight at $220 \mathrm{rpm}$ at $37^{\circ} \mathrm{C}$. The next day, cells were harvested by centrifugation $\left(6000 \mathrm{rpm}\right.$ for $15 \mathrm{~min}$ at $4^{\circ} \mathrm{C}$ in a SS-34 rotor in a Sorval RC $5 \mathrm{~B}$ centrifuge). The supernatant was discarded and the pellet resuspended in $4 \mathrm{~mL}$ of $\mathrm{P} 1$ Buffer (50 mM Tris- $\mathrm{HCl}, \mathrm{pH} 8.0,10 \mathrm{mM}$ EDTA) and incubated at room temperature for 5 min. Four mL of P2 buffer ( $200 \mathrm{mM} \mathrm{NaOH}, 1 \%$ SDS) was added to lyse the cells. The tubes were inverted 6 times and then incubated at room temperature for $5 \mathrm{~min}$. Proteins were precipitated by adding $4 \mathrm{~mL}$ of chilled P3 buffer ( $3 \mathrm{M}$ potassium acetate $\mathrm{pH} 5.5$ ) the samples inverted 6 times to $\mathrm{mix}$, and then incubated on ice for $15 \mathrm{~min}$. Samples were centrifuged at $20,000 \mathrm{rpm}$ for $30 \mathrm{~min}$ at $4^{\circ} \mathrm{C}$. The supernatant was placed in a fresh plastic centrifuge tube and recentrifuged at $20,000 \mathrm{rpm}$ for $15 \mathrm{~min}$ at $4^{\circ} \mathrm{C}$. Meanwhile, the Qiagen-tip 100 was equilibrated with buffer by applying $4 \mathrm{~mL}$ of QBT Buffer $(750 \mathrm{mM}$ $\mathrm{NaCl}, 50 \mathrm{mM}$ MOPS $\mathrm{pH} 7.0,15 \%$ isopropanol, $0.15 \%$ Triton $\mathrm{X}-100$ ), and allowing the ip to empty by gravity flow. The supernatant containing the DNA was then applied to the column and left to empty by gravity flow. The Qiagen-tip was washed with $10 \mathrm{~mL}$ of (OC buffer ( $1 \mathrm{M} \mathrm{NaCl}, 50 \mathrm{mM}$ MOPS, pH 7.0, 15\% isopropanol) and allowed to empty. This step was repeated, and the Qiagen-tip placed above a clean $50 \mathrm{~mL}$ Corex centrifuge tube. The DNA was eluted with $5 \mathrm{~mL}$ of QF Buffer (1.25 M NaCl, $50 \mathrm{mM}$ Tris-HCl, pH 8.5, $15 \%$ isopropanol). The DNA was precipitated by adding $3.5 \mathrm{~mL}$ of room temperature isopropanol, mixing and centrifuging for $30 \mathrm{~min}$ at $15,000 \mathrm{rpm}$ at $4^{\circ} \mathrm{C}$. The supernatant was removed, and the DNA pellet was washed with $2 \mathrm{~mL}$ of $70 \%$ ethanol, and then recentrifuged for $10 \mathrm{~min}$ at $15,000 \mathrm{rpm}$ at $4^{\circ} \mathrm{C}$. The ethanol was removed and the DNA pellet allowed to air dry for 10-20 min before resuspending in $200 \mu \mathrm{L}$ of TE buffer $(1 \mathrm{~mL}$ of $1 \mathrm{M}$ Tris; $200 \mu \mathrm{L}$ of $0.5 \mathrm{M}$ EDTA, pH 8.0). The yield of DNA was determined from its absorbance at $260 \mathrm{~nm}$, as described previously. 


\subsubsection{Midiprep Concert ${ }^{\mathrm{TM}}$ High Pure Plasmid Purification System}

For DNA sequencing, a Concert High Pure Plasmid Purification Kit (Invitrogen), similar to the Qiagen kit, was used to prepare plasmid DNA according to the manufacturer's instructions.

\subsubsection{Gateway BP Reaction}

The BP reaction allows rapid cloning of PCR products synthesised with the appropriate attB tags (Figure 4.3) into a range of GW entry vectors. Once the gene is in the entry clone, it is easily transferred to other GW vectors, each with specific properties. For example, vectors designed for transfection into mammalian expression systems (pDEST 12.2). MOR DNA was cloned into the GW pDONR 201 Vector (Appendix 4). Reagents from the kit (Table 4.4) were added to a $1.5 \mathrm{~mL}$ microfuge tube at room temperature, mixed, and incubated at $25^{\circ} \mathrm{C}$ for $60 \mathrm{~min}$. One $\mu \mathrm{L}$ of proteinase $\mathrm{K}$ solution was added and the solution incubated for $10 \mathrm{~min}$ at $37^{\circ} \mathrm{C}$ according to the manufacturer's instructions. One $\mu \mathrm{L}$ of this reaction was used to transform $50 \mu \mathrm{L}$ of library efficiency DH5 $\alpha$ competent cells as previously described (Section 4.3.4: Transforming bacteria). The transformed bacteria were spread onto LB plates containing $50 \mu \mathrm{g} / \mathrm{mL}$ kanamycin (Sigma). 
Table 4.4 Reagents required for the GW BP reaction.

\begin{tabular}{|l|c|c|c|}
\hline \multicolumn{1}{|c|}{ Component } & Negative control & Positive Control & Sample \\
\hline $\begin{array}{l}\text { PCR product } \\
\text { (with attB tags) } \\
40-100 \text { fmol }\end{array}$ & - & - & $1-5 \mu \mathrm{L}$ \\
\hline $\begin{array}{l}\text { PEXP7- tet } \\
\text { Positive control } \\
(50 \text { ng/ } \mu \mathrm{L})\end{array}$ & - & $1 \mu \mathrm{L}$ & - \\
\hline $\begin{array}{l}\text { pDONR } 201 \text { Vector } \\
(150 \text { ng/ } \mu \mathrm{L})\end{array}$ & $1 \mu \mathrm{L}$ & $1 \mu \mathrm{L}$ & $1 \mu \mathrm{L}$ \\
\hline $\begin{array}{l}\text { BP Reaction Buffer } \\
(5 x)\end{array}$ & $2 \mu \mathrm{L}$ & $2 \mu \mathrm{L}$ & $2 \mu \mathrm{L}$ \\
\hline TE Buffer & $5 \mu \mathrm{L}$ & $4 \mu \mathrm{L}$ & To $8 \mu \mathrm{L}$ \\
\hline
\end{tabular}

The next day, colonies, selected with a toothpick, were grown in $2 \mathrm{~mL}$ of LB medium containing $50 \mu \mathrm{g} / \mathrm{mL}$ kanamycin. The bacteria harbouring the pDONR 201 vector containing MOR were purified using the miniprep alkaline lysis method as previously described. DNA from this reaction was PCR amplified using the MOR primers to see if it contained MOR DNA. Only DNA that could successfully PCR amplify MOR was used for further reactions. The remaining culture was used to make glycerol stocks by adding $10 \%(\mathrm{v} / \mathrm{v})$ glycerol. The stocks were stored at $-80^{\circ} \mathrm{C}$.

\subsubsection{Gateway LR Reaction}

The LR reaction is the second reaction in the GW cloning system. The LR reaction is a recombination reaction between an entry clone and a destination vector and is equivalent to highly specific cutting and ligation reactions. In this reaction proteins cut to the left and right of the gene within the attL site of the entry clone (Figure 4.3). This is then ligated to the appropriate site in the destination vector. The destination vector used in this study (pDEST12.2) is designed for mammalian expression experiments.

The LR reaction requires linearised DNA for optimal results. The entry pDONR 201 vector, with the MOR DNA insert was linearised by cleavage at the Apa I restriction site within the GW cassette. Ten $\mu \mathrm{L}$ of DNA from the BP reaction was added to $1 \mu \mathrm{L}$ of Apa I 
enzyme (Roche), $2 \mu \mathrm{L}$ of Apa I restriction enzyme buffer A (Roche), and $7 \mu \mathrm{L}$ of sterile cd $\mathrm{H}_{2} \mathrm{O}$ in a $1.5 \mathrm{~mL}$ microfuge tube. The solution was mixed, and then incubated for $60 \mathrm{~m} \mathrm{n}$ at $30^{\circ} \mathrm{C}$. This linearised entry clone was used in the following LR reaction.

Following the manufacturer's instructions, reagents were added to $1.5 \mathrm{~mL}$ microfuge tubes (Table 4.5), mixed using a vortex, and incubated for $60 \mathrm{~min}$ at $25^{\circ} \mathrm{C}$. One $\mu \mathrm{L}$ of proteinase $\mathrm{K}$ solution was added to the template reaction and incubated for $10 \mathrm{~min}$ at $37^{\circ} \mathrm{C}$. After the LR reaction was complete, $1 \mu \mathrm{L}$ of this reaction was used to transform $50 \mu \mathrm{L}$ of libracy efficiency DH5 $\alpha$ competent cells (Section 4.3.4). The transformed cells were then plated onto LB plates containing $50 \mu \mathrm{g} / \mathrm{mL}$ ampicillin and grown in an incubator overnight at $37^{\circ} \mathrm{C}$.

Table 4.5 Reagents required for the GW LR reaction

\begin{tabular}{|l|c|c|c|}
\hline \multicolumn{1}{|c|}{ Component } & Negative control & Positive Control & Sample \\
\hline $\begin{array}{l}\text { LR Reaction Buffer } \\
(5 \mathrm{x})\end{array}$ & $2 \mu \mathrm{L}$ & $2 \mu \mathrm{L}$ & $2 \mu \mathrm{L}$ \\
\hline $\begin{array}{l}\text { PENTR-gus } \\
(50 \mathrm{ng} / \mu \mathrm{L})\end{array}$ & - & $1 \mu \mathrm{L}$ & - \\
\hline $\begin{array}{l}\text { Entry Clone } \\
(100-300 \mathrm{ng} / \text { reaction })\end{array}$ & $1 \mu \mathrm{L}$ & $1 \mu \mathrm{L}$ & $0.4 \mu \mathrm{L}$ \\
\hline $\begin{array}{l}\text { Destination Vector } \\
\text { pDEST } 12.2 \\
(150 \text { ng/ } \mu \mathrm{L})\end{array}$ & To $8 \mu \mathrm{L}$ & To $8 \mu \mathrm{L}$ & $1 \mu \mathrm{L}$ \\
\hline \begin{tabular}{l} 
TE Buffer \\
\hline
\end{tabular} & & & To $8 \mu \mathrm{L}$ \\
\hline
\end{tabular}

\subsubsection{DNA Sequencing}

DNA sequencing of the plasmid DNA, purified using the Concert High Pure Plasmid Midiprep Purification System, was performed in the Victoria University of Wellington DNA Sequencing Facility using an ABI Prism 377 DNA sequencer (Perkin Elmer Applied BioSystems, Foster City, CA, USA) and appropriate sequencing primers (Figure 42). Sequencing was carried out to ensure that the MOR DNA was in the correct reading frame and contained no errors. Both strands were sequenced at least once in both directions. 
PCR tubes were placed on ice and $4 \mu \mathrm{L}$ BigDye ${ }^{\mathrm{TM}}$ termination mix (Perkin Elmer), $250 \mathrm{ng}$ of DNA template, and $0.8 \mu \mathrm{L}$ of $1 \mu \mathrm{M}$ primer mix added. The cycle sequencing reaction was carried out in a Gene Amp 9600 PCR machine (Perkin Elmer) and consisted of 25 cycles of $96^{\circ} \mathrm{C}$ for $10 \mathrm{sec}, 50^{\circ} \mathrm{C}$ for $5 \mathrm{sec}$ and $60^{\circ} \mathrm{C}$ for $4 \mathrm{~min}$, with a final hold at $4^{\circ} \mathrm{C}$. The reaction was then transferred to a Sepadex G50 sequencing purification spin column (Appendix 2: Section 12) for desalting. The eluted samples were lyophilised and stored at $-20^{\circ} \mathrm{C}$ until electrophoresis was carried out. Lyophilised samples were supplied to the sequencing technician and analysed on an ABI prism 377 DNA sequencer, following the manufacturer's instructions. 


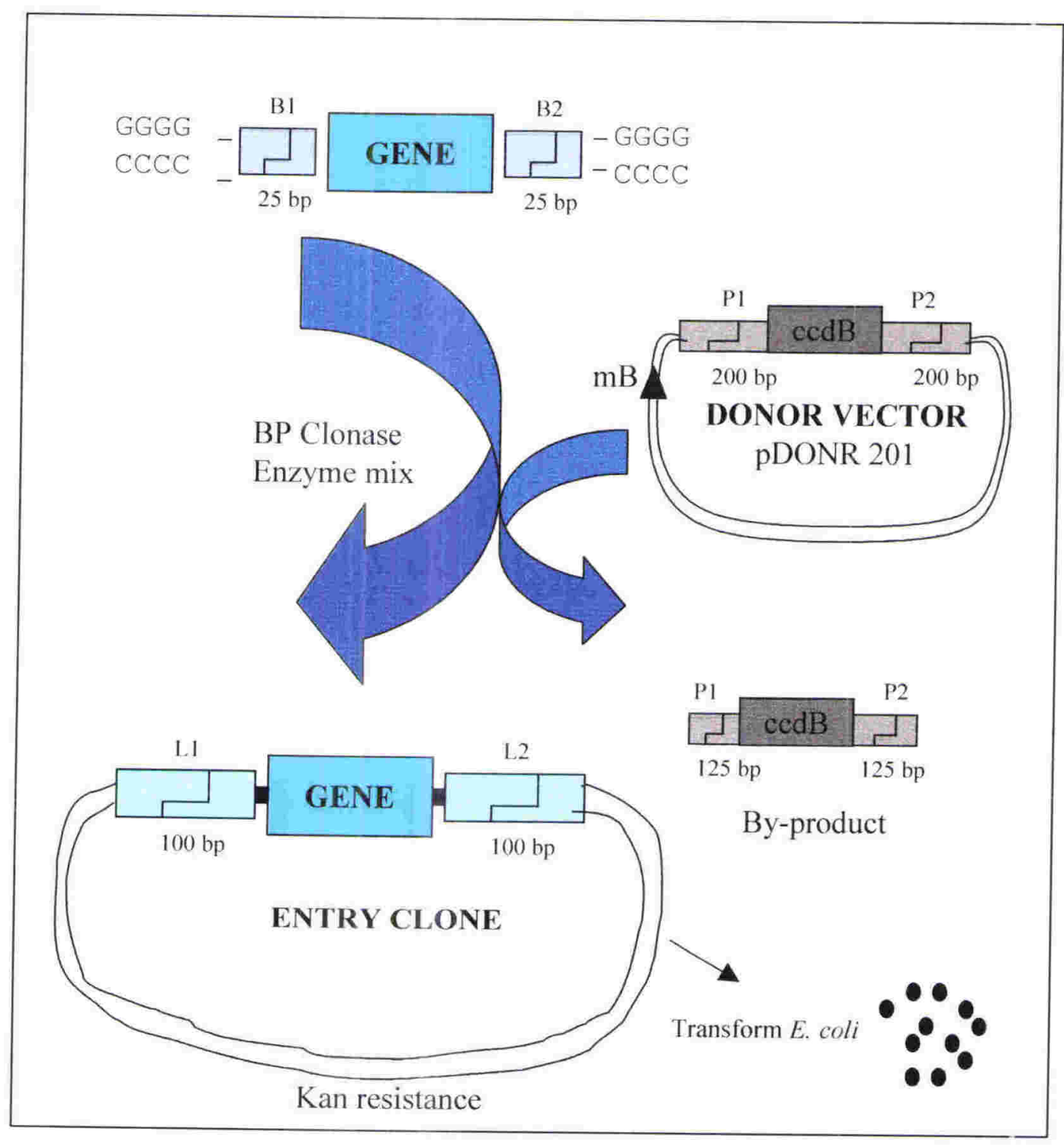

Figure 4.3

\section{Cloning a PCR Product using the Gateway BP Reaction}

A gene flanked by the Gateway attB1 and attB2 sites can be cloned between the attL1 and attL2 sites of an entry vector, and transformed into E. coli. Kanamycin (Kan) resistant colonies can then be selected. 


\subsubsection{TRANSFECTION OF MAMMALIAN CELL LINES}

Two different methods were used to transfect a number of different mammalian cell lines, and several different volumes of transfection reagent were tested to optimise transfection efficiency. Transfected cells that were used for immunocytochemistry were grown on PDL-coated slides as previously described (Chapter 2, Section: 2.3.1.2).

\subsubsection{FuGENETM6 Transfection}

Cells were dissociated using trypsin-EDTA in the same way as for passaging (Chapter 3, Section: 3.3 .1 .2 ), resuspended in $10 \mathrm{~mL}$ culture medium without antibiotics, centrifuged at $1000 \mathrm{rpm}$ for $5 \mathrm{~min}$ and resuspended in $1 \mathrm{~mL}$ of culture medium. Ten $\mu \mathrm{L}$ of the cell suspension was removed and added to $80 \mu \mathrm{L}$ of medium and $10 \mu \mathrm{L}$ trypan blue $(0.2 \%$ in PBS). The proportion of viable cells was determined by dye exclusion using a haemocytometer. Cells were plated at $3 \times 10^{5}$ cells $/ 35 \mathrm{~mm}$ plate, grown overnight, and checked the following day to ensure the cells were $50-80 \%$ confluent. Serum-free medium (94 $\mu \mathrm{L}$ or $97 \mu \mathrm{L}$ ) was placed in a sterile microfuge tube and either $3 \mu \mathrm{L}$ or $6 \mu \mathrm{L}$, respectively, of Fugene ${ }^{\mathrm{TM}} 6$ reagent (Roche) added to give a final volume of $100 \mu \mathrm{L}$. Care was taken to prevent the Fugene ${ }^{\mathrm{TM}_{6}}$ reagent from touching the sides of the plastic microfuge tube. Gentle tapping mixed the tube's contents, $1 \mu \mathrm{g}$ of DNA was added, and the solution mixed again. The tube's contents were incubated at room temperature for 15 min before addition to the culture dish. The medium in the culture dish was gently swirled to mix. Cultures were incubated between $12 \mathrm{hr}$ to $72 \mathrm{hr}$ in a $5 \% \mathrm{CO}_{2}$ atmosphere in a humidified, $37^{\circ} \mathrm{C}$ incubator.

\subsubsection{Lipofectamine Transfection}

Cells were plated the day before transfection as described for Fugene ${ }^{\mathrm{TM}} 6$ transfection. Six $\mu \mathrm{L}$ of Liofectamine reagent (Invitrogen) was added to a $1.5 \mathrm{~mL}$ microfuge tube containing $94 \mu \mathrm{L}$ of serum-free, antibiotic-free, culture medium and the reagent combined with $1 \mu \mathrm{g}$ of DNA within $5 \mathrm{~min}$, according to the manufacturer's instructions. Samples were mixed gently and incubated at room temperature for $20 \mathrm{~min}$ before adding to the culture dish as described above. 


\subsubsection{IMMUNOCYTOCHEMISTRY}

Cells cultured on coverslips were fixed and prepared for ICC by removing endogenous peroxidase activity (Chapter 2; Section; 2.3.2.2) and blocking in TSA TM blocking solution as previously described in Chapter 3 (Section: 3.3.2.1).

\subsubsection{ANTI-FLAG ANTIBODY LABELLING OF TRANSFECTED CELLS}

A FLAG-tag sequence was added to the cloned rat MOR DNA sequence so that expressed protein could be detected using an anti-FLAG IgG mouse antibody (1:1000) (Sigma). This primary antibody was incubated overnight, washed, incubated in goat anti-mouse HRP (1:500) (Sigma) for $1 \mathrm{hr}$, washed, and incubated in TSATM_FITC (or rhodamine) for 3 min as previously described (Chapter 3; Section; 3.3.2.2). Cells were also labelled using the anti-MOR antibody or double-labelled using anti-FLAG and anti-MOR antibodies, the latter using the double-labelling protocol previously described in Chapter 3 (Section: 3.3.5).

\subsubsection{MOR RECEPTOR IN VITRO REGULATION}

MOR regulation was monitored in cell culture using the ICC method of Koch et al. (1998) (Koch et al., 1998). In order to down-regulate MOR, the MOR agonist [D-Ala ${ }^{2}, \mathrm{~N}-\mathrm{Me}$ $\mathrm{Phe}^{4}, \mathrm{Gly}^{-\mathrm{ol}^{5}}$ ] enkephalin (DAMGO) $(1 \mu \mathrm{M})$ was added to cultures for up to $4 \mathrm{hr}$, and cells were fixed for ICC after 1, 2, or 4 hrs. Some cultures were pre-incubated with a sterile solution of $50 \mu \mathrm{M}$ monensin (Sigma) ( $5 \mathrm{mM}$ (100x) stock in ethanol) for $60 \mathrm{~min}$ prior to DAMGO administration. Monensin traps MOR in cytoplasmic vesicles by preventing endosome acidification (Koch et al., 1998). Control cells were cultured at the same time but were not treated with DAMGO. Cytoplasmic and membrane staining of individual cells was scored on a scale of $0=$ no staining, $1=$ light staining, $2=$ medium staining, and $3=$ intense staining. Voltage matched confocal images of serial sections through the cells were scored by blinded observations. Staining intensity measurements of at least 100 cells from 4 coverslips taken from 2 separate experiments were performed blind by 4 different people and the data collated $(n=4$ coverslips). A multiple comparisons test (based on Kruskal-Wallis rank sums, $\mathrm{P}<0.001)$ was carried out according to the criteria outlined in Hollander and Wolfe (1973) (Hollander and Wolfe, 1973b) (Appendix 3). 


\subsection{RESULTS}

\subsubsection{MOR CLONING}

RNA was successfully isolated from the adult rat brainstem at a concentration of 1.25 $\mu \mathrm{g} / \mu \mathrm{L}$. The integrity of the RNA was confirmed by electrophoresing a portion of the sample on an agarose gel. If the RNA was not degraded, the sample was used immediately for RT-PCR reactions. Initial PCR experiments (from the cDNA obtained in the RT reaction) only worked with the short MOR cloning primers, not the longer primers with the GW or FLAG sequences. It was therefore necessary to clone MOR into the pGEM-T plasmid (Figure 4.4A) before PCR with MOR-GW-FLAG primers were successful (Figure 4.4). Following this, MOR was cloned into the pDONR $201 \mathrm{GW}$ vector, then into the pDEST 12.2 vector. Successful MOR clones in the pDEST 12.2 vector were used for transfection experiments. The clones were confirmed by DNA sequencing. Multiple sequencing runs were combined using Seqman ${ }^{\mathrm{TM}}$ II software (DNASTAR Inc.) and a consensus sequence formed from these multiple sequencing runs (Appendix 5). Results, displayed using the Jellyfish program (LabVelocity Inc., San Francisco, CA, USA), showed that the MOR consensus sequence calculated from the multiple sequencing runs did not contain PCR-induced errors (Figure 4.5).

\subsubsection{MOR TRANSFECTION OF MAMMALIAN CELL LINES}

MOR-transfected cell lines were used as controls to check that the guinea pig anti-MOR antibody (Chemicon) was specifically labelling MOR. Double-labelling MOR-transfected cells with anti-FLAG antibodies and directly comparing staining patterns confirmed this. Transfection experiments were also used as a positive control to test whether cultured cells from the rat brainstem express OpRs that were capable of being regulated by opio d agonist exposure. MOR function was assessed by treatment of cells with a MOR agonist, DAMGO, and measuring receptor internalisation (down-regulation).

MOR was successfully transfected into SH-SY5Y cells and H441 cells using both Lipofectamine and FuGENE ${ }^{\mathrm{TM}} 6$ reagents. There were no differences in transfection efficiency between lipofectamine and FuGENE ${ }^{\mathrm{TM}} 6$. Although SH-SY5Y and H441 cells were successfully transfected, transfection into COS-7 and LLC-PK 1 cells was not successful. H441 cells had low transfection efficiencies (about 3\%) under all conditions tested, with varying DNA concentrations and transfection reagent concentrations, and 
were, therefore, not used in further experiments. SH-SY5Y cells were chosen for further MOR transfection experiments because they gave the best transfection efficiency of the cells tested, on the order of $17 \%$. Transfection efficiency was measured using anti-FLAG antibodies (TSA-FITC) on PI-counterstained cells after 5, 24, 48, and $72 \mathrm{hr}$ of transfection. Transfected cells were counted as a percentage of the total number of cells in 3 fields of view, using a $40 \mathrm{X}$ lens on the confocal microscope. The average transfection efficiencies for $5,24,48$, and $72 \mathrm{hr}$, were $3 \%, 15 \%, 17 \%$ and $9 \%$, respectively. For all further experiments the $24 \mathrm{hr}$ transfection period was chosen because it gave the best transfection efficiency, with an average of $17 \%$ and a range of $5-25 \%$. Higher transfection efficiencies are desirable, as they allow a greater number of cells to be studied in each experiment.

SH-SY5Y cells endogenously express MOR; therefore, labelling experiments were performed using the anti-FLAG antibody. This antibody binds to the FLAG sequence that was added to the end of the MOR sequence during cloning. Some experiments using the guinea pig anti-MOR antibody (Chemicon) were also carried out. Transfected cells expressed much higher levels of MOR than non-transfected SH-SY5Y cells and could easily be detected by ICC at lower voltage settings on the confocal microscope. Transfected cells were also double-labelled with both anti-FLAG and anti-MOR antibodies (Figure 4.6), and both antibodies gave identical staining, confirming that the guinea pig anti-MOR antibody from Chemicon was specific for MOR.

No cross-reactivity was seen between DOR and MOR with the DOR specific antibody used in the present study. In some experiments (data not shown) MOR-transfected H441 cells were immunolabelled for DOR, although no H441 cells showed labelling for DOR. MOR-transfected SH-SY5Y cells double labelled with DOR and FLAG specific antibodies, showed no DOR immunoreactivity using the same confocal microscope laser voltage settings (in SH-SY5Y cells, endogenous DOR expression levels could of course be seen at high voltages in the same way as endogenous MOR expression). These results confirm the specificity of the DOR antibody for the DOR receptor. 
MOR PCR from PGEM-T vector

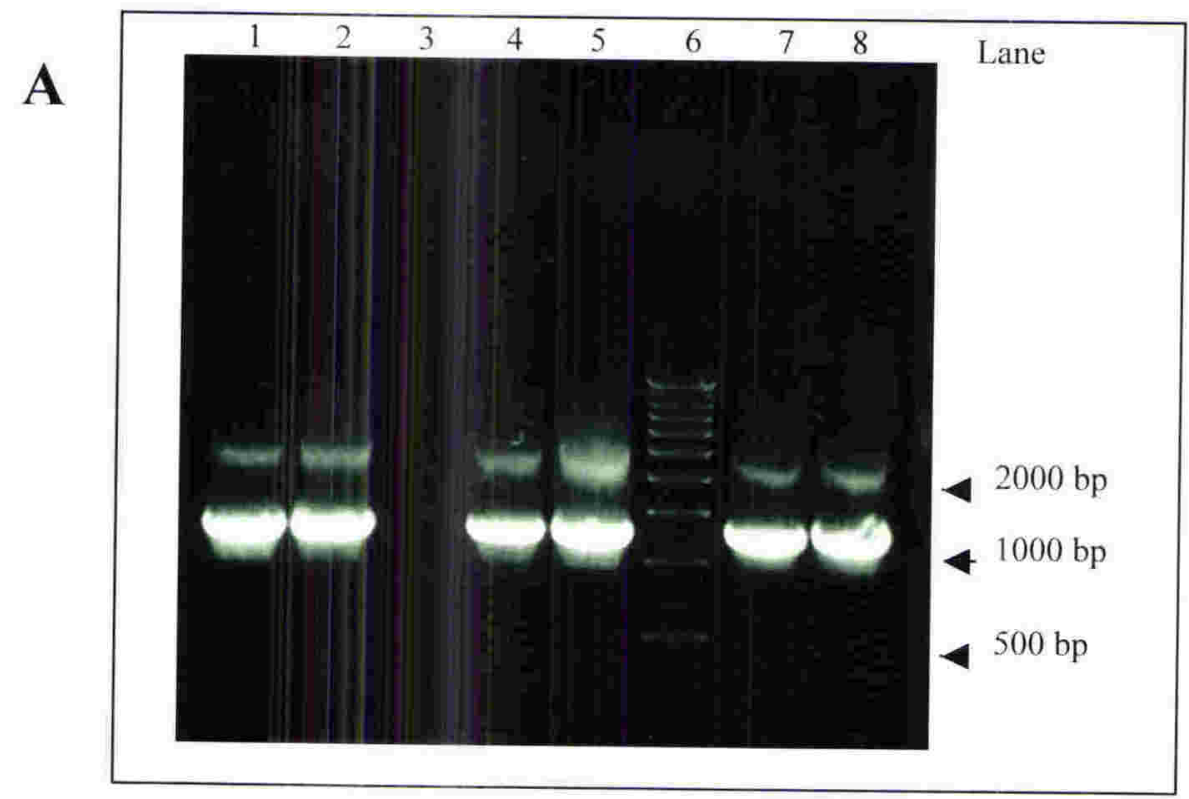

MOR PCR from pDEST 12.2 Gateway mammalian cloning vector

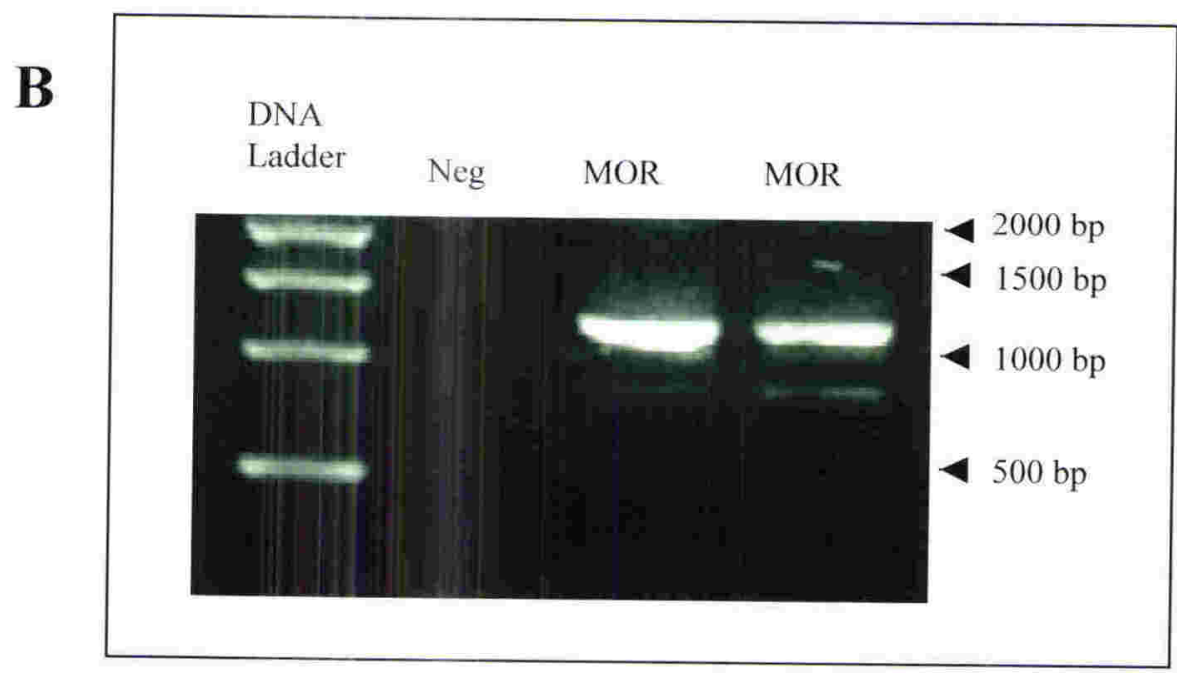

Figure 4.4 MOR PCR

(A) PCR of MOR from the pGEM-T vector using the MOR FWD Gateway (GW) and REV FLAG GW primers, showing a $1200 \mathrm{bp}$ band in several PCR reactions in lanes 1. $2,4,5,7 \& 8$. Another band can be seen at approx 2400 bp and is likely to be a double copy of MOR. The $1200 \mathrm{bp}$ product was purified from the gel and cloned into GW vectors pDONR 201 and pDEST 12.2. Note the negative control in lane 3 and the molecular weight markers in lane 6. (B) MOR PCR primers. Only colonies in which MOR PCR amplification was successful as shown by a 1200 bp product were sequenced and used for transfection experiments. 
Figure 4.5 Sequence Alignment of the Cloned Rat MOR-1 Receptor 
Figure 4.5 Sequence Alignment of the Cloned Rat MOR-1 Receptor

The consensus DNA sequence from the cloned rat MOR and the rat MOR sequence code L22455 from GenBank showing the correct sequence for the MOR-1 receptor 
Cloned MOR AGCACCATGGäCACCACCATGGACAGCAGCACCGGCCCAGGGARCACCAGCGACTGCTCAGACCCCTIAGCTCAGGCARG Rat MOR Sequ -.--.--TTCAGCACCATGGACAGCÄGCACCGGCOCAGGGAACACCAGCGACTGCTCAGACCCCTTAGCTCACGCAAG Consensus cagcaccatggacagcagcaccggcceagggaacaccagcgactgctcagaccecttagctcacgcaag

cloned MoR Rat Mor Sequ

Consensus

cloned Mor

Rat MoR Sequ

Consensus

cloned MoR

Rat MOR Sequ Consensus

Cloned MOR Rat MOR Seqru Consensus

cloned MOR Rat MoR Sequ Consensus

Cloned MOR Rat MOR Sequ Consensus

Cloned MOR Rat MoR Sequ Consensus

Cloned MOR Rat MOR Sequ Consensus

Cloned MOR Rat MOR Sequ Consensus

Cloned MOR Rat MOR Sequ Consenzus

Cloned MOR Rat MoR Sequ Consensus

cloned MOR Rat MOR Segu Consensus

cloned MOR Rat MOR Sequ Consensus

Cloned MoR Rat MOR Sequ Consensus

cloned MoR

Rat MoR Sequ Consensus
31
91
101
111
121
131
141
151

TGCTCLCCAGCACCTGGCTCCTGGCTEAACITGTCCCACGTTGATGGCAACCAGTCCGATCOATGCGMTCTEA CCGCA TTGCTCCCCAGCACCTGGCTCCTGBCTCAACTTGTCCCACGTTGATGGCAACCAGTCCGATC CATGCGGTCTGAג CCGCA ttgctceccagcacctggctcctggctcaacttgtcccacgttgatggcaaccagtccgatccatgcggtctgakccgca

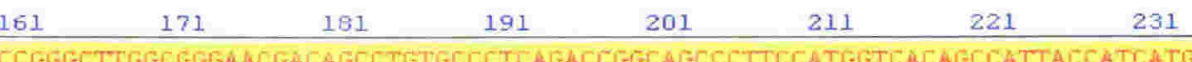
CCGGGCTTGGEGGGAACGACAGOCTGTGCCCTCAGACCGGCAGCCCTTCCATGGTCAC AGCCATTACCATCATGI:CCCTC ccgggcttggcgggaacgacagcctgtgccctcagaccggcagccctccatggtcacagccattaccatcatguccctc
251
261
271
281
291
301
311

241 TACTCTATCGTGTGLGTAGTGGGCCTCTTCGGAAACTTCETGGTCATGTATGTGATTGTAAGÄTACACCAARAT KAAGAC tactctatcgtgtgtgtagtgggcctctcggaaact tcctggtcatgtatgtgattgraagatacaccaaaat jaagac 321 331

341 351 361 381 391 TECCACCAACATCTACATITTCAACCTTGCTCTGGCAGACGCCITAGCGACCAGTACACTGCCCTITCAGACTG TCAACT TGCCACCAACATCTACATTTTCAACLTTGCTCTGGCAGACGCCTTAGCGACCAGTACACTGCCCTTTCAGAGTG TCAACT tgccaccaacatctacatttcaaccttgctctggcagacgccttagcgaccagtacactgcccttcagagtgtcaact 401 411 421 431. 441 451 461 471 ACCTGATGGGAMCATGGCE TTEGGAAICATCCTCTGCAGGATCGTGATCTCAATAGATTACTACAACATGTL ACCAGC ACUTGATGGGAACATGGCCCTTCGGAALTATCCTCTGCAÄATCGTGATCTCAATAGATTACTAC AACATGTTC ACCAGC acctgatgggaacatggccctcggaaccatcctctgcaagatcgtgatctcaatagattactacaacatgttcaccagc 481 491 501 511 521 531 541 551 ATATTCACCCTCTGCACCATGAGCGTGGACNGLTACATTGCTOTCTGCCACCEAGTLAAAGCCTGGATTTCCETACCCC ATATTCACCCTCTGC ACCATGAGCGTGGACCGCTACATTGCTGTCTGECACCCAGTCAAAGCCCTGGATTTCCC TACCCC atattcaccctctgcaccatgagcgtggaccgctacattgctgtctgccacccagtcaaagccetggatttcc tacccc
561
571
581
591
601
511
621 631

CEAAATEECAAAAT TETCAACGTL TGCAACTGGATEETETCTTCTGCEATCGGTCTGCUTGIAATGTTCATG CAACCA CCGAAATGCCAARATCGTCAACGTCTGCAACTGGATCCTCTCTTCTGCCATCGGTCTGCCTGTAATGTTCATG CAACCA ccgaatgccaaaatcgtcaacgtctgcaactggatcctctcttctgccatcggtctgcctgtaatgtcatgucaecca

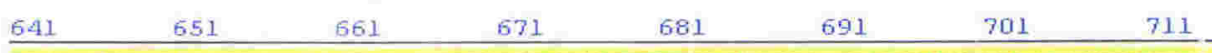

CAAARTACAGGCAGGGGTCCATAGATTGCACCCTCACGTTCTCCCACCCAACCTGGTACTGGGAGAACCTGCTCAAAATC
CAAAATACAGGCAGGGGTCOATAGATTGCACCCTCACGTTCTCCCAICCAACCTGGTACTGGGAGAACCTGCTI 'AAAATC caaaatacaggcaggggtccatagattgcaccctcacgttctcccacccaacctggtactgggagaacc tgctiaaaatc
721
731
741
751
761
771
781
791

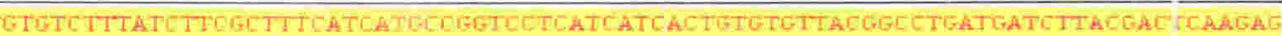
TGTGTCTTTATCTTCGCTTCATCATGCCGGTCCTCATCATCACTGTGTGTTACGGCCTGATGATCTTACGACTCAAGAG tgtgtctttatcttcgcttcatcatgccggtcctcatcatcactgtgtgttacggcetgatgatcttacgac ccaagag
801
811
821
831.
841
851
861
871

CETTCGCATGCTATEGGGC TCCARAGAAAAGGACAGGAATCTGCGCAGGATCACCCGGATGGTGCTGGTGGTC YTGGCTG CGTTCGCATGCTATCGGGTTCLAAGAAAAGGACAGGAATCTGCGCAGGATCACCCGGATGGTGCTGGTGGTC 2TGGCTG cgttcgcatgctategggctccaaagaaaggacaggaatctgcgcaggatcacc cggatggtgctggtggtc gtggctg 881 891 901

911 921 931 941 951 TATTTATCGTCTECTGGACCCOCATCCACATCTACGTCATCATCAAAGCGCTGATTACOATTCCAGAAACCACATTTCAG TATTTATEGTCTGCTGGACCCCCATCLACATCTACGTC ATCATCAMAGCGCTGATCACGATTCCAGARACCACATTTCAG tattratcgtctgctggacccceatccacatctacgtcatcatcaaagcgctgatcacgattccagaaacacatttcag

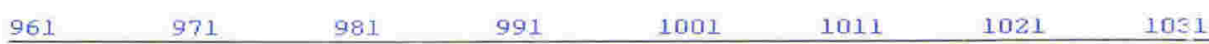

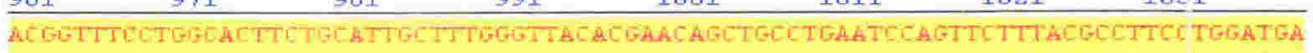
ACGGTTTCCTGGCACTTCTGCATTGCTTGGGTTACACGAACAGCTGCCTCAATCCAGTTCTTTACGCCTTCC TEGATGA acggtttcctggcacttctgcattgcttgggttacacgaacagctgcetgaatccagttctttacgcettce tggatga
1041
1051
1061
1071.
1081
1091
1101
1111

AÄACTTCAAGCGATCCTCAGAGAGTTCTGCATCCCAACCTCOTCCACGATCGAACAGCAAAACTCCACTCOAGTCCGTO AАACTTAAGCGATGCTTCÄGAGAGTTCTGCKTCCCAACCTEGTCCACGATCGAACAECAARACTCCACTEGI GTCCGTC aaacttcaagcgatgcttcagagagttctgcatcccaacctcgtccacgatcgaacagcaaaactccactcgagtccgtc
1121
1131
1141
1151
1161
1171
1181
$11: 1$

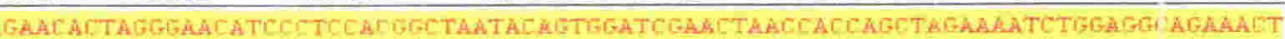
AGAACACTAGGGAACATCECTCCACGGCTAATACAGTGGATEGAACTAACCACCAGCTAGAAAATCTGGAGG AGAAACT agaacactagggaacatccctccacggctaatacagtggatcgaactaaccaccagctagaaaatctggaggcagaaact $\begin{array}{llllllll}1201 & 1211 & 1221 & 1231 & 1241 & 1251 & 1261 & 12 ? 1\end{array}$

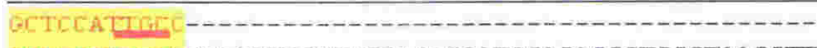
GCTCCATTGCCCTAACTGGGTCTCACACCATCCAGACCCTCGCTAAGCTT getccattgco 

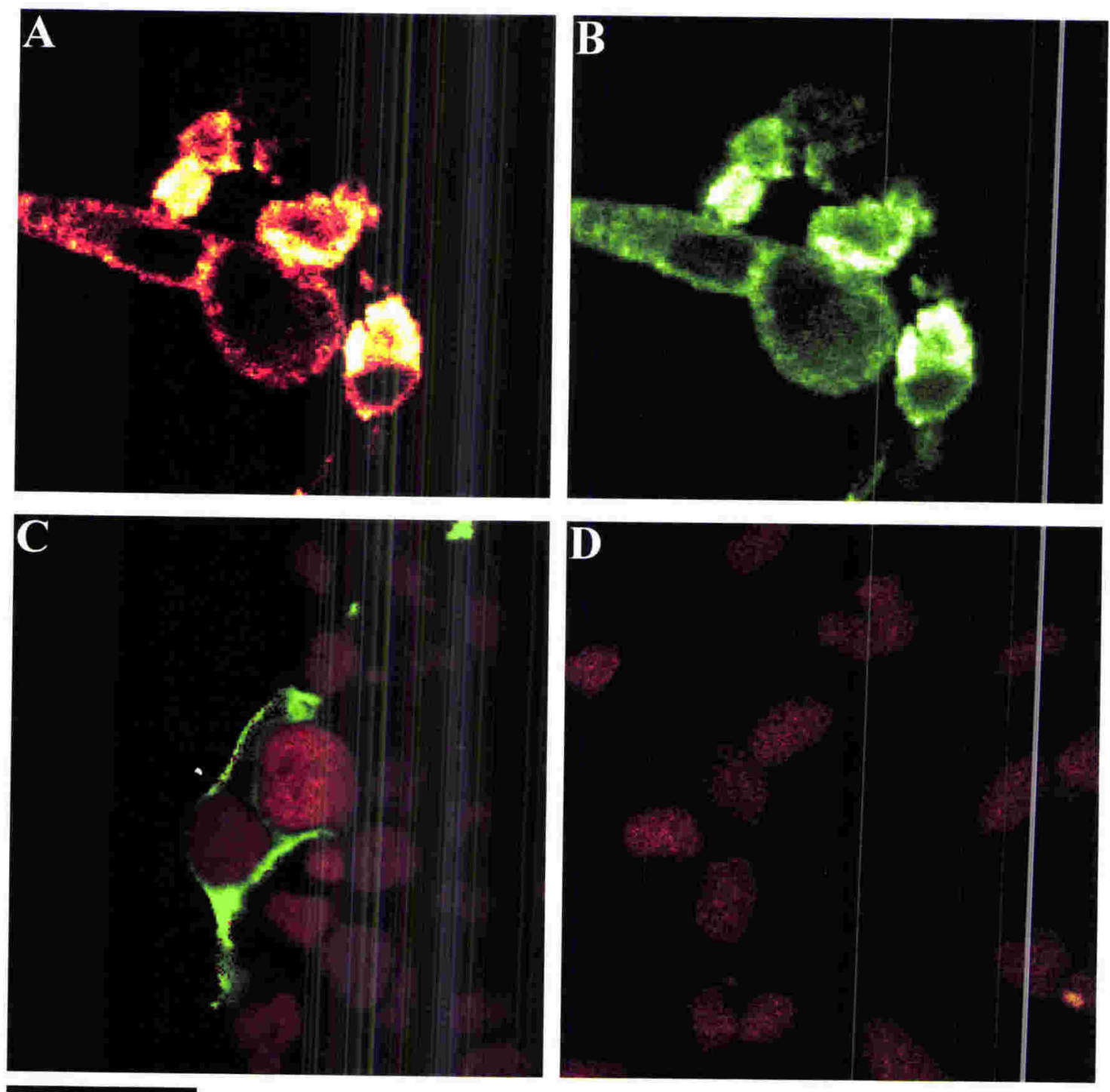

Figure 4.6

\section{Labelling of Transfected SH-SY5Y Cells}

Double-labelling using (A) anti-FLAG (red) and (B) guinea pig anti-MOR (Chemicon) (green) antibodies in MOR transfected, monensin and DAMGO-treated SH-SY5Y cells. FLAG-labelled MOR-transfected SH-SY5Y cells (C) (green) with no treatment with either DAMGO or mon 2 nsin, showing mainly membrane staining. Negative control for (C) with no primary anti-FLAG antibody (D). Figures C and D were counterstained with PI (red). Scale bar $=20 \mu \mathrm{m}$. 


\subsubsection{MOR REGULATION}

\subsubsection{Non-Transfected Cells}

In non-transfected, monensin-pre-treated SH-SY5Y cells, there were significant increases in cytoplasmic staining of MOR in control cells (monensin but no DAMGO) and in cells treated with the MOR agonist DAMGO for $2 \mathrm{hr}$ (Figure 4.7B, D \& F) and 4 hrs (Figure $4.8 \mathrm{~B} \& \mathrm{~F})$. Membrane staining, however, showed no significant differences in intensity between control cells (monensin but no DAMGO) and cells treated with monensin/DAMGO. The increase in cytoplasmic staining occurred within the first hour, with no significant further increases after 4 hrs (Table 4.6). Primary cultures also showed an increase in cytoplasmic MOR staining after treatment with monensin and DAMG() (Figure 4.9), although this difference was not significant due to the inherently large variation in MOR staining patterns in these cultures. In untreated, control cultured brainstem cells (Figure 4.9A, C \& E), the plasma membrane staining appears more intense than the cytoplasmic staining; whereas, in the cells treated with monensin, followed by DAMGO for $2 \mathrm{hr}$ (Figure 4.9B, D \& F), the staining appears more intense in the cytoplasm. 

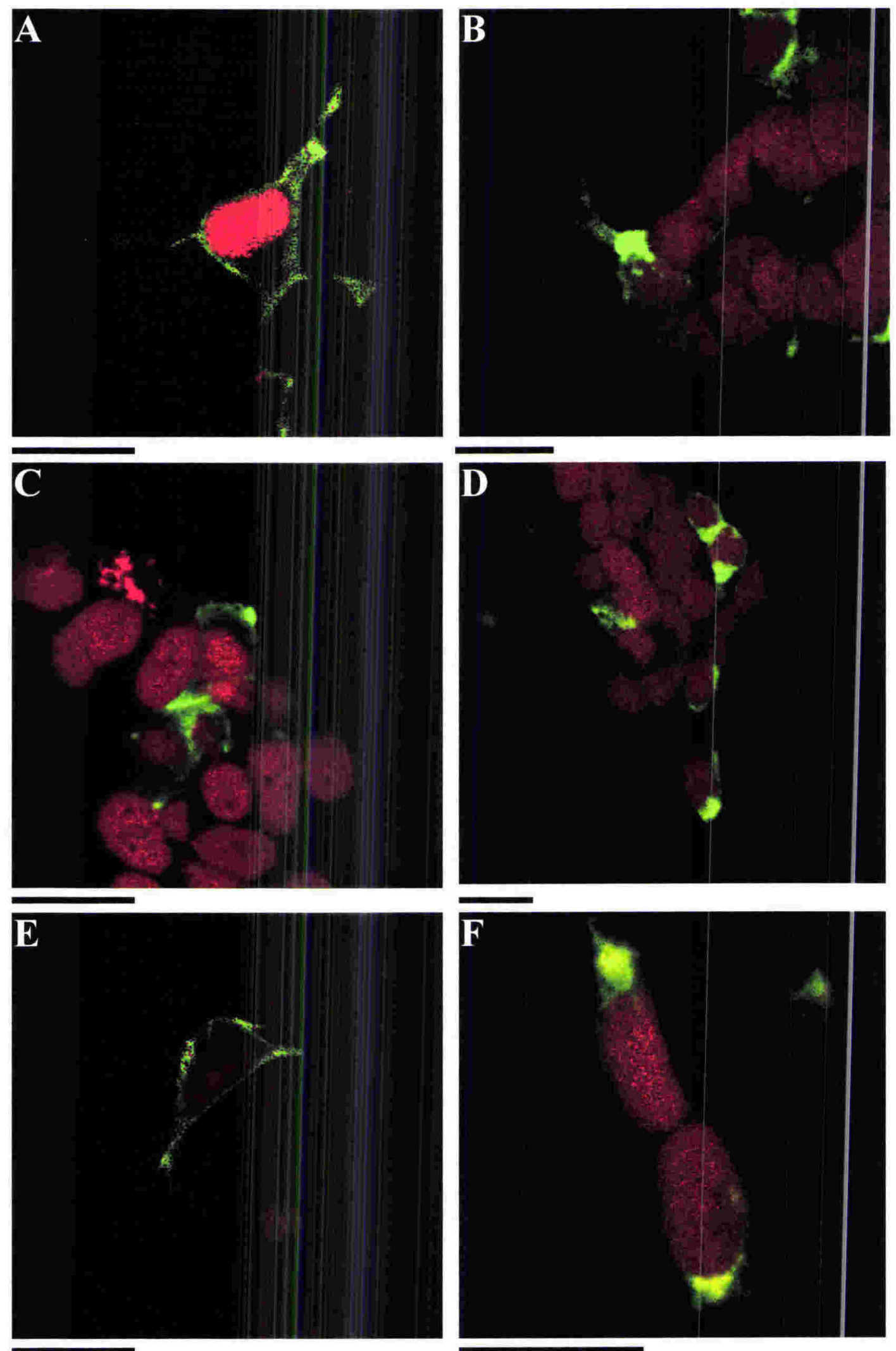

Figure 4.7

\section{MOR Regulation in Non-Transfected SH-SY5Y Cells (A)}

Non-transfected SH-SY5Y cells labelled with antibodies to MOR (guinea pig anti-MOR, Chemicon) (green) and counterstained with PI (red). Control cells (A, C \& E) (monensin (50 $\mu$ $\mathrm{M})$ without DAMGO treatment), and (B, D \& F) after pretreatment with monensin $(50 \mu \mathrm{M})$ followed by $2 \mathrm{hr}$ DAMGO $(1 \mu \mathrm{M})$. Scale bar $=20 \mu \mathrm{m}$. 

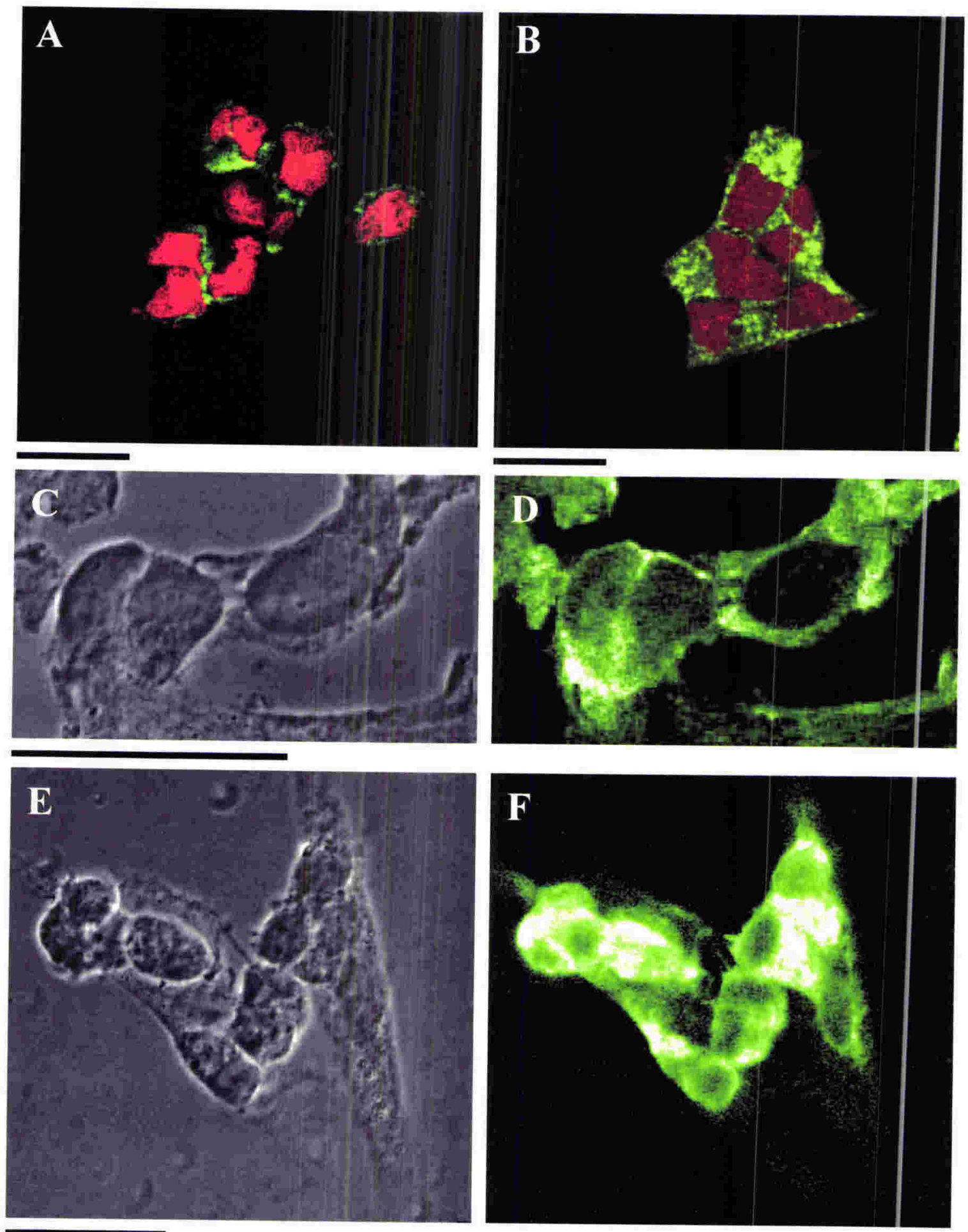

Figure 4.8

MOR Regulation in Non-Transfected SH-SH5Y Cells (B)

Monensin $(50 \mu \mathrm{M})$ treated non-transfected SH-SY5Y cells after (A) $0 \mathrm{hr}$ and (B) $4 \mathrm{hr}$ DAMGO 1 $\mu \mathrm{M})$. Note the increase in punctate cytoplasmic staining with the guinea pig anti-MOR antibody (Chemicon). Non-transfected SH-SY5Y cells shown in phase contrast (C, E) and with MOR labelling after monensin treatment after (D) $0 \mathrm{hr}$ and (F) $4 \mathrm{hr}$ treatment with $1 \mu \mathrm{M}$ DAMGO. Note the intense (white colour) cytoplasmic staining in the DAMGO-treated cells (A \& F). Scale bar $=20$ $\mu \mathrm{m}$. 
Table 4.6 MOR regulation in non-transfected, monensin pretreated SH-SY5Y cells

\begin{tabular}{|c|c|c|c|}
\hline Treatment & $\begin{array}{c}\text { No. of Cells } \\
\text { Counted }\end{array}$ & $\begin{array}{c}\text { Average Membrane } \\
\text { Staining Intensity } \pm \text { SEM }\end{array}$ & $\begin{array}{c}\text { Average Cytoplasmic } \\
\text { Staining Intensity } \pm \text { SEM }\end{array}$ \\
\hline Control & 243 & $1.4 \pm 0.07$ & $1.7 \pm 0.06$ \\
\hline $\begin{array}{c}\text { DAMGO } \\
1 \mathrm{hr}\end{array}$ & 173 & $1.2 \pm 0.07$ & $2.3 \pm 0.06$ \\
\hline $\begin{array}{c}\text { DAMGO } \\
\mathbf{4} \mathrm{hr}\end{array}$ & 194 & $1.3 \pm 0.07$ & $2.3 \pm 0.06$ \\
\hline
\end{tabular}

MOR cytoplasmic and membrane staining of SH-SY5Y cells were ranked on a scale from 0 (no staining) to 3 (intense staining) before and after the addition of the MOR agonist DAMGO $(1 \mu \mathrm{M})$ for $1 \mathrm{hr}$ and $4 \mathrm{hr}$. There was a significant increase in cytoplasmic staining between the control (monensin but no DAMGO) and after $1 \mathrm{hr}$ and $4 \mathrm{hr}$ DAMGO (monensin and DAMGO). Multiple comparisons based on Kruskal-Wallis rank sums $(\mathrm{P}<$ 0.001 ).

\subsubsection{Transfected SH-SY5Y Cells}

MOR-transfected SH-SY5Y cells express much higher levels of MOR than when nontransfected. The localisation of the receptors, however, is the same. MOR-transfected SHSY5Y show an increase in cytoplasmic staining in response to DAMGO and monensin treatment in a similar way to non-transfected SH-SY5Y cells, but the increase is much more apparent in transfected cells (Figure $4.8 \mathrm{D} \& \mathrm{~F}$ ). This difference is probably due to the more intense staining being easier to visually localise in the cell. 

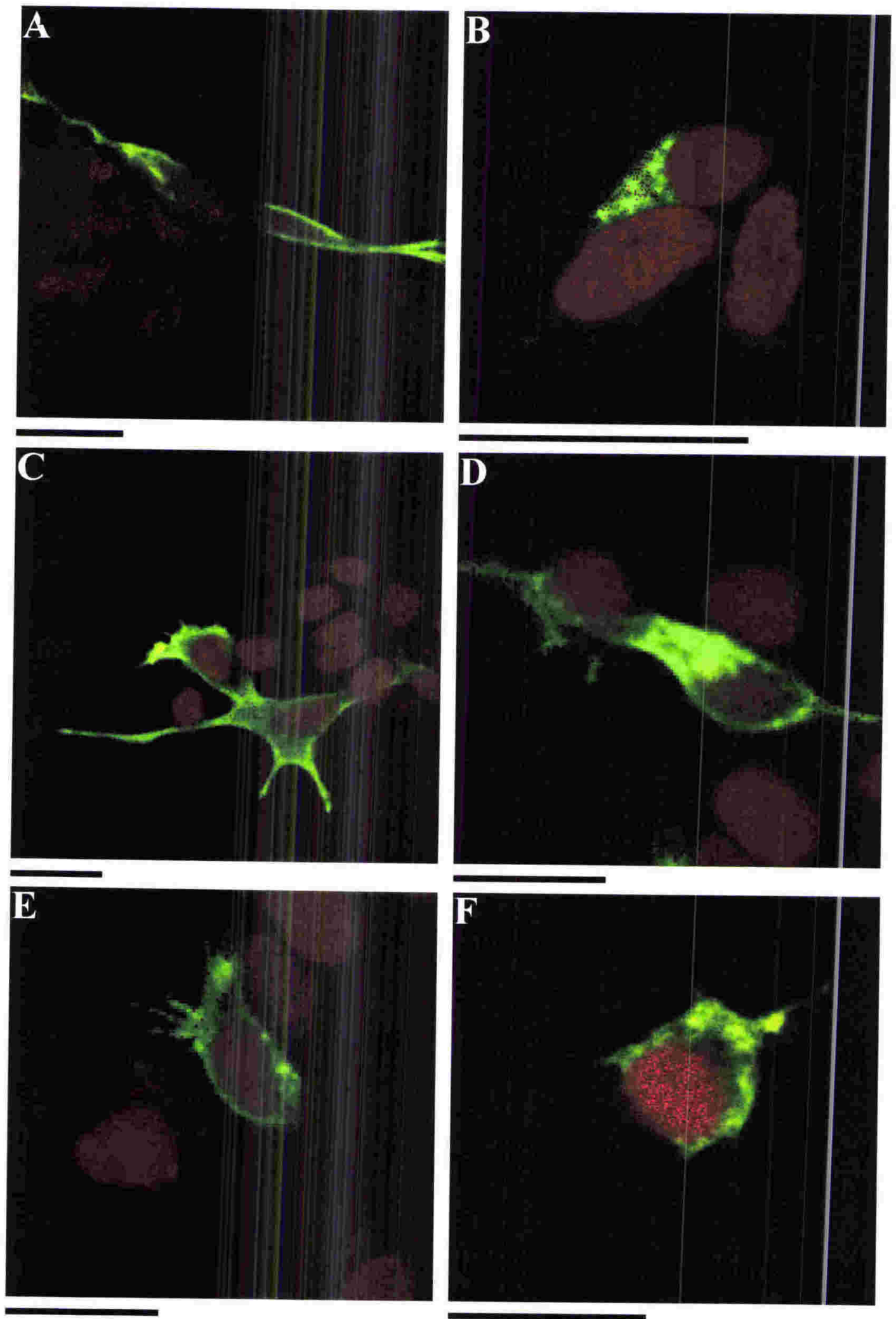

Figure 4.9

OpR Regulation in Dissociated Brainstem Cell Cultures

Primary cultures of rat brainstem P1 cells after 4 days in culture labelled with antibodies to MOR (guinea pig anti-MOR, Chemicon) (green) and counterstained with PI (red). Control cells (A, C \& E) (monensin $(50, \mathrm{M})$ without DAMGO treatment), and (B, D \& F) cells after pretreatment with monensin $(50 \mathrm{fl} M)$ followed by $2 \mathrm{hr}$ DAMGO $(1 / \mathrm{M})$. Scale bars $=20 \mathrm{fm}$. 


\subsection{DISCUSSION}

\subsubsection{MOR CLONING AND TRANSFECTION OF MAMMALIAN CELLS}

The rat mu opioid receptor was cloned in 1993 by Chen et al. (Chen et al., 1993b) ancl Thompson et al. (Thompson et al., 1993), with a deduced polypeptide of 398 amino acid residues. Once cloned, MOR could be transfected into cells that do not express MOR (such as kidney cells), or have low levels of MOR expression (such as neuroblastoma cells). MOR transfection studies have increased our knowledge of how MOR is regulated (Koch et al., 1997; Koch et al., 1998; Law and Loh, 1999). Transfection of mutated or truncated receptors has increased our knowledge of which receptor amino acid residues are responsible for ligand binding selectivity and which are responsible for receptor regulation (Pak et al., 1997; Pak et al., 1999). Opioid receptor regulation in MOR-transfected cel s was not studied in detail in this work because such experiments have been well documented in the literature (Koch et al., 1998; Law et al., 2000c). In the present study, transfected cells were used as controls for interpreting MOR responses in non-transfected primary cultures and SH-SY5Y cells. Changes in receptor localisation patterns in these cells were more difficult to measure due to their lower endogenous expression.

In the present study, transfection experiments helped confirm the specificity of the guinea pig anti-MOR antibody. Identical staining patterns were seen with anti-MOR and antiFLAG antibodies, confirming the specificity of the MOR antibody. Antibody specificity is an essential requirement for meaningful immunocytochemistry and immunohistochemistry. Other studies have used ICC (Keith et al., 1996; Koch et al., 1998; Ingnatova et al., 1999; Koch et al., 2001) or fluorescent ligand binding (Gaudriault et al., 1997) to study OpR regulation on exposure to opioid agonists in transfected kidney cells to monitor the rate of receptor internalisation. In the present study, these techniques were used in an endogenously expressing cell line (SH-SY5Y) and in cultured brainstem cells. Although MOR-transfected cells provide a good model, the results must be interpreted with caution because transfected cells over-express receptors above endogenous levels, and this overexpression may alter the properties of receptor regulation. In the present study, MOR agonist-induced down-regulation in both transfected and non-transfected SH-SY5Y cells was observed, as a result of receptor internalisation. To a lesser extent, this was also seen in rat brainstem cultures, although the ICC data were difficult to interpret due to the high amplification of signal required for the low endogenous MOR expression levels. 


\subsubsection{OPIOID RECEPTOR REGULATION}

Opioid receptor regulation has been described in detail in the Introduction to this thesis (Chapter 1: Section 1.2.3). Monensin is an inhibitor of endosomal acidification and, therefore, blocks receptor recycling to the plasma membrane by trapping internalised receptor proteins in the endosome (Koch et al., 1998). Agonist induced desensitisation involves phosphorylation of intracellular OpR domains and is regulated by protein kinases as has been described in the main Introduction (Chapter 1: Section 1.2.3). In the present study, MOR was rapidly internalised in all the cell lines examined, including primary cultures of brainstem cells. Internalised receptor was generally characterised by punctate cytoplasmic staining. The punctate nature of the staining presumably reflects the packaging of the receptors in endosomes. The significance of diffuse staining is unknown, but is unlikely to be due to fluorescent signal above and below the plane of focus, as confocal microscopy reduces this problem. The staining pattern could however be an artefact of the amplification steps used, in which the enzymatic amplification (TSA) deposits large amounts of fluorophore, potentially limiting the resolution of small Ople containing vesicles.

The increase in cytoplasmic staining occurred within the first hour after $1 \mu \mathrm{M}$ DAMGO treatment, with no further increase seen by $4 \mathrm{hr}$. These results are supported by the work of others who have shown that receptor internalisation occurs rapidly and is complete within $1 \mathrm{hr}$ (Koch et al., 1998; Pak et al., 1999). In studies using forskolin-stimulated intracellular cAMP production to assess receptor activity (Law et al., 2000cS), SH-SY5Y MORs have been shown to be down-regulated in the presence of $1 \mu \mathrm{M}$ DAMGO. Downregulation of OpRs occurs rapidly with internalisation of receptors into endosomes where they are either degraded or recycled to the membrane. Long-term changes in gene expression also occur, but not within the time period used in the present study (Ko et ill., 1999). Receptor phosphorylation has also been described (Xiang et al., ; Chen et al., 1993a Minami, 1995 \#382; Pak et al., 1997; Kramer and Simon, 1999) and is an important step in OpR down-regulation.

MOR and DOR are also regulated differently. A recent study of Xenopus oocyte expression system showed MOR down-regulation occurs at a slower rate than DOR downregulation due to the activation of arrestin (Lowe et al., 2002). Differences in the 
responsiveness to arrestin-mediated desensitisation were due to differences in OpR phosphorylation. Phosphorylation of the C-terminal and $2^{\text {nd }}$ intracellular loop of DOR was required for arrestin-mediated rapid down-regulation, whereas MOR phosphorylation of the $2^{\text {nd }}$ intracellular loop was required. The phosphorylation of DOR at the two regions described leads to more efficient activation of arrestin, and was hypothesised to be the reason for faster down-regulation of DOR. Differences in the regulation properties of different OpR subtypes have not been studied in primary culture. The culture model described in the present study (Chapter 2) expressing both MOR and DOR (Chapter 3) wil provide a useful model in which to study these differences.

It is possible that different cell types within the brain are regulated differently. For example, neurons in the cortex may have different OpR regulation properties than the brainstem. A recent study, the first to demonstrate OpR-mediated internalisation in cultured neurons (cortex), showed dermorphin and deltorphin induced MOR and DOR clathrin-dependent internalisation, which could be blocked by naloxone (Lee et al., 2002). The study by Lee et al (2002) is the first study to determine OpR down-regulation in culture and it remains to be determined if these regulation properties are also found in cultures of other brainstem regions including brainstem neurons cultured in the present study. Down-regulation of MOR was seen in the present study, using DAMGO, although it was not determined if this was via a clathrin-dependent pathway.

Different MOR splice variants demonstrate different mechanisms of regulation (Koch et al., 1998; Koch et al., 2000; Abbadie and Pasternak, 2001; Koch et al., 2001). For example, in a study by Koch et al. (1998) MOR-1 and MOR-1B splice variants, which differ in their C-terminal amino acid composition showed different regulatory patterns. MOR-1B-transfected cells had a slower desensitisation rate and an accelerated recep or reactivation of the internalised receptor in endosomes back to the plasma membrane on exposure to DAMGO (Koch et al., 1998). In the mouse lateral septum in vivo MOR-1 and MOR-1C showed differences in MOR internalisation. MOR-1 was internalised by DAMGO, but not morphine, whereas; MOR-1C was internalised by both DAMGO and morphine (Abbadie and Pasternak, 2001). Differences between the two splice-variants lie in the alternative splicing of the carboxyl terminus. Different OpR agonists, and splicevariants showing different down-regulation properties is an important new area of research whereby the mechanisms of addiction, tolerance and desensitisation are being elucidated. 
The present study is the first to use ICC to demonstrate MOR regulation in non-transfected cells that endogenously express and regulate MOR. The heterogeneous cell populations found in primary cultures makes developmental changes in regulation difficult to determine by ICC techniques, especially when studying the different cells in culture which all show different extents of membrane versus cytoplasmic staining, both with and withou agonist exposure (see MOR localisation Figures in Chapter 3). If there are any overal changes in the regulation of OpRs during development it may be able to be quantified using cAMP measurements.

Receptor up-regulation in response to opioid antagonist exposure was not examined in the present study. An attempt was made to replicate a study by Keith et al. (1996) using $0.4 \mathrm{M}$ sucrose to prevent receptor internalisation, but the neuronal cells were too sensitive to osmotic shock to survive in these experiments (Keith et al., 1996). The original work was carried out on kidney epithelial cells (HEK 293), which are less sensitive to osmolar changes than neuroblastoma (SH-SY5Y cells) and primary neuronal cell cultures.

In summary, developing a primary culture model to study endogenously expressed MOR will contribute to our understanding of MOR regulation in vivo. Using ICC, OpR regulation was assessed in transfected SH-SY5Y cells and, with poorer resolution, in nontransfected SH-SY5Y cells and in primary brainstem cultures from foetal and neonalal animals. This technique was less effective in measuring OpR regulation in primary cultures because of the variations in staining intensity between neurons within a single culture, and between similar cells from different cultures. ICC also has distinct limitations because of its inability to provide quantitative results. A more quantitative method, such as measurement of intracellular cAMP production, could be used to measure OpR regulation, since opioid peptides inhibit cAMP accumulation (Arden et al., 1995; Chan et al., 1995). This method measures cAMP accumulation in cultured cells by measuring basal cAMP levels using $\left[{ }^{32} \mathrm{P}\right] \mathrm{cAMP}$ and synthesised cAMP by measuring $\left[{ }^{3} \mathrm{H}\right] \mathrm{cAMP}$ levels. The ratio between the two radioactive cAMP levels can then be calculated. This method was used by El Kouhen et al. (1999) to measure MOR down-regulation by DAMGO in MORtransfected HEK293 cells (El Kouhen et al., 1999). This same method used in primary cultures may provide a more quantitative measure of $\mathrm{OpR}$ down-regulation in 
endogenously expressing primary cultures, as it would combine the regulation measurements of all cells in culture.

\subsubsection{SUMMARY AND CONCLUSIONS}

Mu opioid receptors are down-regulated in primary rat brainstem cultures established from foetal and early postnatal rats. MOR is rapidly internalised on exposure to the MOR agonist DAMGO in the same way as non-transfected, and MOR-transfected, SH-SY5Y cells, suggesting MOR expressed in cultured brainstem cells is functionally regulated. ICC techniques however, are not sensitive enough to determine if differences occur during development in the rate and level of OpR down-regulation.

The guinea pig anti-MOR antibody (Chemicon) used in the present study specifically labels MOR and this was confirmed by double labelling experiments with antibodies for FLAG and MOR. The rabbit anti-DOR antibody (Chemicon) showed no crossreactivity for MOR (Data not shown).

From this study it can be concluded that the antibodies used are binding specifically for MOR and DOR. In primary brainstem cultures MOR is functionally down-regulated by DAMGO, a MOR selective agonist. 


\section{CHAPTER 5}

\section{MOR and DOR Expression In Purkinje Cells Of The Rat Cerebellum}

\subsection{AIM}

During the course of the present study on rat brainstem opioid receptors, MOR and DOR distributions were determined in brain sections by immunohistochemistry (IHC). During this study, immunoreactivity for both OpR subtypes was also observed in the rat cerebellum in particular in the Purkinje neurons of the molecular layer. The aim of this study was to confirm that both MOR and DOR are present in cerebellar tissue, as it is currently accepted that MOR is not expressed in the rat cerebellum, although MOR expression has been described in cerebellar tissues from most other mammalian species.

\subsection{INTRODUCTION}

\subsubsection{MOR AND DOR EXPRESSION IN THE BRAIN}

MOR and DOR protein and mRNA localisation within the CNS and peripheral tissues have been extensively studied using immunohistochemistry, in situ hybridisation, radiolabelled ligand binding, and RT-PCR (Atweh and Kuhar, 1977; Mansour et al., 1988; Xia and Haddad, 1991; George et al., 1994; Mansour et al., 1994; Arvidsson et al., 1995b; Mansour et al., 1995; Wang and Wessendorf, 2002). Each class of opioid receptor shows distinct expression patterns within the brain (Delfs et al., 1994; Mansour et al., 1994; Bausch et al., 1995a; Mansour et al., 1995; Ding et al., 1996b; Peckys and Landwehrmeyer, 1999; Cahill et al., 2001b). Most brain areas show good correlation between protein and mRNA expression patterns, although some differences are noted. These differences may be due to either the mRNA signal being absent in neuronal processes, or to variations in sensitivity of the different methods used. The latter problem arises because of different binding affinities of the probes for their targets and the use of different signal amplification methods. 


\subsubsection{STRUCTURE OF THE CEREBELLUM}

The cerebellum is a brain region involved in fine control and planning of complex motor activities. It is made up of several lobes, including the anterior lobe, posterior lobe, and flocculonodular lobe, and has three main functional zones, the vermis, lateral, and intermediate zones (Kandel et al., 2000). Regions of the cerebellum are separated by numerous fissures, which divide the tissue into lobules. Cerebellar neurons are segregated into three distinct layers, the outermost molecular layer, the Purkinje cell layer, and the innermost granular layer. The molecular layer contains stellate and basket cell inhibitory interneurons, the dendrites of inhibitory Purkinje cells, and the axons of excitatory granule cells. Purkinje cell axons project to the deep cerebellar nuclei and provide the output of the cerebellar cortex. The inner granular layer contains densely packed, small granule cells, Golgi interneurons, and mossy fibre terminals that provide afferent input to the cerebellum (Kandel et al., 2000).

\subsubsection{CEREBELLAR FUNCTION}

The cerebellum has functions that include learning and planning of complex motor pattens, evaluation of sensory information, modulation of descending motor systems in both the brainstem and cerebral cortex, regulation of postural balance and eye movements, and regulation of body and limb movements (Kandel et al., 2000). Purkinje cells, which are inhibitory in function, project to the inferior olive, vestibular nuclei, and reticular formation in the brainstem, regions that all express both MOR and DOR (Mansour et al., 1994). Purkinje axons project through the white matter to the vestibular nuclei and provide inhibitory ou put that is mediated by the neurotransmitter $\gamma$-aminobutyric acid (GABA). In other regions, for example, the dorsal horn of the cat spinal cord, GABA-containing neurons synapse with MOR-expressing neurons (Gong et al., 1997). In the rat cerebral cortex, many MORexpressing neurons also stain positive for GABA (Taki et al., 2000). Neurons expressing DOR have also been shown to synapse with GABAergic neurons (Commons et al., 2001). These colocalisation studies suggest a possible role for opioids in the cerebellum of modulating Purkinje cell activity. Both opioids and the cerebellum play roles in modulating vestibular function (Kandel et al., 2000). Nystagmus caused by vestibular deafferentation is reduced by administration of naloxone, an OpR antagonist (Dutla et al., 1996). OpRs may act 
by hyperpolarizing Purkinje cells, thus, preventing Purkinje cell inhibition of the vestibular nuclei and other brain regions.

\subsubsection{SPECIES DIFFERENCES IN MOR AND DOR DISTRIBUTIONS IN THE CEREBELLUM}

Species differences exist in opioid receptor expression in the cerebellum, and the OpR subtypes that are present vary. In the rabbit and human, MOR is expressed in the cerebellum at high levels and DOR at low levels (Lu et al., 1993; Peckys and Landwehrmeyer, 1999), with both MOR and DOR expression highest in the Purkinje cell layer. In the rat it is generally accepted that within the cerebellum only DOR is expressed (Ableitner, 1994; Abeyta et al., 2002). In the present study we report strong expression of MOR protein in Purkinje cells of the rat cerebellum, with some MOR protein expression also seen within stellate cells in the external molecular layer. 


\subsection{METHODS}

\subsubsection{IMMUNOHISTOCHEMISTRY}

\subsubsection{Tissue Fixation}

Foetal brain tissue was fixed by removing the whole brain and placing it in paraformaldehyde fixative solution (4\% paraformaldehyde in PBS, pH 7.4) overnight at $4^{\circ} \mathrm{C}$. Adult brain tissue was fixed by cardiac perfusion of a rat that had been deeply anaesthetised with pentobarbital (65 mg/kg ip) (Nembutal). A midline incision was made through the skin at the base of the rib cage, exposing the xiphisternum. A central cut was made along the rib cage and the ribs folded back to expose the heart. The left femoral vein was exposed and cut immediately before inserting a blunted needle into the left ventricle. The needle was taped into place and connected to tubing, which passed through a peristaltic pump (Watson Marlo Ltd, Cornwall, England) and into a reservoir bottle containing ice-cold PBS. The PBS was perfused into the circulation at a flow rate of $11 \mathrm{~mL} / \mathrm{min}$ until the perfusate was free of blood (10-15 $\mathrm{min})$. The perfusate was then replaced with ice-cold 4\% paraformaldehyde in PBS ( $\mathrm{pH} 7.4$ ) and perfused for $30 \mathrm{~min}$ to fix the brain tissue. The brain and rostral spinal cord were carefully removed and placed in fixative for $2 \mathrm{hr}$ at room temperature. The tissue was then transferred to PBS containing $30 \%$ sucrose at $4{ }^{\circ} \mathrm{C}$ and left overnight, or until the brain had sunk to the bottom of the container. This final step prevented tissue damage during freezing. Before sectioning, the brain was stored frozen for up to 60 days at $-70^{\circ} \mathrm{C}$.

\subsubsection{Tissue Sectioning}

Fixed tissue was mounted in Cryo-M-bed freezing compound (Bright Instrument Company Ltd, Huntingdon, UK) and cut using an HM 500 OM series cryostat microtome (Microm International GmbH, Walldorf, Germany). Free-floating $20 \mu \mathrm{m}$ or $30 \mu \mathrm{m}$ serial coronal sections were placed in labelled $1.5 \mathrm{~mL}$ microfuge tubes (or $10 \mathrm{~mL}$ vials for sagittal sections) containing PBS. After sectioning, the tissue was permeabilised in PBS containing $0.1 \%$ Triton $\mathrm{X}-100$ for $30 \mathrm{~min}$, then endogenous peroxidase activity was removed by incubating sections in a solution of $50 \%$ methanol containing $1 \% \mathrm{w} / \mathrm{v}$ hydrogen peroxide. The sections were washed $3 \times 5$ min and stored in PBS containing $0.1 \%$ sodium azide for up 7 days at $4^{\circ} \mathrm{C}$. The sodium azide was washed off before immunolabelling because it inhibits the peroxidase enzyme used in the detection methods. 


\subsubsection{Antibody Labelling}

Several sections were incubated in the same microfuge tube in $200-300 \mu \mathrm{L}$ of primary antibody. For washing, antibody solutions were carefully removed with a micropipette, leaving the sections at the bottom of the tube. The sections were washed in PBS containing $0.1 \%$ Triton $\mathrm{X}-100$ ( $4 \times 5$ min washes), then blocked for $1 \mathrm{~h}$ in blocking solution supplied with the Tyramide amplification kit (Perkin-Elmer). Sections were then incubated overnight at $4{ }^{\circ} \mathrm{C}$ in primary antibody diluted in blocking solution.

\subsubsection{Detection of MOR}

The anti-MOR antibody (guinea pig anti-MOR, 1:1000, Chemicon) was diluted in blocking solution prior to use and incubated overnight at $4^{\circ} \mathrm{C}$ on a rocker platform. The free-floating sections were washed with PBS containing $0.01 \%$ Triton X-100 (4x 5 min each), and the sites of primary antibody binding detected using a rabbit anti-GP IgG-HRP-conjugated secondary antibody, (Zymed Laboratories, San Francisco, CA), diluted 1:3000 in TSA blocking solution, and incubated for $1 \mathrm{~h}$ at room temperature. Unbound anti-GP IgG-HRP was removed by washing $4 \mathrm{x}$ for $5 \mathrm{~min}$ each in PBS containing $0.01 \%$ Triton X-100. An amplification step using TSA-biotin (Perkin-Elmer) was performed following manufacturer's instructions as outlined in Chapter 3 (Section: 3.3.3.2). The sections were then washed in PBS and incubated for $30 \mathrm{~min}$ at room temperature with ExtrAvidin-HRP (Sigma), diluted 1: 2000 in PBS. Immunoreactivity of cells was detected by DAB (Sigma) staining ( $1 \mu \mathrm{g} / \mathrm{mL}$ in $0.1 \mathrm{M}$ phosphate buffer ( $\mathrm{pH} 7.4$ ), containing $0.1 \%$ hydrogen peroxide), with or without nickel sulphate $(0.03 \%)$ until suitable colour development had occurred.

\subsubsection{Detection of DOR}

For immunostaining of DOR, sections were preincubated in TSA blocking solution for $1 \mathrm{~h}$ at room temperature, then incubated overnight at $4^{\circ} \mathrm{C}$ on a rocker platform with DOR antibody, (rabbit anti-DOR, 1:1000, Chemicon) diluted in TSA blocking solution. The next day the samples were washed $4 \times 5 \mathrm{~min}$ each as above. Bound primary antibody was detected by incubating with an anti-rabbit IgG HRP-conjugated antibody (1:2000) for $2 \mathrm{~h}$ at room temperature. Sections were washed, and the signal amplified with TSA- biotin and 
ExtrAvidin-HRP, as described above for MOR. DOR sites were visualised using DAI3 as described above.

\subsubsection{Mounting of Sections and Visualisation of DOR Immunoreactivity}

The immunolabelled, free-floating tissue sections were mounted on slides and air-dried prior to dehydration through increasing ethanol and Histo-clear ${ }^{\circledR}$ histological clearing solution washes (National Diagnostics, Alanta, USA) for 3 min each (70\% ethanol, 70\% ethanol; 95\% ethanol; $95 \%$ ethanol; $100 \%$ ethanol; $100 \%$ ethanol; $25 \%$ histo-clear $/ 75 \%$ ethanol; $50 \%$ histoclear $/ 50 \%$ ethanol; $75 \%$ histo-clear $/ 25 \%$ ethanol; $100 \%$ histo-clear). The sections were mounted onto slides using DePeX mounting solution (BDH Laboratory Supplies, Poole, England). For high magnification digital images, stained sections were photographed using an Olympus AX70 compound photomicroscope (Olympus, Tokyo, Japan) with digital camera (Nikon COOLPIX 9953.34 mega zoom, Tokyo, Japan). For low magnification imaging of coronal and sagittal sections, an inverted series SV11 stereomicroscope (Carl Zeiss Mikroskopie, Jena, Germany) equipped with digital camera (AxioCam HRc, Carl Zeiss Mikroskopie) was used, and images were captured using AxioCam 5.05 and AxioVision 3.1 software (Carl Zeiss Mikroskopie).

\subsection{RESULTS}

\subsubsection{DISTRIBUTION OF MOR AND DOR IN THE RAT BRAIN}

\subsubsection{MOR and DOR Localisation in the Adult Rat Brain}

In the adult rat, MOR and DOR distributions have been well documented in the literature (Delay-Goyet et al., 1987; Mansour et al., 1987, 1988; Delfs et al., 1994; Mansour et al., 1994; Zastawny et al., 1994; Arvidsson et al., 1995a; Mansour et al., 1995; Ding et al., 1996b; Díaz et al., 2000; Cahill et al., 2001b). In the present study, typical staining patterns for these receptors were seen in most brain regions, including the dorsal horn of the spinal cord (DH), olfactory bulb, and in specific brainstem nuclei (Figure 5.1).

\subsubsection{Distribution of MOR and DOR in the Rat Cerebellum}

Previous studies have shown that DOR is expressed in the adult rat cerebellum (Delay-Goyet, 1990; Ableitner, 1994; Bausch et al., 1995a; Abeyta et al., 2002). Using a DOR-specific 
antibody, abundant DOR protein expression was detected in the present study within the large cells of the Purkinje layer, in the dendritic processes of Purkinje cells within the molecular layer, and in some stellate and/or basket cells of the molecular layer in both adult (Figure 5.2A, C, \& E and Figure 5.3A) and P6 rats (Figure 5.4A). Cerebellar sections were also stained for MOR. Figure 5.2 (B, D, \& F) and Figure 5.3B shows the staining obtained in the adult rat cerebellum. The patterns seen for MOR closely resemble the staining pattern seen for DOR, with positive staining in the Purkinje cell layer, the dendritic processes of the molecular layer, and occasionally stellate and/or basket cells within the molecular layer. This staining pattern can also be seen in the cerebellum of P6 rats labelled with the same MOR-specific antibody (Figure 5.4B), although the granular layer in P6 rats shows more intense staining than the granular layer in adult cerebellum.

The distribution of both DOR and MOR in the rat cerebellum obtained by immunohistochemistry is supported by fluorescent in situ hybridisation (FISH) detection using fluorescently-labelled cRNA probes (Eli Mrkusich and Dr Darren Day, personal communication). As with ICC, FISH detection of both MOR and DOR mRNA shows labelling of cells in the Purkinje layer and in some stellate and/or basket cells in the molecular layer. There is little labelling, however, of the Purkinje cell dendrites within the molecular layer. This is consistent with the fact that mRNA is often found only in the cell body where the protein receptors are synthesised. 


\section{ADULT BRAIN}
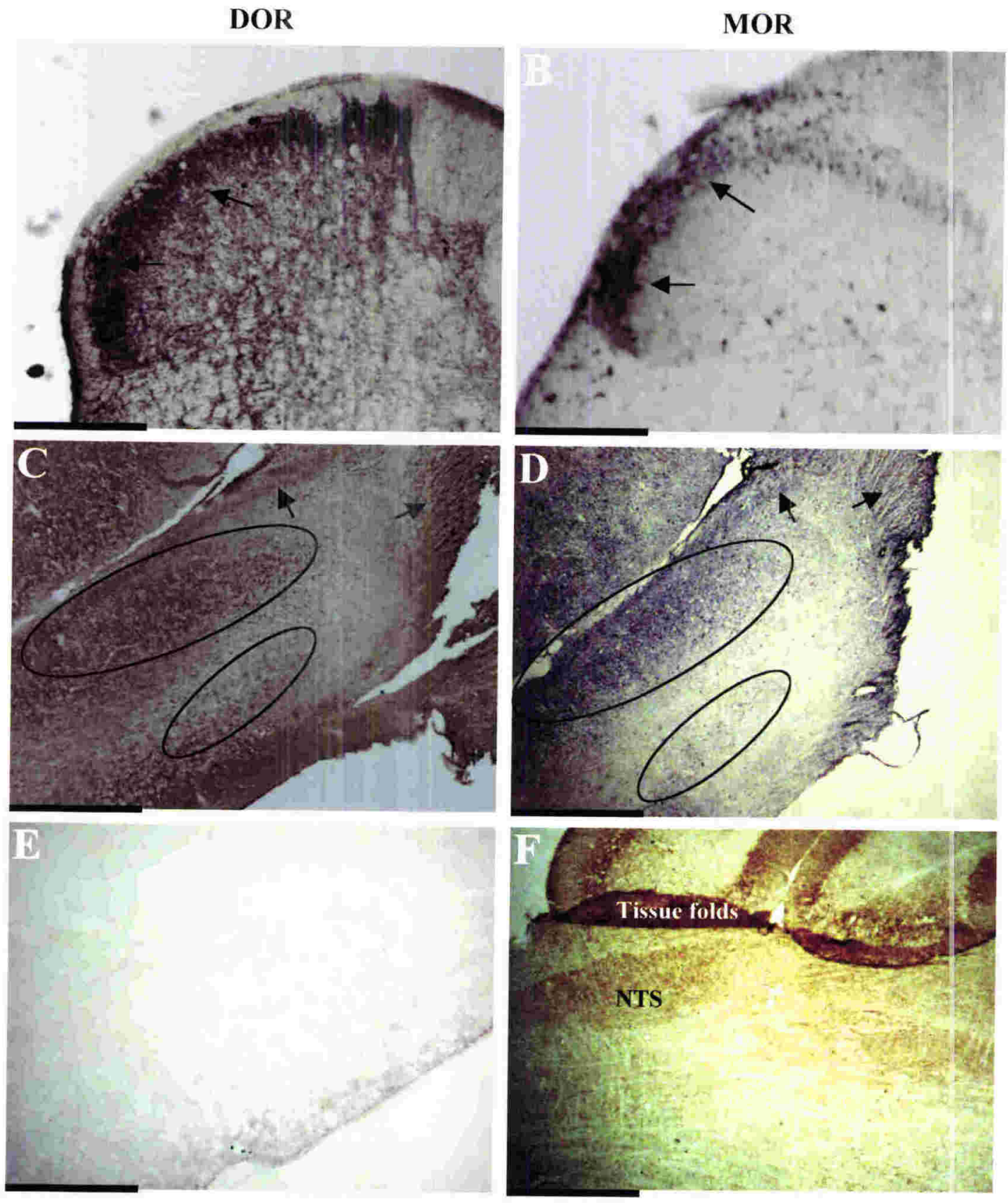

Figure 5.1

\section{DOR And MOR Labelling in Brain Regions}

Adult DOR (A \& C) (rabbit anti-DOR, Chemicon) and MOR (B \& D \& F) (guinea pig antiMOR, Chemicon) immunoreactivity in the adult rat showing similar staining patterns for DOR and MOR: (A \& B) dorsal horn of the spinal cord (coronal section); (C \& D) the olfactory bulb (sagittal section); and (F) brainstem (sagittal section). The negative control (E) with no primary antibody shows no staining in a region of the brainstem. Immunoreactivity was detected with $\mathrm{DAB}$ and visualised using brightfield microscopy. Scale bars $=1 \mathrm{~mm}$ 


\section{ADULT}

DOR
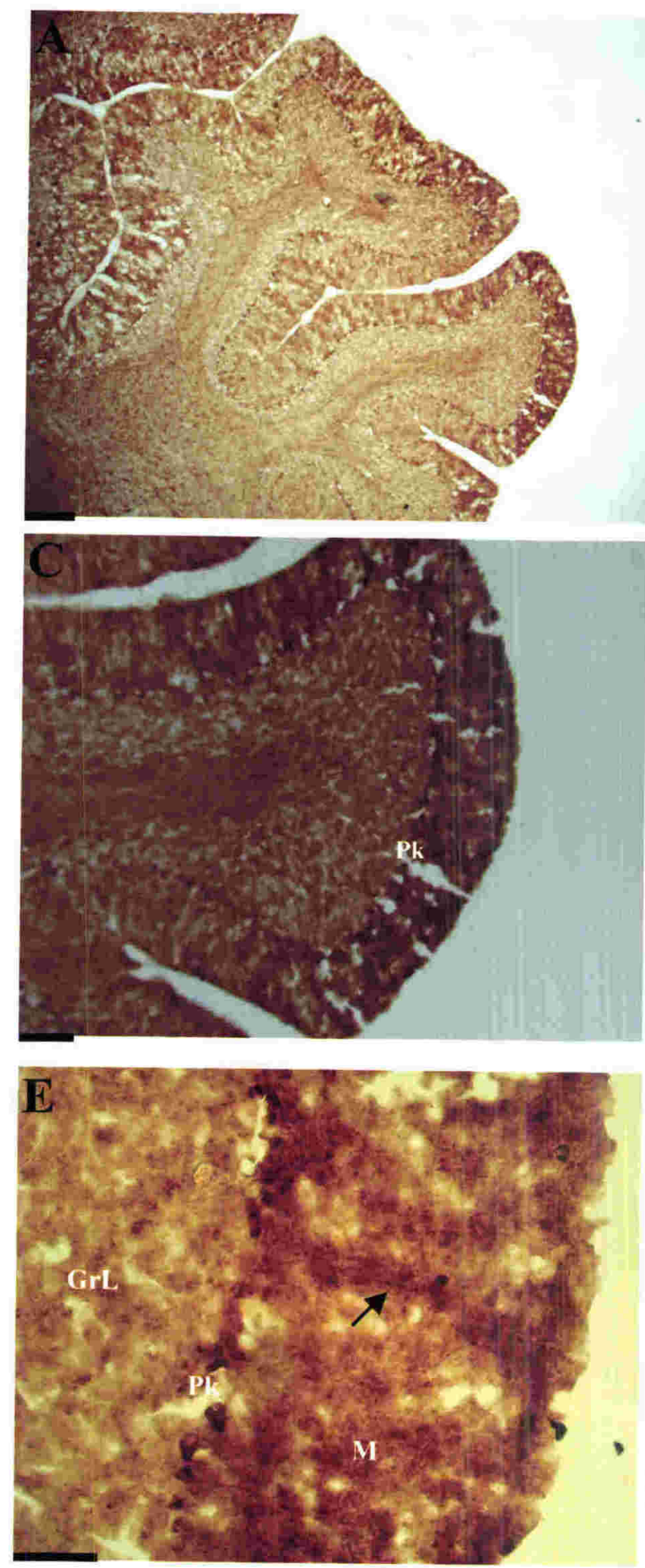

MOR
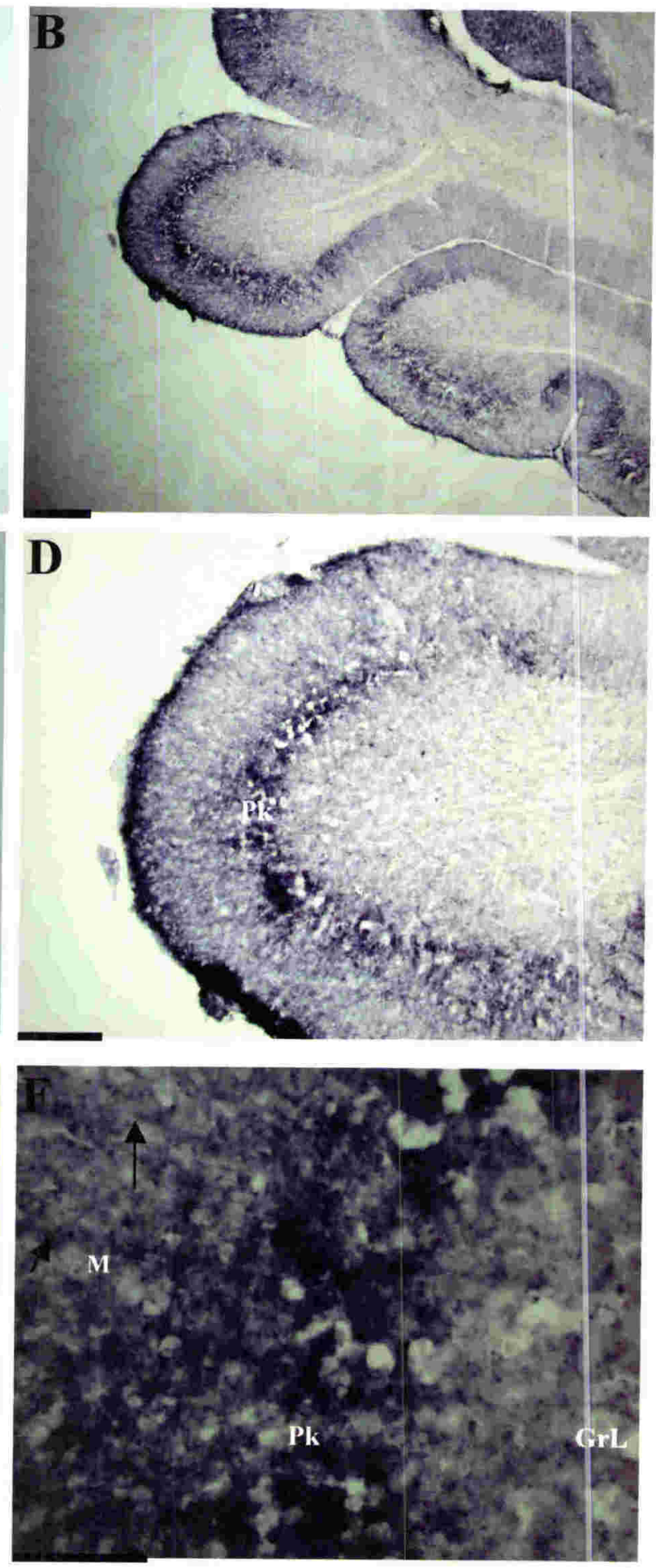

Figure 5.2

Distributions of DOR and MOR immunoreactivity in the Adult Rat Cerebellum

Distribution of DOR (A, C, \& E) (rabbit anti-DOR, Chemicon) and MOR (B, D, \& F) (guinea pig antiMOR, Chemicon) immunoreactivity in the cerebellum of the adult rat. Intense staining of Purkinje cells (Pk) and their dendritic processes (shown by arrows) is seen within the molecular layer (M). The granular layer shows some labelling $(\mathrm{GrL})$. MOR is stained with DAB in the presence of nickel; whereas DOR is labeled without nickel. Some edge staining artifact can be seen. Scale bars $=200 \mu \mathrm{m}$ (A \& B), $100 \mu \mathrm{m}$ (C \& D), and $50 \mu \mathrm{m}$ (E \& F). 


\section{ADULT}

DOR

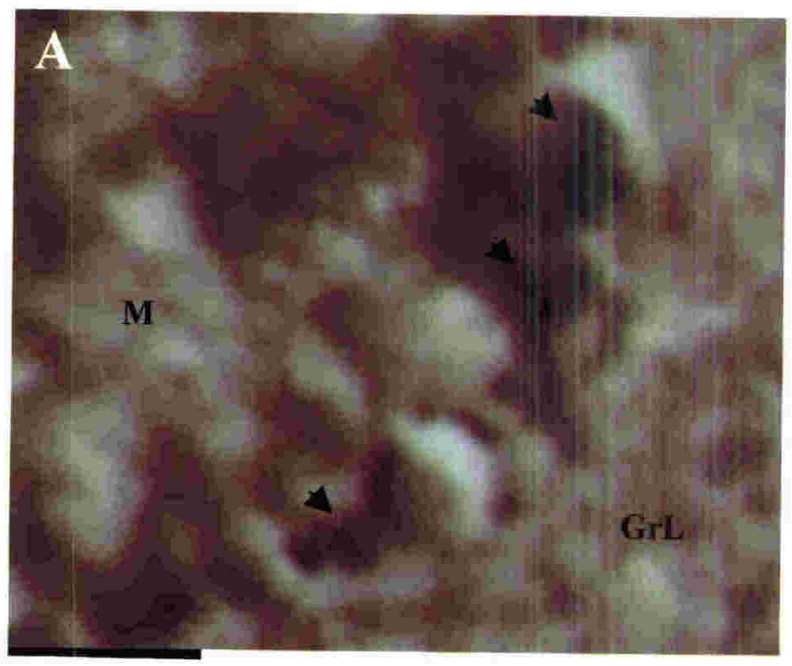

MOR

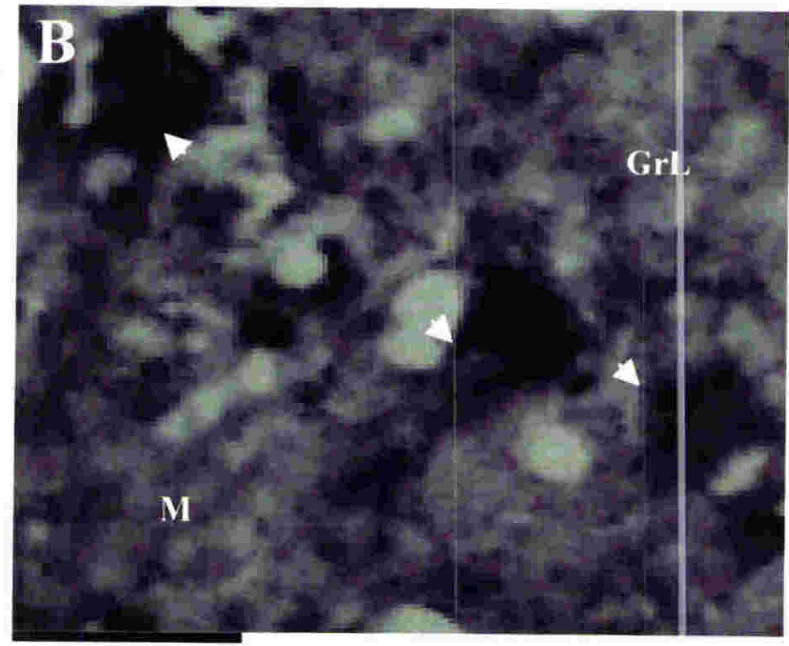

Figure 5.3

\section{DOR and MOR Labelled Purkinje Cells}

High power images of adult rat cerebellum showing (A) DOR (rabbit anti-DOR, Chemicon) and (B) MOR (guinea pig anti-MOR, Chemicon) immunoreactivity in Purkinje cells (arrow heads). Scale bars $=40 \mu \mathrm{m}$.

\section{DOR \\ POSTNATAL (P6)}
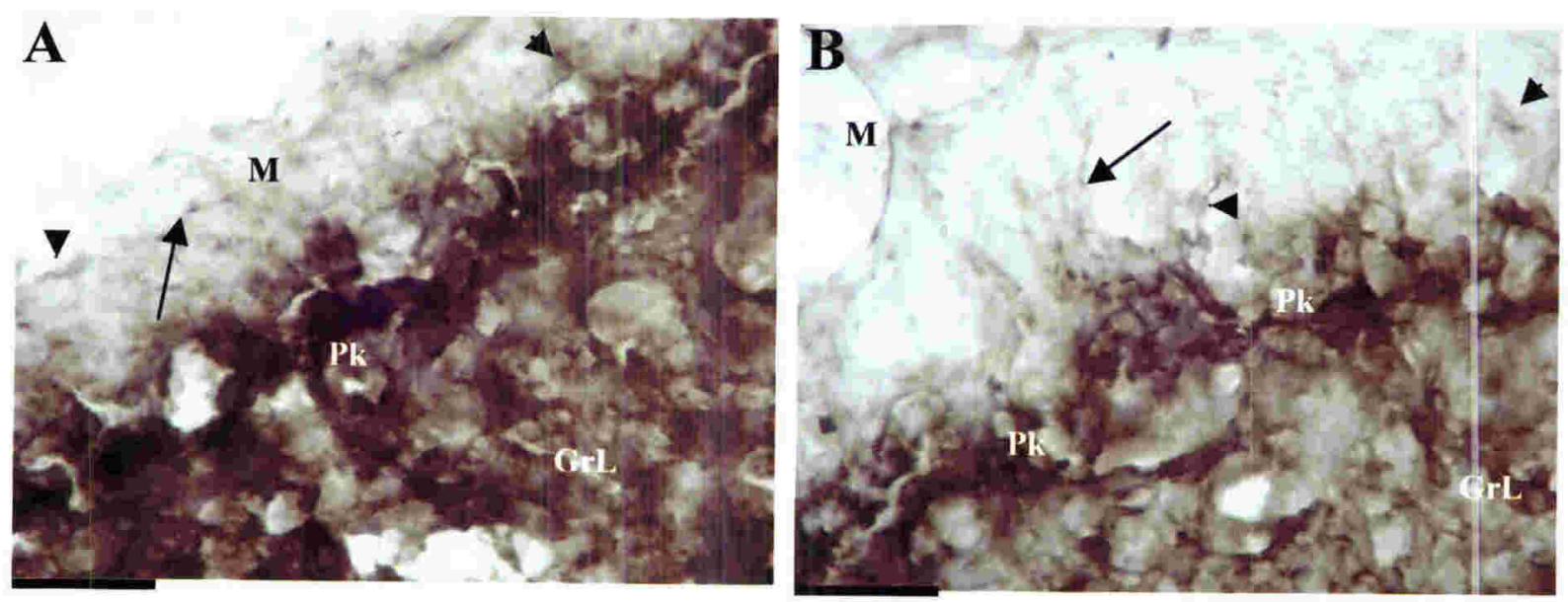

Figure 5.4

\section{Distribution of DOR and MOR immunoreactivity in the Postnatal P6 Rat Cerebellum}

High power pictures of the postnatal P6 rat cerebellum showing (A) DOR (rabbit anti-DOR, Chemicon) and (B) MOR (guinea pig anti-MOR, Chemicon) labelling of the Purkinje cell layer (Pk) with weak labelling of dendrites (shown by arrows) extending from Purkinje cells in the molecular layer (M), Some staining of the granular layer (GrL) below the Purkinje layer is evident. Cell bodies in the molecular layer can be seen and are probably stellate or basket cells (shown by arrow heads). Scale bars $=50 \mu \mathrm{m}$ 


\subsection{DISCUSSION}

\subsubsection{MOR AND DOR DISTRIBUTION IN THE ADULT RAT BRAIN}

Coronal sections of the rat spinal cord, brainstem, and sagittal whole brain sections were labelled with MOR and DOR antibodies. The MOR and DOR staining patterns observed were characteristic of MOR and DOR staining patterns described in the literature (Mansour et al., 1987, 1988; Delay-Goyet, 1990; Delfs et al., 1994; Mansour et al., 1994; Zastawny et al., 1994; Arvidsson et al., 1995a; Mansour et al., 1995; Ding et al., 1996b; Moriwaki et al., 1996; Díaz et al., 2000; Cahill et al., 2001b). The regions stained for both MOR and DOR included the dorsal horn of the spinal cord, olfactory bulb, and the striatum. Similarities with DOR labelling in the mouse brain were seen in the olfactory bulb (Bausch et al., 1995a).

In the present study, the distribution of MOR in the adult rat brain closely resembled the distribution pattern reported in the literature using MOR-1 antibodies (Ding et al., 1996b) and includes MOR detection in the olfactory bulb, striatum, and dorsal horn of the spinal cord (Mansour et al., 1987, 1988; Arvidsson et al., 1995b; Díaz et al., 2000).

The rabbit anti-DOR antibody (Chemicon) used in the present study has been recently well characterised, including one paper giving a detailed comparison with another DOR antibody which gave an almost identical staining pattern in rat brain (Cahill et al., 2001b). This DOR antibody is therefore likely to be specific for DOR. The strongest DOR staining in the study by Cahill et al. (2001) was observed in the caudate putamen, ventral striatum, layers II, III, V and VI of the cerebral frontal cortex, the diagonal band of Broca, and the dorsal horn of the spinal cord (Cahill et al., 2001b). Similar staining patterns were observed in the present study.

\subsubsection{MOR and DOR Expression in the Adult Rat Cerebellum}

It is generally accepted in the literature that MOR is not expressed in the rat cerebellum (Mansour et al., 1987, 1988; Delfs et al., 1994; Mansour et al., 1994; Mansour et al., 1995; Ding et al., 1996b), although a few studies have provided some evidence that MOR is present within the rat cerebellum. For example, Barg, et al. (1989) showed both MOR- and DORspecific ligand binding in the rat cerebellum during development (Barg and Simantov, 1939). In a more recent study, Wittert et al. (1996) demonstrated the presence of MOR mRNA in the 
rat cerebellum using RT-PCR (Wittert et al., 1996). In addition to the above two studies, Bausch et al. (1995) presented an image or photomicrograph of positive MOR immunoreactivity in the cerebellum of the rat within Purkinje cells, although the staining was not mentioned in the text of their paper (Bausch et al., 1995b). There is no controversy that MOR exists in the cerebellum of species other than the rat, including mouse, rabbit and human (Lu et al., 1993; Peckys and Landwehrmeyer, 1999; Hauser et al., 2000). Most OpRs in the cerebellum are found in the granule layer (Hauser et al., 2000) and in Purkinje cells (Bausch et al., 1995b; Peckys and Landwehrmeyer, 1999). MOR and DOR receptors are often found in the same brain regions, especially in brainstem nuclei (Chapter 6). The functional implications of this in the cerebellum, remains to be determined.

In the present study, IHC staining of both MOR and DOR in the cerebellum of postnatal P6 and adult rats showed intense labelling of the Purkinje cell layer, with lighter staining of Purkinje cell dendrites present in the outer molecular layer. IHC techniques are not quantitative; and, therefore, the abundance of MOR relative to DOR could not be assessed. Quantitative immunoblots and confocal immunofluorescent staining of DOR protein in the adult rat cerebellum has recently been reported. Purkinje cells were shown to express DOR at comparable levels to other brain regions, such as the striatum and dendate gyrus, although expression in the granule cells was low (Mansour et al., 1994; Abeyta et al., 2002). DOR expression has also been reported in the rat cerebellar cortex, vermis, dendate nucleus, and interpositus nucleus (Ableitner, 1994). The DOR expression patterns found in the present study were consistent with the above findings. P6 rats showed greater expression of both MOR and DOR in the granular layer than that found in the adult. This is consistent with developmental studies on the cerebellum in guinea pig and rat in which MOR and DOR expression decreased between postnatal and adult animals (Barg and Simantov, 1989).

Purkinje cells, cerebellular astrocytes, and mossy fibres express the endogenous opioid agonist enkephalin within the developing cerebellum (Wiliams and Dockray, 1983; Walker and King, 1989; Spruce et al., 1990; Osborne et al., 1993). Enkephalin is the endogenous ligand for hoth DOR and MOR. Purkinje cells have also recently been shown to contain mRNA for endooligopeptidase (Hayashi et al., 2001), an enzyme that converts enkephalin precursors to 
enkephalin. Preproenkephalin mRNA and its peptide are also found in the cerebellum (Zigon et al., 1985). The presence of endogenous opioid ligands and their receptors in the cerebellum is suggestive of a role for opioids in the development or function of this brain region. The functional significance of opioid receptors and their peptides being localised to specific cell types within the cerebellum has yet to be determined.

\subsubsection{Effects of Opioids on Cerebellar Function and Development}

Purkinje cells play a role in controlling gait, balance, facial movements, and posture. Opioids are known to affect locomotion (for review see)(Olson et al., 1997; Vaccarino et al., 1999; Vaccarino and Kastin, 2001), although no studies have proposed a functional link betwreen opioids and cerebellar motor control. It is possible that Purkinje cells expressing both MOR and DOR may play a role in modulation of some of these complex motor functions.

Opioids play a role in the regulation of cellular proliferation in the developing CNS (Zagon and McLaughlin, 1991; Barg et al., 1993a; Gurwell et al., 1996). In the mouse and rat, opioids inhibit neuroblast replication and differentiation in the developing cerebellum (Zagon and McLaughlin, 1983; Hauser and Mangoura, 1998). Opioids also affect granule cell proliferation and differentiation in a highly selective manner. Since the data from the present study and the work of others has shown that these cells express MOR and DOR as well as the opioid peptide preproenkephalin, it can be concluded that these receptors and their ligands control at least part of granule cell growth and differentiation. Treatment of granule cells cultured from P5 and P6 mice with morphine (a MOR preferential agonist) caused a decrease in cell numbers and inhibition of neuroblast proliferation (Hauser et al., 2000). The localisation of OpRs and peptides within the cerebellum has important developmental implications, particularly in the area of opioid abuse, and this is especially relevant in terms of newborns of drug-addicted mothers. The use of opioids in pain relief during labour may also alter cerebellar function and/or development in the newborn. These speculations need to be investigated, and the cellular distribution of MOR and DOR to specific cells within the cerebellum needs to be refined through the use of high-resolution techniques, such as immunogold electron microscopy or confocal microscopy. Double-labelling with specific cell type markers such as calbindin to specifically label Purkinje cells would also aid in the 
interpretation of the data (Wanner et al., 1997). Calbindin D28k is a calcium-binding protein, which protects neurons against apoptotic cell death. The protein has a distinct distribution in the brain and sensory system and is abundant in specific neuronal cells. It is often used as a positive marker for Purkinje cells within the cerebellum (Puyal et al., 2002).

\subsection{SUMMARY AND CONCLUSIONS}

Immunohistochemical staining of MOR and DOR in the present study demonstrated patterns of expression in regions previously shown to express OpRs, as well as MOR expression in the cerebellum a result not consistent with the consensus in the literature. This study is one of the few to use IHC techniques to describe MOR expression in the rat cerebellum, especially in the Purkinje cell layer. One earlier IHC study has suggested that MOR is expressed in Purkinje cells in the cerebellum (Bausch et al., 1995b), although the authors did not discuss the significance of their finding and may not have realised that others report that MOR is not expressed in this region. The staining patterns observed for MOR in the present study were similar to DOR in the rat cerebellum and to MOR in the human, rabbit, and mouse cerebellum. The most likely reason that MOR was not seen in earlier studies on rat cerebellar tissue was the poor resolution of the methods used. In the present study, high-amplification IHC methods were used, including TSA-biotin amplification. Previous studies using IHC techniques with standard avidin/biotin amplification failed to detect MOR. Given that mRNA FISH labelling has shown a strong MOR mRNA signal in the same areas of the cerebellum (Eli Mrkusich and Dr Darren Day, personal communication), the present results are unlikely to be artefactual. 


\section{CHAPTER 6}

\section{Developmental Expression and Distribution Of Opioid Receptors In The Rat Brainstem}

\subsection{AIM}

The aim of this chapter is to investigate the distribution of MOR and DOR in the rat brainstem during development. To achieve this, total brainstem homogenates from different stages of rat development were used to measure total MOR and DOR protein levels, using SDS-PAGE gel electrophoresis and Western blotting. Immunohistochemistry (IHC) was performed on tissue sections from different developmental ages to determine patterns of expression during development.

\subsection{INTRODUCTION}

\subsubsection{MOR AND DOR EXPRESSION IN THE RAT BRAINSTEM DURING DEVELOPMENT}

Several investigators have studied OpR expression in the rodent brain during development (Coyle and Pert, 1976; Pasternak et al., 1980; Leslie et al., 1982; Tsang et al., 1982; Wohltmann et al., 1982; Spain et al., 1985; Tavani et al., 1985; Kornblum et al., 1987; Petrillo et al., 1987; Attali et al., 1990; Bem et al., 1991; Xia and Haddad, 1991; Barg et al., 1992; Murphey and Olsen, 1995; Zhu et al., 1998; Tong et al., 2000; Beland and Fitzgerald, 2001), but few studies have investigated the distribution and expression patterns of MOR and DOR in the brainstem (Xia and Haddad, 1991; Murphey and Olsen, 1995; Laferrière et al., 1999). Most studies have used ligand-binding methods, and in most cases results reported for the brainstem have been superficial. Often the whole brain is used, or the brain is separated into forebrain and hindbrain regions. The present study concentrates on the lower brainstem regions that were used for primary culture (Chapter 2, 3, \& 4), areas shown to be involved in respiratory rhythm generation and modulation. 


\subsubsection{MOR AND DOR DISTRIBUTION IN THE ADULT BRAIN}

The distribution of MOR and DOR proteins and mRNA expression levels in sections of rat brain have been extensively investigated by autoradiographic techniques (Atweh and Kuhar, 1977; Mansour et al., 1987; Temple and Zukin, 1987; Delay-Goyet, 1990; Díaz et al., 2000; Goody et al., 2002), immunohistochemistry (IHC) (Arvidsson et al., 1995a; Arvidsson et al., 1995b; Ding et al., 1996b; Cahill et al., 200 b), and in situ hybridisation (ISH) (Delfs et al., 1994; George et al., 1994; Mansour et al., 1994; Zastawny et al., 1994). Some of these studies present conflicting distribution patterns, depending on the experimental techniques used. These differences between studies are probably due largely to differences in sensitivity and resolution between the techniques of receptor-ligand binding, antibody binding, and mRNA hybridisation. Results from some of these studies are summarised in Chapter 1 (Table 1.2). Different opioid receptor ligands have unique binding properties, but some ligands and antibodies used in early studies were relatively non-specific, binding more than one receptor subtype. ISH studies often give different labelling patterns to IHC detection; possibly because IHC labels receptors in neural processes as well as in the cell body; whereas, ISH labels mRNA mostly in the cell body. Another factor to consider when interpreting OpR distribution patterns is the presence of multiple molecular forms of MOR and DOR that may be differentially recognised by different antibodies and ligands. Most of these early studies were carried out before these different isoforms of MOR had been described. To further complicate matters, each MOR isoform has a unique distribution within the brain (Schulz et al., 1998; Abbadie et al., 2000b; Abbadie et al., 2000a).

The general consensus from the literature on brainstem and spinal cord is that both MOR and DOR are present in the dorsal horn of the spinal cord (DH) (Arvidsson et al., 1995a; Arvidsson et al., 1995b; Moriwaki et al., 1996; Díaz et al., 2000; Cahill et al., 2001b) and in brainstem areas of the locus coeruleus (Delfs et al., 1994; Zastawny et al., 1994; Arvidsson et al., 1995a; Arvidsson et al., 1995b; Moriwaki et al., 1996), spinal trigeminal tract and nucleus (STT) (Delfs et al., 1994; Mansour et al., 1994; Arvidsson et al., 1995a; Arvidsson et al., 1995b; Bausch et al., 1995a; Mansour et al., 1995; Moriwaki et al., 1996; Cahill et al., 2001b), the dorsal motor nucleus of the vagus (12) (Delfs et al., 1994; Arvidsson et al., 1995a; Arvidsson et al., 1995b; Bausch et al., 1995a), nucleus of the solitary tract (NTS) (Delfs et al., 
1994; Mansour et al., 1994; Zastawny et al., 1994; Arvidsson et al., 1995a; Arvidsson et al., 1995b; Mansour et al., 1995; Moriwaki et al., 1996; Díaz et al., 2000), and the nucleus ambiguus (NA) (Delfs et al., 1994; Zastawny et al., 1994; Arvidsson et al., 1995a; Arvidsson et al., 1995b; Moriwaki et al., 1996; Peckys and Landwehrmeyer, 1999).

\subsubsection{THE EXPRESSION AND DISTRIBUTION OF MOR AND DOR DURING DEVELOPMENT.}

\subsubsection{Early studies in the Developing Brain}

There are many published reports on opioid receptor expression during development that are based on ligand binding of brain homogenate samples in the rat (Coyle and Pert, 1976; Pasternak et al., 1980; Leslie et al., 1982; Tsang et al., 1982; Wohltmann et al., 1982; Span et al., 1985; Kornblum et al., 1987; Petrillo et al., 1987; Attali et al., 1990; Kitchen et al., 1990; Bem et al., 1991), mouse (Tavani et al., 1985; Barg et al., 1992), pig (Laferrière et al., 1999) and guinea pig (Murphey and Olsen, 1994). Often the samples analysed are from whole brain, without separation of brain regions, making regional changes impossible to determine.

A few studies have used ligand-binding autoradiography to show OpR binding to specific brain regions during development. One study showed MOR, DOR, and KOR binding in the postnatal rat brain; however, the brainstem was not included in this study (Kornblum et al., 1987). Another investigation showed binding by naloxone, a non-selective OpR antagonist that binds MOR with a higher affinity than DOR, but again no mention was made of the brainstem (Kent et al., 1982). A third study described the postnatal distribution of MOR and DOR in the brainstem of the pig by autoradiographic binding of the MOR agonist DAMGO and the DOR agonist DPDPE (Laferrière et al., 1999). MOR- and DOR-specific binding was seen in the NTS, NA, and inferior olive (IO). Leslie et al. (1998) have demonstrated KOR mRNA binding in foetal E17 rat brainstem in the region of the dorsal motor nucleus of the vagus, hypoglossal nucleus, and NTS; however, the distribution of KOR was not compared with any other age group (Leslie et al., 1982).

The nature of the ligand used in each study affects the specific distribution patterns observed. For example, naloxone and enkephalin can both bind to MOR and DOR receptors (Kent et al., 
1982). These non-specific ligands show different expression levels compared to DAMGO and DPDPE (Xia and Haddad, 1991). Direct comparisons between studies, however, are difficult because of the different developmental ages used and the fact that the brain sections are not matched.

\subsubsection{Species Differences}

Studies in different species have shown similar opioid receptor distribution patterns to those seen in the rat during development. In the mouse, specific OpR expression levels during train development were first described by Zhu et al. (1998) using ISH techniques (Zhu et al., 1998). Previous studies used ligand binding in forebrain and hindbrain preparations, but did not use specific DOR ligands (Spain et al., 1985). In the mouse, MOR mRNA was first detected at E11.5 and DOR at E13.5, with an increase in expression with increased developmental age (Zhu et al., 1998). In studies of newborn piglets, MOR binding by DAMGO increased with age; whereas, DOR levels, based on DPDPE binding did not change between P2 and P21 (Laferrière et al., 1999). Opioid development in the piglet tends to be ahead of developrnent in the rat, with the highest densities found in the NTS and STT.

\subsubsection{DISTRIBUTION OF MOR AND DOR DURING RAT DEVELOPMENT}

Most studies suggest that MOR and DOR increase during development. DOR appears at a later developmental age than MOR and KOR, which are first detected at E12 and increase during the later foetal and early postnatal developmental stages (Spain et al., 1985; Tavani et al., 1985; Petrillo et al., 1987). DOR is not expressed until early postnatal development (S pain et al., 1985; Tavani et al., 1985; Petrillo et al., 1987), although some studies have detected DOR expression as early as E13.5 in the mouse, using more sensitive ISH techniques (Zhu et al., 1998). Comparisons in expression levels between species are difficult to make in these studies due to the variations in the sensitivities of these techniques. In preparations of whole brain from the rat (minus the cerebellum), changes were observed in opioid receptors during postnatal development. DOR was first present at very low levels at P3, then increased to adult levels at 3.5 months (binding was expressed per g brain). MOR remained high during the postnatal time period between P3 and P21 and in the adult (Petrillo et al., 1987). In the rat 
spinal cord, DOR increased in the 3rd postnatal week (Attali et al., 1990), although the peptide diprenorphine, which binds to both MOR and KOR, was used in this study.

\subsubsection{FUNCTIONAL IMPLICATIONS OF MOR AND DOR EXPRESSION IN THE BRAINSTEM}

The functional implications of OpR expression in the brainstem were discussed in detail in Chapter 1 (Section 1.3). For a review, see (Olson et al., 1997; Vaccarino et al., 1999; Vaccarino and Kastin, 2001). Brainstem nuclei are involved primarily in cardiorespiratory and antinociceptive function. The vagus nerve contains afferent projections to the NTS from the heart, lungs, and abdominal viscera (Lawrence and Jarrott, 1996), and vagal stimulation affects cardiopulmonary reflexes, swallowing, gastrointestinal motility, feedling, antinociception, sleep and respiration. The brainstem areas involved in the control of breathing include the dorsal respiratory group (DRG) in the caudal NTS and the ventral respiratory group (VRG), which includes the pre-Bötzinger Complex (preBötC), Bötzinger Complex (BötC), NA, and ventrolateral medulla (VLM). The NTS is a major afferent integration or relay centre that plays a role in respiratory timing. The VRG, located in and around the NA, includes the preBötC, a region postulated to be responsible for generating the respiratory rhythm (Smith et al., 1991; Rekling and Feldman, 1998). Most brainstem functions become critical at birth, in particular, respiratory function. Studies in the guineit pig have shown that morphine metabolites are more potent at inhibiting respiratory function in the first weeks after birth (Murphey and Olsen, 1994). This may be due to an increase in MOR expression in the brainstem during early postnatal development (Spain et al., 1985; Tavani et al., 1985; Petrillo et al., 1987). Abnormalities in opioid functions during early postnatal development have been implicated in SIDS (Chavez et al., 1979; McMillen, 1986; Storm et al., 1994).

There is a need for a more comprehensive look at OpR localisation and expression during brainstem development because ligand-binding studies do not provide the specificity and resolution required. Early studies lacked the sensitivity that more recent approaches provide, and they should be interpreted with this in mind. Using highly specific antibodies to MOR 
and DOR, the aim of the present study was to provide a more detailed and reliable picture of OpR distributions in the brainstem during development.

\subsection{METHODS}

\subsubsection{QUANTIFICATION OF OPIOID RECEPTOR EXPRESSION}

\subsubsection{Sample Preparation for Western Blots}

Three sets of samples from each developmental age (age-matched samples) were used to measure OpR expression during development. Brainstem tissue from several different developmental ages was dissected from animals sacrificed by a lethal dose of pentobarbital (Pentobarb 300; $100 \mathrm{mg} / \mathrm{kg}$ ip) (Chemstock Animal Health Ltd, Christchurch, NZ). Tissue was placed on ice, washed in PBS, chopped into small pieces, and transferred into Western Sample Buffer (8 M Urea, $3 \mathrm{M}$ thiourea, 4\% CHAPS and $40 \mathrm{mM}$ DTT). The tissue was incubated at room temperature on a vortex mixer for 10-30 min, and samples were stored frozen at $-20^{\circ} \mathrm{C}$ until applied to electrophoretic gels.

In addition to brain tissue pieces, cultured cells from dissociated brainstem tissue were prepared for Western blots. The cultured cells were rinsed in PBS and mechanically detached from the culture dish by trituration with a $1 \mathrm{~mL}$ pipette in $0.4 \mathrm{~mL}$ of PBS. The suspended cells in were placed in a $1.5 \mathrm{~mL}$ microfuge tube and washed in PBS $(2 \mathrm{x})$. The samples were pooled and centrifuged for $5 \mathrm{~min}$ at $3000 \mathrm{rpm}$. The PBS was removed and the cells resuspended in sample buffer, incubated at room temperature with continuous vortexing for 10-30 minutes, and stored at $-20^{\circ} \mathrm{C}$ until required.

\subsubsection{Concentration of Protein by Acetone Precipitation}

Selected samples were concentrated in the Western sample buffer before electrophoresis in order to prevent interference of salts in the buffer when large volumes are loaded. Samples were precipitated for $5 \mathrm{~min}$ in 4 volumes of ice-cold acetone and centrifuged at 14,000 rpm for $10 \mathrm{~min}$ at $4^{\circ} \mathrm{C}$. Acetone was removed by micropipette, and the pellet was re-solubilized in $5 \mathrm{x}$ SDS loading buffer $(62.5 \mathrm{mM}$ Tris- $\mathrm{HCl} \mathrm{pH} 6.8,2 \%$ SDS, $20 \%$ glycerol, $1 \%$ bromophenol blue, $5 \% \beta$-mercaptoethanol) and electrophoresed on $8 \%$ SDS polyacrylamide gels (Appendix 2: Section 13). 


\subsubsection{Protein Determination}

Protein concentrations in brainstem tissue and cell culture samples were carried out according to a modified Bradford method. The protein assay used was first described by Bradford (1976) and modified by Sedmak and Grossberg (1977) to reduce interference by lipids and detergents (Bradford, 1976; Sedmak and Grossberg, 1977). The method, using modfied Bradford reagent $(0.04 \%$ (w/v) Brilliant Blue G 250, in 3.5\% perchloric acid), was further modified for 96-well plates. A standard curve was prepared in $50 \mu \mathrm{L}$, adjusting the sample volume with $\mathrm{dH} 2 \mathrm{O}$. Modified Bradford reagent $(250 \mu \mathrm{L})$ was added, the solution mixed, and the plate incubated at room temperature for $10 \mathrm{~min}$. A microplate reader (VERSAmax, Molecular Devices Corporation, Sunnyvale, CA, USA) was blanked with sample buffer lacking protein, and the absorbance of the samples was measured at $620 \mathrm{nM}$. Sample protein concentrations were estimated from a BSA standard curve $(0-50 \mu \mathrm{g}$ protein $)$ using Softmax ${ }^{\circledR}$ Pro software (Molecular Devices Corporation).

\subsubsection{SDS-PAGE Gel Electrophoresis}

All samples were analysed by electrophoresis on $1.5 \mathrm{~mm}$ thick, $8 \%$ SDS polyacrylamide gels using a 4\% stacking gel (Appendix 2: Section 13). The gels were cast and electrophoresed in a Miniprotean II or III cell (BioRad, Hercules, CA, USA). Appropriate amounts of protein $(10-70 \mu \mathrm{g})$ were added to each lane alongside $7 \mu \mathrm{L}$ of Benchmark prestained molecular weight markers (Invitrogen) and electrophoresed in SDS running buffer $(0.3 \%$ Tris, $1.4 \%$ glycine, $0.1 \%$ SDS, pH 8.3) (Appendix: Section 14) at $150 \mathrm{~V}$ for $1 \mathrm{hr}$. Concentrated protein gel loading buffer (5x stock) (Appendix: section 15) was added to the samples in $1.5 \mathrm{~mL}$ microfuge tubes to give a final concentration of at least $1 \mathrm{x}$ loading buffer. The samples were then boiled for $10 \mathrm{~min}$ on a heated dry block (Barnstead, Dubuque, Iowa, USA).

\subsubsection{Western Transfer}

Proteins were transferred from polyacrylamide gels to polyvinylidene difluoride (PVDF) membrane (BioRad) using a Trans Blot ${ }^{\circledR}$ cell (BioRad). The PVDF membrane was soaked for $5 \mathrm{~min}$ in $100 \%$ methanol, then $5 \mathrm{~min}$ in ddH2O, then placed in Western Transfer Buffer (25 mM Tris, 192 mM glycine, pH 8.3 containing 20\% methanol) (Appendix 2: Section 16). 
The materials used for transfer were stacked in transfer buffer in a Western Transfer Apparatus (Appendix 2: Section 19; Table 1), avoiding air bubbles between layers. A small magnetic stirrer was placed at the bottom of the transfer tank, which was filled with ice cold transfer buffer, and the dark grey side of the assembled cassette was placed on the side nearest the black electrode. The assembled tank with its cooling coil was placed on a magnetic stirrer in a cold room at $4^{\circ} \mathrm{C}$, and proteins in the gel were transferred for $4 \mathrm{hrs}$ at $100 \mathrm{~V}$ into the PVDF membrane.

\subsubsection{Immunostaining of Western Transferred Proteins}

After protein transfer, the membrane was rinsed in $\mathrm{ddH} 2 \mathrm{O}$ and blocked, protein side up, overnight at $4^{\circ} \mathrm{C}$ in $10 \%$ non-fat milk powder (Anchor) in Tris-buffered saline (TBS) $(137 \mathrm{mM}$ $\mathrm{NaCl}, 20 \mathrm{mM}$ Tris, pH 7.6) containing 0.1\% Tween 20 (BioRad, Hercules, CS, USA) (TTBS). All membrane incubations were performed on a rocker platform. The next day, the membrane was washed $(3 \times 5 \mathrm{~min})$ in T-TBS, and then incubated at room temperature for $2 \mathrm{hrs}$ in primary antibody for MOR (1:5000, anti-MOR guinea pig, Chemicon) or DOR (1:5000, anti-DOR rabbit, Chemicon) diluted in T-TBS. The PVDF membranes were rinsed $(2 \times 5$ min and $2 \times 15 \mathrm{~min}$ ) in T-TBS, and incubated for $1 \mathrm{hr}$ at room temperature with secondary antibody conjugated to HRP (for MOR, anti-GP HRP 1:2000, and for DOR, anti-rabbit HRP 1:5000), washing as before.

\subsubsection{Detection And Analysis Of Western Transferred Proteins}

HRP-labelled proteins were visualised using Lumi-light enhanced chemiluminescent substrate (Roche) and developed using Kodak X-OMAT film (Radiographic Supplies Ltd, Christchurch, NZ). The chemiluminescent reaction was performed as per manufacturer's instructions and the film exposed between 1-30 min prior to developing. The location of MW markers was determined and the protein bands analysed by scanning the film using a Molecular Dynamics Personal Densitometer SI (Molecular Dynamics, Sunnyvale, CA, USA). Images were analysed using ImageQuant software (Molecular Dynamics). The band density (minus the average background density of the film) was calculated for statistical analysis. 


\subsubsection{IMMUNOHISTOCHEMISTRY}

Immunohistochemistry was performed by the methods described in Chapter 5 (Section 5.3.1). 


\subsection{RESULTS}

\subsubsection{ANTIBODY QUANTITATION}

MOR and DOR antibodies stained in a quantitative manner. When increasing amounts of protein lysate were run on an SDS-PAGE gel, there was an increase in MOR and DOR band intensity in the Western blots (Figure 6.1).

\subsubsection{MOR AND DOR EXPRESSION IN SH-SY5Y CELLS}

Antibody specificity was confirmed by using SH-SY5Y and LLC-PK1 cells as positive and negative controls, respectively. Undifferentiated SH-SY5Y cells showed MOR-positive protein bands at $50 \mathrm{kD}$ and $70 \mathrm{kD}$, and DOR-positive bands at $30 \mathrm{kD}$ and $60 \mathrm{kD}$ (Figure 6.2). LLC-PK1 cells showed no immunoreactivity for MOR or DOR proteins. A second negative control experiment using no primary MOR or DOR antibody, only secondary antibodies, showed no immunoreactivity; therefore, all immunoreactivity observed was presumed to be due to specific labelling by the primary antibodies. Some SH-SY5Y and brainstem samples gave a band at approx $140 \mathrm{kD}$ for MOR and $130 \mathrm{kD}$ for DOR when the film was exposed for a longer period. Other investigators have attributed this to opioid receptor dimer formation (George et al., 2000). Two MOR antibodies were tested in these experiments to check the specificity of the primary antibodies. Both the guinea pig anti-MOR antibody and the rabbit anti-MOR antibody (Chemicon) gave immunoreactive bands at $50 \mathrm{kD}$ and $70 \mathrm{kD}$, and both produced similar changes in staining intensity during development when the same samples were labelled on a separate Western blot (Figure 6.4).

All band intensity data obtained from the gels were normalised to the postnatal day 3 protein band (P3) to allow comparisons to be made between duplicate samples run on separate ge/s, or protein bands visualised at different chemiluminescent exposure times. 
Figure 6.1

\section{DOR and MOR Labelling of Adult Brainstem Tissue Lysates}

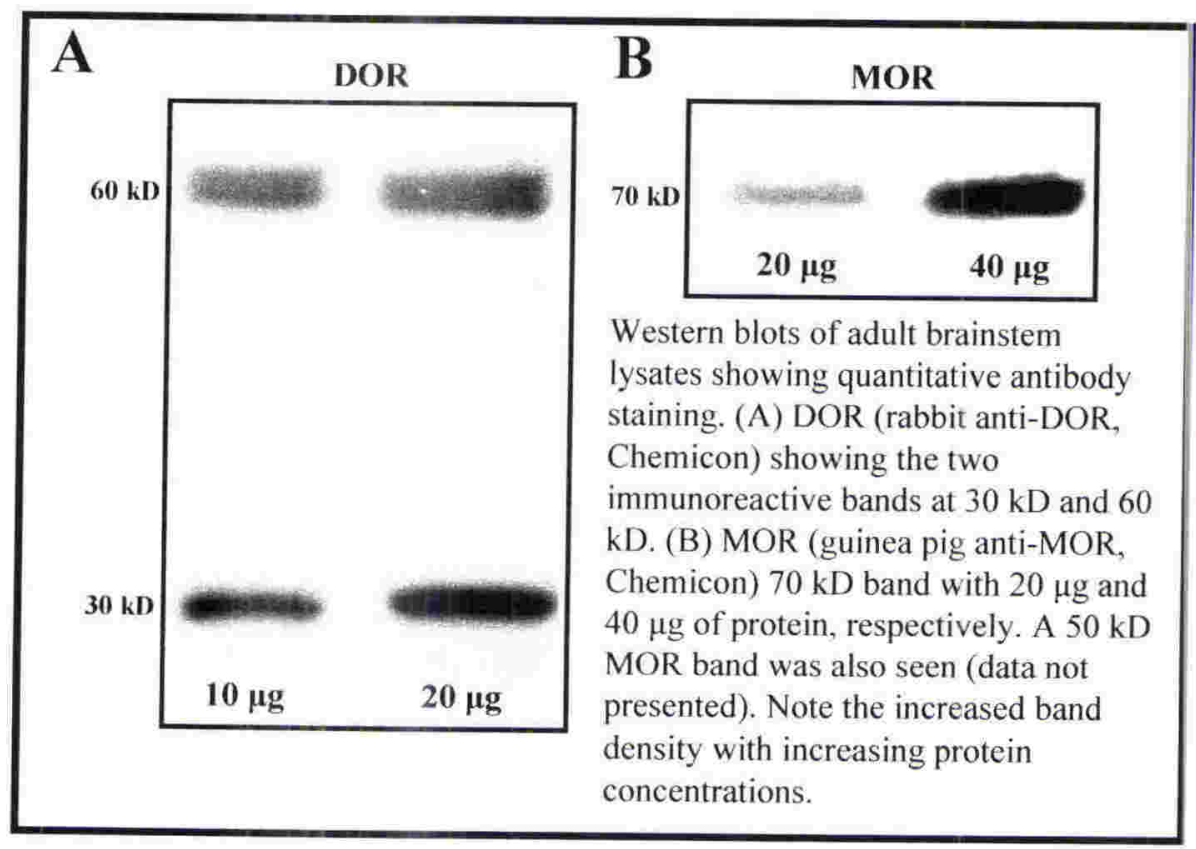

Figure 6.2

DOR and MOR Labelling of SH-SY5Y Cell Lysates

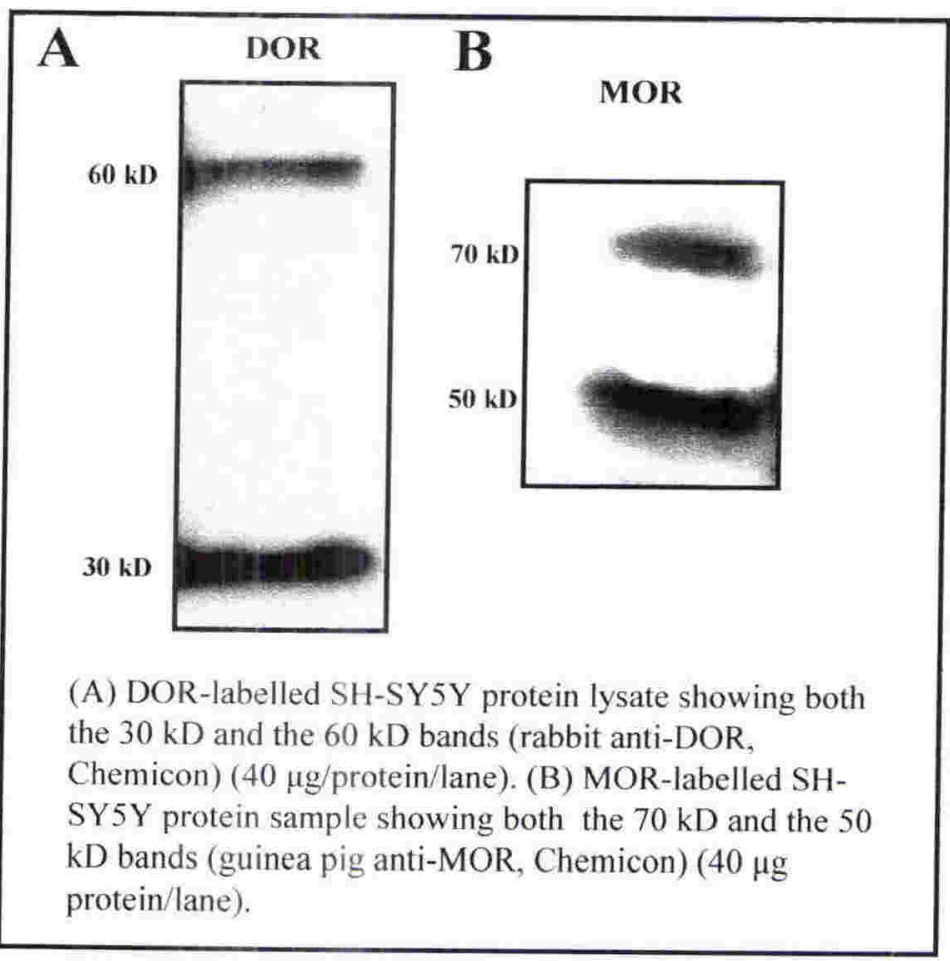




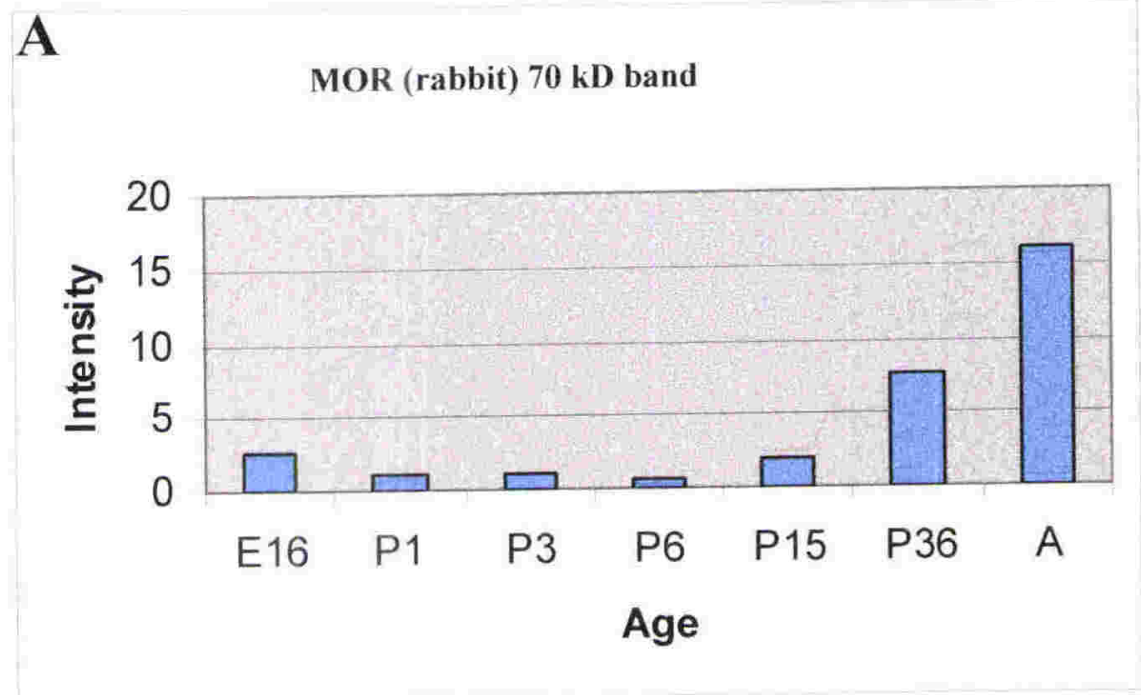

\section{B}

MOR (GP) 70 kD Band

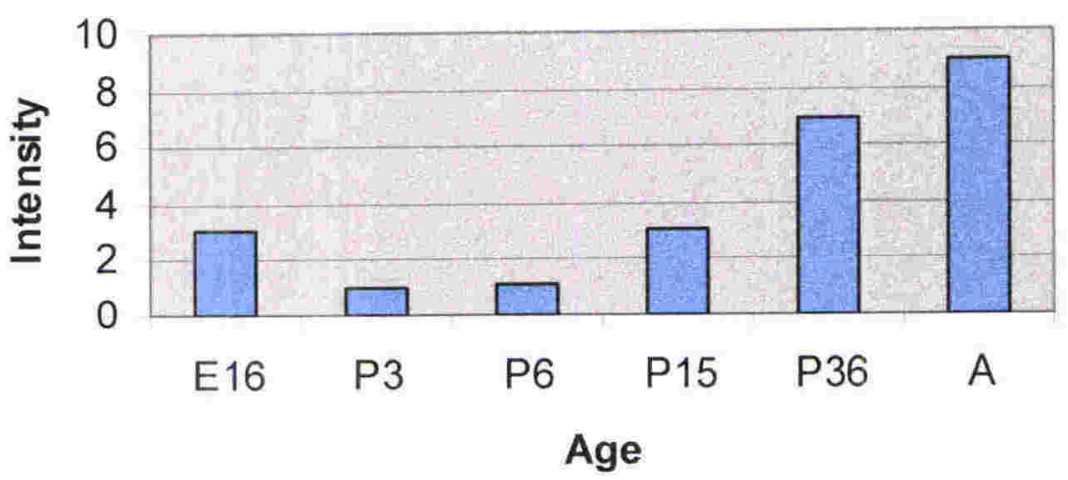

Figure 6.3

\section{Western Blot Comparisons of Two MOR Antibodies}

In one pilot study Western blots were performed using different MOR antibodies. (A) The the rabbit anti-MOR antibody (Chemicon), and (B) the guinea pig (GF) anti-MOR antibody (Chemicon) gave the same size immunoreactive bands and the same staining intensity differences during development. The $50 \mathrm{kD}$ band gave similar results to those described for $70 \mathrm{kD} \mathrm{MOR}$ band (Figure 6.6). 


\subsubsection{MOR AND DOR PROTEIN EXPRESSION DURING BRAINSTEM DEVELOPMENT IN VIVO}

All experiments using brainstem lysates were performed on at least three animals each from the adult and P6 age groups and three preparations of pooled foetal brainstem tissue. Experiments using brain sections were performed at least 3 times. In the adult and P6 aninals, serial sections were taken and alternate sections were stained for MOR and DOR, respectively. In E16 animals, due to the small size of the brainstem, different animals were used for MOR and DOR staining, respectively.

Brainstem preparations from the same animal in each age group were run in duplicate gels (same samples were run at different times) for MOR (Figure 6.4) and DOR (Figure 6.7). Both gels showed similar staining intensity differences between developmental age-matched samples (shown as error bars in Figure 6.4 and 6.7). The average values of these duplicate gels were combined with similar results from two other sets of age-matched developmental samples (also run in duplicate) (Figure 6.4B \& C and 6.7B \& C). These results were then pooled giving an experimental sample of ( $n=3$ preparations) for each developmental age.

As seen in SH-SY5Y cells (Figure 6.2) there are two main MOR immunoreactive bancs in samples of the rat brainstem, one at $50 \mathrm{kD}$ and one at $70 \mathrm{kD}$ (Figure 6.5). These findings are consistent with the findings of Abbadie et al. (2000) who report two MOR immunoreactive bands at 46 and $66 \mathrm{kD}$ (Abbadie et al., 2000a). The intensity of both bands changed during development. The $50 \mathrm{kD}$ band was present in both late foetal (E16) and early postnatal development, but was present in the adult at very low levels (approx 3\% of total) (Figure 6.6A). The $70 \mathrm{kD}$ band was not usually present in the youngest foetal age tested (E16) but appeared during early postnatal development from P6 (depending on the level of chemiluminescent exposure) and was greatest in the adult (approx 97\% of total) (Figure 6.6B). 
MOR Expression

A

Sample 1

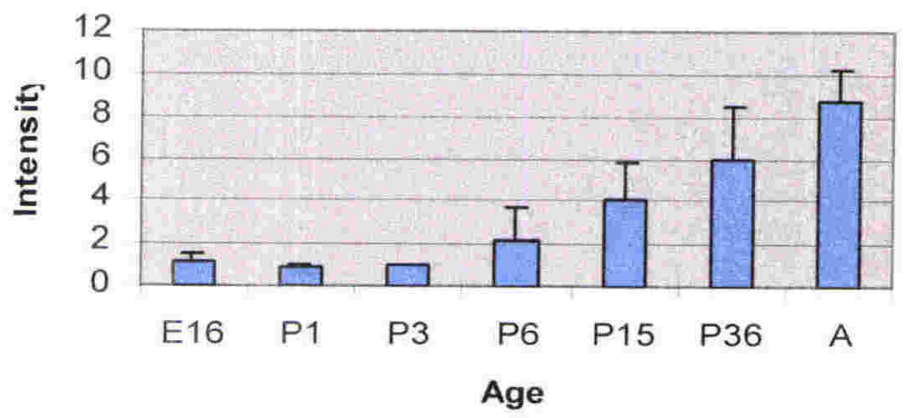

B

Sample 2

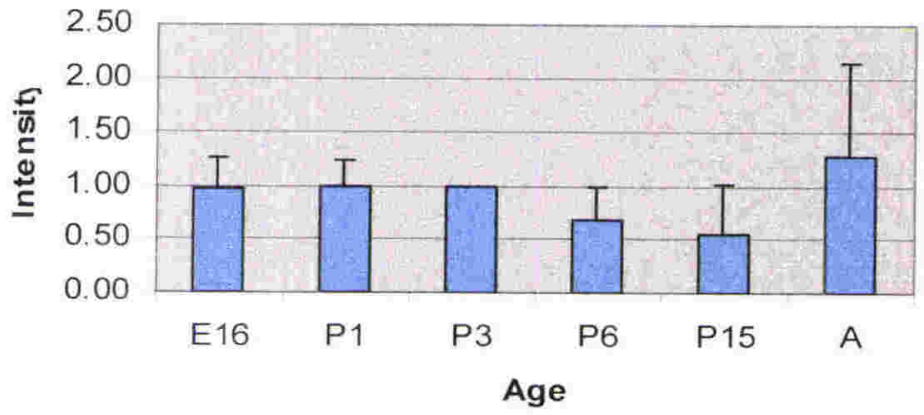

C

Sample 3

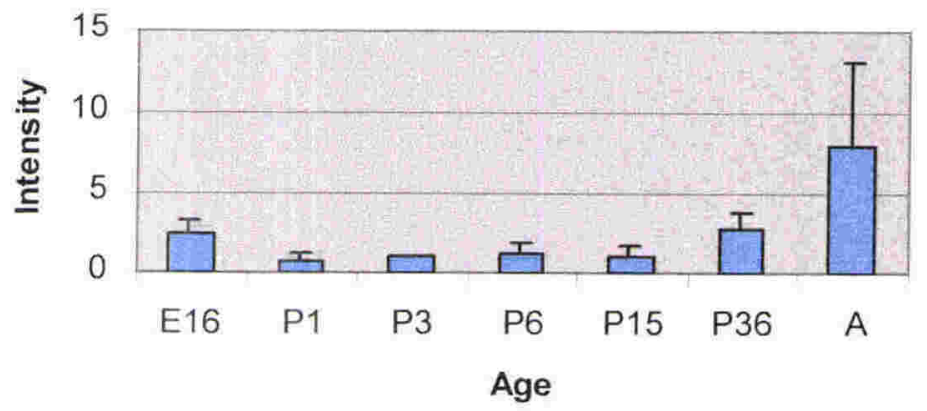

Figure 6.4

\section{MOR Protein Expression During Development in Aged-Matched Samples}

Total brainstem MOR protein levels (guinea pig anti-MOR, Chemicon) from Western blots from three separate sets of age-matched protein samples, each performed in duplicate ( + SEM). Band intensities have been normalised to the P3 sample. Results show a small variation between the same lysate run on different gels (error bars) with a larger variation between different agematched protein samples (A, B, \& C). All aged-matched samples show the highest MOR staining in the adult. 


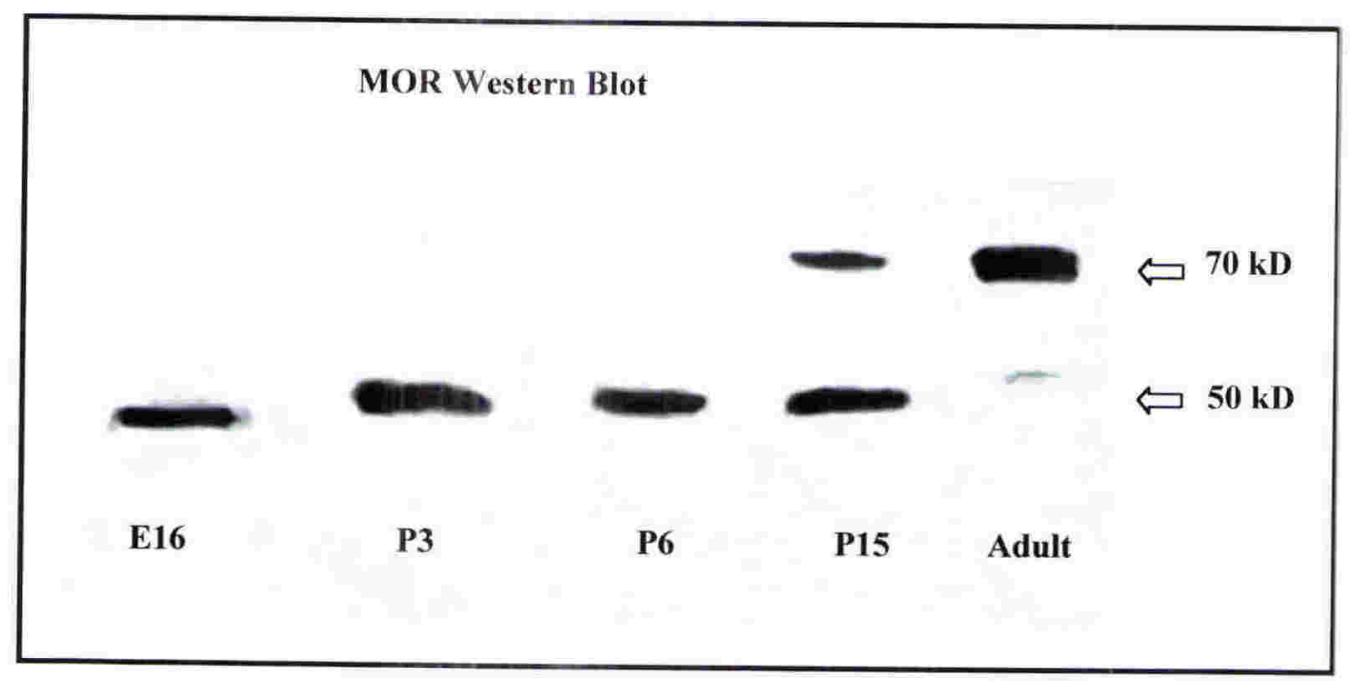

Figure 6.5

Western Blot Showing Developmental Expression of MOR

Western blot showing MOR expression during development using a MOR specific antibody (guinea pig anti-MOR, Chemicon). There are 2 immunoreactive bands present, one at $50 \mathrm{kD}$ and the other at $70 \mathrm{kD}$. The $70 \mathrm{kD}$ band increases in intensity with developmental age; whereas the $50 \mathrm{kD}$ band decreases in intensity with developmental developmental age. $20 \mathrm{~g}$ of protein was added to each lane. 

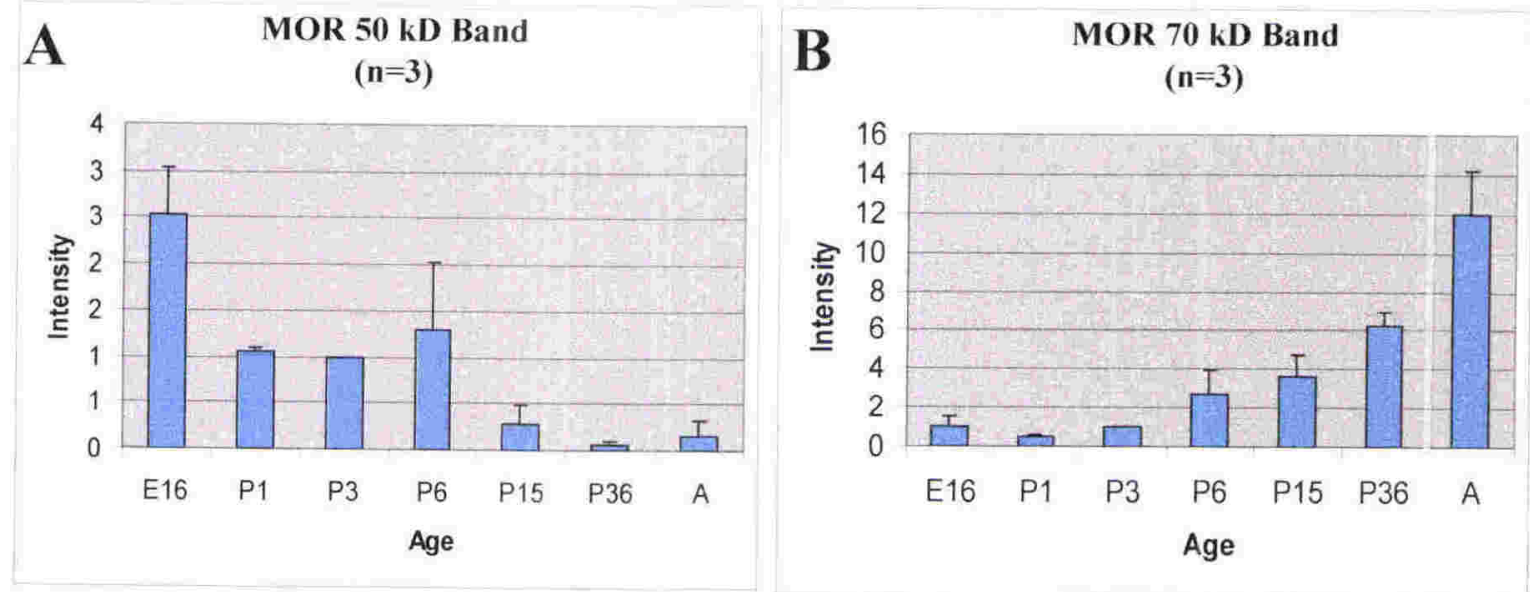

C

Total MOR Protein Expression During Brainstem Development $(n=3)$

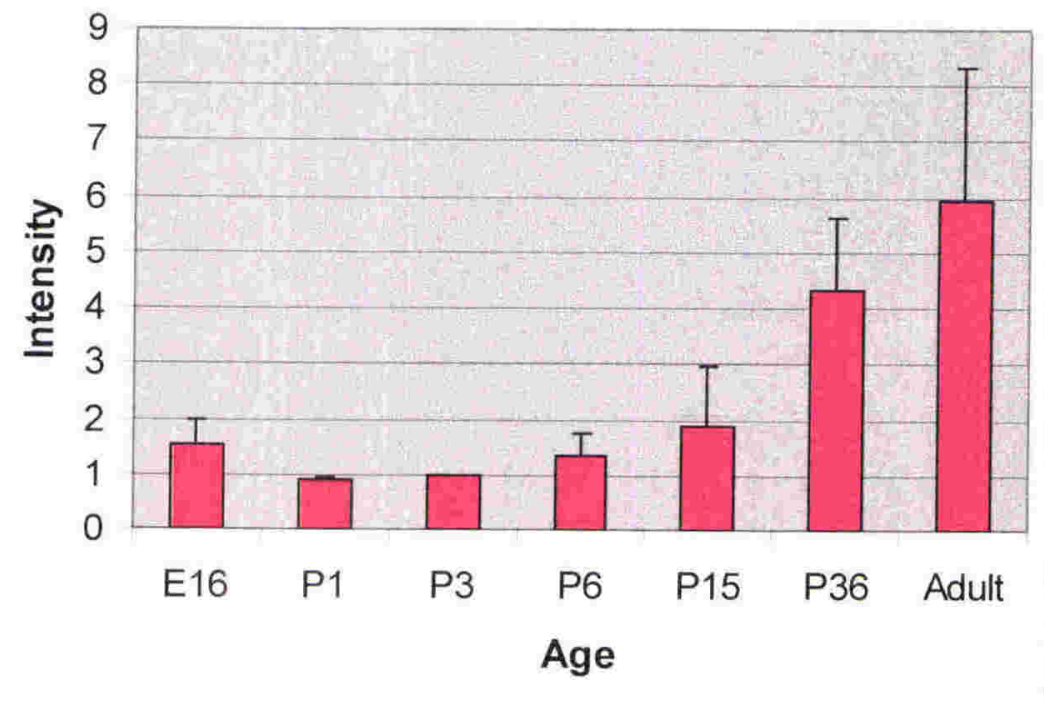

Figure 6.6

\section{Combined Sample Data of MOR Expression During Development}

There are 2 main MOR immunoreactive bands one at $50 \mathrm{kD}(\mathrm{A})$ and another at $70 \mathrm{kD}(\mathrm{B})$ (guinea pig anti-MOR, Chemicon). Band density results showed that the $50 \mathrm{kD}$ band was present at higher levels in early development, whereas the $70 \mathrm{kD}$ band was present at higher levels in late postnatal development and in adult brainstems. (C) Total band intensity for MOR $(50 \mathrm{kD}+70 \mathrm{kD})$ showed an increase in total MOR protein levels during development of the brainstem from late foetal through to adult. 
Combined $50 \mathrm{kD}$ and $70 \mathrm{kD}$ band intensity measurements from 3 separate age-matched sample preparations showed the total MOR protein levels increased with increasing developmental age (Figure 6.6C). MOR protein expression was highest in the adult.

The same age-matched protein samples used for MOR protein expression studies were also used for DOR studies (Figure 6.7). Samples were run on duplicate gels and processed as described for MOR above. There was some variation between duplicate gels (shown as error bars in Figure 6.7), with a larger difference seen between similar lysates from different populations (Figure 6.7A, B, \& C). All samples showed an overall decrease in DOR expression during development.

Two DOR immunoreactive bands were seen in Western blots of brainstem homogenates. one at $30 \mathrm{kD}$ and another at $60 \mathrm{kD}$ (Figure 6.1 and Figure 6.8). The presence of two immunoreactive bands in the present study is consistent with the studies of Carr et al. (1987) who found two DOR immunoreactive bands, one at $30 \mathrm{kD}$ and another at $58 \mathrm{kD}$ (Carr et al., 1987). Both the $30 \mathrm{kD}$ and $60 \mathrm{kD}$ protein bands seen in the present study were more intensely stained at the younger developmental ages (E16-P6) (Figure 6.8 and Figure 6.9A \& B). Total DOR protein levels progressively decreased with increased developmental age through to the adult. This decrease in total DOR expression can be clearly seen when the $30 \mathrm{kD}$ and $60 \mathrm{kD}$ band intensities are combined (Figure 6.9C).

\subsubsection{MOR AND DOR EXPRESSION IN CULTURED BRAINSTEM CELLS FROM LATE FOETAL AND EARLY POSTNATAL ANIMALS}

MOR and DOR immunolabelling was performed by Western blotting of lysates from culcured rat brainstem cells established from foetal E16 and postnatal P1, P3, and P6 developmental ages. MOR and DOR intensity of expression in cell culture lysates reflected what was observed in brainstem lysates. The same two MOR and DOR immunoreactive bands were seen. No difference in MOR and DOR immunoreactivities was seen between cells after 4 and 7 days in culture at any developmental age tested. 
MOR staining of cell culture lysates from E16 rats after 4 and 7 days in culture showed a single band at $50 \mathrm{kD}$ (Figure 6.10A). All postnatal cultures showed two immunoreactive bands, one at $50 \mathrm{kD}$ and another at $70 \mathrm{kD}$. These were the same molecular weights as the bands observed in SH-SY5Y cells (Figure 6.2B) and postnatal and adult brainstem tissue lysates (Figure 6.5). Foetal (E16) cell culture lysates and foetal (E16) tissue lysates showed one MOR immunoreactive band at $50 \mathrm{kD}$ (Figure 6.10A and Figure 6.5). The postnatal cell culture lysates and tissue lysates showed two immunoreactive bands at $50 \mathrm{kD}$ and $70 \mathrm{kD}$ (Figure 6.10A and Figure 6.5).

DOR expression in brainstem cell cultures from E16, P1, P3, and P6 animals showed two immunoreactive bands at $30 \mathrm{kD}$ and $60 \mathrm{kD}$ (Figure 6.10B). These proteins were the same molecular weights as seen in SH-SY5Y cells (Figure 6.2A) and brainstem tissue lysates (Figure 6.1A). DOR expression was higher in foetal and early postnatal (P1) cell culture lysates than in lysates of cultured postnatal (P3 and P6) cells. The expression level observed in cultured cells was similar to that seen in brainstem tissue lysate samples, in which foetal tissue had greater DOR expression than postnatal tissue (Figure 6.10B and Figure 6.8).

Although trends in MOR and DOR expression could be observed in samples prepared from cell culture lysates, a large amount of variation between samples collected from different cell culture preparations was seen. For these reasons results were difficult to interpret, and no statistically significant differences were observed, due to the small sample size used $(n=3$ preparations at each developmental age). 


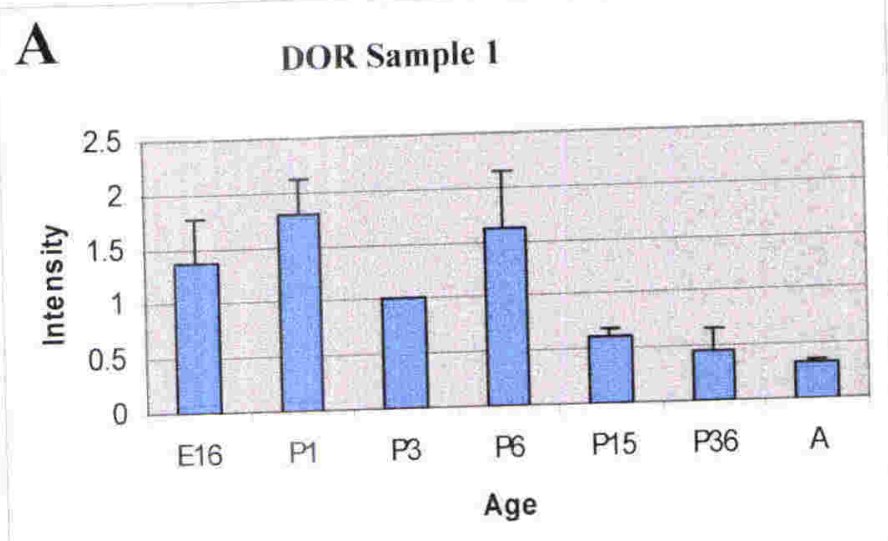

B

DOR Sample 2

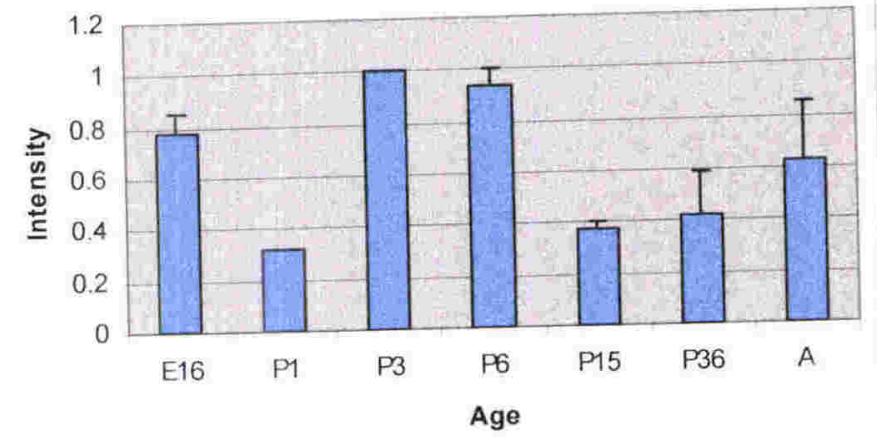

C DOR Sample 3

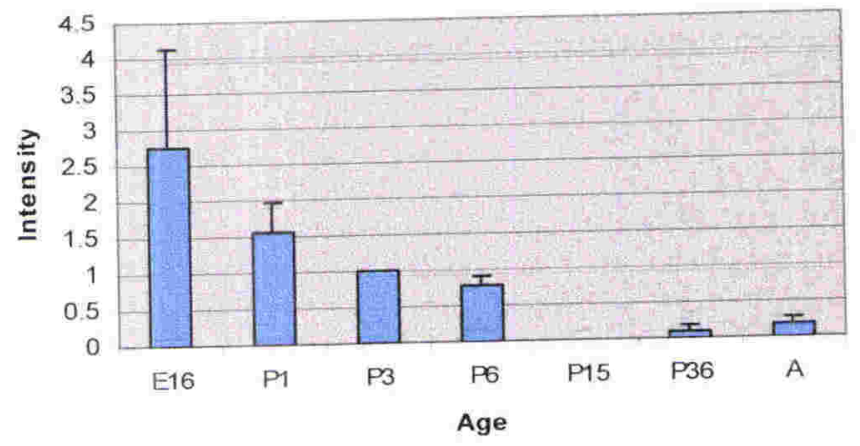

Figure 6.7

DOR Expression During Development in Aged-Matehed Samples

Combined DOR band intensity $(30 \mathrm{kD}+60 \mathrm{kD})$ results $(\mathrm{A}, \mathrm{B}, \& \mathrm{C})$ from 3 separate brainstem samples, performed in duplicate. There was a very small variation between duplicate experiments (error bars) but a larger variation between sample preparations collected from different animals (A, B \& C). Data are presented as Intensity + SEM 


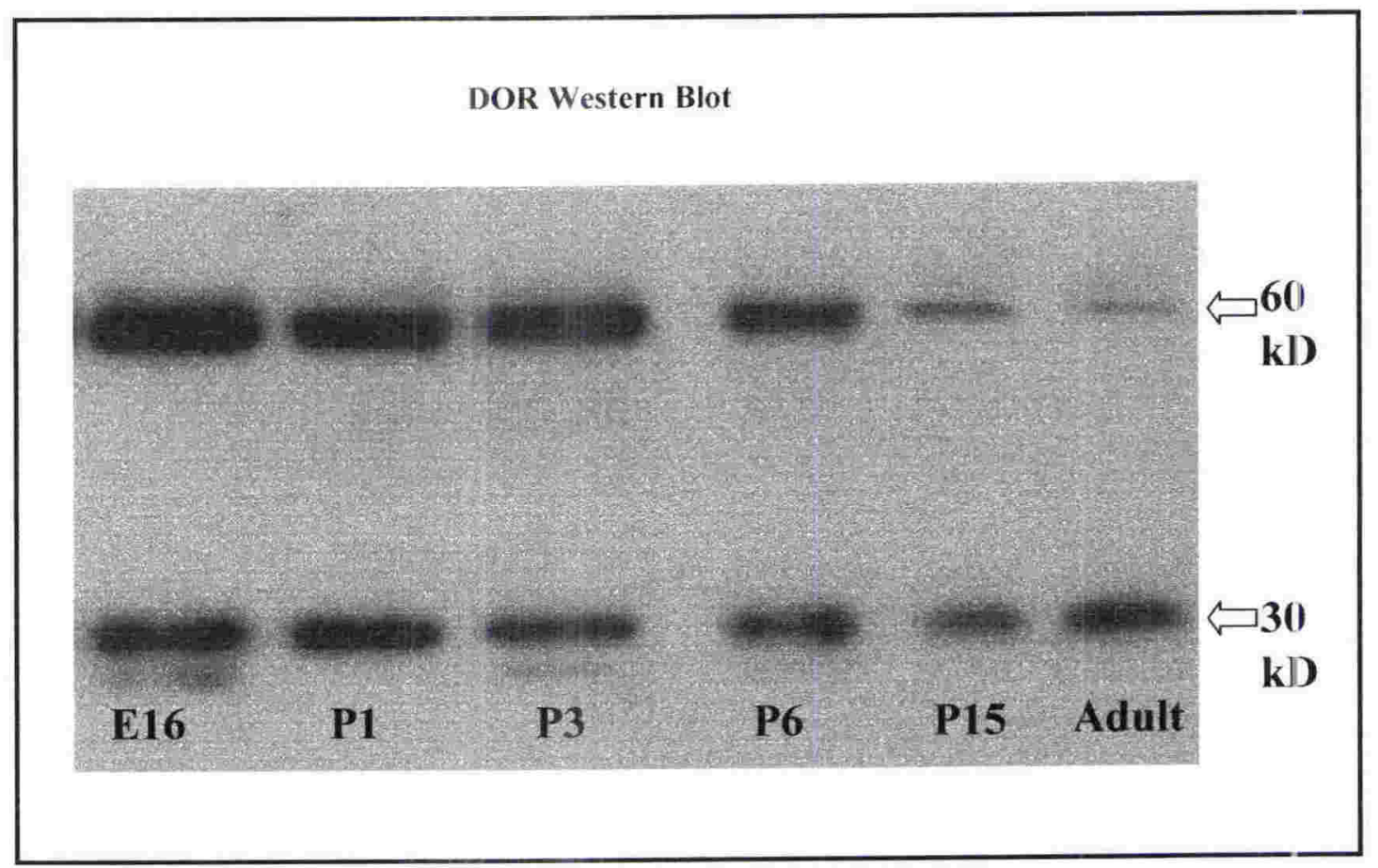

Figure 6.8

\section{Western Blot Showing Developmental Expression of DOR}

Western blots showing that DOR (rabbit anti-DOR, Chemicon) expression levels are highest in foetal tissue (E16) and progressively decrease with increased age. There are two DOR immunoreactive bands, one at $30 \mathrm{kD}$ and the other at $60 \mathrm{kD} .20 \mu \mathrm{g}$ of protein was loaded into each lane. Western blot is from sample 3 . 

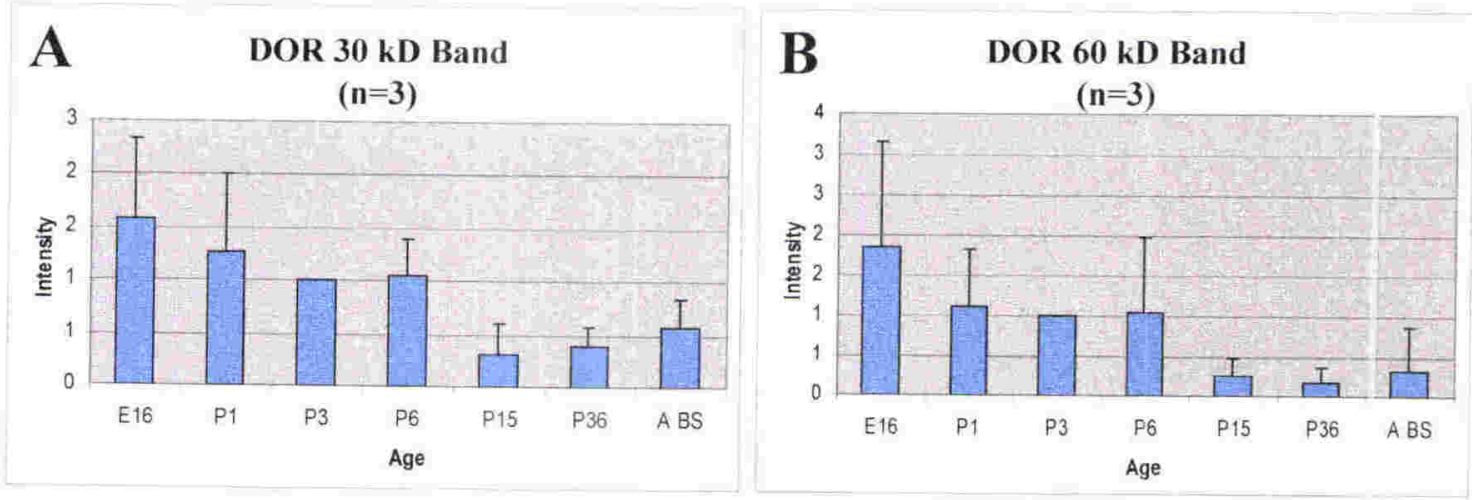

\section{Total DOR Protein Expression During Brainstem Development}

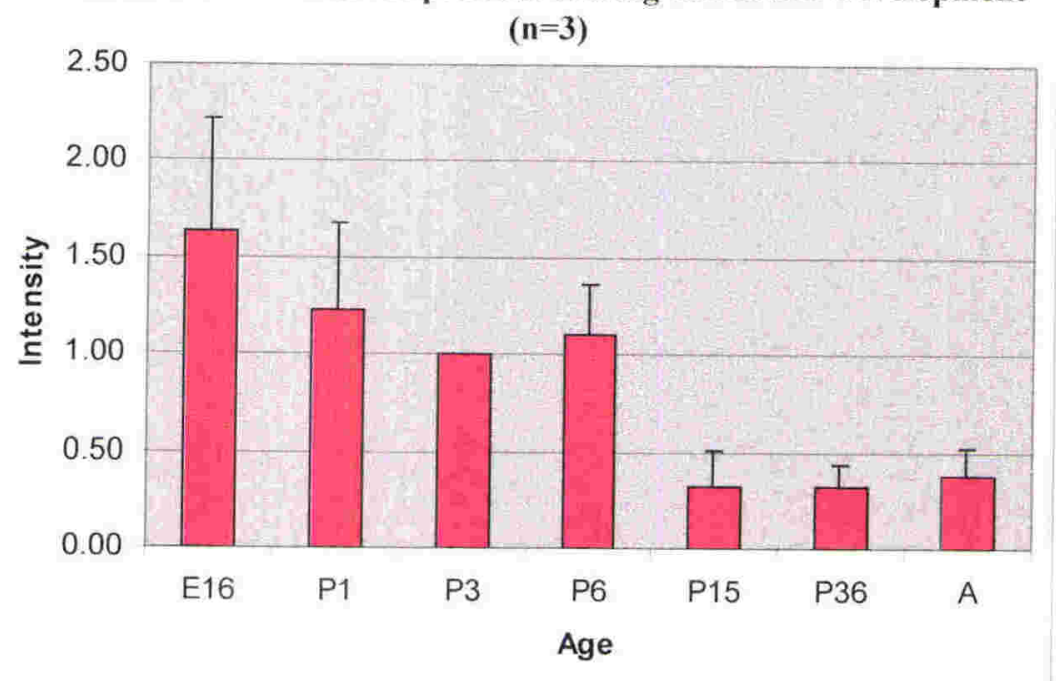

\section{Figure 6.9}

\section{Combined Sample Data of DOR Expression During Development}

DOR Western blot staining intensity pattern during development, using pooled results from 3 separate preparations for the (A) $30 \mathrm{kD}$ band and the (B) $60 \mathrm{kD}$ band. Results are presented as intensity + SEM. (C) Total DOR protein expression levels in the rat brainstem during development showing higher DOR protein expression in the late foetal and early postnatal developmental periods. This figure represents the sum of the $30 \mathrm{kD}$ and $60 \mathrm{kD}$ immunoreactive bands + SEM $(n=3$ separate brainstem preparations with individual preparation values averaged from duplicate gels). 


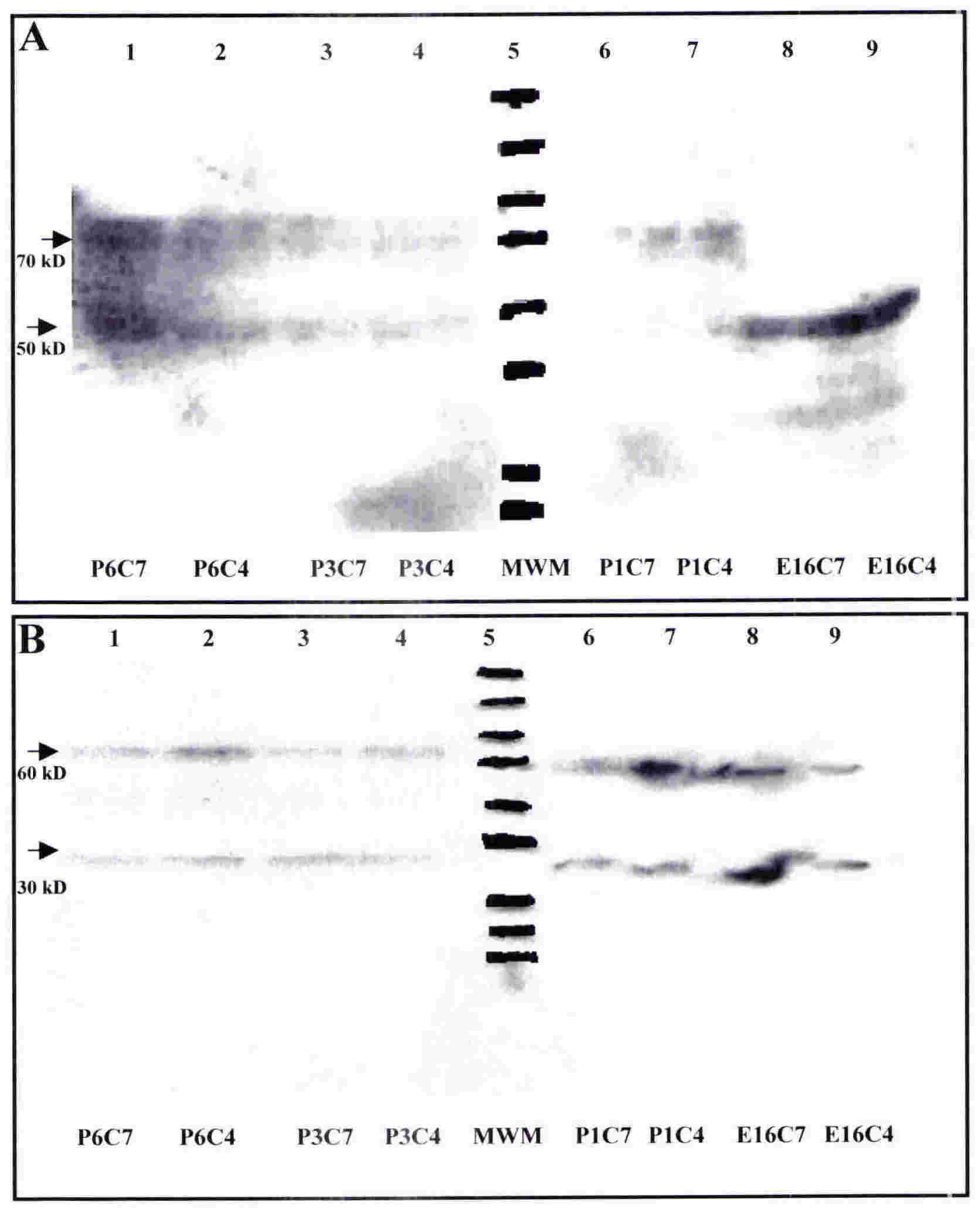

Figure 6.10

\section{MOR and DOR Expression in Cells Cultured from the Rat Brainstem}

(A) MOR (guinea pig anti-MOR, Chemicon) and (B) DOR (rabbit anti-DOR, Chemicon) protein expression in rat brainstem cells cultured from P6, P3, P1 and E16 developmental ages, after 4 (C4) and 7 (C7) days in culture. (A) MOR labelling of E16 cells cultured for 4 and 7 days (lane 9 and 8 respectively) shows a $50 \mathrm{kD}$ band, whereas postnatal cultures show both $50 \mathrm{kD}$ and $70 \mathrm{kD}$ bands, similar to the band staining seen in tissue lysates (Figure 6.5). Staining intensity is highest in E16 and P6 cultures. (B) DOR- labelled (rabbit anti-DOR, Chemicon) bands from cell cultures at all developmental ages show two immunoreactive bands at $30 \mathrm{kD}$ and $60 \mathrm{kD}$. Note the higher intensity of band staining in the cultures isolated from younger brainstems, similar to the intensity seen in brain tissue lysates (Figure 6.8). There are no changes in band sizes or staining intensities of bands between 4 and 7 days in culture in any MOR or DOR labelled samples. ( $40 \mu \mathrm{g}$ of protein per lane). 


\subsubsection{MOR AND DOR DISTRIBUTION DURING DEVELOPMENT OF RAT BRAINSTEM IN VIVO}

The regions of adult brainstem coronal sections taken for immunostaining are shown in Figure 6.11. Staining patterns were very similar for both MOR and DOR (Figure 6.12). The regions showing the most intense staining for MOR and DOR included the nucleus of the solitary tract (NTS), nucleus ambiguus (NA), inferior olive (IO), the spinal trigeminal nuclei and tracts (STT), area postrema (AP), hypoglossal nuclei (10), and the dorsal motor nucleus of the vagus (12). Other areas that also showed staining included the raphe nuclei and VRG surrounding the NA. High power images show staining of these regions in more detail (Figure 6.13).

\section{Figure 6.11}

Regions of brainstem used for immunolabelling of MOR and DOR in Figure 6.12

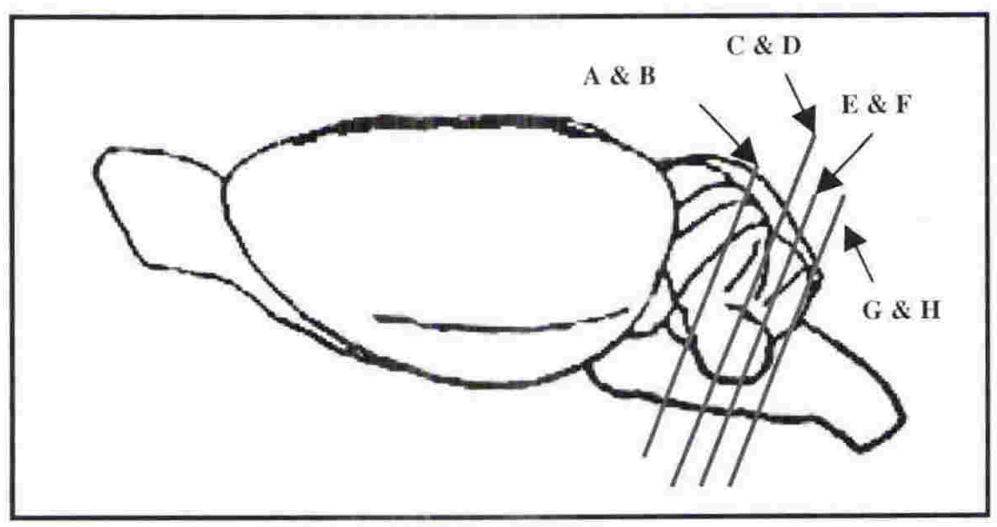


Figure 6.12 DOR and MOR Distribution in the Adult Rat Brainstem 


\section{Figure 6.12}

\section{Distribution of DOR and MOR immunoreactivity in the Adult Rat Brainstem}

Distribution of DOR (A, C, E, \& G) (rabbit anti-DOR, Chemicon) and MOR (B, D, $\mathrm{F}, \& \mathrm{H}$ ) (guinea pig anti-MOR, Chemicon) immunoreactivity in matched sections of the adult rat brainstem. Specific brainstem regions and nuclei are highlighted in the MOR sections. Note the identical staining patterns for both MOR and DOR in the NTS, NA, IO and spinal STT. Scale bar $=3.5 \mathrm{~mm}$. Abbreviations: nucleus of the solitary tract (NTS); nucleus ambiguus (NA); inferior olive (IO); spinal trigeminal tract and nucleus (STT); medial longitudinal fasciculus (mlf); rostro ventrolateral reticular nucleus (RVL); gracile nucleus (Gr); lateral reticular nucleus (LR);

hypoglossal nucleus $(12)=$ dorsal motor nucleus of the vagus (10); external cuneate nucleus (ECu); medial vestibular nucleus (MVe); raphe nuclei (R); area postrema (AP); linear nucleus ( $\mathrm{Li}$ ). 


\section{ADULT BRAINSTEM}

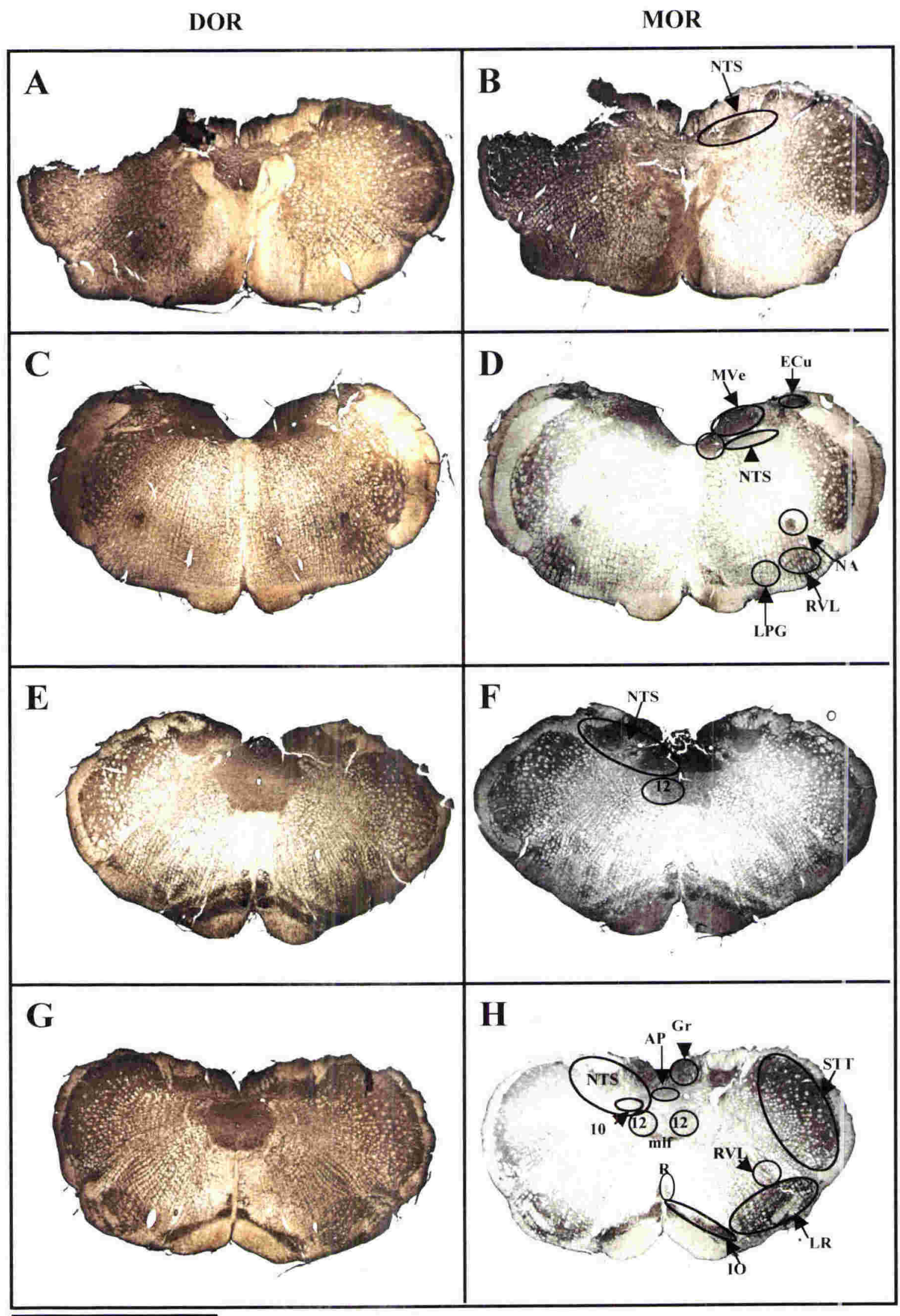




\section{ADULT BRAINSTEM \\ DOR \\ MOR}
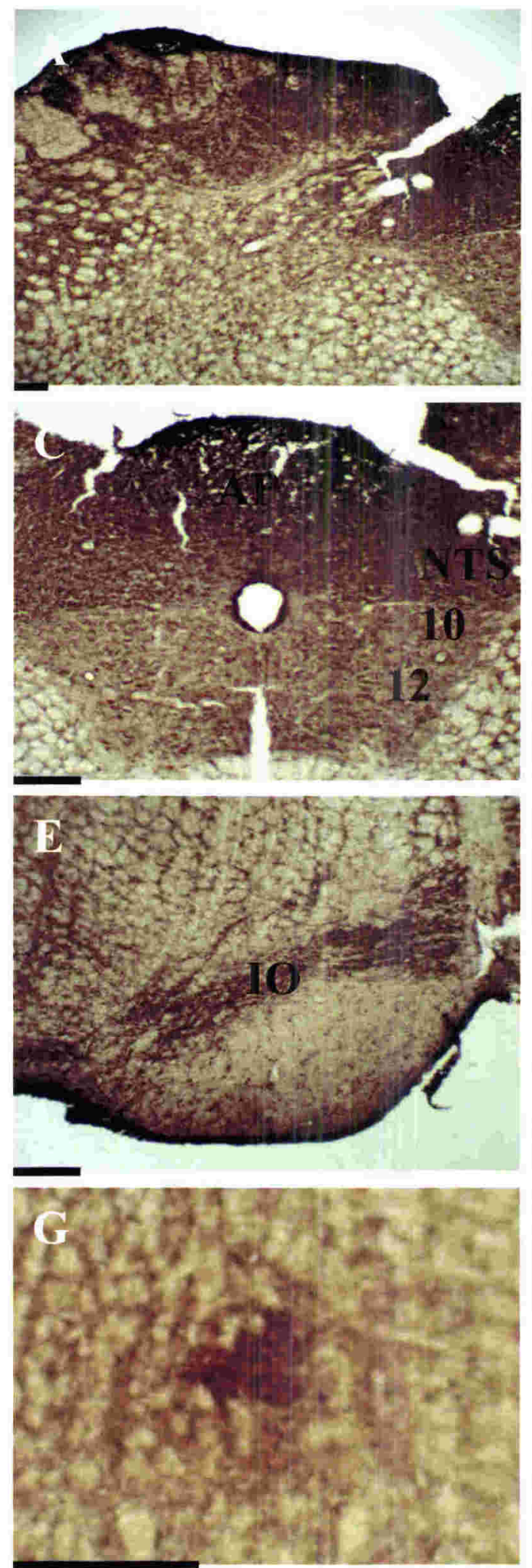
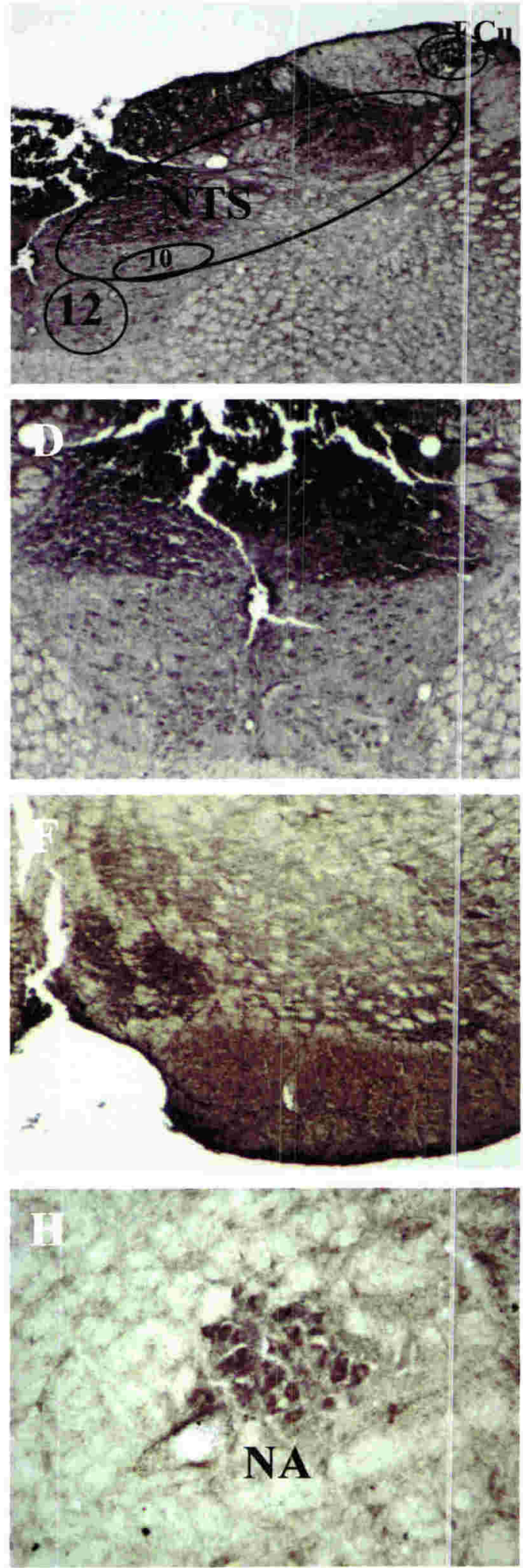

Fig 6.13

High Power Images of Adult DOR and MOR Immunoreactivity in the Brainstem

High-power images of DOR ( A, C, E, \& G) (rabbit anti-DOR, Chemicon) and MOR (B, D, F, \& H) (guinea pig anti-MOR, Chemicon) immunoreactive regions of the brainstem including the infer or olive (IO), nucleus ambiguus (NA), nucleus of the solitary tract (NTS), area postrema (AP), dorsalmotor nucleus of the vagus (10), and hypoglossal nucleus (12). Scale bar $=100 \mu \mathrm{m}$. 
In P6 rat pups, the distributions of MOR and DOR in brainstem areas (Figure 6.14) were very similar to the expression patterns seen in the adult (Figure 6.12). Intense staining was seen in the NA, IO, STT, and the NTS. High power images of these areas (Figure 6.15) show this staining in more detail.

In E16 foetal animals, MOR and DOR labelling gave a much more diffuse staining patern compared with P6 or adult animals (Figure 6.16). Staining could be seen in the dorsal motor nucleus of the vagus (10) and hypoglossal nucleus (12), as well as the areas of the NTS, STT, and NA. Higher power images show clearer staining of these regions, particularly the MOR and DOR staining seen in the NA (Figure 6.17). 
POSTNATAL BRAINSTEM (P6)

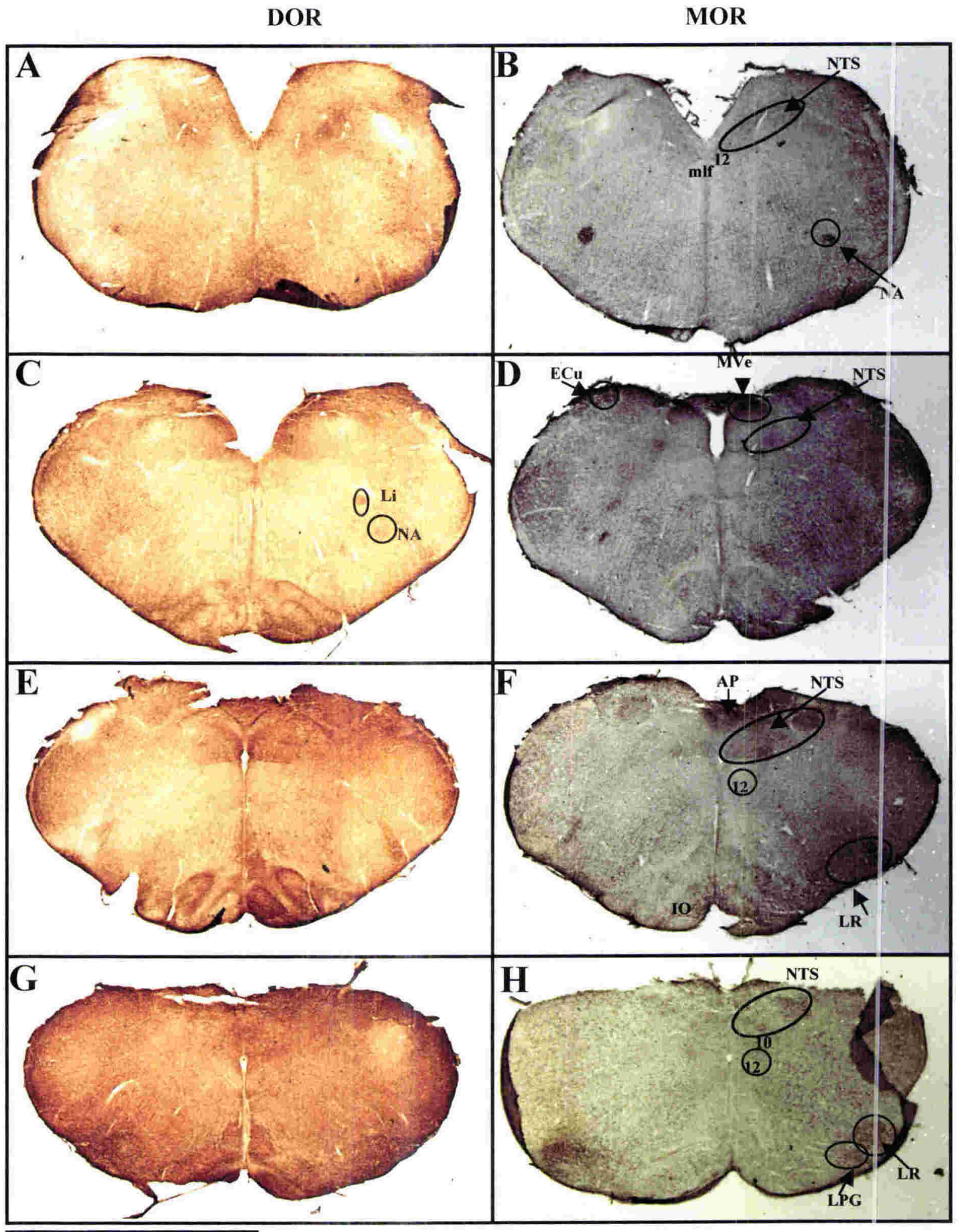

Figure 6.14

DOR and MOR Labelling in the Postnatal Rat Brainstem

DOR (A, C, E, G) (rabbit anti-DOR, Chemicon) and MOR (B, D, F, H) (guinea pig anti-MOR, Chemicon) labelled P6 rat brainstem sections showing staining of the NTS, NA, IO, mlf, and STT. The regions taken for sectioning are similar to those shown for the adult (Figure 6.12) and are displayed from rostral (A \& B) to caudal $(\mathrm{G} \& \mathrm{H})$. Scale bar $=2.5 \mathrm{~mm}$. 


\section{POSTNATAL BRAINSTEM (P6)}

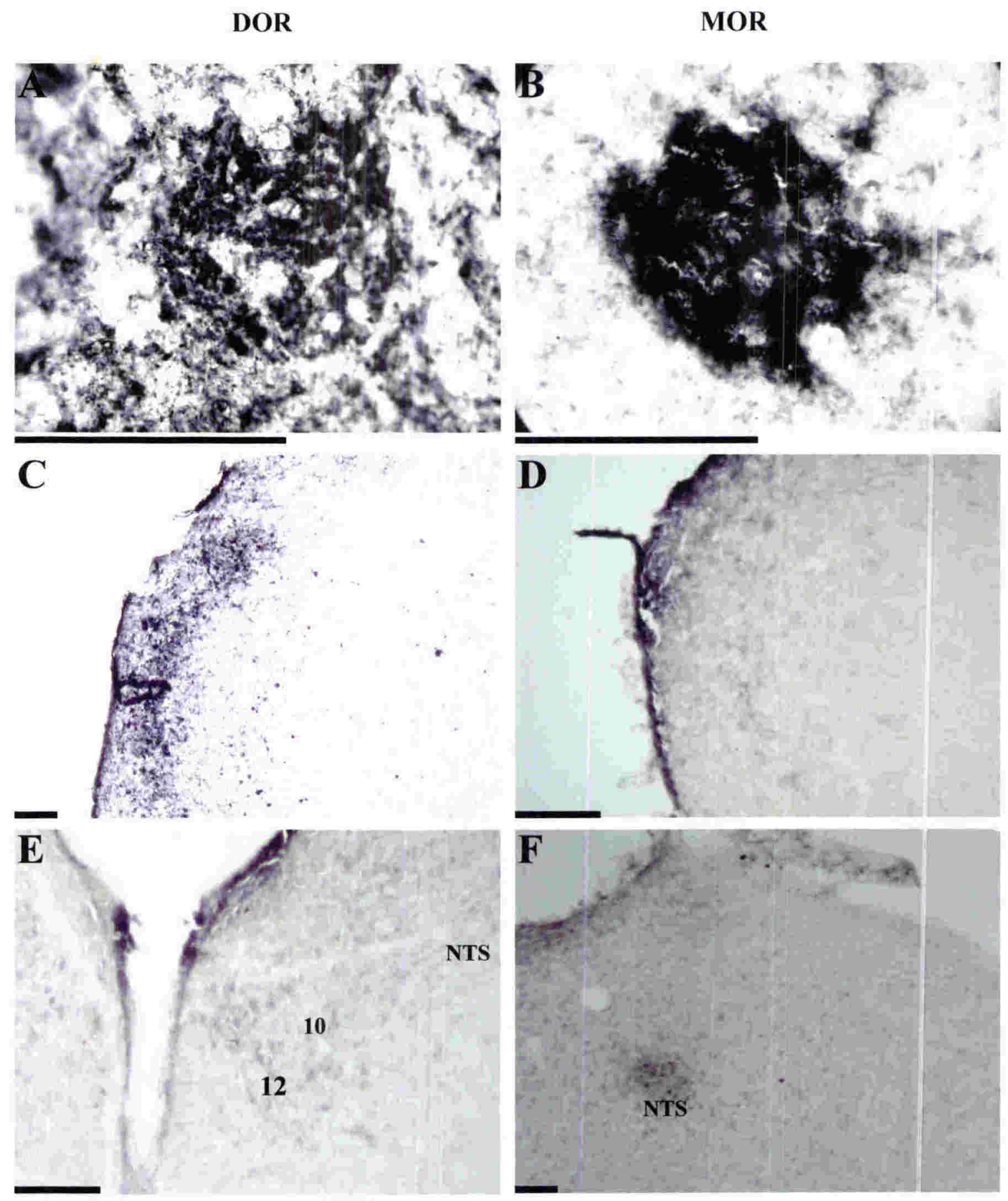

Figure 6.15

High Power Images of Postnatal DOR and MOR labelled Brainstem Regions

High power magnification of DOR (A, C \& E) (rabbit anti-DOR, Chemicon) and MOR (B, D, \& F) (guinea pig anti-MOR, Chemicon) labelling of P6 rat brainstem showing staining of the NA ( $\& \& B$ ), STT (C \& D), nucleus 10, 12, and the NTS (E \& F). Scale bar $=100 \mu \mathrm{m}$. 


\section{FOETAL BRAINSTEM (E16)}

DOR MOR

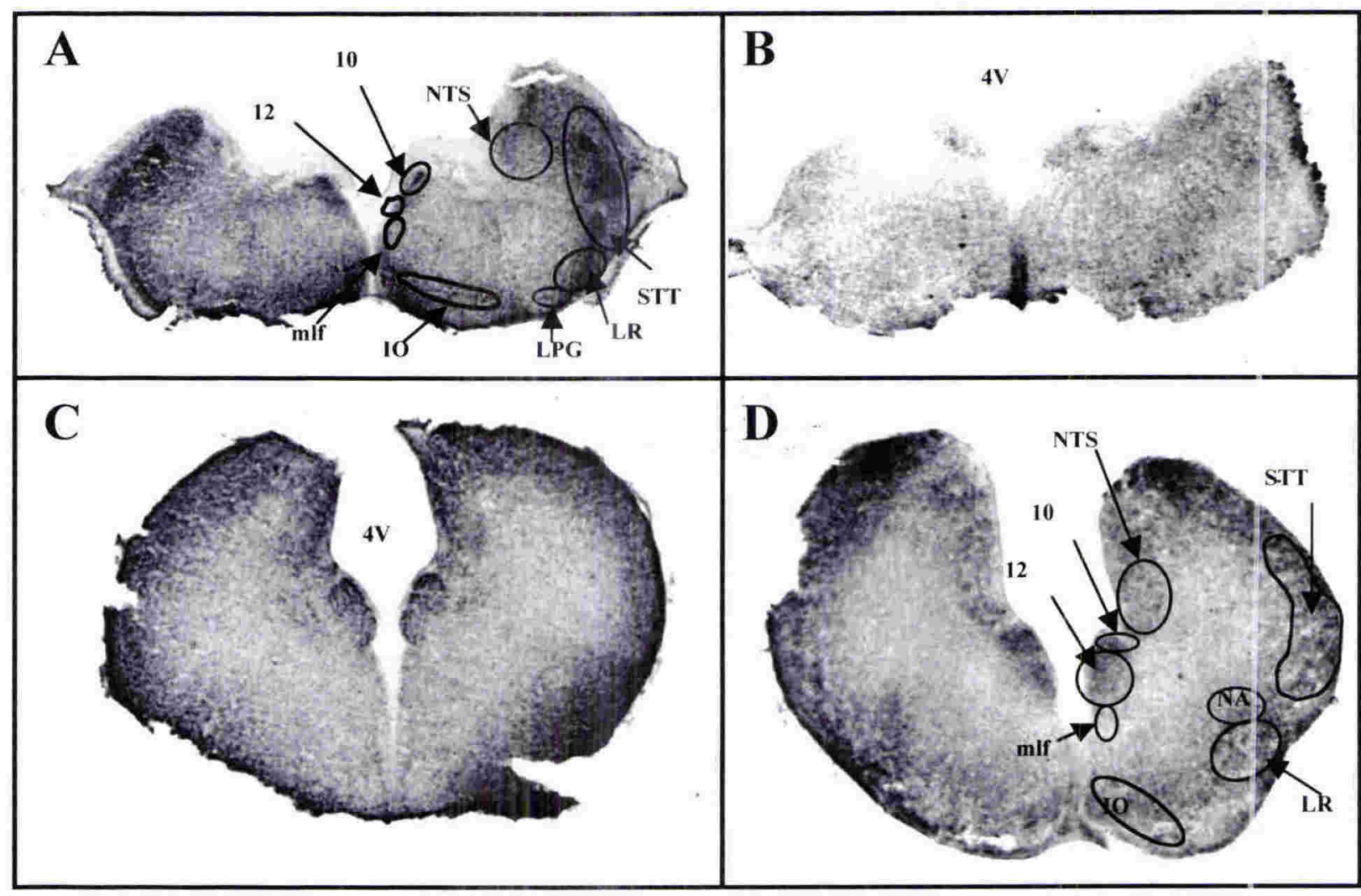

Figure 6.16

DOR and MOR Immunoreactivity in the Foetal Brainstem

Diffuse DOR (A \& C) (rabbit anti-DOR, Chemicon) and MOR (B \& D) (guinea pig anti-MOR, Chemicon) immunoreactivity in the nucleus of the solitary tract (NTS), nucleus ambiguus (NA), inferior olive (IO), spinal trigeminal trigeminal tract (STT), medial longitudinal fasciculus (mlf), dorsalmotor nucleus of the vagus (10), and hypoglossal nucleus (12). Sections are presented in rostral (A \& B) and caudal (C \& D) brainstem regions. Scale bar $=1 \mathrm{~mm}$ 


\section{FOETAL BRAINSTEM (E16)}

DOR
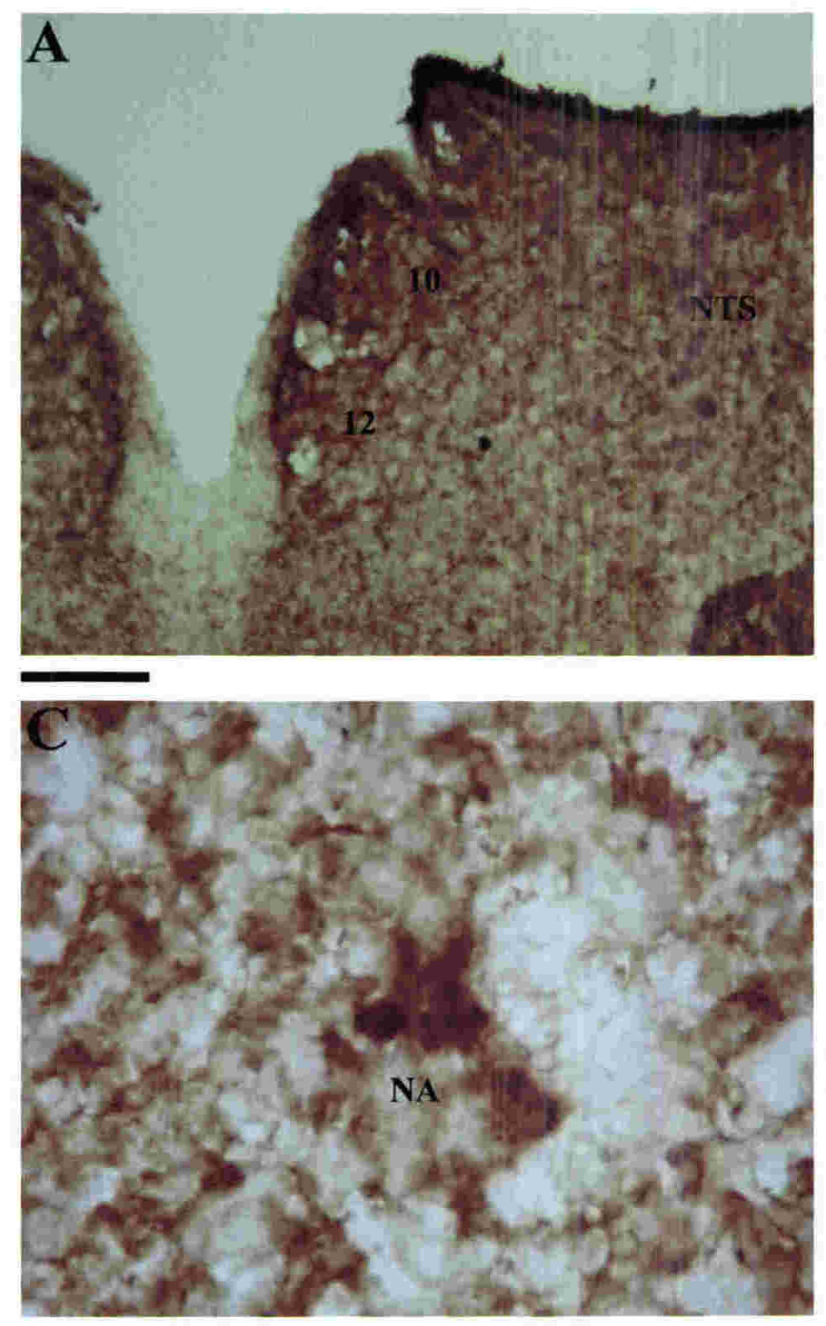

MOR
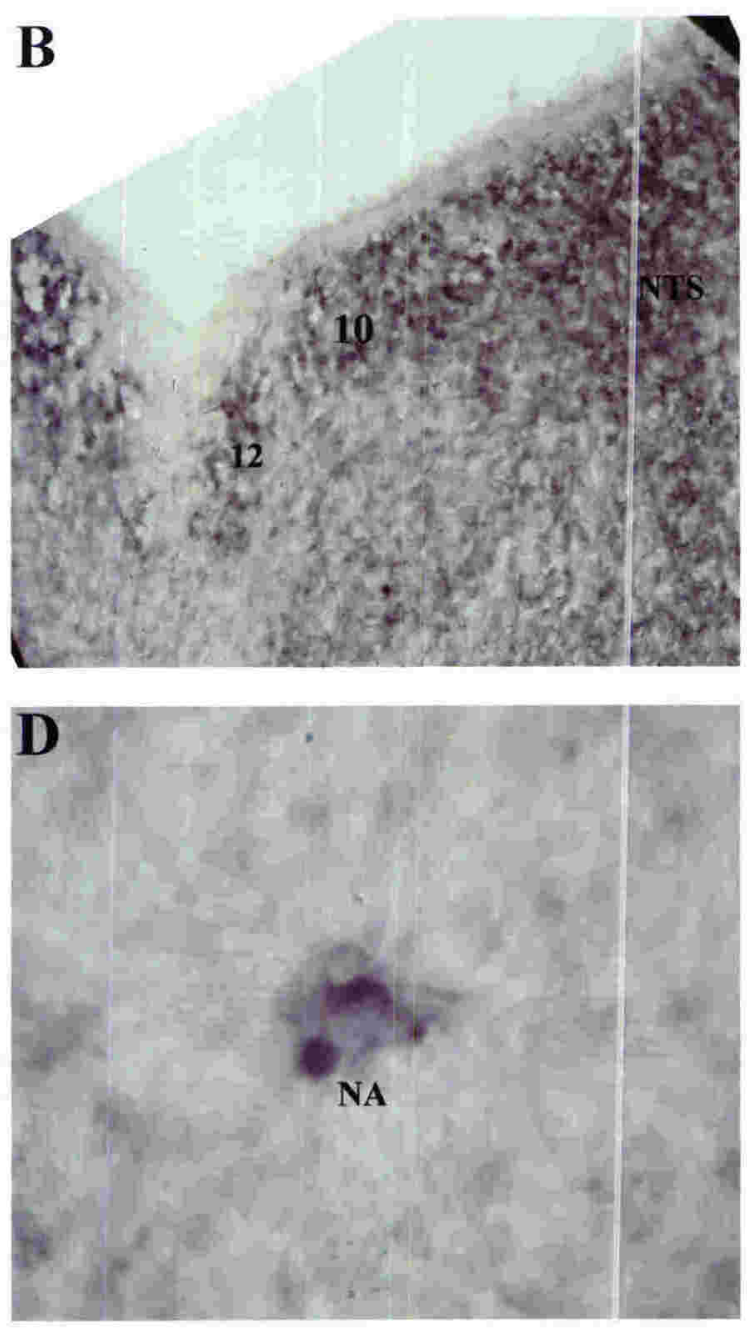

Figure 6.17

\section{High Power Images of of DOR and MOR Labelled Brainstem Regions}

High power pictures of E16 brainstem stained for DOR (A \& C) (rabbit anti-DOR, Chemicon) and MOR (B \& D) (guinea pig anti-MOR, Chemicon). Staining is seen in the region of the nucleus of the solitary tract (NTS) (A \& B), dorsalmotor nucleus of the vagus (10), hypoglossal nucleus (12), and nucleus ambiguus (NA) (C \& D). Scale bars $=100 \mu \mathrm{m}$. 


\subsection{DISCUSSION}

\subsubsection{METHODOLOGY}

\subsubsection{Western Blotting}

Both MOR and DOR antibody staining were shown to be quantitative on Western-transferred whole brain, brainstem, and spinal cord lysates. Increased protein loading on the gel caused the predicted increase in band intensity. Negative controls included the use of LLC-PK1 cell lysates, which showed no immunoreactivity for either MOR or DOR antibodies, as expected. Positive controls included SH-SY5Y cell lysates, which showed immunostained protein bands of the expected sizes for MOR and DOR that were identical in size to those seen in whole brain and brainstem lysates. From these control experiments, it is reasonable to conclude that MOR- and DOR-specific antibodies were binding to their respective receptors and not binding non-specifically to other proteins.

Several lysis buffer solutions were used in preliminary studies. The lysis buffer (Western Sample Buffer) described in the Methods (Section: 6.3.1.1) was chosen because the buffer was highly reducing and helped prevent the action of proteases from degrading proteins within the samples. This enabled the samples to be stored at $-20^{\circ} \mathrm{C}$ for several months and allowed samples of different developmental ages to be collected and electrophoresed on the same SDSPAGE gel. Brainstem lysates had a higher concentration of proteins $(1 \mu \mathrm{g} / \mu \mathrm{L}$ to $10 \mu \mathrm{g} / \mu \mathrm{L})$ compared to cultured cell lysates $(0.5 \mu \mathrm{g} / \mu \mathrm{L}$ to $1 \mu \mathrm{g} / \mu \mathrm{L})$. Thus, cultured cell lysates needed proportionally more lysis buffer to lyse the sample. This caused several problems when large volumes of sample were loaded on the gel because salts in the lysis buffer interfered with the running of the SDS-PAGE gel. This was overcome by precipitation of the proteins with acetone and reconstitution in a small volume in order to remove the salts. This concentration and de-salting procedure was carried out immediately prior to electrophoresis of the samples on a gel.

In preliminary experiments, brainstem lysates from different developmental ages were dotblotted onto PVDF membrane at increasing protein concentrations and immunolabelled using antibodies specific for MOR and DOR. Although these experiments provided some general trends, the protein samples tended to wash off the membrane when the membrane was 
rehydrated in methanol. The PVDF membrane also became saturated if too much sample was added, making it difficult to accurately determine increases in OpR expression. Other types of membranes such as pitrocellulose could not he used hecanse of their, namsnecific hindino 


\subsubsection{OPIOID RECEPTOR EXPRESSION DURING BRAINSTEM DEVELOPMENT}

\subsubsection{MOR Expression During Development}

Two MOR antibodies specific for the MOR-1 protein were used in the pilot experiments (guinea pig anti-MOR and rabbit anti-MOR, both from Chemicon). Both gave the same MOR-positive bands at $50 \mathrm{kD}$ and $70 \mathrm{kD}$. Both antibodies also gave the same staining intensity patterns during development, suggesting that the staining patterns reflected true MOR expression in the tissues. The presence of two immunoreactive bands of different MW is not due to non-specific antibody binding. All samples analysed in this chapter were performed using the guinea pig anti-MOR antibody (Chemicon). The presence of two immunoreactive bands for MOR in the present study $(50 \mathrm{kD}$ and $70 \mathrm{kD})$ is consistent with the literature findings. For example, Abbadie et al. (2000), have described two MOR bands at (46 $\mathrm{kD}$ and $66 \mathrm{kD}$ ) (Abbadie et al., 2000a). MOR-1 cDNA predicts a protein with a mass of 45 $\mathrm{kD}$. Other reports in the literature describe a MOR protein band between $67-72 \mathrm{kD}$ (Arvidsson et al., 1995b). Studies by Kaneko et al. (1995) showed MOR proteins bands at 50 kD, $65 \mathrm{kD}$ and $94 \mathrm{kD}$ (Kaneko et al., 1995). Further evidence suggests that the difference in size of MOR protein bands is a result of post-transcriptional modification of the receptor by glycosylation (Eppler et al., 1993; Garzón et al., 1995; Kaneko et al., 1995; Abbadie et al., 2000a). The N-terminal of MOR has 5 potential sites for N-linked glycosylation (Chapter 1: Figure 1.1) (Minami and M., 1995). Some deglycosylation experiments of brainstem lysates were performed using the method of Garzon et al. (1995) who reported deglycosylation of the MOR protein from mouse brain homogenates (Garzón et al., 1995). In the present study, endoglycosydase F enzyme (Sigma) was used to treat SH-SY5Y cell lysates and rat brain homogenates, but deglycosylation was not observed, even when higher concentrations of enzyme were used (data not presented). It is possible that the deglycosylation enzyme used was inactive. These experiments were not pursued further due to the high cost of the enzyme.

In the present study, MOR protein expression during development of rat brainstem showed that there was a progressive decline in the amount of the $50 \mathrm{kD}$ MOR protein with increasing age; whereas, there was a progressive increase in the amount of the $70 \mathrm{kD}$ MOR protein. The functional implications of differences in MOR glycosylation during development remain to be elucidated. It is possible that the different states of MOR glycosylation reflect different 
binding properties for opioid ligands. If this is true, the $\mathrm{OpR}$ glycosylation state may explain differences in binding affinity of opioid ligands to receptors that occurs during development (Spain et al., 1985). These possibilities, along with alterations in OpR expression levels that have been shown to occur during development (Coyle and Pert, 1976; Pasternak et al., 1980; Leslie et al., 1982; Tsang et al., 1982; Wohltmann et al., 1982; Spain et al., 1985; Tavani et al., 1985; Kornblum et al., 1987; Petrillo et al., 1987; Attali et al., 1990; Bem et al., 1991; Xia and Haddad, 1991; Barg et al., 1992; Murphey and Olsen, 1995; Zhu et al., 1998; Tong et al., 2000; Beland and Fitzgerald, 2001) provide possible mechanisms for the differences seen in opioid sensitivities during development (Grunstein and Grunstein, 1982; Moss et al., 1993a; Murphey and Olsen, 1994; Greer et al., 1995; Colman and Miller, 2001). This information will be useful when considering the link between opioids and developmentally-related diseases such as SIDS (Chavez et al., 1979; McMillen, 1986; Coqueral et al., 1992; Storm et al., 1994).

Total MOR protein expression levels increased in the present study approximately 5 -fold with developmental age from E16 to P6. The increase in total MOR expression during development has also been described in the literature, based on binding studies in whole brain and spinal cord lysates (Coyle and Pert, 1976; Pasternak et al., 1980; Leslie et al., 1982; Tsang et al., 1982; Wohltmann et al., 1982; Spain et al., 1985; Tavani et al., 1985; Kornblum et al., 1987; Petrillo et al., 1987; Attali et al., 1990; Kitchen et al., 1990; Bem et al., 1991; Barg et al., 1992), as well as in the brainstem of several species (Xia and Haddad, 1991; Murphey and Olsen, 1995). In guinea pig brainstem, there was a $42 \%$ increase in MOR binding between the P3 and P7 age groups (Murphey and Olsen, 1995); however, results from other species such as guinea pig need to be interpreted with caution. For example, the guinea pig is more developmentally advanced than the rat at birth. In the present study of the rat brainstem, an approximate $30 \%$ increase was seen between P3 and P6, with a 50\% increase between P3 and P15 (Figure 6.6), and these results support the studies in guinea pig brainstem. Other studies have shown that MOR expression increases in different brain regions at different developmental ages. For example, MOR in the brainstem appears at an earlier age than MOR expression in the cortex (Xia and Haddad, 1991). In some brain regions, for example, the olfactory bulb, MOR expression increases during late foetal and early postnatal development, 
and then decreases to adult levels (Kent et al., 1982). In the brainstem, however, MOR expression remains highest in the adult, especially in the brainstem regions involved in cardiorespiratory function (Xia and Haddad, 1991). The present study, is the first to describe developmental $\mathrm{OpR}$ expression in the rat lower brainstem using antibody labelling.

\subsubsection{DOR Expression During Development}

DOR immunoreactive proteins on Western-transferred SDS-PAGE gels consisted of two bands, one at $30 \mathrm{kD}$ and the other at $60 \mathrm{kD}$. This pattern is consistent with the findings of Carr et al. (1987) who showed DOR bands at $58 \mathrm{kD}$ and $30 \mathrm{kD}$ in rat brain lysates (Carr et al., 1987). Other studies have reported a single $29 \mathrm{kD}$ band (Harada et al., 1992). DORtransfected Cos-7 cells gave bands between 110 to $135 \mathrm{kD}$ and between 65 to $80 \mathrm{kD}$ (Cahill et al., 2001b), whereas spinal cord preparations from the rat gave three bands of $65 \mathrm{kD}, 52 \mathrm{kD}$ and $125 \mathrm{kD}$, corresponding to different forms of DOR (Cahill et al., 2001b). The $125 \mathrm{kD}$ DOR band was most likely a result of dimer formation (George et al., 2000; Ramsay et al., 2002) and could be seen in the samples of the present study if the X-ray film was exposed for longer periods. DOR protein sizes differed in various studies depending on the types of cell sample and the methods used, including the lysis buffers, the species, percentage acrylamide, and electrophoretic running conditions. The predicted size of DOR based on its amino acid content is $39 \mathrm{kD}$, although it is known to exist in multiple glycosylation states (Petäjä-Repo et al., 2001). DOR is synthesized as a core-glycosylated $45 \mathrm{kD}$ precursor that is converted to the fully mature $55 \mathrm{kD}$ glycosylated receptor within the golgi apparatus (Petäja-Repo et al., 2000).

The low molecular weight form of DOR reported in the present study (30 kD) is consistent with the findings of Belcheva et al (1996), who reported a $26 \mathrm{kD}$ DOR band that proved to be a truncated, internalised form of DOR associated with the nuclear matrix (Belcheva et al., 1996). The existence of a truncated, internalised DOR may explain why high levels of DOR were found in early development in the present study. Others, however, report little DOR ligand binding during development. Perhaps the differences between these studies can be attributed to the differences between total DOR detection (including functional and nonfunctional receptors) and detection of functional receptors only. The decrease in total DOR protein levels during development in the present study is supported by a study on rat dorsal 
root ganglion (Beland and Fitzgerald, 2001). In this study, greater DOR immunoreactivity was found at P0 than at P21. A down-regulation with age was found mainly in the nonnociceptive neurofilament-200 positive, large diameter sensory neurons. Most other studies using binding in whole brain lysates report an increase of DOR during the late foetal and early postnatal period that rises to maximum levels in the adult (Kent et al., 1982; Petrillo et al., 1987; Attali et al., 1990). Others report little change in expression of DOR during development in hindbrain lysates when DADL, in the presence of DAMGO, was used to specifically label DOR (Spain et al., 1985).

\subsubsection{MOR And DOR Expression In Cells Cultured From The Rat Brainstem During Development}

There were no differences observed in the staining intensity of MOR and DOR protein bands in lysates prepared from cells cultured from rat brainstems with increased time in culture. This suggests that OpR levels are maintained in culture and that the developmental changes that occur in vivo do not occur in vitro. This is probably due to the absence of cell signalling (neurotransmitter or trophic support) that is required for developmentally programmed changes in receptor expression. This property of cell culture suggests that the developmental stage the cells were isolated at is maintained when cells are cultured for up to 7 days. The present study, thus, provides a stable culture system with which to study factors affecting OpR expression. Unfortunately, a large amount of variation was seen between cultures prepared from animals of the same developmental stage in different experiments. This variation made analysis difficult, given the small sample size used in this study $(\mathrm{n}=3$ preparations from each developmental age). Interpretation of the data was also complicated due to the heterogeneity of cells present in primary cultures. For example, neuron survival decreases with increased developmental age from which cultures are established, and certain populations of cells present at E16 may not be present in P6 cultures.

\subsubsection{Comparisons With Literature Findings}

Total OpR binding increases with increasing developmental age (Clendeninn et al., 1976; Coyle and Pert, 1976), and differences between MOR, DOR and KOR expression levels during development have been reported (Leslie et al., 1982; Tsang et al., 1982; Tavani et al., 
1985). These studies looked at whole brain preparations (usually with the cerebellum removed) and often used non-specific, or poorly selective, opioid receptor ligands such as naloxone or enkephalin (Kent et al., 1982). Other studies have shown that binding affinity of the receptor for certain ligands also changes during development. For example, DOR binding by DADL decreases 14 fold from P1 to adult (Spain et al., 1985). For these reasons, the results from these experiments cannot be accurately extrapolated to the present findings for the lower brainstem, using antibodies that specifically label MOR and DOR proteins. Binding studies often use tissue lysates, usually of membrane fractions, that do not take into account the internalised OpRs present in endosomes. In binding studies, only functional protein expression is being assessed, and ligand binding or cAMP responses to agonist may be affected by a number of factors, including post-translational modifications and the state of receptor phosphorylation. These factors play a major role in regulating opioid receptor activity (Law et al., 1983; Haynes, 1988; Kieffer et al., 1992; Chan et al., 1995; MonteilletAgius et al., 1996; Lefkowitz, 1998; Law and Loh, 1999; Liu et al., 1999; Belcheva et al., 2000; Kraus et al., 2001; Tsao et al., 2001; Sun, 2002). Ligand binding studies during development were also investigated before different splice-variants of the opioid receptors were discovered. In the present study, the MOR-1 receptor was specifically labelled by an antibody; whereas, the studies described in the literature looked at binding to all MOR proteins, including the splice variants that have the same binding site as MOR-1. In addition, differences have been shown to occur in the binding studies described, depending on whether binding is expressed as a measure of binding per brain, or per mg of protein. When results are expressed as a measure of total protein, the differences that occur during development are much less obvious (Barg et al., 1992). This is probably due to changes in protein concentration during development and changes in functional OpR densities at the cell surface. A study by Petrillo et al. (1987) showed that there was an increase in protein concentration in the brain from $25 \mathrm{mg} / \mathrm{g}$ brain at P3 to $56 \mathrm{mg} / \mathrm{g}$ brain at P21 (Petrillo et al., 1987). These findings complicate data interpretation because a decrease in specific content $(\mathrm{OpR} / \mathrm{mg}$ protein) may not reflect a decrease in the total amount of OpR present (OpR/g brain). Comparisons between different studies that present their data in different ways must be taken into account. 


\subsubsection{Implications of Developmental Changes in Opioid Receptor Expression}

Differences in opioid receptor levels during development have implications for the use of opioids as labour analgesics during childbirth (Mardirosoff et al., 2002) and for analgesia in newborns. In the rat spinal cord, binding studies have shown that there is little DOR binding at birth and that DOR analgesia is not functional at birth (Rahman et al., 1998). Changes in the distribution of opioid receptors during early postnatal development have implications for respiration control. Timing of developmentally-related $\mathrm{OpR}$ expression may play a role in sudden infant death syndrome, since abnormal opioid function has been implicated in this disease (Chavez et al., 1979; McMillen, 1986; Storm et al., 1994). The role of opioids in SIDS has been described in detail in Chapter 1(Section 1.4.1.1).

\subsubsection{MOR AND DOR DISTRIBUTION IN THE ADULT RAT BRAIN}

Earlier studies into opioid receptor distributions in the late 1970's and 1980's used ligandbinding techniques, which have several limitations, including the non-specificity of the ligands and poor sensitivity. Mansour et al. (1988) used autoradiographic ligand binding techniques to compare the distribution of MOR and DOR in the rat. Results of their study showed no DOR binding in the STT or lateral reticular nucleus (LR), areas shown by others to have DOR (Arvidsson et al., 1995a; Cahill et al., 2001b). Mansour et al. (1988) also showed low DOR staining levels in the NTS (Mansour et al., 1988), an area that has high levels of MOR and DOR binding (Delay-Goyet, 1990; Arvidsson et al., 1995a). These differences have been attributed to differences in sensitivity between the methods

A breakthrough in opioid receptor distribution in the brain came in 1993 with the cloning of the OpR. It then became possible to make specific antibodies to each receptor and to look at the mRNA expression patterns by ISH. A review in 1995 on OpR expression in the rat using mRNA probes showed that DOR mRNA was present in the STT, LR and NA but was absent in the NTS, IO, and hypoglossal nuclei (10) (Mansour et al., 1994). These results differ from the present study and the study of Arvidson et al. (1995) who found that the DOR was present in the IO and NTS (Arvidsson et al., 1995a). Most ISH studies use radioactive mRNA probes that do not label axons and dendrites where the opioid receptor proteins reside; therefore, their distribution patterns are expected to be slightly different from protein localisation studies. The 
discovery of multiple isoforms of MOR has also made it possible to subdivide MOR expression into unique isoform distributions within the brain (Schulz et al., 1998; Pan et al., 1999; Abbadie et al., 2000b; Abbadie et al., 2000a). For example, MOR-1N, M, and L are only present in the spinal cord, but not other brain regions. MOR-1 is present in the mouse brainstem; however, the splice-variant isoforms MOR-1-G, H, I, J, K, L, M, and N were not (Pan et al., 1999). MOR-1 and MOR-1C were present in the same brainstem regions in the rat, but MOR-1C was absent in several brain regions, including most thalamic nuclei and the dendate gyrus, areas expressing high levels of MOR-1 (Abbadie et al., 2000a). Because ligands and antibodies may bind to several splice variants of MOR, the significance of the distributions described is not clear.

\subsubsection{DOR Distribution in the Adult Rat Brainstem}

DOR distribution patterns in lower brainstem nuclei have been determined by ICC by Arvidsson et al. (1995), who reported staining in the locus coeruleus, NA, NTS, pontine reticular nucleus, gigantocellular nucleus, facial motor nucleus, raphe nuclei, STT, hypoglossal nuclei (12), vagal nuclei (10), IO, and the medial and lateral reticular nuclei (Arvidsson et al., 1995a). Another study showed DOR localised to the brainstem regions of the spinal trigeminal nucleus (STT), the reticular nuclei (LR), RVLM, and olivary nuclei (Cahill et al., 2001b); however, the lower brainstem areas, including the NTS and NA, were not mentioned in this study. The staining of these other brainstem regions is consistent with the staining patterns described in the present study.

\subsubsection{MOR Distribution in the Adult Rat Brainstem}

The MOR distribution found in the adult rat brain in the present study closely resembles the distribution pattern reported in the literature using MOR-1 antibodies (Ding et al., 1996b) and includes positive MOR immunoreactivity in the NA, NTS, IO, and STT of the brainstem. Studies using mRNA probes in the rat brain have found some differences in MOR distribution in the rat brain, including an absence of signal in the IO, area postrema, and external cuneate nucleus (Delfs et al., 1994), regions that were positively labelled for MOR protein. The external cuneate nucleus also stained positive for MOR-1 receptor in an IHC study by Abbadie et al. (2000) (Abbadie et al., 2000b). All other regions labelled by mRNA probes were also 
stained in the present study. As previously stated, differences in staining that were observed were probably due to differences in sensitivity between the techniques, as well as differences between mRNA and protein distributions at the cellular level.

\subsubsection{DEVELOPMENTAL DISTRIBUTION OF MOR AND DOR RECEPTORS IN THE RAT BRAINSTEM}

Few differences were seen between MOR and DOR staining in the rat brainstem between late foetal (E16), postnatal (P6), and adult. In the adult brainstem there was intense staining in the dorsal respiratory areas of the NTS, and the ventral respiratory areas in and around the NA, as well as the hypoglossal nuclei (10), the dorsal motor nucleus of the vagus (12), IO, and STT. These same areas were also stained in the P6 rat brainstem. The brainstem regions of P6 rats were anatomically similar to the adult rat, although proportionally smaller; however, in foetal (E16) brainstem, the nuclei were not fully developed, and staining was much more diffuse. This may be due to the immaturity of the boundaries between brainstem nuclei at this early developmental age (Lidov and Molliver, 1982). The brainstem nuclei of interest (NA, NTS, STT, and nuclei 10 and 12) were proportionally larger in the foetal (E16) brainstem than the older postnatal and adult brainstems (Paxinos and Watson, 1986; Paxinos et al., 1994). Similar MOR protein patterns were found in the present study to mRNA distributions during development reported in other brain areas. For example, in foetal (E17.5) mouse embryos, the MOR expression pattern was characteristic of the adult brain, although the pattern was more homogeneous and the nuclei were less discretely labelled. In the striatum, the characteristic patch-like patterns seen in the adult appeared diffuse in the foetus (Zhu et al., 1998). This difference was attributed to the absence of cellular differentiation within the striatum at this early stage of development.

Few studies have looked at or commented on the intracellular distribution of opioid receptors during development (Kornblum et al., 1987; Zhu et al., 1998; Tong et al., 2000), and only one study investigated the lower brainstem region in the rat (Xia and Haddad, 1991). Some developmental studies on opioid receptor distributions in the brainstem have been carried out on other species (Murphey and Olsen, 1995; Zhu et al., 1998; Moss and Laferrière, 2002). For example, in the piglet, the distribution patterns are linked to acquisition of respiratory function 
during development, including the attenuated response to hypoxia caused by an increase in MOR levels in respiratory-related brainstem areas during development (Moss and Laferrière, 2002). In the present study MOR and DOR immunoreactivities were usually colocalised within the same brainstem nuclei at all stages of development, although the relative density of staining could not be compared in tissue sections when different antibodies were used. In some individual sections, if signal amplification was low, some poorly expressing MOR and DOR nuclei were not always co-labelled. Others have also reported colocalisation for MOR and DOR in the adult rat brainstem (Goodman et al., 1980).

\subsection{SUMMARY AND CONCLUSIONS}

The expression levels of MOR and DOR change during development. MOR is expressed at low levels during foetal (E16) development and progressively increases during postnatal development to reach adult expression levels. These findings are supported by the findings of others (Zhu et al., 1998; Laferrière et al., 1999). DOR is expressed at high levels in early development (E16) and progressively decreases during postnatal development to reach adult levels. In previous studies of whole brain homogenates from the rat (Spain et al., 1985; Petrillo et al., 1987) and mouse (Tavani et al., 1985) DOR was found to increase, although another study on the brainstem of piglets showed that DOR levels did not change in postnatal development (P2 to P21) (Laferrière et al., 1999). Both MOR and DOR are colocalised in specific brainstem regions during embryonic, postnatal, and adult development. The areas of highest expression were in the NTS, NA, STT, and IO, a distribution pattern consistent with FISH experiments on mRNA expression (Eli Mrkusich and Dr Darren Day, personal communication).

The presence of two immunoreactive bands for MOR and DOR is consistent with what others have found for MOR (Abbadie et al., 2000a) and DOR (Carr et al., 1987). The difference in size of the bands is probably a result of receptor glycosylation and, in some cases, homodimer (or heterodimer) formation. The present study is the first to investigate developmental changes in expression levels of these individual protein bands. The results suggest that MOR and DOR are colocalised to the same brainstem regions but are regulated differently during development. 


\section{Chapter 7}

\section{Discussion}

\subsection{General Discussion and Conclusions}

One aim of the present study was to develop a primary culture system in which opioid receptor expression could be monitored in isolated cells from late foetal and early postnatal animals. It is well known that opioids play an important role in many physiological processes within the body (Olson et al., 1997; Vaccarino et al., 1999; Vaccarino and Kastin, 2001). Opioid receptors are particularly important in the brainstem where they are involved in the control of pain (Fields and Heinricher, 1985; Gutstein et al., 1998; Commons et al., 2001) and in the modulation of respiratory activity (Pfeiffer et al., 1984; Punnen and Sapuru, 1986; Yeadon and Kitchen, 1989; Shook et al., 1990; Pokela, 1993; Rutherfurd and Gundlach, 1993; Kato, 1998; Colman and Miller, 2001; Morin-Surun et al., 2001; Sarton et al., 2001) and cardiovascular function (Hassen et al., 1982; Pfeiffer et al., 1984; Punnen and Sapuru, 1986; Schultz and Gross, 2001). Previous studies have reported high densities of OpRs in the brainstem regions of the nucleus of the solitary tract (NTS), nucleus ambiguus (NA), spinal trigeminal nuclei and tract (STT), dorsal motor nuclei of the vagus, and hypoglossal nuclei (Mansour et al., 1988; Delfs et al., 1994; Mansour et al., 1994; Arvidsson et al., 1995a; Bausch et al., 1995a; Ding et al., 1996b; Moriwaki et al., 1996; Díaz et al., 2000). These staining patterns were confirmed in the present study. Each different study, however, has presented slightly different levels of expression, primarily due to differences in sensitivity between experimental techniques. The present investigation, using highly specific MOR and DOR antibodies and a TSA amplification method, is the first to show MOR and DOR distributions specifically in the rat brainstem during development. It is also the first study to show DOR immunoreactivity in the rat foetus. One interesting result was that MOR and DOR during late foetal (E16), postnatal (P6), and adult development are generally colocalised within the same brainstem nuclei. Most previous studies have looked at OpR expression within the whole brain, often using non-selective ligands in receptor binding assays (Coyle and Pert, 1976; Pasternak et al., 1980; Leslie et al., 1982; Tsang et al., 1982; Wohltmann et al., 1982; Spain et al., 1985; Kornblum et al., 1987; Petrillo et al., 1987; Attali et al., 1990; Kitchen et al., 1990; Bem et al., 1991). The expression of OpRs within the brain is developmentally regulated as shown by the results of this study and also the work of others. Both MOR and DOR expression 
levels change during development in a consistent way (Liu et al., 1999; Beland and Fitzgerald, 2001; Ko et al., 2002). The role opioids play during development, however, is not clearly understood. A primary culture method developed in the present study allows brainstem cells to be investigated later in postnatal developmental than has previously been possible. This method uses a defined, serum-free culture medium that would be ideal for studying the effects of growth factors on neuronal development and differentiation (Kivell et al., 2000, 2001). OpR expression was determined in cells cultured from different developmental ages, and expression levels in culture were compared to normal development in vivo. MOR, in primary culture, down-regulates from the membrane to lysosomes on exposure to DAMGO, a MOR agonist. This down-regulation occurs in a similar way to cloned MOR when it is expressed in cell lines, suggesting that cultured neurons from the rat brainstem retain OpR functions. Thus, the culture system provides a valuable in vitro model for determining brainstem OpR function.

Detailed aspects of this study have been discussed previously in each chapter. This chapter aims to explore some of the remaining issues that were not discussed earlier, and to draw some conclusions based on the overall results of the study.

\subsubsection{PRIMARY CELL CULTURE}

OpR expression was investigated using a unique serum-free primary culture method that allows brainstem cells to be cultured from late foetal to early postnatal development, up to six days postpartum (Kivell et al., 2000, 2001). The types of cells present in culture were monitored over time and in cultures from different developmental ages and the cell types at each developmental age compared. More bipolar neurons were present in developmentally younger cultures; whereas, older postnatal cultures had proportionally more multipolar neurons (Chapter 2 Section 2.4.4). Cultured cells seemed to retain their differentiated characteristics over time in culture. For example, the proportion of glial cells, bipolar, and multipolar neurons was the same between 4, 7, and 9 days in culture for each developmental age. Lysates from cells cultured from the rat brainstem also showed the same amounts of MOR and DOR protein at 4 and 7 days in culture from each developmental age tested (Chapter 6: Section 6.4.4). MOR and DOR expression in culture was also measured by immunocytochemistry. No changes in intensity of staining were seen when foetal (E16) and postnatal (P6) brainstems were cultured for 4, 7, and 12 days. 
This finding suggests that brainstem cells in culture remain in the developmental state in which they were isolated. No up- or down-regulation of OpR expression was seen with time in culture. One advantage of this stability of the culture system would be to allow more flexible experimental timing in which to study $\mathrm{OpR}$ regulation. If expression rapidly changed with time in culture, experiments would need to be carried out immediately after isolation and attachment of the cells.

Cell culture provides a simplified system for studying cellular functions. OpR regulation has been extensively examined in neural cell lines and in cells transfected with the OpR (Prathert et al., 1994; Capeyrou et al., 1997; Gaudriault et al., 1997; Gies et al., 1997; Yabaluri and Medzihradsky, 1997; Koch et al., 1998; Kramer and Simon, 1999; Arttamangkul et al., 2000; Remmers et al., 2000; Tsao and von Zastro, 2000; Gray et al., 2001; Levac et al., 2001; Shapira et al., 2001; Jenab and Inturrisi, 2002; Wallington et al., 2002). OpR regulation, however, has not been studied during brainstem development. The present study shows for the first time in cultured brainstem cells internalisation of MOR on exposure to the OpR agonist DAMGO. This result indicates that the process of down-regulation occurs in the same way as seen in transfected cells and non-transfected SH-SY5Y cells, with rapid (within $1 \mathrm{hr}$ ) internalisation of receptors. It remains to be determined, however, if the same pathways of internalisation are occurring in these different cellular systems. It is possible that OpR regulation may be modified by the developmental expression of other proteins involved in down-regulation, for example $\beta$ arrestin and various isoforms of G-proteins and adenylyl cyclase. Immunocytochemistry and Western blotting of cell lysates with antibodies against regulatory proteins could be used if this proves to be the case. In vitro culture also provides a method for examining the interaction between neurons and glial cells and the effects of their interactions on OpR expression.

In some brain regions MOR is reported to be colocalised with $\mathrm{K}^{+}$channels (Bausch et al., 1995b) where it acts on postsynaptic sites to inhibit neuronal firing by opening the $\mathrm{K}^{+}$ channels; however, the brainstem was not reported on in these studies. Primary cultures of brainstem neurons could be doubled-labelled with an antibody specific for the $\mathrm{K}^{+}$channel to help determine if OpRs and $\mathrm{K}^{+}$channels are colocalised within the brainstem. If this is the case, properties of $\mathrm{OpR}$ inhibition of neuronal firing could be monitored. Membrane 
potentials with and without opioids, changes in potassium permeability, and $\mathrm{K}^{+}$-channel blockers could be used to study OpR regulation specifically in cultured brainstem neurons.

MOR is also colocalised with glutamate receptors in the NTS where it may be involved in the decrease in arterial blood pressure and heart rate seen on exposure to opioid ligands (Miyawaki et al., 2002). MOR acts as a general brake on numerous systems by inhibiting adenylyl cyclase, decreasing cAMP levels, increasing potassium conductance, and decreasing the conductance of voltage-gated calcium channels, as described in detail in Chapter 1 (Section 1.2.3). The specific localisation of opioid receptors to particular neurons helps elucidate their diverse functions in distinct brain regions. In the preBötC and NTS brainstem regions involved in respiratory modulation, MOR is colocalised with neurokinin 1 receptors (NK1r) (Gray et al., 1999; Laferrière et al., 2003). In the preBötC, rhythmogenic type 1 neurons are depolarised by substance P (acting on the NK1r) a peptide that increases respiratory frequency and hyperpolarised by MOR agonist DAMGO, a peptide that causes respiratory depression (Gray et al., 1999). Both NK1r and OpRs are Gprotein coupled receptors, although NK1r is excitatory and MOR inhibitory. In the dorsal horn of the spinal cord, substance $\mathrm{P}$, acting on the NK1r is involved in pain transmission (Marvisón et al., 1997); whereas, activation of MOR by opioids inhibits the transmission of pain (Fields and Heinricher, 1985; Tershner and Helmstetter, 2000). Substance P and opioids are involved in the control of breathing, with opioids having inhibitory (Shook et al., 1990; Yeadon and Kitchen, 1990; Freye et al., 1992; Greer et al., 1995; Takita et al., 1997; Gray et al., 1999; Colman and Miller, 2001) and substance P excitatory effects (Chen et al., 1990; Gray et al., 1999). In a recent paper by Laferriere et al. (2003) NK1r in the adult rat NTS showed receptor down-regulation after short-term intermittent (6x $5 \mathrm{~min}$ interval) exposure to hypoxia (depleted oxygen); whereas MOR receptors remained the same (Laferrière et al., 2003). Results suggest that down-regulation of the NK1r on exposure to short-term intermittent hypoxia results in relatively more available MORs to opioid peptides resulting in attenuated respiratory depression. The culture system described, if shown to express NK1 receptors, could be used to study the function of these specialised cells, which have been suggested to be the preBötC neurons responsible for generating respiratory rhythm.

The process of internalisation of opioid receptors on exposure to opioid ligands has been linked to the ability of opioid drugs to induce tolerance (Keith et al., 1998). This 
association is supported by the fact that different opioid drugs cause different internalisation kinetics and responses. For example, methadone and morphine differ in their abilities to induce both internalisation and addiction (Zhang et al., 1998; Whistler et al., 1999), and mice lacking $\beta$-arrestin, a protein required for receptor internalisation, show a reduced tolerance to morphine (Bohn et al., 2000). It remains a mystery why morphine does not lead to OpR internalisation. One possibility is that morphine does not induce the conformational change in the receptor required for phosphorylation, and phosphorylation of the C-terminal of MOR and DOR is a requirement for endocytosis (Dohlman, 1991; Koch et al., 1998; El Kouhen et al., 1999; El Kouhen et al., 2000; El Kouhen et al., 2001).

The study of OpR regulation in primary culture is a new field, and the present study is the first to measure MOR regulation in brainstem neurons at the single cell level. A recent study by Lee et al. (2002) has described OpR regulation in primary cultures of rat cortex. This study used the OpR ligands dermorphin and deltorphin to label MOR and DOR, respectively. Although these ligands have high affinities for their targeted receptors, binding to other receptors is still possible. In addition, this technique does not take into account different splice variants of the OpRs that have been shown to be regulated differently (Koch et al., 1998; Koch et al., 2001). This study by Lee et al. (2002) is unique in that it uses confocal microscopy to examine OpR expression at high levels of resolution similar to that used in the present study.

Primary cell culture models may provide simplified systems in which to study cell function and regulation in a manner that more closely resembles the in vivo state compared to use of established cell lines. Cell culture models are helpful for identifying individual components involved in $\mathrm{OpR}$ regulation in vitro system before more complex OpR regulatory pathways can be elucidated in vivo. Primary cultures however, have disadvantages and limitations, as described previously in Chapter 2. Briefly, cultured brainstem cells do not necessarily function in the same way as the same cells in vivo. Alterations in gene expression and differential cell survival in different brainstem nuclei may occur once the cells are dissociated for culture. It is possible that some neuronal populations within the brainstem do not survive in culture at all the developmental ages tested. Therefore, if this primary culture system is to be used to study receptors in vitro, it may be necessary to first determine if the population of cells has been substantially 
modified from the in vivo population at each developmental age. Despite these limiting factors, primary cell culture provides a useful approach in the process of understanding complex cellular functions, despite the fact that extrapolation of findings to the in vivo situation must be made with caution.

\subsubsection{OPIOID RECEPTOR DISTRIBUTION AND EXPRESSION DURING BRAINSTEM DEVELOPMENT}

Immunohistochemical labelling of OpRs by specific antibodies and TSA amplification techniques was used to determine OpR distributions in coronal and sagittal sections of rat brainstem during development. In the foetal brainstem, DOR was found in the same brainstem regions as in postnatal (P6) and adult animals. Earlier studies of DOR expression in the developing brainstem used radioligand binding, which was unable to detect significant levels of DOR in P1 rats, and the labelling that was seen at this age was very diffuse (Xia and Haddad, 1991). Earlier studies of MOR expression also showed binding of MOR ligand in the brainstem nuclei of the NTS, NA and STT (Xia and Haddad, 1991). Similar distributions were found in the present study (Chapter 6: Section 6.4.5).

Autoradiographic techniques have been used to measure $\mathrm{OpR}$ expression and to localise the expression to particular brainstem regions. One study used DALDE to label DOR; however, this OpR ligand has only slight selectivity for DOR over other OpRs (Gacel et al., 1980). Autoradiography also does not have the resolution or the sensitivity characteristic of highly selective antibody techniques and cannot take into account the changes in OpR binding affinity that occur during development (Spain et al., 1985). Ligand binding in whole brain homogenates of the rat (Spain et al., 1985; Tavani et al., 1985; Petrillo et al., 1987) and mouse (Zhu et al., 1998) indicate that DOR is expressed at low levels during late foetal and early postnatal development. The present study, however, found that DOR was expressed at its highest levels in brainstem lysates of foetal rats, with a subsequent decrease in expression to adult levels (Chapter 6: Section 6.4.3). This finding has several possible implications. One is that DORs are expressed early in development; however, their binding properties are different in the foetus, not binding to the ligand, and change later in development to a form that does interact with the ligand. This possibility is supported by reports that there is an increase in binding affinity of DOR ligands during postnatal development (Spain et al., 1985). Another possibility is that the brainstem region has different expression levels during development compared to other brain regions. 
Therefore, when whole brain lysates are measured for OpR binding, the lower expression levels in other brain regions dilute out the high expression levels found in the brainstem. IHC results from the present study, however, provided a different picture to the brainstem lysate immunoblot results. There was no difference in DOR expression in cells cultured from foetal (E16) and postnatal (P6) brainstems (Chapter 3: Section 3.4.4). It is conceivable that changes in expression occurred that were not statistically significant due to the small sample size used and the minimum level of change required for detection using this technique.

The significance of different molecular weight forms of DOR seen in Western blots is unknown. The $30 \mathrm{kD}$ band is believed to be a truncated form of DOR that is unlikely to reside in the plasma membrane and is therefore likely to be non-functional, although OpR binding experiments will need to be undertaken to determine this. The detection of this protein along with the complete $70 \mathrm{kD}$ form may explain why higher expression of $\mathrm{DOR}$ was seen during early development using $\mathrm{IHC}$ than that reported from ligand binding studies that only measure complete protein with functional binding sites. It is also possible that the $30 \mathrm{kD}$ DOR protein is a splice variant. DOR splice variants have been described (Gavériaux-Ruff et al., 1997), and one of these splice variants encodes a truncated DOR protein with a single transmembrane domain. The DOR antibody used in the present study was specific for the N-terminal region of DOR, and therefore is likely to recognise the Nterminal of the truncated DOR receptor; whereas, an antibody specific for the C-terminal region would not. In future, development of antibodies and mRNA probes specific for each subtype of OpR will be able to determine if the truncated DOR is specifically found in a particular cell type or if both molecular weight forms are present in all DOR-positive cells. Opioid receptor splice variants are known to have different spatial distributions in the brain (Pan et al., 1999; Abbadie et al., 2000b; Abbadie et al., 2000a), and their regulatory properties differ (Abbadie and Pasternak, 2001; Koch et al., 2001). In one study of mouse splice variants in transfected cells showed MOR-1 and MOR-1C exhibit phosphorylation and internalisation in response to DAMGO but not morphine exposure. MOR-1D and MOR-1E, however, exhibit phosphorylation and internalisation in response to both morphine and DAMGO (Koch et al., 2001). In another study of the mouse splice variants in vivo MOR-1C showed internalisation with morphine exposure; whereas, MOR1 did not (Abbadie and Pasternak, 2001). Splice variants are also likely to bind OpR ligands with varying affinities, and this may account for some of the different 
pharmacological opioid receptor subtypes described in the literature (Lutz and Pfister, 1992; Satoh and Minami, 1995; Dhawan et al., 1996).

The expression of MOR during late foetal and early postnatal development resembled the expression levels described by others in vivo. MOR is expressed at low levels in the brainstem during early development, and expression levels increase through later stages to adult levels (Spain et al., 1985; Petrillo et al., 1987; Zhu et al., 1998). Several molecular weight MOR protein bands have been described and the size differences attributed to different glycosylation states of MOR (Garzón et al., 1995). The MOR-1 cDNA predicts a protein with a mass of $45 \mathrm{kD}$, so it is likely that both MOR bands seen in the present study have some glycosylation.

The present study is the first to describe changes in the expression level of different forms of MOR during brainstem development (Chapter 6: Section 6.4.3). The $70 \mathrm{kD}$ band is found predominantly in the adult, with decreasing levels seen earlier in brainstem development. The $50 \mathrm{kD}$ band, however, is present at its highest levels in the foetal (E16) brainstem, with decreasing levels seen at later ages. The two bands were also seen in Western blots of lysates prepared from primary brainstem cell cultures, and the same developmental trends for the two bands were seen. The functional significance of these developmental changes in the glycosylation state of MOR remains to be determined. Glycosylation may change ligand binding properties or the mechanisms of receptor regulation, thus, like DOR, contributing to the multiple pharmacological receptor subtypes that have been described.

MOR agonists appear to cause a reduction in respiratory frequency via $\mathrm{mu}_{1}$ receptors and a decrease in tidal volume via $\mathrm{mu}_{2}$ receptors (Colman and Miller, 2001; Colman and Miller, 2002). Different MOR agonists also cause respiratory depression via either $m_{1}$ or $m u_{2}$ activation (Ling et al., 1985; Paakkari et al., 1990; Chen et al., 1996). One theory that could be put forward is that the pharmacological and physiological uniqueness of different MOR agonists could be due to the glycosylated receptors having different binding affinities for their ligands. For example, $m_{1}$ may be glycosylated at one site or sites and $\mathrm{mu}_{2}$ at another or other sites. These different glycosylation forms of MOR may have unique spatial distributions within the brain, which would also cause different physiological responses to agonist exposure. Changes in expression of the glycosylated 
receptors during development may therefore account for the variation in sensitivity reported for young neonates to opioid agonists and antagonists compared to older neonates and adult animals. For example, in a study of neonatal piglets, administration of OpR agonist naloxone raised respiratory output, an effect that decreased with age (Long and Lawson, 1983). Administration of the MOR agonists dermorphin and fentanyl caused a decrease in respiratory output in neonatal and adult rats. This decrease in respiratory output was greater in younger pups (Colman and Miller, 2001).

MOR and DOR were found to be distributed in the same brainstem regions. In addition, cultured cells from brainstem showed MOR and DOR colocalisation in the same neurons, suggesting that MOR and DOR have closely related or overlapping functions or that they interact with each other, possibly by formation of heterodimers with unique binding properties and signal transduction pathways. The extent of colocalisation of MOR and DOR in cultured brain cells is only beginning to be investigated. A recent study in cultured cortical neurons by Lee et al. (2002) reported binding of fluorescent ligands to MOR and DOR in the same cells, although the exact proportions were not determined in their study (Lee et al., 2002). This recent study by Lee et al. is the first to show fluorescent OpR localisation in primary brain cultures. The present study shows colocalisation of MOR and DOR in cultures of brainstem cells and within brainstem nuclei indicating that MOR and DOR colocalisation may be more prevalent than anticipated. It remains to be determined if MOR and DOR form heterodimers in vivo.

\subsection{FUTURE EXPERIMENTS}

\subsubsection{CELL CULTURE}

A significant advance would be to establish cell cultures from more defined regions of the brainstem. For example, a specific brainstem region, such as the NTS or NA, could be micropunched from thick coronal sections and cultured in microplates. The disadvantage of using this technique is the small number of cells available for further analysis; however, it would be ideal for studying the properties of single neuronal cells during development using ICC or electrophysiology.

The use of primary cultures of individual brain regions would be essential for understanding OpR regulation in those regions. Different neuronal populations have 
different neurotransmitters, receptors, and isoforms of regulatory proteins such G-proteins (Harrison et al., 1998; Connor and Christie, 1999) or adenylyl cyclase isoforms (Standifer and Pasternak, 1997). A review by Law and Loh (1999) suggested a model for OpR regulation that involves the receptor complex changing conformation upon agonist binding, with each opioid agonist inducing a different conformational change in the OpR and thereby altering the receptor's other regulatory properties (Law and Loh, 1999). A model involving a static OpR does not account for all the known effects of the receptors and their ligands. If this is the case, the interaction of the $\mathrm{OpR}$ complex with other proteins such as $\beta$-arrestin, calmodulin and different isoforms of adenylyl cyclase is an important area for future research.

\subsubsection{Molecular Biology}

Another interesting area of research is to study the ability of different OpR splice variants to be phosphorylated (Koch et al., 1998), an important feature of agonist-induced desensitisation. Spatial localisation of splice variants (Pan et al., 1998; Abbadie et al., 2000b; Abbadie et al., 2000a), along with differences in their ability to be phosphorylated (Koch et al., 1998), are interesting areas that may help investigators design splice variantspecific opioid peptides. Splice variants that are resistant to desensitisation offer the opportunity to provide pain relief without the need for increasing the doses of opiates. It is also possible that opioid drugs without unwanted side effects could be developed.

Opioid receptor down-regulation involves phosphorylation of the opioid receptor. Antibodies could be made against phosphorylated MOR or DOR, and Western blots could be used to directly determine receptor phosphorylation on exposure to opioid agonists, such as DAMGO (MOR) and DPDPE (DOR) or antagonists such $\beta$-FNA (MOR) and natrindole (DOR). Immunogold electron microscopy could help determine if internalised OpRs are actually in endosomes. Double-labelling of cells in culture could also help determine if MOR and DOR share the same endocytotic pathways, or whether they are in separate endocytotic vesicles, as has been previously studied in OpR transfected cells (Gaudriault et al., 1997). 


\subsubsection{Electrophysiology}

The cell culture model described in this study (Chapter 2) provides an ideal system to study firing of brainstem neurons. Previous studies by Rigatto et al. (1992 and 1994) using a cell culture method described by Fitzgerald et al. (1992) (Fitzgerald et al., 1992) have described spontaneous neuronal firing in foetal rat neurons cultured from the brainstem regions of the NTS and NA (Rigatto et al., 1992; Rigatto et al., 1994). These spontaneous action potentials may originate from the preBötC neurons that are postulated to be responsible for generating respiratory rhythm in vivo (Smith et al., 1991; Rekling and Feldman, 1998). Some of the cultured neurons in the study by Rigatto et al. (1994) acted like pacemaker cells with regular or single bursting activities. These neurons also had chemosensitive properties. For example, when these cells were exposed to pulses of $\mathrm{CO}_{2}$ or low $\mathrm{pH}$, an increase in frequency and a decrease in amplitude of action potentials were seen. Irregularly firing cells and silent cells showed no response to $\mathrm{CO}_{2}$ or low $\mathrm{pH}$ (Rigatto et al., 1994). The firing properties of these cells, however, have not been studied during postnatal developmental because of the limitations of culture methods for older postnatal brainstem cells. The culture method described in this thesis opens the door to further investigation of $\mathrm{CO}_{2}$ - and $\mathrm{pH}$-responsive brainstem neurons in postnatal animals. Cell culture is an ideal method to study the electrical activity and receptor properties of individual neurons. It may be possible to study the effects of opioid agonists and antagonists on the electrophysiological properties of these neurons, and the same cells could then be isolated and their OpR expression profiles determined. Novel methods have been developed for examining mRNA expression in single cells using PCR techniques (Phillips and Lipski, 2000).

\subsubsection{MOR and DOR Colocalisation}

MOR and DOR colocalisation was often seen in primary culture of brainstem cells (Chapter 3: Section 3.4.6), although not all regions of individual cells expressing one or other receptor showed colocalisation. MOR and DOR were also colocalised within brainstem nuclei in vivo, although the localisation of MOR and DOR to the same cell was not determined. Future experiments using double-labelling techniques or immunogold electron microscopy would be able to assess whether MOR and DOR are colocalised in the same cells in vivo. Labelling of mRNA by fluorescence in situ hybridisation (FISH) could also be used to determine if the message and protein are found within the same cells. 
Preliminary FISH studies carried out by Eli Mrkusich and Dr Darren Day (Victoria University of Wellington, personal communication) show similar staining patterns to IHC antibody labelling presented in this study. A preliminary experiment using double FISH labelling of MOR and DOR mRNA showed that MOR and DOR mRNA are present in the same cells in most brainstem regions. Labelling with FISH and IHC, however, is yet to be performed on the same sections cut from the same rat brain. It seems likely that at least some MOR and DOR protein will be colocalised to the same neurons in vivo. Quantitative FISH techniques currently being developed make it possible to determine the relative distributions in brain regions.

It is unknown if MOR and DOR form heterodimers in vivo in the same way as have been found in cotransfected cells. Heterodimer formation could be assessed through the use of time resolved fluorescence resonance energy transfer (FRET), bioluminescence resonance energy transfer (BRET), or immunoprecipitation. Immunoprecipitation experiments have already been carried out on MOR- and DOR-transfected cells by George et al. (2000) (George et al., 2000), and homodimer formation has been examined by McVey (2001) in DOR transfected cells (McVey et al., 2001).

Knockout models of OpRs have been generated and studied (Matthes et al., 1996; Kieffer, 1999; Chen et al., 2000; Kieffer and Gavériaux-Ruff, 2002); however, the development of conditional knockout mice in which OpR or peptide genes are inactivated in a regionalspecific manner may help elucidate opioid function in specific brain regions during development. The effects of opioids on respiration have also not been determined in all OpR knockout mice. Studies on respiratory function in the triple MOR, DOR, and KOR knockout mouse may help investigators further elucidate the role played by opioids in respiration.

\subsubsection{Opioids and Cerebellar function}

The present study is the first to demonstrate MOR localisation in Purkinje cells in the rat cerebellum (Chapter5). A clear determination of the roles played by opioids in cerebellar development and function would help determine the significance of the receptor distributions described in the present study. Quantitative assessment of the differences in 
expression levels between species, using both functional activation of opioid receptors and protein expression by Western blotting, would be helpful in this regard.

\subsection{OVERALL CONCLUSIONS}

Primary cultures of brainstem cells of the rat can be established up to postnatal day 6 . Cells in brainstem cultures of late foetal and early postnatal development express both MOR and DOR, with some colocalisation of receptors seen. MOR in cultured cells is functionally down-regulated on exposure to the MOR agonist DAMGO in a similar way as has been described for SH-SY5Y cells and MOR-transfected cells (Arden et al., 1995; Law and Loh, 1999; Law et al., 2000c; Afify, 2002); thus, providing a suitable model to study OpR regulation in culture. Brainstem cultures have advantages over use of established cell lines in that the cell populations express receptors at endogenous expression levels, unlike the over-expression seen in transfected cells. Thus, cells in primary culture are more likely to resemble the in vivo cell population in which the cells were isolated from.

Opioid receptor expression is developmentally and spatially regulated. MOR and DOR were found in the same brainstem regions in foetal, postnatal and adult rats with DOR seen for the first time in foetal rat brainstem coronal sections. Several different molecular weight forms of both MOR and DOR were seen in Western blots. This study shows for the first time developmental changes in the molecular weight forms of MOR and DOR. The developmental changes are likely to be due to differences in receptor glycosylation and/or splice-variant expression.

Changes in the developmental expression and distribution of opioid receptors is likely to play a key role in the growth, differentiation and physiological activity of brainstem neurons involved in respiration and cardiovascular function 


\section{Appendix 1 Hibernate Medium Composition}

The only difference between Hibernate $\mathrm{E}$ and Hibernate $\mathrm{A}$ medium is the $\mathrm{NaCl}$ concentration which alters osmolality.

\begin{tabular}{|c|c|c|}
\hline & $\begin{array}{c}\text { Hybernate E } \\
\mathrm{mg} / \mathrm{L}\end{array}$ & $\begin{array}{c}\text { Hybernate A } \\
\text { mg/L }\end{array}$ \\
\hline \multicolumn{3}{|l|}{ INORGANIC SALTS } \\
\hline $\mathrm{CaCl}_{2}$ & 200.000 & 200.000 \\
\hline $\mathrm{Fe}\left(\mathrm{NO}_{3}\right)_{3} \cdot 9 \mathrm{H}_{2} \mathrm{O}$ & 0.100 & 0.100 \\
\hline $\mathrm{KCl}$ & 400.000 & 400.000 \\
\hline $\mathrm{MgCl}_{2}$ & 77.300 & 77.300 \\
\hline $\mathrm{NaCl}$ & 3000.000 & 4000.000 \\
\hline $\mathrm{NaH}_{2} \mathrm{PO}_{4} \cdot \mathrm{H}_{2} \mathrm{O}$ & 125.000 & 125.000 \\
\hline $\mathrm{NaHCO}_{3}$ & 74.000 & 74.000 \\
\hline $\mathrm{ZnSO}_{4} \cdot 7 \mathrm{H}_{2} \mathrm{O}$ & 0.190 & 0.190 \\
\hline \multicolumn{3}{|l|}{ AMINO ACIDS } \\
\hline L-Alanine & 2.000 & 2.000 \\
\hline L-Arginine $\mathrm{HCl}$ & 84.000 & 84.000 \\
\hline L- Asparagine. $\mathrm{H}_{2} \mathrm{O}$ & 0.830 & 0.830 \\
\hline L-Cysteine & 31.500 & 31.500 \\
\hline Glycine & 30.000 & 30.000 \\
\hline L-Histeine $\mathrm{HCl} \mathrm{H}_{2} \mathrm{O}$ & 42.000 & 42.000 \\
\hline L-Isoleucine & 105.000 & 105.000 \\
\hline L-Leucine & 105.000 & 105.000 \\
\hline L-Lysine & 146.000 & 146.000 \\
\hline L-Methionine & 30.000 & 30.000 \\
\hline L-Phenylalanine & 66.000 & 66.000 \\
\hline L-Proline & 7.760 & 7.760 \\
\hline L-Serine & 42.000 & 42.000 \\
\hline L-Threonine & 95.000 & 95.000 \\
\hline L-Tryptophan & 16.000 & 16.000 \\
\hline L-Tyrosine & 72.000 & 72.000 \\
\hline L-Valine & 94.000 & 94.000 \\
\hline \multicolumn{3}{|l|}{ OTHER COMPONENTS } \\
\hline D-Glucose & 4500.000 & 4500.000 \\
\hline MOPS & 209.000 & 209.000 \\
\hline Phenol Red Solution & 8.100 & 8.1 .000 \\
\hline Sodium Pyruvate & 25.000 & 25.000 \\
\hline \multicolumn{3}{|l|}{ VITAMINS } \\
\hline Choline Chloride & 4.000 & 4.000 \\
\hline D-Ca-Pantothenate & 4.000 & 4.000 \\
\hline Folic Acid & 4.000 & 4.000 \\
\hline I-Inositol & 7.200 & 7.200 \\
\hline Niacinamide & 4.000 & 4.000 \\
\hline Pyrodoxal $\mathrm{HCl}$ & 4.000 & 4.000 \\
\hline Riboflavin & 0.400 & 0.400 \\
\hline Thiamine $\mathrm{HCl}$ & 4.000 & 4.000 \\
\hline Vitamin B12 Solution & 0.007 & 0.007 \\
\hline
\end{tabular}




\section{Appendix 2}

\section{Solutions, Buffers and Miscellaneous Methods}

$\begin{array}{lc}1 \quad \text { BALANCED SALT SOLUTION (BSS) } \\ \mathrm{NaCl} & 148.0 \mathrm{mM} \\ \mathrm{KCl} & 5.0 \mathrm{mM} \\ \mathrm{CaCl} 2 & 1.3 \mathrm{mM} \\ \mathrm{NaHCO} 3 & 4.2 \mathrm{mM} \\ \mathrm{MgSO} 4 & 0.9 \mathrm{mM} \\ \mathrm{Na} 2 \mathrm{HPO} 4 & 0.3 \mathrm{mM} \\ \mathrm{KH} 2 \mathrm{PO} 4 . & 0.4 \mathrm{mM}\end{array}$

2 PHOSPHATE BUFFERED SALINE (PBS) 0.15M PH 7.4

$\begin{array}{lc} & \mathrm{g} / \mathrm{L} \\ \mathrm{NaCl} & 0.80 \mathrm{~g} \\ \mathrm{KCl} & 0.20 \mathrm{~g} \\ \mathrm{Na}_{2} \mathrm{HPO}_{4} & 1.15 \mathrm{~g} \\ \mathrm{KHPO}_{4} & 0.20 \mathrm{~g}\end{array}$

PBS was made by dissolving the salts in $\mathrm{ddH} 2 \mathrm{O}$. The $\mathrm{pH}$ was adjusted to 7.4 using concentrated HCL or $\mathrm{NaOH}$.

\section{REMOVAL OF PEROXIDASE ACTIVITY}

Samples are incubated in hydrogen peroxide solution for 15 minutes Hydrogen Peroxide Solution

$\begin{array}{ll}\text { Methanol } & 5.000 \mathrm{~mL} \\ 30 \% \mathrm{H}_{2} \mathrm{O}_{2} & 0.333 \mu \mathrm{L} \\ \mathrm{dH}_{2} \mathrm{O} & 4.660 \mathrm{~mL}\end{array}$




\section{DAB SOLUTION}

DAB

$12.50 \mathrm{mg}$

$\mathrm{dH}_{2} \mathrm{O}$

$18.75 \mathrm{~mL}$

$0.4 \mathrm{M} \mathrm{PO}_{4}$ buffer

$6.25 \mathrm{~mL}$

$1 \% \mathrm{H}_{2} \mathrm{O}_{2}$

$9.00 \mu \mathrm{L}$

DAB was dissolved in $\mathrm{dH}_{2} \mathrm{O}$ and the phosphate buffer added. Immediately prior to use $\mathrm{H}_{2} \mathrm{O}_{2}$ was added and the samples incubated for 1-20 min until the desired brown colour was reached. For increased staining $1 \mathrm{~mL}$ of $1 \%$ Nickel sulphate was added to the mixture above to produce intense black staining.

\section{0.4 M PHOSPHATE BUFFER}

$\begin{array}{lr} & \mathrm{g} / \mathrm{L} \\ \mathrm{Na}_{2} \mathrm{HPO}_{4} 12 \mathrm{H}_{2} \mathrm{O} & 111.3 \mathrm{~g} \\ \mathrm{NaH}_{2} \mathrm{PO}_{4} & 9.1 \mathrm{~g}\end{array}$

Salts were dissolved in $\mathrm{ddH}_{2} \mathrm{O}$, and the $\mathrm{pH}$ adjusted to $\mathrm{pH} 7.4$ before making to the final volume.

\section{GLYCERIN JELLY}

Gelatin

$$
5.00 \mathrm{~g}
$$

$\mathrm{dH}_{2} \mathrm{O}$

$60.00 \mathrm{~mL}$

Pure glycerin $70.00 \mathrm{~mL}$

Phenol Crystals

$0.25 \mathrm{~g}$

Gelatin was dissolved in $\mathrm{dH}_{2} \mathrm{O}$ in a beaker, in a waterbath. When gelatin was dissolved glycerin and phenol were added and melted in a hot waterbath or oven.

\section{ANTIFADE MOUNTING MEDIUM}

Antifade reagent, $0.2 \mathrm{~g}$ of 1,4-diazabicyclo[2.2.2]octane (DABCO) was added to $9 \mathrm{~mL}$ glycerol and heated to $50^{\circ} \mathrm{C}$ until dissolved. When dissolved, $1 \mathrm{~mL}$ of $0.2 \mathrm{M}$ Tris buffer containing $0.02 \%$ sodium azide was added. 


\section{DEPC TREATED WATER}

One $\mathrm{mL}$ of diethyl-pyro carbonate (DEPC) was added to $1 \mathrm{~L}$ of $\mathrm{d} \mathrm{H}_{2} \mathrm{O}$ and shaken vigously. The treated water is placed in a fume hood overnight. The next day the DEPC treated water is autoclaved for $30 \mathrm{~min}$.

\section{LB MEDIUM}

To make $1 \mathrm{~L}$

The following reagents are mixed together until they have dissolved. The $\mathrm{pH}$ adjusted to pH 7 with $\mathrm{NaOH}$ and made up to the final volume of $1 \mathrm{~L}$, then the mixture sterilised by autoclaving.

$\begin{array}{lc}\text { Bacto-tryptone } & 10 \mathrm{~g} \\ \text { Bacto-yeast extract } & 5 \mathrm{~g} \\ \mathrm{NaCl} & 10 \mathrm{~g} \\ \mathrm{dH} 2 \mathrm{O} & 950 \mathrm{~mL}\end{array}$

\section{LB-AGAR}

LB agar plates were made by adding agar $(15 \mathrm{~g} / \mathrm{L})$ to $\mathrm{LB}$ medium before $\mathrm{pH}$ adjustment. After autoclaving the media was cooled and antibiotics added. For LB-amp plates 50 $\mu \mathrm{g} / \mathrm{mL}$ of ampicillin, and for LB-kan plates $50 \mu \mathrm{g} / \mathrm{mL}$ of kanamycin was added. The LB Agar is then poured into $10 \mathrm{~cm}$ diameter petri dishes and stored at $4^{\circ}$ until required.

\section{SOC MEDIUM}

$950 \mathrm{ml}$

$\mathrm{dH}_{2} \mathrm{O}$

bacto-tryptone

$20.0 \mathrm{~g}$

bacto-yeast extract

$50 \mathrm{~g}$

$\mathrm{NaCl}$

$0.5 \mathrm{~g}$

The reagents listed above are added together and shaken until all the solutes were dissolved. Ten $\mathrm{mL}$ of $\mathrm{KCl}(250 \mathrm{mM})$ was added and the $\mathrm{pH}$ adjusted to $\mathrm{pH} 7$ with $\mathrm{NaOH}$. The solution is made up to $975 \mathrm{~mL}$ with $\mathrm{dH}_{2} \mathrm{O}$, and the mixture sterilised by autoclaving. Following sterilisation $5 \mathrm{~mL}$ of sterile $\mathrm{MgCl}(2 \mathrm{M})$ and $20 \mathrm{~mL}$ of $1 \mathrm{M}$, filter sterilised glucose solution was added. 


\section{SEQUENCING PURIFICATION SPIN COLUMN}

G50 Stock: Ten g of Sephadex-G50 (Pharmacia, Uppsala, Sweden) was added to $100 \mathrm{~mL}$ of sterile $\mathrm{dH} 2 \mathrm{O}$ and allowed to hydrate for $30 \mathrm{~min}$. Water was decanted from the beads, them more water was added. This step was repeated 3 times and the beads stored at $4^{\circ} \mathrm{C}$ until required.

Spin column preparation: Clean reusable microfuge tube columns were washed 5 times in $\mathrm{dH} 2 \mathrm{O}$ and once in $70 \%$ ethanol and the column allowed to air dry. To the bottom of the column $0.75 \mathrm{~mL}$ of $\mathrm{G} 50$ stock was added and centrifuged (2000 rpm for $1 \mathrm{~min}$ ). After centrifugation $200 \mu \mathrm{L}$ of $\mathrm{d} \mathrm{H}_{2} \mathrm{O}$ was added to the middle of the column centrifuged as above. Prepared columns were sealed and stored at $4{ }^{\circ} \mathrm{C}$ for up to 1 week. Before use, the column was washed by addition of $50 \mu \mathrm{L}$ of sterile $\mathrm{dH}_{2} \mathrm{O}$ to the centre of the column and centrifuged.

\section{SDS-POLYACRYLAMIDE GEL ELECTROPHORESIS}

Gel plates and combs were cleaned with $70 \%$ ethanol, allowed to dry, and assembled onto gel casting apparatus (BioRad).

\section{Stock Solutions}

1.5 M Tris $\mathrm{pH} 8.9$

0.5 M Tris $\mathrm{pH} 6.8$

$10 \%$ SDS

Water saturated butanol

\section{8\% Separating Gel}

(2 gels $1.5 \mathrm{~mm}$ thick)

$\begin{array}{lr}\text { Acrylamide/bis (30\% stock) } & 5.32 \mathrm{~mL} \\ 1.5 \mathrm{M} \text { Tris pH } 8.8 & 5.00 \mathrm{~mL} \\ \text { dd } \mathrm{H}_{2} \mathrm{O} & 9.38 \mathrm{~mL} \\ 10 \% \text { SDS in } \mathrm{H}_{2} \mathrm{O} & 200.00 \mu \mathrm{L} \\ \text { TEMED } & 10.00 \mu \mathrm{L} \\ 10 \% \text { ammonium persulfate } & 100.00 \mu \mathrm{L}\end{array}$

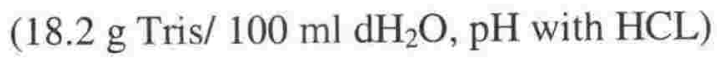

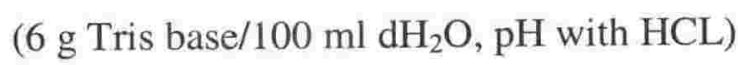

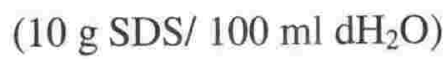


The solutions were added in the order shown. TEMED and $10 \%$ ammonium persulphate were added last to initiate polymerisation. The gel solution was added to the casting apparatus and overlayed with water-saturated butanol. The gel was left to polymerise for 30 min. The butanol was removed from the gel and the gel rinsed with $\mathrm{ddH}_{2} \mathrm{O}$. The stacking gel was then made and cast on top of the washed separating gel.

\section{4\% Stacking Gel}

Acrylamide/bis (30\% stock) $1.33 \mathrm{~mL}$

0.5 M Tris pH 6.8

$2.50 \mathrm{~mL}$

$10 \%$ SDS

$0.10 \mathrm{~mL}$

$\mathrm{ddH}_{2} \mathrm{O}$

$6.10 \mathrm{~mL}$

TEMED

$10.00 \mu \mathrm{L}$

10\% Ammonium persulfate

$50.00 \mu \mathrm{L}$

(Stored frozen)

Total

$1000 \mathrm{~mL}$

The stacking gel reagents were combined, mixed and overlayed on top of the separating gel. A comb was added to form well for the samples and the gel was allowed to polymerise for approximately $15 \mathrm{~min}$. The comb was removed and the gel placed in the electrophoresis tank with running buffer. Samples were added to the wells and electrophoresed at $150 \mathrm{~V}$ for 1 hour.

\section{5X RUNNING BUFFER (TRIS-GLYCINE PH 8.3)}

$600 \mathrm{~mL}$

Tris base

$9.0 \mathrm{~g}$

Glycine

$43.2 \mathrm{~g}$

SDS

$3.0 \mathrm{~g}$

The reagents listed above were added together with $500 \mathrm{~mL}$ of $\mathrm{ddH}_{2} \mathrm{O}$, mixed, and made up to $600 \mathrm{~mL}$ and stored at $4^{\circ} \mathrm{C}$ until required.

\section{PROTEIN GEL LOADING BUFFER 5 X (10 ML)}

glycerol

SDS
$5.62 \mathrm{~mL}$

$1.15 \mathrm{~g}$ 
bromophenol blue

$5 \mathrm{mg}$

0.5 M Tris- $\mathrm{HCl} \mathrm{pH} 6.8$

$4.38 \mathrm{~mL}$

The following reagents were added, mixed and stored at -20 until use. Immediately before e use $10 \mu \mathrm{L}$ of 2 -mercaptoethanol per $0.1 \mathrm{~mL}$ gel loading buffer was added.

\section{WESTERN TRANSFER BUFFER (3 L)}

Tris $9.09 \mathrm{~g}$

glycine $43.2 \mathrm{~g}$

SDS

methanol

$600 \mathrm{~mL}$

The following reagents are combined and the volume made up to $3 \mathrm{~L}$ with $\mathrm{ddH}_{2} \mathrm{O}$.

17 TABLE 1: ARRANGEMENT OF WESTERN TRANSFER APPARATUS

Table Appendix 2 Arrangement of western transfer apparatus

\begin{tabular}{|l|l|}
\hline Bottom & Grey Side of Cassette \\
& Fibre Pad \\
& 3MM Paper \\
& Electrophoresed Gel \\
& PVDF Membrane \\
& 3MM Paper \\
& Fibre Pad \\
& Clear Side of Cassette \\
\hline
\end{tabular}




\section{Appendix 3}

\section{Statistical Analysis}

\section{MULTIPLE COMPARISONS (BASED OF KRUSKAL-WALLIS TEST)}

The statistical significance of differences between pairs of values was determined using Mann Whitney (2 groups) or Kruskal-Wallis (3 or more groups) for each developmental age group or treatment, followed by Multiple comparisons test (Hollander and Wolfe, 1973a) (Hollander and Wolfe, 1973a))

For unequal sample sizes a significant difference was analysed using the following equation.

Results are significant when

$$
\left(R_{\mathrm{u}}-\mathrm{R}_{\mathrm{v}}\right) \mu \mathrm{Z}_{(\alpha /[\mathrm{k}(\mathrm{k}-} \quad\left[\frac{\mathrm{N}(\mathrm{N}+1)}{12}\right]^{1 / 2}\left(\frac{1}{\mathrm{~N}_{\mathrm{u}}}+\frac{1}{\mathrm{~N}_{\mathrm{v}}}\right)^{1 / 2}
$$

$$
\begin{array}{ll}
\mathrm{R}_{\mathrm{u}}, \mathrm{R}_{\mathrm{v}} & =\text { Mean ranks of samples } \mathrm{u}, \mathrm{v} \\
\mathrm{Z} & =\text { Upper quartile of normal distribution } \mathrm{N}(0,1) \text { (statistical table) } \\
\alpha & =\text { Acceptable experimental error rate }(0.001) \\
\mathrm{N} & =\text { Mean rank } \\
\mathrm{k} & =\text { Number of groups } \\
\mathrm{N}_{\mathrm{u}}, \mathrm{N}_{\mathrm{v}} & =\text { Total number in group u, } \mathrm{v} \\
\mathrm{N} &
\end{array}
$$




\title{
Appendix 4 Cloning and Sequencing of MOR
}

\author{
Vector properties
}

\section{pGEM-T}

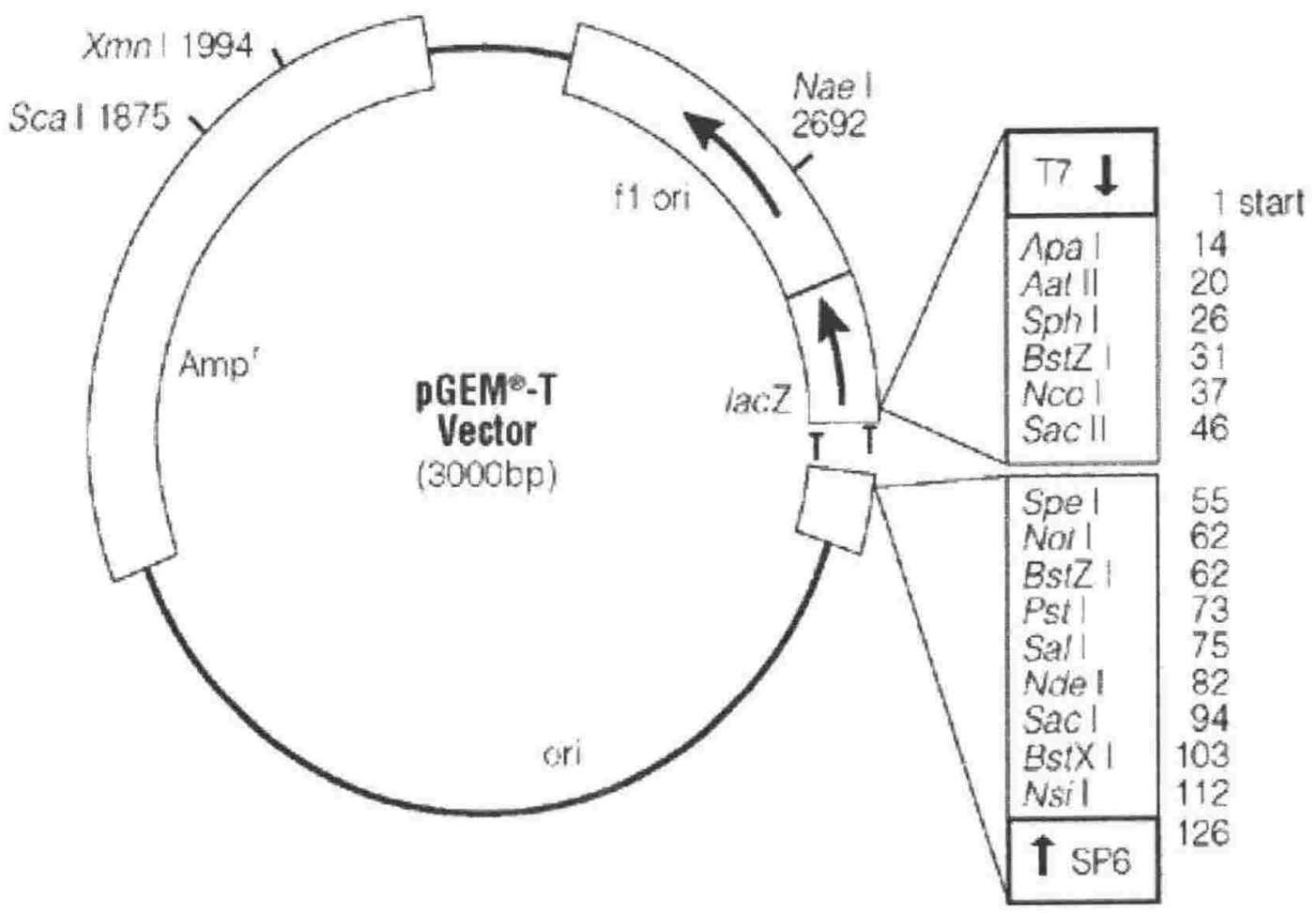



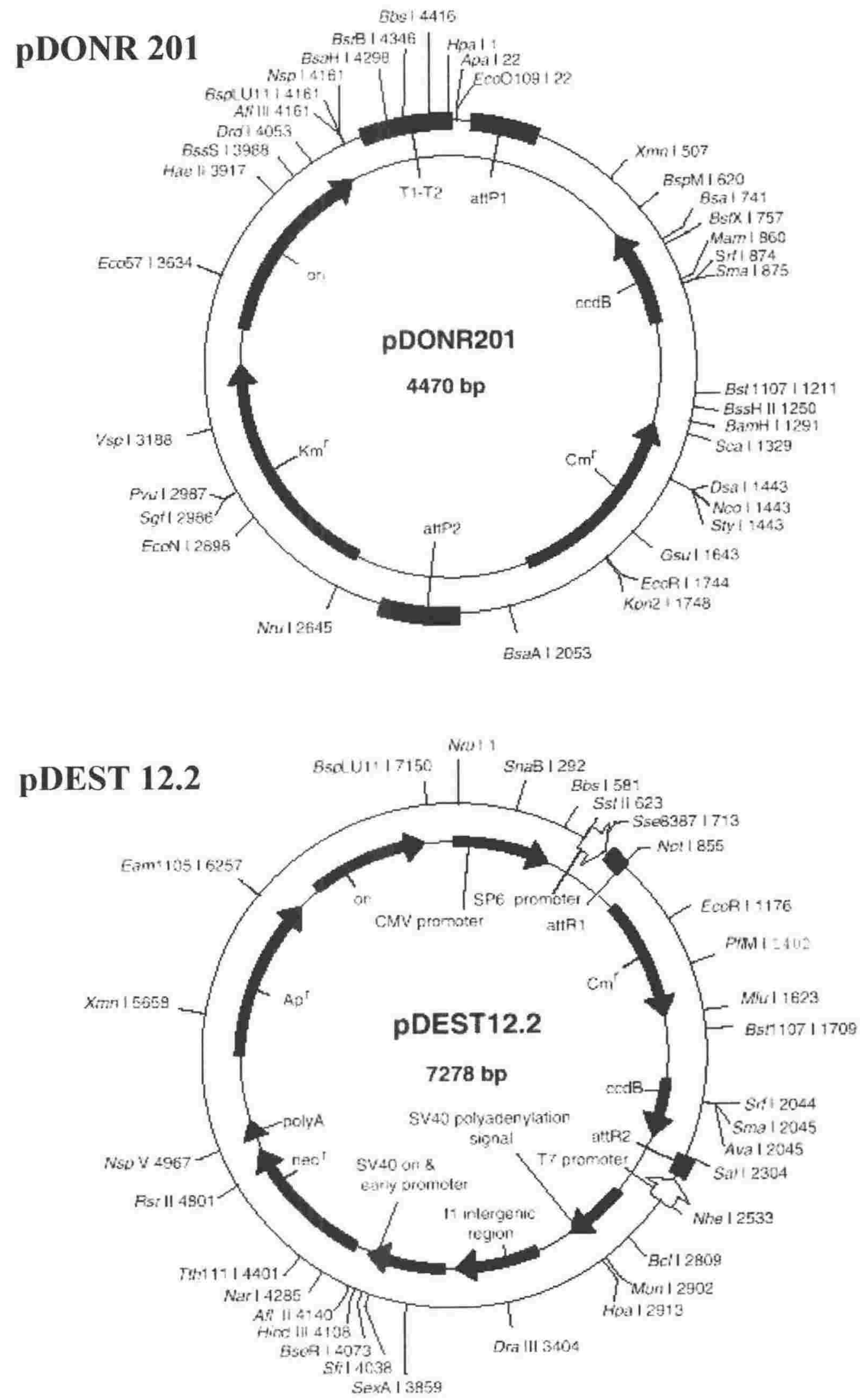

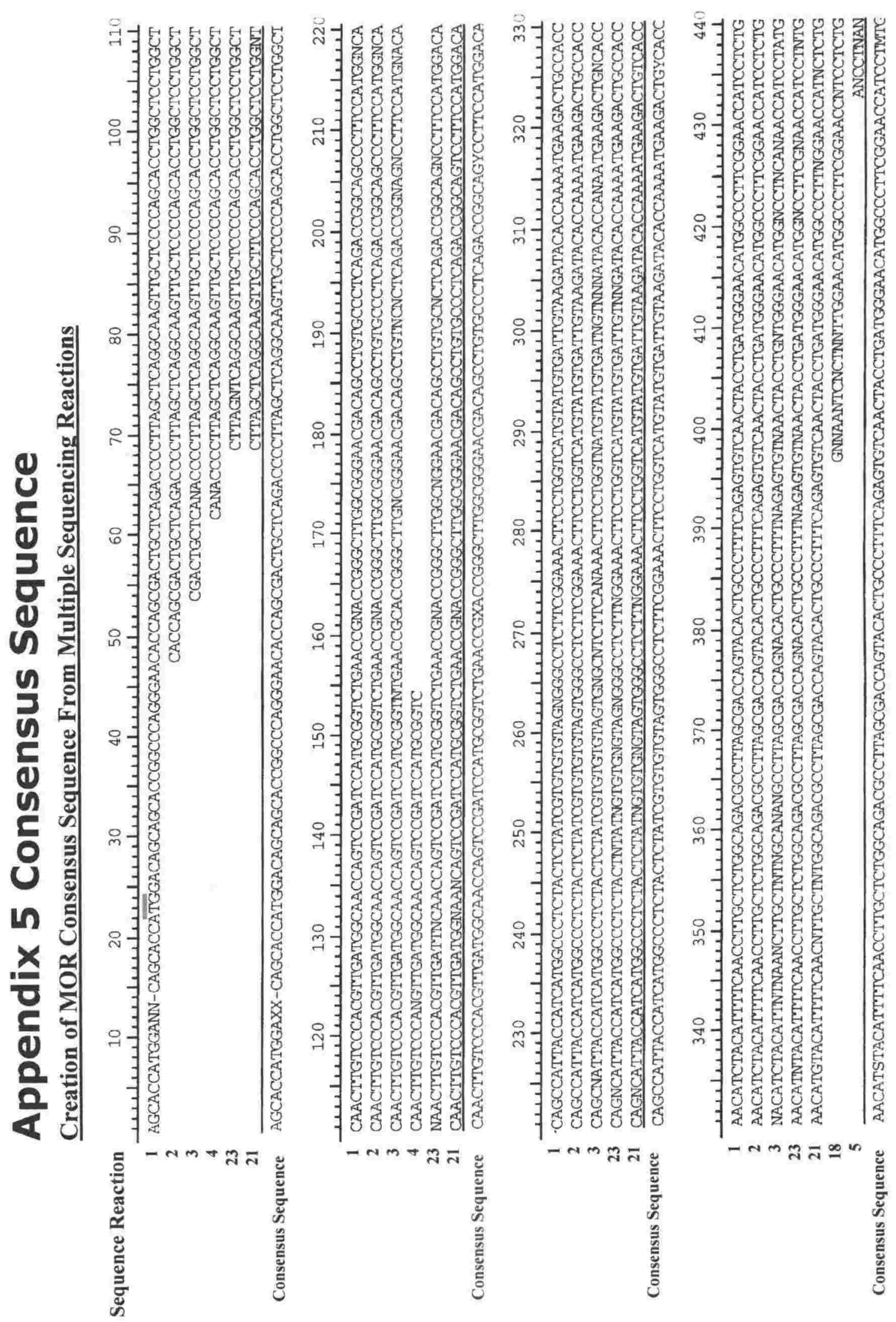


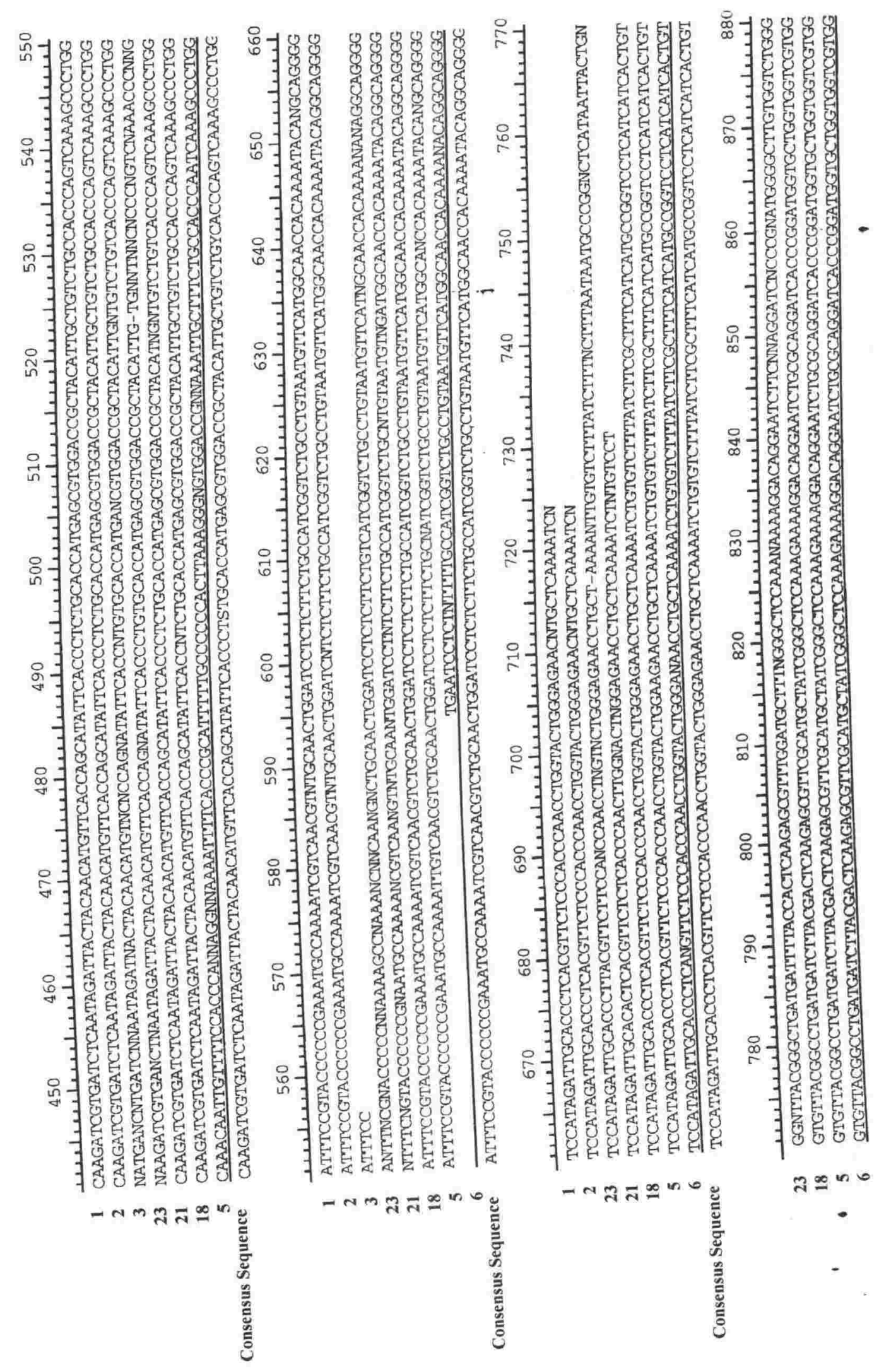




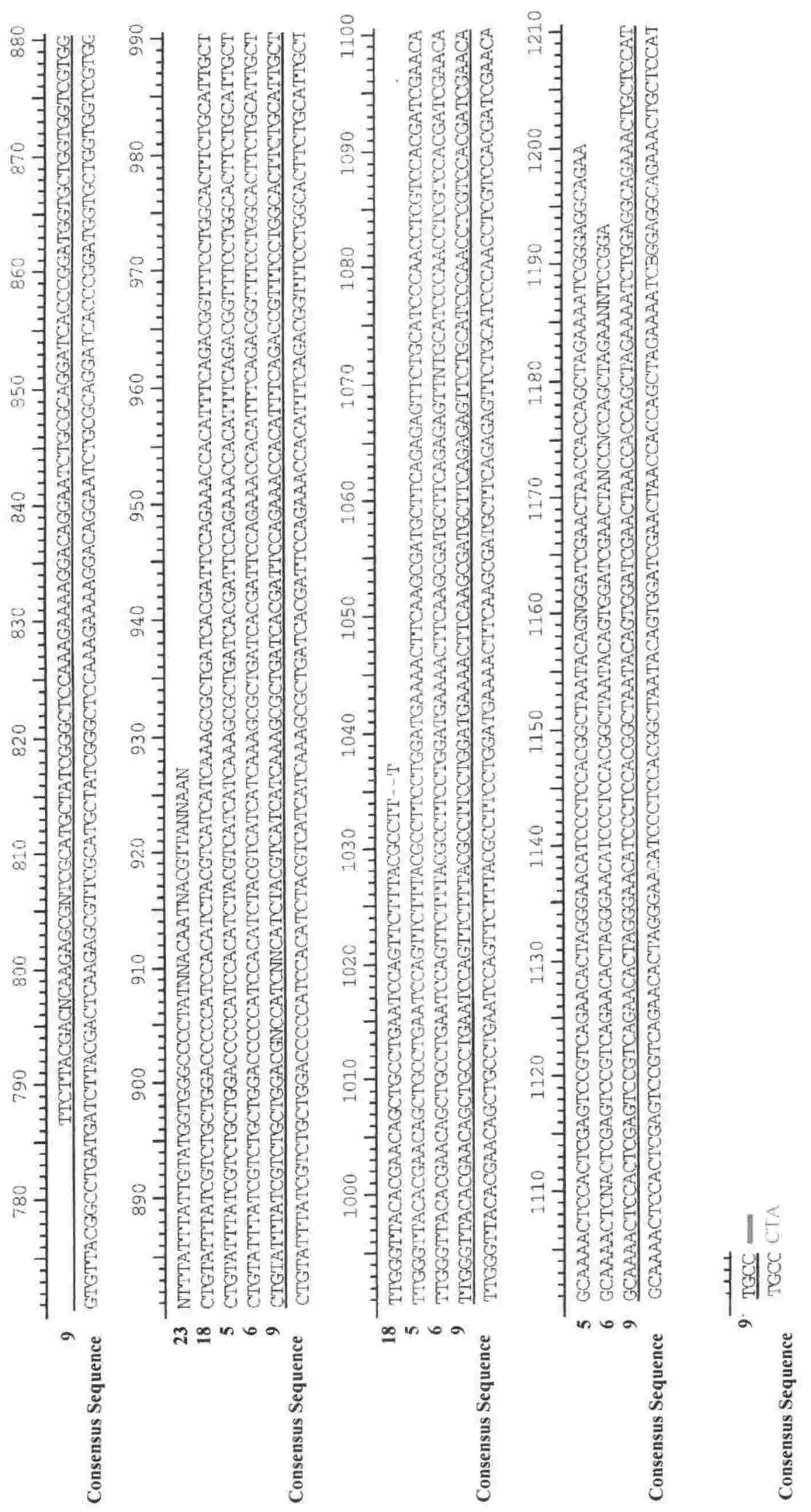




\section{References}

Abbadie C, Pasternak G (2001) Differential in vivo internalization of MOR-1 and MOR$1 \mathrm{C}$ by Morphine. NeuroReport 12:3069-3072.

Abbadie C, Pan Y, Pasternak G (2000a) Differential distribution in rat brain of Mu opioid receptor carboxy terminal splice variants MOR-1C-like and MOR-1-like immunoreactivity: evidence for region specific processing. The Journal of Comparative Neurology 419:244-256.

Abbadie C, Pan Y, Drake C, Pasternak G (2000b) Comparative immunohistochemical distributions of carboxy terminus epitopes from the mu-opioid receptor splice variants MOR-1D, MOR-1 and MOR-1C in the mouse and rat CNS. Neuroscience 100:141-153.

Abbadie C, Lombard M, Besson J, Trafton J, Basbaum A (2002) Mu and delta opioid receptor-like immunoreactivity in the cervical spinal cord of the rat after dorsal rhizotomy or neonatal capsaicin: an analysis of pre- and postsynaptic receptor distributions. Brain Research 930:150-162.

Abeyta A, Dettmer T, Barnes A, Vega D, Carta M, Gallegos N, Raymond-Stintz M, Savage D, Valenzuela C, Saland L (2002) Delta opioid receptor localization in the rat cerebellum. Brain Research 931:100-105.

Ableitner A (1994) Brain sites involved in $\delta$-opioid receptor-mediated actions. European Journal of Pharmacology 271:213-222.

Abood M, Noel M, Farnsworth J, Tao Q (1994) Molecular cloning and expressionn of a $\delta$ opioid receptor from rat brain. Journal of Neuroscience Research 37:714-719.

Adler M, Geller E (1988) The opioid system and temperature regulation. Annual Review of Pharmacology and Toxicology 28:429-449.

Afify E (2002) Turnover of $\mu$-opioid receptors in neuroblastoma cells. Molecular Brain Research 106:83-87.

Aicher S, Punnoose A, Goldberg A (2000a) m-opioid receptors often colocalise with the substance $\mathrm{P}$ receptor (NK1) in the trigeminal dorsal horn. The Journal of Comparative Neurology 20:4345-4354.

Aicher S, Goldberg A, Sharma S, Pickel V (2000b) $\mu$-opioid receptors are present in vagal afferents and their dendritic targets in the medial nucleus tractus solitarius. The Journal of Comparative Neurology 422:181-190.

Aicher S, Schreihofer A, Kraus J, Sharma S, Milner T, Guyenet P (2001) $\mu$-opioid receptors are present in functionally identified sympathoexctiatory neurons in the rat rostral ventrolateral medulla. The Journal of Comparative Neurology 433:34-47.

Akil H, Watson S, Young E, Lewis M, Khachaturian H, Walker J (1984) Endogenous opioids: Biology and function. Annual Review of Neuroscience 7:223-255.

Akil H, Meng F, Mansour A, Thompson R, Xie G, Watson S (1996) Cloning and characterization of multiple opioid receptors. In: Molecular aproaches to drug abuse research (Lee T, ed). Rockville: NIDA Research Monograph 161.

Altman J, Bayer S (1980) Development of the brain stem in the rat. IV. thymidineradiograph study of the time of origin of neurons in the pontine region. Journal of Comparative Neurology 194:905-929.

Anders P, Persson I, Thorlin T, Rönnbäck L, Hansson E, Eriksson P (2000) Differential expression of delta opioid receptors and mRNA in proliferating astrocytes during the cell cycle. Journal of Neuroscience Research 61:371-375. 
Andria M, Simon E (1999) Localisation of promotor elements in the human mu-opioid receptor gene and regulation by DNA methylation. Molecular Brain Research 70:54-65.

Andria M, Simon, EJ. (2001) Identification of a neurorestrictive supressor element (NRSE) in the human $\mu$-opioid receptor gene. Molecular Brain Research 91:73-80.

Appleyard S, Celver J, Pineda V, Kovoor A, Wayman G, Chavkin C (1999) Agonistdependent desensitization of the $\kappa$ opioid receptor by $\mathrm{G}$ protein receptor kinase and $\beta$-arrestin. Journal of Biological Chemistry 274:23802-23807.

Arden J, Segredo V, Wang Z, Lameh J, Sadée W (1995) Phosphorylation and agonistspecific intracellular trafficking of an epitope-tagged $\mu$ opioid receptor expressed in HEK 293 cells. Journal of Neurochemistry 65:1636-1645.

Arias C, Arrieta I, Massieu L, Tapia R (1997) Neuronal damage and MAP2 changes induced by the glutamate transport inhibitor dihydrokainate and by kainate in rat hippocampus in vivo. Experimental Brain Research 116:467-476.

Armstead W (1995) Opioids and nitric oxide contribute to hypoxia-induced pial arterial vasodilation in newborn pigs. American Journal of Physiology 268:H226-H232.

Arttamangkul S, Alvarez-Maubecin V, Thomas G, Williams J, Grandy D (2000) Binding and internalization of fluorescent opiate peptide conjugates in living cells. Molecular Pharmacology 58:1570-1580.

Arvidsson U, Dado R, Riedl M, Lee J, Law P, Loh H, Elde R, Wessendorf M (1995a) $\delta$ opioid receptor immunoreactivity: Distribution in brainstem and spinal cord, and relationship to biogenic amines and enkephalin. The Journal of Neuroscience 15:1215-1235.

Arvidsson U, Riedl M, Chakrabarti S, Lee J, Nakano A, Dado R, Loh H, Law P, Wessendorf M, Elde R (1995b) Distribution and targeting of a $\mu$-opioid receptor (MOR1) in brain and spinal cord. The Journal of Neuroscience 15:3328-3341.

Attali B, Saya D, Vogel Z (1990) Pre- and postnatal development of opiate receptor subtypes in rat spinal cord. Developmental Brain Research 53:97-102.

Attila M, Salvadori S, Balboni G, Bryant S, Lazarus L (1993) Synthesis and receptor binding analysis of dermorphin hepta-, hexa- and pentapeptide analogues. International Journal of Peptide Protein Research 42:550-559.

Atweh S, Kuhar M (1977) Autoradiographic localization of opiate receptors in rat brain. I. Spinal cord and lower medulla. Brain Research 124:53-67.

Azmitia E (1990) Microcultures of dissociated primary central nervous system neurons. Methods in Neuroscience 2:263-275.

Ballantyne D, Scheid P (2000) Mammalian brainstem chemosensitive neurones: linking them to respiration in vitro. Journal of Physiology 525:567-577.

Barbacid M (1995) Neurotrophic factors and their receptors. Current Opinion In Cell Biology 7:148-155.

Barde Y (1989) Trophic factors and neuronal survival. Neuron 2:1525-1534.

Barg J, Simantov R (1989) Developmental profile of kappa, mu and delta opioid receptors in the rat and guinea pig cerebellum. Developmental Neuroscience 11:428-434.

Barg J, Rius A, Bem W, Belcheva M, Loh Y, Coscia C (1992) Differential development of $\beta$-endorphin and $\mu$ opioid binding sites in mouse brain. Developmental Brain Research 66:71-76.

Barg J, Belcheva M, McHale R, Levy R, Vogel Z, Cosica C (1993a) $\beta$-endorphin is a potent inhibitor of thymidine incorporation into DNA via $\mu$ - and $\kappa$ - opioid receptors in fetal rat brain cell aggregates in culture. Journal of Neurochemistry 60:765-767. 
Barg J, Belcheva M, Rowinski J, Ho A, Burke W, Chung H, Schmidt C, Coscia C (1993b) Opioid receptor density changes in alzheimer amygdala and putamen. Brain Research 632:209-215.

Barnard E, Demoliou-Mason C (1983) Molecular properties of opioid receptors. British Medical Bulletin 39:37-45.

Barnes D, Sato G (1980) Serum-free cell culture: a unifying approach. Cell and Tissue Research 22:649-655.

Barnes P (1986) Asthma as an axon reflex. The Lancet 1:242-245.

Barry M, Halsell C, Whitehead M (1993) Organisation of the nucleus of the solitary tract in the hamster: Acetylcholinesterase, NADH dehydrogenase, and cytochrome oxidase histochemistry. Microscopy Research and Technique 26:231-244.

Barry M, Haglund S, Savoy L (2001) Association of extracellular acetylcholinesterase with gustatory nerve terminal fibres in the nucleus of the solitary tract. Brain Research 921:12-20.

Bausch S, Patterson T, Appleyard S, Chavkin C (1995a) Immunocytochemical localisation of delta opioid receptors in mouse brain. Journal of Chemical Neuroanatomy 8:175189.

Bausch S, Patterson T, Ehrengruber M, Lester H, Davidson N, Chavkin C (1995b) Colocalisation of mu opioid receptors with GIRK1 potassium channels in the rat brain: an immunocytochemical study. Receptors and Channels 3:221-241.

Bayliss D, Viana F, Talley E, Berger A (1997) Neuromodulation of hypoglossal motorneurons: cellular and developmental mechanisms. Respiration Physiology 110:139-150.

Bedell E, DeWitt D, Prough D (1998) Fentanyl infusion preserves cerebral blood flow during decreased arterial blood pressure after traumatic brain injury in cats. Journal of Neurotrauma 15:985-992.

Befort K, Kieffer B (1997) Structure-activity relationships in the $\delta$-opioid receptor. Pain Reviews 4:100-121.

Befort K, Matteí M, Roeckel N, Kieffer B (1994) Chromosomal localization of the delta opioid receptor gene to human IP34.3-36.1 and mouse 4D bands by in situ hybridisation. Genomics 20:143-145.

Beland B, Fitzgerald M (2001) Mu and Delta-opioid receptors are downregulated in the largest diameter primary sensory neurons during postnatal development in rats. Pain 90:143-150.

Belcheva M, Wong Y, Coscia C (2000) Evidence for transduction of mu but not kappa opioid modulation of extracellular signal-regulated kinase activity by $G_{z}$ and $G_{12}$ proteins. Cellular Signalling 12:481-489.

Belcheva M, Ignatova E, Young E, Coscia C (1996) Agonist-induced desensitization and down-regulation of $\delta$-opioid receptors. 35:14818-14824.

Bem W, Yeung S, Belcheva M, Barg J, Cosica C (1991) Age-dependent changes in the subcellular distribution of rat brain $\mu$-opioid receptors and GTP binding regulatory proteins. Journal of Neurochemistry 57:1470-1477.

Berger A (1977) Dorsal respiratory group neurons in the medulla of cat: spinal projections, responses to lung inflation and superior laryngeal nerve stimulation. Brain Research 135:231-254.

Berger A, Averill D, Cameron W (1984) Morphology of inspiritory neurons located in the ventrolateral nucleus of the tractus solitarius of the cat. Journal of Comparative Neurology 224:60-70. 
Besse D, Lombard M, Zajac J, Roques B, Besson J (1990) Pre- and postsynaptic distributions of $\mu, \delta$, and $\kappa$ opioid receptors in the superficial layers of the cervical dorsal horn of the rat spinal cord. Brain Research 521:15-22.

Bianchi A, Denavit-Saubíe M, Champagnat J (1995) Central control of breathing in mammals: neuronal circuitary, membrane properties, and neurotransmitters. Physiological Reviews 75:1-45.

Bidlack J (2000) Detection and function of opioid receptors on cells from the immune system. Clinical and Diagonistic Laboratory Immunology 7:719-723.

Binder A, Frankfurter A, Kim H, Cáceres A, Payne M, Rebhun L (1984) Heterogeneity of microtubule-associated protein 2 during rat brain development. Proceedings of the National Academy of Science USA 81:5613-5617.

Blake A, Bot G, Reisine T (1996) Structure-function analysis of the cloned opiate receptors: peptide and small molecule interactions. Chemistry and Biology 3:967972.

Blake A, Bot G, Freeman J, Reisine T (1997) Differential opioid agonist regulation of the mouse $\mu$ opioid receptor. The Journal of Biological Chemistry 272:782-790.

Bohn L, Gainetdinov R, Lin F, Lefkowitz R, Caron M (2000) $\mu$-opioid receptor desensitization by $\beta$-arrestin- 2 determines morphine tolerance but not dependance. Nature 408:720-723.

Bond C, LaForge K, Tian M, Melia D, Zhang S, Borg L, Gong J, Schuluger J, Strong J, Leal S, Tischfield J, Kreek M, Yu L (1998) Single-nucleotide polymorphism in the human mu opioid receptor gene alters $\beta$-endorphin binding and activity: possible implications for opiate addiction. Proceedings of the National Academy of Science USA 95:9608-9613.

Bonham A (1995) Neurotransmitters in the CNS control of breathing. Respiration Physiology 101:219-230.

Bouret S, Prevot V, Croix D, Viltart O, Stefano G, Mitchell V, Beauvillain J (2000) $\mu$ opioid receptor mRNA expression in neuronal nitric oxide synthaseimmunopositive preoptic area neurons. Molecular Brain Research 80:46-52.

Bouvier M (2001) Oligomerization of G-protein-coupled transmitter receptors. Nature 2:274-286.

Bradford M (1976) A rapid and sensitive method for the quantitation of microgram quantities of protein utilizing the principle of protein-dye binding. Analytical Biochemistry 72:248-254.

Bragg P, Zwass M, Lau M, Fisher D (1995) Opioid pharmacodynamics in neonatal dogs: differences between morphine and fentanyl. Journal of Applied Physiology 79:1519-1524.

Brewer G (1995) Serum-free B27/neurobasal medium supports differentiated growth of neurons from the striatum, substantia nigra, septum, cerebral cortex, cerebellum and dentate gyrus. Journal of Neuroscience Research 42:674-683.

Brewer G (1997) Isolation and culture of adult rat hippocampal neurons. Journal of Neuroscience Methods 71:143-155.

Brewer G, Cotman C (1989) Survival and growth of hippocampal neurons in defined medium at low density; advantages of a sandwich culture technique or low oxygen. Brain research 494:65-74.

Brewer G, Price P (1996) Viable cultured neurons in ambient carbon dioxide and hibernation storage for a month. Neuroreport 7:1509-1512.

Brewer G, Torricelli J, Evege E, Price P (1993) Optimized survival of hippocampal neurons in B27-supplemented Neurobasal ${ }^{\mathrm{TM}}$, a new serum-free medium. Journal of Neuroscience Research 35:567-576. 
Brewer G, Torricelli J, Evege E, Price P (1994) Neurobasal ${ }^{\mathrm{TM}}$ Medium/B27 supplement: A new serum-free medium combination for survival of Neurons. Focus 16:6-9.

Brody M (1986) Central nervous system mechanisms of arterial pressure regulation. Federation Proceedings 45:2700-2706.

Bryant T, Yoshida S, De Castro D, Lipski J (1993) Expiratory neurons of the Bötzinger complex in the rat: a morphological study following labeling with biocytin. The Journal of Comparative Neurology 335:267-282.

Bunzow J, Zhang G, Bouvier C, Saez C, Rønnekleiv O, Kelly M, Grandy D (1995) Chacterization and distribution of a cloned rat $\mu$-opioid receptor. Journal of Neurochemistry 64:14-24.

Burton M, Nouri M, Kazemi H (1995) Acetylcholine and central respiratory control: perturbations of acetylcholine synthesis in the isolated brainstem of the neonatal rat. Brain Research 670:39-47.

Buse E (1985) A method for the collection of defined areas from the embryonic rat brain for cell and tissue culture. Journal of Neuroscience Methods 14:177-186.

Butera R, Rinzel J, Smith J (1999) Models of respiratory rhythm generation in the preBötzinger complex. I. bursting pacemaker neurons. Journal of Neurophysiology 82:131-147.

Cáceres A, Binder L, Payne M, Bender P, Rebhun L, Steward O (1983) Differential subcellular localisation of tubulin and the microtubule-associated protein MAP2 in brain tissue as revealed by immunocytochemistry with monoclonal hybridoma antibodies. The Journal of Neuroscience 4:394-410.

Cahill C, ZLee M, Vincent J, Collier B, Beaudet A (2001a) Prolonged morphine treatment targets $\delta$ opioid receptors to neuronal plasma membranes and enhances $\delta$-mediated antinociception. The Journal of Neuroscience 21:7598-7607.

Cahill C, McClellan K, Morinville A, Hoffert C, Hubatsch C, O'Donnell D, Beaudet A (2001b) Immunohistochemical distribution of delta opioid receptors in the rat central nervous system: Evidence for somatodendritic labeling and antigen-specific cellular compartmentalization. The Journal of Comparative Neurology 440:65-84.

Capeyrou R, Riond J, Corbani M, Lepage J, Bertin B, Emorine L (1997) Agonist-induced signalling and trafficking of the $\mu$-opioid receptor: role of serine and threonine residues in the third cytoplasmic loop and c-terminal domain. FEBS Letters 415:200-205.

Carr D (1988) Opioids. International Anesthesiology Clinics 26:273-278.

Carr D, Bost K, Blalock J (1986) An antibody to a peptide specified by an RNA that is complementary to $\gamma$-endorphin mRNA recognizes an opiate receptor. Journal of Neuroimmunology 12:329-337.

Carr D, De Costa B, AE J, Bost K, Rice K, Blalock J (1987) Immunoaffinity-purified opiate receptor specifically binds the $\delta$-class opiate receptor ligand, cis-(+)-3mrthylfentanylisothiocynate, SUPERFIT. FEBS Letters 224:272-276.

Cavun S, Resch G, Evec A, Rapacon-Baker M, Millington W (2001) Blocade of delta opioid receptors in the ventrolateral periaqeductal gray region inhibits the fall of blood pressure evoked by hemorrhage. Journal of Pharmacology and Experimental Therapeutics 297:612-619.

Ceccatelli S, Millhorn D, Hökfelt T, Goldstein M (1989) Evidence for the occurance of an enkephalin-like peptide in adrenaline and noradrenaline neurons of the rat medulla oblongata. Experimental Brain Research 74:631-640.

Ceccatelli S, Seroogy K, Millhorn D, Terenius L (1992) Presence of a dynorphin-like peptide in a restricted subpopulation of catecholaminergic neurons in rat nucleus tractus solitarii. Brain Research 589:225-230. 
Chan J, Chiu T, Wong Y (1995) Activation of type II adenylyl cyclase by the cloned muopioid receptor: coupling to multiple $\mathrm{G}$ proteins. Journal of Neurochemistry 65:2682-2689.

Chang K, Eckel R, Blanchard S (1982) Opioid peptides induce reduction of enkephalin receptors in cultured neuroblastoma cells. Nature 296:446-448.

Chao C, Hu S, Shark K, Sheng W, Gekker G, Peterson P (1997) Activation of mu opioid receptors inhibits microglial cell chemotaxis. The Journal of Pharmacology and Experimental Therapeutics 281:998-1004.

Chaturvedi K, Shahrestanifar M, Howells R (2000) mu opioid receptor: role for the amino terminus as a determinant of ligand binding affinity. Molecular Brain Research 76:64-72.

Chaturvedi K, Bandari P, Chinen N, Howells R (2001) Proteosome involvement in agonist-induced down-regulation of $\mu$ and $\delta$ opioid receptors. Journal of Biological Chemistry 276:12345-12355.

Chauhan N, Siegel G (1997) Age-dependent organotypic expression of microtubuleassociated protein (MAP1, MAP2, and MAP5) in rat brain. Neurochemical Research 22:713-719.

Chavez C, Ostrea E, Stryker J, Smialek Z (1979) Sudden infand death syndrome among infants of drug-dependent mothers. The Journal of Pediatrics 95:407-409.

Chen B, Szabolcs M, Matsushima A, Erlanger B (1996) A strategy for immunohistochemical signal enhancement by end-product amplification. The Journal of Histochemistry and Cytochemistry 44:819-824.

Chen H, Seybold V, Loh H (2000) An autoradiographic study in mu opioid receptor knockout mice. Molecular Brain Research 76:170-172.

Chen J, Dymshitz J, Vasko M (1997) Regulation of opioid receptors in rat sensory neurons in culture. Molecular Pharmacology 51:666-673.

Chen L, LC W, Liu H, Qiu Y, Chan Y (2001) Cholinergic neurons expressing substance P receptor (NK1) in the basal forebrain of the rat: a double immunocytochemical study. Brain Research 904:161-166.

Chen Y, Mestek A, Li J, Yu L (1993a) Molecular cloning of rat $\kappa$ opioid receptor reveals sequence similarities with $\mu$ and $\delta$ opioid receptors. Biochemistry Journal 295:625628.

Chen Y, Mestek A, Liu J, Hurley J, Yu L (1993b) Molecular cloning and functional expression of a $\mu$-opioid receptor from rat brain. Molecular Pharmacology 44:8-12.

Chen Z, Hedner J, Hedner T (1990) Local effects of substance P on respiratory regulation in rat medulla oblongata. Journal of Applied Physiology 68:693-699.

Cheng J, Standifer K, Tublin P, Su W, Pasternak G (1995a) Demonstration of k3-opioid receptors in the SH-SY5Y human neuroblastoma cell line. Journal of Neurochemistry 65:170-175.

Cheng P, Liu-Chen L, Pickel V (1996) Immunolabeling of mu opioid receptors in the rat nucleus of the solitary tract: Extrasynaptic plasmalemmal localization and association with $\mathrm{Leu}^{5}$-enkephalin. The Journal of Comparative Neurology 371:522536.

Cheng P, Liu-Chen L, Pickel V (1997) Dual ultrastructural immunocytochemical labelling of $\mu$ and $\delta$ opioid receptors in the superficial layers of the rat cervical spinal cord. Brain Research 778:367-380.

Cheng P, Decena J, Wu D, Cheng Y, Szeto H (1991) The effects of selective $\mathrm{mu}_{1}$ opiate receptor blocade on breathing patterns in the fetal lamb. Pediatric Research 30. 
Cheng P, Wu D, Decena J, McCabe S, Szeto H (1993a) Opioid-induced stimulation of fetal respiratory activity by $\left[\mathrm{D}-\mathrm{Ala}^{2}\right.$ ]deltorphin I. European Journal of Pharmacology 230:85-88.

Cheng P, Wu D, Decena J, Cheng Y, McCabe S, Szeto H (1992) Central opioid modulation of breathing dynamics in the fetal lamb: effects of [D-Pen ${ }^{2}$, D-Pen $\left.{ }^{5}\right]-$ enkephalin and partial antagonism by naltrindole. Journal of Pharmacology and Experimental Therapeutics 262:1004-1010.

Cheng P, Wu J, Soong Y, McCabe S, Decena, Szeto H (1993b) Role of $\mu 1$ - and $\delta$-opioid receptors in modulation of fetal EEG and respiratory activity. American Journal of Physiology 265:R433-438.

Cheng P, Svingos A, Wang H, Clarke C, Jenab S, Beczkowska I, CE I, Pickel V (1995b) Ultrastructural immunolabelling shows prominent presynaptic vesicular localization of $\delta$-opioid receptor within both enkephalin- and nonenkephalincontaining axon terminals in the superficial layers of the rat cervical spinal cord. The Journal of Neuroscience 15:5976-5988.

Cheung N, Hickling Y, Beart P (1997) Development and survival of rat embryonic dopaminergic neurons in serum-free, antioxidant-rich primary cultures. Neuroscience Letters 233:13-16.

Chu P, Murray S, Lissin D, von Zastrow M (1997) $\delta$ and $\kappa$ opioid receptors are differentially regulated by Dynamin-dependent endocytosis when activated by the same alkaloid agonist. The Journal of Biological Chemistry 272:27124-27130.

Clendeninn N, Petraitis M, Simon E (1976) Ontological development of opiate receptors in rodent brain. Brain Research 118:157-160.

Colman A, Miller J (2001) Modulation of breathing by $\mu_{1}$ and $\mu_{2}$ opioid receptor stimulation in neonatal and adult rats. Respiration Physiology 127:157-172.

Colman A, Miller J (2002) Lack of involvement of $\mu 1$ opioid receptors in dermorphininduced inhibition of hypoxic and hypercapnic ventilation in rat pups. Respiratory Physiology and Neurobiology 131:199-212.

Commons K, Van Bockstaele E, Pfaaft D (1999) Frequent colocalization of mu opioid and NMDA-type glutamate receptors at postsynaptic sites in periaqueductal gray neurons. The Journal of Comparative Neurology 408:549-559.

Commons K, Beck S, Rudoy C, Van Bockstaele E (2001) Anatomical evidence for presynaptic modulation by the delta opioid receptor in the ventrolateral periaqueductal gray of the rat. The Journal of Comparative Neurology 430:200208.

Connor M, Christie M (1999) Opioid receptor signalling mechanisms. Clinical and Experimental Pharmacology and Physiology 26:493-499.

Copray J, Leim R (1993) Survival and neurite formation of mesencephalic trigeminal neurones of the rat in vitro. Archives of Oral Biology 38:547-557.

Copray J, Bastiaansen M, Gibbons H, van Roon W, Comer A, Lipski J (1999) Neurotrophic requirements of rat embryonic catecholaminergic neurons from the rostral ventrolateral medulla. Developmental Brain Research 116:217-222.

Coqueral A, Buser M, Taynot J, Matray F, Proust B (1992) Beta-endorphin and neurotensin in brainstem and cerebrospinal fluid in the sudden infant death syndrome. Neurochemistry International 20:97-102.

Coyle J, Pert C (1976) Ontogenic development of $\left[{ }^{3} \mathrm{H}\right]$ naloxone binding in rat brain. Neuropharmacology 15:555-560.

Cvejic S, Devi L (1997) Dimerization of the $\delta$-opioid receptor. The Journal of Biological Chemistry 272:26959-26964. 
Cvejic S, Devi, LA. (1997) Dimerization of the opioid receptor: implication for a role in receptor internalization. The Journal of Biological Chemistry 272:26959-26964.

Dado R, Law P, Loh H, Elde R (1993) Immunoflurorescent identification of a delta $(\delta)$ opioid receptor on primary afferent nerve terminals. NeuroReport 5:341-344.

Dashwood M, Muddle J, Spyer K (1988) Opiate receptor subtypes in the nucleus tractus solitarii of the cat: the effect of vagal section. European Journal of Pharmacology 155:85-92.

de Boer-van Huizen R, ten Donkelaar H (1999) Early delvelopment of descending supraspinal pathways: a tracing study in fixed and isolated rat embryos. Anatomy and Embryology 199:539-547.

De Haas R, Verwoerd N, Van Der Corput M, Van Gijlswijk R, Siitari H, Tanke H (1996) The use of peroxidase-mediated deposition of biotin-tyramide in combination with time-resolved fluorescence imaging of euripium chelate label in immunohistochemistry and in situ hybridisation. The Journal of Histochemistry and Cytochemistry 44:1091-1099.

De Lima A, Merten M, Voigt T (1997) Neuritic differentiation and synaptogenesis in serum-free neuronal cultures of the rat cerebral cortex. The Journal of Comparative Neurology 382:230-246.

Del Negro C, Morgado-Valle, Feldman J (2002) Respiratory rhythm: an emergent network property. Neuron 34:821-830.

Delay-Goyet P, Zajac J, Javoy-Agid F, Agid Y, Roques B (1987) Regional distribution of $\mu, \delta$, and $\kappa$ opioid receptors in human brains from controls and parkinsonian subjects. Brain Research 414:8-14.

Delay-Goyet P, Zajac, JM, Roques, BP. (1990) Improved quantitative radioautography of rat brain $\delta$-opioid binding sites using $\left[{ }^{3} \mathrm{H}\right]$ DSTBULET, a new highly potent and selective linear enkephalin analogue. Neurochemistry International 16:341-368.

Delfs J, H K, A M, Y C, L Y, T R, MF. C (1994) Expression of mu opioid receptor mRNA in rat Brain: An in situ hybridization study at the single cell level. The Journal of Comparative Neurology 345:46-68.

Denavit-Saubíe M, Champagnat J, Zieglgansberger W (1978) Effects of opiates and methionine-enkephalin on pontine and bulbar respiratory neurons of the cat. Brain Research 155:55-67.

Dhawan B, Cesselin F, Raghubir R, Reisine T, Bradley P, Portoghese P, Hamon M (1996) International union of pharmacology. XII. Classification of opioid receptors. Pharmacological Reviews 48:567-592.

Di Scala-Guenot D, Strosser M, Richard P (1990) Expression of vasopressin and opiates but not of oxytocin genes studied by in situ hybridisation in embryonic rat brain primary cultures. Developmental Brain Research 56:35-39.

Díaz A, Pazos A, Flórez, Hurlé M (2000) Autoradiographic mapping of mu-opioid receptors during opiate tolerance and supersensitivity in the rat central nervous system. Naunyn-Schmiedeberg's Arch Pharmacol 362:101-109.

Díaz A, Pazos J, Flórez J, Ayesta F, Santana V, Hurlé M (2002) Regulation of $\mu$-opioid receptors, G-protein-coupled receptor kinases and $\beta$-arrestin 2 in the rat brain chronic opioid receptor antagonism. Neuroscience 112:345-353.

Ding M, Robel L, James A, Eisenstat D, Leckman J, Rubenstein J, Vaccarino F (1996a) Dlx-2 homeobox gene controls neuronal differentiation in primary cultures of developing basal ganglia. Journal of Molecular Neuroscience 8:93-113.

Ding Y, Nomura S, Kaneko T, Mizuno N (1995) Colocalization of $\mu$-oppioid receptor-like and substance P-like immunoreactivities in axon terminals within the superficial 
layers of the medullary and spinal dorsal horns of the rat. Neuroscience Letters 198:45-48.

Ding Y, Kaneko T, Nomura S, Mizuno N (1996b) Immunohistochemical localization of $\mu$ opioid receptors in the central nervous system of the rat. The Journal of Comparative Neurology 367:375-402.

Dohlman H (1991) Model systems for the study of seven-transmembrane-segment proteins. Annual Review of Biochemistry 60:653-688.

Dutla M, Gilchrist D, Sansom A, Smith P, Darlington C (1996) Experimental Neurobiology. Experimental Neurobiology 141:141-144.

El Kouhen R, El Kouhen O, Law P, Loh H (1999) The absence of a direct correlation between the loss of [D-Ala $\left.{ }^{2}, \mathrm{MePhe}^{4}, \mathrm{Gly}^{5}-\mathrm{ol}\right]$ enkephalin inhibition of adenylyl cyclase activity and agonist-induced $\mu$-opioid receptor phosphorylation. The Journal Of Biological Chemistry 274:9207-9215.

El Kouhen R, Wang G, Solberg J, Erikson L, Law P, Loh H (2000) Hierarchical phosphorylation of $\delta$-opioid receptor regulates agonist-induced receptor desensitization and internalization. The Journal of Biological Chemistry 275:36659-36664.

El Kouhen R, Burd A, Erickson-Herbrandson L, Chang C, Law P, Loh H (2001) Phosphorylation of $\mathrm{Ser}^{363}$, $\mathrm{Thr}^{370}$, and $\mathrm{Ser}^{375}$ residues within the carboxyl tail differentially regulates $\mu$-opioid receptor internalization. The Journal of Biological Chemistry 276:12774-12780.

Elde R, Arvidsson U, Riedl M, Vulchanova L, Lee J, Dado R, Nakano A, Chakrabarti S, Zhang X, Loh H, Law P, Hökfelt T, Wessendorf M (1995) Distribution of neuropeptide receptors. Annuals New York Academy of Science 757:390-404.

Elliott J, Smart D, Lambert D, Traynor J (1994) Characterisation of $\mu$ opioid receptors on SH-SY5Y cells using naloxonazine and B-funaltrexamine. European Journal of Pharmacology 268:447-450.

Entwistle A, Noble M (1992) The use of lucifer yellow, bodipy, FITC, TRITC, RITC and texas red for dual immunofluorescence visualized with a confocal scanning laser microscope. Journal of Microscopy 168:219-238.

Eppler M, Hulmes J, Wang J, Johnson B, Corbett M, Luthin D, Uhl G, Linden J (1993) Purification and partial amino acid sequence of a $\mu$ opioid receptor from rat brain. The Journal Of Biological Chemistry 268:26447-26451.

Erikson P, Hansson E, Rönnbäck L (1990) Opiate receptors in neuronal primary cultures. Neuropharmacology 29:799-804.

Erikson P, Carlsson B, Isaksson O, Hansson E, Rönnbäck L (1992) Altered amounts of Gprotein $\mathrm{mRNA}$ and $\mathrm{cAMP}$ accumulation after long-term opioid receptor stimulation of neurons in primary culture from the rat cerebral coetex. Molecular Brain Research 14:317-325.

Evans C, Keith Jr D, Morrison H, Magendzo K, Edwards R (1992) Cloning of a delta opioid receptor by functional expression. Science 258:1952-1955.

Faden A, Holaday J (1979) Opiate antagonists: a role in the treatment of hypovolemic shock. Science 205:317-318.

Fan S, Crain S (1995) Dual regulation by $\mu, \delta$ and $\kappa$ opioid receptor agonists of K+ conductance of DRG neurons and neuroblastoma x DRG neuron hybrid F11 cells. Brain Research 696:97-105.

Fan S, Shen K, Scheider M, Crain S (1992) F11 neuroblastoma x DRG neuron hybrid cell express inhibitory $\mu$ and $\delta$ opioid receptors which increase voltage-dependent $\mathrm{K}+$ currents upon activation. Brain Research 590:329-333. 
Ferber C, Sanchez P, Lemoine P, Mouret J (1988) Successful treatment of sleep apnoeas with naltrxone. A clinical, polysomnographic and oxymetric study. Academie des Sciences, Paris 307:695-700.

Feuerstein G (1985) The opioid system and central cardiovascular control: analysis of controversies. Peptides 6:51-56.

Fields H, Heinricher M (1985) Anatomy and physiology of a nociceptive modulatory system. Philosophy Transcripts Royal Society London B308:361-374.

Finley J, Katz D (1992) The central organisation of carotid body afferent projections to the brainstem of the rat. Brain Research 572:108-116.

Fitzgerald S, Willis M, Rigatto H (1992) In search of the central respiratory neurons: I. Dissociated cell cultures of respiratory areas from the upper medulla. Journal of Neuroscience Research 33:579-589.

Flórez J, Hurlé M (1993) Opioid in respiration and vomiting. In: Opioids II Chaper 41 (Herz A, ed), pp 263-292. Berlin Heildeberg: Spinger-Verlag.

Freeman J, Nicholson D (2001) Ontogenic changes in the neural mechanisms of eyeblink conditioning. Integrative Physiological and Behavioural Science 36:15-35.

Freye E, Latasch L, Portoghese P (1992) The delta receptor is involved in sufentanilinduced respiratory depression-opioid subreceptors mediate different effects. European Journal of Anaesthesiology 9:457-462.

Fukuda K, Kato S, Mori K, Nishi M, Takeshima H (1993) Primary structures and expression of rat opioid receptor $\delta$-and $\mu$-subtypes. FEBS Letters 327:311-314.

Funk G, Feldman J (1995) Generation of respiratory rhythm and pattern in mammals: insights from developmental studies. Current Opinion in Neurobiology 5:778-785.

Gacel G, Fournie-Zaluski M, Roques B (1980) D-Tyr-Ser-Gly-Phe-Leu-Thr, a highly preferential ligand for delta-opiate receptors. FEBS Letters 118:245-247.

Gamboa-Esteves F, Tavares I, Imeida A, Batten T, PN M, Lima D (2001) Projection sites of superficial and deep spinal dorsal horn cells in the nucleus tractus solitarii of the rat. Brain Research 921:195-205.

Garzón J, Juarros J, Castro M, Sanchez-Blazquez P (1995) Antibodies to the cloned $\mu$ opioid receptor detects various molecular weight forms in areas of mouse brain. Molecular Pharmacology 47:739-744.

Gaudriault G, Nouel D, Farra C, Beaudet A, Vincent J (1997) Receptor-induced internalization of selective peptidic $\mu$ and $\delta$ opioid ligands. The Journal of Biological Chemistry 272:2880-2888.

Gavériaux-Ruff C, Peluso J, Befort K, Simonin F, Zilliox C, Kieffer B (1997) Detection of opioid receptor mRNA by RT-PCR reveals alternative splicing for the $\delta$ - and $\kappa$ opioid receptors. Molecular Brain Research 48:298-304.

George S, Fan T, Xie Z, Tse R, Tam V, Varghese G, O'Dowd B (2000) Oligomerization of $\mu-$ and $\delta$-opioid receptors. Generation of novel functional properties. The Journal of Biological Chemistry 275:26128-26135.

George S, Zastawny R, Briones-Urbina R, Cheng R, Nguyen T, Heiber M, Kouvelas A, Chan A, O'Dowd B (1994) Distinct distributions of mu, delta, and kappa, opioid receptors mRNA in rat brain. Biochemical and Biophysical Research Communications 205.

Georges F, Normand E, Bloch B, Moine C (1998) Opioid receptor gene expression in the rat brain during ontogeny, with special reference to the mesostriatal system: an in situ hybridisation study. Developmental Brain Research 109:187-199.

Gies E, Peters D, Gelb C, Knag K, Peterfreund R (1997) Regulation of mu opioid receptor mRNA levels by activation of protein kinase C in human SH-SY5Y neuroblastoma cells. Anesthesiology 87:1127-1138. 
Gingras-Leatherman J, McNamara M, Lawson E (1986) Age-dependent influence of hypoxia and methionine-enkephalin concentration within rabbit brainstem nuclei. Pediatric Research 20:655-657.

Glasel J, Bradbury W, Venn R (1983) Properties of murine anti-morphine antibodies. Molecular Immunology 20:1419-1422.

Gluckman P, Marti-Henneberg C, Kaplin S, Li C, Grumbach M (1980) Hormone ontogeny in the ovine fetus. $X$. The effects of $\beta$-endorphin and naloxone on circulating growth hormone, prolactin, and chorionic somatomamotropin. Endocrinology 107:76-80.

Goldstein A, Naidu A (1989) Multiple opioid receptors: ligand selectivity profiles and binding site signatures. The American Journal of Pharmacology and Experimental Therapeutics 36:265-272.

Goldstein A, Fischli W, Lowney L, Hunkapiller M, Hood L (1981) Porcine pituitary dynorphin: complete amino acid sequence of the biologically active heptadecapeptide. Proceedings of the National Acadamy of Science USA 78:72197223.

Gomes I, Gupta A, Trapaidze N, Nagy V, Devi L (2000) Heterodimerization of $\mu$ and $\delta$ opioid receptors: A role in opiate synergy. The Journal of Neuroscience 20:1-5.

Gong L, Ding Y, Wang D, Zheng H, Qin B, Li J, Kaneko T, Mizuno N (1997) GABAergic synapses on $\mu$-opioid receptor-expressing neurons in the superficial dorsal horn: an electron microscope study in the cat spinal cord. Neuroscience Letters 227:33-36.

Gonzalez J, Brogden R (1988) Naltrexone, A review of its pharmacodynamics and pharmacokinetic properties and therapeutic efficacy in the management of opioid dependence. Drugs 35:192-213.

Goodman C, Heyliger S, Emilien B, Partilla J, Yang Y, Cadet J, Rothman R (1999) Chronic exposure to antibodies directed against anti-opiate peptides alter $\delta$-opioid receptor levels. Peptides 20:1419-1424.

Goodman R, Snyder S, Kuhar M, Young W (1980) Differentiation of delta and mu opiate receptor localisations by light microscopic autoradiography. Proceedings of the National Acadamy of Science USA 77:6239-6243.

Goody R, Oakley S, Filliol D, Kieffer B, Kitchen I (2002) Quantitative autoradiographic mapping of opioid receptors in the brain of $\delta$-opioid receptor gene knockout mice. Brain Research 945:9-19.

Górski P, Ulinski S (1996) Effect of occupational exposure to opiates on the respiratory system. International Journal of Occupational Medicine and Environmental Health 9:245-253.

Gouldson P, Snell C, Bywater R, Higgs C, Reynolds C (1998) Domain swapping in Gprotein coupled receptor dimers. Protein Engineering 11:1181-1193.

Gracy K, Svingos A, Pickel V (1997a) Dual ultrastructural localization of mu-opioid receptors and NMDA-type glutamate receptors in the shell of the rat nucleus accumbens. The Journal of Neuroscience 17:4839-4848.

Gracy NK, Svingos A, Pickel M (1997b) Dual ultrastructural localisation of m-opioid receptors and NMDA-type glutamate receptors in the shell of the rat nucleus accumbens. The Journal of Neuroscience 17:4839-4848.

Gray P, Rekling J, Bocchiaro C, Feldman J (1999) Modulation of respiratory frequency by peptidergic input to rhythmogenic neurons in the preBötzinger complex. Science 286:1566-1568.

Gray R, Munks M, Haynes R, Olsen G (2001) Mu opioid receptor efficacy and potency of morphine-6-glucuronide in neonatal guinea pig brainstem membranes: Comparison with transfected CHO cells. Brain Research Bulletin 54:499-505. 
Greer J, Carter J, Al-Zubaidy Z (1995) Opioid depression of respiration in neonatal rats. Journal of Physiology London 485:845-855.

Groneberg D, Fischer A (2001) Endogenus opioids as mediators of asthma. Pulmonary Pharmacology \& Therapeutics 14:383-389.

Grunstein M, Grunstein J (1982) Maturational effect of enkephalin on respiratory control in newborn rabbits. Journal of Applied Physiology 53:1063-1070.

Gulledge C, Mann P, Bridges R, Bialos M, Hammer Jr R (2000) Expression of $\mu$-opioid receptor mRNA in the medial preoptic area of the juvenile rats. Developmental Brain Research 119:269-276.

Gurwell J, Duncan M, Maderspach K, Stiene-Martin A, Elde R, Hauser Kh (1996) אopioid receptor expression defines a phenotypically distinct subpoppulation of astroglia: relationship to $\mathrm{Ca}^{2+}$ mobilization, development, and the antiproliferative. Brain Research 737:175-187.

Gutstein H, Mansour A, Watson S, Akil H, Fields H (1998) Mu and kappa opioid receptors in periaqueductal gray and rostral ventromedial medulla. NeuroReport 9:17771781.

Guyenet P, Stornetta R, Riley T, Norton F, Rosin D, Lynch K (1994) Alpha 2 A-adrenergic receptors are present in lower brainstem catecholaminergic and serotonergic neurons innervating spinal cord. Brain Research 638:285-294.

Guyenet P, Stornetta R, Schreihofer A, Pelaez N, Hayar A, Aicher S, Llewellyn-Smith I (2002) Opioid signalling in the rat rostral ventrolateral medula. Clinical and Experimental Pharmacology and Physiology 29:238-242.

Hampson R, Mu J, Deadwyler S (2000) Cannabinoid and kappa opioid receptors reduce potassium $\mathrm{K}$ current via activation of $\mathrm{G}_{\mathrm{s}}$ proteins in cultured hippocampal neurons. The Americal Journal of Physiology 84:2356-2364.

Harada J, Ueda H, Iso Y, Satoh M (1992) Affinity cross-linked $\delta$-opioid receptor in NG108-15 cells is low molecular weight $(25 \mathrm{kD})$ and coupled to GTP- binding proteins. European Journal of Pharmacology 227:301-307.

Harlan R, Shivers B, Romano G, Howells R, Pfaff D (1987) Localisation of preproenkephalin mRNA in the rat spinal cord by in situ hybridisation. The Journal of Comparative Neurology 258:159-184.

Harlow E, Lane D (1999) Using Antibodies A Laboratory Manual. Cold Springs Harbour, New York: Cold Springs Harbour Laboratory Press.

Harraway J (1997) Introductory Statistical Methods for Biological, Health and Social Sciences. Dunedin: University of Otago Press.

Harrison L, AJ K, Zadina J (1998) Opiate tolerance and dependence: receptors, G-proteins, and antopiates. Peptides 19:1603-1630.

Hassen A, Feuerstein G, Faden A (1982) $\mu$ Receptors and opioid cardiovascular effects in the NTS of rat. Peptides 3:1031-1037.

Hauser K, Mangoura D (1998) Diversity of the endogenous opioid system in development: novel signal transduction translates multiple extracellular signals into neural cell growth and differentiation. Perspectives in Developmental Neurobiology 5:337449.

Hauser K, Osborne J, Stiene-Martin, Melner M (1990) Cellular localization of proenkephalin mRNA and enkephalin peptide products in cultured astrocytes. Brain Research 522:347-353.

Hauser K, Houdi A, Turbek C, Elde R, Maxson W (2000) Opioids intrinsically inhibit the genesis of mouse cerebellar granule neuron precursors in vitro: differential impact of $\mu$ and $\delta$ receptor activation on proliferation and neurite elongation. European Journal of Neuroscience 12:1281-1293. 
Hayashi M, Pires R, Reboucas N, Britto L, Camargo A (2001) Expression of endooligopeptidase $\mathrm{A}$ in the rat central nervous system: a non-radioactive in situ hybridisation study. Molecular Brain Research 89:86-93.

Haynes L (1988) Opioid receptors and signal transduction. 9:309-311.

Hazinski T, Grunstein M, Schlueter M, Tooley W (1981) Effect of naloxone on ventilation in newborn rabbits. Journal of Applied Physiology 50:713-717.

He L, Fong J, von Zastrow M, Whistler J (2002) Regulation of opioid receptor trafficking and morphine tolerance by receptor oligomerization. Proposes that oligomerization of the MOR influences the endocytic properties of the receptor. Cell 108:271-282.

He X, Lee P, Pennypacker K, Tuominen R, Mar E, Thai L, Hong J (1992) Characterisation of dynorphin-containing neurons on dissociated dendate gyrus cell cultures. Brain Research 594:91-98.

Henderson L, Keay K, Bandler R (2002) $\delta$ - and K-opioid receptors in the caudal midline medulla mediate haemorrhage-evoked hypotension. NeuroReport 13:729-733.

Hollander M, Wolfe D (1973a) Nonparametrical Statistical Methods. New York: John Wiley \& Sons.

Hollander M, Wolfe D (1973b) Distribution-free multiple comparisons based on KruskalWallis rank sums. In: Nonparametric Statistical Methods. New York: John Wiley \& Sons.

Hsu S, Raine L, Fanger H (1981) Use of avidin-biotin-peroxidase complex (ABC) in immunoperoxidase techniques: A comparison between $\mathrm{ABC}$ and unlabeled antibody (PAP) proceedures. The Journal of Histochemistry and Cytochemistry 29:577-580.

Huang J, Wang H, Pickel V (2000) Rostrocaudal variation in targeting of N-methyl-Daspartate and mu-opioid receptors in the rat medial nucleus of the solitary tract. The Journal of Comparative Neurology 421:400-411.

Hughes J, Smith T, Kosterlitz H, Fothergill L, Morgan B, Morris H (1975) Identification of two related pentapeptides from the brain with potent opiate agonist activity. Nature 258:577-580.

Hunyady B, Krempels K, Harta G, Mezey E (1996) Immunohistochemical signal amplification by catalyzed reporter deposition and its application in double immunostaining. The Journal of Histochemistry and Cytochemistry 44:1353-1362.

Hutcheson D, Matthes H, Valjient E, Sánchez-Blázquez P, Rodríguez-Díaz M, Garzon J, Kieffer B, Maldonado R (2001) lack of dependence and rewarding effects of deltorphin II in mu-opioid receptor-deficient mice. European Journal of Neuroscience 13:153-161.

Ikeda K, Kobayashi T, Kumanishi T, Yano R, Sora I, Niki H (2002) Molecular mechanisms of analgesia induced by opioids and ethanol: is the GIRK channel one of the keys? Neuroscience Research 44:121-131.

Ingnatova E, Belcheva M, Bohn L, Neuman M, Coscia C (1999) Requirement of opioid receptor internalization for opioid stimulation of mitogen-activated protein kinase; biochemical and imunoflourescence confocal microscopic evidence. The Journal of Neuroscience 19:56-63.

Iscoe S (1988) Central control of the upper airway. In: Lung Biology in Health and Disease (Oommen P, Giuseppe S, eds), pp 125-192. New York: Marcel Dekker Inc.

Iwamoto E, Martin W (1981) Multiple opioid receptors. Medicinal Research Reviews 1:411-440.

James I, Goldstein A (1984) Site-directed alkylation of multiple opioid receptors. I. Binding selectivity. Molecular Pharmacology 25:337-342. 
Javitch J, Fu D, Liapakis G, Chen J (1997) Constitutive activation of the $\beta_{2}$ adrenergic receptor alters the orientation of its sixth membrane-spanning segment. The Journal of Biological Chemistry 272:18546-18549.

Jenab S, Inturrisi C (2002) Retonic acid regulation of mu opioid receptor and c-fos mRNAs and AP-1 DNA binding in SH-SY5Y neuroblastoma cells. Molecular Brain Research 99:34-39.

Johnson S, Smith J, Feldman J (1996) Modulation of respiratory rhythm in vitro: role of $\mathrm{G}_{\mathrm{i} / \mathrm{o}}$ protein-mediated mechanisms. Journal of Applied Physiology 80:2120-2133.

Jordan B, Devi L (1999) G-protein-coupled receptor heterodimerization modulates receptor function. Nature 399:697-700.

Jordan B, Devi L (2001) Oligomerisation of opioid receptors with $\mathrm{B}_{2-}$ adrenergic receptors: a role in trafficing and mitogen-activated protein kinase activation. Proceedings of the National Acadamy of Science USA 98:343-348.

Kadota Y, Niiya A, Masaki R, Yamamoto A, Araki M, Taketani S (1997) A newly identified membrane protein localized exclusively in intracellular organelles of neurons. Molecular Brain Research 46:265-273.

Kalyuzhny A, Arvidsson U, Wu W, Wessendorf M (1996) $\mu$-opioid and $\delta$-opioid receptors are expressed in brainstem antinociceptive circuits: Studies using immunocytochemistry and retrograde tract-tracing. The Journal of Neuroscience 16:6490-6503.

Kandall S, Gaines J, Habel L, Davidson G, Jessop D (1993) Relationship of maternal substance abuse to sudden infant death syndrome in offspring. The Journal of Pediatrics 123:120-126.

Kandel E, Schwartz J, Jessell T (2000) Principles of Neural Science, 4 Edition. USA: McGraw-Hill Companies Inc.

Kaneko T, Minami M, Satoh M, Mizuno N (1995) Immunocytochemical localization of $\mu$ opioid receptor in the rat caudate-putamen. Neuroscience Letters 184:149-152.

Kassof G, Mahanty N, Miller L, Curran T, Connor J, Morgan J (1995) Spontaneous and evoked glutamate signalling influnces Fas-lacZ expression and pyramidal cell death in hippocampal slice cultures from transgenic rats. Molecular Brain Research 34:197-208.

Kato F (1998) Suppression of inspiratory fast rhythm, but not bilateral short-term synchronization, by morphine in anesthetized rabbit. Neuroscience Letters 258:8992.

Kavaliers M, Colwell D, Choleris E (1998) Sex differences in opioid and N-methyl-Daspartate mediated non-opioid biting fly exposure induced analgesia in deer mice. Pain 77:163-171.

Kazmi S, Mishra R (1986) Opioid receptors in human neuroblastoma SH-SY5Y cells: evidence for distinct morphine $(\mu)$ and enkephalin (?) binding sites. Biochemical and Biophysical Research Communications 137:813-820.

Keith D, Murray S, Zaki P, Chu P, Lissin D, Kang L, Evans C, von Zastrow M (1996) Morphine activates opioid receptors without causing their rapid internalization. The Journal of Biological Chemistry 271:19021-19024.

Keith D, Anton B, Murray S, Zaki P, Chu P, Lissin D, Monteillet-Aguis G, Stewart P, Evans C, von Zastrow M (1998) $\mu$-opioid receptor internalization;opiate drugs have differential effects on a conserved endocytic mechanism in vitro and in the mammalian brain. The Americal Society for Pharmacology and Experimental Therapeutics 53:377-384. 
Kent J, Pert C, Herkenham M (1982) Ontogeny of opiate receptors in rat forebrain: visualisation by in vitro autoradiography. Developmental Brain Research 2:487504.

Kieffer B (1999) Opioids: first lessons from knock out mice. Trends in Pharmacological Sciences 20:19-26.

Kieffer B, Gavériaux-Ruff C (2002) Exploring the opioid system by gene knockout. Progress in Neurobiology 66:285-306.

Kieffer B, Befort K, Gavériaux-Ruff C, Hirth C (1992) The $\delta$-opioid receptor: Isolation of a cDNA by expression cloning and pharmacological characterization. Proceedings of the National Acadamy of Science USA 89:12048-12052.

Kinney H, Filiano J, Sleeper L, Mandell F, M V-D, Frost W (1995) Decreased muscarinic receptor binding in the arcuate nucleus in sudden infant death syndrome. Science 269:1446-1450.

Kinney H, Filiano J, Assmann S, Mandell F, Valdes-Dapena M, Krous H, O'Donnell T, Rava L, Frost W (1998) Tritiated-naloxone binding to brainstem opioid receptors in the sudden infant death syndrome. Journal of the Autonomic Nervous System 69:156-163.

Kitchen I, Kelly M, Viveros M (1990) Ontogenesis of $\kappa-$-opioid receptors in rat brain using $\left[{ }^{3} \mathrm{H}\right] \mathrm{U}-69593$ as a binding ligand. European Journal of Pharmacology 175:93-96.

Kitchen I, Slowe S, Matthes H, Kieffer B (1997) Quantitative autoradiographic mapping of $\mu-, \delta$, and $\kappa$-opioid receptors in knockout mice lacking the $\mu$-opioid receptor gene. Brain Research 778:73-88.

Kivell B, FJ MD, JH. M (2000) Serum-free culture of rat postnatal and fetal brainstem neurons. Developmental Brain Research 120:199-210.

Kivell B, FJ MD, JH. M (2001) Method for serum-free culture of late fetal and early postnatal rat brainstem neurons. Brain Research Protocols 6:91-99.

Knapp R, Malatynska E, Collins N, Fang L, Wang J, Hruby V, Roeske W, Yamamura H (1995) Molecular biology and pharmacology of cloned opioid receptors. Faseb Journal 9:516-524.

Knusel B, Hefti F (1988) Development of cholinergic pedunculopontine neurons in vitro: comparison with cholinergic septal cells and response to nerve growth factor, ciliary neurotrophic factor, and retinoic acid. Journal of Neuroscience Research 21:365-375.

Ko J, Chen H, HH. L (2002) Differential promotor usage of mouse $\mu$-opioid receptor gene during development. Molecular Brain Research 104:184-193.

Ko J, U A, FG W, PY L, R E, HH. L (1999) Visualization of time-dependent redistribution of $\delta$-opioid receptors in neuronal cells during prolonged agonist exposure. Molecular Brain Research 69:171-185.

Koch T, Kroslak T, Mayer P, Raulf E, Höllt V (1997) Site mutation in the rat $\mu$-opioid receptor demonstrates the involvement of calcium/calmodulin-dependent protein kinase II in agonist-mediated desentization. Journal of Neurochemistry 69:17671770.

Koch T, Schulz S, Schröder H, Wolf R, Raulf E, Höllt V (1998) Carboxyl-terminal splicing of the rat $\mu$ opioid receptor modulates agonist-mediated internalization and receptor resensitization. The Journal of Biological Chemistry 273:13652-13657.

Koch T, Kroslak T, Averbeck M, Mayer P, Schröder H, Raulf E, Höllt V (2000) Allelic variation $\mathrm{S} 268 \mathrm{P}$ of the human $\mu$-opioid receptor affects both desensitization and $\mathrm{G}$ rotein coupling. Molecular Pharmacology 58:328-334. 
Koch T, Schulz S, Pfeiffer M, Klutzny M, Schröder H, Kahl E, Höllt V (2001) C-terminal splice varients of the mouse $\mu$-opioid receptor differ in morphine-induced internalization and receptor resensitization. The Journal of Biological Chemistry 276:31408-31414.

Kolb V, Koman A, Terenius L (1983) Fluorescent probes for opioid receptors. Life Sciences 33:423-426.

Kolb V, Koman A, Neil A (1985) Synthesis and pharmacological chacterisation of fluorescent opioid receptor probes. Pharmaceutical Research 6:266-271.

Koman A, Einarsson M, Terenius L (1985) Agonist-antagonist properties of flourescent opioid probes in the guinea-pig myenteric plexus-longitudinal muscle preparation. Archives of Pharmacology 331:355-358.

Koob G, Moal M (1997) Drug abuse: hedonic homeostatic dysregulation. Science 278:5257.

Kornblum H, Hurlbut D, Leslie F (1987) Postnatal development of multiple opioid receptors in rat brain. Developmental Brain Research 37:21-41.

Kotz C, Billington C, Levine A (1997) Opioids in the nucleus of the solitary tract are involved in feeding in the rat. American Journal of Physiology 272:R1028-R1032.

Kovoor A, Henry D, Chavkin C (1995) Agonist-induced desensitization of the mu opioid receptor-coupled potassium channel (GIRK1). Journal of Biological Chemistry 270:589-595.

Kramer K, Simon E (1999) Role of protein kinase C (PKC) in agonist-induced $\mu$-opioid receptor down regulation: I. PKC translocation to the membrane of SH-SY5Y neuroblastoma cells is induced by $\mu$-opioid agonists. Journal of Neurochemistry 72:585-604.

Kramer K, Simon E (2000) $\mu$ and $\delta$-opioid receptor agonists induce mitogen-activated protein kinase (MAPK) activation in the absence of receptor internalization. Neuropharmacology 39:1707-1719.

Kraus J, Horn G, Zimprich A, Simon T, Mayer P, Höllt V (1995) Molecular cloning and functional analysis of the rat $\mu$ opioid receptor gene promotor. Biochemical and Biophysical Research Communications 215:591-597.

Kraus J, Börner C, Giannini E, Hickfang K, Braun H, Mayer P, Hoehe M, Ambrosch A, König W, Höllt V (2001) Regulation of $\mu$-opioid receptor gene transcription by interleukin-4 and influence of an allelic variation within a STAT6 transcription factor binding site. Journal of Biological Chemistry 276:43901-43908.

Kuipers I, Maertzdorf W, De Jong D, Hanson M, Blanco C (1997) Initiation and maintenance of continuous breathing at birth. Pediatric Research 42:163-168.

Kulkarni-Narla A, Brown D (2001) Opioid, cannabinoid and vanilloid receptor localization on porcine cultured myentric neurons. Neuroscience Letters 308:153-156.

Laferrière A, Liu J, Moss I (1999) $\mu$ - and $\delta$-opioid receptor densities in respiratory-related brainstem regions of neonatal swine. Developmental Brain Research 112:1-9.

Laferrière A, Liu J, Moss I (2003) Neurokinin-1 versus mu-opioid receptor binding in rat nucleus tractus solitarius after single and recurrent intermittent hypoxia. Brain Research Bulletin 54:307-313.

Lagane B, Gazibelet G, Meihoc E, Masson J, Cèzanne L, Lopez A (2000) Role of sterols in modulating the human $\mu$-opioid receptor function in Saccharomyces cervisiae. Journal of Biological Chemistry 275:33197-33200.

Lakke E (1997) Advances in anatomy embryology and cell biology: The projections to the spinal cord of the rat during development: A timetable of descent. Advances in Anatomy and Cell Biology 135:1-139. 
Law P, Loh H (1999) Regulation of opioid receptor activities. The Journal of Pharmacology and Experimental Therapeutics 289:607-624.

Law P, Hom D, Loh H (1983) Opiate receptor down-regulation and desentization in neuroblastoma x glioma NG108-15 hybrid cells are two separate cellular adaptation processes. Molecular Pharmacology 24:413-424.

Law P, Wong Y, Loh H (1999) Mutational analysis of the structure and function of opioid receptors. Biopolymers 51:440-455.

Law P, Wong Y, Loh H (2000a) Molecular mechanisms and regulation of opioid receptor signaling. Annual Review of Pharmacology and Toxicology 40:389-430.

Law P, Kouhen O, Soberg J, Wang W, Erickson L, Loh H (2000b) Deltorphin II-induced rapid desensitization of $\delta$-opioid receptors requires both phosphorylation and internalization of the receptor. Journal of Biological Chemistry.

Law P, LJ E, El-Kouhen R, L D, Soberg J, Wang W, Miller E, AL B, Loh H (2000c) Receptor density and recycling affect the rate of agonist-induced desensitization of $\mu$-opioid receptor. Molecular Pharmacology 58:388-398.

Lawrence A, Jarrott B (1996) Neurochemical modulation of cardiovascular control in the nucleus tractus solitarius. Progress in Neurobiology 48:21-53.

Lee M, Cahill C, Vincent J, Beaudet A (2002) Internalization and trafficking of opioid receptor ligands in rat cortical neurons. Synapse 43:102-111.

Lefkowitz R (1998) G protein-coupled receptors. The Journal Of Biological Chemistry 273:18677-18680.

Leslie F, Tso S, Hurlbut D (1982) Differential appearance of opiate receptor subtypes in neonatal rat brain. Life Sciences 31:1393-1396.

Levac B, O'Dowd B, SR. G (2001) Oligomerization of opioid receptors: generation of novel signaling units. Current Opinion In Pharmacology 2:76-81.

Levi-Montalcini R (1995) The nerve growth factor: it's mode of action on sensory and sympathetic nerve cells. Trends in Pharmacological Science 16:344-350.

Lewis D (1998) Ventral medullary chemoreceptor inputs to neurons within the nucleus ambiguus. Clinical and Experimental Pharmacology and Physiology 25:479-482.

Li C, Chang D (1976) Isolation and structure of an untriakontapeptide with opiate activity from camel pituitary glands. Proceedings of the National Acadamy of Science USA 73:1145-1148.

Li S, Zhu J, Chen C, Chen Y, JK D, LY. L-C (1993) Molecular cloning and expression of a rat $\kappa$ opioid receptor. Biochemistry Journal 295:629-633.

Lidov H, Molliver M (1982) Immunocytochemical study of the development of serotonergic neurons in the rat CNS. Brain Research Bulletin 9:559-604.

Ling G, Clark J, Pasternak G (1986) Naloxonazine actions in vivo. European Journal of Pharmacology 129:33-38.

Ling G, Spiegel K, Lockheart S, Pasternak G (1985) Seperation of opioid analgesia from respiratory depression: evidence for different receptor mechanisms. The Journal of Pharmacology and Experimental Therapeutics 232:149-155.

Lipski J, Zhang X, Kruszewska B, Kanjhan R (1994) Morphological study of long axonal projections of ventral medullary inspiratory neurons in the rat. Brain Research 640:171-184.

Lipski J, Lin J, Teo M, van Wyk M (2002) The network vs. pacemaker theory of the activity of RVL presympathetic neurons - a comparison with another putative pacemaker system. Autonomic Neuroscience: Basic and Clinical 98:85-89.

Liu H, JT S, Augustin L, JL K, HH. L (1999) Transcriptional regulation of mouse $\delta$-opioid receptor gene. The Journal of Biological Chemistry 274:2367-23626. 
Liu J, Laferrière A, Moss R (2000) Repeated prenatal cocaine increases met-enkephalin immunoreactivity in respiratory-related medulla of developing swine. Brain Research Bulletin 51:419-424.

Llewellyn-Smith (2000) Organisation of inputs to barosensitive pre-sympathetic neurons in rostral ventrolateral medulla. Society of Neuroscience Abstracts 26:1185.

Loeschcke H (1982) Central chemosensitivity and the reaction theory. Journal of Physiology 332:1-24.

Long W, Lawson E (1983) Developmental aspects of the effect of naloxone on control of breathing in piglets. Respiration Physiology 51:119-129.

Loughlin S, Massamiri T, Kornblum H, Leslie F (1985) Postnatal development of opioid systems in rat brain. Neuropeptides 5:469-472.

Lowe J, Celver J, Gurevich V, Chavkin C (2002) Mu opioid receptors desensitize less rapidly than delta opioid receptors due to less efficient activation of arrestin. Journal of Biological Chemistry 277:15729-15735.

Lu Y, Li G, Chi Z (1993) Characterization and distribution of mu opioid receptors in rabbit cerebellum. Zhongguo Yao Li Xue Bao 14:489-491.

Lutz R, Pfister H (1992) Opioid receptors and their Pharmacological profiles. Journal of Receptor Research 12:267-286.

MacDonald R, Werz M (1986) Dynorphin A decreases voltage-dependent calcium conductance of mouse dorsal root ganglion neurons. Journal of Physiology 377:237-249.

Mack K, Killian A, Weyhenmeyer J (1984) Comparison of mu, delta, and kappa opiate binding sites in rat brain and spinal cord. Life Sciences 34:281-285.

MacManus J, Rasquinha I, Black M, Laferrière N, Monette R, Walker T, Morley P (1997) Glutamate-treated rat cortical neuronal cultures die in a way different from the classical apoptosis induced by staurosporine. Experimental Cell Research 233:310320.

Madsen B, Beglan C, Spivak C (2000) Fluorescein-labelled naloxone binding to mu opioid receptors on live chinese hamster ovary cells using confocal fluorescent microscopy. Journal of Neuroscience Methods 97:123-131.

Maekawa K, Minami M, Yabuuchi K, Toya T, Katao Y, Hosoi Y, Onogi T, Satoh M (1994) In situ hybridisation study of $\mu$ - and $\kappa$ - receptor mRNAs in the rat spinal cord. Neuroscience Letters 168:97-100.

Mains R, Patterson P (1973) Primary cultures of dissociated sympathetic neurons. The Journal of Cell Biology 59:329-345.

Maneckjee R, Archer S, Zukin S (1988) Characterization of a polyclonal antibody to the m opioid receptor. Journal of Neuroimmunology 17:199-208.

Mansour A, Fox C, Akil H, Watson S (1995) Opioid-receptor mRNA expression in the rat CNS: anatomical and functional implications. Trends In Neuroscience 18:22-29.

Mansour A, Khachaturian, Lewis M, Akil H, Watson S (1987) Autoradiographic differentiation of $\mathrm{mu}$, delta, and kappa opioid receptors in the rat forebrain and midbrain. The Journal of Neuroscience 7:2445-2464.

Mansour A, Khachaturian, Lewis M, Akil H, Watson S (1988) Anatomy of CNS opioid receptors. Trends in Neuroscience 11:308-314.

Mansour A, Fow C, Burke S, Meng F, Thompson R, Akil H, Watson S (1994) Mu, Delta and Kappa Opioid receptor mRNA expression in the rat CNS: an in situ hybridization study. The Journal of Comparative Neurology 350:412-438.

Mardirosoff C, Dumont L, Boulvain M, Tramèr M (2002) Fetal bradycardia due to intrathecal opioids for labour analgesia: a systematic review. British Journal of Obstetrics and Gynaecology 109:274-281. 
Marriott D, Hirst W, Ljunberg (1995) Astrocytes. In: Neural Cell Culture (Wilkin, ed), pp 85-96. New York: Oxford University Press.

Martin N, PL. P (2001) Interaction of co-expressed $\mu$ - and $\delta$-opioid receptors in transfected rat pituitary $\mathrm{GH}_{3}$ cells. Molecular Pharmacology 59:774-783.

Martin W, Eades C, Thompson J, Huppler R, Gilbert P (1976) The effects of morphine and nalorphine like like drugs in the nondependent and morphine-dependent chronic spinal dog. The Journal of Pharmacology and Experimental Therapeutics 197:517532.

Marvisón J, Martínez V, Grady E, Bunnett E, Mayer E (1997) Neurokinin 1 receptor internalization in spinal cord slices induced by dorsal root stimulation is mediated by NMDA receptors. The Journal of Neuroscience 17:8129-8136.

Massotte D, Kieffer B (1998) A molecular basis for opiate action. Essays in Biochemistry:65-77.

Masuko S, Nakajima Y, Yamaguchi K (1986) Noradrenergic neurons from the locus cerleus in dissociated cell culture: culture methods, morphology, and electrophysiology. The Journal of Neuroscience 6:3229-3241.

Matsumoto B, Wilson L, Matsudaria P (1993) Cell Biological Applications of Confocal Microscopy. In: Methods in Cell Biology (Leslie Wilson PM, ed). New York: Academic Press Inc.

Matthes H, Smadja C, Valverde O, Vonesch J, Foutz A, Boudinot E, Danavit-Saubie M, Severini C, Negri, Roques B, Maldonado R, Kieffer B (1998) Activity of the $\delta$ opioid receptor is partially reduced, whereas activity of the $\mathrm{K}$-receptor is maintained in mice lacking the $\mu$-receptor. The Journal of Neuroscience 18:72857295 .

Matthes H, Maldonado, R Simonin F, Valverde O, Slowe S, Kitchen I, Befort K, Dierich A, Le Meur M, Dollè P, Tzavara E, Hanoune J, Roques B, Kieffer B (1996) Loss of morphine induced analgesia, reward effect and withdrawl symptoms in mice lacking the mu opioid receptor gene. Nature 383::819-823.

Matus A (1988) Microtubule-Associated proteins: Their potential role in determining neuronal morphology. Annual Review of Neuroscience 11:29-44.

Maus M, Premont J, Glowinski J (1990) In vitro effects of 17 beta-oestradiol on the sensitivity of receptors coupled to adenylyl cyclase on striatal neurons in primary culture. Ciba Foundation Symposium 153:145-153.

Mayer P, Höllt V (2001) Allelic and somatic variations in the endogenous opioid system of humans. The Journal of Pharmacology and Experimental Therapeutics 91:167-177.

Mayfield K, Kozak G, Malvin G, Porreca F (1996) Hypoxia decreases opioid delta receptor expression in mouse brain. Neuroscience 72:785-789.

Mazzone S, Canning B (2002) Central nervous system control of the airways: pharmacological implications. Current Opinion in Pharmacology 2:220-228.

McDonald A, Mascagni F (2001) Colocalisation of calcium-binding proteins and GABA in neurons of the rat basolaterral amygdala. Neuroscience 105:681-693.

McMillen I (1986) Opioid peptides: A role in sudden infant death syndrome. Australian Paediatrics Journal Suppl.:75-76.

McVey M, Ramsay D, Kellett E, Rees S, Wilson S, Pope A, Milligan G (2001) Monitoring receptor oligomerization using time-resolved fluorescence resonance energy transfer and bioluminescence resonance energy transfer. Journal of Biological Chemistry 276:14092-14099.

Meng F, Ueda Y, Hoversten M, Thompson R, Taylor L, Watson S, Akil H (1996) Mapping the receptor domains critical for the binding selectivity of $\delta$-opioid receptor ligands. European Journal of Pharmacology 311:285-292. 
Meunier J, Mouledous L, Topham C (2000) The nociceptin (ORL1) receptor: molecular cloning and functional architecture. Peptides 21:893-900.

Mihara H, Lee S, Shimohigashi Y, Aoyagi H, Kato T, Izumiya N, Costa T (1985) $\delta$ and $\mu$ opiate receptor probes: fluorescent enkephalins with high receptor affinity and specificity. FEBS Letters 193:35-38.

Miller J, Azmitia E (1999) Growth inhibitory effects of a mu opioid on cultured cholinergic neurons from fetal rat ventral forebrain, brainstem, and spinal cord. Developmental Brain Research 114:66-77.

Minami M, M. S (1995) Molecular biology of the opioid receptors: structures, functions and distributions. Neuroscience Research 23:121-145.

Minamoto Y, Ogawa K, Abe H, Iochi Y, Mitsugi K (1991) Development of a serum-free and heat sterilizable medium and continuous high-density cell culture. Cytotechnology 5:S35-51.

Mitchel R, Berger A (1975) Neural regulation of respiration. American Review of Respiratory Disease 111:206-224.

Miyawaki T, Goodchild A, Pilowsky P (2002) Activation of mu-opioid receptors in rat venterolateral medulla selectively blocks baroreceptor reflexes while activation of delta opioid receptors somato-sympathetic reflexes. Neuroscience 109:133-144.

Mollereau C, Mouledous L (2000) Tissue distribution of the opioid receptor-like (ORL1) receptor. Peptides 21:907-917.

Momose-Sato Y, Sato K, Kamino K (2001) Optical approaches to embryonic development of neural functions in the brainstem. Progress in Neurobiology 63:151-197.

Monteillet-Agius G, Fein J, Phan T, Anton B, Lam H, Zaki P, Miotto K, Evans C (1996) Regulation and distribution of members of the opioid receptor family. Proceedings: Western Pharmacological Society 39:69-70.

Morin-Surun M, Boudinot E, Fournie-Zaluski M, Champagnat J, Roques B, DenavitSaubié M (1992) Control of breathing by endogenous opioid peptides: possible involvement in sudden infant death syndrome. Neurochemistry International 20:103-107.

Morin-Surun M, Boudinot E, Dubois C, Matthes H, Kieffer B, Denavit-Saubié M, Champagnat J, Foutz A (2001) Respiratory function in adult mice lacking the $\mu$ opioid receptor: role of $\delta$-receptors. European Journal of Neuroscience 13:17031710 .

Moriwaki A, Wang J, Svingos A, van Bockstaele E, Cheng P, Pickel V, Uhl G (1996) $\mu$ opiate receptor immunoreactivity in rat central nervous system. Neurochemical Research 21:1315-1331.

Mortola J (1984) Breathing pattern in newborns. Journal of Applied Physiology 56:15331540 .

Moss I, Scarpelli E (1979) Generation and regulation of breathing in utero: fetal $\mathrm{CO}_{2}$ response test. Journal of Applied Physiology 47:527-531.

Moss I, Scarpelli E (1981) $\beta$-endorphin central depression of respiration and circulation. Journal of Applied Physiology 50:1011-1016.

Moss I, Inman J (1989) Proopiomeloanocortin opioids in brain, CSF, and plasma of piglets during hypoxia. Journal of Applied Physiology 66:2280-2286.

Moss I, Laferrière A (2000) Prenatal cocaine raises $\mu$-opioid receptor density in piglet cardiorespiratory medulla. Neurotoxicology and Teratology 22:3-10.

Moss I, Laferrière A (2002) Central neuropeptide systems and respiratory control during development. Respiratory Physiology and Neurobiology 131:15-27.

Moss I, Scott S, Inman J (1993a) Hypoxia, sleep and respiration in relation to opioids in developing swine. Respiration Physiology 92:115-125. 
Moss I, Scott S, Inman J (1993b) $\mu$ - vs $\delta$-opioid influence on respiratory and sleep behaviour during development. The Americal Journal of Physiology:R754-R760.

Mossberg H, Hurst R, Hruby V, Gee K, Yamamura H, Galligan J, Burks T (1983) Bispenicillamine enkephalins possess highly improved specificity toward $\delta$ opioid receptors. Proceedings of the National Acadamy of Science USA 80:5871-5874.

Mullin J, Weibel J, Diamond L, Kleinzeller A (1980) Sugar transport in the LLC-PK 1 renal epithelial cell line: similarity to mammalian kidney and the influence of cell density. Journal of Cellular Physiology 104:375-389.

Murphey L, Olsen G (1994) Morphine-6- $\beta$-D-Glucuronide respiratory pharmacodynamics in the neonatal guinea pig. The Journal of Pharmacology and Experimental Therapeutics 268:110-116.

Murphey L, Olsen G (1995) Developmental change of mu opioid receptors in neonatal guinea pig brainstem. Developmental Brain Research 85:146-148.

Murray S, Evans C, von Zastrow M (1998) Phosphorylation is not required for dynamindependent endocytosis of a truncated mutant opioid receptor. Journal of Biological Chemistry 273:24987-24991.

Myer E, Morris D, Brase D, Dewey W, Zimmerman A (1990) Naltrexone therapy of apnea in children with elevated cerebrospinal fluid beta-endorphin. Annal of Neurology 27:75-80.

Myers W, Glasel J (1986) Subclass specificity of anti-idiotypic anti-opiate receptor antibodies in rat brain, guinea pig cerebellum, \& neuroblastoma X glioma (NG 108-15). Life Sciences 38:1783-1788.

Nakamura Y, Katakura N, Nakajima M (1999) Generation of rhythmical food injestive activities of the trigeminal, facial, and hypoglossal motorneurons in in vitro CNS preparations isolated from rats and mice. Journal of Medical and Dental Science 46:63-73.

Negri L, Falconieri Erspamer G, Severini C, Potenza R, Melchiorri P, Erspamer V (1992) Dermorphin related peptides from the skin of Phyllomedusa bicolor and their amidated analogs activate two $\mu$-opioid receptor subtypes which modulate antinociception and catalepsy, in the rat. Proceedings of the National Acadamy of Science USA 89:2703-2707.

Nestler E, Aghajanian G (1997) Molecular and cellular basis of addiction. Science 278:5863.

Neubauer J, Posner M, Santiago T, Edelman N (1987) Naloxone reduces ventilatory depression of brain hypoxia. Journal of Applied Physiology 63:699-706.

Nomura S, Ding Y, Kaneko T, Li J, Mizuno N (1996) Localization of $\mu$-opioid receptorlike immunoreactivity in the central components of the vagus nerve: a light and electron microscope study in the rat. Neuroscience 73:277-286.

Ojcius D, Niedergang F, Subtil A, Hellio R, Dautry-Varsat A (1996) Immunology and the confocal microscope. Research Immunology 147:175-188.

Olsen G, Less M (1980) Ventilatory response to carbon dioxide of infants following chronic prenatal methadone exposure. The Journal of Pediatrics 96:983-989.

Olsen G, Olson R, Kastin A (1989) Endogenous opiates:1987. Peptides 10:205-236.

Olsen G, Olsen R, Kastin A (1995) Endogenous opiates: 1994. Peptides 16:1517-1555.

Olson G, Olson R, Kastin A (1997) Endogenous opiates: 1996. Peptides 18:1651-1688.

Osborne J, Kindy M, Spruce B, Hauser K (1993) Ontogeny of proenkephalin mRNA and enkephalin peptide expression in the cerebellar cortex of the rat: spatial and temporal patterns of expression follow maturation gradients in the external granular layer and in purkinje cells. Developmental Brain Research 76:1-12. 
Paakkari P, Paakkari I, Siren A, Feuerstein G (1990) Respiratory and locomotor stimulation by low doses of dermorphin, a $\mathrm{mu}_{1}$ receptor-mediated effect. The Journal of Pharmacology and Experimental Therapeutics 252:235-238.

Påhlman S, Ruusala A, Abrahamsson L, Mattsson M, Esscher T (1984) Retinoic acidinduced differentiation of cultured humen neuroblastoma cells: a comparison with phorbolester-induced differentiation. Cell Differentiation 14:135-144.

Påhlman S, Mamaeva S, Meyerson G, Mattsson M, Bjelfman C, Örtoft E, Hammerling U (1990) Human neuroblastoma cells in culture: a model for neuronal cell differentiation and function. Acta Physiologica Scandanavia 592:25-37.

Pak Y, O'Dowd, George S (1997) Agonist-induced desensitization of the $\mu$ opioid receptor is determined by threonine 394 preceded by acidic amino acids in the COOHterminal tail. The Journal of Biological Chemistry 272:24961-24965.

Pak Y, O'Dowd, Wang J, George S (1999) Agonist-induced, G protein-dependent and independent down-regulation of the $\mu$ opioid receptor. The Journal of Biological Chemistry 274.

Pan Y, Wan J, Zuckeerman A, Pasternak G (1998) Identification and differnetial regional expression of KOR-3/ORL-1 gene splice variants in mouse brain. FEBS Letters 435:65-68.

Pan Y, Mahurter L, Bolan E, Xu M, Pasternak G (2001) Generation of the mu opioid receptor (MOR-1) protein by three new splice variants of oprm gene. Proceedings of the National Acadamy of Science USA 98:14084-14089.

Pan Y, Bolan J, Abbadie C, Chang A, Zuckeerman A, Rossi G, Pasternak G (1999) Identification and characterization of three new alternatively spliced $\mu$-opioid receptor isoforms. Molecular Pharmacology 56:396-403.

Pan Z (1998) $\mu$-opposing actions of the K-opioid receptor. Trends In Physiological Science 19:94-98.

Park Y, Ho I, Jang C, Tanaka S, Ma T, Loh H, Ko K (2001) Effects of morphine on pentobarbital-induced responses in $\mu$-opioid receptor knockout mice. Brain Research Bulletin 54:527-531.

Parks D, Hardy R, LA. H (1983) Dual immunofluorescence - new frontiers in cell analysis and sorting. Immunology Today 4:145-150.

Pasternak G (1993) Pharmacological mechanisms of opioid analgesics. Clinical Neuropharmacology 16:1-18.

Pasternak G, Zhang A, Tecott L (1980) Developmental differences between high and low affinity opiate binding sites: their relationship to analgesia and respiratory depression. Life Sciences 27:1185-1190.

Paul, D, Bodner R, Gistrak M, Pasternak G (1989) Different $\mu$ receptor subtypes mediate spinal and supraspinal analgesia in mice. European Journal of Pharmacology 168:307-314.

Pavord I, Hall I, Wahedna I, Cooper S, Tatterafield A (1994) Effect of 443c81, an inhaled $\mu$-opioid receptor agonist in asthma. Clinical and Experimental Allergy 24:144148.

Paxinos G, Watson C (1986) The Rat Brain In Stereotaxic Coordinates, 2nd edition. New York: Academic Press Inc.

Paxinos G, Ashwell K, Törk I (1994) Atlas Of The Developing Rat Nervous System. New York: Academic Press Inc.

Pecci Saavedra J, Brusco A, Lopez-Costa J, Gomez L, Lopez E (1993) Antibodies as molecular probes in neurobiology. Molecular Neurobiology 6:387-405. 
Peckys D, Landwehrmeyer (1999) Expression of mu, kappa, and delta opioid receptor messenger RNA in the human CNS: a ${ }^{33} \mathrm{P}$ in situ hybridisation study. Neuroscience 88:1093-1135.

Pert C, Snyder S (1974) Opiate receptor binding of agonists and antagonists affected differentially by sodium. Molecular Pharmacology 11:735.

Pert C, Pasternak G, Snyderm S (1973) Opiate agonists and antagonists discriminated by receptor binding in rat brain. Science 182:1357-1361.

Petäja-Repo U, Hogue M, Lapèrrière A, Walker P, Bouvier M (2000) Export from the endoplasmic reticulum represents the limiting step in the maturation and cell surface expression of the human $\delta$ opioid receptor. The Journal of Biological Chemistry 275:13727-13736.

Petäjä-Repo U, Hogue M, Lapèrrière A, Bhalla S, Walker P, Bouvier M (2001) Newly synthesized human $\delta$-opioid receptors retained in the endoplasmic reticulum are retrotranslocated to the cytosol, deglycosylated, ubiquitinated, and degraded by the proteasome. Journal of Biological Chemistry 276:4416-4423.

Petrillo P, Tavani A, Verotta D, Robson L, Kosterlitz H (1987) Differential postnatal development of $\mu$-, $\delta$ - and $\kappa$-opioid binding sites in rat brain. Developmental Brain Research 31:53-58.

Pfeiffer A, Pfeiffer D, Feuerstein G, AI, Kopin I (1984) An increase in opiate receptor-sites is associated with enhanced cardiovascular depressant, but not respiratory depressant action of morphine. Brain Research 296:305-311.

Pfeiffer M, Koch T, Schröder H, Laugsch M, Höllt V, Schulz S (2002) Heterodimerization of somatostatin and opioid receptors cross-modulates phosphorylation, internalization and desensitization. Journal of Biological Chemistry 277:1976219772.

Phelps P, Brennan L, Vaughn J (1990) Generation patterns of immunocytochemically identified cholinergic neurons in the rat brainstem. Developmental Brain Research 56:63-74.

Phillips J, Lipski J (2000) Single-cell RT-PCR as a tool to study gene expression in central and peripheral autonomic neurons. Autonomic Neuroscience 86:1-12.

Pick C, Paul D, Pasternak G (1991) Comparisons of naloxonazine and $\beta$-funaltrexamine antagonism of $\mu 1$ and $\mu 2$ opioid actions. Life Sciences 48:2005-2001.

Pilowsky P, Jiang C, Lipski J (1990a) An intracellular study of respiratory neurons in the rostral ventrolateral medulla of the rat and their relationship to catecholaminecontaining neurons. The Journal of Comparative Neurology 4:604-617.

Pilowsky P, Jiang C, Lipski J (1990b) An intracellular study of respiratory neurons in the rostral ventrolateral medulla of the rat and their relationship to catecholaminecontaining neurons. The Journal of Comparative Neurology 301:604-617.

Pilowsky P, Lipski J, Prestidge R, Jiang C (1991) Dual fluorescence combined with a twocolour immunoperoxidase technique: a new way of visualizing diverse neuronal elements. Journal of Neuroscience Methods 36:185-193.

Pilowsky P, Llewellyn-Smith I, Arnolda L, Lipski J, Minson J, Chalmers J (1993) Are the ventrally projecting dendrites of respiratory neurons a neuroanatomical basis for the chemosensitivity of the ventral medulla oblongata? Sleep 16:S53-55.

Piros E, Charles A, Song L, Evans C, Hales T (2000) Cloned $\delta$-opioid receptors in $\mathrm{GH}_{3}$ cells inhibit spontaneous $\mathrm{Ca}^{2+}$ oscillations and prolactin release through $\mathrm{K}_{\mathrm{IR}}$ channel activation. Journal of Neurophysiology 83:2691-2698.

Pokela M (1993) Effect of opioid-induced analgesia on $\beta$-endorphin, colrosol and glucose responses in neonates with cardiorespiratory problems. Biology of the Neonate 64:360-367. 
Porreca F, Takemori A, Sultana M, Portoghese P, Bowen W, Mosberg H (1992) Modulation of mu-mediated antinociception in the mouse involves opioid delta-2 receptors. Journal of Pharmacology and Experimental Therapeutics 263:147-152.

Prathert P, Tsai A, Law P (1994) Mu and delta opioid receptor desensitization in undifferentiated human neuroblastoma cell lines. The Journal of Pharmacology and Experimental Therapeutics 270:177-184.

Pugsley M (2002) The diverse molecular mechanisms responsible for the actions of opioids on the cardiovascular system. Pharmacology and Therapeutics 93:51-75.

Punnen S, Sapuru H (1986) Cardiovascular responses to medullary microinjections of opiate agonists in urethane-anesthetized rats. Journal of Cardiovascular Pharmacology 1986:950-956.

Puttfarcken P, Cox B (1989) Morphine-induced desensitization and down-regulation at mu-receptors in 7315C pituitary tumor cells. Life Sciences 45:1937-1942.

Puyal P, Devau G, Venteo S, Sans N, Raymond J (2002) Calcium-binding proteins map the postnatal development of rat vestibular nuclei and their vestibular and cerebellar projections. Journal of Comparative Neurology 415:374-391.

Quock R, Burkey T, Varga E, Hosohata T, Hosohata K, Cowell S, Slate C, Ehlert F, Roeske W, Yamamura H (1999) The $\delta$-opioid receptor: molecular pharmacology, signal transduction, and the determination of drug efficacy. Pharmacological Reviews 51:503-518.

Rabkin S (1991) D-Ala-2-Me-Phe-4-Met- $(O)$-ol-enkephalin in the nucleus tractus solitarius of the rat produces cardiorespiratory depression. Genetics and Pharmacology 22:479-484.

Raff M, Abney E, Cohen J, Lindsay R, Noble M (1983) Two types of astrocytes in cultures of developing rat white matter: differences in morphology, surface gangliosides, and growth chacteristics. The Journal of Neuroscience 3:1289-1300.

Raff M, Fields K, Hakomori S, Mirsky R, Pruss R, Winter J (1979) Cell type specific markers for distinguishing and studying neurons and the major classes of glial cells in culture. Brain Research 174:283-308.

Rahman W, Dashwood M, Fitzgerald M, Aynsley-Green A, Dickenson A (1998) Postnatal development of multiple opioid receptors in the spinal cord and development of spinal morphine analgesia. Developmental Brain Research 108:239-254.

Ramsay D, Kellett E, McVey M, Rees S, Milligan G (2002) Homo- and hetero-oligomeric interactions between $\mathrm{G}$ protein-coupled receptors in living cells monitored by two variants of bioluminescence resonance energy transfer. Journal of Biological Chemistry.

Randich A, Thurston C, Ludwig P, Robertson J, Rasmussen C (1992) Intravenous morphine-induced activation of vagal afferents: peripheral, spinal, and CNS substrates mediating inhibition of spinal nociception and cardiovascular responses. Journal of Neurophysiology 68:10271045.

Reisine T (1995) Opiate receptors. Neuropharmacology 34:463-472.

Rekling J, Feldman J (1997) Bidirectional electrical coupling between inspiratory motoneurons in the newborn mouse nucleus ambiguus. Journal of Neurophysiology 78:3508-3510.

Rekling J, Feldman J (1998) PreBötzinger complex and pacemaker neurons: hypothesized site and kernal for respiratory rhythm generation. Annual Review of Physiology 60:385-405.

Rekling J, Champagnat J, Denavit-Saubié M (1996) Thropin-releasing hormone (THR) depolarise a subset of inspiratory neurons in the newborn mouse brain stem in vitro. Journal of Neurophysiology 75:811-819. 
Remmers A, Clarke M, Medzihradsky F, Woods J, Traynor J (2000) Activation of Gprotein by opioid receptors: role of receptor number and G-protein concentration. European Journal of Pharmacology 396:65-75.

Rhim H, Glaum S, Miller R (1993) Selective opioid agonists modulate afferent transmission in the rat nucleus tractus solitarius. The Journal of Pharmacology and Experimental Therapeutics 264:795-800.

Rigatto H (1996) Regulation of fetal breathing. Reproduction Fertilisation and Development 8:23-33.

Rigatto H, Fitzgerald S, Willis M, Yu C (1992) In search of the central respiratory neurons: II. Electrophysiologic studies of medullary fetal cells inherently sensitive to carbon dioxide and low $\mathrm{pH}$. Neuroscience Research 33:590-597.

Rigatto H, Fitzgerald S, Willis M, Yu C (1994) In search of the real respiratory neurons: culture of medullary fetal cells sensitive to carbon dioxide and low $\mathrm{pH}$. Biology of the Neonate 65:149-155.

Rios C, Jordan B, Devi L (2001) G-protein-coupled receptor dimerisation: modulation of receptor function. Pharmacology and Therapeutics 92:71-87.

Rocheville M, Lange D, Kumar U, Patel S, Patel Y (2000) Receptors for Dopamine and somatostatin: Formation of Hetero-ologomers with enhanced functional activity. Science 288:154-157.

Rogers D, Barnes P (1989) Opioid inhibition of neurally mediated mucus secretion in the human bronchi. The Lancet:930-931.

Ruegg H, Hahn B, Koch J, Bodnar R (1994) Differential modulation of angiotensin II and hypertonic saline-induced drinking by opioid receptor subtype antgonists in rats. Brain Research 635:203-210.

Rutherford R, Azher T, Gilmartin J (2002) Dramatic response to nebulized morphine in an asthmatic patient with severe chronic cough. Irish Medical Journal 95:113-114.

Rutherfurd S, Gundlach A (1993) Opioid peptide gene expression in the nucleus tractus solitarus of rat brain and increases induced by unilateral cervical vagotomy: implications for role of opioid neurons in respiratory control mechanisms. Neuroscience 57:797-810.

Saito Y, Tanaka I, Ezure K (2002) Morphology of the decrementing expiratory neurons in the brainstem of the rat. Neuroscience Research 44:141-153.

Salim K, Fenton T, Bacha J, Urien-Rodriguez H, Bonnert T, Skynner H, Watts E, Kerby J, Heald A, Beer M, McAllister G, Guest P (2002) Oligomerisation of G-proteincoupled receptors shown by selective co-immunoprecipitation. Journal of Biological Chemistry 277:15482-15485.

Sarnat H, Nochlin D, Born D (1997) Neuropathology of developmental nervous systen. Brain Pathology 7:1269-1272.

Sarton E, Teppema L, Oliver C, Nieuwenhuijs D, Matthes H, Kieffer B, Dahan A (2001) The involvement of the $\mu$-opioid receptor in ketamine-induced respiratory depression and antinociception. Anesthesiology Analgesia 93:1495-1500.

Satoh M, Minami M (1995) Molecular pharmacology of the opioid receptor. Pharmacology and Therapeutics 68:344-364.

Schultz J, Gross G (2001) Opioids and cardioprotection. Pharmacology and Therapeutics 89:123-137.

Schulz S, Schreff M, Koch T, Zimprich A, Gramsch C, Elde R, Höllt V (1998) Immunolocalization of two mu-opioid receptor isoforms (MOR1 and MOR1B) in the rat central nervous system. Neuroscience 82:613-622.

Sedmak J, Grossberg S (1977) A rapid, sensitive, and versatile assay for protein using coomassie brilliant blue G250. Analytical Biochemistry 79:544-552. 
Sessel B, Ball G, Lucier G (1981) Suppressive influences from periaqueductal gray and nucleus raphe megnus on respiration and related reflex activities and on solitary tract neurons, and effect of naloxone. Brain Research 216:145-161.

Sessle B (1973) Excitatatory and inhibitory inputs to single neurons in the solitary tract nucleus and adjacent reticular formation. Brain Research 53:319-331.

Sessle B, Henry J (1985) Effects of enkephalin and 5-hydroxytryptamine on solitary tract neurons involved in respiration and respiratory reflexes. Brain Research 327:221230.

Sessle B, Henry J (1989) Neural mechanisms of swallowing: neurophysiological and neurochemical studies on brain stem neurons in the solitary tract region. Dysphagia 4:61-75.

Shannon D, Kelly D (1977) Impared regulation of aveolar ventilation and the sudden infant death syndrome. Science 197:367-368.

Shapira M, Keren O, Gafni M, Sarne Y (2001) Divers pathways mediate $\delta$-opioid receptor down regulation within the same cell. Molecular Brain Research 96:142-150.

Shimoda K, Sauve Y, Marini A, Schwartz J, Commissiong J (1992) A high percentage yield of tyrosine hydroxylase-positive cells from rat E14 mesencephalic cell culture. Brain Research 586:319-331.

Shimosegawa T, Foda H, Said S (1990) [Met]enkephalin-Arg6-Gly7-Leu8immunoreactive nerves in guinea pig and rat lungs: distribution, origin, and coexistence with vasoactive intestinal polypeptide immunoreactivity. Neuroscience 36:737-750.

Shook J, Watkins W, Camporesi E (1990) Differential roles of opioid receptors in respiration, respiratory disease, and opiate-induced respiratory depression. American Review of Respiratory Disease 142:895-909.

Simonin F, Slowe S, Becker J, Matthes H, Filliol D, Chluba J, Kitchen I, Kieffer B (2001) Analysis of $\left[{ }^{3} \mathrm{H}\right]$ bremazocine binding in single and combinatorial opioid receptor knockout mice. European Journal of Pharmacology 414:189-195.

Slowe S, Clarke I, Goody R, Lattanzi R, Negri L, Simonin F, Matthes H, Filliol D, Kieffer B, Kitchen I (2001) Autoradiographic mapping of the opioid receptor-like 1 (ORL1) receptor in the brains of $\mu, \delta$, or $\kappa$-opioid receptor knockout mice. Neuroscience 106:469-480.

Smith A, Low H (1981) The opiate receptor. Hormonal Proteins and Peptides 10:89-170.

Smith J, Morrison D, Ellenberger H, Otto M, Feldman J (1989) Brainstem projections to the major respiratory neuron populations in the medulla of the cat. The Journal of Comparative Neurobiology 281:69-96.

Smith J, Ellenberger H, Ballayanyi K, Richter D, Feldman J (1991) Pre-Bötzinger complex: A brainstem region that may generate respiratory rhythm in mammals. Science 254:726-729.

Spain J, Roth B, Coscia C (1985) Differential ontogeny of multiple opioid receptors $(\mu, \delta$, and $\kappa)^{1}$. The Journal of Neuroscience 5:584-588.

Spruce B, Curtis R, Wilkin G, Glover D (1990) A neuropeptide precursor in cerebellum: proenkephalin exists in subpopulations of both neurons and astrocytes. EMBO Journal 9:1787-1797.

St. John W (1996) Medullary regions for neurogenesis of gasping:noeud vital ornoeuds vitals? Journal of Applied Physiology 81:1865-1877.

St. John W (1998) Neurogenesis of patterns of autonomic ventilatory activity. Progress in Neurobiology 56:97-117.

Standifer K, Pasternak G (1997) G protein and opioid receptor-mediated signalling. Cellular Signalling 9:237-248. 
Stasinopoulos T, Goodchild A, Christie M, Chalmers J, Pilowsky P (2000) Delta opioid receptor immunoreactive boutons appose bulbospinal $\mathrm{C} 1$ neurons in the rat. NeuroReport 11:887-891.

Stiene-Martin A, Hauser K (1990) Opioid-dependent growth of glial cultures: supression of astrocyte DNA synthesis by met-enkephalin. Life Sciences 46:91-98.

Stiene-Martin A, Hauser K (1991) Glial growth is regulated by agonists selective for multiple opioid receptor types in vitro. Journal of Neuroscience Research 29:538548.

Storm H, Rognum T, Reichlt K (1994) Inverse relationship between beta-endorphin immunoreactivity in cerebrospinal fluid and nucleus tractus solitarius in sudden infant death. European Journal of Pediatrics 153:381-386.

Stornetta R, Schreihofer A, Pelaez N, Sevigny C, Guyenet P (2001) Preproenkephalin mRNA is expressed by $\mathrm{C} 1$ and non-C1 barosensitive bulbospinal neurons in the rostral ventrolateral medulla of the rat. The Journal of Comparative Neurology 435:111-126.

Suarez-Roca H, Maixner W (1995) Morphine produces a biphasic modulation of substance $P$ release from cultured dorsal root ganglion neurons. Neuroscience Letters 194:4144.

Sun P, Loh H (2001) Transcriptional regulation of mouse $\delta$-opioid receptor gene. Journal of Biological Chemistry 276:45462-45469.

Sun P, Loh, HH. (2002) Transcriptional regulation of mouse $\delta$-opioid receptor gene: Role of Ikaros in the stimulated transcription of mouse $\delta$-opioid receptor gene in activated $\mathrm{T}$ cells. Journal of Biological Chemistry in press.

Suzuki S, Chuang L, Yau P, Doi R, Chuang R (2002) Interactions of opioid and chemokine receptors: Oligomerization of mu, kappa and delta with CCR5 on immune cells. Experimental Cell Research 280:192-200.

Svingos A, Clarke C, Pickel V (1998) Cellular sites for activation of $\delta$-opioid receptors in the rat nucleus accumbens shell: relationship with $\mathrm{Met}^{5}$-enkephalin. The Journal of Neuroscience 18.

Svingos A, Moriwaki A, Wang J, Uhi G, Pickel V (1996) Ultrastructural immunocytochemical localization of $\mu$-opioid receptors in rat nucleus accumbens: extrasynaptic plasmalemmal distribution and association with Leu ${ }^{5}$-enkephalin. Journal of Neuroscience 16:4162-4173.

Szeto H, Zhu Y, Umans J, Dwyer G, Clare S, Amione J (1988) Dual action of morphine on fetal breathing movements. The Journal of Pharmacology and Experimental Therapeutics 245:537-542.

Szilagyi J (1998) Endogenous opiate modulation of baroreflexes in normotensive and hypotensive rats. The American Journal of Physiology:H987-J911.

Taki K, Kaneko T, Mizuno N (2000) A group of cortical interneurons expressing $\beta \gamma$-opioid receptor-like immunoreactivity: A double immunofluorescence study in the rat cerebral cortex. Neuroscience 98:221-231.

Takita K, Herlenius E, Lindahl S, Yamamoto Y (1997) Actions of opioids on respiratory activity via activation of brainsten $\mu-, \delta$-, and $\kappa$-receptors; an in vitro study. Brain Research 778:233-241.

Takita K, Herlenius E, Lindahl S, Yamamoto Y (1998) Age- and temperature-dependent effects of opioids on medulla oblongata respiratory activity: an in vitro study in newborn rat. Brain Research 800:308-311.

Tallent M, Dichter M, Bell G, Reisine T (1994) The cloned Kappa opioid receptor couples to an N-type calcium current in undifferentiated PC-12 cells. Neuroscience 63:1033-1040. 
Tanowitz M, von Zastrow M (2002) Ubiquitination-independent trafficing of G-proteincoupled receptors to lysosomes. Journal of Biological Chemistry 277:50219-50222.

Tavani A, Robson L, Kosterlitz H (1985) Differential postnatal development of $\mu$-, $\delta$ - and $\kappa$-opioid binding sites in mouse brain. Developmental Brain Research 23:306-309.

Tefano G, Goumon Y, Bilfinger T, Welters I, Cadet P (2000) Basal nitric oxide limits immune, nervous and cardiovascular excitation: human endothelia express a mu opiate receptor. Progress in Neurobiology 60:513-530.

Temple A, Zukin R (1987) Neuroanatomical patterns of the $\mu, \delta$, and $\kappa$, opioid receptors of rat brain as determined by quantatative in vitro autoradiography. Proceedings of the National Acadamy of Science USA 84:4308-4312.

Temple A, Habas J, Paredes W, Barr G (1988) Morphine-induced downregulation of $\mu$ opioid receptors in neonatal rat brain. Developmental Brain Research 41:129-133.

Ternaux J, Portalier P (1993) Culture of hypoglossal cells, dissociated from foetal and new-born rats. Journal of Neuroscience Methods 49:33-47.

Tershner S, Helmstetter F (2000) Antinociception produced by mu opioid receptor activation in the amygdala is partly dependent on activation of mu opioid and neurotensin receptors in the ventral periaqueductal gray. Brain Research 865:17-26.

Thompson R, Mansour A, Akil H, SJ. W (1993) Cloning and pharmacological characterization of a rat $\mu$ opioid receptor. Neuron 11:903-913.

Thorlin T, P A, I P, PS E, E H, L. R (1999) Delta-opioid receptor immunoreactivity on Astrocytes is upregulated during mitosis. GLIA 25:370-378.

Titus J, Haugland R, Sharrow S, Segal D (1982) Texas red, a hydrophilic, red emitting fluropore for use with fluorescein in dual parameter flow microfluorometric and fluorescence microscopic studies. Journal of Immunological Methods 50:193-204.

Tong Y, Chabot J, Shen S, O'Dowd, George S, Quirion R (2000) Ontogenic profile of the expression of the mu opioid receptor gene in the rat telencephalon and diencephalon: an in situ hybridization study. Journal of Chemical Neuroanatomy 18:209-2522.

Toth G, Kramer T, Knapp R, Lui G, Davis P, Burks T, Yamamura H, Hurby V (1990) [D$\mathrm{Pen}^{2}$, D-Pen ${ }^{5}$ ]enkephalin analogues with increased affinity and selectivity for $\delta$ opioid receptors. Journal of Medical Chemistry 33:249-253.

Trapaidze N, Keith D, Cvejic S, Evans C, Devi L (1996) Sequestration of the $\delta$ opioid receptor. The Journal Of Biological Chemistry 271:29279-29285.

Trapaidze N, Gomes I, Cvejic S, Bansinath M, Devi L (2000) Opioid receptor endocytosis and activation of MAP kinase pathway. Molecular Brain Research 76:220-228.

Traynor J, Elliott J (1993) $\delta$-opioid receptor subtypes and cross-talk with $\mu$-receptors. Trends in Pharmacological Science 14:84-86.

Trenkner E (1992) Cerebellar cells in culture. In: Culturing Nerve Cells (Banker G, Goslin, K, ed), pp 283-330. USA: MIT.

Tryoen-Toth P, Gavériaux-Ruff, Maderspach K, G. L (2000) Down-regulation of muopioid receptor expression in rat oligodendrocytes during their development in vitro. Journal of Neuroscience Research 60:10-20.

Tsang D, Ng S, Ho K (1982) Development of methionine-enkephalin and naloxone binding sites in regions of rat brain. Developmental Brain Research 3:637-644.

Tsao P, von Zastro M (2000) Type-specific sorting of G-protein-coupled receptors after endocytosis. The Journal Of Biological Chemistry 275:11130-11140.

Tsao P, Cao T, von Zastrow M (2001) Role of endocytosis in mediation downregulation of G-protein-coupled receptors. Trends in Pharmacological Science 22:91-96. 
Tso P, Yung L, Wong Y (2000) Regulation of adenylyl cyclase, ERK1/2, and CREB by $\mathrm{G}_{z}$ following acute and chronic activation of the $\delta$-opioid receptor. Journal of Neurochemistry 74b:1685-1693.

Vaccarino A, Kastin A (2001) Endogenous opiates: 2000. Peptides 22:2257-2328.

Vaccarino A, Olson G, Kastin A (1999) Endogenous opiates: 1998. Peptides 20:15271574.

Van Den Hoogen R, Colpaert F (1996) Respiratory effects of morphine in awake unrestrained rats. The Journal of Pharmacology and Experimental Therapeutics 237:252-259.

Vanderah T, Ossipov M, Lai J, Malan T, Porreca F (2001) Mechanisms of opioid-induced pain and antinociceptive tolerance: descending facilitation and spinal dynorphin. Pain 92:5-9.

Varon S, Adler R (1980) Nerve growth factors and control of nerve growth. In: Current Topics in Developmental Biology, pp 207-247: Academic Press, Inc.

Vaughan C, Christie M (1997) Presynaptic inhibitory action of opioids an synaptic transmission in the rat periaqueductal grey in vitro. Journal of Physiology 498:463472.

Vaysse P, Zukin R, Fields K, Kessler J (1990) Chacterisation of opioid receptors in cultured neurons. Journal of Neurochemistry 55:624-631.

Villiger J, Taylor K, Gluckman P (1982) Otogenesis of opiate receptors in regions of the ovine brain. Pediatric Pharmacology 2:349-356.

Vinay L, Brocard F, Pflieger J, Simeoni-Alias J, Clarac F (2000) Prenatal development of lumbar motoneurons and their inputs in the rat. Brain Research Bulletin 53:635637.

Volsen S (1984) A boltin-avidin technique for the localisation of membrane-bound monoclonal antibodies by low power transmission electron microscopy. The Journal of Immunological Methods 72:119-126.

Walker J, King J (1989) Ontogensesis of enkephalinergic afferent systems in the opossum cerebellum. Developmental Brain Research 48:35-58.

Wallace T, Johnson E (1989) Cytosine arabinoside kills post-mitotic neurons: evidence that deoxycytidine may have a role in neuronal survival that is independent of DNA synthesis. Neuroscience 9:115-124.

Wallington C, Peters D, Gelb C, Peterfreund R (2002) Orphan FQ/nociceptin and $\mu$-opioid receptor mRNA levels in human SH-SY5Y neuroblastoma cells: effects of activating the cAMP-PKA signal transduction pathway. Brain Research 944:73-81.

Wamsley J (1983) Opioid receptors: autoradiography. Pharmacological Reviews 35:69-83.

Wang D, Sadée W, JM. Q (1999) Calmodulin binding to G protein-coupling domain to opioid receptors. The Journal Of Biological Chemistry 274:22018-22088.

Wang D, Surratt C, Sadée W (2000) Calmodulin regulation of basal and agonist-stimulated $\mathrm{G}$ Protein coupling by the $\mu$-opioid receptor $\left(\mathrm{OP}_{3}\right)$ in morphine-pretreated cell. Journal of Neurochemistry 75:763-771.

Wang H, Pickel V (2001) Preferential cytoplasmic localisation of $\delta$-opioid receptors in rat striatal patches: comparison with plasmalemmal $\mu$-opioid receptors. The Journal of Neuroscience 21:3242-3250.

Wang $H$, Wessendorf $M(2002) \mu$ - and $\delta$-opioid receptor mRNAs are expressed in periaqueductal gray neurons projecting to the rostral venteromedial medulla. Neuroscience 109:619-634.

Wang H, A M, Wang J, Uhl G, Pickel V (1996) Ultrastructural immunocytochemical localization of $\mu$ opioid receptors and $\mathrm{Leu}^{5}$-enkephalin in the patch compartment of 
the rat caudate-putamen nucleus. The Journal of Comparative Neurology 375:659674.

Wang J, Johnson P, Persico A, Hawkjins A, Griffin C, Uhl G (1994) Human $\mu$ opiate receptor: cDNA and genomic clones, pharmacologic characterization and chromosomal assignment. FEBS Letters 338:217-222.

Wang Q, Li P (1988) Inhibition of baroreflex following microinjection of GABA or morphine into the nucleus tractus solitarii in rabbits. Journal of the Autonomic Nervous System 25:165-172.

Wang W, Pizzonia J, Richerson G (1998) Chemosensitivity of rat medullary raphe neurons in primary tissue culture. Journal of Physiology 511:433-450.

Wang W, Shahrestanifar M, Jin J, Howells R (1995) Studies on $\mu$ and $\delta$ opioid receptor selectivity utilizing chimeric and site-mutagenized receptors. Proceedings of the National Acadamy of Science USA 92:12436-12440.

Wanner I, Baader S, Brich M, Oberdick J, Schilling K (1997) Subcellular localisation of specific mRNAs and their protein products in Purkinje cells by combined fluorescence in situ hybridisation and immunocytochemistry. Histochem Cell Biology 108:345-357.

Wei L, Low H (2002) Regulation of opioid receptor expression. Current Opinion In Pharmacology 2:69-75.

Werz M, Grega D, MacDonald R (1987) Actions of mu, delta and kappa opioid agonists and antagonists on mouse primary afferent neurons in culture. Journal of Pharmacology and Experimental Therapeutics 243:258-263.

Wess J (1998) Molecular basis of receptor/G-protein-coupling selectivity. Pharmacology and Therapeutics 80:231-264.

Wessendorf M, Dooyema J (2001) Coexistence of kappa- and delta-opioid receptors in rat spinal cord axons. Neuroscience Letters 298:151-154.

Whistler J, Tsao P, von Zastrow M (2001) A phosphorylation-regulated brake mechanism controls the initial endocytosis of opioid receptors but is not required for postendocytic sorting to lysosomes. Journal of Biological Chemistry 276:34331-34338.

Whistler J, Chuang H, Chu P, Jan L, von Zastrow M (1999) Functional dissociation of m opioid receptor signaling and endocytosis: implications for the biology of opiate tolerance and addiction. Neuron 23:737-746.

Whistler J, Enquist J, Marley A, Fong J, Tsuruda P, Murray S, von Zastrow M (2002) Modulation of postendocytic sorting of $\mathrm{G}$ protein-coupled receptors. Science 297:615-620.

White J, Irvine R (1999) Mechanisms of fatal opioid overdose. Addiction 94:961-972.

Wiliams R, Dockray G (1983) Distribution of enkephalin-related peptides in rat brain: immunohistochemical studies using antisera to met-enkephalin and met-enkephalin $\mathrm{Arg}^{6} \mathrm{Phe}^{7}$. Neuroscience 9:563-586.

Wilkin G, Marriott D, Pearce B (1995) Peptide receptors on astrocytes. In: Neuroglia (Kettenmann H, ed), pp 367-376. New York: Oxford University Press.

Wilson M, Burt, AR, Milligan, G, Anderson, NG (1997) Mitogenic signalling by $\delta$ opioid receptors expressed in Rat-1 fibroblasts involves activation of the $\mathrm{p} 70^{\mathrm{s} 6 \mathrm{k}} / \mathrm{p} 85^{\mathrm{s} 6 \mathrm{k}} \mathrm{S} 6$ kinase. Biochemistry Journal 325:217-222.

Wind R, Kuhin C (1995) Increased sensitivity to mu opiate antinoception in the neonatal rat despite weaker receptor-guanyl nucleotide binding protein coupling. The Journal of Pharmacology and Experimental Therapeutics 273:1353-1360.

Wittert G, Hope P, Pyle D (1996) Tissue distribution of opioid receptor gene expression in the rat. Biochemical and Biophysical Research Communications 218:877-881. 
Wohltmann M, Roth B, Coscia C (1982) Differential postnatal development of mu and delta opiate receptors. Developmental Brain Research 3:679-684.

Xia Y, Haddad G (1991) Ontogeny and distribution of opioid receptors in the rat brainstem. Brain Research 549:181-193.

Xiang B, Yu G, Guo J, Hu W, Pei G, Ma L Heterologous activation of protein kinase C stimulates phosphorylation of $\delta$-opioid receptor at serine 344 and this results in $\beta-$ arrestin-and clathrin-mediated receptor internalization. The Journal Of Biological Chemistry.

Yabaluri N, Medzihradsky F (1997) Regulation of $\mu$-opioid receptor in neural cells by extracellular sodium. Journal of Neurochemistry 68:1052-1061.

Yan S, Zhang C, Laferrière A, Moss I (1995) Met-enkephalin-like immunoreactivity in microdialysates from nucleus tractus solitarii in piglets during normoxia and hypoxia. Brain Research 687:217-220.

Yasuda K, Espinosa R, Takeda J, Lebeau M, Bell I (1994) Localization of the kappa opioid receptor gene to human chromosome band 8Q11.2. Genomics 19:596-597.

Yeadon M, Kitchen I (1989) Opioids and respiration. Progress in Neurobiology 33:1-16.

Yeadon M, Kitchen I (1990) Multiple opioid receptors mediate the respiratory depressant effects of fentanyl-like drugs in the rat. Genetics and Pharmacology 21:655-664.

Yew D, Luo C, Guan Y, Chen W (1990) Development and localization of enkephalin and substance $\mathrm{P}$ in the nucleus of tractus solitarius in the medulla oblongata of human fetuses. Neuroscience 34:491-498.

Yoburn B, Lutfy K, Candido J (1991) Species differences in $\mu$ - and $\delta$-opioid receptors. European Journal of Pharmacology 193:105-108.

Yoshikawa M, Nakayama H, Ueno S, Hirano M, Hatanaka H, Furuya H (2000) Chronic fentanyl treatments induce the up-regulation of mu opioid receptor mRNA in rat pheochromocytoma cells. Brain Research 859:217-223.

Yu V, Sadée W (1988) Efficacy and tolerance of Narcotic analgesics at the Mu opioid receptor in differentiated human neuroblastoma cells. The Journal of Pharmacology and Experimental Therapeutics 245:350-355.

Yu V, Richards M, Sadée W (1986) A Human Neuroblastoma cell line Expresses $\mu$ and $\delta$ opioid receptors. The Journal Of Biological Chemistry 261:1065-1070.

Yu Y, Zhang L, Yin X, Sun H, Uhl G, Wang J (1997) $\mu$ opioid receptor phosphorylation, desensitization, and ligand efficacy. The Journal of Biological Chemistry 272:28869-28874.

Zadina J, Hackler L, Ge L, Kastin A (1997) A potent and selective endogenous agonist for the mu opiate receptor. Nature 386:499-502.

Zadina J, Martin-Schild S, Gerall A, Kastin A, Hackler L, Ge L, Zhang X (1999) Endormorphins: novel endogenous $\mu$-opiate receptor agonists in regions of high $\mu$ opiate receptor density. Annals - New York Academy of Sciences 897:136-144.

Zagon I, McLaughlin (1983) Increased brain size and cellular content in infant rats treated with an opiate antagonist. Science 221:1179-1180.

Zagon I, McLaughlin P (1991) Identification of opioid peptides regulating proliferation of neurons and glia in the developing nervous system. Brain Research 542:318-323.

Zagon I, Rhodes R, PJ. M (1985) Opioid recepor and peptide mRNA expression in proliferative zones of fetal rat central nervous system. Science 227:1049-1051.

Zagon I, Verderame M, McLaughlin P (2002) The biology of the opioid growth factor receptor (OGFr). Brain Research Reviews 38:351-376.

Zagon I, Verderame M, Allen S, McLaughlin P (1999) Cloning, sequencing, expression and function of a cDNA encoding a receptor for the opioid growth factor, $\left[\mathrm{Met}^{5}\right]$ enkephalin. Brain Research 849:147-154. 
Zastawny R, George S, Nguyen T, Cheng R, Tsatsos J, Briones-Urbina R, O'Dowd B (1994) Cloning, characterization, and distribution of a $\mu$-opioid receptor in rat brain. Journal of Neurochemistry 62:2099-2105.

Zhang C, Moss I (1995) Age related $\mu-, \delta$-, and $\kappa$-opioid ligands in respiratory-related brain regions of piglets: effects of prenatal cocaine. Developmental Brain Research 87:188-193.

Zhang J, Ferguson S, Barak L, Bodduluri S, Laporte S, Law P, Caron M (1998) Role for G protein-coupled receptor kinase in agonist-specific regulation of $\mu$-opioid receptor responsiveness. Proceedings of the National Acadamy of Science USA 95:71577162.

Zhu Y, Hsu M, Pintar J (1998) Developmental expression of the $\mu, \kappa$, and $\delta$ Opioid receptor mRNAs in mouse. The Journal of Neuroscience 18:2538-2549.

Zimprich A, Simon T, Höllt V (1995) Cloning and expression of an isoform of the rat $\mu$ opioid receptor (rMOR1B) which differs in agonist induced desensitization from rMOR1. FEBS Letters 359:142-146. 